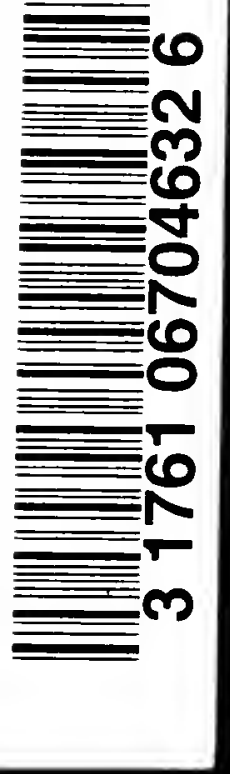




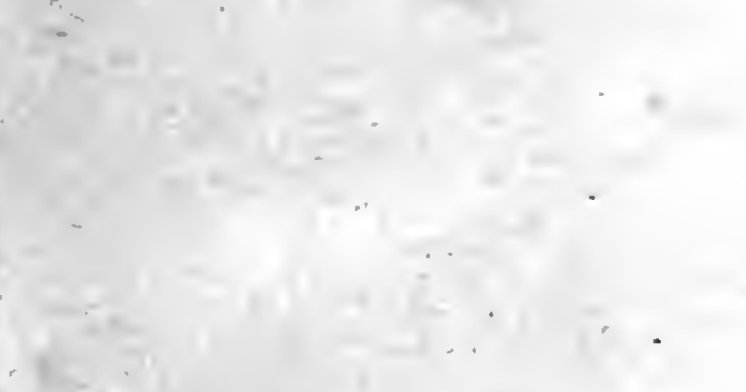

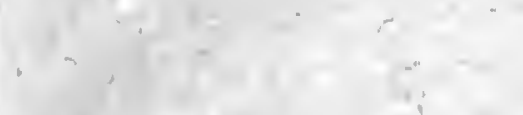
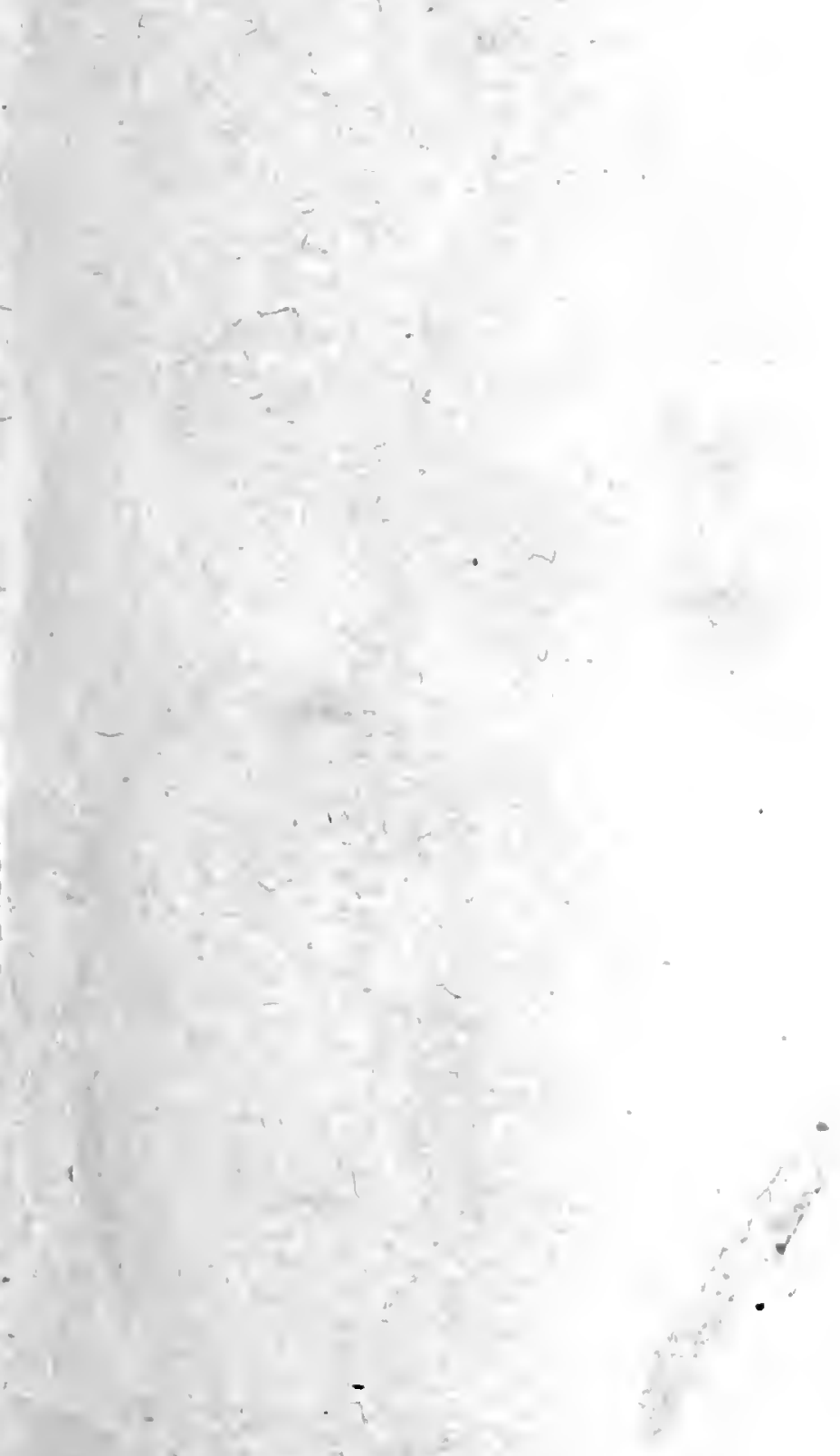
Digitized by the Internet Archive in 2007 with funding from Microsoft Corporation 


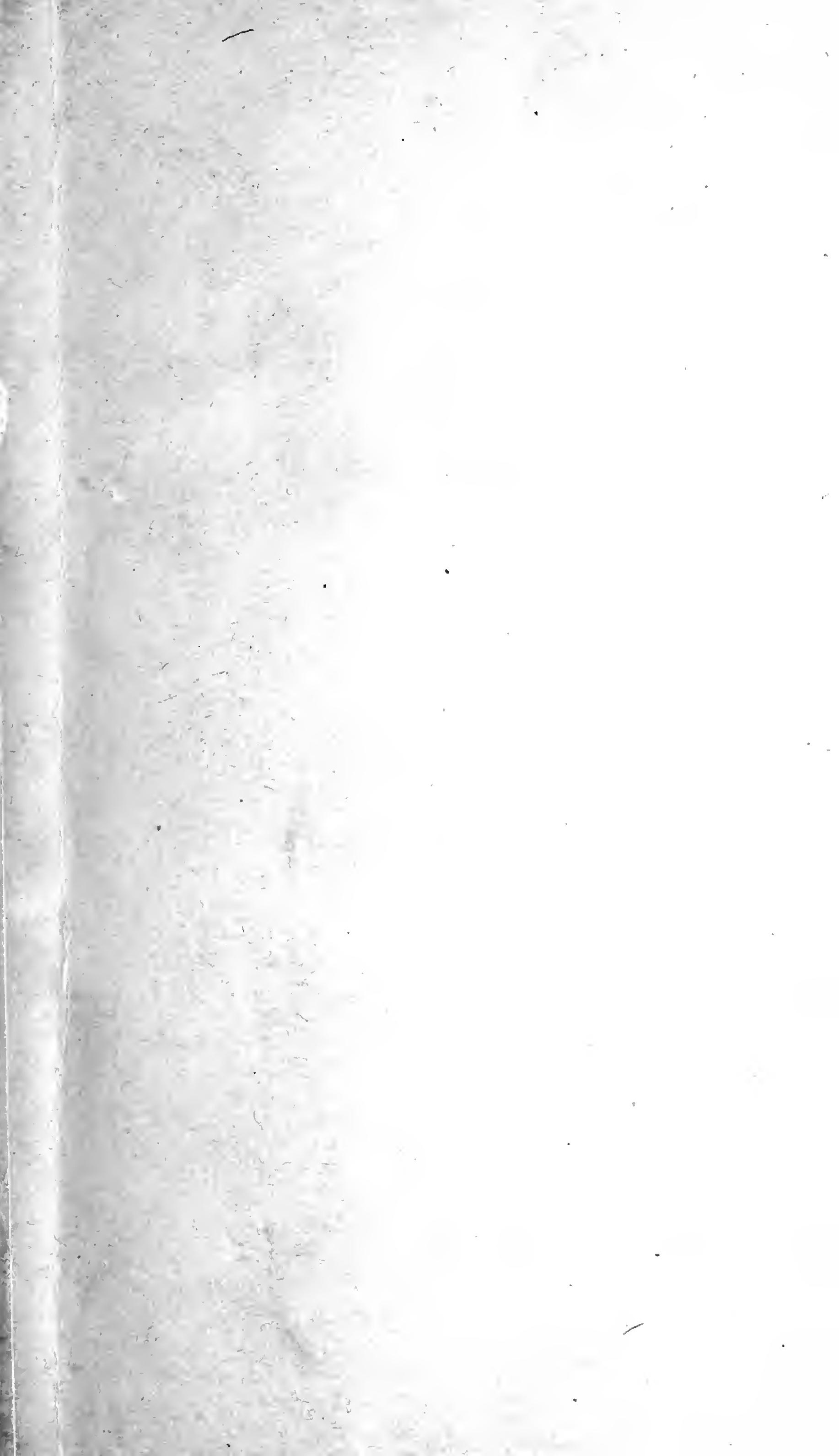



THE ANATOMY OF

THE HUMAN ORBIT 



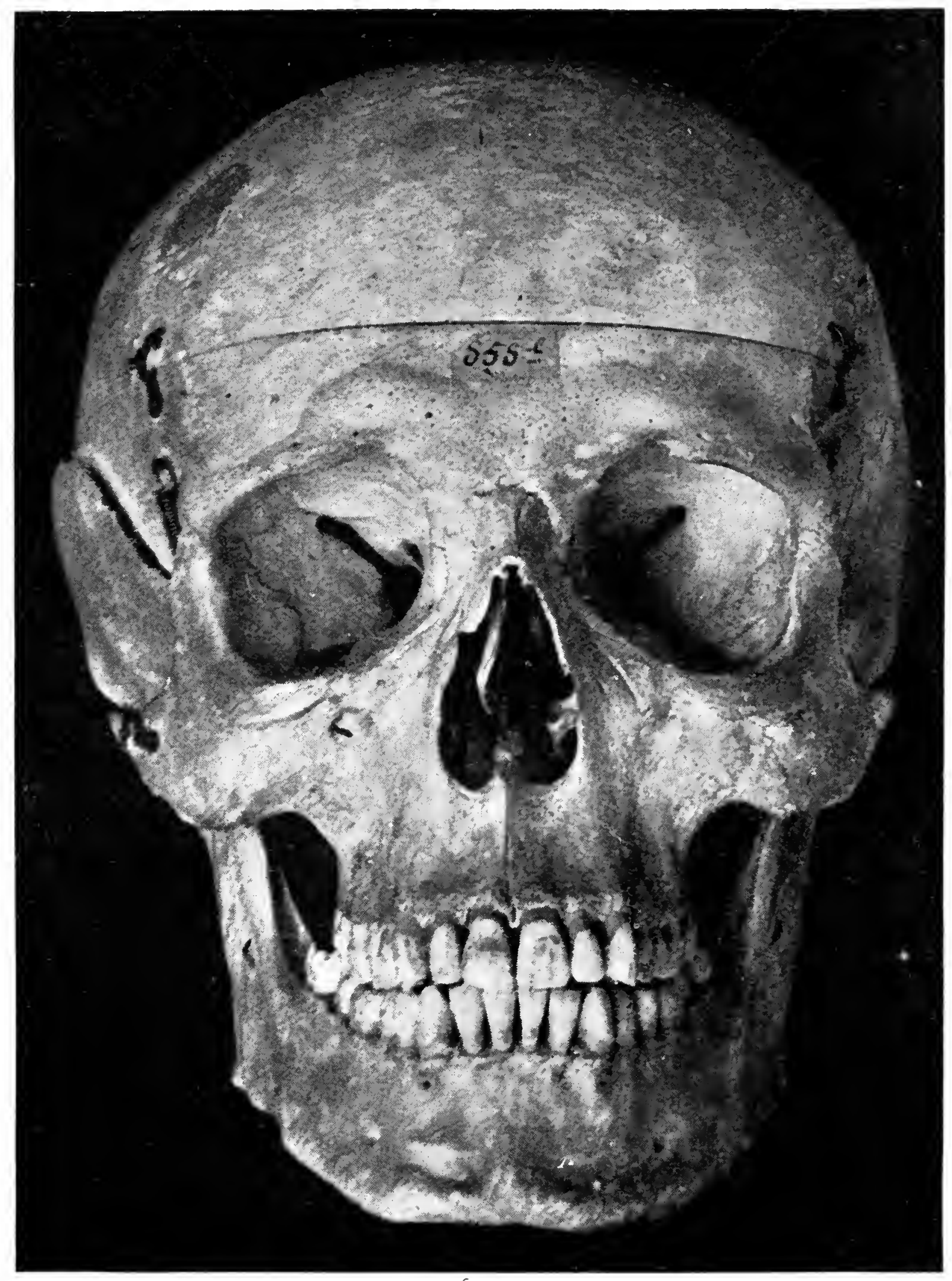

Fig. I. -Adult Male skull, English. Cephalie index, 83 ; orbital index, 86.3; for other orbital measurements see p. 9I. $\times \frac{2}{3}$. 
THE OXFORD M MEDICAL PUBLICATIONS

\title{
THE ANATOMY OF
}

\section{THE HUMAN ORBIT}

AND

\section{ACCESSORY ORGANS \\ OF VISION}

\author{
BY \\ S. ERNEST WHITNALL \\ M.A., M.D. B.CH. (OXON.), M.R.C.S., L.R.C.P. (LOND.) \\ PROFESSOR OF ANATOMY, MCGILL UNIVERSITY, MONTREAL \\ LATE UNIVERSITY DEMONSTRATOR OF HUMAN ANATOMY, OXFORD
}

ILLUSTRATED LARGELY BY PHOTOGRAPHS OF ACTUAL DISSECTIONS

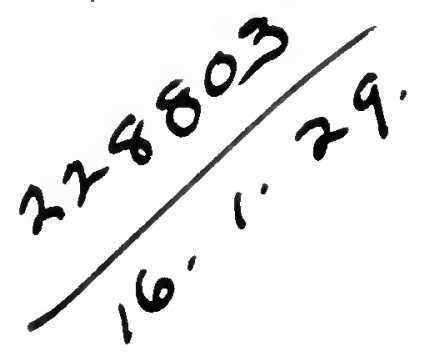

LONDON

HENRY FROWDE AND HODDER \& STOUGHTON . THE LANCET BUILDING I \& 2 BEDFORD STREET, STRAND, W.C.2 
First printed 1921.

Printed in Great Britain by R. \& R. ClaRK, LIMITED, Edinburgh. 



\section{PREFACE}

THE subject-matter of this work originally formed the substance of a series of lectures given to candidates for the Oxford Diploma of Ophthalmology, and is here presented in an amplified and completed form. The work has suffered many interruptions since its initiation in $\mathrm{x} 9 \mathrm{I} 3$, which must be held as an excuse for any fluctuations in the manner of its treatment. Primarily intended for the use of ophthalmologists, it is hoped that the necessary incorporation of matter relative to the neighbouring nasal region will also prove of interest to the rhinologist, and that as a regional study the whole will commend itself to the general anatomist.

It was intended that the chief value of the lectures should lie in the demonstration of the large series of dissections and preparations (now in the Oxford anatomical museum) which by the kindness of Professor Arthur Thomson the author was able to make while working under him. Similarly, it is hoped that the chief feature of value in this book will be the photographic illustrations of such of these preparations as lend themselves to the purpose. For any success this object may attain I am greatly indebted to the technical skill and tireless interest of the departmental assistant, Mr. Chesterman, who took all the photographs, with the exception of Nos. 3, 23, 36, 50, 90, 97, 130, which were prepared at McGill. The source of the borrowed illustrations 
is acknowledged in the legends. The dates following the authors' names in the text refer to the year of publication of articles specially bearing upon the subject which will be found in the bibliography.

To my Associate Professor of Histology and Embryology, Professor J. C. Simpson, I am indebted for the redrawing of the majority of my diagrams, and their presentation in a more artistic form. It is regretted that the cost of colour illustration prohibits the more general use of this means of simplifying the diagrams. To my wife I wish to express my indebtedness for the careful transcribing of my pencil MSS.

The final completion of the work has been greatly facilitated by a grant from the Cooper Fund of McGill University, to the Trustees of which Fund I tender my thanks. 


\section{CONTENTS}

\section{PART I \\ OSTEOLOGY}

The Bones forming the Orbit, its Relations, and THE AcCessory Air-sinuses of the Nose.

\section{PART II}

\section{EYELIDS}

The Eyebrows, Eyelins, Conjunctiva, and Lacrimal Apparatus

\section{PART III \\ CON'TENTS OF THE ORBI'T}

The Globe (External Configuration), Ocular Muscles, BloOd-VESSEls, AND Nerves . . . . $25 \mathrm{I}$

\section{PART IV}

APPENDIX

The Cerebral Connections of the Nerves 



\section{PART I \\ OSTEOLOGY}

ThE Orbits (orbital cavities, fossae orbitales) are two large and deep cavities situated one on each side of the root of the nose. They lie between and are formed by the bones of the face and those of the calvaria (Fig. 2). The wide anterior opening of the orbit on to the face is called the base or entrance (aditus orbitae), and is circumscribed by the margin (margo orbitalis); the cavity is enclosed by four walls, the roof, floor, lateral and medial walls, connected by rounded angles, and it is terminated posteriorly by the apex. The continuity of the walls is interrupted in the skull by one large orifice, the optic foramen (foramen opticum), and by several smaller foramina, by two wide fissures, the superior and inferior orbital fissures (or sphenoidal and spheno-maxillary), and by the opening of the nasolacrimal canal (canalis nasolacrimalis) ; all these open-

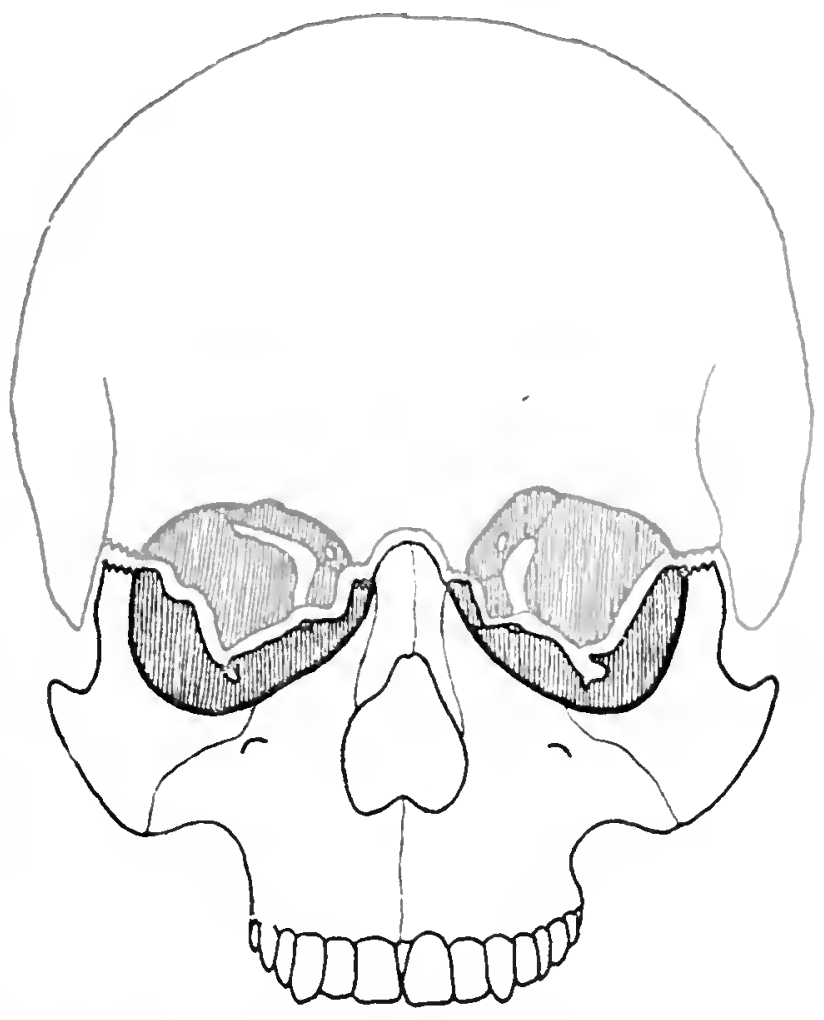

Fig. 2.-Diagram to illustrate the position of the Orbits between the bones of the Calvaria (red) and those of the Face (black). (After Poirier, modified.) ings are closed in life by structures which pass through them. The walls are crossed by sutures between the bones which form them, and are smoothly lined with periosteum, here termed the periorbita. 
Above the orbit is situated the anterior cranial fossa of the skull, containing the frontal lobe of the brain; below

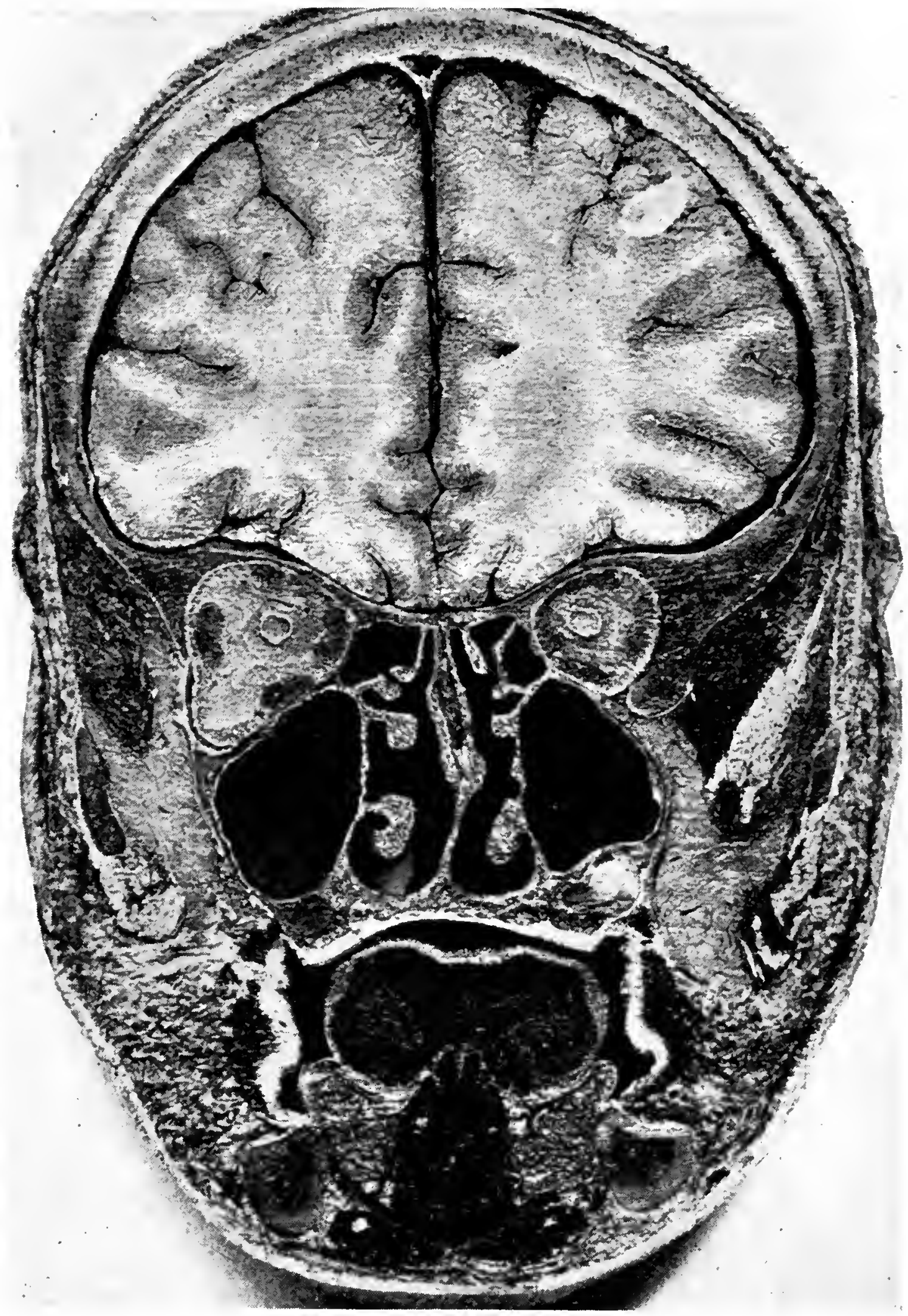

FIG. 3.--Coronal section of an Adult Male Head to show the general relations of the Orbits ; the section cuts through them at about the junction of their posterior third and anterior two-thirds. Compare with the horizontal section through the head seen in Fig. I30, p. 252. From the McGill Anatomical Museum. $\times \frac{2}{3}$.

lies the maxillary air-sinus; medially the lateral mass of the ethmoidal bone together with the lacrimal bone intervene 
between it and the nasal fossa in front, whilst behind the two orbits are separated from one another by the body of the sphenoidal bone ; the temporal region and middle cranial fossa are in relation to its lateral aspect; it is bounded in front by the eyelids.

It is convenient to study the osteology of the orbit on the following plan :

I. The form of the cavity.

2. The orbital margin.

3. The walls of the orbit.

4. The accessory sinuses of the nose in their relation to the orbit.

5. The osseous lacrimal passages.

6. Table of apertures of the orbit.

7. The periorbita.

8. Mensuration.

9. Development and growth.

Io. Variations, Asymmetry, and Deformities.

\section{THE FORM OF THE ORBIT}

The form of the orbit is usually likened to either a cone or a four-sided pyramid with rounded angles, of which figure the base corresponds to the wide opening directed forwards on to the face and circumscribed by the orbital margin, and the apex to the narrowed portion leading backwards within the skull.

An examination of plaster casts of the orbit (Fig. 4) shows that neither of these descriptions is accurate as regards the shape. No common geometrical figure will illustrate it without missing several important features; moreover, it was found by Gayat (I873) that the mathematical volume of a corresponding pyramid is less by one-third than the volume of the orbit calculated empirically by means of filling it with lead pellets.

The first point to be noticed is that owing to the overhang of the orbital walls in front the largest part of the cavity does not lie at the facial entrance, but is just behind it, and in consequence of this narrowing of the margin a cast cannot 

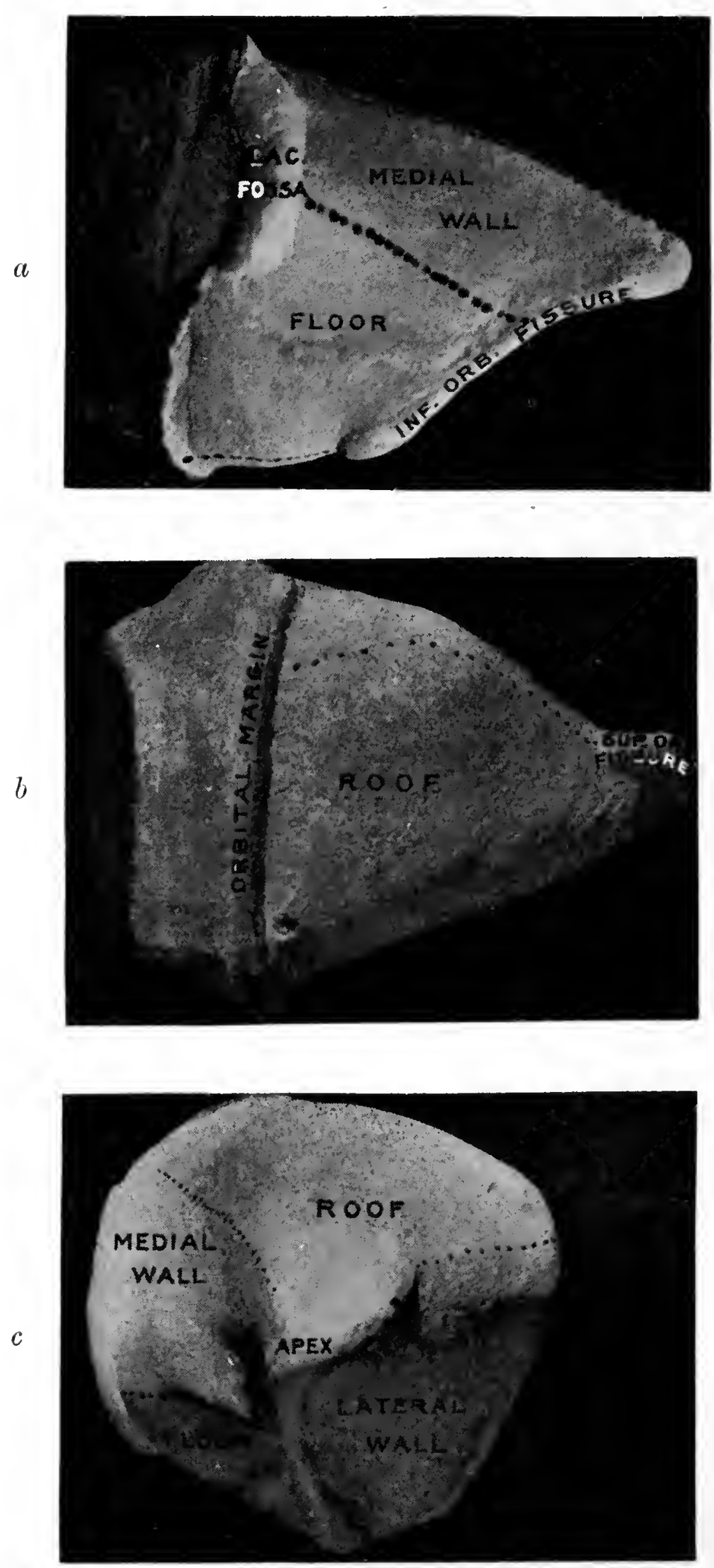

FIG. 4. - Casts of Right Orbit. Adult male, natural size.
(a) Medial aspect.
(b) Superior aspect.
(c) Posterior aspect. 
be withdrawn without partial removal of the bones. Thus the roomiest part of the orbit is found about one centimetre behind the margin, and since here is lodged the eyeball the walls are roughly moulded upon it. The primitive spheroidal shape is, however, distorted by outward bulgings of the walls in several regions, to accommodate the lacrimal gland, the lacrimal sac, and the pulley of the superior oblique muscle. The growth of these structures within the orbit, together with the development of the surrounding parts, causes the bulk of the cast to present a quadrilateral shape with rounded angles, as is seen in a section through the skull itself taken just behind the orbital margin (Fig. I7). The shape of this forepart of the cavity allows the orbit to be conveniently described as contained by four walls, but the cast emphasises the important differences between them as they are traced from the base to the apex. The medial wall is oblong in outline; the roof and lateral wall are the shape of an isosceles triangle; the floor is also triangular (equilateral), but is shorter than the other boundaries, extending only as far back as the junction between the anterior two-thirds and posterior third of the orbit. Behind this point is the region of the apex, and here the absence of the floor is compensated by the inclination of the lateral towards the medial wall. There are therefore only three walls forming the apex, as seen in the cast, and also shown by the more triangular outline of a section of the skull through this region (Fig. 3). This three-sided pyramidal form of the apex is accentuated by the presence of the superior and inferior orbital fissures, between which lies the posterior extremity of the lateral wall. The apex lies wholly behind the eyeball, and becomes narrow in conformity with its less bulky contents, which are chiefly the optic nerve and the origin of the ocular muscles.

The comparison of the form of the orbital cavity to that of a cone or four-sided pyramid then misses the facts shown so well by a cast, namely, that the figure has a constricted base, a roughly four-sided anterior portion in which the eyeball is lodged, and a well-defined three-sided pyramidal apex wherein lie the optic nerve and muscles. If the form 
must be likened to some common object, it may be said to resemble that of a pear with the thin end (apex) and stalk (optic nerve) curving slightly towards the mesial plane.

Although not sharply marked, the division of the cavity into an anterior two-thirds or ocular portion and a posterior third or retro-ocular part is to be emphasised, since these two regions differ not only in regard to their chief contents, but also in their external relations.

\section{THE ORBITAL MARGIN}

From a practical standpoint the superficial position and prominence of the margin of the orbit are sufficient justifications for its detailed description apart from the walls of the cavity. The surface anatomy is better considered after such description, when the features which can be made out by digital examination will more readily be recognised.

The shape of the margin may be considered first. Since the orbital walls are moulded upon the previously formed eyeball, the primitive outline of the margin is circular and comparatively flat, as is seen in the earlier foetal skulls up to the age of six months, where also the margin is more closely applied to the globe and only extends as far as its equator (Fig. 42, p. 95). After this period the outline usually assumes an oval contour, due probably to the more rapid expansion of the brain-containing part of the skull, and later on the striking changes in the infantile face, consequent to the development of the nose and jaws, react upon the margin, which becomes altered in both frontal and sagittal planes. In the former plane the circular outline may be altered to oval, square, or oblong (as seen in the photographs on pp. 89, Ior, ro3), the quadrangular contour being found the generalised type in modern male skulls by Cameron (I920); in the latter plane the flattened margin becomes more undulating. (See also under fronto-sphenoidal process, p. I3, and refer p. 97.)

It should be noticed that even in foetal skulls the orbital margin does not form a completed circle, owing to the presence of the fossa for the lacrimal sac on the nasal side. 
This fossa is bounded by two crests, of which the anterior is clearly the unbroken continuation of the infra-orbital margin, whilst the posterior can be traced, though less definitely, as a downward prolongation of the supra-orbital margin. The whole outline therefore assumes the form of one coil of an undulating spiral, the two ends of which overlap on the nasal side and enclose the fossa. This overlap is not only obvious from the lateral aspect but in most cases can be seen from the front, owing to the pushing outward of the upper overlap of the spiral, that is the posterior lacrimal crest, by the development of the ethmoidal air-cells, as is seen in Figs. 6, 9.

It is convenient for descriptive purposes to subdivide the margin into four segments corresponding to the four walls of the orbit, namely, the supra-orbital, lateral, infraorbital, and medial margins, though the limits of these regions can be defined less strictly than the walls, owing to the fact that they may be united by either curves or wellmarked angles, according to type.

Four bones form the orbital margin: the frontal, zygomatic (malar), maxilla, and lacrimal:

(i.) The supra-orbital margin is formed entirely by the frontal.

(ii.) The lateral margin by frontal and zygomatic.

(iii.) The infra-orbital margin by zygomatic and maxilla.

(iv.) The medial margin by maxilla and frontal, with the addition of the lacrimal posterior to the former.

Three sutures interrupt its continuity :

(a) The zygomatico-frontal'(fronto-malar) laterally.

(b) The fronto-maxillary on the medial side.

(c) The zygomatico-maxillary on the infra-orbital margin.

In the following description attention will also be drawn to certain features of immediate interest which lie beyond the margin itself.

(i.) The supra-orbital margin (margo supraorbitalis) is curved to a degree which varies in individuals and races. It is sharp in the lateral two-thirds, that is, as far as the supra-orbital notch, but is rounded in the medial third, where it is crossed by vessels and nerves. It is formed wholly by 




FIG. 5. - The Orbital Margin. Frontal section of left half of skull passing just behincl orbital margin (the lacrimal bone has broken away). Natural size. Orbital height $=32 \mathrm{~mm}$., orbital width $=4 \mathrm{I} \mathrm{mm}$., orbital index, $78 \cdot 05$.

S.O.G. $=$ Supra-orbital grooves.

S.O.K. = Supra-orbital ridge.

S.O.F. = Supra-orbital foramen.

$\mathrm{G} .=$ the Glabella.

M.A.P. = Medial angular process of frontal bone.

L.A.P. = Lateral angular process of frontal bone.
F.S.P. = Fronto-sphenoidal process of zygomatic bone F.P. $=$ Frontal priscess of maxilla.

O.T. = Orbital tubercle of zygomatic bone, just cut. Z.F. = Zygomatico-facial foramen.

T.M. = Tuberositas malaris.

I.O.F. = Infra-orbital foranen, surmounted by an infra-orbital tubercle.

The dotted lines show the position of the minute osseous canals through which run the anterior superior alveolar (dental) nerve and accompanying artery. The ascending canal contains an arterial twig only and it emerges from a foramen in the sutura notha to anastomose with the facial artery at $\mathrm{F}$. The horizontal canal contains both nerve and arterial twigs which supply the nasal mucous membrane, and the foramen through which they emerge on the nasal aspect of the frontal process of the maxilla can be seen in Figs. 32 and 33 . Compare with Fig. I7. 
the frontal bone, and corresponds to the junction of its vertical and horizontal portions (pars frontalis and pars orbitalis), as seen in Fig. I2, $a$. It forms an arcade extending between the medial and lateral angular processes of that bone (pars nasalis and processus zygomaticus), which processes form also the upper parts of the medial and lateral orbital margins respectively.

At the junction of the medial third with the lateral two-thirds the supra-orbital margin is cut by the supraorbital noteh (incisura supraorbitalis), which in about a quarter of cases is converted into a foramen (foramen supraorbitale). The notch is bridged in life by the thickened edge of the septum orbitale, and transmits from within the orbit to the forehead the supra-orbital nerve and vessels, as shown in Fig. 54, p. I23; it is situated about $25 \mathrm{~mm}$. or an inch (varying from I3 to $40 \mathrm{~mm}$.; Schwegel, I859) from the mid-line of the skull, is from 3 to $5 \mathrm{~mm}$. broad, and its lateral boundary is sharper than the medial; the size, form, and depth are very variable. In rare cases the supra-orbital margin is unmarked by grooves, a smooth condition (" pithekoid") typical of the higher apes. On the other hand, the nerve sometimes cuts the margin so deeply that it lies in a supra-orbital canal (canalis supraorbitalis), which may lie from 5 to $30 \mathrm{~mm}$. deep to the rim, and be from 5 to $15 \mathrm{~mm}$. long, as seen in Figs. 5, 6 , Io. Leading from the supra-orbital notch, there are occasionally to be seen long grooves, which pass upwards and lateralwards over the face of the frontal bone, as in Fig. 5 . These "supra-orbital grooves" contain the nerve, artery, and vein, or may lodge only one of them; they are not dependent upon the presence or absence of the supraorbital notches, and they may be found at all ages (even in the foetal skull, Fig. 43, p. 96), and their relative frequency is marked in some races, but not in others. Zoja (I884) found them in $I 6.4$ per cent of 908 skulls, and they have been seen roofed over to form long canals. Dixon (I904), who has described them at length, considers them caused by the growth of the nerves not having been proportionate to the expansion of the cranium. Opening into 
or near the supra-orbital notch there is nearly always a minute foramen called the "supra-ciliary canal " by Ward (I858), who found it constant in the foetal skull and in 72 out of r05 adult skulls. Zweibach (I900) found it present in 50 per cent of I062 skulls. It may be single or double, and it transmits a nutrient artery into the bone and allows exit of the anterior or frontal diploic vein, which drains blood from the diploë of the frontal bone and mucous membrane of the frontal air-sinus. A nerve filament (" nerve of Kobelt ") also enters it ; it may open into the frontal sinus and afford a channel for the transmission of disease; an example is seen in Fig. 27 (second bone), p. 6I.

A short distance to the medial side of the supra-orbital notch there occurs in about 50 per cent of cases a second one, the frontal notch (incisura frontalis), which likewise, though much more rarely, may either be converted into a foramen by ossification of the periosteal ligament which bridges it ( $2 \frac{1}{2}$ per cent), or even form a short canal, as in Fig. 6, or be continued into a groove over the frontal bone: It transmits the medial frontal division of the supra-orbital nerve when the latter has divided within the orbit, together with corresponding blood-vessels. Both these notches have been studied specially by Zweibach (I900), from whose account, as cited by both Merkel'(Igor) and Ledouble (I906), the following table is taken.

On examination of ro62 skulls there were found-

\begin{tabular}{|c|c|c|c|c|c|}
\hline & \multicolumn{2}{|c|}{ Orbits. } & & \multicolumn{2}{|c|}{ Orbits. } \\
\hline & Right. & Left. & & Right. & Left. \\
\hline $\begin{array}{l}\text { Supra-orbital notch } \\
\qquad, \quad \text { foramen } \\
\text { Margin smooth }\end{array}$ & $\begin{array}{c}\% \\
70 \cdot 5 \\
22 \cdot 2 \\
2 \cdot 8 \\
I\end{array}$ & $\begin{array}{c}\% \\
67 \cdot 7 \\
22 \cdot 3 \\
2 \cdot 8 \\
5\end{array}$ & $\begin{array}{l}\text { Frontal notch } \\
\qquad, \quad \text { foramen } \\
\text { Neither. . }\end{array}$ & $\begin{array}{r}\% \\
47 \cdot 5 \\
2 \cdot 5 \\
48 \cdot 3\end{array}$ & $\begin{array}{c}\% \\
47 \\
2 \cdot 4 \\
48 \cdot 5\end{array}$ \\
\hline
\end{tabular}

with numerous variations and combinations on either side. From Ledouble's figures, quoting various other authors, there was found a frontal notch in $25^{\circ} 7$ per cent and a frontal foramen in $I \cdot 7$ per cent of 1738 orbits. 
There may also be found instances of supernumerary notches, foramina, and canals which are usually smaller than the normal ones and lie to their lateral side. There appear to be no sexual or racial peculiarities in their dis-

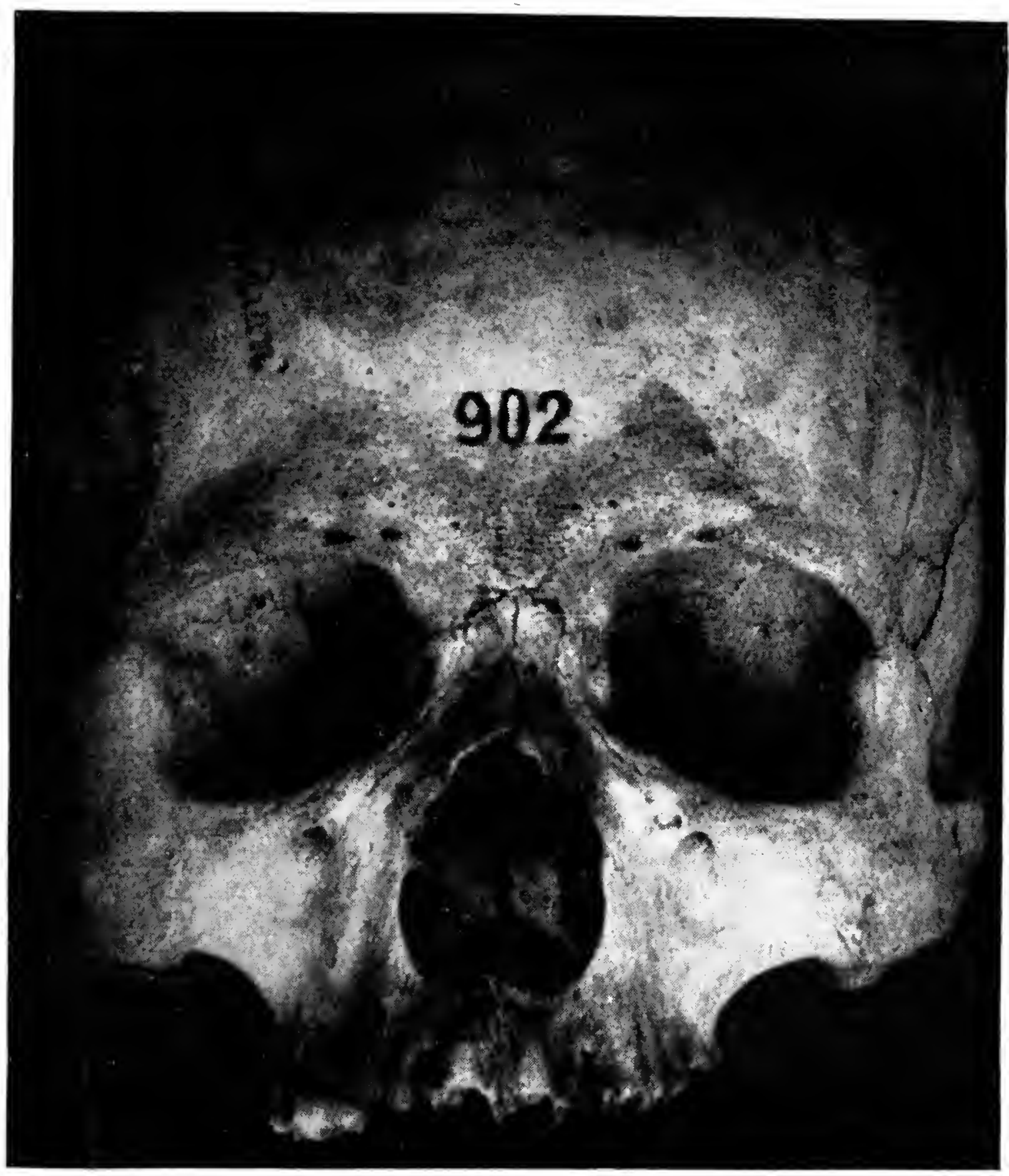

Fig. 6. - Skull showing both Frontal (medial) and Supra-orbital (lateral) Foramina, well-developed Supra-orbital Ridges, two Infra-orbital Foramina on left side, and a well-marked Orbital Tubercle of the Zygomatic bone. Orbital width $=42 \mathrm{~mm}$., orbital height $=33.5 \mathrm{~mm}$, orbital index $=79 \cdot 76$. Kansas Indian, adult male. $\times$ about $\frac{2}{3}$.

position; varieties are seen in different figures. As in the case of the supernumerary infra-orbital canals, the variation is no doubt due to the nerve dividing abnormally into separate branches within the orbit instead of after leaving that cavity. Practically it is important to remember that 
the nerve can thus cross the orbital margin in separate divisions, and it should be noted that there is yet another branch of the frontal nerve (from which the supra-orbital is derived), namely, the supra-trochlear, which leaves the orbit and crosses the margin still nearer the mid-line of the skull (Fig. 8).

Above the inner half of each supra-orbital margin in the male skull there is usually a prominence of the bone called the supra-orbital ridge (arcus superciliaris) or superciliary arch. These ridges, which are variable in size, form a characteristic difference between the sexes (see Fig. 44, p. IOI); at the mid-line they meet to form a low eminence, the "glabella" (from the Latin glaber, smooth, hairless), and between them and the outstanding lateral angular processes lie shallow and obliquely placed depressions, along which there sometimes pass the supra-orbital nerves and vessels, and, when present, the supra-orbital - grooves, as seen in Fig. 5, p. 8. Whilst the size of the supraorbital ridges is due to the degree of development of the underlying frontal air-sinuses, it does not follow that the sinuses are small or absent when the ridges are slightly marked.

(ii.) The lateral orbital margin (outer, external, or temporal border, margo latevalis) is the strongest part of the orbital rim; it is curved with a backwardly directed concavity, more especially marked in the lower part, thereby permitting a greater extension of the lateral range of vision on the ground where it is most required, whereas in the upper region the prominence of the lateral angular process of the frontal bone serves to protect the eye from injury on the side where blows are most likely to fall (Humphry). It is formed by parts of two bones, the lateral angular or zygomatic process of the frontal above, and the frontosphenoidal process and half of the orbital border of the zygomatic bone below. The zygomatico-frontal suture uniting these processes has been found duplicated in I7 out of 400 skulls by Nicola (I903), a condition explained by non-union of the lateral angular process, for which a secondary centre of ossification is described (Fig. 4I, p. 94). This margin may also be cut by a second suture in those very rare cases where 
the zygomatic bone consists of two pieces (mala bipartita or os japonicum, found present by Ledouble in $0^{\cdot} \mathrm{I} 6$ per cent of European and in 2I.I per cent of Japanese skulls).

The lateral angular or zygomatic process of the frontal bone is strong and prominent in the male, narrow and more delicate in the female; just beneath it, and between it and the globe over which it arches, is lodged the lacrimal gland. The sharp posterior margin of this process marks the beginning of the temporal ridge (linea temporalis), to which is attached the fascia covering the temporal muscle. Just below the zygomatico-frontal suture the margin is sometimes marked by fine hair-like grooves corresponding in position to the terminal branches of the lacrimal nerve which supply the skin in this region.

The breadth of the fronto-sphenoidal process of the zygomatic bone, as seen from the side, varies greatly. Its degree of recession, which appears to be a racial trait in man, is combined with a proportionately pronounced erection in the horizontal sense, and so has an effect upon the frontal inclination of the orbital margin (Oetteking, I9I9). On its free or temporal border there may be developed to a variable degree the processus marginalis of Sömmering, also called the spina zygomatica by Broca (Fig. 20, p. 49). According to Ledouble (I906), this process is more pronounced on the right side and in the male; it increases in old age, and is a muscular process, but is not well developed in Australian skulls (A. Thomson).

On the orbital surface of the fronto-sphenoidal process of the zygomatic bone, just within the lateral orbital margin at its centre and about II mm. below the suture, is a small tubercle, which may be termed the lateral palpebral or " orbital tubercle" (tuberculum orbitale) of the zygomatic bone; it is illustrated in Fig. 7, and is well seen in the orbits shown in Figs. 5, 6, 39. Often inconspicuous, it can generally be appreciated more readily by the touch ; it is of importance as marking the point of attachment of $(a)$ the " check ligament" of the lateral rectus muscle; (b) the suspensory ligament of the eyeball; (c) the lateral palpebral ligament; and $(d)$ the aponeurosis of the levator palpebrae superioris 
muscle (these confluent attachments forming the retinaculum oculi laterale of Hesser, I9I3; shown in Fig. 55, p. I24). It was found present in more than 95 per cent of 2000 skulls of all races (Whitnall, IgII).

The facial aspect of the zygomatic bone may present at its centre a slight tuberosity (tuberositas malaris). This underlies the most prominent part of the cheek and marks the attachment of the zygomaticus major muscle, as indicated in Fig. 59, p. I3I; it can sometimes be felt in life, and is in line with the infero-lateral angle of the orbital

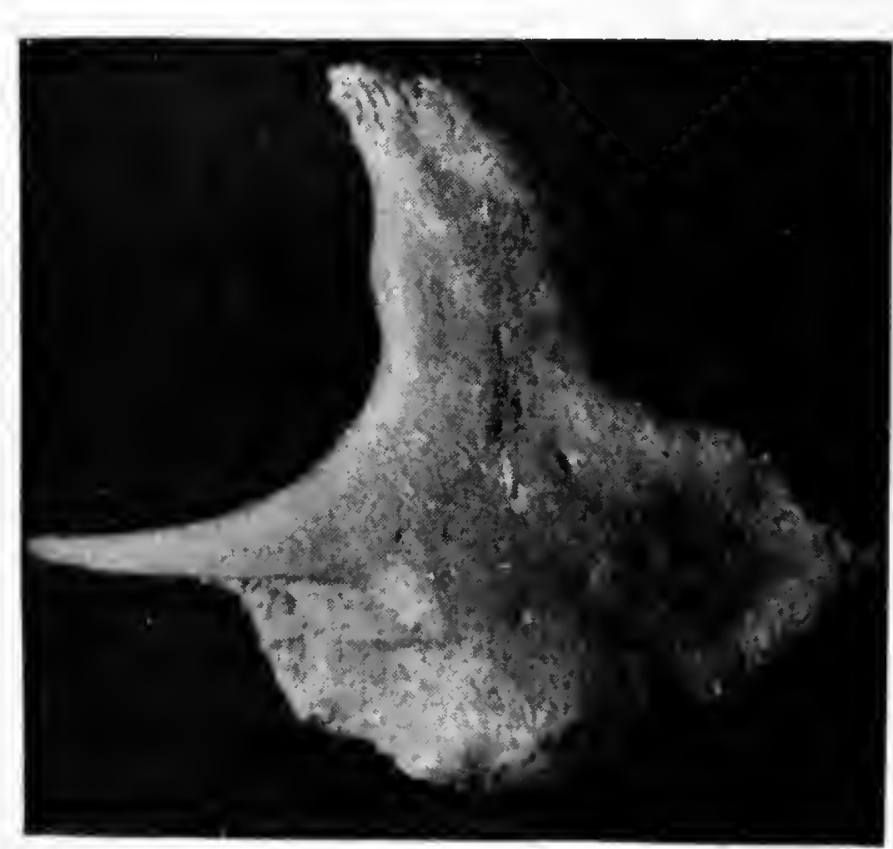

FIG. 7.-Left Zygomatic Bone seen from in front in natural position, showing the Orbital Tubercle (tuberculum orbitale). Natural size. margin and the long axis of the inferior orbital fissure. On this surface of the bone there is also to be seen the single or double opening of the zygomatic canal (foramen zygomatico-faciale, Figs. 7 , IO, 20, 2I), through which there passes from within the orbit to the skin of the cheek the zygomatico-facial branch of the zygomatic nerve from the maxillary division of the fifth cranial nerve, seen in Fig. 53, p. I22, together with an arterial twig. (iii.) The infra-orbital margin (margo infraorbitalis) is flattened, its edge is blunt and projects but little over the orbital cavity. Laterally it curves widely round into the lateral margin; medially it slopes upwards, to become continuous with the anterior lacrimal crest of the frontal process of the maxilla. It is formed by the confluent " orbital borders" of two bones, the zygomatic on the lateral side, the maxilla on the medial side, each contributing a half, so that the zygomatico-maxillary suture uniting them lies at about the middle of the margin (p. 4I). At this point there is frequently an elevation which can be felt in life, the "infra-orbital tubercle" (tubercuium infraorbitale 
marginale, Fig. 5); Zuckerkandl found it more frequently present in Malays (I9 out of 30 skulls) than in Europeans. The infra-orbital part of the orbital border of the zygomatic bone usually forms a long thin spur, the " marginal process," which rests upon the maxilla lateral to the suture (Figs. 7, I3) ; it may be broken off by injury, or, very rarely, exist as a separate ossiculum infraorbitale marginale (Grüber, I877).

Directly below the suture, and about Io mm., but varying from 4 to $I 2 \mathrm{~mm}$. ( $\frac{1}{4}$ to $\frac{1}{2}$ inch) from the margin and in rare cases even less, is an opening on the face of the maxilla, the infra-orbital foramen (foramen infraorbitale), which leads into the infra-orbital sulcus on the floor of the orbit; this foramen varies in shape from a circular opening to a vertically placed slit; usually it is oval in outline, from 4 to $5 \mathrm{~mm}$. deep by 3 to $4 \mathrm{~mm}$. wide; its lateral edge is sharp, and its opening is directed downwards and medially, as is seen in Fig. 2I, p. 5o. There is to be found rarely a second or "supernumerary infra-orbital foramen" (foramen infraorbitale supernumerarium of Grüber), as in the case of the supraorbital notch, and similarly explained by an early division of the nerve (Fig. 6) ; the two foramina may lie as far as I cm. apart; also " supplementary infra-orbital foramina" (foramina infraorbitalia anomala) may occur lateral or medial to and smaller than the main foramen. Finally, there may be seen minute "false infra-orbital foramina" on the anterior face of the maxilla which do not lead into the orbit but into either the maxillary antrum, the nasal fossa, or a nutrient dental canal. Ledouble found a double infra-orbital foramen in about II per cent of nearly 3000 skulls, and more often on one side than on both; three, four, or even five extra openings have been noted; Poirier (IgI2) in $2 \mathrm{I} 7 \mathrm{skulls}$ found 35 double, 2 triple, and I case of quadruple foramina.

Through the foramen pass the infra-orbital nerve the main continuation of the maxillary division of the fifth cranial nerve) and artery (a branch of the internal maxillary). At this point of emergence upon the face the nerve lies in a 
hollow much deeper from the surface than might be supposed, an average depth being Io $\mathrm{mm}$. or nearly $\frac{1}{2}$ inch ; it is embedded in fat and covered by the levator labii superioris muscle (caput infraorbitale of the quadratus labii superioris), the origin of which is sometimes marked by a slight ridge just above the foramen, as well as by the orbicularis oculi muscle and the skin. Below the foramen arises the levator anguli oris (caninus) muscle (Fig. 59, p. I3I). The bridge of bone between the infra-orbital margin and the foramen may be marked by a fine vertically directed suture (sutura infraorbitalis verticalis vel sagittalis), indicating the line of fusion of the maxilla as it closed up round the nerve in development. It usually cuts the margin at a point medial to the zygomatico-maxillary suture, and in most cases is obliterated early in life ; an example is seen in the female skull, Fig. 44, p. Ior. The face of the maxilla below the foramen forms the anterior wall of the maxillary air-sinus (antrum of Highmore), and embedded in a minute canal in its substance is the anterior superior alveolar (dental) nerve. The nerve arises from the lateral aspect of the forepart of the infra-orbital nerve, and in company with a small artery winds round below the foramen to gain its medial side before bending downwards at an angle to reach the two incisors and canine teeth which it supplies. From the artery and nerve twigs pass through minute bony channels to the mucous membrane lining the nasal cavity, and a communication is effected with the facial artery by a vessel which lies in a groove (the sutura notha) on the face of the frontal process of the maxilla at the infero-medial angle of the orbital margin, as indicated in Fig. 5, p. 8. By these intra-osseous paths it is possible for disease of dental origin, especially from the canine tooth, to spread to ocular or lacrimal regions (see also the paper by Hardy, I9I7). Occasionally the infra-orbital margin may be faintly grooved by the passage over it of a branch of the infra-orbital artery. Other variations in the bones forming this border concern the medial half, and may result in exclusion of the maxilla from it, either by extension of the marginal process of the zygomatic as a 
long spur up to the lacrimal bone, as is constant in lower mammals with enclosed orbits (the extremely rare condition in man of a " lacrimo-jugal suture " so formed was found present by. Reid (I9IO) in two out of 4500 skulls at Cambridge), or by the intervention of a hamular process of the lacrimal bone (Fig. I9, p. 48). These abnormalities have been studied by Grüber, and are tabulated by Merkel (I9OI). The varieties of the infra-orbital suture will be found in Ledouble's treatise (I906), and are also the subject of papers by Turner (I885) and Davida (I9I3).

(iv.) The medial orbital margin (internal or nasal border, margo medialis) is the most ill-defined segment of the margin, since it is not only rounded and indistinct in its upper part, but is complicated below by the presence of the fossa for the lacrimal sac (fossa sacci lacrimalis). It is formed by means of three bones: in its upper part by the medial angular process (pars nasalis) of the frontal bone; in its lower part by the lacrimal bone behind, together with the frontal (nasal or ascending) process of the maxilla in front. The supra-orbital margin medial to the notch loses its sharpness, but though interrupted by slight vascular grooves is still traceable as a rough line passing downwards and slightly backwards into the sharp posterior lacrimal crest (crista lacrimalis posterior) of the lacrimal bone: on the other hand, the infra-orbital margin traced medially passes upwards, to become continuous with the anterior lacrimal crest (crista lacrimalis anterior) of the frontal process of the maxilla. Hence, as explained on p. 7, the ring formed by the whole orbital margin is broken on the medial side, and the two ends which form the lacrimal crests separate to enclose the fossa of the lacrimal sac. Opinions differ as to whether the anterior or posterior lacrimal crest should be described as forming the exact orbital margin in this region, the fixation being chiefly important for purposes of mensuration (p. 86). Merkel took the posterior crest, since to it is attached the septum orbitale, a membrane which arises from the lip of the whole margin, and forms the true limit to the orbital contents (Fig. 59, p. I3I): the fossa then is extra-orbital in position, with which opinion Evatt 
concurs; Topinard, on the other hand, considered the whole lacrimal apparatus to lie within the orbit. Morphologically, the position of the fossa is interesting, since in most mammals it is undoubtedly placed outside the orbit, but of the primates in lemurs alone is it so placed, being considered to lie within it in all others (Flower). From the attachments of the septum orbitale, however, the posterior lacrimal crest must be taken as the limit of the orbital cavity on the medial side, and the fossa for the lacrimal sac in man is situated on the margin, which is broadened out or divides to accommodate it; so also it will be found that parts of the chief constituents of the eyelids (the medial palpebral ligament of the tarsal plate and the orbicularis oculi muscle) divide, and are attached to the margin both in front and behind the fossa. The fossa for the lacrimal sac is the chief feature of interest in this margin, but in view of the fact that it is partly formed by the lacrimal bone, and, moreover, presents important deep relations to the ethmoidal air-cells, which structures come under the heading of the medial wall, its description, together with that of its continuation the naso-lacrimal canal, is best deferred until later (p. 64).

There remain a few minor points to be noticed. At the junction of the medial and inferior orbital margins, that is, at the base of the anterior lacrimal crest, there is in many cases a small " lacrimal tubercle," also considered later in its relation to the lacrimal fossa on p. 64 . On the lateral aspect of the frontal process of the maxilla, in front of the lower part of the lacrimal crest and running parallel with it, there is constantly to be seen a fine groove, the sutura notha or sutura longitudinalis imperfecta of Weber (Fig. Io). It is a vascular groove lodging a branch of the infra-orbital artery, from which minute twigs pass through a row of small foramina into the bone and to the nasal mucous membrane. In rare instances it divides off a strip of bone from the margin to form the ossiculum maxillo-frontale of Macalister (who found it in I per cent of English skulls), or, since the fragment helps to form the lacrimal fossa, the os de la gouttière lacrymale of Ledouble (I.6 per cent of 2723 
skulls), who suggests a possible secondary centre of ossification for this piece. Ledouble also records and figures three cases of separation of the medial angular process of the frontal bone to form an os maxillo-naso-lacrimofrontal; this he regards as a Wormian bone, but it has been held to represent the pre-frontal of lower vertebrates; it may be explained by non-union of a part of the bone developed from a secondary centre of ossification (Fig. 4I, p. 94).

The relations of the whole orbital margin to the eyeball are considered under the section devoted to the latter and will be found on p. 256 , and its dimensions are given under mensuration on p. 87 .

\section{The Surface Anatomy of the Orbital Margin.-} The whole margin can be defined in life and its main features identified by the finger. In the mid-line above the hollow at the root of the nose the glabella can be felt, and lateral to this on either side the prominence of the supra-orbital pidge ; overlying the bone between the ridge and the actual orbital margin is the " head " of the eyebrow. The medial third of the supra-orbital margin is rounded and indistinct as it passes downwards into the medial angular process of the frontal bone, but may present a frontal notch about a finger's breadth from the mesial plane; lateral to this, at the junction of the medial third and lateral two-thirds of the margin and about an inch from the mid-line of the skull, the supra-orbital notch may be felt, and the presence of the corresponding nerve realised by pressure above the margin. A line drawn downwards from this notch to the interval between the upper two bicuspid teeth crosses the infra-orbital foramen. From the supra-orbital notch the sharp lateral two-thirds of the margin can be traced round the supero-lateral angle to the zygomatico-frontal suture and the upper part of the lateral orbital margin. This suture can always be felt owing to a notch on its hinder edge. It lies at a slightly higher level than the corresponding fronto-maxillary suture on the opposite margin of the orbit. Above the suture the prominent lateral angular process of the frontal bone can be felt, and the 
temporal ridge traced upwards from its posterior edge ; the "tail" of the eyebrow lies over this process and the lacrimal gland lies beneath it, though nothing can be felt of this organ. Below the suture the lateral margin is formed by the fronto-sphenoidal process of the zygomatic bone; on its outer side there may be felt the processus marginalis

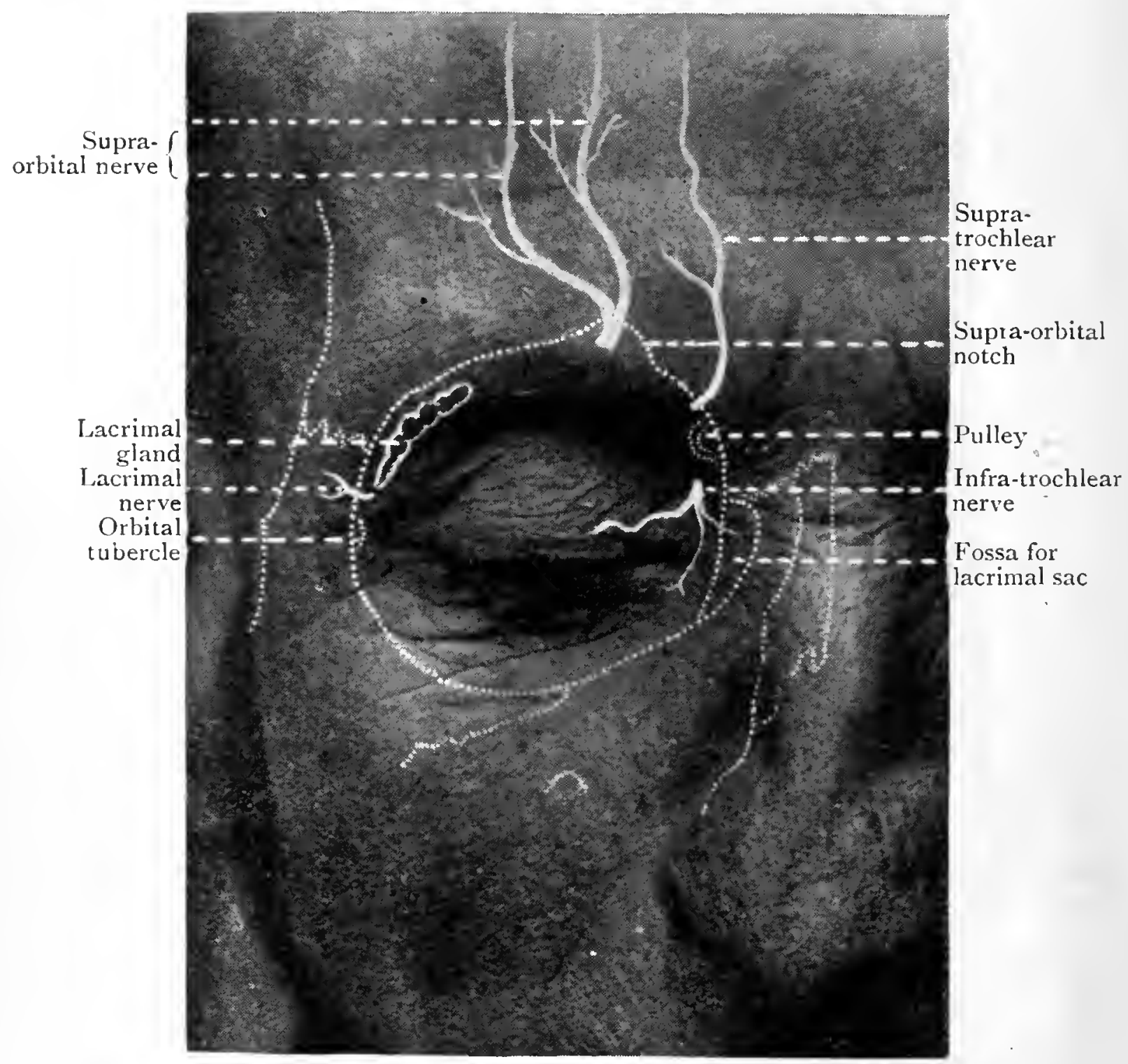

FIG. 8. - To illustrate the surface anatomy of the Orbital Margin.

projecting backwards towards the temporal fossa; just below it the zygomatico-temporal branch of the zygomatic nerve reaches the skin. On the orbital aspect of the process, and just within the centre of the lateral margin, lies the orbital tuberele of the zygomatic bone: it can be felt by inserting the finger-tip deeply between the orbital margin and the eyeball opposite to the lateral commissure (external canthus) of the eyelids and rubbing the finger up and down. This margin is backwardly curved to such 
an extent that it lies on a plane $22 \mathrm{~mm}$. posterior to the root of the nose, I2 $\mathrm{mm}$. behind the anterior lacrimal crest, and $7 \mathrm{~mm}$. behind the fovea trochlearis (Maddox). Below the suture the lateral margin can be traced as a well-defined edge downwards round the infero-lateral angle of the base and along the infra-orbital margin until at, or a little medial to, its centre the zygomatico-maxillary suture is felt. The site of the suture may be more obvious owing to the presence of an infra-orbital tubercle, and about I cm. below it and the same distance from the skin there is situated the infra-orbital foramen. Medial to this suture, the margin slopes upwards into the anterior lacrimal crest, at the commencement of which there may be felt a lacrimal tubercle. It is generally easy to feel with the finger-nail the depression of the fossa for the lacrimal sac behind this crest, and behind and above the tubercle if present, and also to identify the posterior lacrimal crest which bounds the fossa behind. In the upper part of the fossa the outlines are rendered indistinct by the medial palpebral ligament (iendo oculi internus) which crosses it. The fronto-maxillary suture cannot be felt; above it the margin curves up into the supra-orbital margin, so forming the supero-medial angle of the base. It is full and rounded, and is crossed by the supra-trochlear nerve and by the important anastomoses between the ophthalmic blood-vessels and the angular vessels of the face. By pressing the end of the thumb within this angle the trochlea or pulley of the superior oblique muscle can readily be felt on the orbital roof.

As regards the relation of the orbital margin to the brain, it may be noted that the anterior pole of the temporal lobe lies about 2 to $2 \frac{1}{2} \mathrm{cms}$. or I inch behind the zygomaticofrontal suture of the lateral margin, and about the same distance above the zygoma; the inferior surface of the frontal lobe lies about half an inch above the suture, and its medial lower edge is on a level with the fronto-nasal suture.

The position of the vessels and nerves which cross the orbital margin in various regions is shown in Fig. 8 and Fig. 83, p. I62. Overlying the whole margin is the orbital part of the orbicularis oculi muscle, covered by superficial fascia 
and skin, which are thickened to form the eyebrow in the supra-orbital region.

\section{THE WALLS OF THE ORBIT}

For convenience of description the orbital cavity is regarded as being bounded by four walls, but the four angles formed between them are so rounded off, especially in front, that certain features may be described as belonging to either one or the other of two adjacent walls. The walls are :

(i.) The roof or superior wall (paries superior).

(ii.) The lateral or external wall (paries lateralis).

(iii.) The floor or inferior wall (paries inferior).

(iv.) The medial or internal wall (paries medialis).

Of these, the medial wall alone is quadrilateral in shape ; the others are triangular in outline, with the apices behind and the bases in front at the margin; the floor is the shortest and extends only to two-thirds of the depth of the cavity. The lateral wall is best demarked owing to the presence of the superior and inferior orbital fissures above and below its posterior part; the medial wall may be limited above and below by prolonging forwards and backwards the lines of the upper and lower sutures of the ethmoidal bone.

The bones forming the walls are chiefly those which have already been seen to form the margin, namely, the frontal, zygomatic, maxilla, and lacrimal ; in addition, portions of the sphenoidal, ethmoidal, and palatine help to complete the walls posteriorly; of these bones the frontal, sphenoidal, and ethmoidal are common to both orbits.

(i.) The roof is formed by the frontal, and the lesser wing (ala parva) of the sphenoidal bone.

(ii.) The lateral wall is formed by the zygomatic, and greater wing (ala magna) of the sphenoidal, together with a small part of the frontal bone.

(iii.) The floor is formed by the maxilla, together with the palatine and part of the zygomatic bone.

(iv.) The medial wall is formed by the frontal, lacrimal, ethmoidal, and body of the sphenoidal bone.

(i.) The Roof (superior or cerebral wall, vault, paries 
superior) is separated from the medial wall in greater part by the fronto-ethmoidal suture; a backward prolongation of this line passes just below the optic foramen (which lies, therefore, in the apex of the roof) and cuts into the widest part of the superior orbital fissure, whilst its forward prolongation runs across the medial angular process of the frontal bone and approximately hits off that point at the root of the nose where the nasal bones articulate with the

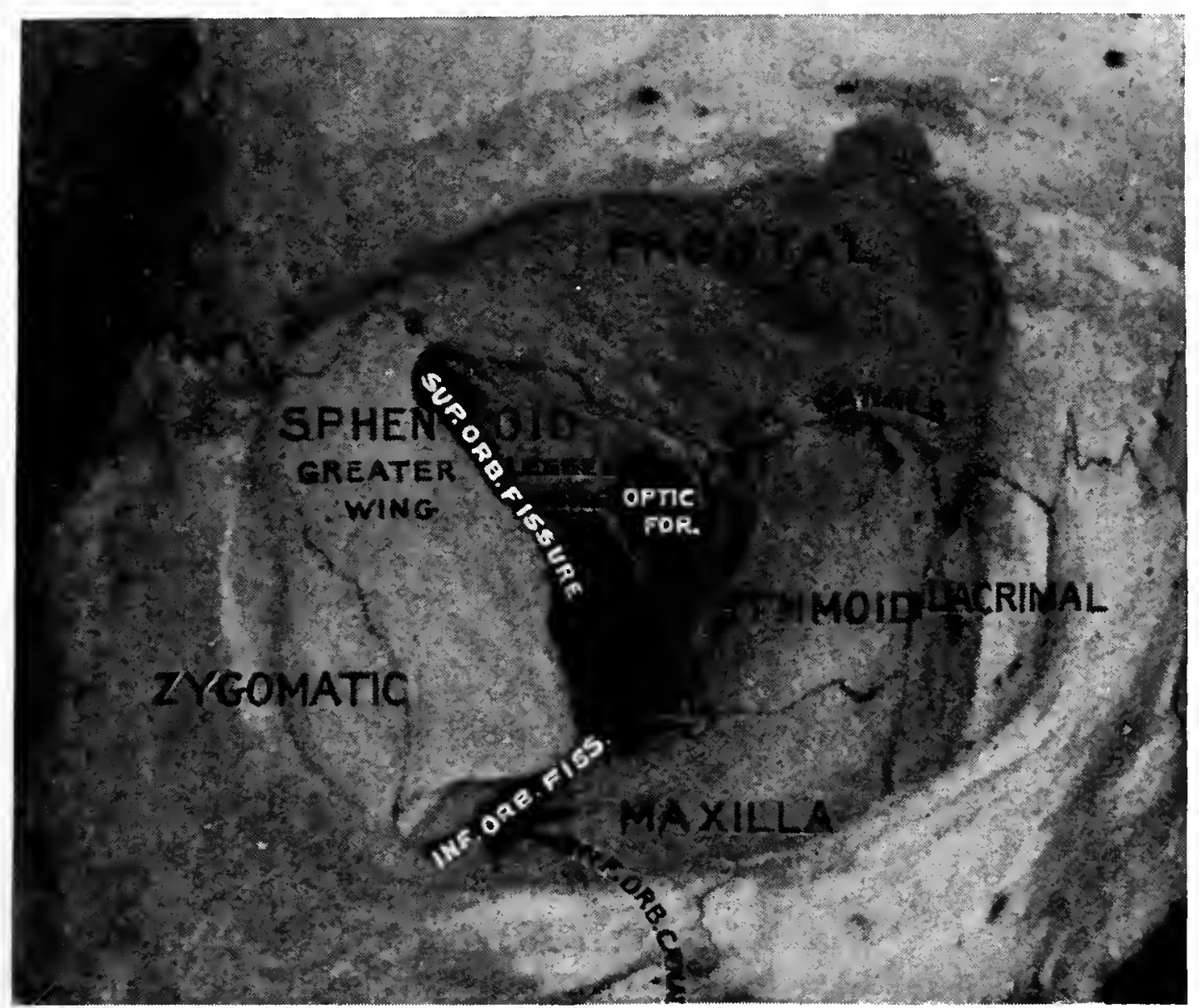

FIG. 9. - Right Orbit, showing the bones which form it and the apertures. $\times 2$.

frontal (known in craniometry as the point nasion). The line of separation from the lateral wall is indicated by continuing the direction of the upper limb of the superior orbital fissure until it cuts the supero-lateral angle of the margin at the most prominent point of the lateral angular process of the frontal, a short distance above its articulation with the zygomatic bone. The roof presents in outline the shape of an isosceles triangle. It is nearly flat at the apex behind, and of increasing concavity in front; it forms a smooth dome, well arched from side to side, but less so from behind forwards. It is formed in greater part by the orbital 
plate (pars orbitalis) of the frontal bone, and is completed behind at the apex by a small triangular piece formed by the lesser or orbital wing (ala parva) of the sphenoidal bone. This pieceis about a half to three-quarters of an inch in length and is pierced by the optic foramen (Fig. II). The features to be noticed in the roof are - the fossa for the lacrimal gland, the fovea trochlearis, the fronto-sphenoidal suture, the optic foramen, the architecture, and the relations to adjacent parts.

The fossa for the lacrimal gland (fossa glandulae lacrimalis) is a depression in the frontal bone at the anterolateral angle of the roof of the orbit; it is overhung by the lateral angular process. In some skulls the fossa is nearly conical in shape, as in the foetal condition, but generally a uniform hollow is found, the fossa being merely a deeper part of the general concavity of the roof and best appreciated by feeling the bone with the finger; its limits are defined below only by the marked ridge running along the zygomatico-frontal suture on the lateral orbital wall, as seen in the right orbit of Fig. Io. Whether the marked difference in depth found in various skulls (not necessarily in the two sexes) is accounted for by a corresponding development of the gland is questionable, since the fossa not only lodges the gland but is filled up by a mass of orbital fat behind it; this posterior part of the fossa is called by Rochon-Duvigneaud (1903) the loge accessoire, and in some cases a faint undulation of the surface may be felt to demark the two regions.

The fovea trochlearis is a small circular pitting or dimple of the bone in the antero-medial angle of the roof, 4 to $5 \mathrm{~mm}$. from the orbital margin; its position is said to vary according to the shape of this angle of the orbit, being found higher when the angle is narrow, lower when the angle is broader (Stanculeanu, I902). It is present in 80 per cent of cases on one or both sides, and marks the point of attachment of the cartilaginous pulley (trochlea) of the superior oblique muscle of the globe. Sometimes it is surmounted by a small spine, the spina trochlearis, as seen in Fig. 2I, p. 50, for which a secondary centre of ossification has 
been described (Keibel and Mall, I9I2). Sperino (I904) pointed out that it may occupy a variable position with regard to the fovea, though most often developed above and behind it; in the observations quoted by Ledouble the spine was found present in nearly I6 per cent of 15 I 6 skulls, more often on one side only and then on the right, and in all races, at any age, and in either sex. In I to 2 per cent of skulls there is a second spine situated below the fovea, and a complete bony ring has even been observed. These spines are formed by ossification of the fibrous bands which attach the ends of the semilunar cartilage of the pulley to the edges of the fovea, and the more frequent occurrence of the upper one is explained by the greater traction exerted upon the corresponding liga-

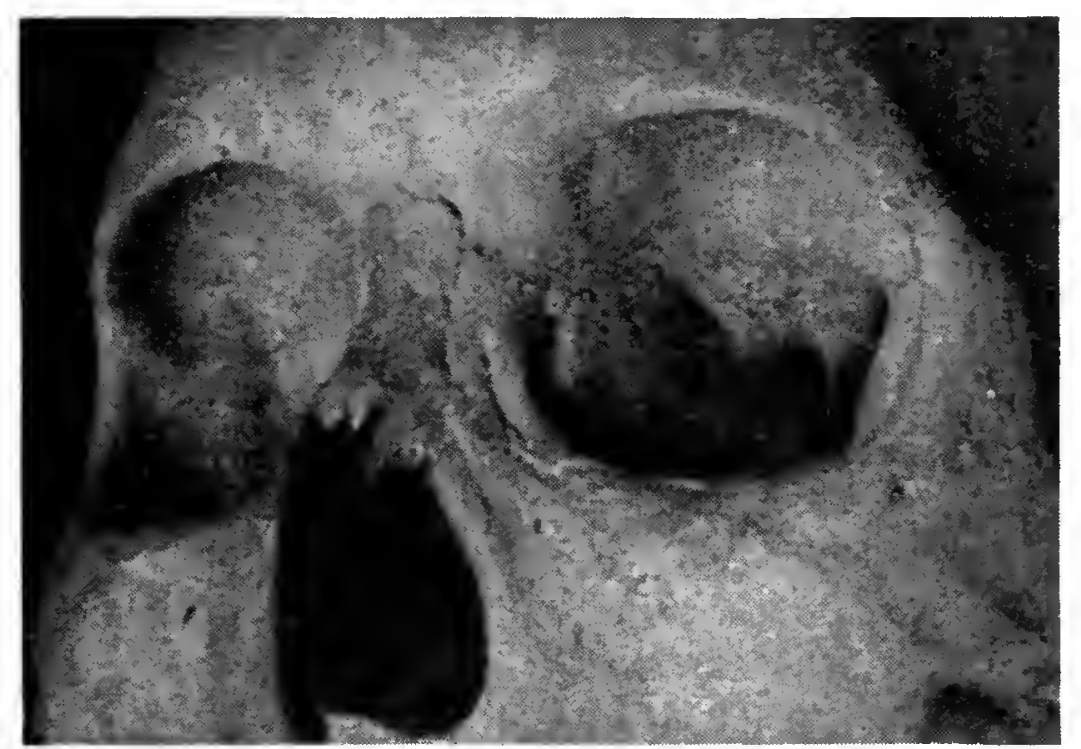

FIG. I0.-Features in the orbital walls. The Left Orbit shows the forea trochlearis as a dimple in the antero-medial corner of the roof; there is a supraorbital canal, a well-marked sutura notha, and triple zygomatico-facial foramina. The Right Orbit shows the fossa for the lacrimal gland beneath the lateral angular process of the frontal bone limited below by a ridge along the line of the zygomaticofrontal suture. Calcutta Indian sliull. $\times \frac{1}{2}$. ment, giving rise to an activity of the osteogenetic periosteum. The bone may be grooved above the fovea trochlearis by the supra-trochlear nerve, and below by the infra-trochlear nerve and terminal branches of the ophthalmic blood-vessels. A description of the pulley itself will be found on p. 274. The bony wall on which it is situated is undermined in one-third of cases by the frontal air-sinus, as is well shown in Fig. 63, p. I35.

The fronto-sphenoidal suture (posterior limb) crosses the apex of the roof obliquely from the anterior end of the superior orbital fissure on the lateral side to a point just behind the posterior ethmoidal foramen on the medial wall; it cuts off the lesser wing of the sphenoidal bone with the 
enclosed optic foramen, and is the only suture which normally crosses the roof, but is usually obliterated in the adult.

The optic foramen (foramen opticum). - This name is commonly applied to the anterior or orbital opening of a short canal (canalis nervi optici, canalis opticus), which has also a posterior or cerebral opening into the middle cranial fossa. It lies in the apex of the roof of the orbit at the hinder end of the angle between the roof and the medial wall ; it is nearly in line with the two ethmoidal foramina, which lie along this angle, and the nasion, and a horizontal plane drawn through this last point at the root of the nose would pass just above it. The distance of this opening from the supra-orbital margin is about $50 \mathrm{~mm}$. or 2 inches, but the precise measurements from this and other points on the margin will be found under mensuration on p. 90, since the optic foramen is the point from which the lengths of the orbital walls are usually measured.

The optic canal is formed by the union of the two roots of the lesser wing of the sphenoidal bone which spring from the side of its body. The floor projects posteriorly, the roof anteriorly: that is 'to say, its two ends are obliquely cut from above downwards and backwards, as shown in Fig. 28 , p. 65 , but the bony roof is prolonged behind for a few millimetres, or in rare cases even entirely formed, by a fibrous arcade or "falciform fold" of the dura mater. The length of the canal varies from 4 to $9 \mathrm{~mm}$., and its long axis is inclined posteriorly and medially (continuing the line of the lateral orbital wall) so that the axes of the two canals, which are separated at their orbital openings by a distance of about $25 \mathrm{~mm}$. or I inch, would, if projected backwards, meet at the centre of the dorsum sellae of the body of the sphenoid; this central meeting-point lies 27 to $29 \mathrm{~mm}$. behind the orbital end of the canal along its projected long axis. The canal lies opposite the middle and widest part of the zygoma and a finger's breadth above it ; its horizontal distance from the centre and most prominent part of this arch was $40 \mathrm{~mm}$. in a skull of five years, $43 \mathrm{~mm}$. in one of twelve years, 49.5 $\mathrm{mm}$. in one of sixteen years, $52.5 \mathrm{~mm}$. in one of nineteen years, $52 \mathrm{~mm}$. in an adult female, and 60.5 in an adult male 
skull, as found by Weiss (I890, I897); the position relative to the zygoma is seen in Fig. I84, p. 364. The anterior opening is oval in outline and measures 5 to $6 \mathrm{~mm}$. in its longest (the vertical) diameter ; the central part of the canal is circular, and the posterior opening is generally considerably flattened from above downwards.

The contents of the canal are the optic nerve and the ophthalmic artery, the latter being accompanied by minute branches from the carotid plexus of the sympathetic nervous system. The optic nerve is enclosed in sheaths prolonged from the pia mater, arachnoidea, and dura mater of the brain. The artery is embedded in the dural sheath on the lower and lateral side of the nerve, and ossification of the fibrous septum between them accounts for the rare abnormality of a "doubled optic foramen" (Fig. 2I, p. 50), the vessel or one of its branches passing through the lower and smaller opening; more commonly it grooves the hinder part of the floor of the canal.

The relations of the optic canal are as follows : superiorly a fairly strong plate of bone separates it from the base of the olfactory tract on the under surface of the frontal lobe of the brain. In some instances the roof of the canal is hollowed out by an extension of the sphenoidal air-sinus; indeed, according to Howell Evans (I908), this "optic projection " of the sinus into the lesser wing or the clinoid process of the sphenoidal bone occurs as often as I in 3 . On the infero-lateral aspect the optic canal is separated from the superior orbital fissure by a bridge of bone formed by the lateral root of the lesser wing of the sphenoid; this bridge, which also may be hollowed out by an extension of the sphenoidal sinus or of a posterior ethmoidal air-cell, sometimes presents a minute tubercle in its lower part (Fig. 9), to which is attached the tendon of Zinn (p. 263). Ledouble has seen two cases in which the bridge was absent, so that the optic canal communicated with the superior orbital fissure, a condition found in birds, marsupials, and whales. On the medial side the canal is in relation with the sphenoidal air-sinus or occasionally with a posterior ethmoidal air-cell. According to Loeb (I909) the optic 
chiasma is nearly always related in front to both the sphenoidal sinuses and never to the posterior ethmoidal cells, whilst the optic nerve is usually related to the sphenoidal sinus but in a few cases to an ethmoidal cell. Onodi (I903) even classifies thirty-eight different forms and twelve groups of relations of these air-spaces to the optic canal, which indicates the great variation that may occur in their arrangement.

Fig. II shows a sphenoidal bone in which the optic canal is

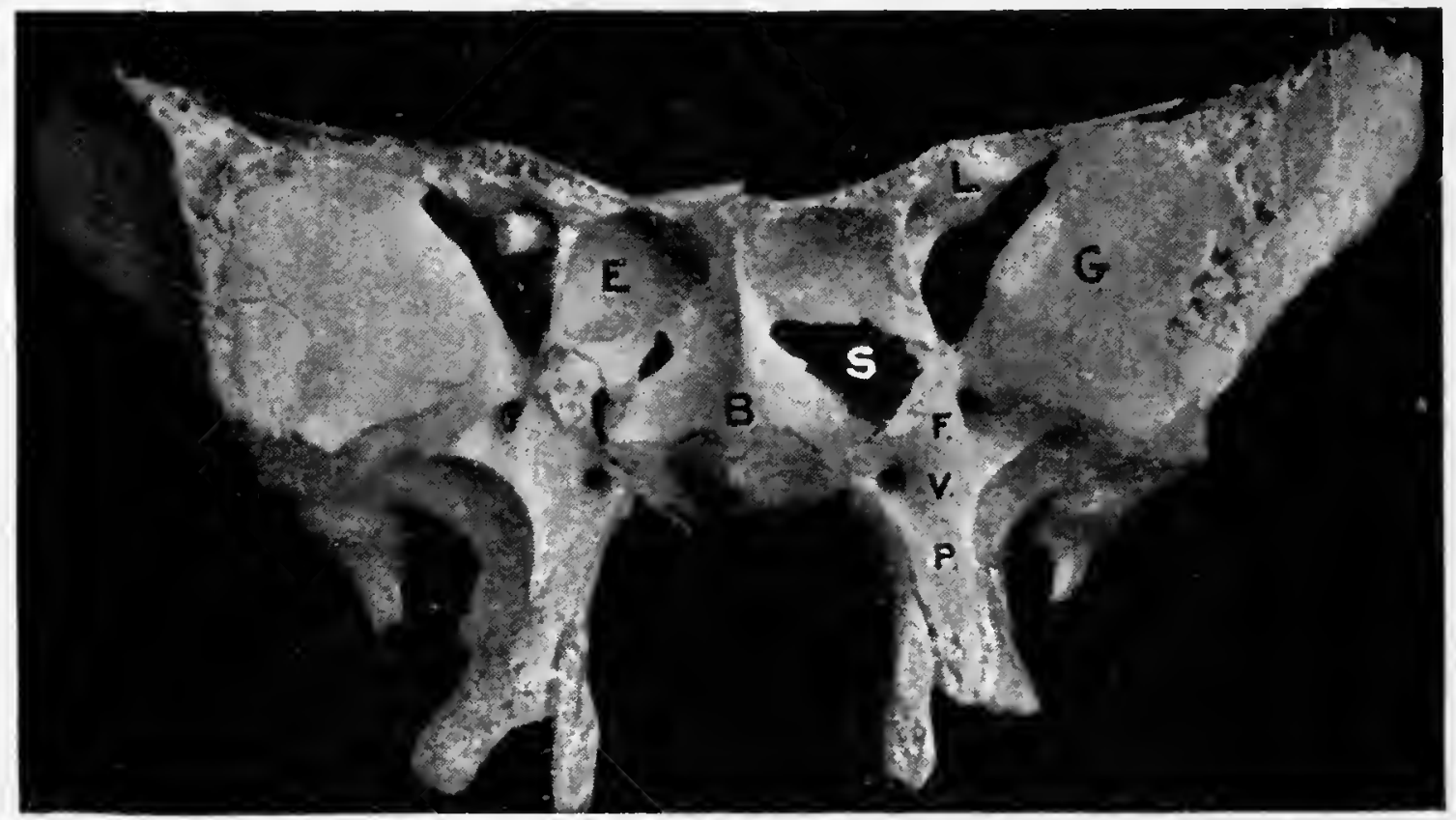

FIG. I I. - Sphenoidal Bone from in front (orbital aspect). Adult. $\times \frac{2}{3}$

$\mathrm{B}_{.}=$Body of sphenoidal bone.

$G_{.}=$Orbital surface of greater wing separated

from the lesser wing by the superior orbital fissure.

$L_{0}=$ Lesser wing pierced by the optic canal, through which a rod has been passed on the right side.

$\mathrm{P} .=$ Pterygoid process.

V. $=$ Vidian canal.

E. = Posterior ethmoidal cell into which the. optic canal projects.

$\mathrm{S} .=$ Sphenoidal sinus showing relation to $\mathrm{F}$, the foramen rotunamm.

related to the hindermost ethmoidal cell. In one specimen of the Oxford collection the sphenoidal sinus surrounds the optic canal on all sides, so that the latter passes freely through the middle of the cavity as a bony tube; and in the M'Gill Museum is one specimen in which a large posterior ethmoidal cell presents a similar relation, the sphenoidal sinus being much reduced in size, and another specimen where a large right sphenoidal sinus is related to both optic canals. The frontal sinus has been seen to undermine the roof of the orbit so. far back as to come close to the optic canal, but the maxillary sinus has never been seen by the writer to extend nearer than $7 \mathrm{~mm}$. to it. 
The most important fact in regard to these relations is the delicacy of the bony wall which separates the optic canal from the neighbouring air-space, especially when this is formed by an ethmoidal cell; it is most often as thin as $0.5 \mathrm{~mm}$., but has been found from 5 to $7 \mathrm{~mm}$. thick (Stanculeanu, I902). Francis and Gibson (I9II) found a very thin wall in 38 per cent of cases; this wall, moreover, may be the site of senile atrophy, dehiscences having been recorded by Barraguer (I896), Berger (I894), Holmes, C. R. (I896; two cases in 50 skulls), and Gallemaerts (two in 200), the optic nerve in its sheath being then in contact with the mucous membrane lining the sinus; one such case has been seen by the writer.

The relations of the ocular muscles at their origin to the anterior optic foramen are illustrated in Fig. I35, p. 260, and described on p. 262 ; here it may be noted that the superior and medial recti muscles are closely related to the superomedial side of the foramen, with the superior oblique and levator palpebrae superioris muscles lying just above them.

The architecture of the roof of the orbit.- The roof presents more variations in its thickness than any other wall ; it is usually about I mm. thick, but is often of such paper-like delicacy that a tap with the finger-nail will break through it, and dehiscences due to atrophy of the bone in old age may be present, the periorbita then lying in contact with the dura mater; on the other hand, in one case it was found to be $4 \mathrm{~mm}$. thick (Fig. I2, b). It may be doubled in lesser or greater degree by extensions from the medial side of the frontal and ethmoidal air-cells between the two tables of the bone, as indicated in Fig. 27, p. 6r. Witt (Igo8) finds such extensions of air-cells over the roof in one-third of cases and describes three types- $(a)$ formed by the frontal sinus alone, (b) by the frontal sinus and one ethmoidal cell, $(c)$ by the frontal sinus and two ethmoidal cells, each cell having its own opening into the nose; he terms them " orbito-ethmoidal cells." Cryer (I907) calls them "supra-orbital sinuses" and notes that the posterior ethmoidal cells also may occasionally form them. The two walls thus formed may be very thin or fairly thick, and 
any variation between these extremes, which are illustrated
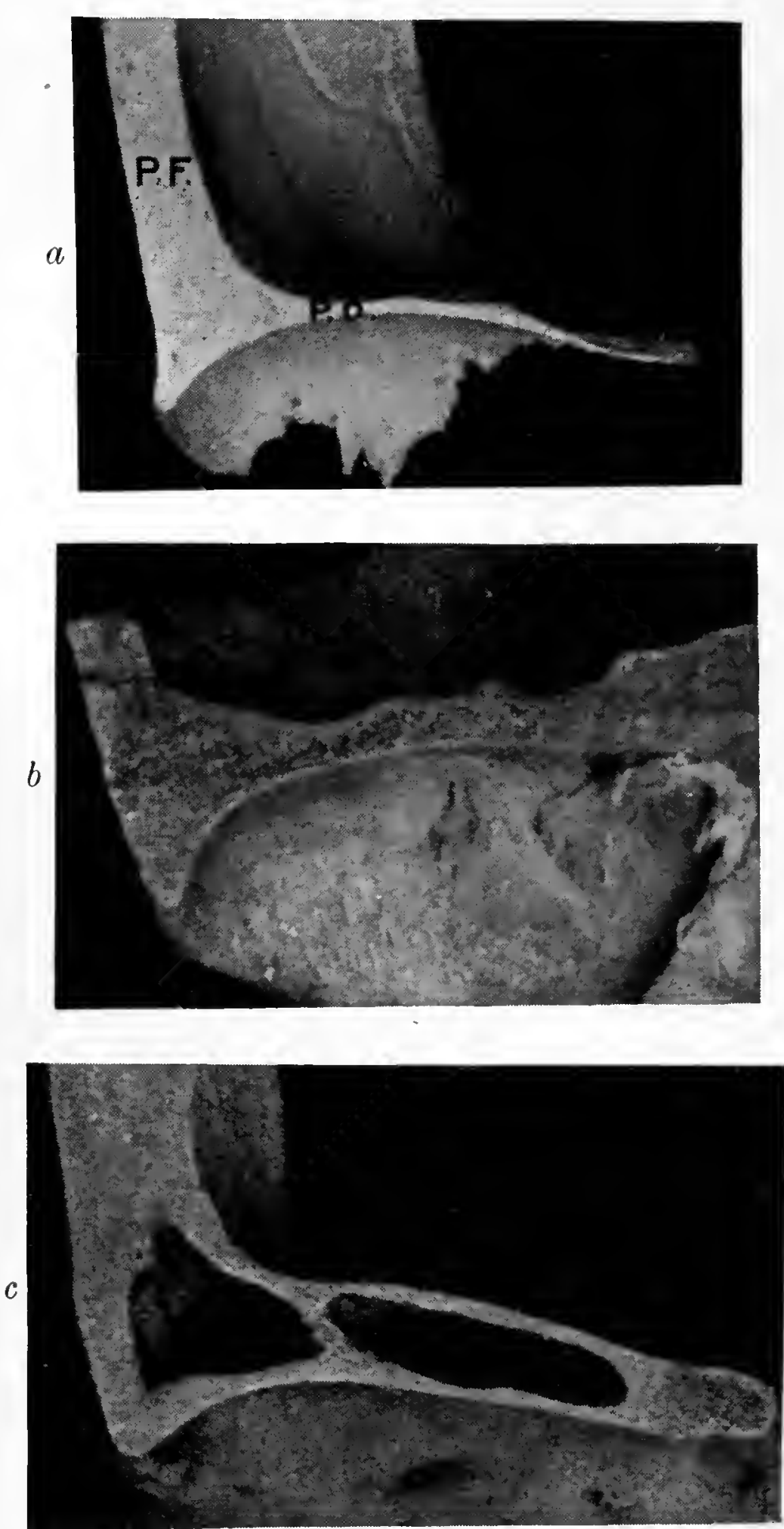

FIG. I 2. - Architecture of Orbital Roof, three types. $\times \frac{2}{3}$.

$a=$ Thin, normal. P.F. = pars frontalis, P.O. = pars orbitalis of frontal bone.

$b=$ Thick, solid.

$c=$ Doubled by extensions of air-cells with thick walls (compare Fig. 28, where the walls are thin, making a fourth type). in Fig. I2 and Fig. 28 , p. 65, may be found on examination of a series of bones.

The posterior two - thirds of the roof is usually the thinnest part, and on holding the bone up to the light there may be seen the lines of certain ridges formed on the cerebral aspect. These ridges (juga cerebralia) and the hollows between them (impressiones digitatae) are moulded by the sulci and convolutions of the superimposed inferior surface of the frontal lobe of the brain (Fig. I66, p. 3I9). The anterior third of the roof is always thicker and is not transparent.

Abnormalsutures may divide the roof into many pieces, and under the designation. of "accessory frontal bones" Augier (I9I2) classifies six varieties. Tenchini (I905) has observed an abnormal "orbito-frontal emissary vein," which passed 
from the superior longitudinal sinus of the brain through the roof of the orbit to join the superior ophthalmic vein.

The bone in the antero-lateral region of the roof is sometimes marked by an area of numerous fine holes, termed cribra orbitalia by Welcker (I887), which may even extend medially across the greater part of the roof. This porous condition affects only the-outer table of the bone, and, according to Toldt, is caused not by a pathological process but by a fault in development of the bone whereby the venous blood of the diploë, instead of being collected by the

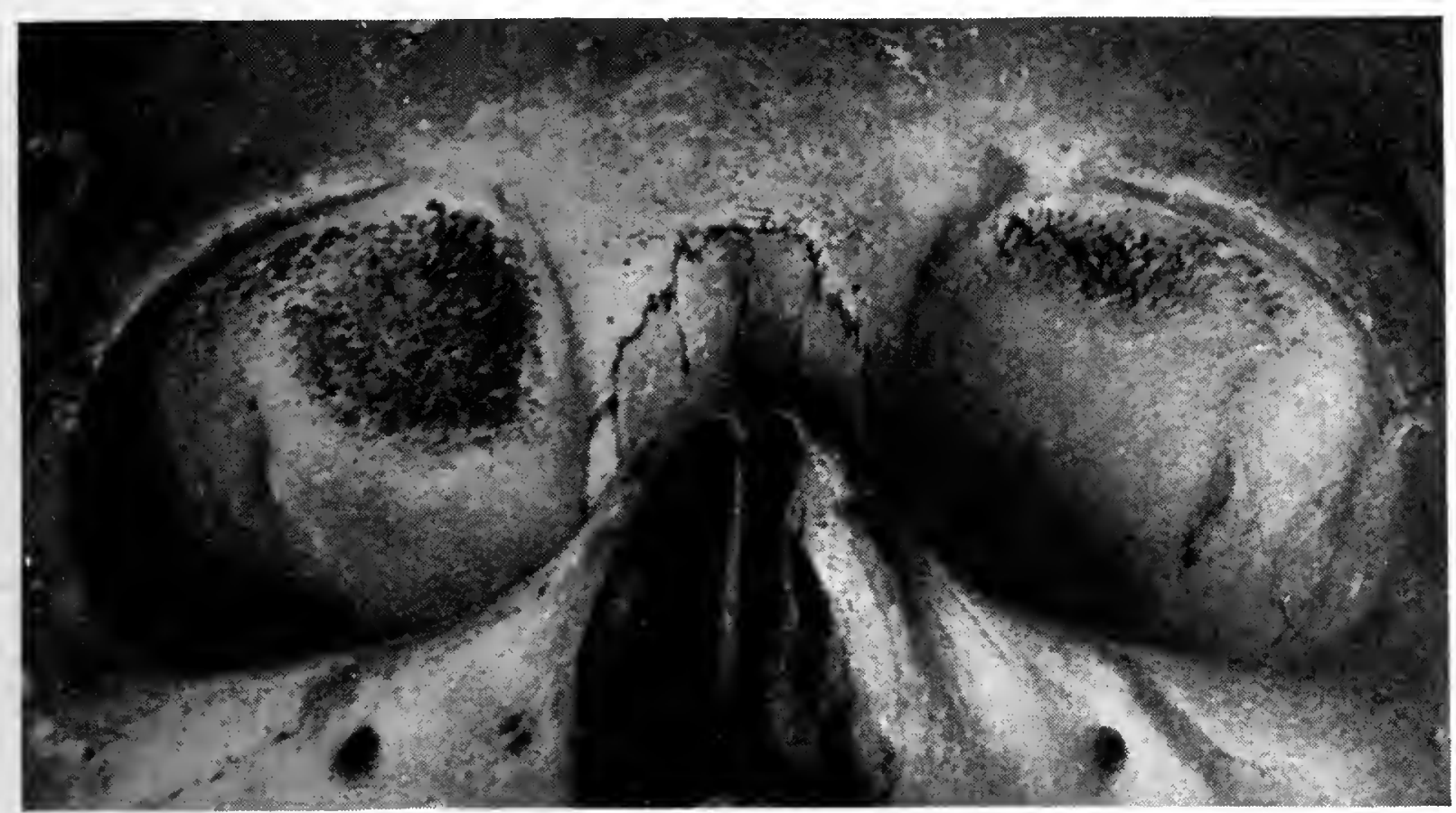

FIG. I3.-Roofs of Orbits showing condition of cribra orbitalia. The right orbit shows an advanced condition forming an exostosis. Compare with the slight condition shown in.Fig. 27 (second and third bones). New Zealand skull. $\times \frac{2}{3}$.

normal diploic channels, passes into the veins of the periorbita by numerous venules; hence the bony tissue of the orbital wall cannot develop as a compact layer and becomes cribriform. In rare cases a spongy swelling may be formed which projects into the orbital cavity and resembles an osteophyte. Ahrens (I904) found cribra orbitalia chiefly in young adults and in about 25 per cent of 86I skulls of all races, in I7 per cent of Europeans, but more commonly in lower races. Koganei (I9I3), in a more detailed account, differentiates three types according to the degree of development. He found them present more often in children $\left(24^{\circ} 4\right.$ per cent of 84 skulls) than in adults (II per cent of 372 
skulls), and always on both sides. A well-marked type is illustrated in Fig. I3, where in the right orbit it forms a well-marked osteophyte; less developed examples are seen in two of the orbital roofs in Fig. 27, p. 6r. It may be added that the condition is also found, though much more rarely, in the parietal and occipital bones of the skull.

The relations of the roof.-Internally, the periorbita is thin and easily detachable, except along the orbital margin and at the fovea trochlearis, where it binds the trochlea down to the bone. It is also firmly attached along the frontosphenoidal suture, so that a fluid introduced between it and the bone will not pass backwards beyond this line to reach the optic nerve or enter the cranial cavity. Closely applied to the roof and traversing its whole length is the frontal nerve, but the supra-orbital artery comes into relation with its anterior half only. Beneath the nerve the levator palpebrae superioris and underlying superior rectus muscles lie close against the roof, with the possible intervention of a little fat. Along the medial margin lies the superior oblique muscle, separated posteriorly by the fourth cranial nerve, and against the anterior and lateral part is lodged the lacrimal gland. Externally, the roof separates the orbital cavity from the anterior cranial fossa of the skull in which rests the frontal lobe of the brain, separated from it only by the meninges; so close is the relation between them that the bone is moulded by the cerebral convolutions and fissures, causing the "digital impressions" noted above; on the other hand, this region of the brain is slightly hollowed by the domed roof of the orbit and presents on its surface the composite fissure termed the sulcus orbitalis (Fig. I68, p. 323). The olfactory tract crosses obliquely the extreme posterior end of the roof on its medial side. The air-spaces which may enter into relation with the roof have been described under the architecture.

(ii.) The Lateral Wall (outer, external, or temporal wall; paries lateralis).-The limits of this wall are well defined, since the posterior part is bounded by the two orbital fissures, the superior dividing it from the roof, the 
inferior from the floor, and by prolonging their long axes forwards to the orbital margin the outline is completed; the upper line so taken ends on the most prominent part of the lateral angular process of the frontal bone, the lower one at the infero-lateral angle of the margin. The wall is flat, save for a slight concavity just behind the margin, and triangular in shape, with a free apex directed superiorly and medially and pointing towards the base of the mastoid process of the opposite side of the skull (see p. 40). It is formed chiefly by two bones, the greater wing (ala magna) of the sphenoidal in its posterior two-thirds and the upperhalf of the orbital surface of the zygomatic in its anterior third, but a small part of the frontal completes this wall above

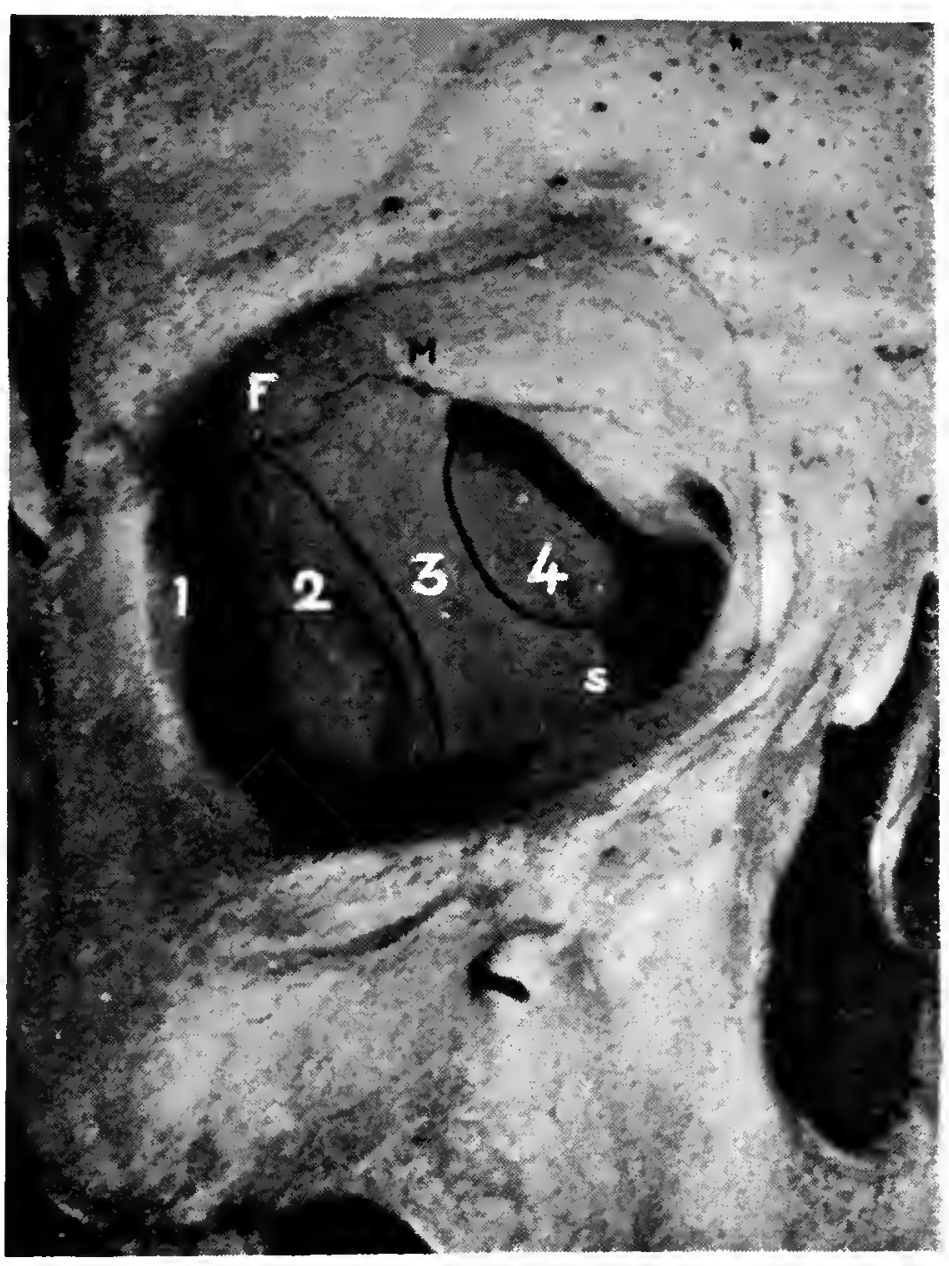

Fig. I 4.--Architecture of Lateral Wall of Right Orbit. The four numbers show areas which cliffer in strength and external relations (compare with the horizontal section of this wall shown in Fig. I 5). Natural size.

the zygomatic area. F.=Fossa for lacrimal gland. M.=Meningeal formen. The features to be noticed are-three sutures, the orbital tubercle, the spina recti lateralis, the openings of the zygomatic canals and the meningeal foramen, the architecture and relations.

The sutures are three in number, two placed horizontally and one vertically, and they meet at a point towards the upper limit of the wall not far from its centre. (i.) The zygomatico-frontal is a finely denticulated suture running backwards from the orbital margin and usually raised to form a ridge which marks the lower limit of the fossa for the lacrimal gland, as seen in Fig. Io. Nicola (I903) has 
studied the variations of these sutures and states that an obliquity from above downwards and from behind forwards of this one in particular is most often found in lower races. (ii.) The fronto-sphenoidal suture (anterior limb) continues the line of the zygomatico-frontal suture backwards to the anterior extremity of the superior orbital fissure. It divides the frontal bone from the greater wing of the sphenoidal, but is usually obliterated in the adult. (iii.) The sphenozygomatic suture runs up the lateral wall from the anterior end of the inferior orbital fissure to the point of junction of the above two sutures, meeting their line nearly at right angles; it lies between the orbital plate of the zygomatic bone and greater wing of the sphenoidal, and is often serrated and marked by small isolated ossicles analogous to the wormian bones of the cranium.

The orbital tubercle of the zygomatic bone has been described above on p. I3 with reference to the lateral orbital margin, close to which it is situated.

The spina recti lateralis (Merkel) is a small spur frequently found at or near the apex of the lateral wall, and projecting backwards towards the widest part of the superior orbital fissure opposite the optic foramen; it is slightly marked in the above figure, but is exceptionally well developed in Fig. I36, p. 26r. It may be rounded or spinous, and affords an attachment to the lateral rectus muscle. Below and in front of this spine there may occasionally be found a second small tubercle or rugosity possibly affording additional origin to the same muscle. W. Krause (I879) refers to these spines as spinae orbitales, superior and inferior, and regards the former as an extra origin of the lateral rectus and the latter as the origin of the orbital muscle of Müller.

The zygomatic canals.-On the orbital surface of the zygomatic bone there is usually to be seen the opening of a single canal (canalis zygomatico-orbitalis), which divides in the substance of the bone into two divergent branches, one of which opens on the facial aspect at the zygomatico-facial foramen, whilst the other ascends to open into the temporal fossa behind the frontal process at the zygomatico-temporal foramen. These canals contain the zygomatic or orbital 
nerve with its two terminal divisions, accompanied by branches of the infra-orbital artery. Though seldom absent, they are very irregular in size, number, and course. In one-third of 800 skulls Ledouble found two complete canals, and in rare cases three or four have been seen; one or more may end blindly within the bone and contain only nutrient vessels; there may be a single canal containing the facial branch of the nerve, the temporal branch of which, accompanied by a twig from the lacrimal artery, then runs up the surface of the wall, sometimes in a groove along the spheno-zygomatic suture, to reach the temporal fossa through a foramen in its. upper part (the spheno-zygomatic foramen, which in the adult may be wholly confined to the zygomatic bone). The lower edge of the greater wing of the sphenoidal bone may also be grooved by the main stem of the orbital nerve before it divides; the canals are formed in early development by an upgrowth of the zygomatic bone round the nerves (Fawcett), and indicate the line of fusion between the two chief ossific centres (Morris).

The meningeal foramen appears fairly constantly near the anterior end of the superior orbital fissure in or near the anterior fronto-sphenoidal suture. It transmits the orbital branch of the middle meningeal artery and is accompanied, according to Testut, by a vein. Examples are seen in Figs. 9, I3, I4.

The architecture. - The lateral is the strongest of the orbital walls, though it is by no means of uniform thickness. It is strong anteriorly, where the two processes of the frontal and zygomatic bones form the orbital margin; behind the margin the anterior half of the wall up to the sphenozygomatic suture is fairly thick, and separates the orbit from the temporal fossa. Posterior to the suture the wall is strengthened on its outer side by its confluence with the lateral wall of the cranium, and still further back the apex of the wall consists of a thin plate of bone which separates the orbit from the middle cranial fossa of the skull. Thus there are two strong regions alternating with two weak ones, and the lateral wall may be divided into four areas (Figs. 
I4, I5). The weaker temporal and cranial areas are often the site of senile atrophy, the wall being then exceedingly thin in these regions.

The relations of the Lateral Wall.-Internally the lateral

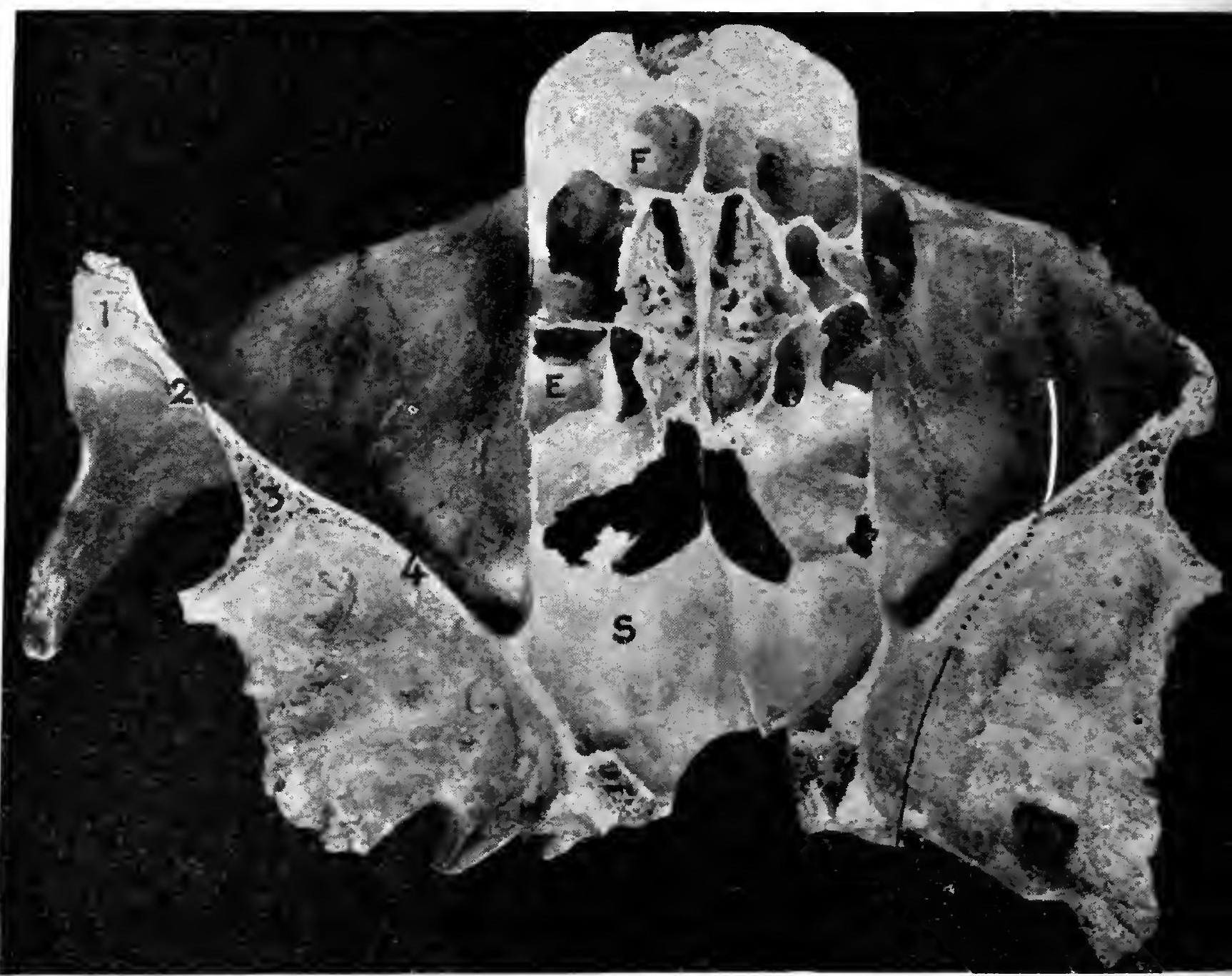

FIG. I 5. - Horizontal section through Orbits to illustrate architecture and relations of walls; lower half seen from above. The preparation shows the parallelism of the medial walls and their relation to the frontal sinus (F.) in iront (the infundibulum is seen on the right side), the ethmoidal cells (E.) in the middle, anr the sphenoidal sinus (S.) behind. Between the ethmoidal cells the cribriform plate is well displayed.

'The lateral walls show the difference of thickness in the four regions: (i.) thick at the orbital margin, (ii.) thin in relation to the ten poral fossa, (iii.) thick at the confluence with the lateral wall of the cranium, and (iv.) thin in relation to the niddle cranial fossa. The lateral wall forms an angle with the middle wall of approximately $45^{\circ}$, and with its fellow of the opposite side of $90^{\circ}$ (compare with Figs. 40 and 130 ).

The dotted line on the right side follows the curved course of the maxillary nerve through the foramen rotundum and its continuation as the infra-orbital nerve along the infra-orbital sulcus of the orbital floor. Natural size.

rectus muscle is in close contact with this wall from the spina recti lateralis posteriorly to the orbital tubercle anteriorly, where its "check ligament" is attached. The lacrimal artery and nerve run along the upper border of the muscle 
close to the wall. The relations of the zygomatic or orbital nerve and its two branches are indicated in the description of the zygomatic canals: there is an anastomotic loop between the lacrimal nerve and the zygomatico-temporal branch which is closely applied to the bone (Fig. 7I, p. I49). The lacrimal gland extends as low down as the fronto-zygomatic suture on the forepart of this wall, and is in close contact with it. Externally the lateral wall presents different relations in its anterior and posterior regions. The forepart of the wall or temporal area separates the orbit from the temporal fossa, in which lies the temporal muscle and the middle temporal artery with its zygomatico-orbital branch. It may be of interest to note that the orbital plate of the zygomatic bone which forms this partition is developed in higher primates only, and its double relation to the frontal and sphenoidal bones is a distinctive character of the primates as opposed. to all other mammals, since in the latter the orbit communicates openly with the temporal fossa (as indeed it does in the early human foetus). A more important relation is that of the posterior part of the wall behind the strong junction which it forms with the cranium. Here the orbit is separated from the fore end of the middle cranial fossa in which is lodged the temporal lobe of the brain, the apex of the wall lying against its middle temporal gyrus. These relations of the lateral wall to the soft parts are well displayed in Fig. I30, p. 252. The distance of this wall from the skin surface is about I5 $\mathrm{mm}$. just behind the margin, and from 45 to $50 \mathrm{~mm}$., or nearly two inches, at its posterior free end, as measured in the preparation just referred to.

The superior orbital fissure (sphenoidal fissure; fissura orbitalis superior; foramen lacerum anterius) may most fittingly be described here since it separates the roof and lateral walls in their posterior thirds. It is the gap between the greater and lesser wings of the sphenoidal bone and is closed at its anterior extremity by the frontal bone. It consists of two segments : a narrow lateral $\operatorname{limb}$ lying between the lateral wall and roof of the orbit, and a 
medial broader part lying between the apex of the lateral wall and the bridge of bone which bounds the optic foramen. Below the lower extremity of the fissure is the foramen rotundum, a relation clearly seen on looking from inside a sectioned skull but not so obvious on looking into the orbit from in front, since at this point the superior orbital fissure becomes confluent with the inferior and the foramen lies deep to their junction. The shape varies greatly in individuals, being dependent upon the formation of its anterior boundary. It is said to be longer in dolichocephalic than in brachycephalic skulls. It may be asymmetrical in the two orbits and is larger in the aged through absorption of the edge of the greater wing of the sphenoid. Gallemaerts (I897) summarises three types in I6o skulls of various races: (a) The commonest type, with two segments, one narrow, the other broad, as described above; and as seen in Fig. I 4 ; (b) a type with both segments broad; and (c) a triangular type, as in Fig. II. The lateral segment is said to be smaller in negroes, and in two skulls of this type the fissure was found reduced to a round hole, as in the gorilla and gibbon. He finds the length of the fissure to be most often $\mathrm{I} 5$ or $\mathrm{I} 6 \mathrm{~mm}$. (Panas and also Krause give a mean of $22 \mathrm{~mm}$.), and that of its lateral segment 9 or Io $\mathrm{mm}$. ; the distance of its fore end from the orbital margin was found by the writer to vary from I2 to $I 8 \mathrm{~mm}$., with an average of $15.5 \mathrm{~mm}$. in ro male skulls, and from I2 to I9 $\mathrm{mm}$. with an average of $\mathrm{I}_{4} \cdot 3$ in Io female skulls. It puts the orbit in communication with the middle cranial fossa, and on looking at the orbit from in front there can be seen through the widest part of the fissure the depression on the apex of the petrous bone which lodges the semilunar: or Gasserian ganglion of the fifth nerve.

The following structures pass through the superior orbital fissure :

(i.) The third, fourth, and sixth cranial nerves.

(ii.) The ophthalmic division of the fifth nerve in three branches: nasal, frontal, and lacrimal.

(iii.) The ophthalmic vein.

(iv.) The orbital branch of the middle meningeal artery 
(when it does not pass through the meningeal foramen).

(v.) The sympathetic root of the ciliary ganglion, and some filaments from the cavernous plexus of the sympathetic system.

The central part of the fissure is crossed by the " annulus of Zinn," from which arise the recti muscles of the eyeball, and the position of the above nerves relative to it is shown diagrammatically in Fig. I35, p. 260. The importance of the superior orbital fissure is to be emphasised since, with the exception of the optic and maxillary, there pass through it all the nerves in the orbit-nerves which control the movements of the eyeball, the pupil, the accommodation of the lens, and the levator palpebrae superioris muscle, and nerves conveying sensation from the cornea, iris, chorioid, conjunctiva, lacrimal apparatus, frontal region, upper eyelid, and forepart of nose.

The inferior orbital fissure (spheno-maxillary fissure, fissura orbitalis' inferior) lies along the infero-lateral angle of the orbit, and separates the lateral wall from the floor in their posterior two-thirds. It is bounded above by the inferior margin of the greater wing of the sphenoid, and below by the lateral margins of the orbital surface of the palatine bone behind and that of the maxilla in front. Posteriorly, the foramen rotundum opens into it, and just above this point it is continuous with the lower end of the superior orbital fissure. It is narrower in the centre than at the extremities, the constriction being sometimes emphasised by a tongue-like process of the floor of the orbit hollowed out by an extension of the maxillary sinus or a posterior ethmoidal cell (Fig. 2I, p. 50) ; its anterior end is the wider, and is completed by the zygomatic bone in 60 to 65 per cent of cases (Ledouble), this bone being otherwise excluded by the junction of the sphenoidal bone with the maxilla.

The fissure is about $20 \mathrm{~mm}$. long, extending further forwards than does the superior fissure, and reaching to within 15 to $20 \mathrm{~mm}$. or $\frac{3}{4}$ inch of the orbital margin. It lies on a level with the upper edge of the zygoma, is directed 
forwards and outwards from the apex of the orbit, and its long axis is in line with the zygomatic tuberosity on the cheek in one direction, the optic foramen in the other. This line corresponds with the long axis of the optic canal, and beyond this can be followed in a sectioned skull along the superior petrosal groove of the opposite side to a surface point at the centre of the base of the mastoid process; the line also indicates the inclination of the lateral wall of the orbit.

By the inferior orbital fissure communication is established with the pterygo-palatine (spheno-maxillary) fossa behind and the infra-temporal (zygomatic) fossa in front. In life it is closed by the periorbita, here containing the involuntary orbitalis muscle (Fig. 26). Along its posterior part pass the maxillary nerve and the infra-orbital artery on their way to the infra-orbital sulcus on the floor of the orbit; it also transmits the zygomatic or orbital nerve, a few twigs from the spheno-palatine ganglion, and vessels passing from the inferior ophthalmic vein to the pterygoid plexus. According to Treves, after violent blows upon the temple, blood may enter the orbit through this fissure and cause a sub-conjunctival ecchymosis; on the other hand, the writer has found a small hernia of the orbital fat protruding through it into the infra-temporal fossa.

In man and the higher mammals this fissure represents the wide communication between the orbit and the temporal fossa found in lower vertebrates; it is relatively larger in the skull at birth, as seen in Fig. 43, p. 96, and also in the aged from absorption of its bony margins; either from this cause or from mal-development, its anterior end may be abnormally large and encroach upon the lateral wall of the orbit, as in an Australian skull illustrated by Duckworth (I904) ; in rare instances a " spheno-zygomatic fissure" may thus be formed along the line of the corresponding suture.

(iii.) The Floop of the Orbit (maxillary or inferior wall, paries inferior).-The floor is limited on the lateral side by the inferior orbital fissure with a line prolonged from its anterior extremity to the orbital margin ; medially it curves 
smoothly into the medial wall, but may be demarked from it by extending the lower border of the ethmoidal bone, a line marked from before backwards by the edge of the naso-lacrimal canal, and the sutures between the lacrimal and maxilla, ethmoidal and maxilla, and sphenoidal and palatine bones. It is the shortest wall, extending only two-thirds of the depth of the orbit; the outline is that of an equilateral triangle, and the surface is slightly concave anteriorly and convex posteriorly, owing to the upward expansion of the maxillary sinus. It is formed in greater part by the orbital plate (facies orbitalis) of the maxilla, completed antero-laterally by part of the orbital surface of the zygomaticand posteriorly by a small triangular area . (the processus orbitalis) of the palatine bone (Fig. 9). Of the sutures which unite these bones, that between the maxilla and the palatine bone is usually obliterated in the adult skull, and in con-

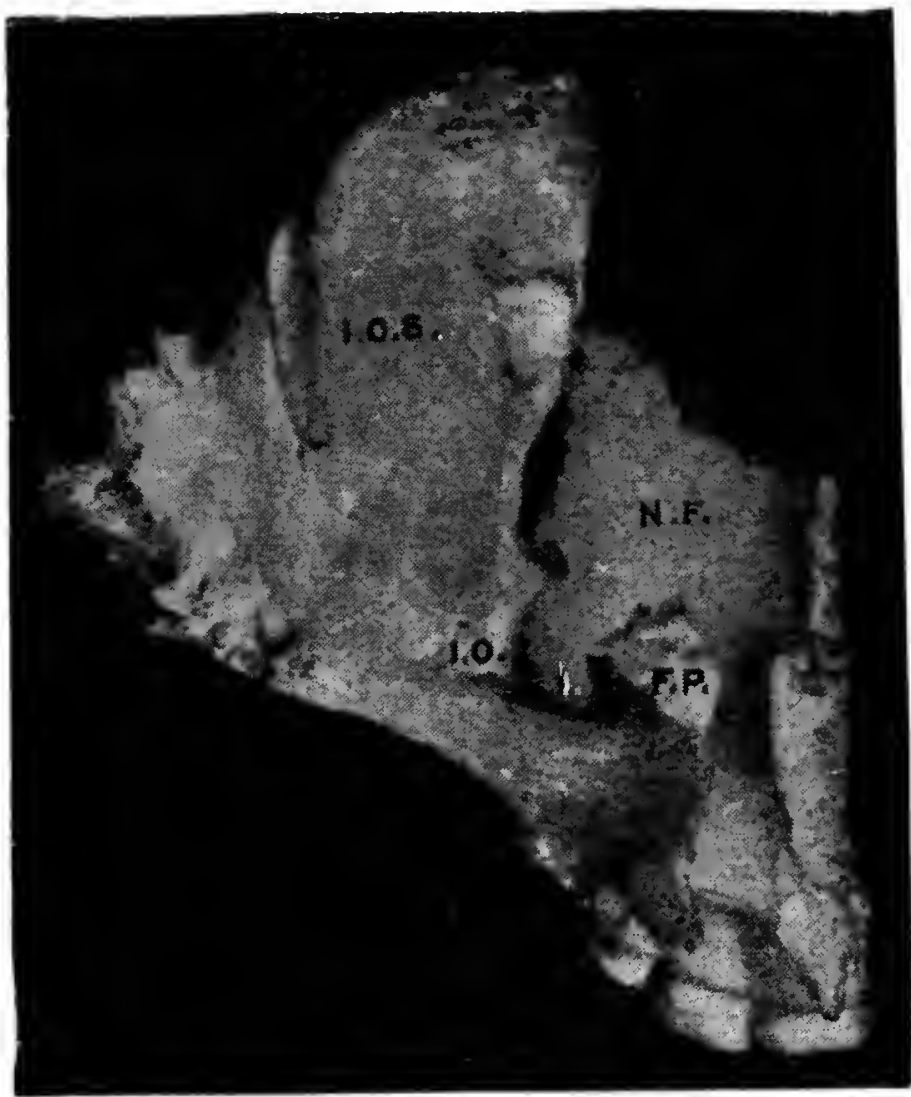

F1G. I6. - Right Maxilla seen from above to illustrate features on floor of Orbit. Natural size.
I.O.S. $=$ Infra-orbital sulcus.
I. O. = Fossa of origin of inferior oblique muscle.
I. = Incisura lacrimalis of maxilla, forming part of superior orifice of naso-lacrimal canal. F.P. $=$ Frontal process of maxilla, pointing up towards one. N.F. $=$ Floor of nasal fossa. sequence the palatinal area of the floor is hard to define. The suture between the maxilla and zygomatic bone appears both on the orbital floor and on the cheek, and is $V$-shaped, with its apex at the middle of the infra-orbital margin; the orbital limb is inclined forwards and medially from the fore end of the inferior orbital fissure, running wide of the infra-orbital sulcus, to cut the margin at its mid-point, where it has already been noticed (p. I4); the facial limb slopes from this point in 
the opposite direction, backwards and then abruptly downwards to cut the lower border of the zygomatic arch at the fore end of the infra-temporal fossa (Fig. 5, p. 8). The features to be noted on the floor are the infra-orbital sulcus and canal, the fossa of origin of the inferior oblique muscle, and the architecture and relations.

The infra-orbital sulcus or groove (sulcus infraorbitalis) is a shallow gutter running nearly the whole length of the floor from the lateral side of its apex towards the centre of the margin. Its anterior half or two-thirds is converted into a canal (canalis infraorbitalis), the roofing over being caused in greater part by an extension of its lateral wall in the form of a very thin plate (the lamina infraorbitalis of Henle), as seen in Fig. I7; this union of the walls is marked along the supero-medial angle of the roof of the canal by a fine line, the sutura canalis infraorbitalis longitudinalis, which is continuous with the sutura infraorbitalis verticalis of the orbital margin. Anteriorly the canal sinks down beneath the margin, to emerge on the face at the infraorbital foramen. The sulcus and canal lie entirely in the maxilla, and indicate the line of confluence between the maxillary and malar centres of the bone (Morris, I898). The sulcus runs parallel to the mesial plane of the skull and not to the long axis of the orbital floor, which latter has an antero-lateral inclination, owing to the development of the underlying maxillary sinus (Fig. I5). The total length of both sulcus and canal is about $20 \mathrm{~mm}$., with an average width of $4 \mathrm{~mm}$. and a depth of $\mathrm{x} \cdot 5$ to $2 \mathrm{~mm}$. From the lateral aspect arise the superior alveolar (dental) canals, the middle leaving the sulcus far back and running to the bicuspid teeth, whilst the anterior (usually double) leads out of the canal not far from its facial opening, and is continued on to the canine and incisor teeth (p. I6).

The contents are the infra-orbital nerve, the infra-orbital artery, and rarely (Stanculeanu, I902) a vein. Czermak (I895), in an atlas of sections through the frozen orbit, figures the artery lateral to the nerve and the vein medial to it, but in a few cases examined by the writer the artery lay above and medial to the nerve, and no vein was found. 
The sulcus and canal are lined, and the former with its contents is also covered, by the periorbita; the infra-orbital nerve therefore does not lie freely within the periorbital boundary, as does the supra-orbital nerve.

The variations are explained by reference to the mode of development. In the embryo the infra-orbital nerve lies at first above the orbital surface of the maxilla, and passes freely over the orbital margin; it comes in contact with them towards the end of the second month, forming a groove on the bone, the walls of which finally close over it anteriorly to form the canal (Fawcett, IgII). The sutura infraorbitalis, with its longitudinal (orbital) and vertical (marginal) portions, marks the line of junction of this overlap. Later on the nerve cannot accommodate itself quickly enough to the more rapidly growing underlying maxillary sinus, and sinks into its roof; the groove is curved laterally by this expansion, instead of lying in a straight line between the foramen rotundum and infra-orbital foramen as in the skull at birth. In rare cases the anterior part of the sulcus is not ultimately transformed into a canal, but persists as an open groove passing over the infra-orbital margin on to the face, as seen in three adult skulls by von Langer (Merkel) ; on the other hand, the degree of ossification of the periorbital roof of the sulcus determines the length of the canal, which may occasionally be found to extend backwards the whole length of the floor of the orbit. An early or intraorbital division of the nerve into its terminal branches accounts for the presence of branching or supernumerary infra-orbital canals; a medial canal containing the nasal and palpebral branches was found in eighteen, and a lateral one containing the labial division of the nerve in twelve out of 2700 skulls by Ledouble; other variations in number will be found in conformity with those of the infra-orbital foramen (p. I5); finally, Ledouble has seen the canal duplicated in its whole extent like a doublebarrelled gun, an anomaly caused by ossification of the fibrous tissue which separates the nerve from the artery.

The fossa of origin of the inferior oblique muscle may occasionally be seen, but is usually better felt. It is a 
very shallow depression, situated in the extreme anteromedial angle of the floor just behind the margin and lateral to the orifice of the naso-lacrimal canal (see also p. 284). This orifice is sometimes described as a feature of the floor of the orbit, but will be dealt with under a later section in conjunction. with the osseous lacrimal passages. (pp. 70, 74). Its lateral margin, formed by the incisura lacrimalis of the maxilla, notches the antero-medial angle of the floor, and is seen in Fig. I6 above.

The architecture.-The floor is always composed of a thin ( $\frac{1}{2}$ to $I \mathrm{~mm}$.) plate of bone, though it is stronger just behind the margin. Its thinnest part is along the floor of the infra-orbital sulcus and canal, and here dehiscences may occur, whereby the contents are only separated from the mucous membrane lining of the maxillary sinus by the periorbita; gaps are also to be seen in rare cases along the medial margin of the orbital floor between the maxilla and the ethmoidal bone. The zygomatic portion of the floor sometimes contains small air-cells or a cavity (sinus zygomaticus), which may or may not communicate with the maxillary sinus (Ledouble). A small vein has been noted by Gaillard to pass through the floor from the maxillary sinus to the orbital venous system (not found by Stanculeanu).

The relations of the floor: internally, the periorbita both lines and roofs over the infra-orbital sulcus, so enclosing the infra-orbital nerve and artery; on the lateral side the orbitalis muscle is incorporated with it (Fig. 37, p. 82). The inferior oblique muscle arises from and lies across the floor anteriorly; the inferior rectus muscle is in close contact with it behind at the apex, but is separated from it in front by the inferior oblique muscle and a mass of orbital fat. Externally, that is inferiorly, the floor is related in nearly the whole of its extent to the maxillary sinus, as seen in the next figure and also in Fig. I8 ; behind, at the apex, the orbital process of the palatine bone may be hollowed out by an air-cell, as is well shown in Fig. I8. Abnormal posterior ethmoidal cells in rare instances undermine the maxillary part of the floor along its posteromedial border. 


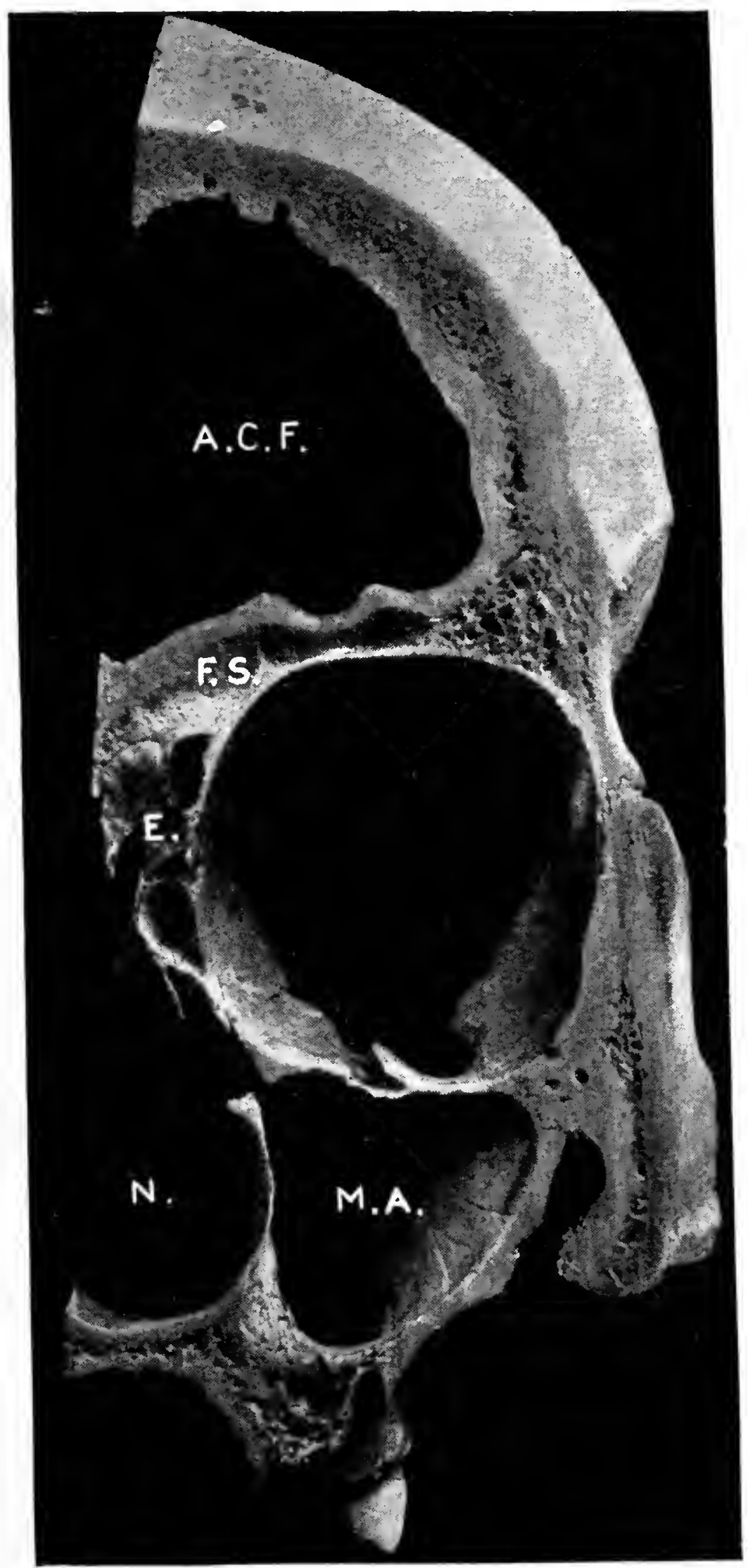

Ftg. 17. - Frontal Section of Left Half of skull just behind Orbital Margin and passing through largest part of cavity (greatest orbital height $=37.5 \mathrm{~mm}$.); seen from in front. Natural size. The frontal section removed is seen in Fig. 5 .

The floor of the orbit is cleeply grooved by the infra-orbital sulcus.
A.C.F. $=$ Anterior cranial fossa.
F.S. $=$ Frontal sinus.
N. = Nasal cavity.
E. $=$ Ethmoidal air-cells.
M.A. = Naxillary sinus (showing the ostium maxillare).


(iv.) The Medial Wall (inner, nasal, or ethmoidal wall; paries medialis).-This is the smallest of the orbital boundaries. Its limits lie broadly between lines parallel with the upper and lower borders of the ethmoidal bone. It is marked off from the roof by the fronto-ethmoidal

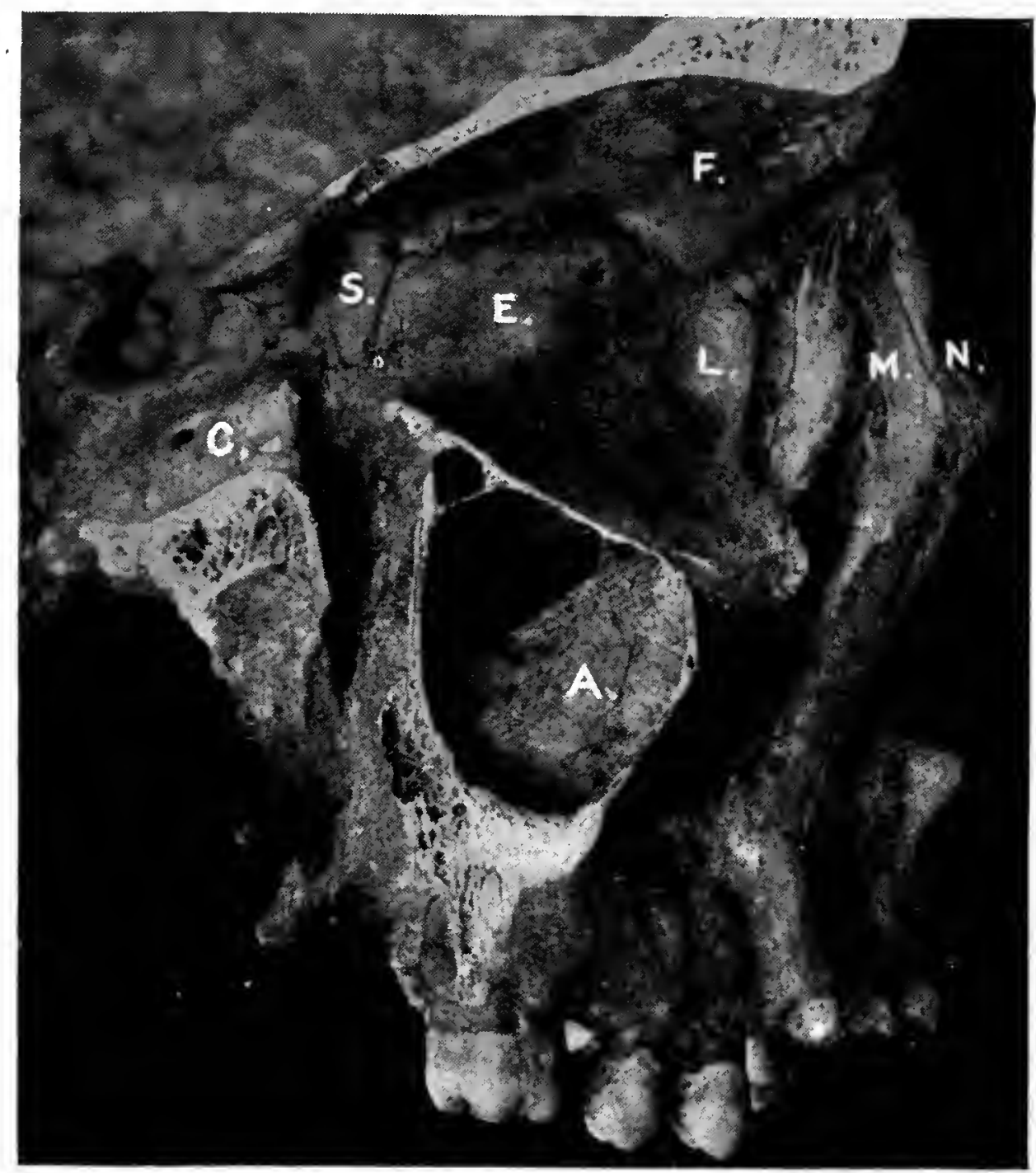

FIG. I 8. - Sagittal section through Right Orbit to display the Medial Wall. Natural size.

$\mathrm{S} .=$ Sphenoidal bone, and is placed just in front of the optic foramen.

E. = Ethmoidal bone; L. = Lacrimal bone; M.= Frontal process of maxilla. Between L. and $M$. is the fossa for the lacrimal sac, bounded behind by the sharp posterior lacrimal crest, in front by the blunt anterior lacrimal crest.

N. = Nasal bone $; \mathbf{F} .=$ Frontal bone.

A. = Maxillary antrum opened into, and at its postero-superior angle the palatinal air-cell is seen.

C. = The position of the cavernous sinus at the side of the body of the sphenoidal bone; above it lies the sella turcica; the lower limb of the letter $\mathrm{C}$ is pointing towards the foramen rotundum, through which the section passes.

suture prolonged forwards towards the point nasion at the root of the nose, and from the floor by the line of sutures already referred to ; anteriorly it is bounded by the posterior lacrimal crest of the lacrimal bone. The surface is convex about the centre, and concave in front in harmony with the 
contour of the eyeball, though these curves are but slightly marked ; it lies almost parallel to the mesial plane and to its fellow of the opposite side, as shown in Fig. I5, but curves into the floor below. It is formed by four bones, which are from before backwards: (I) the lower part of the medial angular process of the frontal lying above (2) the lacrimal bone, (3) the orbital plate (lamina papyracea or os planum) of the ethmoidal, and (4) a small part of the lateral aspect of the body of the sphenoidal bone. The individual bones take part in the formation of this wall to a variable degree, but the largest and most important is the lamina papyracea of the ethmoidal ; it is oblong in shape, and articulates with the sphenoidal bone behind by the spheno-ethmoidal suture and with the lacrimal in front by the ethmo-lacrimal suture. These sutures are both vertically placed, but, like the bones; they are subject to much variation. Posteriorly the palatine bone commonly extends upwards on to this wall; sometimes a descending process from the frontal meets it and completely separates the ethmoidal from the sphenoidal bone. In this region also a part of the sphenoidal turbinated bone has been seen. Anteriorly the ethmoidal surface may be separated from the lacrimal by prolongations of the frontal and maxilla, an "orbito-maxillo-frontal suture" being formed by their union (A. Thomson, I890). Small ethmo-lacrimal ossicles may occupy the upper or lower ends of the ethmo-lacrimal suture (Macalister, I884). The lamina papyracea may be subdivided into two or more pieces by additional vertically placed sutures, a condition found by Bianchi (quoted by Ledouble) to occur more frequently in the skulls of imbeciles. Poirier (IgI2) figures the very rare " orbital Wormian bone" behind the ethmoidal and between the palatine and sphenoidal bones. It may be noted that in mammals below the primates the ethmoidal bone does not enter into the formation of the orbit, its place being taken by the frontal.

The lateral or orbital surface of the lacrimal bone is divided into two parts by a vertical ridge, the posterior lacrimal crest (crista lacrimalis posterior); the bone behind the crest is flat and forms the forepart of the orbital wall, 
- the area in front is excavated by a gutter, the sulcus lacrimalis, which joins a similar groove on the frontal process of the maxilla to form the fossa for the lacrimal sac. The lower end of the posterior lacrimal crest usually terminates in a forwardly directed and hook-like process, the hamulus lacrimalis. This. process articulates with the lacrimal notch of the maxilla, and defines the opening of the naso-

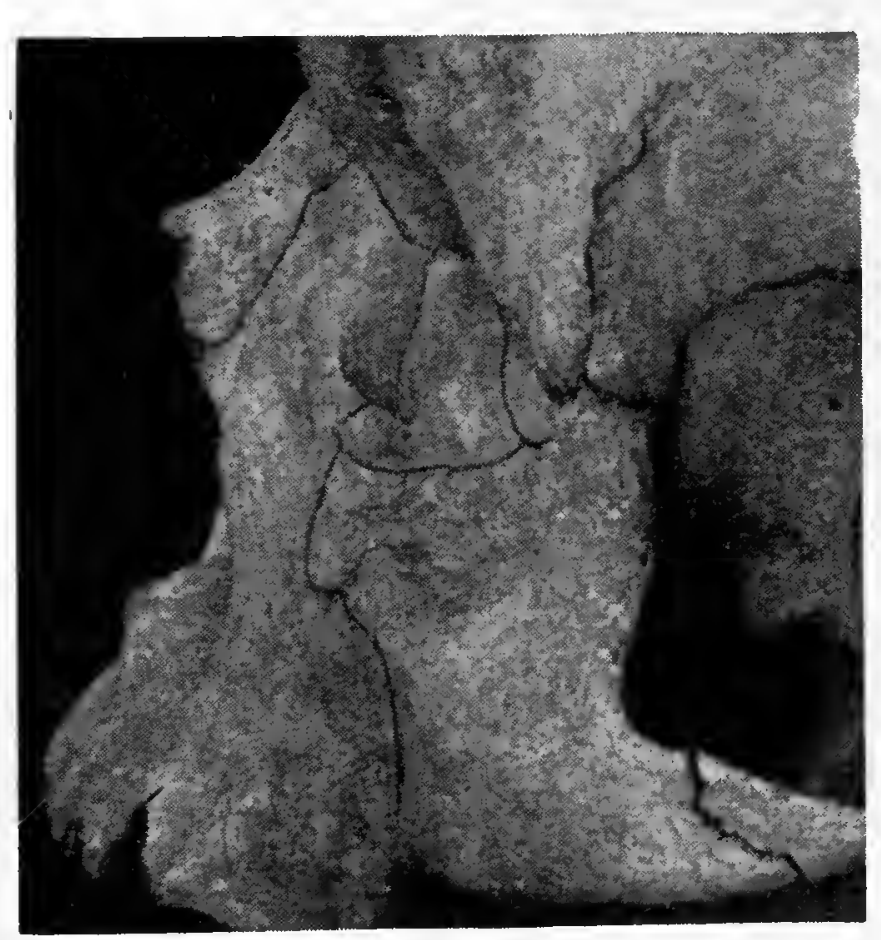

FIG. I9. - Well-developed Lacrimal Bone with a large hamular process extending forwards on to the orbital margin as the pars facialis. The naso-lacrimal canal in this specimen is of large calibre $(7 \mathrm{~mm} . \times 5.5$ nm.). Peruvian Indian skull. $\times \frac{1}{2}$. Compare with Fig. 20. lacrimal canal.

Much variation is found in this hamular process : it is more often present in males than in females, and may either form a short spur, as in most cases, or reach the lacrimal tubercle of the anterior crest, or even extend on to the facial aspect of the maxilla as the pars facialis (Gegenbaur, I88I), a condition which exists in lower mammals and primates generally, and was found in 4.25 per cent of IOOO human skulls by Macalister. In rare instances it forms a separate ossicle (the "os hamulus" or lesser lacrimal bone), as found in $\mathrm{I}_{2}^{\frac{1}{2}}$ per cent of subjects by Macalister and Thomson, or it may be separated from the adjacent floor of the orbit by an ossicle (the ossiculum canalis naso-lacrimalis of Grüber, I877). When the hamulus is well developed, the level of the upper opening of the naso-lacrimal canal is raised accordingly. It may be notched or pierced by a branch of infra-orbital artery on its passage to the lacrimal sac, as seen in the above figure.

Numerous variations are to be found in the lacrimal bone, which is evidently undergoing retrogressive changes in man. Either half in front or behind its crest may be undeveloped, the deficiency being made up in the one case by the frontal 
process of the maxilla, as seen in Fig. 20, and in the other by an extension of the lamina papyracea, though this condition is probably due to an early union of the bones, such fusion with adjacent bones being often found, especially with the frontal process of the maxilla. It may be absent altogether ( $\mathrm{I} \cdot 2$ per cent of 202I skulls of all races-Ledouble). On the other hand, as just noted, it may develop a hamular process, extending forwards on to the orbital margin.

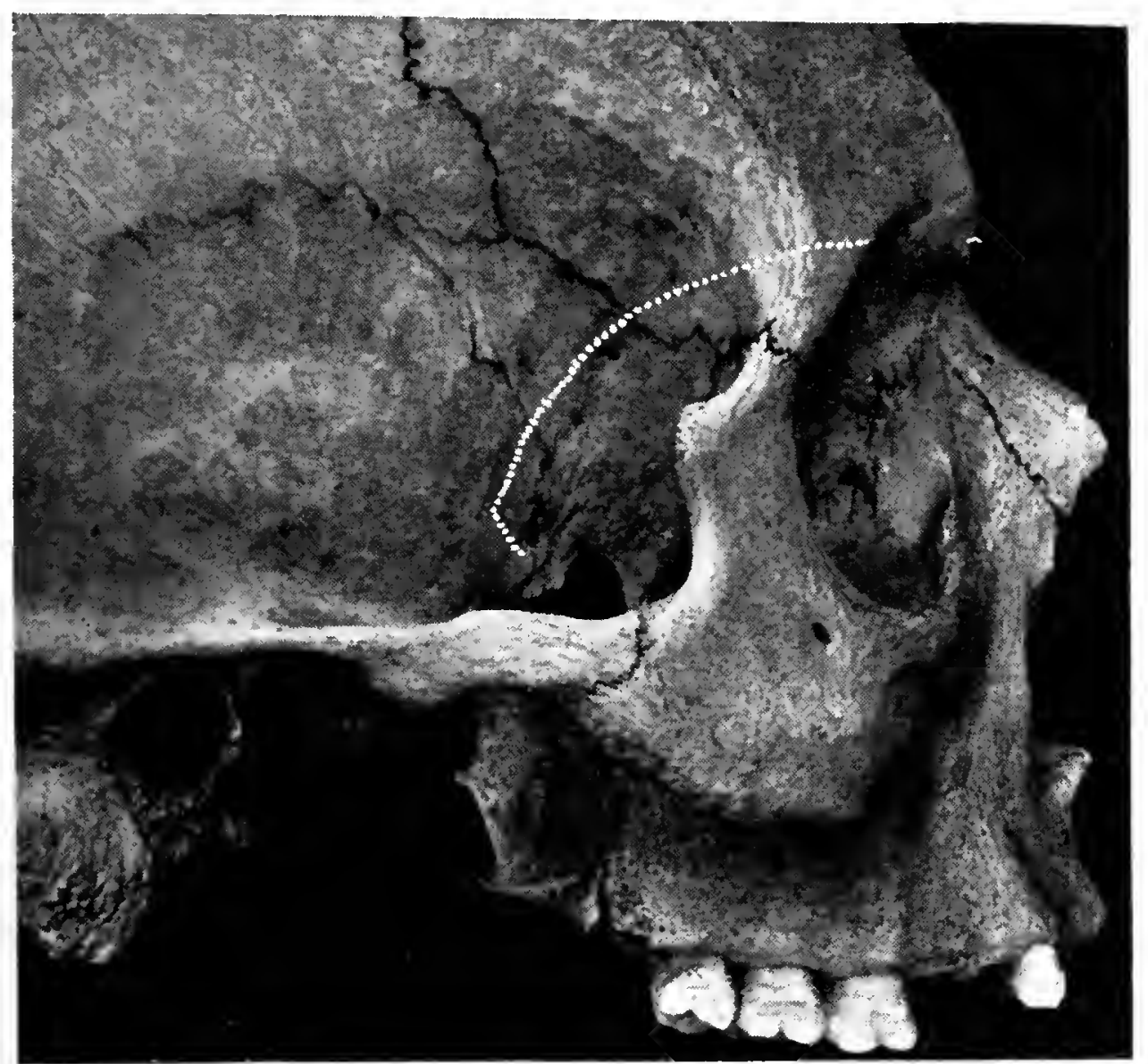

Fig. 2o. - Absence of Lacrimal Bone, the whole fossa for the lacrimal sac being formed by the maxilla. The outline of the orbit is dotted in. 'The lateral margin presents a well-developed processus marginalis on its temporal border. $\times \frac{1}{2}$.

Occasionally the bone is bipartite, indicating an accessory centre of ossification, and finally "peri-lacrimal ossicles" sometimes occur in relation with it behind, in front, and below, as many as twelve varieties having been described by Flecker (I9I3), though only one of them, representing a separated hamulus, appears to be derived from the lacrimal bone itself.

The lacrimal is the most fragile of all the bones, and is usually perforate owing to incomplete ossification or to absorption in old age; it is fenestrated in the foetus 
(Macalister, I884), and Merkel considers a complete bone to be an anatomical curiosity. Its strongest part is along the line of the posterior lacrimal crest.

The features of this wall to be noticed are the ethmoidal foramina, the architecture, and relations. It will be convenient to describe later in a separate section

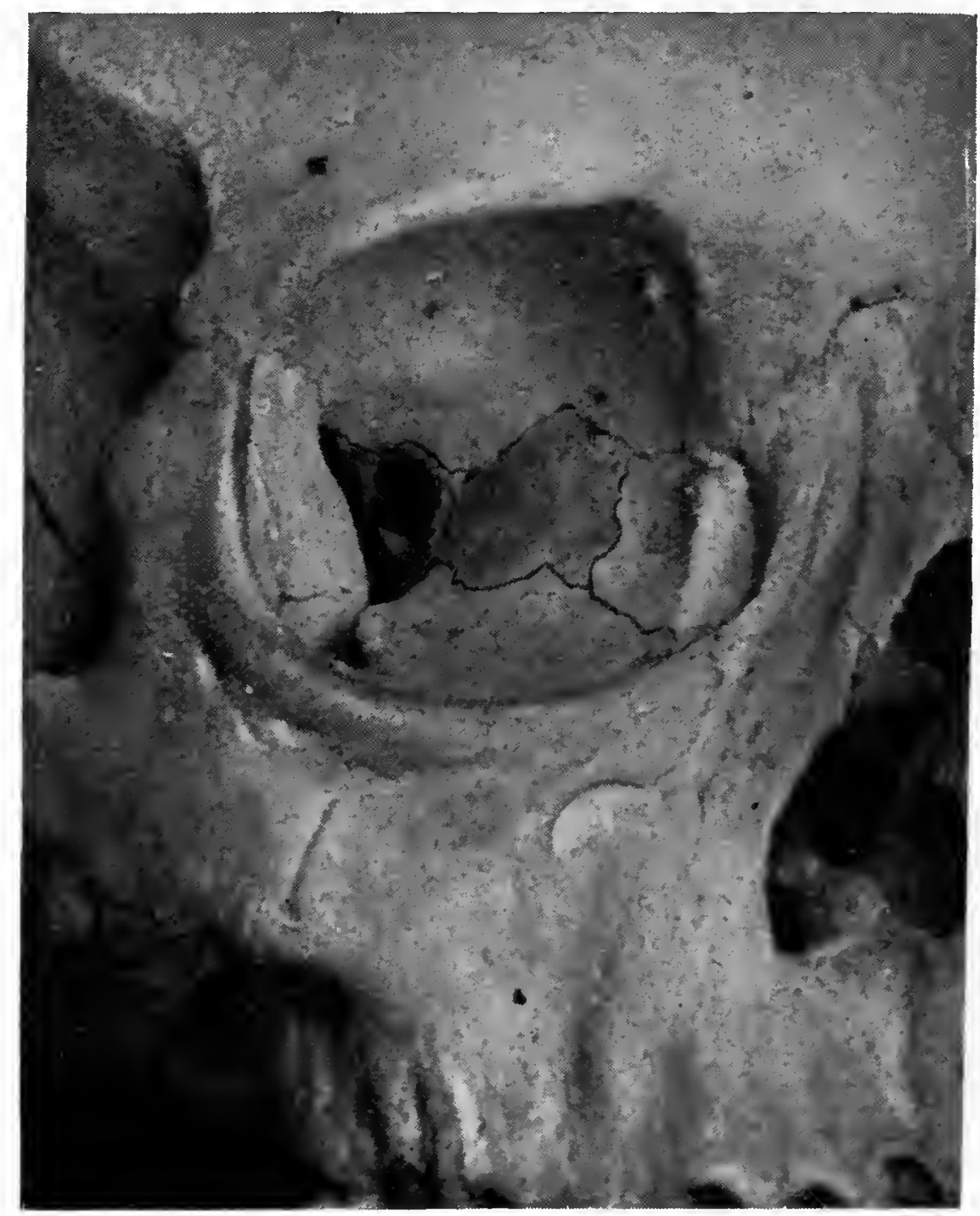

FIG. 2r.-Right Orbit to show features of Medial Wall. The specimen also illustrates a doubled optic foramen, a spina trochlearis, the tubercle of attachment of the pars lacrimalis (Horner's) muscle at upper end of posterior lacrimal crest, and the projection of orbital floor over posterior extremity of inferior orbital fissure. Double zygomatico-facial foramina are also present, and the curved edge of the infra-orbital foramen is well marked. Natural size.

the fossa for the lacrimal sac (p. 64), which is sometimes considered as a feature of this wall.

The ethmoidal foramina (internal orbital foramina, foramen ethmoidale anterius vel posterius).-These small oval orifices lie in the angle between the roof and the medial wall of the orbit, along the line of the fronto-ethmoidal suture. They are two in number, distinguished as anterior 
and posterior, and lead into corresponding canals, which are formed above and in greater part by the frontal bone (and so would more appropriately be termed frontoethmoidal canals), as seen in Fig. 27. They are directed anteriorly and medially, and open into the middle cranial fossa just above and at the sides of the lamina cribrosa of the ethmoidal bone; in rare instances the posterior foramen is duplicated, or there may be a third foramen intermediate in position, and even four have been noted.

The anterior ethmoidal canal is the larger of the two, and slopes more forward. Its orifice lies $20 \mathrm{~mm}$. from the orbital margin and $30 \mathrm{~mm}$. behind the point nasion (on an average of twenty skulls measured by the writer). It contains the naso-ciliary nerve (here called the anterior ethmoidal) and the anterior ethmoidal artery.

The posterior ethmoidal canal is usually placed from Io to $\mathrm{I} 5 \mathrm{~mm}$. behind the anterior one, sometimes at a slightly lower level and in the spheno-ethmoidal suture. Through it pass the posterior ethmoidal artery, and occasionally a nerve filament (the nervus spheno-ethmoidalis of Luschka) derived from the naso-ciliary nerve. Both canals are lined by the periorbita, and in addition possibly transmit lymphatic vessels.

The architecture. - The medial wall, save for the small comparatively thick sphenoidal area, is the thinnest of the orbital boundaries; the orbital plate of the ethmoidal bone (os planum) is of such paper-like delicacy (as is well seen in Fig. I5) that it is termed the lamina papyracea, but in spite of its thinness $(0.2$ to $0.4 \mathrm{~mm}$.), which may allow of the outlines of the divisions between the underlying air-cells to be seen through it, the bone is tough, and is very rarely the site of senile atrophy. Congenital dehiscences, however, along the ethmo-maxillary suture, or at the site of the orbital Wormian bone, have been noticed by several authors; Zuckerkandl found fifteen cases and Onodi (I9I3) saw eighteen, three of which.communicated with the frontal sinuses as well as with the ethmoidal cells.

With regard to the dehiscences found in various parts of the orbital walls, it may be noted that when due to absorption or atrophy of the bone in old age they are 
usually situated in the middle of a bony plate, and the edges are irregular, whereas when due to lack of development they lie between the sutures, and the margin is smooth and regular in outline. Congenital dehiscences are found specially in those parts of the orbital walls which are developed by ossification of the primitive cartilaginous nasal capsule, namely, the lamina papyracea and the orbital wall of the maxilla (Bull). Dehiscences of a third kind are pathological, and may also be seen on the medial wall, where they are caused by extension of disease from the ethmoidal cells. An instance is seen in Fig. II8, p. 228.

"The relations of the medial wall. - Internally, the lining periorbita is continuous through the ethmoidal canals with the dura mater of the anterior cranial fossa. The medial rectus muscle is in close contact with the greater part of this wall, and just behind the posterior lacrimal crest is the attachment of its " check ligament," and also of the pars lacrimalis (Horner's muscle) of the orbicularis oculi muscle; against the anterior half of the upper part of the wall and lying in the interval between the adjacent borders of the medial rectus and superior oblique muscles are the naso-ciliary nerve and ophthalmic artery. Externally, the medial wall is the most important from a practical point of view, owing to the fact that it is related in its whole extent to the accessory air-sinuses of the nose, from which disease may invade the orbit; posteriorly a small area is in contact with the sphenoidal sinus, but in the rest of its extent this wall is in direct relation to the ethmoidal cells, which underlie not only the lamina papyracea but the lacrimal bone as well, and may even encroach backwards upon the sphenoidal region. By these aircells the orbital cavity is' separated on its medial aspect from the nasal cavity. These relations are well displayed in Figs. 3, I5, I7, 29, 37, and especially I30, p. 252.

It is fitting at this point to review the accessory sinuses of the nose as a whole before describing the lacrimal fossa and naso-lacrimal duct, to which, in common with the orbital walls, they present such important relations. 


\section{THE ACCESSORY AIR-SINUSES OF THE NOSE IN THEIR RELATION TO THE ORBIT}

From the above account of the relation of the walls, the orbit is seen to be surrounded by air-sinuses (simus paranasales) on three sides, the lateral wall alone being exempt. The roof in its medial and anterior region is usually related to the frontal sinus. The floor is always undermined by the maxillary sinus, behind which there may be present the small palatinal air-cell. The medial wall is generally related to the sphenoidal sinus and always to the ethmoidal cells, which latter may extend over the roof, or beneath the medial edge of the floor, or replace the sphenoidal sinus in position.

The massing of these air-sinuses round the orbit is illustrated in the above-enumerated figures, and also in Fig. I84, p. 364. They may be divided into two groups: an anterior, comprising the maxillary and frontal sinuses together with the bulk of the ethmoidal cells, and related to the fore part of the orbit wherein is lodged the globe of the eye, and a posterior group, consisting of the posterior ethmoidal, the palatinal cell, and the sphenoidal sinus, which are related more closely to the optic nerve. The openings of these sinuses into the nasal cavity are arranged around the middle concha, those of the anterior group lying below its line of origin, those of the posterior group above it (Logan Turner, I9o8); on the orbital aspect the two groups may approximately be demarked by a line running downwards and backwards from the anterior ethmoidal foramen to the floor of the orbit (Ranglaret, I896). All have in their venous system branches which pass into the orbit, and thence through the ophthalmic veins to the cavernous blood sinus; some of their lymph channels possibly take the same course, but this is not certain.

These air-spaces are all derived as outgrowths of the primitive nasal cavities; they appear in early childhood, increase most actively in size at puberty, and continue to grow until about the age of thirty, with a slight increase in 
old age through atrophy of their bony walls. They retain communication with the nasal cavity and are lined by a direct continuation of its mucous membrane, here blended so closely with the periosteum as to form a "muco-periosteum," a fact which explains how readily disease can

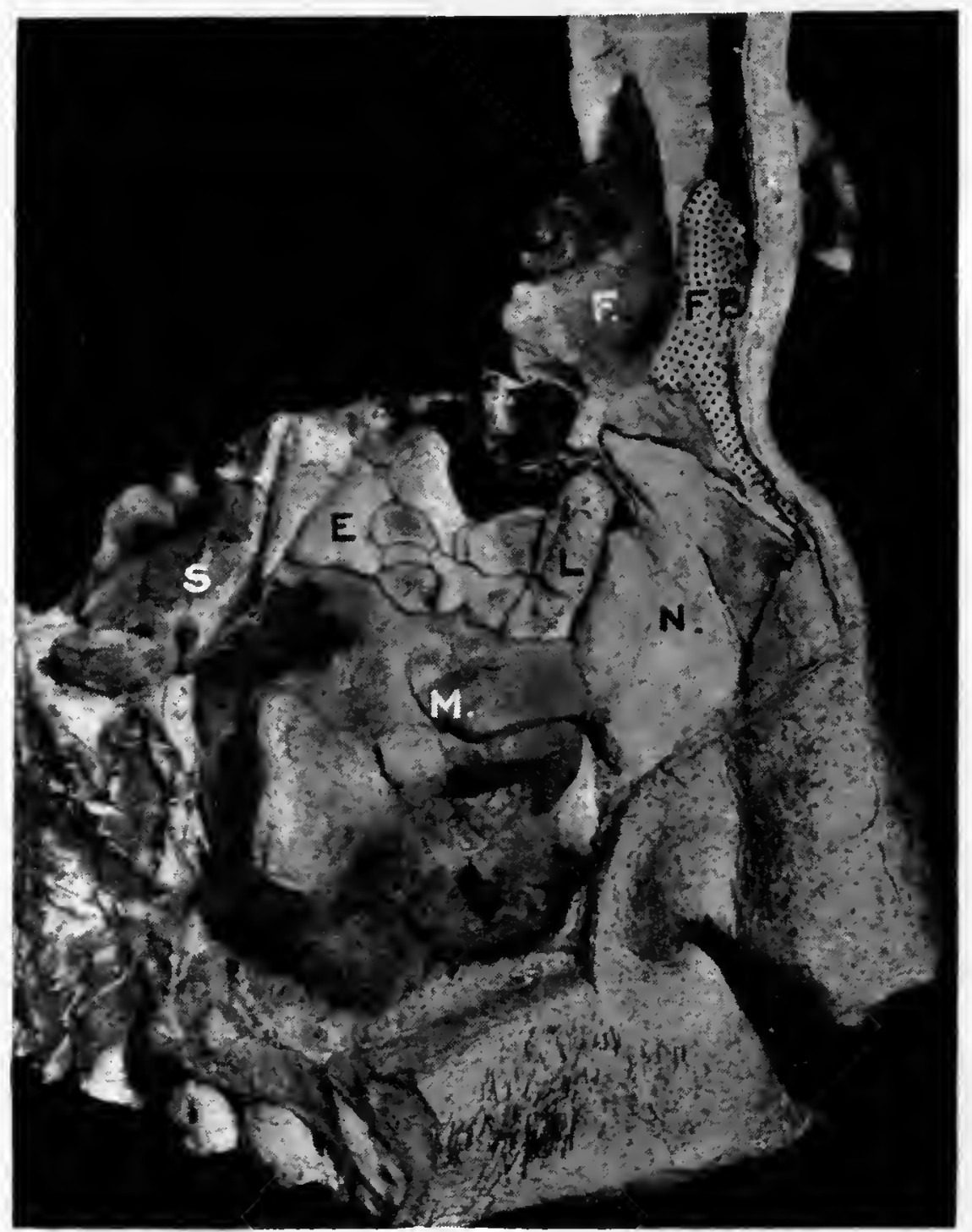

Fig. 22. - The Accessory Air Sinuses of the Nose. Prepared by removing the bones piecemeal from around the nuco-periosteal lining of the air spaces after hardening in formalin. (Preparation by Dr. E. J. Curran of Harvard.) $\times \frac{2}{3}$.

The anterior ethmoidal cell in relation to the upper part of the lacrimal sac (L.) has been blackened.

$\begin{array}{lll}\text { F. }=\text { Frontal sinus. } & \text { F. B. }=\text { Frontal bone. } & \text { N. }=\text { Nasal mucous membrane. } \\ \text { L. = Lacrimal sac. } & M_{\text {I. }}=\text { Maxillary sinus. } & \text { S. }=\text { Sphenoidal sinus } \\ & \text { E. }=\text { Ethmoidal cells. } & \end{array}$

spread into them from the nose. Their collective capacity is more than twice that of the nasal cavity (Braune), and a good idea of their extent can be obtained from such a dissection as has been photographed in Fig. 22. The method, for which the writer is indebted to Dr. E. J. Curran of 
Harvard University, consists in removing the bones piecemeal with chisel and forceps from around and between the sinuses after soaking the specimen for two weeks in strong formalin solution; the lining muco-periosteum is left intact, and will be found sufficiently hardened to retain the shape of the cells. Their form can also be well shown either by wax models (Jackson and Connor, I9I7) or by metal casts (Fig. 23).

The complex arrangement and variable extent of these sinuses, together with the irregular modelling of the lateral nasal wall to which they are related, will be understood better by a consideration of their mode of growth, before proceeding to a brief description of the adult formation.

Development of the air-sinuses. - Before cartilage has been formed in the walls of the primitive nasal cavities, linear outgrowths of the lining epithelium (which is ectodermal in origin) occur in the side and roof of each.

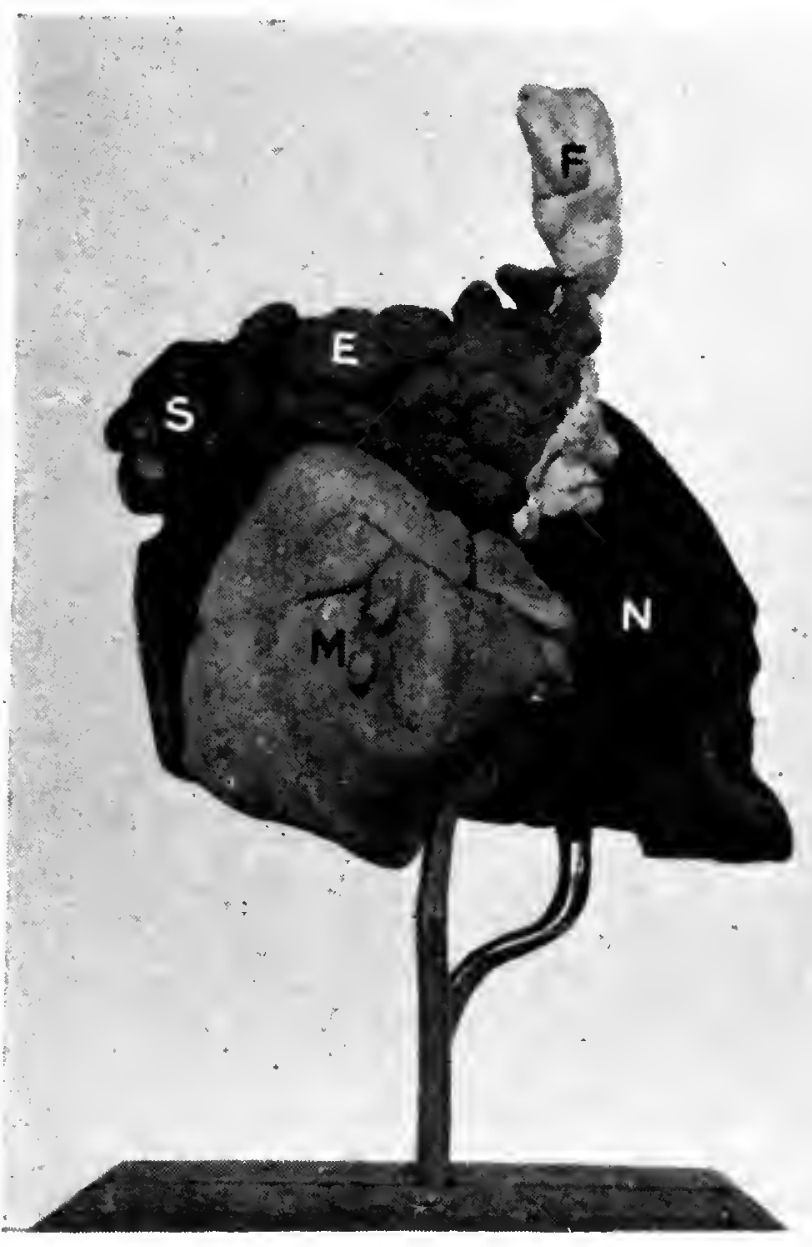

Fig. 23. - Fusible metal cast of the Accessory Sinuses of the Nose (made by Sir Auckland Geddes, in the McGill Anatomical Museum).

F. $=$ Frontal sinus. $\quad$ S. $=$ Sphenoidal sinus. E. $=$ Ethmoidal cells. M. Maxillary antrum N. = Nasal mucous membrane.

These gutters are the future meatuses of the nose, and the ridges left between them eventually form the three conchae or turbinated processes, superior, middle, and inferior: three is the number usually described, though Zuckerkandl (I892) found four such processes in 87 per cent of 267 skulls, a number which agrees with the description given by Sappey ( 1867 ), the additional process being found above, or below and hidden by, the one usually designated superior. The superior concha is the shortest, 
the middle is nearly twice its length, and the inferior extends still further forwards; the first two are processes of the ethmoidal, the last is formed by a separate inferior turbinated bone (concha nasalis inferior). Above the superior concha is a narrow space, the "spheno-ethmoidal recess." In front of the attached margin of the middle concha there

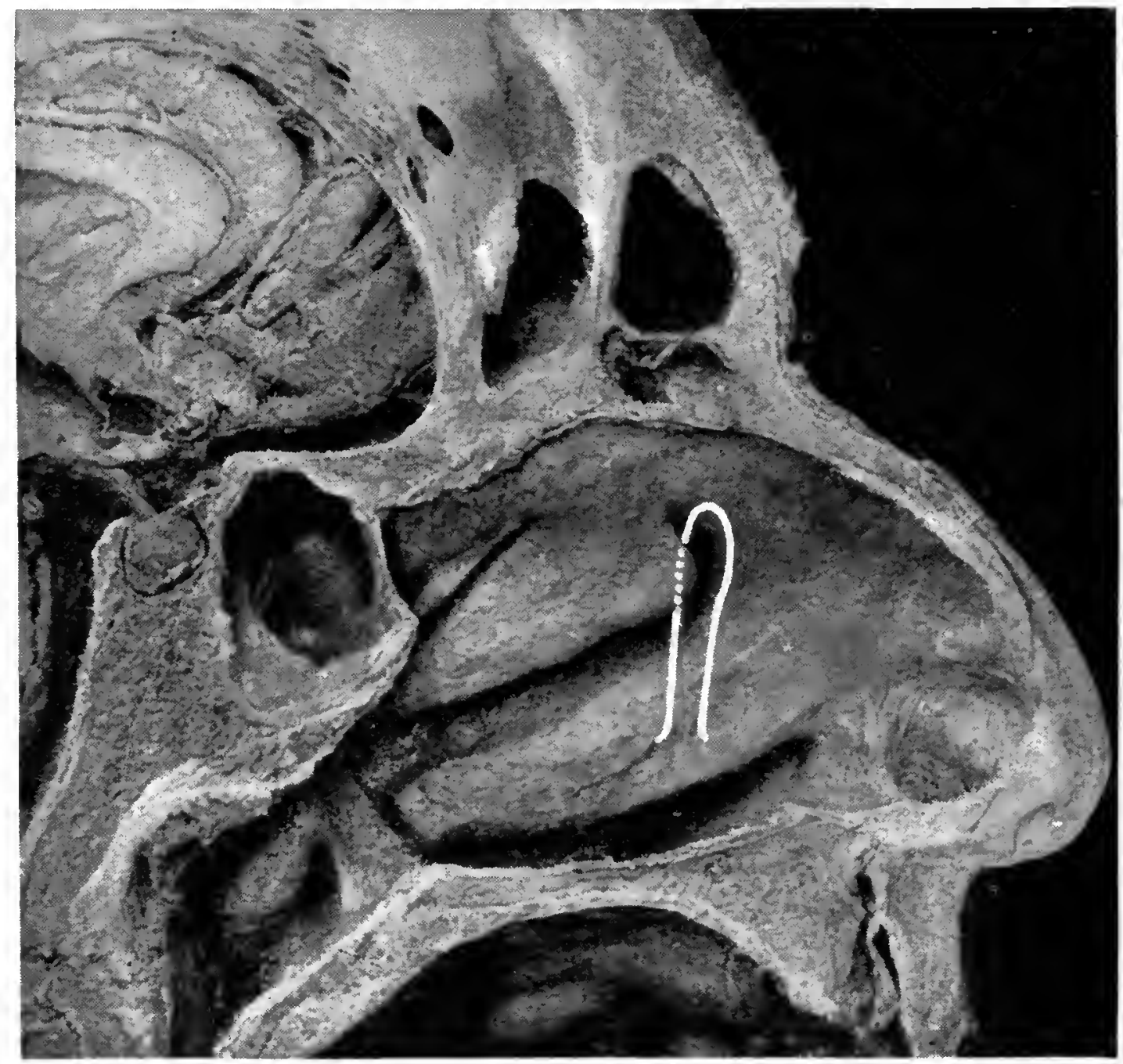

FIG. 24. - Sagittal section of Head of Adult Female. I,eft half viewed from within to show the left lateral nasal wall. See Fig. 25 for guide to parts. The relative positions of the fossa for the lacrimal sac and the naso-lacrimal canal are indicated by the white line. $x$ about $\frac{2}{33}$.

is often a prominence on the frontal process of the maxilla, the agger nasi; this prominence (named from the Latin agger, a mound or rampart) forms the upper boundary of a shallow depression, the atrium, which is an anterior continuation of the middle meatus, and lies above the "vestibule" or cartilaginous dilatation at the entrance of the nose (Fig. 25). On the lateral wall of the middle meatus a secondary linear depression develops, forming in the adult a curved gutter, the hiatus semilunaris, which is bounded 
below by a sharp crest, the processus uncinatus, and overhung by a prominence, the bulla ethmoidalis; it leads into a curved and upwardly directed channel, the infundibulum ethmoidale. These features on the meatal wall are hidden

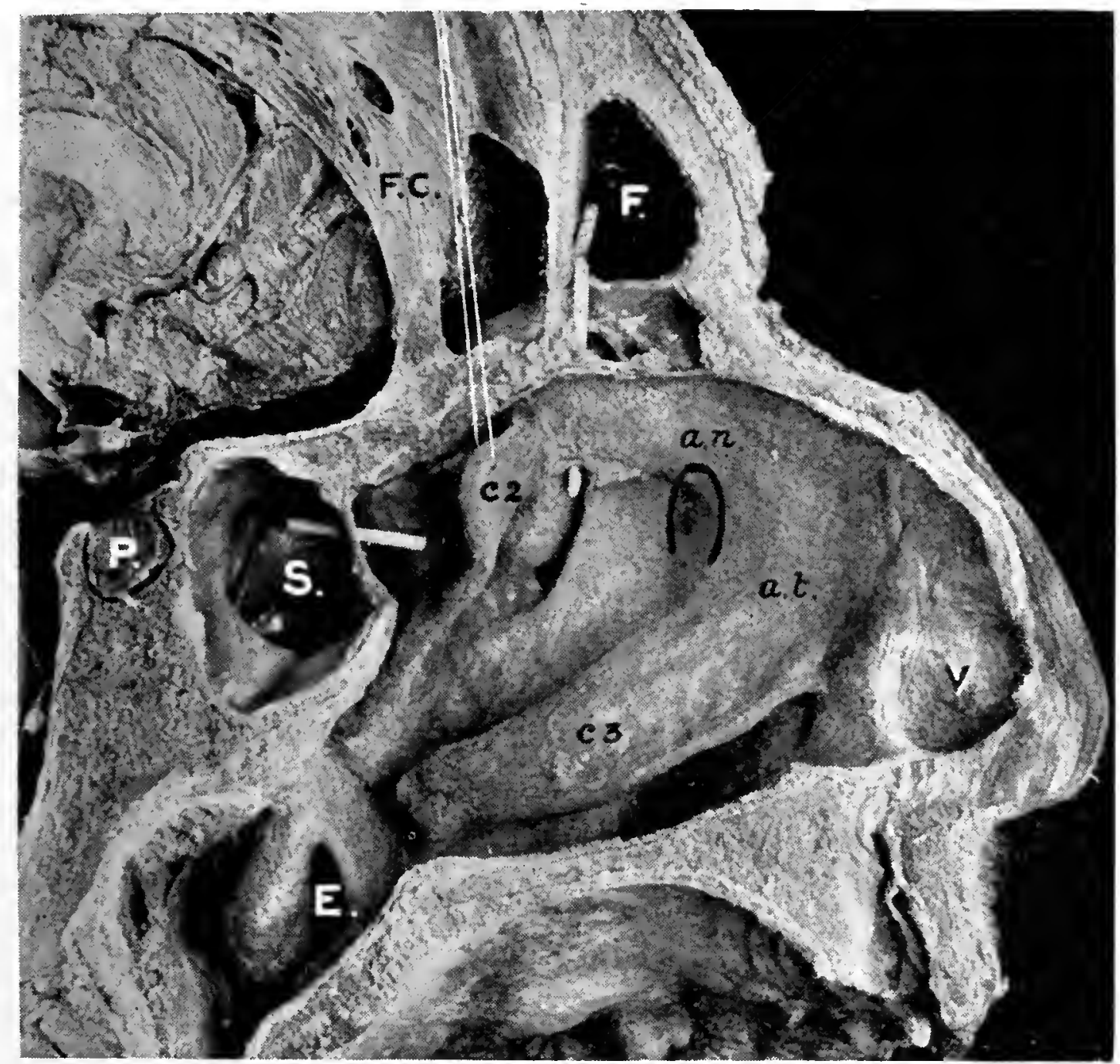

FIG. 25.--The same preparation as in Fig. 24. The middle concha has been pullert upwards to expose the middle meatus. The position of the fossa for the lacrimal sac relative to this wall was ascertained by driving pins through from the opposite side and is outlined in black. Rods have been passed through the opening of the sphenoidal sinus and down the infundibulum of the frontal simus; the latter leads into the hiatus semilunaris, which is bounded above by the rounded bulla ethmoidalis and below by the processus uncinatus; the ostium maxillare of the antrum is also seen. $\times$ about $\frac{8}{4}$.

Compare with the deeper stage of a similar dissection seen in Fig. I22, p. 233.

$\mathrm{P}_{\mathrm{b}}=$ Hypopinysis or pituitary body lying in the sella turcica. S. $=$ Sphenoidal sinus. F.C. $=$ Fal. cerebri. $\mathrm{F} .=$ Frontal sinus. $c 2,3=$ The middle and inferior concha. $a . n .=$ Agger nasi, here little developed. a.t. $=$ Atrizm, and $\mathrm{V} .=$ Vesibule, of the nose. $\mathrm{E}_{0}=$ Orifice of auditory or Eustachian tube.

by the overhanging middle concha, but are well displayed in Fig. 25.

The nasal mucous membrane continues to grow outwards in various directions, and its projections develop into the accessory sinuses of the nose. Thus the frontal sinus 
is formed at the upper end of the infundibulum by a bud of mucous membrane which grows upwards through the ethmoid, and pushes its way between the outer and inner lamellae of the frontal bone to a very variable extent. This outgrowth appears in the first or second year of life, but is nascent until the fifth or sixth; at the end of the seventh it is about the size of a pea, and reaches its full development at twenty-five years. The stalk of this outgrowth forms the "fronto-nasal duct," and either retains its original continuity with the infundibulum or, as in half the number of cases, opens independently into the middle meatus, the infundibulum then ending blindly above, owing to the anterior end of the uncinate process fusing with the forepart of the bulla. The ethmoidal cells bud out into the nasal wall above and below the middle concha, and are present at birth as little culs-de-sac. They multiply until each lateral mass of the bone is composed of a number of cells (8-ro), the "ethmoidal labyrinth," an increase in growth which causes the greater width between the eyes in the adult. The bulla ethmoidalis is inflated by one or more such cells, and the term, derived from the Latin bulla, a bubble, well expresses the formation of these cellular outgrowths. The sphenoidal sinus is primarily formed by a similar budding from the region of the spheno-ethmoidal recess into the body of the bone; present as a round depression in the first to third year, it develops more rapidly after the sixth or seventh. The maxillary sinus is formed by an outgrowth from the middle meatus in the region of the future hiatus semilunaris, which bursts through the lateral nasal plate of cartilage and distends the maxillary process of the foetal face. This is the only one of the sinuses that is more than a mere rudiment at birth, at which period it forms a shallow depression above the germ of the first milk tooth; at the age of ten it is well formed, and during adolescence it expands and fills out the whole of the maxilla, reaching full size after completion of the secondary dentition.

In the adult, as might be expected from the mode of development, much variation in the size and extensions 
and consequently in the relations of these air-spaces, especially of the ethmoidal group, is found. The ethmoidal group, together with the maxillary sinus, are constantly present, the others may in rare instances be undeveloped even in the adult. The frontal, sphenoidal, and palatine sinuses are homologous with, and may be succeeded or displaced by, the ethmoidal cells, the growth of which, indeed, may be so exuberant as to encroach upon the area usually occupied by any of the others.

The frontal sinuses (sinus frontales) lie above the upper

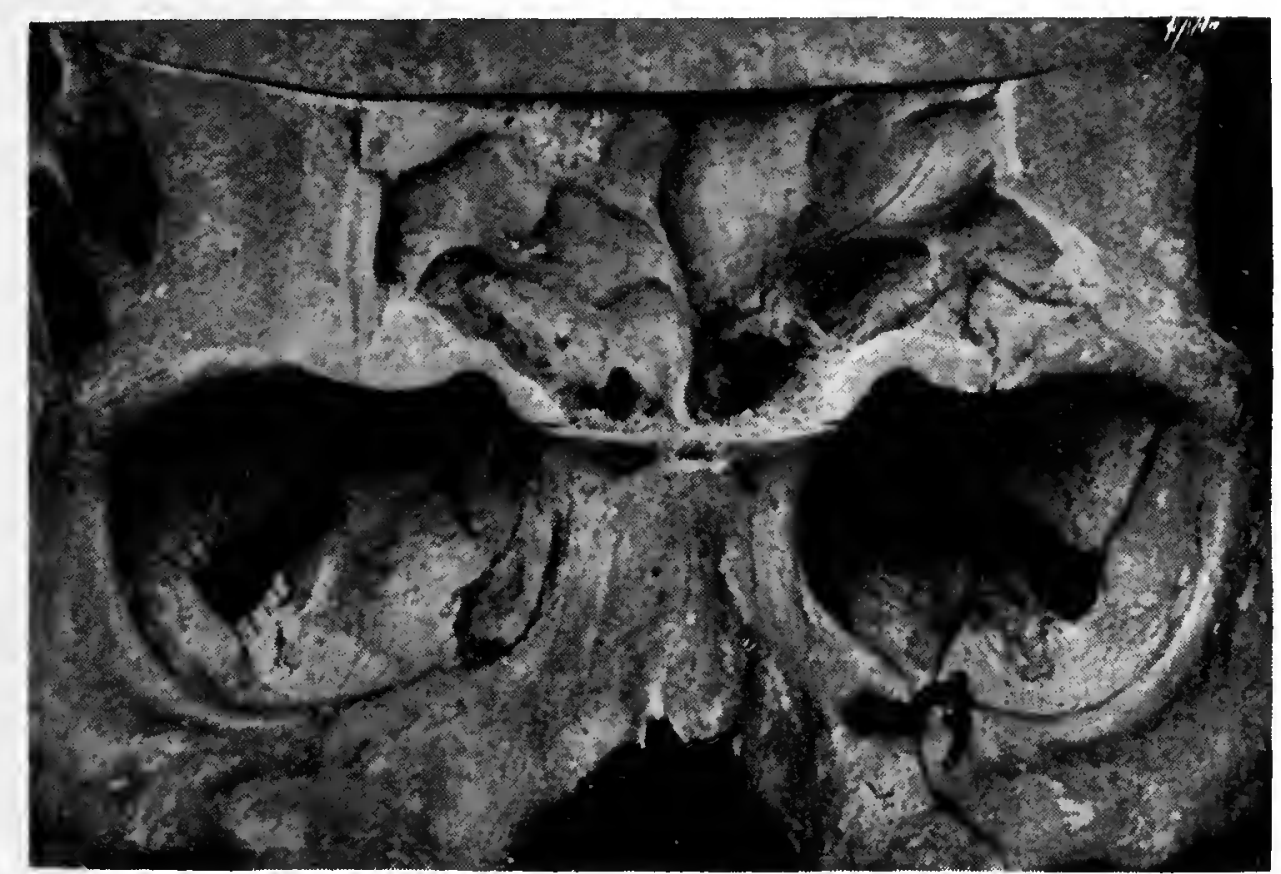

FIG. 26. - The Frontal Sinuses exposed by removal of their anterior walls. The irregularity and roughness of the cerebral wall is abnormal, but the preparation shows a median dividing septum and the openings at the back and lower part. (The position of the orbitalis muscle of Müller is indicated by the shading across the right inferior orbital fissure.) $\times \frac{2}{3}$.

and inner angles of the orbital entrances and behind the supra-orbital ridges. Usually two in number, they are rarely of equal size, the dividing septum being generally displaced to one side of the mid-line; they may be undeveloped, as in the upper two bones seen in Fig. I2, p. 30. Logan Turner gives normal dimensions of $\mathrm{I} \frac{1}{4}$ inches high, $\mathrm{I}$ inch wide, and I inch deep from before backwards, or 3I, 25, 25 $\mathrm{mm}$. respectively, and the average capacity is from 3 to 5 c.c. in males, but much less in females. Schaeffer found the extreme capacity of both to vary from I to 45 c.c. The opening (ostium frontale) of eachlies posteriorly and near the 
median plane (Fig. 26), and leads through the fronto-nasal duct into the middle meatus of the nose, either separately, or through the infundibulum (as found in 56 per cent of cases by Schaeffer and as illustrated in Fig. 25) ; the duct is separated from the lacrimal fossa by ethmoidal cells. The width of the sinus is its most variable feature, and, as already noted on p. 29 , it may extend to a considerable distance over the roof of the orbit (orbital part of the sinus), as shown in the opposite figures.

The ethmoidal cells (cellulae ethmoidales) form a mass, the ethmoidal labyrinth, lying between the upper part of the nasal fossa and the orbit, from the latter of which it is separated by a smooth thin plate of bone, the lamina papyracea. These cells are variable in number; usually there are eight or ten, with extremes of four to fifteen (Rollet), and averaging the size of a pea, though smaller in front than behind; separated from one another by delicate bony partitions, they open either individually or through one another into the nasal cavity. They may be subdivided into three groups, anterior, middle, and posterior, in accordance with their relative positions, the site of their openings, and the bones completing their walls. The anterior cells, one to three in number, open into the middle meatus, either into the infundibulum or into the recess above (or more rarely beneath) the bulla ethmoidalis, and their walls are completed by the frontal, lacrimal, and maxillary bones; they may project into the frontal sinus to form a frontal bulla, or hollow out the anterior extremity of the middle concha and also the agger nasi, and as "lacrimo-ethmoidal cells" they present important relations to the fossa for the lacrimal sac (see p. 66) Those of the middle and largest group open abové and below the bulla ethmoidalis, which they also inflate; their walls are all formed by the ethmoidal bone itself, save where they project like the frontal sinus above the orbit between the lamellae of the frontal bone. The posterior cells, unlike the other two groups, open into the superior meatus and above the middle, concha; usually three in number, their walls are completed by the ethmoidal, sphenoidal, and palatine bones; they may project or even 
open into the sphenoidal sinus or replace the air-cell of the
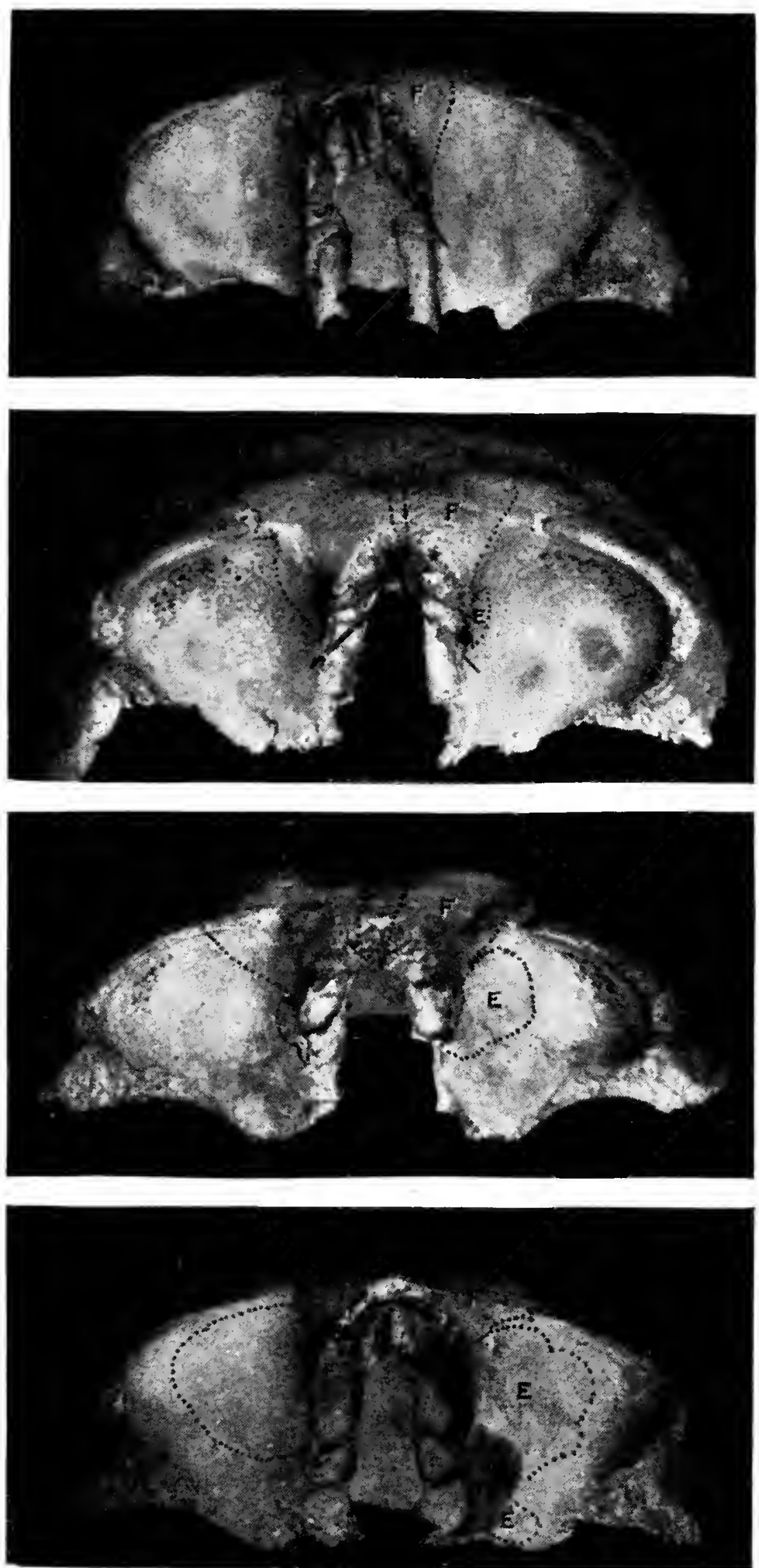

FiG. 27. - Four Frontal Bones showing varying degrees of extension of frontal sinuses (F.) and ethmoidal cells (E.) over the orbital roof. In the upper two specimens bristles have been passed through the anterior ethmoidal canals. $\quad \times \frac{1}{2}$.

palatine bone, and one or more cells may present important 
relations to the optic canal, as has been seen on p. 27 . The palatinal air-cell hollows out the orbital process of the palatine bone, and so is related to the apex of the floor of the orbit (Fig. I8, p. 46) ; it usually opens into either the sphenoidal sinus or a posterior ethmoidal cell.

The sphenoidal sinus (sinus sphenoidalis) is one of a pair of air-spaces hollowed out in the body of the sphenoidal bone; they are generally completely, but rarely equally, divided from one another by a septum, and are frequently encroached upon by ethmoidal cells; an average size is I2 $\mathrm{mm}$. high, I5 mm. wide, $22 \mathrm{~mm}$. deep, with a capacity of 7.5 c.c., but the extent to which they hollow out the basi-sphenoid is most variable, Schaeffer, for example, giving a range of from 5 to 30 c.c. each. Cope (I9I6, I9I7) classifies three types according to their degree of development; out of 292 sinuses examined he found that I55 extended backwards beneath the fossa hypophyseos (sella turcica), 72 were confined to the pre-sphenoid in front of the olivary eminence, and 65 were intermediate in type and only slightly encroached upon the basi-sphenoid; examples are seen in Figs. 25, 33, I22, I86. They lie at the back and upper part of the nasal cavities; in the skull, the distance of the midpoint of the anterior wall of the sinus from the point nasion is about $48 \mathrm{~mm}$. or 2 inches (varying from 42 to 60 $\mathrm{mm}$. in 20 cases measured by the writer), and from the lateral margin of the anterior nares is about $70 \mathrm{~mm}$. or $2 \frac{3}{4}$ inches (varying from 64 to $74 \mathrm{~mm}$. in 13 preparations). Each communicates by an opening (apertura simus sphenoidalis) situated above the centre of its anterior wall with the corresponding spheno-ethmoidal recess. They are related to the cavernous sinus laterally (where the bony wall has been found deficient by St. Clair Thomson, I906) and to the hypophysis cerebri or pituitary body superiorly and posteriorly; their important relations to the optic nerves and chiasma are described on pp. 27,370; and further it is to be noted that the pterygoid or Vidian canal, containing the sympathetic and motor roots of the spheno-palatine or Meckel's ganglion, may form a prominence in the floor, as also may the internal carotid artery on the side wall (Schaeffer). 
The maxillary sinus (sinus maxillaris, antrum of Highmore) occupies the body of the maxilla, lying beneath the orbital floor, to the lateral side and extending a little below the level of the nasal cavity; its average dimensions are $\mathrm{I} \frac{1}{2}$ inches high, $\mathrm{I}$ inch wide, $\mathrm{I} \frac{1}{4}$ inch deep, with a capacity of I5 c.c. Its opening, the ostium maxillare, which is duplicated in nearly half the number of cases (i.e. in $42 \cdot 4$ per cent of I25, as found by Schaeffer), is situated at the upper part of its nasal wall (Figs. I7, II8), and leads into the middle meatus through the hiatus semilunaris (Fig. I22, p. 233); in those cases where the fronto-nasal duct leads directly into the hiatus semilunaris, this gutter could drain the frontal sinus more or less completely into the antrum, a condition seen in Fig. 25. On the fore part of this nasal wall of the antrum there is a thin-walled "lacrimal protuberance "' formed by the naso-lacrimal canal (Fig. I23, p. 234). The upper or orbital wall is thin and ridged in its fore part by the infra-orbital canal and by bony channels containing the superior alveolar or dental nerves. The cavity may thus be partly or completely subdivided by septa, and part of it circumscribed anteriorly to form a "prelacrimal recess" (p. 72). This sinus, like the others, may present abnormal extensions passing into any of the processes of the bone, such as the frontal or the zygomatic (with, but very rarely, a continuation into the zygomatic bone itself, the sinus malaris vel zygomaticus), or it may replace the palatine air-cell, or even be prolonged under the medial wall of the orbit.

For further details of the anatomy and development of these accessory sinuses the reader should consult the works of Zuckerkandl (I882), Logan Turner (Igor), Killian (I903), Rollet (I903), Onodi (I9I3), Skillern (I9I3), Schaeffer (I920), and papers by Coffin (I905), Underwood (I9I0), Schaeffer (IgIo), and Fawcett (I9II).

\section{THE OSSEOUS LACRIMAL PASSAGES}

These comprise the lacrimal fossa together with a downward continuation, the naso-lacrimal canal. 
(i.) The Fossa for the Lacrimal Sae (fossa sacci lacrimalis) is a broad groove occupying the lower two-thirds of the medial orbital margin, which, as explained on p. I7, divides to enclose it; shallow above, where it usually extends up to the frontal bone, it becomes deeper at its lower end, where it is continued downwards into a bony channel, the naso-lacrimal canal, the two combined resembling the cut end of a quill pen seen sideways (Fig. 34). It is formed by two bones, the frontal process of the maxilla in front and the lacrimal behind; each is grooved by a sulcus lacrimalis; the approximation of the two sulci forms the fossa, and the vertically disposed "lacrimomaxillary suture" between them divides it into halves; it is bounded in front and behind by two "crests" (Fig. I8, p. 46$)$.

The rounded anterior lacrimal crest (crista lacrimalis anterior) of the maxilla is inconspicuous above but well marked below, where it becomes directly continuous with the infra-orbital margin; here it may present, as in I7 out of 5 o skulls examined by the writer, a " lacrimal tubercle" which can be felt in life, and from its position may be taken as a guide to the lacrimal sac which lies just behind and above it. Gérard (I907) found that in 28 per cent of I40 orbits the tubercle projected backwards over the lower part of the fossa as an "anterior lacrimal spur" (Fig. 28), and so might form an obstruction to catheterisation of the lacrimal passages. Attached to the anterior lacrimal crest is the medial palpebral ligament of the eyelids above, with fibres of the orbicularis oculi muscle below.

The posterior lacrimal crest (crista lacrimalis posterior), formed by the lacrimal bone, is much more sharply defined, and may even be prolonged as a thin plate of bone curving forwards over the fossa, as is well seen in foetal skulls towards term or at birth (Fig. 43, p. 96). It is thickened above at the site of attachment of the pars lacrimalis (Horner's) muscle, as seen in Fig. 2I, and at its lower extremity it usually ends in a projection, the hamulus lacrimalis (described above on p. 48), which curves round laterally and so defines the upper opening of the 
naso-lacrimal canal. Just behind the posterior lacrimal crest is affixed the septum orbitale.

The fossa has three walls: an anterior, narrow and well marked only in its lower part; a medial, broad and divided vertically by the lacrimo-maxillary suture ; and a posterior, variable in width. As regards the medial wall, it is important to note that there is a striking difference in the archi-

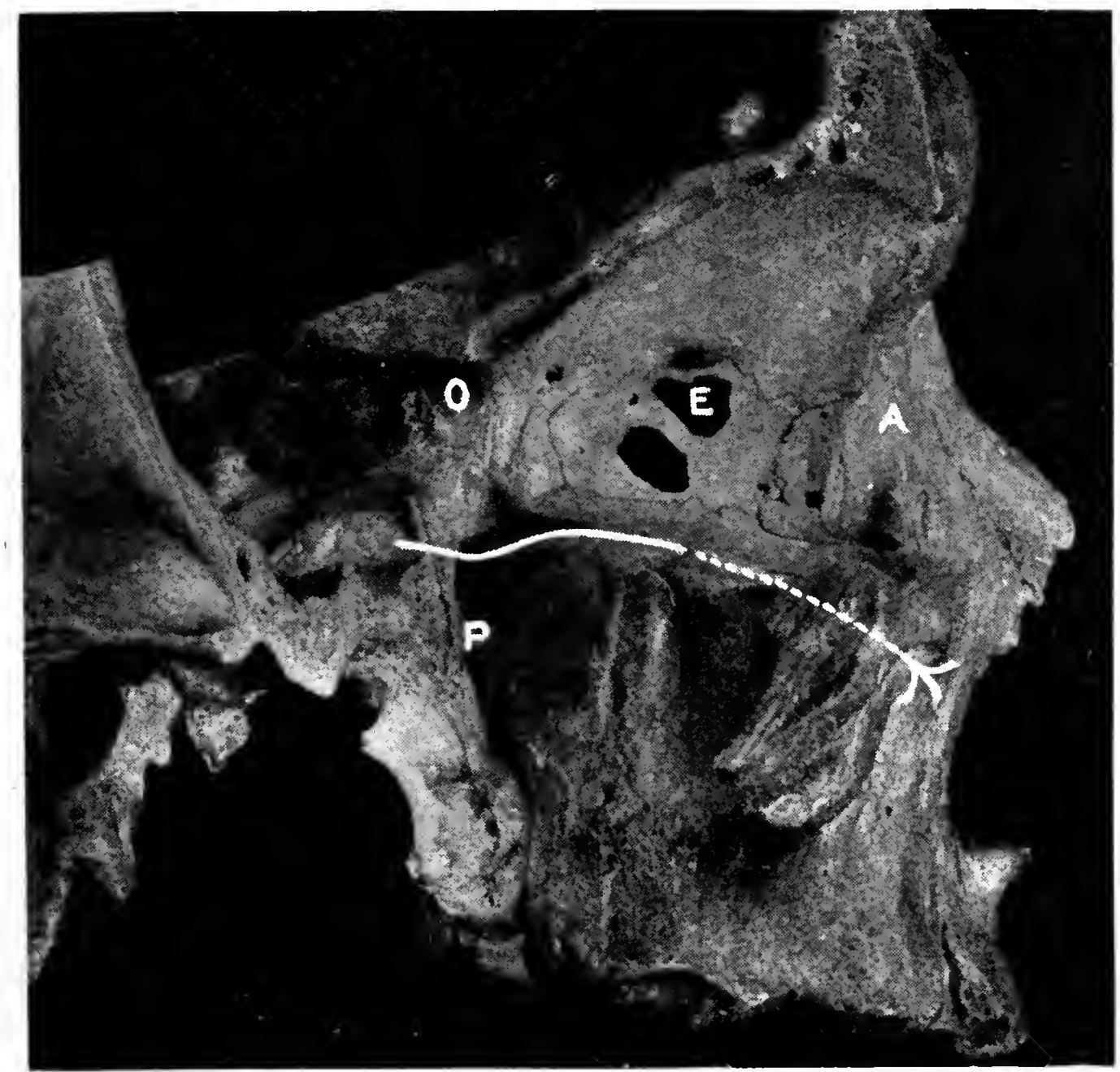

FIG. 28. - Sagittal section through Right Orbit to show medial wall. There is a well-marked "lacrimal tubercle" at the base of the anterior lacrimal crest, and a slender hamular process at the base of the posterior lacrimal crest.

The orbital roof is doubled throughout by extensions from ethmoidal cells. $\times \frac{3}{4}$.

A. = Anterior lacrimal crest.

E. shows an ethmoidal cell broken into; above it is a large anterior ethmoidal foramen.

$O$. is the optic canal through which the section passes.

$P . \quad$ is placed in the pterygo-palatine or spheno-maxillary fossa.

The white line shows the course of the infra-orbital nerve.

tecture of the two regions demarked by the suture, the anterior or maxillary half being strong and resistant, the posterior lacrimal half being thin and fragile; the subdivision is not always equal, for the maxillary portion may encroach upon the other, and in very rare instances even form the whole of the medial wall of the fossa, in which 
cases not only is the fossa found much shallower and smaller than usual, but the calibre of the naso-lacrimal canal is correspondingly reduced, as is seen so well in the casts figured by Zabel (I900), Fig. 34. The posterior wall, always formed by thin bone, may be comparatively broad and conspicuous when the skull is viewed from the front, giving depth to the fossa, or it may be narrow and less prominent, the fossa then being shallow; these differences are most noticeable in the upper region, and depend upon the degree of development of the underlying ethmoidal cells; they are illustrated in the left orbits of the skulls shown in Fig. 45, p. I03. At the upper end of the lacrimalmaxillo suture (i.e. at the point dacryon) there is present in 20 per cent of cases a foramen which transmits to the ethmoidal cells an arteriole from the nasal branch of the ophthalmic artery, as well as a filament from the infra-trochlear branch of the nasal nerve (Macalister, Ledouble). In 2 per cent of cases small twigs from the angular and infra-orbital arteries pass through small orifices lower down in the suture. Zuckerkandl, moreover, describes a "lacrimo-facial vein" into which blood passes from the anterior ethmoidal cells through the lacrimal bone.

The relations of the lacrimal fossa are as follows: on the lateral or orbital aspect it is covered by the lacrimal fascia of the periorbita passing from one crest to the other; across the front of its upper part lies the medial palpebral ligament and skin; whilst behind it in the same region there is found the pars lacrimalis or Horner's muscle; the lower part of the fossa has the skin and orbicularis muscle related to it in front, the septum orbitale behind. These relations are illustrated and referred to further on p. 229.

On the medial or nasal side the fossa is related to ethmoidal air-cells, which intervene to a variable extent between it and the nasal cavity (Fig. II2, p. 220). These "lacrimo-ethmoidal cells" are usually two in number, superior and inferior, and belong to the anterior group; their walls are completed by the lacrimal and frontal bones and sometimes in addition by the frontal process of the maxilla, and they open into the infundibulum. When well 
developed they may bound the fossa on its posterior, medial, and anterior aspects. Their precise relations are important in connection with the above emphasised difference in the thickness of the walls where formed by the lacrimal bone behind, or by maxilla in front, a difference strongly marked even when the frontal process of the latter also is undermined by cells. In an examination of IOO orbits by the writer, approximately the lower half of the whole fossa was found in every instance directly related to the middle meatus of the nose, which space could always be entered easily through the thin postero-inferior quadrant of the fossa formed by the lacrimal bone (a relation found generally by Toti, Dieulafé (I905), and Aubaret (Igro),

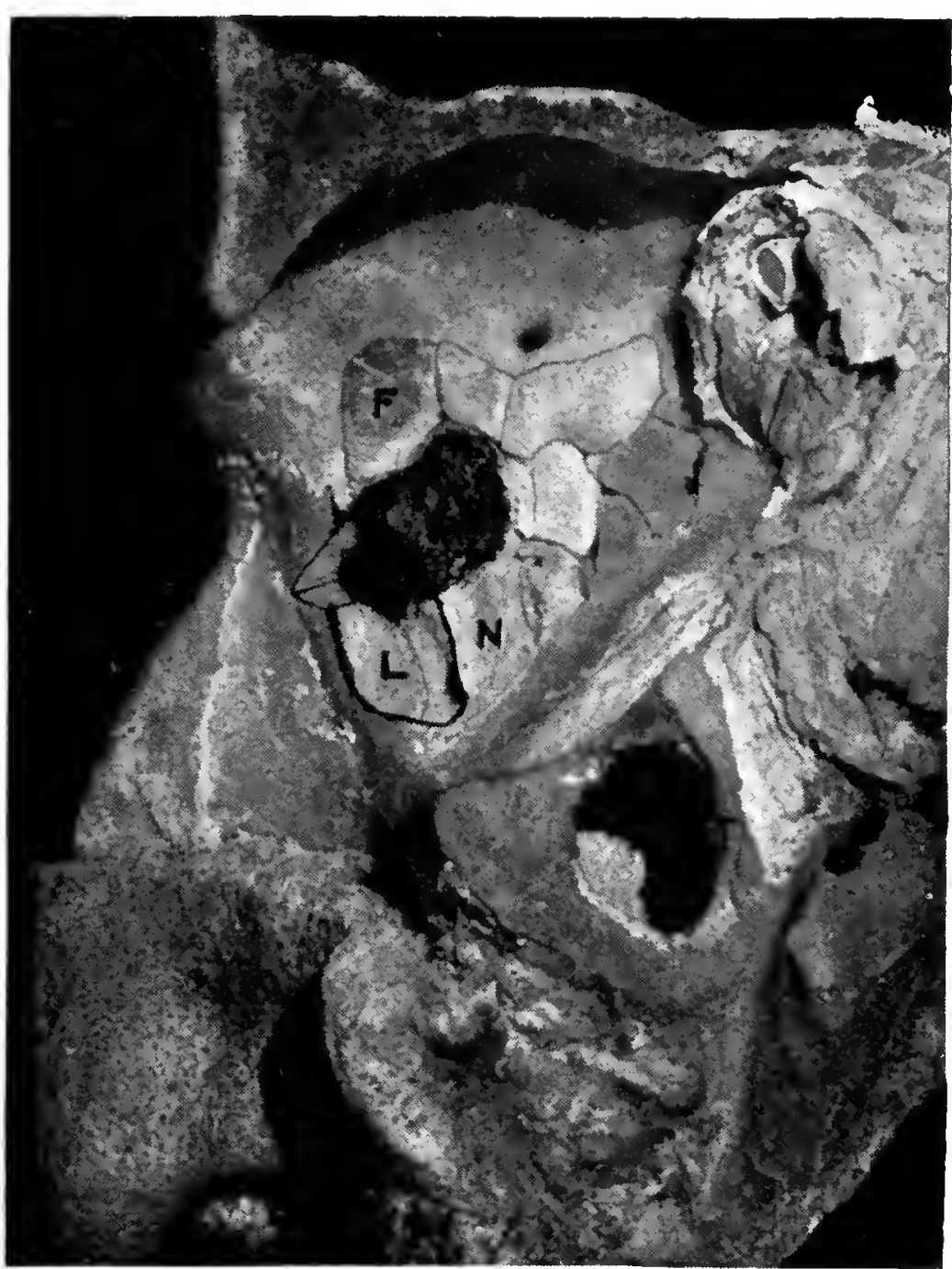

FIG. 29.-Dissection of Medial Wall of I eft Orbit to show the relation of the fossa for the lacrimal sac (L.) in its upper part to an anterior ethnoidal cell (in black) above which is seen the frontal sinus $(F$.$) , and in its lower part to the nasal$ mucosa (N.). The muco-periosteal lining of the air-cells has been exposed by removal of their bony orbital walls. A wedge-shaped piece has been cut out of the anterior lacrimal crest to show its thickness. Natural size. and in the proportion of 80 per cent of 82 skulls by Thorsch (I909), and in I8 out of 29 by Salus). On the other hand, the upper half of the fossa presented relations to either one or two anterior ethmoidal cells, which extended (i.) to the posterior wall only in I4 orbits; (ii.) as far as the suture in the centre of the medial wall in 32 ; (iii.) completely across the fossa and up to, or even into, the 
frontal process of the maxilla in 54 (Whitnall, I9II). These variations are shown in the following figure.

There was in most cases a single intervening cell situated in the fore end of the processus uncinatus of the ethmoid in the region of the agger nasi (cellula lacrimalis, or "cell of the agger nasi," or the "cellule fronto-ethmoido-ungueale"

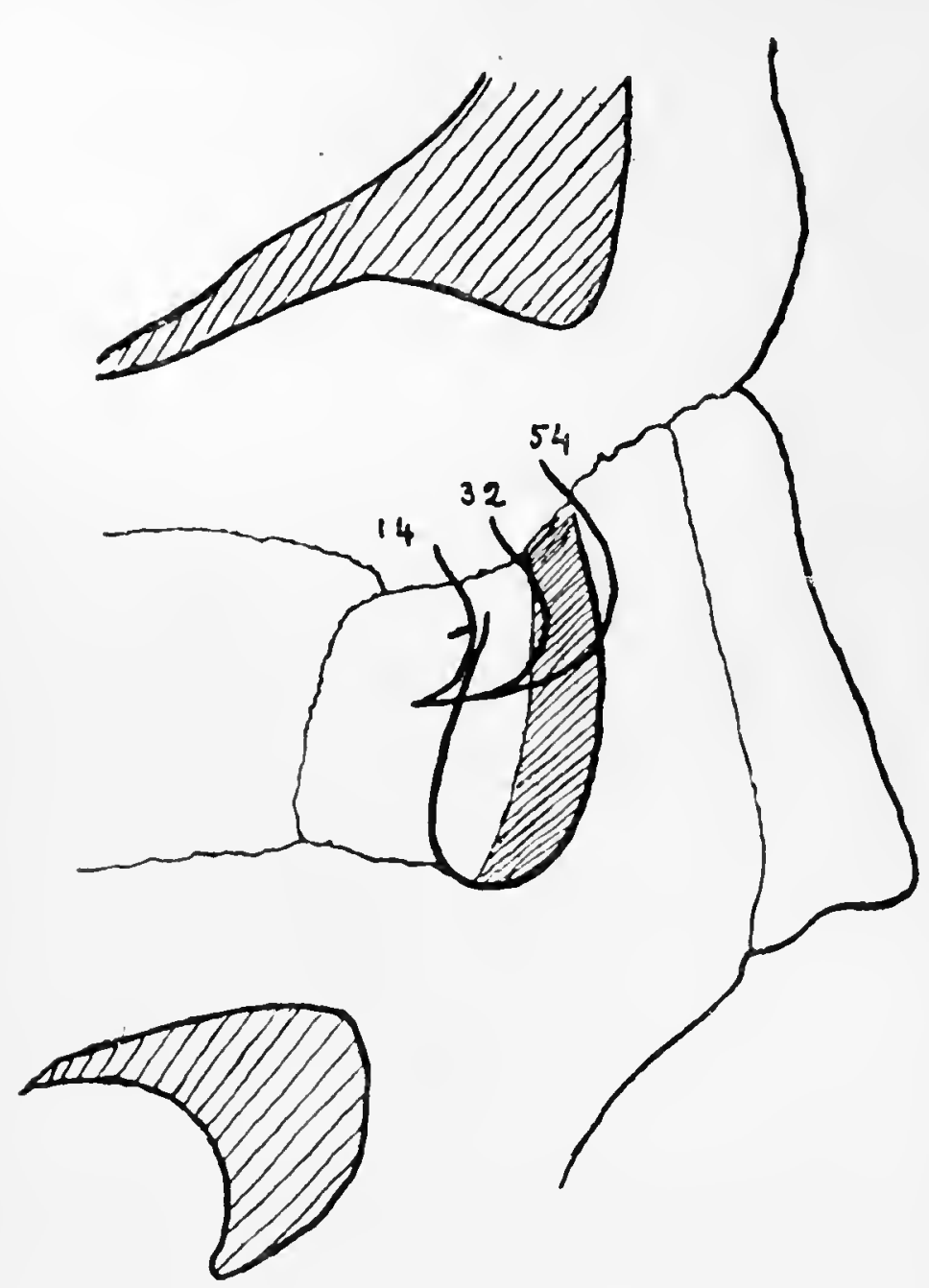

FIG. 30.-To show the varying relations of an Anterior Ethmoidal Cell to the upper half of the fossa for the lacrimal sac. 'The figures give the percentages of cases found in roo orbits examined. The lower limb of the lines corresponds approximately to the free margin of the iniddle concha. Compare with Fig. 3r opposite. of Aubaret), as is well shown in Figs. 22 and 29 above, and in Fig. I22, p. 233, and it should be particularly noticed that this aircell separated the upper half of the lacrimal fossa from the nasalcavity, and íwould first be opened into on attempting to enter the cavity by this route, or, on the other hand, in obtaining access to the lacrimal sac by the nasal route. In no case was the frontonasal duct found in direct relation to the lacrimal fossa, but there was always a cellular interval between them, as seen in Fig. 22, though Onodi (I9r3) has seen an enlarged frontal sinus itself extending downwards to the upper end of the fossa. Finally, it should be mentioned that an extension from the upper part of the hiatus semilunaris reaching to the posterior wall of the lacrimal fossa is often present; such an "ethmolacrimal recess" was found by Grünwald (IgIO) in 56 out of 79 preparations.

As regards the deeper relations to the lateral wall of the 
nasal cavity, the upper half of the lacrimal fossa lies opposite the fore end of the attached border of the middle concha and its forward continuation, the agger nasi, an air-cell usually intervening, as described above. The lower half of the fossa

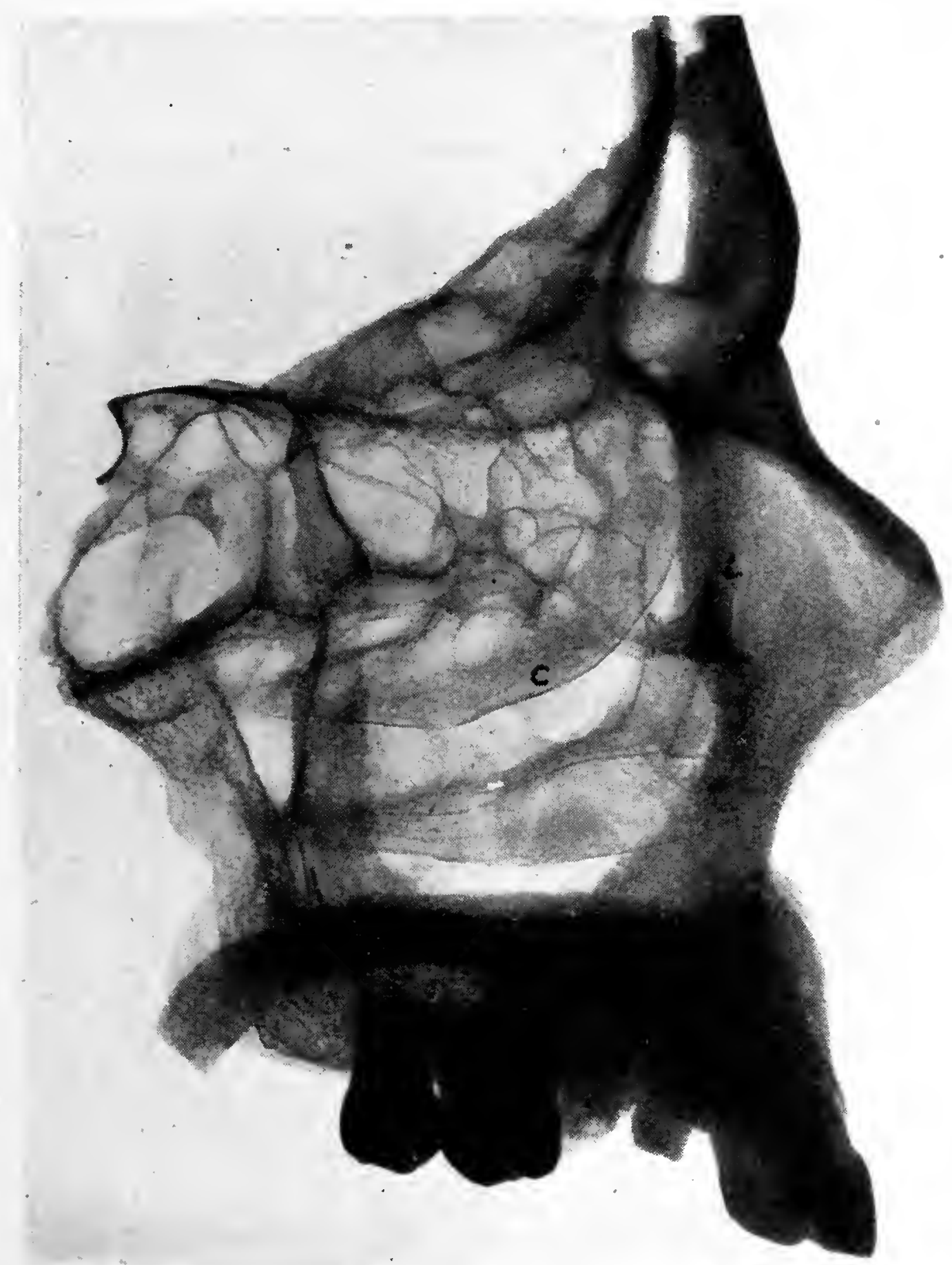

FIG. 3I. - Radiograph through the left lateral nasal wall scen from the medial aspect, to show the position of the fossa for the lacrimal sac (L.), on the further side, relative to the fore end of the middle concha (C.) on this side. The outline of the naso-lacrimal canal is also well shown. Compare with Fig. I22, p. 233. Natural size. (By Dr. J. D. Morgan.)

is directly related to the upper part of the middle meatus, just below the attachment of the middle concha (the remainder of the meatus in vertical height being related to its continuation, the naso-lacrimal canal). In Figs. 24, 25. 
and I 86 the position of the fossa relative to this wall was indicated by pins driven through its outlines from the orbital aspect, and in Fig. $3 I$ the exact relation is clearly displayed. The free extremity of the middle concha does not usually project as far forwards as the fossa, since Thorsch (Ig09) found that an opening made through the

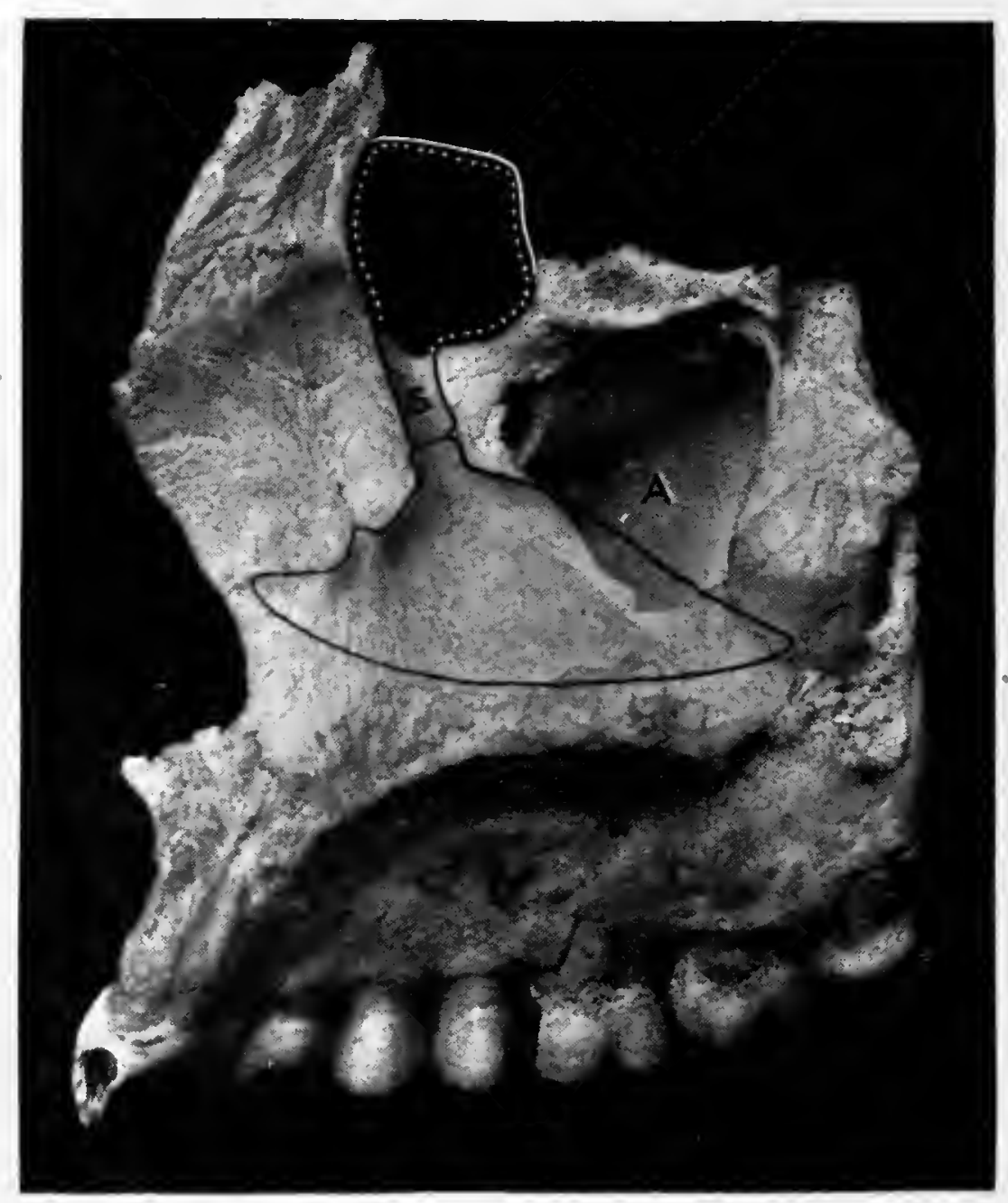

FIG. 32.-Formation of the naso-lacrimal canal. Right Maxilla seen from Nasal Side, showing outlines of lacrimal bone, above, and inferior concha or turbinated bone, below, and the formation of the sulcus lacrimalis of the maxilla, $\mathrm{S}$., into the naso-lacrinal canal by the junction of processes of these two bones on its medial side. The extent to which the lips of the sulcus normally form the medial wall of the canal is here seen. Compare with Fig. 33 opposite.

The fore and upper part of the antrum, A., presents a "well-defined "prelacrimal recess." Natural size.

fossa into the nose was covered by the middle concha in $\mathrm{I}_{4}$, partly covered in I3, but completely uncovered in 52 out of 79 cases.

(ii.) The Naso-lacrimal Canal (canalis naso-lacrimalis) is a short bony tube leading downwards from the lacrimal fossa to the inferior meatus of the nose, and containing the 
membranous naso-lacrimal duct. It is formed by three bones, the maxilla, lacrimal, and inferior nasal concha (inferior turbinated bone), of which the greater part is contributed by the maxilla. On the forepart of the nasal surface of the body of this last bone is a deep groove, the sulcus lacrimalis, the downward continuation of the same sulcus on the posterior aspect of the frontal process which

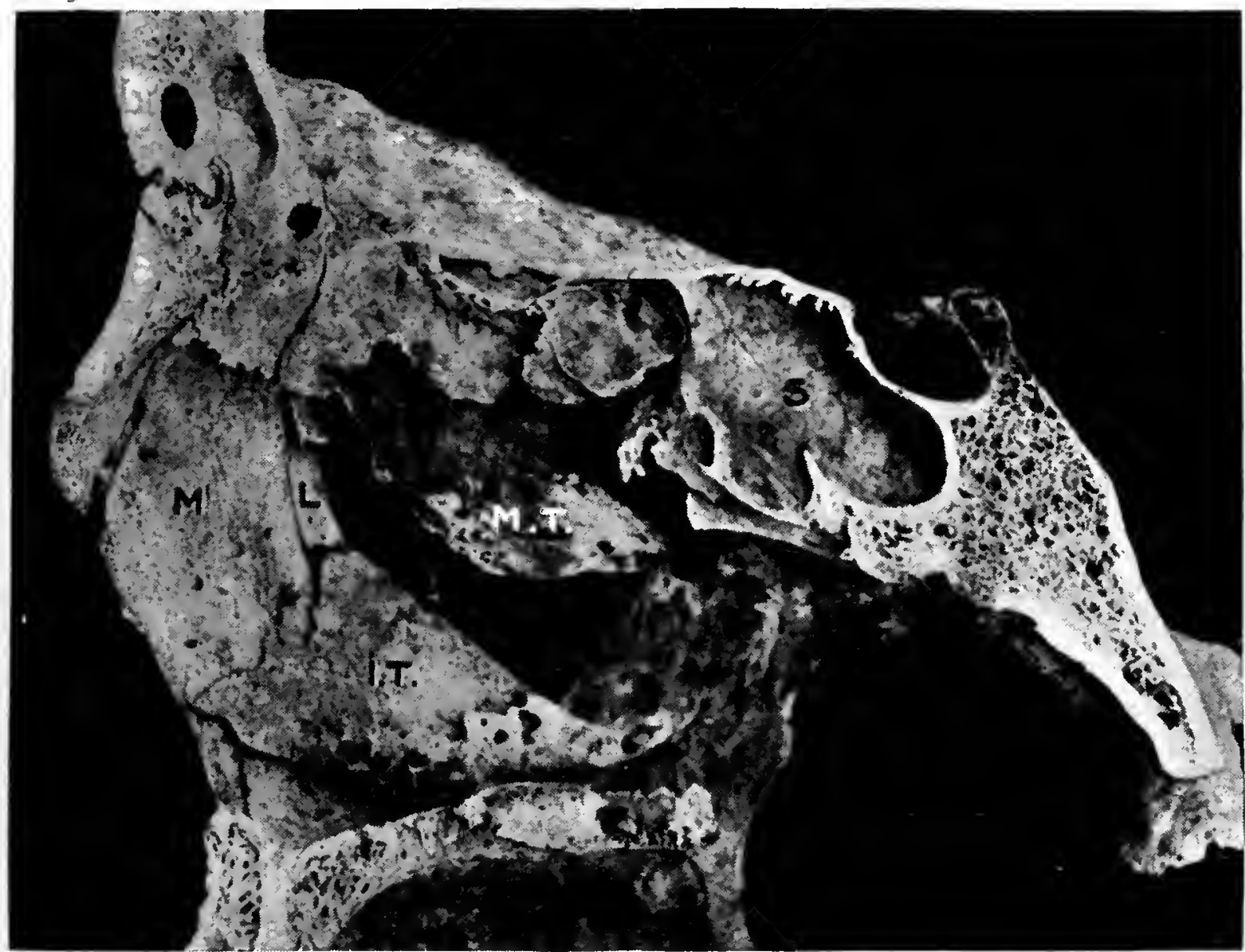

Fig. 33.--The Bones of the Lateral Nasal Wall, slowing the descending process of the lacrimal bone, L., and the lacrimal process of the inferior concha or turbinated bone, I.' $\Gamma$., which together form the medial wall of the naso-lacrimal canal. Compare with Fig. 32. Natural size.

\footnotetext{
M. = Frontal process of maxilla.

M. T. = Middle concha or turbinated process of ethmoidal bone. S. $=$ Sphennidal sinus.
}

helps to form the lacrimal fossa, and the gap between its lips on the nasal side is completed by the articulation of the descending process of the lacrimal bone from above with the lacrimal process of the inferior turbinated (concha nasalis inferior) bone from below, as shown in Figs. 32, 33.

These two processes, of paper-like delicacy, as are the bones to which they belong, complete the medial wall of the canal and shut it off from the nasal cavity; the extent 
to which they take part in the completion of the canal is variable, and is compensated by the degree of development of the lips of the sulcus lacrimalis. The upper aperture of the canal is situated in the antero-medial angle of the orbital floor, and its lateral margin, the incisura lacrimalis, is circumscribed by the hamulus of the lacrimal bone when this process is developed; the inferior oblique muscle of the eyeball arises from the orbital floor just wide of this margin (Fig. I6, p. 4I). The lower or nasal opening of the canal lies in the apex of the roof of the inferior meatus and near the junction of its anterior and middle thirds; it is distant from $I 5$ to $20 \mathrm{~mm}$. or $\frac{3}{4}$ inch (average $\mathrm{I} 6 \cdot \mathrm{I} \mathrm{mm}$.) from the fore end of the inferior concha, and from II to $23 \mathrm{~mm}$. (average $17.3 \mathrm{~mm}$.) above the floor of the nasal fossa (Swerschewsky, IgIo). (This opening of the bony canal is not to be confused with that of the contained membranous duct, which commonly lies at a lower level beneath the mucous membrane lining the side wall of the meatus, and the position of which is described on p. 236).

The naso-lacrimal canal lies between the adjacent foreparts of the maxillary sinus and the middle meatus of the nose, projecting into either or both spaces. As regards the former, it has already been noted that the canal usually projects more or less boldly into the inner and forepart of the sinus to form the "lacrimal protuberance" of Zuckerkandl (Fig. I23, p. 234); this columnar prominence extends from the roof of the sinus, where an abnormal ethmoidal cell may present relations to it, to nearly half-way down the medial wall, that is, as far as the line of attachment of the inferior turbinated bone; the thinness of its wall, about I mm., is to be remembered in curetting the sinus; Fein (I9I2) found a lacrimal protuberance well developed in 9 but absent in I7 out of 37 bones, and noted that the alveolus of the first bicuspid tooth may be prolonged to meet it. Part of the maxillary sinus may be related more intimately to the canal by the formation of a " prelacrimal recess" (Killian, Onodi) or " infra-orbital recess" (Zuckerkandl), the upper, inner, and fore corner of the cavity being then circumscribed by the lacrimal protuberance on the 
medial side, with projecting ridges due to the infra-orbital canal laterally, and the anterior superior alveolar or dental canal inferiorly, the roof of the sinus bounding the space above. Out of $2 \mathrm{I}$ maxillae, 5 presented such a recess about as large as a pea, curving round the front of the lacrimal protuberance and separated off from the rest of the sinus by a constricted neck (Whitnall, I9I3) ; its formation and position are well shown in Fig. 32 and are also seen in Fig. I70, p. 326.

On the lateral wall of the nasal cavity, the line of the naso-lacrimal canal, which may be indicated by a protuberance similar to that seen within the sinus, extends downwards with a backward slope from the attached end of the middle concha, as indicated in Fig. 24 and shown in Fig. $3 \mathrm{I}$, and bounds the atrium posteriorly. The relations to this wall should be considered in conjunction with those of the lacrimal fossa given above.

The form, dimension, and direction of the bony lacrimal passages all show considerable variation, chiefly owing to the extent to which the individual bones participate in their formation, in a lesser degree according to the type of skull, and also from the influence of certain intrinsic factors (see p. 235). The form of the passages is shown best by the fusible metal casts taken by Zabel (I90o), as seen in Fig. 34, and is indicated, though less accurately, by the plaster moulds of the interior of the sac and duct prepared by Aubaret (I9IO).

The lacrimal fossa is deepest at its base, where it is confluent with the circular opening of the naso-lacrimal canal ; it.presents an antero-posterior oval section towards its middle and tapers off at its upper end; in the infantile skull it is almost cylindrical throughout, and is extensively overlapped by the posterior lacrimal crest. It is shallower in degree as it is formed in greater extent by the maxilla, and its breadth varies with the shape of the skull, being wider in the brachycephalic and narrower in the dolichocephalic type. The width varies from 4 to $9 \mathrm{~mm}$. (with an average of $6.5 \mathrm{~mm}$. in I22 orbits; Gérard, I907), and its length averages $16.5 \mathrm{~mm}$. in males and a millimetre shorter in 
females (Ledouble, I906). Facial asymmetry explains variation in the two fossae of the same skull, found Io times in 138 orbits by the latter author.

The naso-lacrimal canal is usually flattened from side to side, but is sometimes cylindrical. Gérard found it narrowest at the orbital opening ; Zabel, on the other hand, described a slight constriction towards the middle with dilatations at the upper and lower openings, the planes of which apertures are parallel. The upper or orbital opening is generally oval and much more cleanly cut, especially when defined by the presence of a hamular process, than the wider, almost funnel-shaped inferior or nasal aperture.

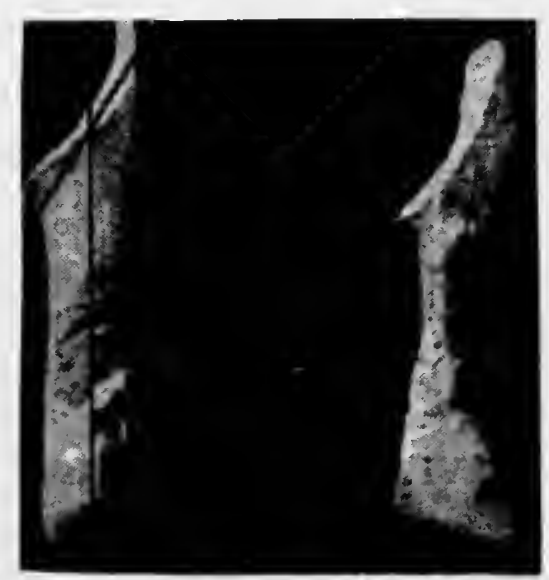

$a$

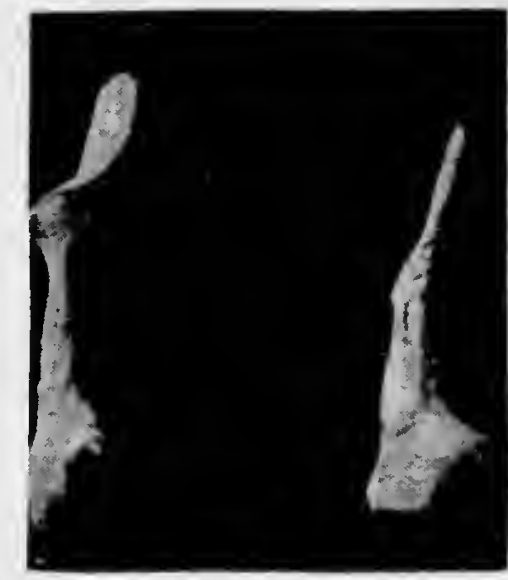

$b$

FIG. 34-Casts of Osseous Lacrimal Passages by Zabel, modified. Taken from right orbits and seen from anterior aspect. About natural size.

$a=$ From two skulls with normal lacrimal bones, showing variation in calibre of naso-lacrimal canal. The black lines show the inclinations of the long axes of the fossa and canal.

$b=$ From two skulls with badly developed lacrimal bones, showing marked reduction in calibre of canal.

The dimensions of the naso-lacrimal canal vary considerably. The length, generally less in dolichocephalic and greater in brachycephalic skulls, is affected by the development of the hamular process at the upper end and by the level of the articulation of the inferior concha at the lower end. From its upper to its lower opening an average length is $12 \cdot 4 \mathrm{~mm}$. or $\frac{1}{2}$ inch, combining the following extreme ranges : Zabel, from $2.5 \mathrm{~mm}$. to $15 \mathrm{~mm}$.; Merkel, from to to I2 mm.; Swerschewsky, from 6 to $15 \mathrm{~mm}$. (average 9.2) ; Schwalbe, from $\mathrm{I} 2$ to $24 \mathrm{~mm}$.; Power, an average of $15.3 \mathrm{~mm}$. in 287 European skulls (see Fig. II6, p. 225).

The height of the upper or orbital opening of the canal 
from the level of the nasal floor was found by Power (I886) to average $27.4 \mathrm{~mm}$. in 292 European skulls; Gérard's figure is $23.3 \mathrm{~mm}$., or nearly an inch, with extremes of 25 and $40 \mathrm{~mm}$. in 69 skulls. Theobald (I9O0) found that the average combined length of the fossa and canal was 30.7 $\mathrm{mm}$. in 286 skulls. The transverse width (the narrower dimension) of the upper orifice of the canal is $4.6 \mathrm{~mm}$., on an average made from the following figures:

(i.) Zabel, from 4.3 to $6.8 \mathrm{~mm}$. (sagittal width from $5^{\circ} 9$ to II.9 $\mathrm{mm}$.).

(ii.) Onodi, from 3 to $4 \mathrm{~mm}$.

(iii.) Merkel, $5 \mathrm{~mm}$.

(iv.) Gérard, from 3 to $6 \mathrm{~mm}$.

(v.) Power, an average of $3.77 \mathrm{~mm}$. in 205 European skulls (with an average antero-posterior diameter of $4 . \mathrm{I} 4 \mathrm{~mm}$.). The right duct was slightly narrower than the left in both dimensions.

(vi.) Swerschewsky, from 3 to $6 \mathrm{~mm}$. (average $5 \cdot \mathrm{I}$ ), and transverse width of lower opening from 4 to $8 \mathrm{~mm}$. (average 7 ).

(vii.) Theobald, 3 to $7 \mathrm{~mm}$. (average $4 . \mathrm{II}$ ) in 70 adult canals measured by rigid probes; with a difference in the measurements on the two sides in I8 cases.

The calibre of the canal appears to be affected by the degree of development of the lacrimal bone, being narrowed when this bone is rudimentary; the extent to which it is then formed by the maxilla compensates for this deficiency. In an examination of 50 maxillae by the writer, it was found that in 3I the lacrimal sulcus formed about three-quarters of the circumference of the canal, the lips being separated by about $4 \mathrm{~mm}$., and the gaps between completed as usual by processes of the lacrimal bone and inferior concha (Fig. $32)$; in these cases the canal was always roomy, an anteroposterior diameter of $8 \mathrm{~mm}$. being found near the middle of several, and there was nowhere any narrowing; in I2 bones in which the lips of the sulcus were only one or two millimetres apart, the canal was a little narrower, but without constriction; in 7 bones the lips met and fused 
(Fig. 35), and the central part of the canal thus formed entirely by maxilla was much narrower, no less than four of these cases presenting a decided constriction of the lumen in this region (Whitnall, I9I2).

Whilst the naso-lacrimal duct enclosed in such a narrowed canal may have been permeable, it is obvious that a slight cause would lead to its occlusion. In these cases the part contributed to the formation of the canal by the lacrimal bone was naturally reduced; and Zabel has noted that in

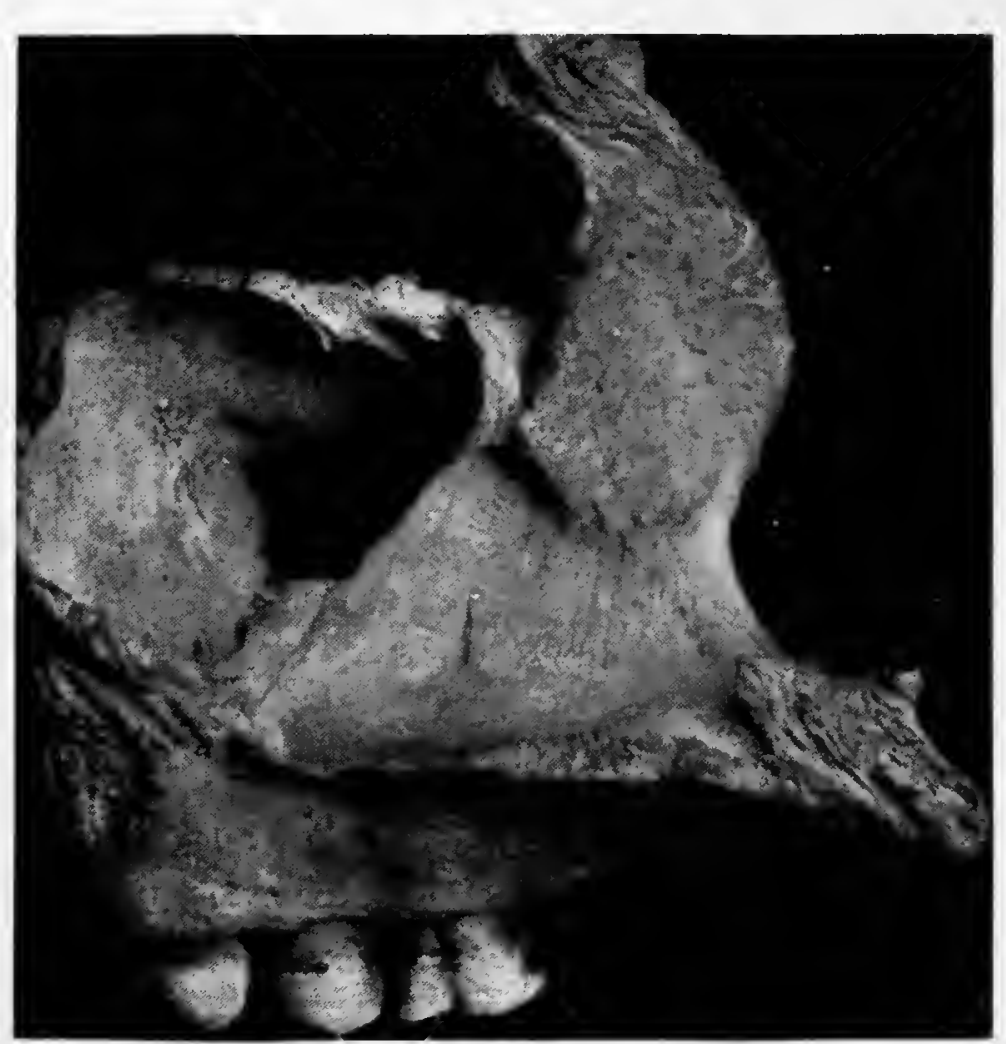

FIG. 35.-Left Maxilla viewed from Nasal Side, showing approximation of lips of sulcus lacrimalis to form completely the mid-part of the naso-lacrimal canal, with constriction of lumen to $3 \mathrm{~mm}$. Compare with Figs. I6, 32 . Natural size. some skulls where the lacrimal bone is rudimentary or absent not only is the lacrimal fossa shallow and small, but the canal is narrowed transversely. In $\mathrm{I} 2$ complete skulls with rudimentary lacrimal bones seen by the writer, 7 presented shallow fossae, with decided narrowing of the orbital openings of the canals. The constriction can well be seen by holding the skull with the vertex towards one and bringing the orifice of the canal into view by glancing over the supraorbital margin, as illustrated in the following figure.

The above dimensions may be compared with those of the membranous lacrimal sac and duct given on p. 237, where Onodi's measurement of the duct in children will be found.

The direction and inclination of the lacrimal passages stand next to the dimensions in importance from a practical point of view; they are dependent on the configuration of 
the facial skeleton, being influenced by the breadth of the bridge of the nose and of the nasal aperture, and by the development of the maxillary air-sinus. Seen in profile, both fossa and canal slope slightly backwards as they pass downwards, the long axis of the canal forming an angle of from I $5^{\circ}$ to $25^{\circ}$ (Testut), with the frontal plane (Figs. 3I, I I9, I22). Seen from in front, the inclinations differ, the fossa showing a lateral deviation inferiorly, whilst the canal on the other
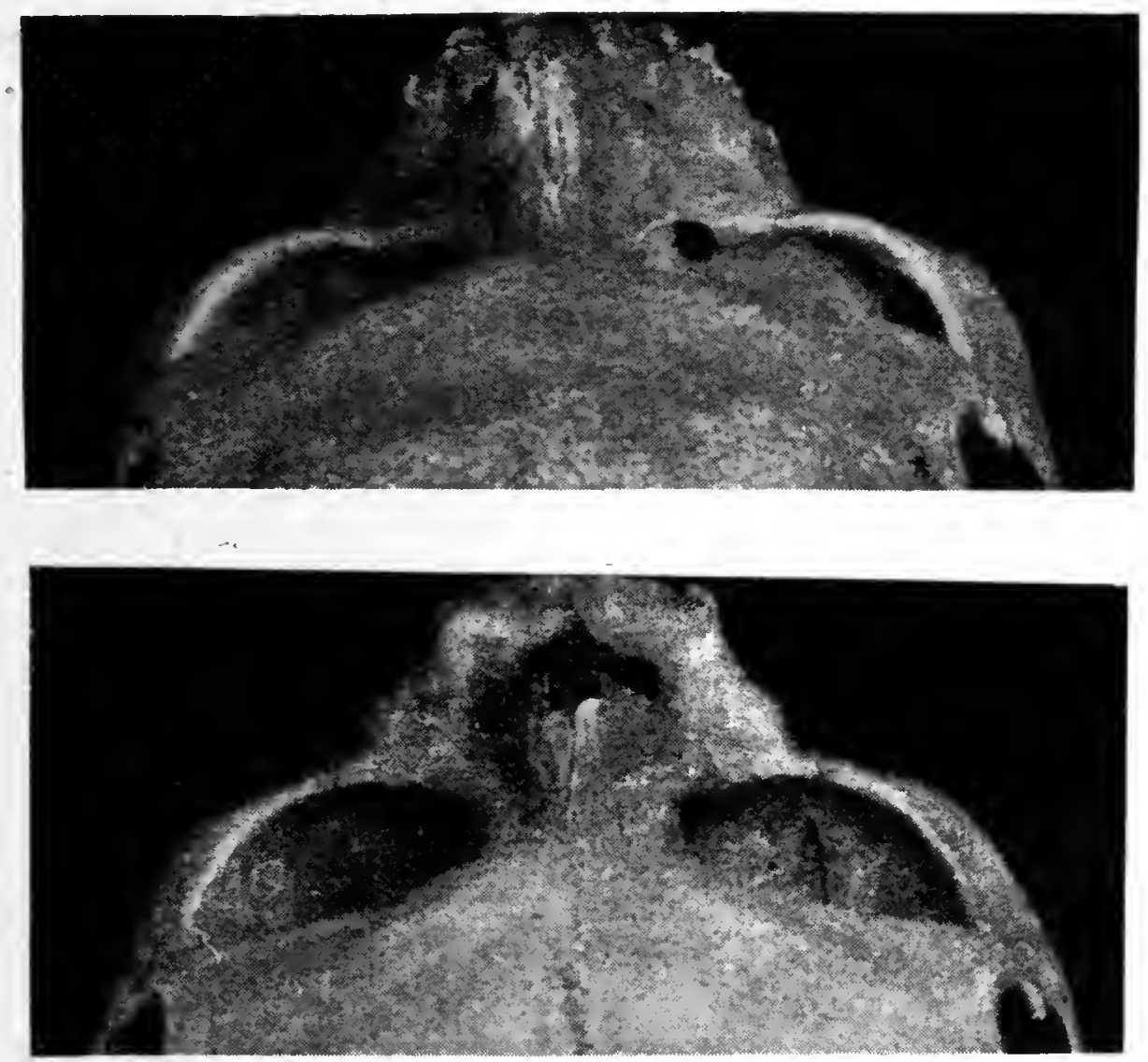

Fig. 36. - Fore Parts of two Skulls, viewed from above to show orbital opening of naso-lacrimal canal, in the upper specimen of normal calibre, in the lower one narrowed. The latter is the skull shown in Fig. 20 with absence of lacrimal bone. $\times \frac{1}{2}$.

hand inclines slightly medially towards its lower end; hence the long axis of the fossa and canal together form an angle, or rather a wide parabolic curve, with a concavity directed towards the nose and a summit near the upper orifice of the canal (Fig. 34a). The lateral obliquity of the medial wall of the fossa is more pronounced in leptorhine or narrow-nosed skulls than in the platyrhine or broad-nosed type; it forms an angle with the median sagittal plane open below and on the average of $26^{\circ}$ (with extremes of $13^{\circ}$ and $37^{\circ}$ ) according to Picou. The long axis of the 
canal forms a similar angle, but open above, and of $12^{\circ}$; the two canals therefore converge inferiorly, in agreement with the observations of Schwalbe, Merkel, Zabel, and Picou, though a divergence of direction is described as normal by Henle, Hyrtl, Arlt, and Onodi. Theobald in 275 European skulls found the canals were parallel in 37 , sub-parallel in II7, converging below in. I2I. The nasolacrimal canal in the negro has been studied by Santos Fernandos (I903), who finds it shorter, broader, and straighter than in white races.

In the living subject, the line of the long axis of the fossa is indicated by the upper part of the naso-jugal fold of the skin (Fig. 8, p. 20; Fig. 48, p. II4; and Fig. I42, p. 265). According to Arlt (I855), the deviation of the canal, which he considers is a lateral one, is shown by a line drawn from the medial canthus of the eyelids to the lower part of the naso-labial fold. In his words (quoted by Onodi), "when the interval between the alae nasi, measured at the place where they pass on to the cheek, is equal to the interval between the middle points of the two medial palpebral ligaments, then there is no lateral deviation of the nasal duct. But when the first-mentioned interval is the wider, as is usually the case, then the lateral deviation of the duct is represented by half this difference. In exceptional cases the alae nasi approach each other so closely that the space between them is less than that between the middle points of the palpebral ligaments. When this is the case the lateral deviation is reversed. The deviation can readily be ascertained by applying a straight sound to the base of the ala nasi below and to the middle of the palpebral ligament above." In life it should be remembered that a probe passed down the lacrimal sac and duct has to accommodate itself to the oppositely inclined long axes of their osseous boundaries, though its passage is no doubt facilitated by the elasticity of the delicate bones forming the medial wall of the channel, which it must touch in the middle of its course. "It is commonly said that probes passed one into each nasal duct meet above on the glabella; this is certainly not often the case during life" (Fisher, I904). 
From this point of view the inclination of the fossa is negligible, since its contained sac is confined laterally by soft and extensible structures, whereas the direction of the duct lying in its osseous canal is of greater importance.

The backward inclination as seen in profile is indicated by a line passing from the medial commissure of the eyelids towards the first molar tooth. The line passes just in front of this tooth, according to Onodi and Testut, but behind it according to Luschka and Merkel. In children the direction is towards the first temporary molar tooth (Onodi).

Of the authors quoted in this section, the work by Onodi (IgI3) will be found of special interest, in that he discusses the various operations performed on the nasal wall of the lacrimal passages, and illustrates their relations to the surrounding parts by numerous photographs of fresh preparations in the child as well as in the adult.

\section{TABLE OF APERTURES OF THE ORBIT}

AND THE STRUCTURES WHICH TRAVERSE THEM

The orbit communicates with the Cranium by the

r. Optic canal, transmitting

the optic nerve;

the ophthalmic artery;

the sympathetic fibres from the carotid plexus;

the orbital prolongations of the meninges as sheaths round the optic nerve.

2. Superior orbital fissure, transmitting

the III., IV., VI., and ophthalmic division of V. cranial nerves;

the ophthalmic vein;

the sympathetic, and sometimes also sensory root of the ciliary ganglion, and some filaments from the cavernous plexus of the sympathetic system ;

the orbital branch of the middle meningeal artery.

3. Ethmoidal canal, anterior, transmitting the internal nasal nerve and anterior ethmoidal artery.

4. Ethmoidal canal, posterior, transmitting the nerve of Luschka and posterior ethmoidal artery; probably also lymphatics through both canals. 
With the Nose by the

5. Naso-lacrimal canal transmitting the naso-lacrimal duct.

Ethmoidal canal (posterior).

With the Face by the

6. Inferior orbital fissure (to infra-temporal fossa), transmitting the infra-orbital nerve and blood-vessels;

the veins connecting the ophthalmic and pterygoid plexuses.

7. Zygomatico-temporal canal (to temporal fossa), transmitting the corresponding branch of the zygomatic or orbital branch of the superior maxillary division of $\mathrm{V}$. nerve, together with an artery.

8. Zygomatico-facial canal (to cheek), transmitting the corresponding branch of the zygomatic or orbital branch of the superior maxillary division of $\mathrm{V}$. nerve, together with an artery.

9. Infra-orbital canal (to cheek), transmitting the infra-orbital nerve and vessels.

Io. Supra-orbital notch and frontal notch (to frontal region), transmitting the branches of supra-orbital nerve and vessels.

It is of interest to note how much more closely connected the orbit is with the brain than are the other cavities of the face. The orbit communicates with the cranial cavity by several apertures; its lining periorbita is a direct continuation of the dura mater, which, together with the other two cerebral membranes, the pia and arachnoidea, are prolonged around the optic nerve into the orbit; the blood comes from the internal carotid artery just before this vessel terminates in branches which supply the brain, and is drained into the cranial venous sinus system; and, finally, the chief contents of the orbit are the globe and optic nerve, outgrowths from the brain.

A distant means of connection between the two orbits is afforded by the cavernous sinuses into which the ophthalmic veins drain, since they are joined together by inter-cavernous sinuses; and possibly also by the sympathetic nerves (p.356).

\section{THE PERIORBITA}

is the name given to the periosteal lining of the orbital cavity. Like the periosteum elsewhere, with which it is 
continuous in many places, it consists essentially of a sheet of dense fibrous tissue supporting the minute nutrient vessels of the bone; it is not of uniform strength throughout, but is exceedingly thin where it lines the roof and comparatively thick at certain other points. It is easily detachable from the surface of the bones, and therefore can be raised from them by sanguineous or pathological effusions; but it is adherent (i.) along the sutures, where in early life it is continuous with the periosteum covering the opposite sides of the bones; (ii.) at the fovea trochlearis, where it binds down the trochlea of the superior oblique muscle; (iii.) at the fissures and foramina through which it becomes continuous with the lining of the cavities into which they lead; and (iv.) at the orbital margin, where it is confluent with the periosteum covering the bones of the face; here it is thickened to form a ridge, the arcus marginale, which marks the exact line of the orbital margin, and from which arises the septum orbitale of the eyelids.

By removing the surrounding bones piecemeal in a formalin-hardened preparation, the whole contents are retained in the periorbita as a cast of the orbit ; the trochlea is left with the membrane, but the lacrimal sac requires severing from the naso-lacrimal duct; posteriorly the continuity of the periorbita with the dura mater of the cranial cavity is demonstrated (Fig. 184, p. 364).

Internally, save for numerous fine loose strands which connect it with the stroma of the orbital fat, muscle sheaths, and lacrimal gland (the last constituting the so-called ligamenta glandulae lacrimalis), the periorbita will be found, on removal of the entire contents of the orbit and so viewing it from within, to line the space smoothly, hiding the sutures, and so continually maintaining the contour of the medial wall as it bridges across the lacrimal fossa to form the fascia lacrimalis that the position of the enclosed sac is only indicated by the severed points of its canaliculi (Fig. II8, p. 228). As in the case of the lacrimal fossa, the membrane not only lines but roofs over the infra-orbital sulcus, and in both these places its deeper osteogenetic layer may develop bone to a variable degree, giving an accentuated posterior 
lacrimal crest in the one case and a more or less complete bony roofing to the infra-orbital sulcus in the other. Further. reference is made to the lacrimal fascia on p. 227.

The blood-vessels of the periorbita are derived from various branches of those which traverse the orbit, and it is innervated by the sympathetic system, possibly through

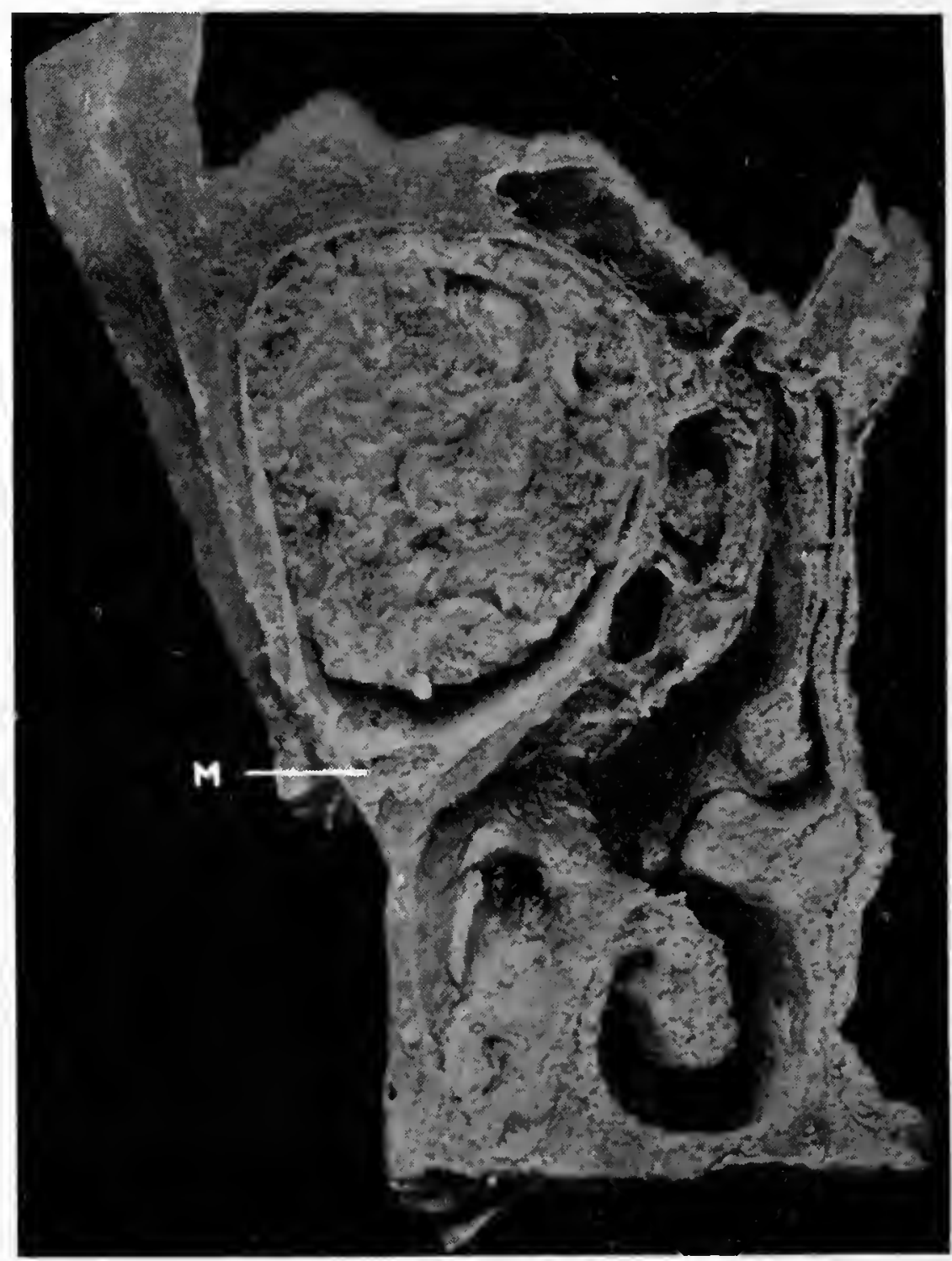

FIG. 37.-Frontal section through the Right Orbit seen from in front, showing the musculus orbitalis or "orbital muscle" of Müller, M., in the inferior orbital fissure, and its connection with the lining periorbita. Natural size.

filaments from the spheno-palatine ganglion, though Gray (I920) figures a supply from a long ciliary nerve.

The periorbita presents a point of especial interest in the region of the inferior orbital fissure, where a mass of smooth.or involuntary muscle fibres is found incorporated upon its superficial aspect. This muscle, described by $\mathrm{H}$. Müller in $185^{8}$, is known as the "orbita! muscle of Müller," or musculus orbitalis, and should not be confused with the 
involuntary palpebral muscles also discovered by him. The term " periorbital muscle" is suggested as a better name. It completely fills the inferior orbital fissure, and is narrow, but two or three millimetres deep, in the centre, and fanshaped at the extremities, where it spreads in a thin layer over the orbital floor; its position and extent are indicated in Fig. 26, p. 59. Anteriorly it is continuous, according to Charpy (I9I2), with the orbital expansion of the inferior oblique muscle, a relation the writer has been unable to confirm ; posteriorly it can be followed beneath the annulus of Zinn to the lower extremity of the superior orbital fissure, and even up to the wall of the cavernous sinus; whilst inferiorly it is in contact with the fat of the pterygopalatine fossa (Hesser, I9I3). Through it there pass only small venules and nerves; some of its fibres accompany the veins out of the orbit (W. Krauss, I9II), and the inferior ophthalmic vein is related to it beneath the annulus of Zinn (see Fig. I6I, p. 3Io). Like other vestigial structures, it shows much variation in size.

It is of twofold interest: first, in that it represents in man the larger muscular sheet (membrana orbitalis musculosa) which completes the lateral wall of the orbit in lower animals where the bone is absent. It exists as a smoothfibred muscle in all mammals save in the aquatic group, where it is cross-striped as in amphibians, lizards, and birds, and where, according to Groyer (I903), who, like Burkard (I902), has studied it from the point of view of comparative anatomy, it appears to play an important rôle as an organ accessory to the lacrimal apparatus. Its function in this respect is not clear, and any use it may have in animals beyond keeping tense the orbital wall is to protrude the eyeball, since Müller found that irritation of the cervical sympathetic nerve causes protrusion of the globe in beasts where the muscle is well developed, and in them it is antagonised by a special " retractor bulbi " muscle. In man it is purely vestigial, and excitation of the nerve does not cause protrusion of the globe (Turner, I862) ; moreover, a retractor bulbi muscle is not developed. The second point of interest is a pathological one, an explanation of, the 
exophthalmos of Basedow's disease being sought in the relation of the periorbital muscle, firstly, to the orbital contents as a whole, and secondly, to the venous system. It is hardly possible either that contraction of such a small muscular mass, for the most part deeply confined in the inferior orbital fissure, as seen in the above figure, and elsewhere blended with the closely adherent periorbita, could affect the position of the eyeball by compression of the mobile orbital fat (i.e. that the muscle in its vestigial condition could retain the possible action of its full development), or that occlusion of such venules as traverse the muscle could affect the volume of the orbital venous system which communicates so freely with the vessels of the face. W. Krauss (I9I2), who has studied the muscle in the newborn, and Fründ (IgI2) consider both these explanations as sufficient. Hesser (I9I3) expressly denies that an exophthalmos could be produced by compression of the veins, since only in exceptional cases did he find either the inferior or superior ophthalmic vein in a position to be so affected, and he agrees that the free anastomoses between the veins of the orbit and those of the face would obviate the questionable engorgement. It may be of interest to note that other explanations of the condition are : contraction of the peribulbar involuntary musculature (Landström, I9o8; see p. 296), an orbital oedema (Sättler, I9II), hypertrophy of the retro-ocular tissue (Basedow), elongation and relaxation of the ocular muscles (Cooper, Egeberg, Lemke, etc.); but the most probable cause of the condition is dilatation of the orbital blood-vessels by excitation of the sympathetic nervous system (Fuchs, I9I7). The exophthalmos is said to disappear after death, and in a dissection of two orbits taken from a subject who had died from the disease and had presented the well-marked sign, nothing abnormal could be found by the writer.

\section{MENSURATION}

The dimensions of the orbit considered here are the volume, the size of the entrance, the depth and the length 
of the walls, and certain angles; measurements of the smaller features have been given in the text.

Since the form of the orbital cavity varies according to race, age, sex, and in individuals, and since the exact points from which the dimensions are taken are neither identical nor definitely specified in all cases, it is not surprising that there is a great lack of agreement in the figures given by different authors. In measuring the orbit, it is of primary importance to fix the points clearly; they should, as Gayat notes, be well marked, easily recognisable in ancient as well as in recent skulls, constantly present at all ages, and the progress of growth should neither accentuate some nor obscure others. It is to be remembered also that the presence of the-periorbita makes a considerable difference in the measurements, it being stated that the depth, for instance, is diminished by from 3 to $6 \mathrm{~mm}$. after removal of the membrane; further, it is hardly necessary to point out that great accuracy is required, since a difference of one millimetre in the height converts an orbital index of .86 .3 into 88.9 or $83^{\circ} 7$, and one millimetre difference in the width converts the index into $84^{\cdot} \mathrm{I}$ or 88.6 , according as the dimension is increased or decreased by that amount.

The volume has been obtained by filling the orbit with lead pellets, by which means Gayat (I873) found it to be 22 c.c. in a child of ten, and 29 c.c., with extremes of 25 and 33 , in eleven adult skulls; Weiss gives 29.74 c.c. for adults. Relative to the size of the whole skull, it is larger in females.

The dimensions of the orbital entrance or base are conveniently obtained by measuring along threads fixed across it ; the vertical diameter or height should be parallel to the lateral and medial margins, and the horizontal diameter or width parallel to the upper and lower margins; these two lines are nearly at right angles to one another, but, owing to the greater projection of the supra- and infraorbital margins, they do not touch where they cross, but are separated by an " axial distance," which varies from 2 to Io $\mathrm{mm}$. (Ambialet, I905). The line of height at its upper extremity falls usually to the lateral side of the supraorbital notch, but should they coincide the distance is taken 
to an imaginary line crossing the edges of the groove; inferiorly a point should be chosen which avoids the undue prominence of an infra-orbital tubercle at the suture. The line of width is on a level with the lateral orbital tubercle (p. I3), so being opposite the commissure of the eyelids, and if the margin here presents no distinct edge, one can be indicated by drawing the flat of a lead-pencil down it; the point

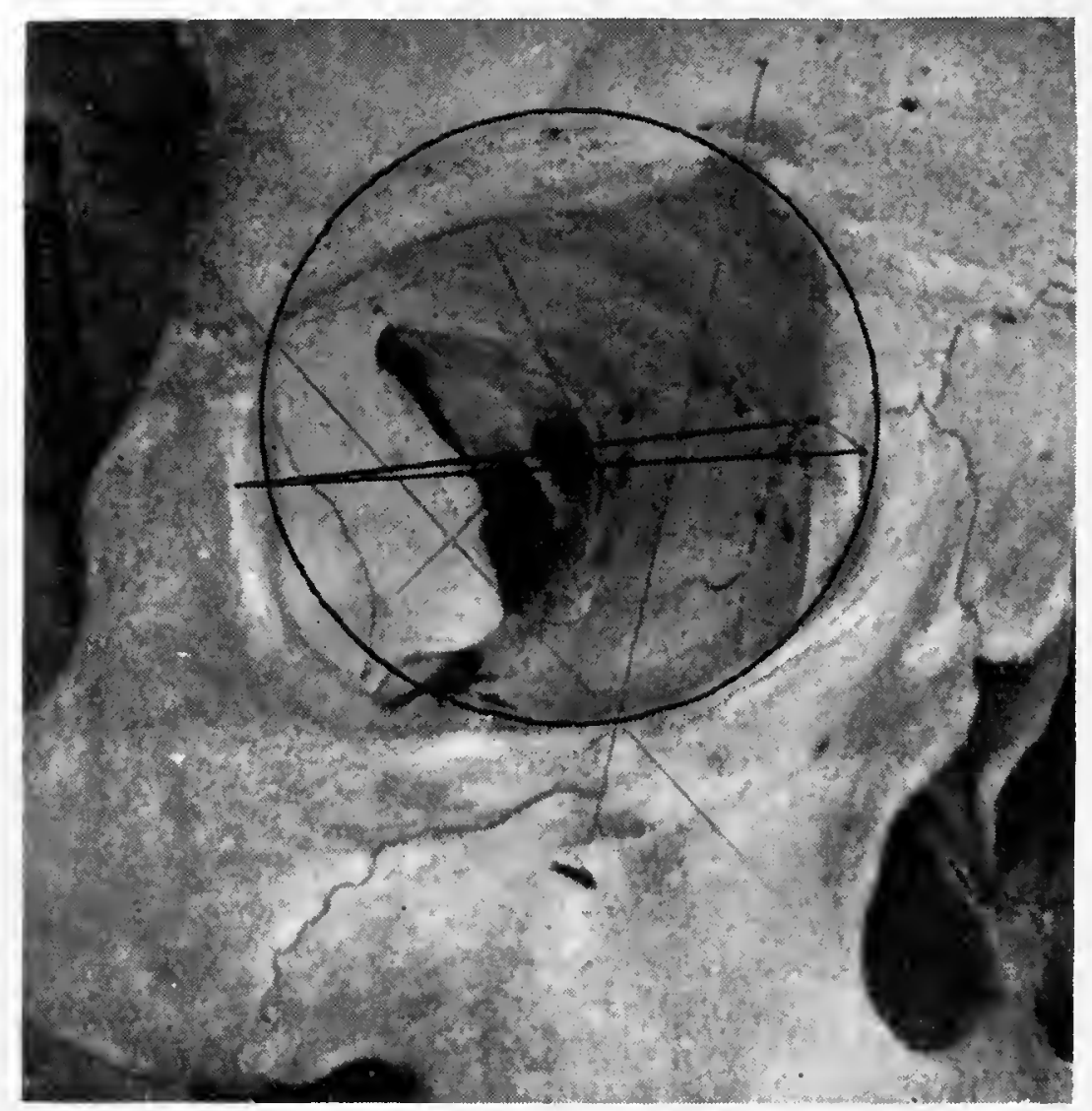

FIG. 38. - Right Orbit to illustrate $(a)$ the difference in orbital width $(2.9 \mathrm{~mm}$.) according as taken to Flower's lacrimal point (upper horizontal line), or to the point dacryon (lower line) on the medial side. The orbital index in the former case is $86 \cdot 3$, and in the latter $80 \cdot 3$. (b) The figure also indicates the directions in which the primitive circular outline of the orbital margin has been altered by growth in the surrounding parts. The circle is drawn from a central point equidistant from the supra-orbital notch, the zygomatico-frontal and the zygomatico-maxillary sutures.

where it abuts on the medial margin is less easy to define owing to the presence of the fossa for the lacrimal sac: Topinard measured to the upper part of the anterior lacrimal crest, the width thus being affected by the configuration of the frontal process of the maxilla and the nasal bones at the root of the nose; Broca chose the point dacryon, where the upper end of the lacrimo-maxillary suture meets the frontal bone, but whilst marking accurately the centre of the orbital margin, it is often obscured by fusion of 
the bones. Flower marked a "lacrimal point " further back, where the upper end of the posterior lacrimal crest meets the frontal bone, and although its position is affected by the degree of projection of the underlying ethmoidal cells, yet this variation is shared by the orbital wall and gives the dimension a truer value as indicative of the width of the forepart of the cavity. The exact point taken is important, since a difference of as much as three millimetres may exist between the above three points in the horizontal direction (Fig. 38), so affecting the orbital index.

The height of the orbital entrance is approximately $35 \mathrm{~mm}$. or $\mathrm{I} \frac{3}{8}$ inch, and the width $40 \mathrm{~mm}$. or $I \frac{1}{2}$ inch, but precise figures vary greatly-the height from $30 \mathrm{~mm}$. (Arlt) to $40 \mathrm{~mm}$. (Richet, Luschka), and the width from 36 to $50 \mathrm{~mm}$. as given by the same authors. According to Baker (I900), the figures given by Emmert (I880) are on the whole the most extensive, and were very carefully taken in a series of skulls free from abnormalities; the race, however, is not noted. His figures are :

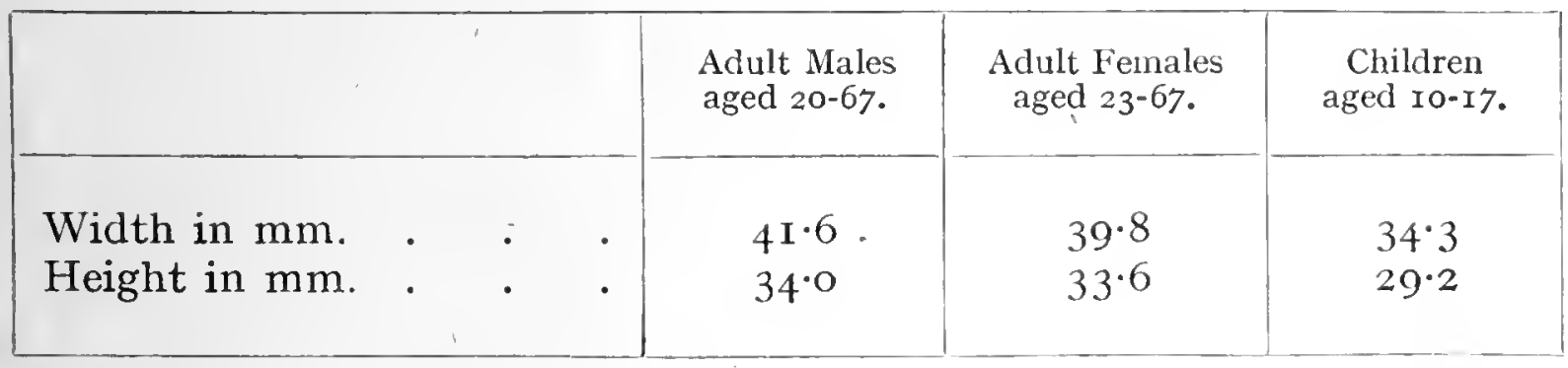

Other figures are :

\begin{tabular}{|c|c|c|c|c|c|}
\hline & De Wecker. & Arlt. & Legrange. & Richet. & Weiss. \\
\hline $\begin{array}{l}\text { Width in } \mathrm{mm} \text {. } \\
\text { Height in } \mathrm{mm} \text {. }\end{array}$ & $\begin{array}{l}39 \\
35\end{array}$ & $\begin{array}{l}36 \\
30\end{array}$ & $\begin{array}{l}40 \\
35\end{array}$ & $\begin{array}{c}4^{0-4^{6}} \\
40\end{array}$ & $\begin{array}{l}39 \cdot 7 \\
35\end{array}$ \\
\hline
\end{tabular}

The distance from the lateral margin of the one orbit to that of the other (i.e. the extra-orbital width) is given by Emmert as $99^{\circ} 7 \mathrm{~mm}$., or 4 inches, in males, $96 \mathrm{~mm}$. in females, $80.8 \mathrm{~mm}$. in children, and the inter-orbital width at the margins is, according to Broca, $25 \mathrm{~mm}$.; diagonal together with other measurements are given in the table on p. 99. 
At birth the height of the orbital entrance is almost equal to the width, but during growth the width predominates, more in man than in woman, and, it is interesting to note, more or less according to race. Broca gave this relation a mathematical value by expressing the proportion of the orbital height to the orbital width, using the formula :

\section{Orbital height $\times$ Ioo
Orbital width}

the result being termed the orbital index; for example, in the skull illustrated in the frontispiece :

Marginal height $33.4 \times 100$

Marginal width $\frac{334 \times 100}{38 \cdot 7}=86 \cdot 3$ the orbital index.

Three classes of orbit are obtained by this means :

Megaseme, with an orbital index of 89 and over; this high numerical value is remarkably constant in the yellow races, excepting the Esquimaux, and the opening is usually round (Fig. 45, second skull, p. I03).

Mesoseme, with an index between 89 and 83 , found in the white races, where Flower gives a mean figure of 87 for 208 European and $88 \cdot 4$ for 33 English skulls.

Microseme, with an index of 83 and less, characteristic of the black races, where the opening is rectangular (Fig. 39).

Cameron (I920) has devised a composite " naso-orbitoalveolar index," according to which he divides modern races into two great groups, to which, broadly speaking, he applies the terms Eurasiatic and Negro. The orbital measurement concerned is the height from the level of the inferior orbital margin to the point nasion.

The vertical height is proportionately greater in the yellow and in females of all races. The orbital index is greater in infants than in adults of either sex, and is higher in females than in males. This character is of anthropological interest, and will be found fully considered in the works of Topinard (1885), Broca (1875), and Flower (1907). Duckworth (I904), however, concludes that its range of variation is too great to render accurate information in most instances, since so many different records exist, and it has been accorded quite a secondary place in the list of selected indices. 
Broca states that the difference due to sex alone amounts to as much as $3 \mathrm{I}$ per cent of the total racial variation.

The depth of the orbit and the length of its walls. - The point at the apex from which these dimensions are taken is best considered to lie at the centre of the bridge of bone which separates the optic foramen from the superior orbital

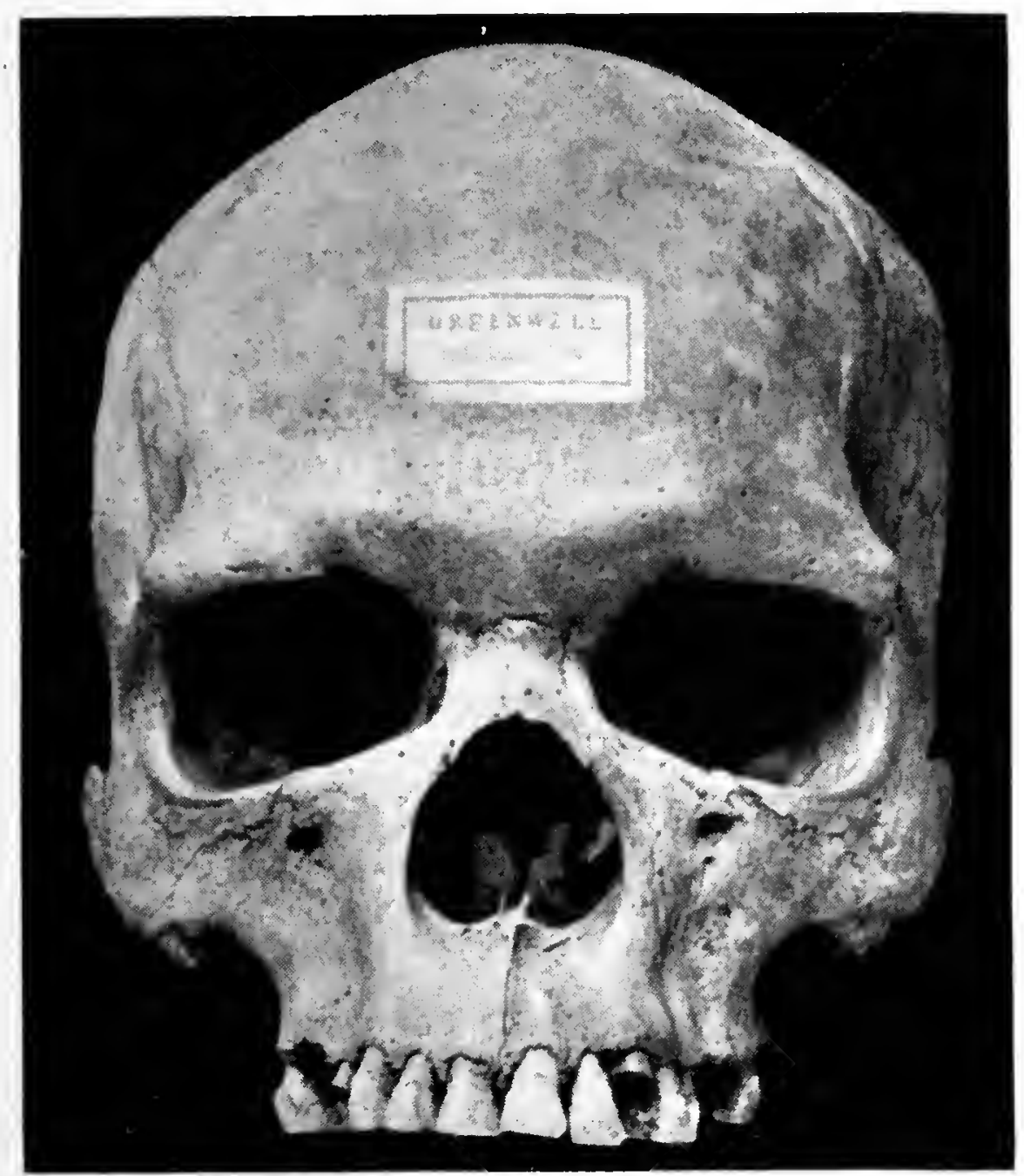

FIG. 39. - Australian Skull, showing rectangular orbital margin. Orbital height, $27 \mathrm{~mm}$.; orbital width (to Flower's point), $39 \mathrm{~mm}$. Orbital index, 69.2. If the width is taken to the point dacryon $(42 \mathrm{~mm}$.), the index becomes $64 \cdot 3$. The orbital height is unusually low. $\times \frac{1}{2}$.

fissure; this point is accurate in that it is situated in the middle of the annulus of Zinn from which the recti muscles of the globe arise, and lies between the optic nerve and the bulk of the other nerves which enter the orbit; and it is convenient, since it can be straddled by the forked extremity of a graduated rod along which a cross-piece may' be slid against the desired point on the margin and the distance between them read off, as suggested by Gayat. This is the central point of the apex or "apical point" of the 
orbit, and it is separated from its fellow of the opposite side by $25 \mathrm{~mm}$. or an inch.

The orbital depth is usually measured to various points on the margin, though actually such figures give the length of the corresponding walls or the distance of the anterior opening of the optic canal from the margin; theoretically, the true depth is that of the long axis measured to the centre of the axial distance (i.e. to the mean of the distance which separates the centre of the vertical from the centre of the horizontal plane of the entrance), but practically the centre of the vertical plane or marginal height is easier to define, and, moreover, corresponds to the normal position of the most prominent part of the cornea.

The figures given show again much variation: the orbital depth is about $40 \mathrm{~mm}$. or $\mathrm{I}_{\frac{1}{2}}$ inch, but the extremes in literature are from $39 \mathrm{~mm}$. (Merkel) to $50 \mathrm{~mm}$. (Richet, Tillaux); Emmert's distances of $39.8 \mathrm{~mm}$. in males, 39.4 $\mathrm{mm}$. in females, $34.75 \mathrm{~mm}$. in children are taken to the centre of the breadth of the entrance; Merkel's figure of $39 \mathrm{~mm}$. is taken to where the breadth and height cross; Arlt gives $42 \mathrm{~mm}$.; Gerlach $43 \mathrm{~mm}$.; De Wecker and Lagrange 40-50 $\mathrm{mm}$.; the depth is less in females.

The walls are longer on the temporal side and floor than on the nasal side and roof; their lengths from the apical point to the margin (which, it may be noted, are measured in a straight line and do not follow the curves) give also the distance of the optic foramen from the latter. The figures which follow are in millimetres:

Medial wall :

Emmert : $4 \mathrm{I} \cdot 4$ in males, $40 \cdot 3$ in females, 36 in children (apical and marginal points not stated).

Gayat : in $5^{6}$ adult skulls, $5^{0} \cdot 6$ from apical point to anterior tip of fovea trochlearis and $47 \cdot 4$ to crest of lacrimal bone.

Roof :

Broca : $50 \cdot 9$, from apical point to supra-orbital margin.

Zanda and Geissler (Gayat), 53.

De Wecker (Testut), 43 from optic foramen to centre of margin ; 40-4I from optic foramen to supero-medial angle of margin; 43 from optic foramen to supero-lateral angle. 
Lateral wall :

Emmert : $46 \cdot 4$ in males, 46 in females, $39 \cdot 4$ in children.

Gayat: $48 \cdot 2$ from apical point to zygomatico-frontal suture. Floor :

Gayat: $49 \cdot$ I from apical point to zygomatico-maxillary suture. De Wecker (Testut) : 46 from optic foramen to centre of margin. Zander and Geissler (Lagrange): 53 from optic foramen to margin ; 47 from optic foramen to medial angle ; 56 from optic foramen to lateral angle.

The dimensions of the right orbit in the adult male skull shown in the frontispiece, and used as type in Figs. 9 , I4, 38 , and 44 , are :

Orbital height, $33.4 \mathrm{~mm}$. ; orbital width to lacrimal point, $38.7 \mathrm{~mm}$.

(to dacryon, $4 \mathrm{I} \cdot 6$ ).

Orbital index, $86 \cdot 3$.

Lateral wall, $47 \mathrm{~mm}$. long; floor, $53 \mathrm{~mm}$. long; roof, $5 \mathrm{I} \cdot 5 \mathrm{~mm}$. long. Medial wall, length to dacryon, $39 \mathrm{~mm}$. ; to anterior lacrimal crest, $45 \mathrm{~mm}$.

Anterior end of inferior orbital fissure to orbital margin, $16 \mathrm{~mm}$. Anterior end of superior orbital margin to orbital margin, $29 \mathrm{~mm}$. Inter-orbital width between dacryon on each side, 2I $\mathrm{mm}$.

Inter-orbital width between lacrimal point on each side, $25.3 \mathrm{~mm}$. Extra-orbital width between lateral orbital margins, $98 \mathrm{~mm}$.

In the female skull illustrated in Fig. 44 the measurements are :

Orbital height, $34 \mathrm{~mm}$.; width to lacrimal point, $35.8 \mathrm{~mm}$.; orbital index, $94 \cdot 9$.

Length of roof from apical point, $45 \mathrm{~mm}$. ; floor, $43.5 \mathrm{~mm}$. ; lateral wall, $42 \mathrm{~mm}$.

Length of medial wall from apical point to posterior lacrimal crest, $34 \mathrm{~mm}$. ; to the dacryon, $36 \mathrm{~mm}$.

The angles to be described are those of $(a)$ the planes of the orbital entrances ; $(b)$ the walls; $(c)$ the long axis of the cavity.

(a) The horizontal planes of the two orbital entrances form an angle with one another, the summit of which is placed at the root of the nose and the extremities at each margin just below the lateral angular process of the frontal bone. This "naso-malar" angle is given by Flower as $\mathrm{I} \mathrm{I}^{\circ}$ in $\mathrm{I} 30$ Europeans, and is much more open in yellow 
races, where the root of the nose is flattened. Weiss gives about $\mathrm{I} 40^{\circ}$, Lagrange $\mathrm{I} 45^{\circ}-\mathrm{I} 50^{\circ}$. Merkel gives angles of $\mathrm{I} 44^{\circ} \cdot 6$ in children, $\mathrm{I} 46^{\circ} .5$ in adult females, $\mathrm{I} 47^{\circ}$ in adult males. Evatt (I907) has devised a means of plotting the position of the orbital entrances on paper by joining up

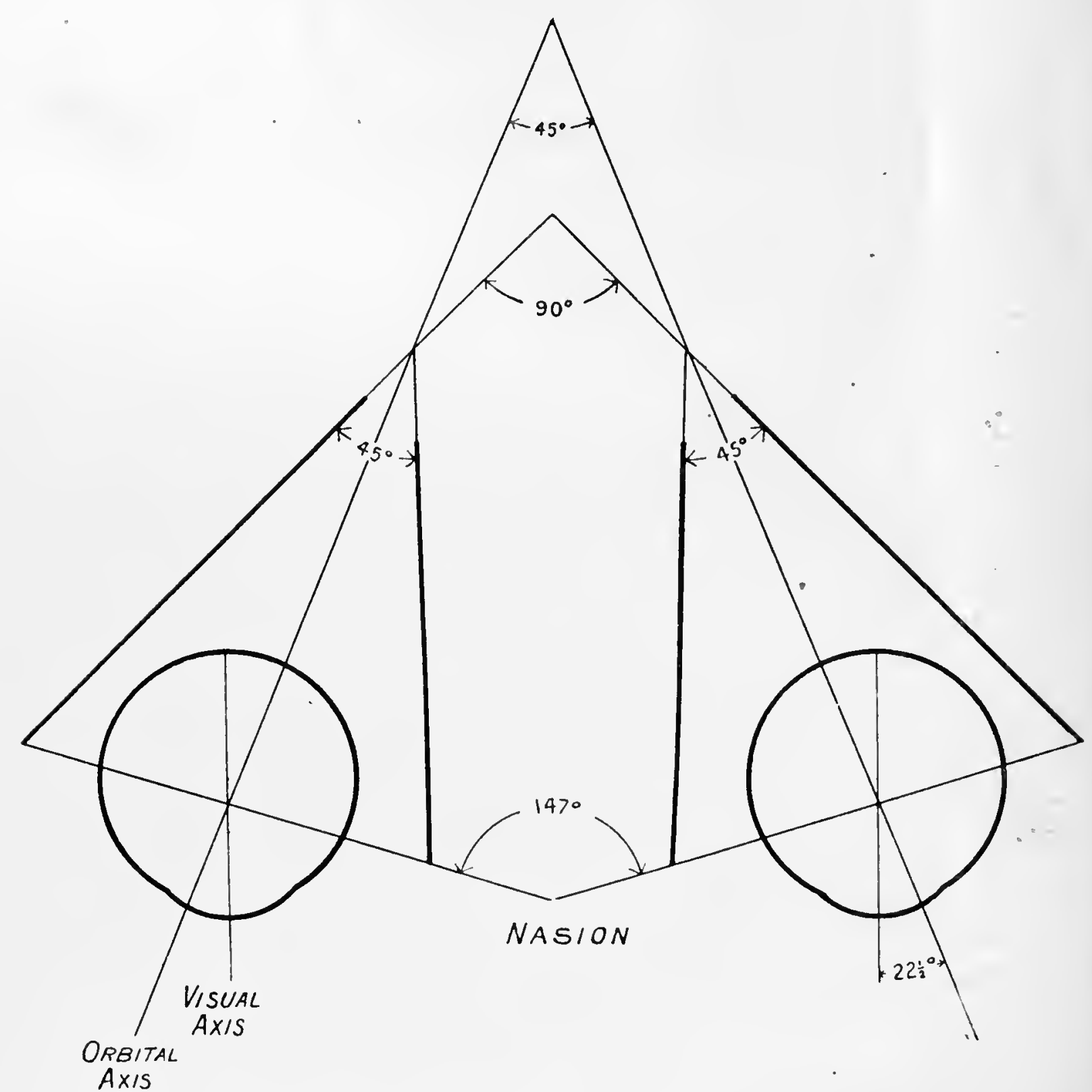

Fig. 40. - Linear Reconstruction of Horizontal Section through the Orbits, to illustrate the angles formed by the walls. Compare with Figs. ${ }_{5}$, p. $3^{6}$, and I 30, p. 252. Natural size.

points on the zygomatic bone just below the lateral marginal suture with the lacrimal points; he thus obtains a "basic angle," which is another measure of their obliquity, and classifies "ithybasic," "mesobasic," and "loxobasic" types accordingly.

(b) The angles formed between the lateral and medial walls are from $45^{\circ} \cdot 9$ to $48^{\circ} \cdot 6$ (Lagrange), and between the 
lateral wall of each orbit, from $87^{\circ} \cdot 4$ to $90^{\circ} \cdot 6$ (Lagrange), or $89^{\circ} \cdot 9$ in males, $89^{\circ} \cdot 9$ in females, $87^{\circ} \cdot 4$ in children (Emmert). The two medial walls are rarely parallel (only in II cases out of Ioo; Merkel, I9OI), but are usually about $3 \mathrm{~mm}$. wider apart behind (see Fig. I30, p. 252).

(c) The long axis of the orbital cavity is level in infants, but in adults is depressed anteriorly to form an angle of $15^{\circ}$ to $20^{\circ}$ with the horizontal plane (Fig. I8, p. 46). With its fellow of the opposite side it forms rather less than half a right angle, or more precisely an angle, according to Emmert, of $43^{\circ} .4$ in males, $44^{\circ} .7$ in females, $42^{\circ} \cdot 4$ in children; other figures given are $40^{\circ} \cdot 6$ to $44^{\circ} \cdot 7$ (Lagrange), $40^{\circ}$ (Poirier), $46^{\circ}$ (Testut). The orbital and ocular axes therefore do not coincide, but differ by $18^{\circ}$ (Testut), or by $20^{\circ} \cdot 3$ to $23^{\circ} \cdot 2$ (Lagrange), or $23^{\circ}$ to $25^{\circ}$ (Druault). The distances between the orbital axes at their anterior extremities is given by Emmert as $60 \mathrm{~mm}$. in males, $58.3 \mathrm{~mm}$. in females, $48 \mathrm{~mm}$. in children. These axes and angles are illustrated in Fig. 40 opposite.

As regards the measurements given by the various authors cited above, it is noteworthy that, according to Adachi (I904), they were made upon only 20 skulls by Merkel and Kallius, 25 by Emmert, Ioo European by Weiss. Adachi himself, in a most careful and detailed examination of Japanese orbits, took 94 skulls of that race and 30 European.

\section{DEVELOPMENT AND GROWTH}

Towards the end of the third week of foetal life five processes arise from the base of the primitive cerebral capsule, and by the end of the second month they have united to form the facial part of the head. Of these processes, two are placed on each side and are called the maxillary and mandibular, whilst the remaining process, the nasal (or fronto-nasal), is median in position, and is composed of symmetrical right and left halves, each of which subdivides later into lateral and mesial nasal processes. The chief structures developed from the original five pro- 
cesses are the nose from the nasal, the cheek and upper jaw from the maxillary, and the lower jaw from the mandibular (see Figs. 9I, 92, p. I84).

The orbital walls are formed superiorly by the capsule of the fore-brain, in which the frontal bone is developed, laterally and inferiorly by the maxillary process, in which the

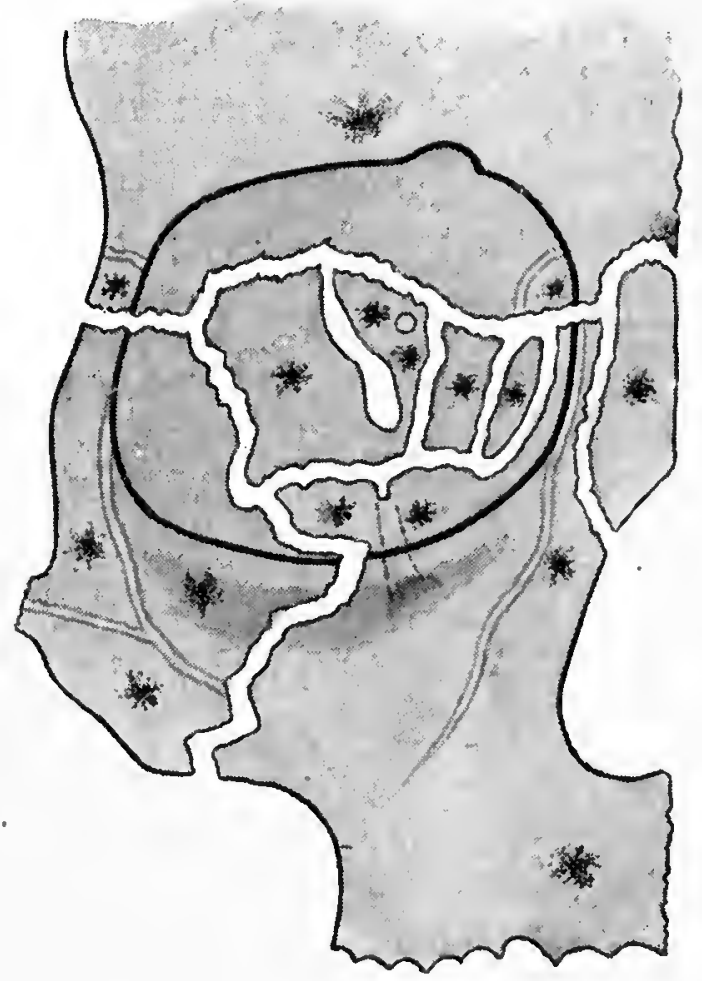

FIG 4r.--Diagram to show the approximate position and number of centres of ossification in the bones which form the Orbit. The centres appear between the sixth and eighth weeks of foetal life, and, except for the sphenoidal, have fused into the component bones before birth $\left(6\right.$ th to $7^{\text {th }}$ month). In very rare cases one of the secondary centres may remain ununited. zygomatic bone and maxilla (with the exception of its frontal process) arise, and medially by the lateral nasal process, which gives origin to the frontal process of the maxilla, nasal, lacrimal, and lateral mass of the ethmoidal bones. Posteriorly the orbit is completed by a separately developed part of the sphenoidal bone derived from the base of the skull; between the primitive pre- and orbitosphenoidal parts of this bone the optic nerve enters the cavity, and gaps left. on each side of its greater wing form the orbital fissures. The nasolacrimal canal is formed between that part of the lateral nasal process which gives rise to the frontal process of the maxilla and the maxillary process. The approximate number and positions of the centres of ossification are given in Fig. 4I above.

Primitively the orbital boundaries must tend to be moulded upon the optic cup, the precursor of the eyeball, since this is formed before the matrices of the bones are laid down; in foetal skulls the margin, for example, is circular up to the age of six or seven months. Later on the various adnexa of the globe cause the orbital shape to be modified in the regions they occupy in the adult. The 
globe, lacrimal apparatus, and oblique muscles may therefore be considered to be the chief internal factors which determine the form of the orbital cavity; there are also external influences to be noted due to the growth of the surrounding parts, but their effect is more marked after birth. In agreement with the precocious development of the eyeball the dimensions of the orbit are considerable relative to those of the other cavities of the face, and seem to be correlated also to the size of the cranial cavity Even so, the

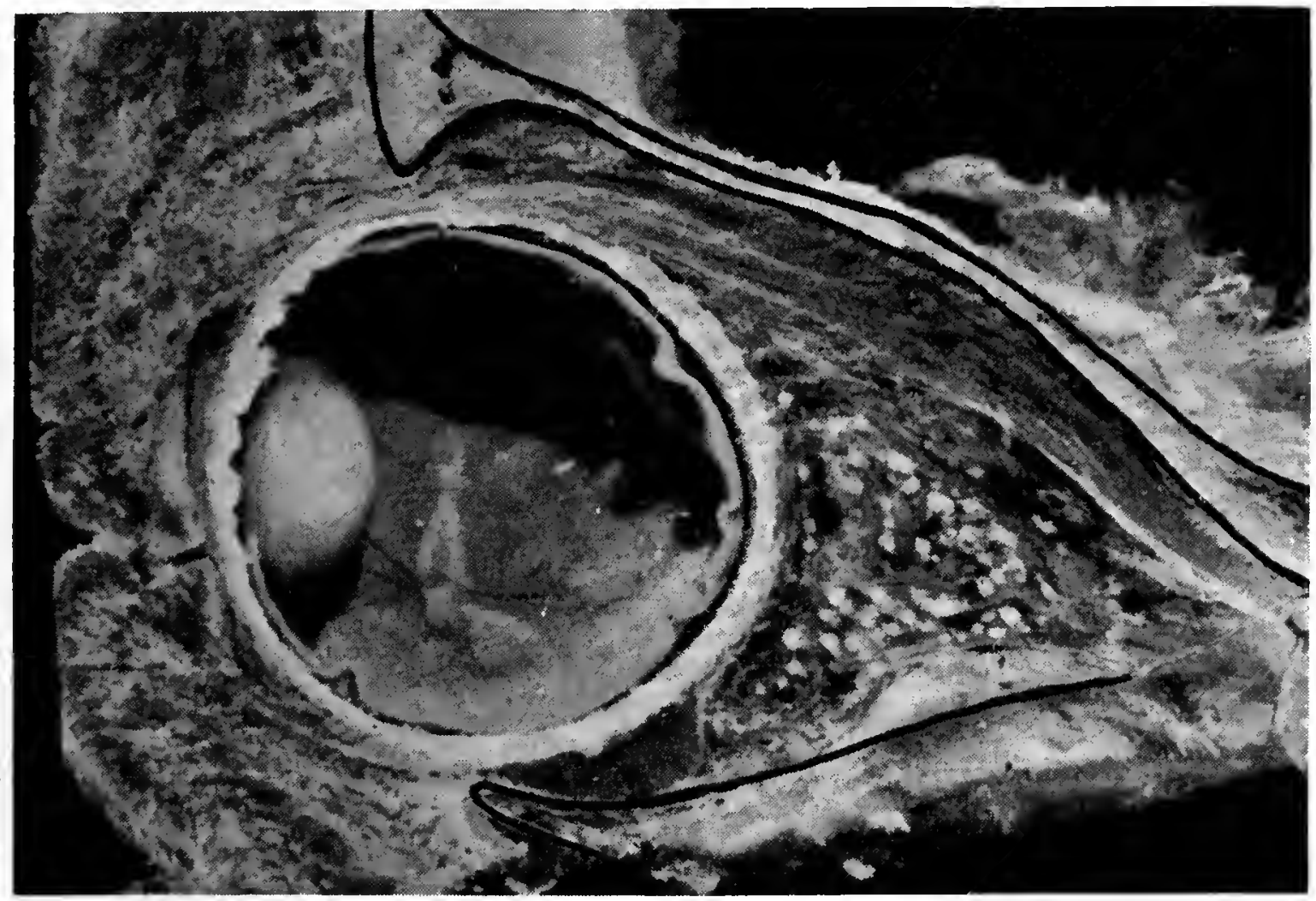

FIG. 42. - Sagittal section through the Orbit of a 6-7 months' foetus, showing the small extent to which the walls enclose the globe. Note the thickness of the eyelids. $\times 4$.

(The hyaioid artery is faintly seen, but throws a well-marked shadow on to the retina below.)

orbit at first is outpaced in growth by the globe, as seen in the transverse section of a six-months' foetal head in Fig. 42, where the margin is closely applied to, and only extends as far as, the equator of the globe, instead of arching well over and standing away from it as in the adult.

At birth the chief features to be noted are as follows: the large size of the orbital entrance, its sharp and strongly developed margin, and the close position of the infraorbital foramen below it. The infantile margin naturally conforms to that of the primitive form, fitting closely round 
the globe, and the development of the surrounding parts has not yet affected it to an appreciable extent; the circular outline tends to be retained, or to recur, during early life up to puberty, after which time the changes occurring in the secondary period of skull growth affect this region to a much more marked degree than the cavity itself. The height and breadth of the entrance at birth are almost equal. The top of the nasal aperture bears the same relation

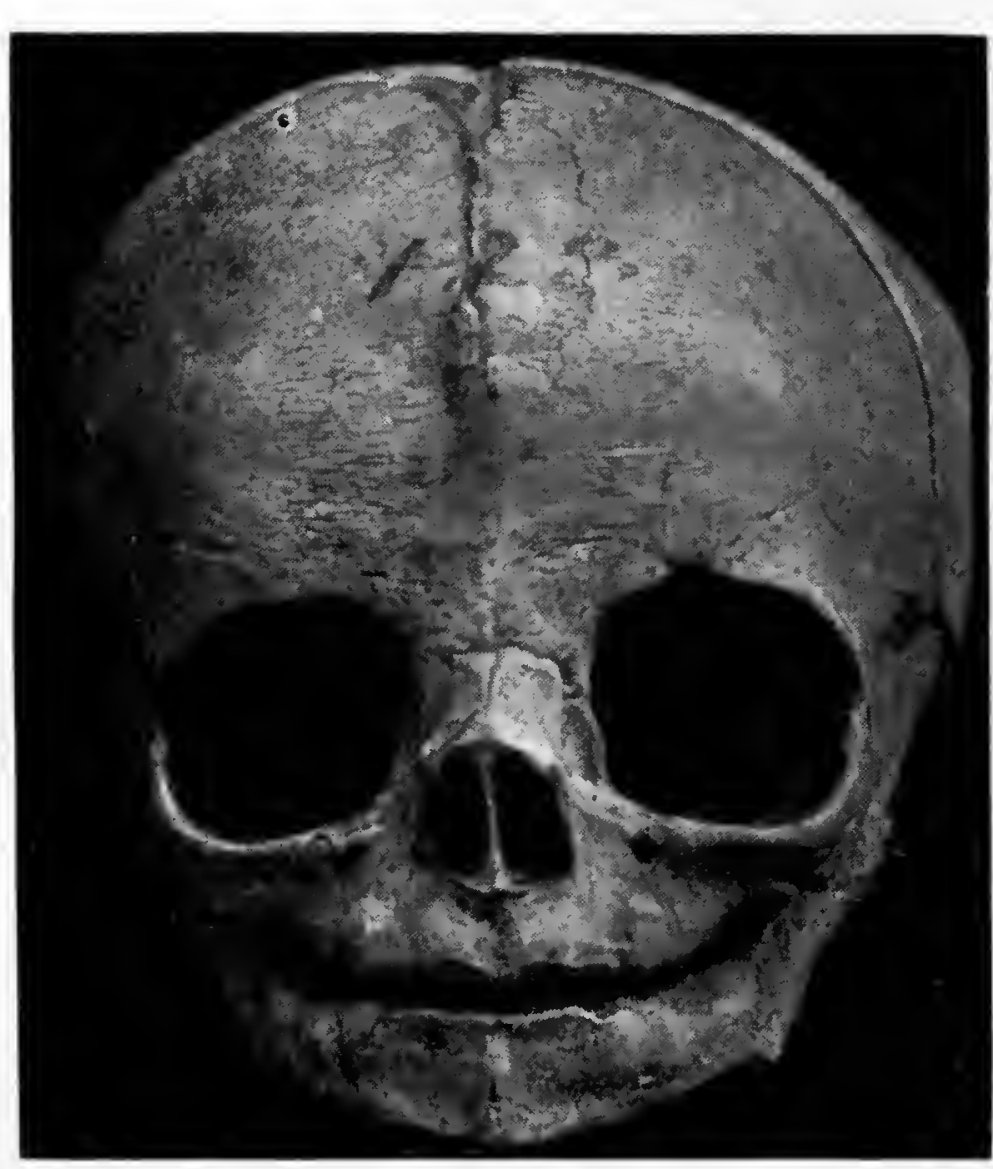

FIG. 43.-The Skull at Birth. Note the broad posterior wall of the fossa for the lacrimal sac and the accentuation of the posterior lacrimal crest. $\times \frac{2}{73}$.

to the orbit at all ages, but its lower border is nearly on a level with the infra-orbital margin at birth, and is much below it in the adult. The orbit bears nearly the same proportion to the cranium at all ages, but at birth the marginal height is about half that of the face, and in theadult rather less than one-third; as the face grows, the vertical diameter of the orbit increases rapidly, so that, according to Merkel, at five years the entrance lacks only two or three millimetres of the adult height, which it gains in the next two years. The bones at birth are still widely separated, and the walls are completed by membrane; the roof is proportionately large, and flat or even convex; the medial wall is low and not definitely separate from the floor, which is likewise small; the greater wing of the sphenoidal bone forms a relatively larger part of the lateral boundary than it does in the adult. The fossa for the lacrimal gland is a deep conical recess, that for the lacrimal sac is cylindrical, and 
faces anteriorly instead of laterally owing to the large development of the posterior lacrimal crest. The long axis of the cavity is horizontal, instead of being inclined downwards as in the adult; a frontal section behind the margin presents a long oval outline, with a blunt pole directed super-laterally and a pointed pole directed infero-medially, whereas in the adult a similar section is circular or square.

In the child the features noticed at birth are gradually modified by the growth of the adjacent parts. The orbits lie between the bones of the calvaria and those of the face, as shown in Fig. 2 ; the former are influenced by the expansion of the brain, the latter by the development of the nasal cavity with its accessory air-sinuses and of the teeth. Hence there are regions of great developmental activity above, between, and below the orbits, which react upon their form. The margin becomes more oval, but retains in early years, and always in the female, its sharp edge; the directions in which the primitive round opening becomes thus modified can be seen in the frontal plane by drawing a circle round it from the centre, as in Fig. 38. The development of the bridge of the nose carries both margins forward on their adjacent sides. That the shape of the anterior nasal orifice has an influence upon the contour of the medial part of the infra-orbital margin, and so is correlated with the shape of the orbital entrance, will be apparent on a comparison of Figs. 6 and 39 with Fig. 44. On the other hand, the rectangular shape of such orbits as are seen in Fig. 39 suggests the compression of the more delicate facial bones between the calvaria and the mandible through the action of the muscles of mastication in the strong-jawed type of skull. The growth of the nasal fossae and ethmoidal cells, together with the development of that part of the frontal lobe of the brain which rests upon the lamina cribrosa of the ethmoidal bone, increases the width between, and the height of, the medial orbital walls.

A further point of interest is the determination of the period at which growth may be considered to have ceased. The practical importance of this lies in regard to the changes which may follow enucleation of the eyeball. The orbit 
conforms to the law of adaptation of the organ to the function which it is called upon to fulfil, and if the globe be removed before the orbit is fully formed, growth of the latter is checked. E. Thomson (I9Oo) found in experiments upon rabbits that a uniform deficiency in orbital size amounting to Io per cent of all dimensions followed enucleation of the globe. Merkel states that in early childhood the changes occur in as soon as ten weeks after operation, the result being that in the adult the marginal height is reduced, the upper wall is flattened, and the lower slightly raised, and the cavity is narrowed in all its diameters, and more or less facial asymmetry is found according to the stage of growth which had been reached.

The full dimensions of the orbit are variously stated to be attained as early as at three years, or at from five to seven, or not until eighteen or twenty. From the following measurements of a series of skulls from birth to the adult condition, it appears that at three years the dimensions fall considerably short of those found in the adult, at six there is still a difference, whilst at twelve certain measurements approach those of the adult, but others are not fully attained until puberty. Although the eye, like the brain and the ear, attains its full structure and dimensions much earlier than the rest of the body, one would expect to find that the full size of the orbit is not reached until the end of the first and most active period of cranial growth, namely, until puberty. 


\begin{tabular}{|c|c|c|}
\hline \multicolumn{2}{|c|}{ 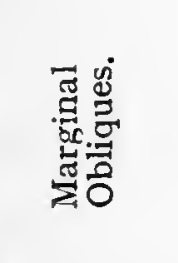 } & 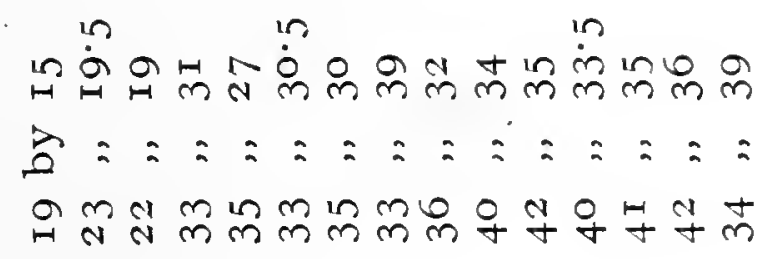 \\
\hline \multirow{4}{*}{ مُ } & 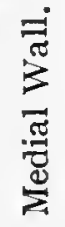 & : \\
\hline & 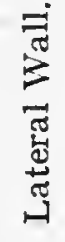 & 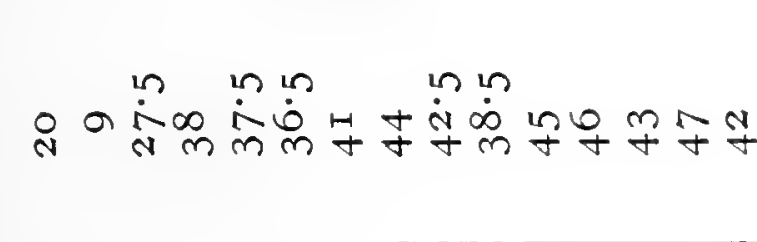 \\
\hline & 岁 & 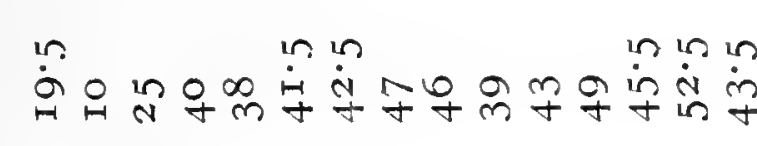 \\
\hline & 峞 & 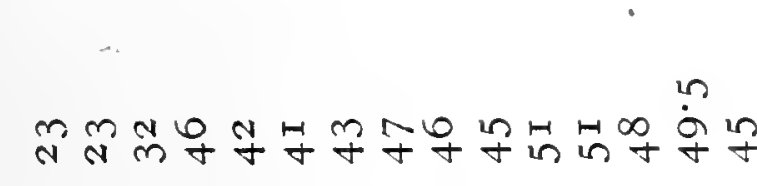 \\
\hline \multicolumn{2}{|c|}{ 焉 } & 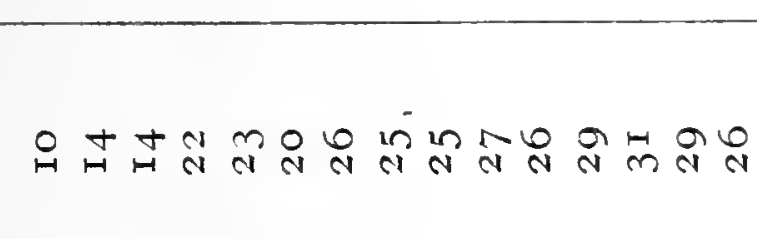 \\
\hline \multicolumn{2}{|c|}{ 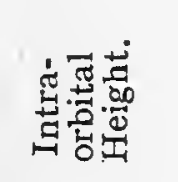 } & 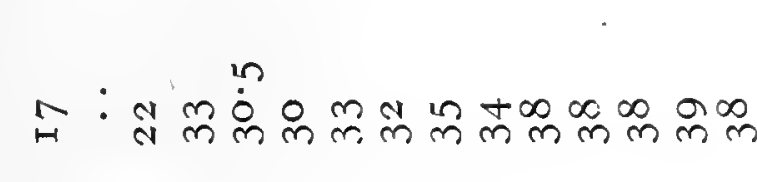 \\
\hline \multirow{2}{*}{ 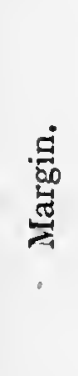 } & 总 & \\
\hline & 竞 & 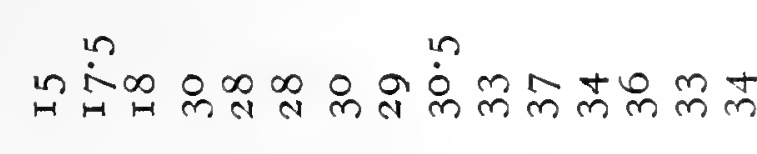 \\
\hline \multicolumn{2}{|c|}{$\stackrel{8}{\stackrel{0}{* 0}}$} & 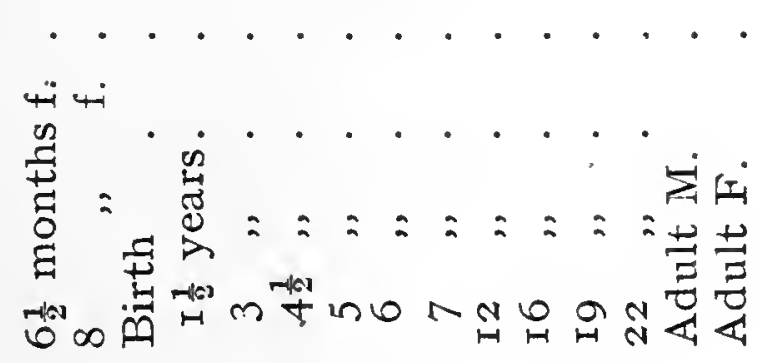 \\
\hline
\end{tabular}


The reader may also refer to the various dimensions given by Koenigstein (r896).

At puberty the sexual differences become marked, the bones in the male being stouter, the supra-orbital ridges. more prominent, and the marginal edge, especially superiorly, more rounded. The development of these ridges, and of the frontal sinus above and the maxillary sinus below, throws the margin forward in these two regions.

In the aged the margin is flatter, the entrance broader and especially higher than in the adult, owing to atrophy of the bones, a factor which causes also a loss of substance in the walls, chiefly in the lateral ; the sutures are obliterated.

\section{VARIATIONS, ASYMMETRY, AND DEFORMITIES}

The chief variations in the orbit as a whole are found according to the sex and type of skull.

Sexual Differences.-In the female the bones forming the orbit, as elsewhere in the skeleton, show a greater delicacy of construction; there is a tendency towards the retention of the early circular margin, de Wecker finding perfectly round openings in female skulls only. This opening is larger relative to the face, being broader by $I \cdot 5$ $\mathrm{mm}$. and higher by $\cdot 5$ or $\cdot 7 \mathrm{~mm}$., and the orbital index is greater than in the male; the edge is sharper, especially in the supra-orbital region where the ridges are less developed or even absent, and the lateral angular process is more slender and pointed than in the male, features which are characteristic differences between the sexes. The female orbit is more elongated and its cavity relatively larger (Merkel).

In the male the margin as a whole is more strongly developed, so affording greater protection to the eyeball, and is correlated with the more powerfully developed jaws. The walls are longer, the upper and lower are more curved owing to the greater prominence of their margins; the margin is less flat and the axial distance is consequently. longer; the absolute depth of the cavity is greater, but 
relative to the size of the entrance and length of the walls it is less.

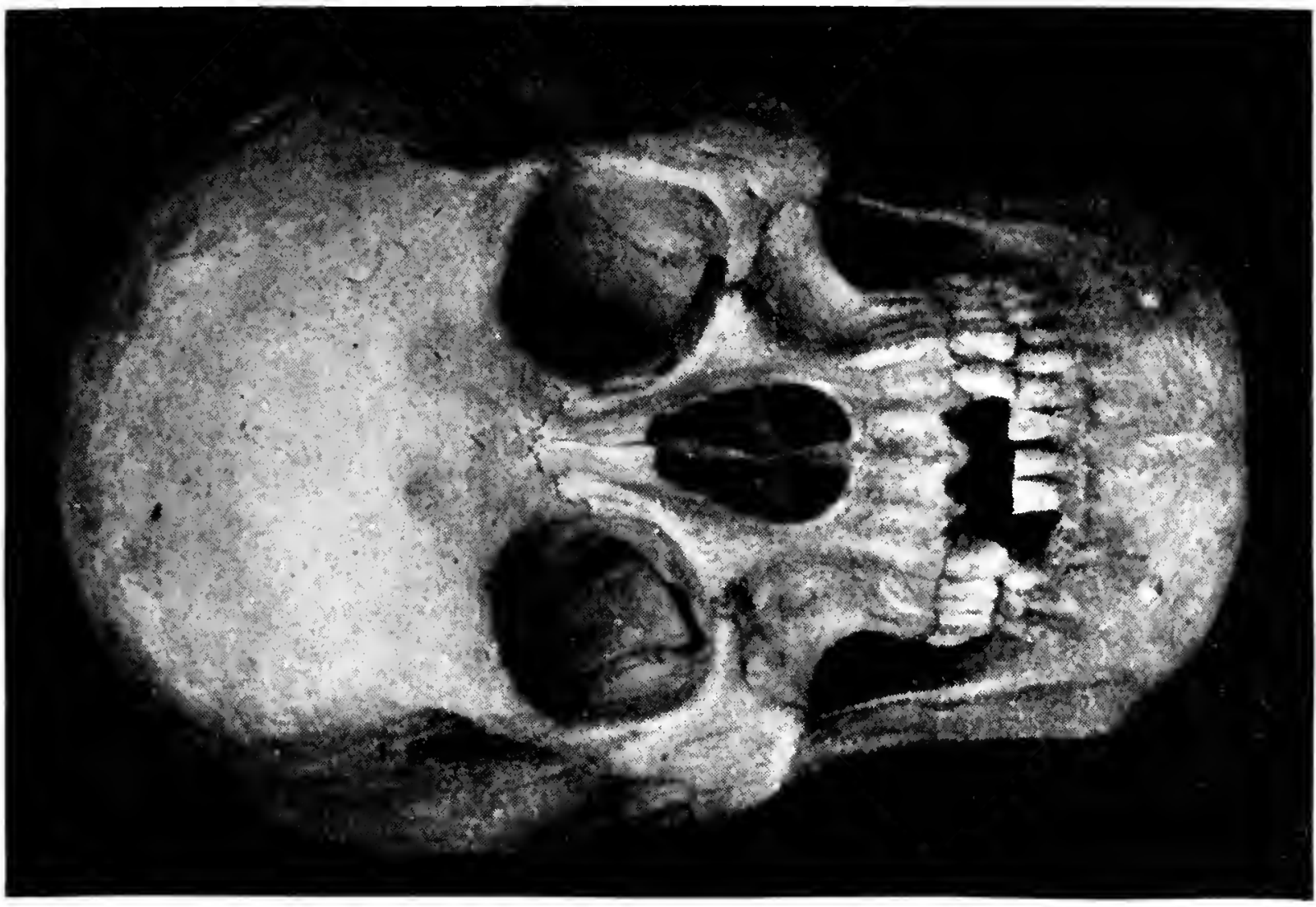

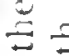

$\stackrel{2}{2}$

$\ddot{\Xi}$

$\Xi$

$\Xi \tilde{5}$

芯言

n?

$\dot{\mathscr{2}}$

$=7$

$\Xi$

$\stackrel{\Xi}{\Xi}$

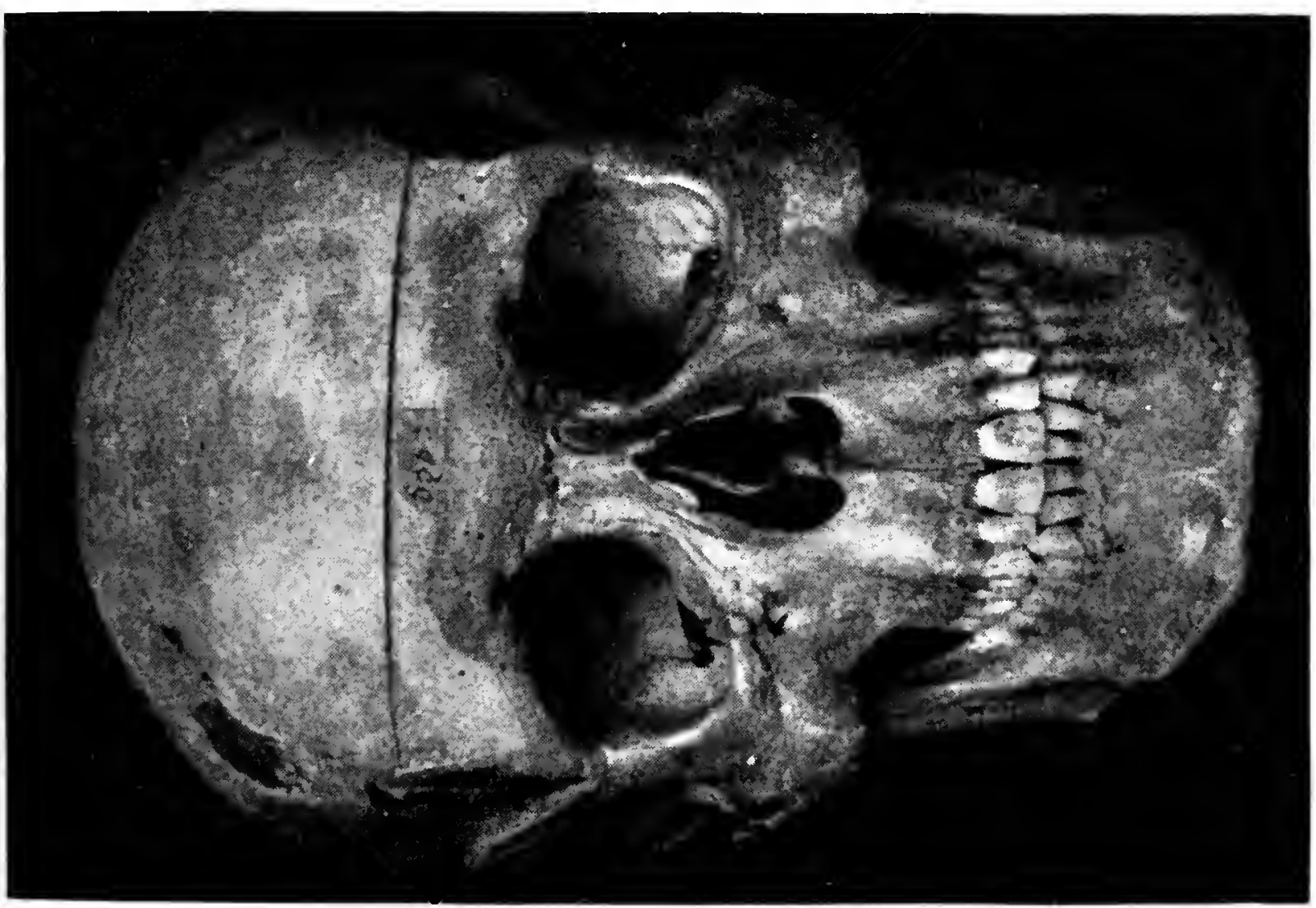

Type of Skull.- It is said that in the long or dolichocephalic skull the margin is more circular, the naso-malar angle and also the orbital index less, but the cavity deeper 
than in the short or brachycephalic type, where the margin is oval. The shape of the orbit has been considered to have an influence upon the power of vision, deep orbits being associated with myopic eyes and shallow ones with hypermetropic, but this is denied by Roy (I9rg). Also Stilling (I888) stated that short-sightedness was commonly associated with a low orbital entrance, the explanation suggested being that in such microsemic skulls the superior oblique muscle was placed at a lower level from the orbital floor than usual, and by compressing the globe caused it to become elongated in an antero-posterior direction with consequent myopia. His theory was supported by Seggel (I890) and Ask (I906), but disputed by Schmidt-Rimpler (I889), who found nearly the same orbital index in myopic and emmetropic subjects, and Hamburger (I904), who observed that on experimental raising of the intra-ocular pressure by injection of the globe in the cadaver there is no furrow caused by the tendon of the muscle. Moreover, the position of the pulley of the superior oblique muscle, that is, the point from which its action in compressing the globe against the inferior oblique muscle would originate, does not appear to be correlated to either the height, shape, or index of the orbital entrance; indeed the distance between the fovea trochlearis, where the pulley is attached, and the site of origin of the inferior oblique muscle, both of which points lie in the same vertical plane and deep to the actual margin, was found remarkably constant in 30 skulls of different type and race, and much less variable (28-30 mm.) than the height of the orbit either at the margin $(30 \cdot 3-38 \cdot 5 \mathrm{~mm}$.) or at the roomiest part of the cavity $(35-43$ $\mathrm{mm}$.). This. "inter-oblique distance" of about $29 \mathrm{~mm}$. may be compared with the vertical height of the globe, $23.5 \mathrm{~mm}$. (Whitnall, Igr3).

Birnbaum (I9I5), by taking exophthalmometric measurements of normal eyes, found that prominent eyes coincide with large and oval orbital openings, whilst the globes are deeply seated when the openings are small and round.

Asymmetry.-There is usually a slight difference in the shape and position of the orbits and their contents, due, 
according to Elliot Smith (I907), to an unequal development of the frontal areas of the brain. This inequality is only part of a general asymmetry which is a characteristic difference between man, especially in the higher races, and apes. Ambialet found that the right or left orbit indifferently is displaced, and Dwight states that one orbit and cheek are commonly anterior to the other, whilst the right is usually placed higher. The margin of the right orbit is said to be weaker and sharper than that of the left Emmert

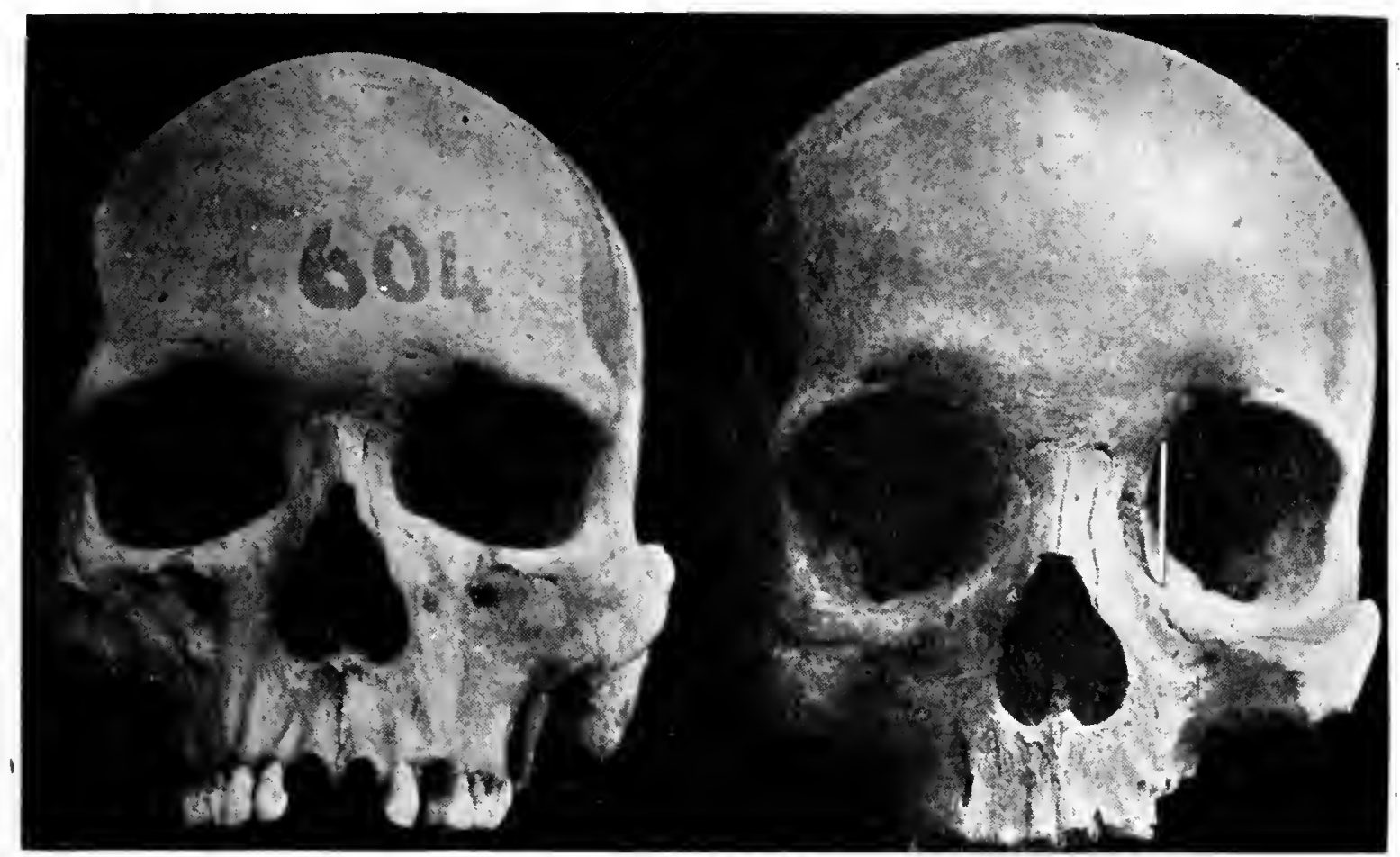

FIG. 45. - Two Skulls, to illustrate criticism on Stilling's theory of influence of orbital height upon shape of globe. $x$ about $\frac{1}{2}$.

Difference between marginal orbital heights ( $31 \mathrm{~mm}$. in first

skull, $38 \mathrm{~mm}$. in second) . $\cdot=7 \mathrm{~mm}$.

Detween greatest intra-marginal heights ${ }^{\circ} \cdot=2.5 \mathrm{~mm}$

Difference between "inter-oblique distances" (as shown by line in second figure, which was $28 \mathrm{~mm}$. in each skull),$=0$.

found that the absolute depth of the left orbit is less than that of the right. Tubby (I9I9) draws attention to the asymmetrical growth of the skull, especially marked in the face and orbits, secondary to congenital torticollis, and leading to the condition known as "ocular torticollis" (Stephenson, I9I3); in a right-sided torticollis the superciliary ridges are not level, and the right orbital aperture is lower and often smaller than the left. Also Lowman (IgI8) and Lloyd Mills (I9I9) have written on the effects of faulty skeletal alignment upon the eyes, such as exophoria, and 
remedial by appropriate orthopaedic treatment of the primary cause. The latter writers found, indeed, that about three-quarters of fifty eye cases met with in regular orthopaedic practice showed marked muscular imbalance of the eyes. The effects of such imbalance upon the facial expression are noted by Stevens (I892).

Deformities, due either to artificial causes, as in some Indian skulls where compression of the brain causes a slight protrusion of the apex of the lateral wall of the orbit, or to pathological processes, may be found. Of the latter, the condition known as "tower skull" (Turmschädel) or oxycephaly is interesting. It is a deformity due to premature synostosis of the coronal or the sagittal suture following a meningitis, and of all cranial malformations this one is most frequently associated with optic neuritis, due, however, not to the intra-cranial pressure, but to the same primary affection (Brav, I9I2). Good descriptions of the condition are given by Dorfman (Igo8), Sharpe (Igr6), and Dock (I9I9), and Swanzy (Igoo) gives the literature up to that date. Power (I894) published details of a remarkable case, Holloway (I9I5) has recorded two cases of this deformity associated with exophthalmos, divergent squint, and post-optic neuritis, and instances are also reported by Bedell, Lewin, Ischreyt, and Goldenburg (I9I8). 


\section{PART II}

\section{THE EYELIDS}

\section{THE EYEBROWS}

THE eyebrows (supercilia) are formed by muscle fibres and thick skin, surmounted by strong hairs; they are not facial but cranial appendages, being a modified part of the hairy scalp, the continuity with which is indicated both structurally and also by an accentuation of down or fine hair on the region between the temple and the end of the eyebrow. Each has a head, a body, and a tail or lateral extremity, and in extent is limited by the hairs which it bears (Fig. 48). The heads are usually separated by an "inter-superciliary region " corresponding to the glabella (from the Latin glaber, smooth, hairless). The exact position is between the supraorbital ridge and the orbital margin, but they are a little less curved than the latter, and in abnormal cases lie at a higher level. They overlie the frontal sinuses, and on a deeper plane the second frontal convolutions of the brain ; the tail lies upon the lateral angular process and extends to the zygomatico-frontal suture; they are traversed by the supra-orbital vessels and nerves. They serve to divert the sweat of the brow from running into the eyes, and also shade them from strong light, and they add greatly to the expression of the face.

In each eyebrow four principal layers can be identified in a mid-vertical section. These are :

(i.) The skin, intimately united to a dense superficial fascia.

(ii.) A muscle layer, the fibres of which end in the skin.

(iii.) A cellulo-adipose layer.

(iv.) The galea aponeurotica (epicranial aponeurosis). 
So constituted by the close union of these layers, the eyebrow glides freely over the periosteum of the bone, from which it is separated by a loose cellular " sub-epicranial tissue," an arrangement that is the exact counterpart of the scalp proper (Fig. 46).

(i.) The skin is thicker than in the surrounding regions, and is rich in sebaceous and sweat glands and large hair follicles, between which are numerous striated muscle fibres. The

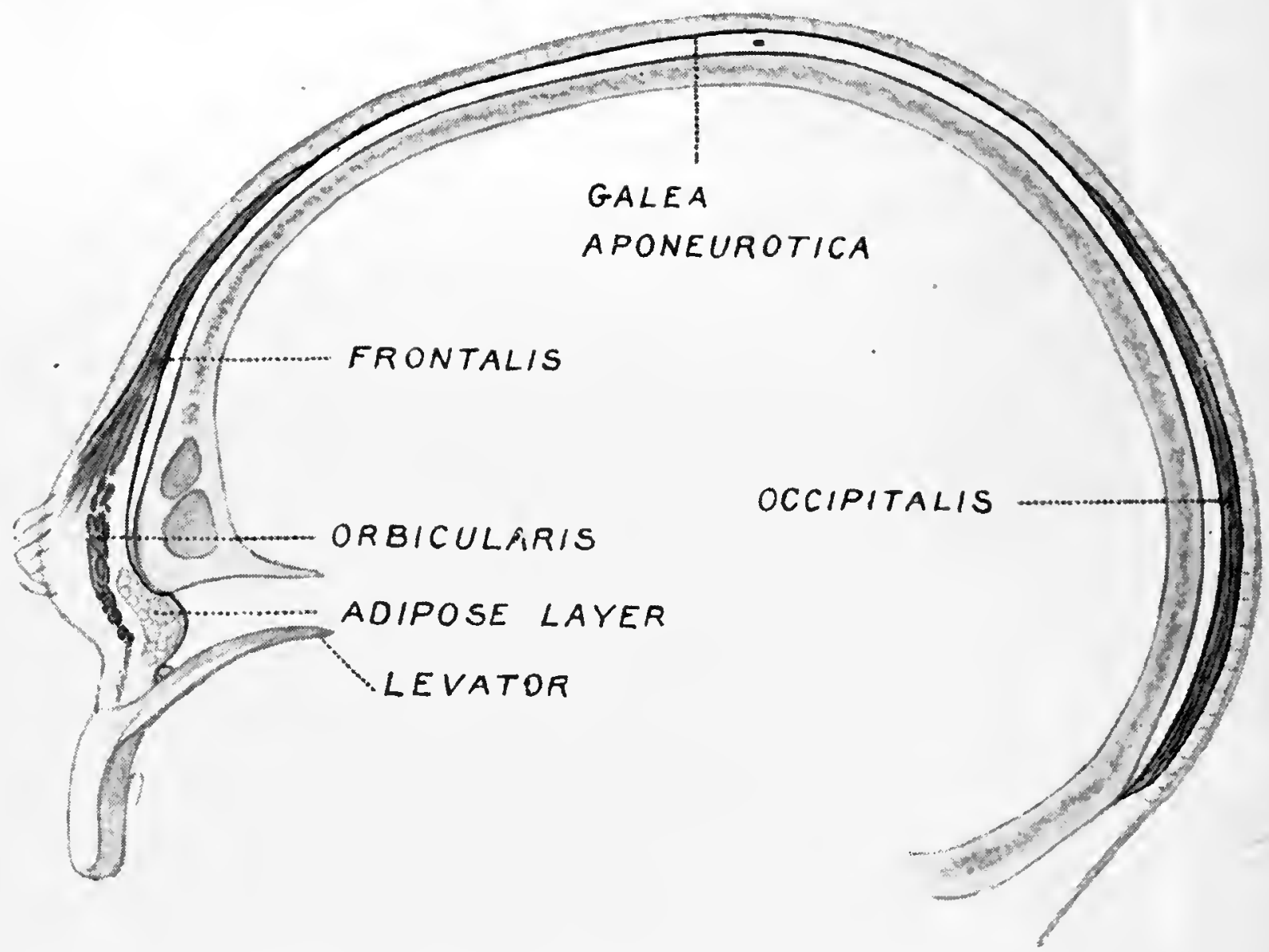

FIG. 46. - The Structure of the Eyebrow and its connection with the Scalp.

hairs are of interest as being the first to develop in the embryo, appearing at three months (Contino, I907) ; generally they are directed obliquely and laterally, but those of the eyebrow are often inclined forwards and upwards, owing to the action of the corrugator supercilii muscle; there may therefore be a break in the stream of the hairs, the more frequent occurrence of which in the left eyebrow (I53 out of 200) is ascribed by Kidd (I904) to the greater strain placed upon the left muscle in our practice of reading from left to right. The occasional presence of a tuft of longer hairs near the middle recalls the tactile hairs or 
"supra-orbital vibrissae" seen in many mammals, and the well-marked sensitiveness to touch of the hairs of the eyebrow is doubtless due to the presence amidst their roots of large sensory bulbs, such as are present in lower animals and have been found in the human foetus by Friedenthal (Keith, I9I3). The hairs are more numerous, larger, and more irregularly planted in males than in females, and are less developed in northern than in southern races, in which latter they usually meet in the mid-line. The colour is generally the same as that of the hair of the scalp, but may be lighter in tint; it may differ on the two sides, and instances of piebald or wholly white (in albinos), and, indeed, of absence of the eyebrows are recorded. The superficial fascia is composed of dense connective tissue containing fat lobules in its meshes, and it intimately unites the skin to the muscle layer.

(ii.) The muscular layer is comprised by three orders of fasciculi : the vertically directed fibres of the frontalis, the concentrically arranged fasciculi of the orbicularis oculi, and the obliquely placed corrugator supercilii. These fibres intermingle and are inseparable at their insertion into the skin, where they can be traced through the dense superficial fascia to end between the sweat glands and hair follicles.

The Frontales muscles are the anterior bellies of the epicranius or occipito-frontalis. This muscular and aponeurotic sheet covers the whole cranium, and is differentiated into two flat posterior bellies, the occipitales muscles, attached to the superior curved line of the occipital bone behind, an intervening tendinous portion, the galea aponeurotica, and two anterior bellies, the frontales muscles. The latter have no bony attachments, but blending together at their medial borders extend across the full width of the forehead, and at the supra-orbital margin each intermingles with the corresponding orbicularis oculi to be inserted into the skin of the eyebrow. The medial parts of the muscles between the eyebrows appear to extend downwards, to gain attachment to the nasal bones, but these fibres really constitute an independent muscle, the procerus or pyramidalis nasi, as shown by Duchenne, who termed it the "muscle of aggression" ; it has, moreover, an innervation 
from the infra-orbital branch of the facial nerve distinct from that of the frontalis (Frohse, I895), and it contracts synergetically with the corrugator and orbicularis in the presence of strong light.

The Orbicularis Oculi (orbicularis palpebrarum) muscle is a nearly circular sheet of fasciculi placed over each eye, covering the eyelids and orbital margin. The muscle is described at length in connection with the eyelids, and is figured on P. I2I; here it is sufficient to note that it is the upper fibres of the peripheral or orbital part that enter into the composition of the eyebrow, where they mingle with, though lying mainly superficial to, the frontalis. Elsewhere round the orbital circumference the skin can readily be dissected from the orbicularis to display its extent and contour, but in the eyebrow the blending with the frontalis is so intricate and its connections with the skin so intimate that neither muscle can be isolated as a definite layer.

The Corrugator Supercilii muscle lies deep to the abovementioned muscles: it is seen upon reflecting the whole eyebrow downwards from the bone in the form of a definite band of muscle fibres arising from the most medial part of the supra-orbital arcade, and running laterally for 2 or $3 \mathrm{cms}$. to blend with the overlying orbicularis; it is inserted into the skin of the medial half of the eyebrow, the site being marked by a depression when the muscle is contracted (Fig. 90, p. I78). Sometimes distinguished as a separate muscle, the " depressor of the head of the eyebrow" (Arlt, I863), it is more generally regarded as a special fasciculus of the orbicularis (Henle, Merkel, Virchow, Kidd), though its origin is quite separate, and it can be definitely isolated for most of its course, as seen in the figure opposite.

The corrugator is differentiated late in the human embryo, after all the other muscles of the face (Futamura, Ig06); it may be absent, in which case the eyebrows remain level on lowering the brows and a frown does not appear.

(iii.) The cellulo-adipose layer underlies the muscles, and facilitates the movement of the eyebrow over the orbital 
margin; it is fairly loose, and contains fat in its meshes; it is abundant in the lower part of the eyebrow, where Charpy (I909) describes it as forming le coussinet adipeux $d u$ sourcil, an elongated fatty mass I cm. high and $5 \mathrm{~mm}$. thick, placed over the supra-orbital margin and extending a little downwards into the eyelid, but it does not always appear so definite a structure as he describes.

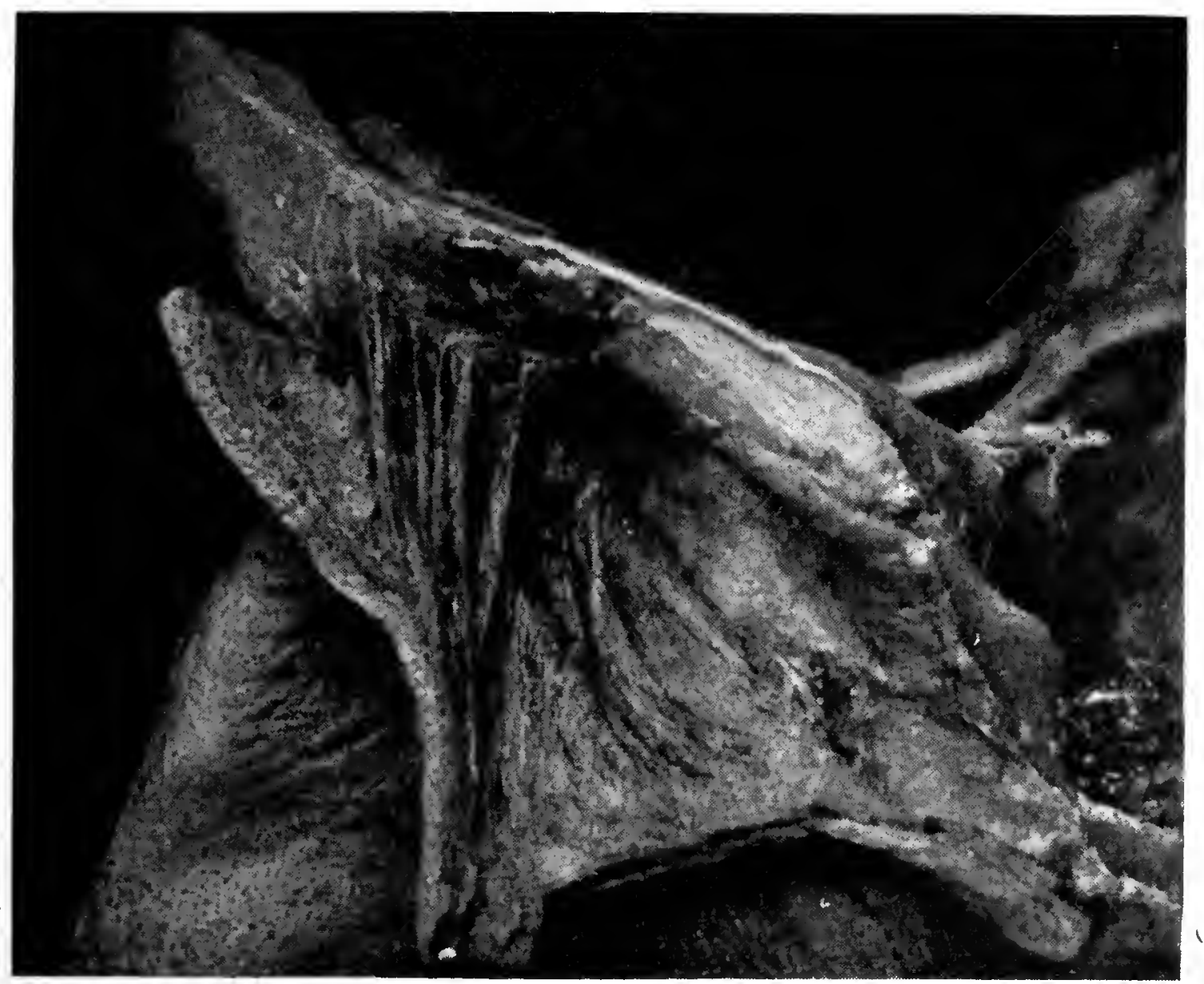

FIG. 47.-Dissection to expose the Corrugator Supercilii Muscle. Left orbit seen from the side. The skin has been turned down from the supra-orbital margin carrying the muscle with it ; the origin of the muscle from the bone medial to the supra-orbital notch and separate from the rest of the orbicularis is clearly seen. Natural size. Compare with Fig. 90, p. I78.

(iv.) The Galea Aponeurotica (epicranial aponeurosis) : the aponeurotic central portion of this structure splits anteriorly to enclose the frontalis muscle; the superficial layer is very thin and passes with the muscle fibres to the skin of the eyebrow, whilst the deeper and more definite layer passes beneath the muscles and is firmly attached to the supra-orbital margin (Fig. 46).

These four layers of skin, muscle, connective tissue, and aponeurosis then constitute the eyebrow proper, and their 
close union is exactly similar to what is found in the scalp proper, of which this region is, as noted above, merely a specialised portion. In stripping the scalp off the cranial vault the reflection can be carried forwards to the supraorbital margin, taking the eyebrow with it, and the plane of separation is in the " sub-epicranial " layer of delicate connective tissue lying beneath and facilitating movement of the scalp over the periosteally covered bone; it is in this space that a traumatic haematoma, suppuration, or emphysema of the frontal sinus extends, and such sub-aponeurotic effusions are prevented from passing down into the eyelids by the above-mentioned attachment of the deeper layer of the aponeurosis to the supra-orbital margin. Charpy (I9II) has also demonstrated by means of gelatine injections that the barrier formed by the dense eyebrow mass prevents fluids from extending upwards from the eyelids on to the brow (Fig. 64, p. I37).

The blood-vessels of the superciliary region will be described in detail together with those of the eyelids ( $p$. I6I) ; here it may briefly be noted that there are two chief arteries in this region, the supra-orbital, which passes directly upwards from the supra-orbital notch about an inch from the mid-plane and lies deep to the eyebrow against the bone, and the superficial temporal, which runs parallel with and on the temporal side of the tail of the eyebrow; though some distance from it. The supra-orbital vein does not accompany the artery of the same name upwards over the brow, but runs along the lower border of the eyebrow and forms a marginal arcade (vena marginalis), lying deep to the orbicularis. There is, however, a frontal vein which passes directly upwards beneath the head of the eyebrow (p. I65).

The lymphatic vessels drain chiefly into the parotid. nodes, but a small number from the head of the eyebrow join those of the medial half of the eyelids and pass directly into the sub-maxillary nodes (see p. I70).

The muscles of the eyebrows are innervated by the facial nerve, in which respect they present certain points of clinical interest which, together with their actions, will be considered later (p. I79). The sensory supply to the skin is 
derived chiefly from the supra-orbital nerve, which accompanies the artery in its course, and, like it, would be severed by a deep horizontal incision along the eyebrow. The head of the eyebrow receives filaments from the external nasal nerve, the tail from the lacrimal nerve ; each part may have a further supply, the head by ascending branches of the infra-orbital nerve, the tail from the zygomatico-temporal nerves; all of these are sensory branches of the trigeminal nerve.

\section{THE EYELIDS}

The eyelids (palpebrae, from the Latin palpare, to stroke) are two musculo-membranous folds of modified skin placed in front of the orbit, which they close; they are closely applied to the globe of the eye, for which they act as organs of protection, and they also serve as barriers to the orbital contents between it and the orbital margin. It may be noted, moreover, that they fulfil the rôle of resting the brain from the rays of light which would otherwise create cortical pictures and maintain cerebral activity; it is only when the eyelids are closed that the visual area of the brain obtains rest (Wood Jones). They are distinguished as superior and inferior. The former is more extensive and more mobile than the latter, covering the greater part of the anterior surface of the globe, and being provided with a special elevator muscle. In each eyelid there are to be noted two faces, anterior and posterior, two borders, adherent and free, and two extremities, medial and lateral, uniting to form two corresponding commissures (commissura palpebrarum). They are opposed by their free borders or margins, which limit between them a transverse opening, the palpebral fissure or rima palpebrarum; the borders of this opening join together at each side at an acute angle to form the medial and lateral canthi or " angles " of the eye (angulus oculi). (It is preferable to apply the term "canthus" to the angle of the opening between the lids, and the name "commissure" to the actual conjoint ends of the lids themselves.) They are modified folds of skin, in the thickness of which is developed a thin sheet of 
muscle fibres (the orbicularis oculi); the deeper skin layer becomes converted into a mucous membrane, which lines the lids and is reflected on to the anterior part of the globe, and is called the "conjunctiva." The membrane is kept moist by the secretion of the lacrimal gland, which is an outgrowth from it, and excess fluid is drained away through a series of channels, the lacrimal passages. The normal skin elements of the free edges of these folds, that is, of the lid margins, becomes greatly developed for protective and sensory purposes; the hairs are developed as cilia or eyelashes, the sebaceous glands become huge in size and are called "tarsal or meibomian glands," and the connective tissue supporting them is condensed to form the "tarsal plates."

The description of the eyelids will be in the following order :

(I) The Surface Anatomy,

(2) Structure,

(3) Vessels,

(4) Nerve-supply,

(5) Movements and Action,

(6) Development, and

(7) Abnormalities.

The conjunctiva will be described in a separate section, as also the lacrimal apparatus.

(I) The Surface Anatomy.-The eyelids pass normally into the neighbouring parts of the face without apparent line of demarcation, but their limits are clearly outlined in pathological conditions such as oedema, since the extravasation spreads beneath the skin in the loose connective tissue devoid of fat which represents the superficial fascia of the lids, whereas in the surrounding region of the cheek or eyebrow the corresponding tissue is dense and fatty and connected to the periosteum; thus defined, the upper lid extends to the lower edge of the eyebrow and coincides with the supra-orbital margin, but in the lower lid a swelling can be seen to extend beyond the bony margin as low as the naso-jugal and malar skin-folds. 
The form of the lids is essentially correlated to the volume and position of the globe, though also partly dependent upon the shape of the orbital entrance. In infants and subjects of delicate skin the prominence of the cornea is observable through the closed lids. The margin of the upper tarsal plate can readily be felt with the finger.

The colour of the skin is generally in harmony with that of the face, but the redness of the cheek does not extend beyond the skin-folds noted above as limiting the extent of the lower lid region, nor usually above the level of the lateral canthus; the lower lid is generally darker, but the tint varies according to health and local conditions. The " palpebral areola," which may be black or white, and is so noticeable in fatigue, is attributed by Waldeyer to a reflex of the lymph from the connective tissue spaces beneath the skin, but it is also caused by venous stasis, the vessels showing up through the delicate skin; it is usually most marked in the lateral part of the lower lid region, where, it may be noted, there are no cutaneous arteries of any size. A permanent darker tint commonly noticeable in the medial part of the lower lid or sometimes at the lateral commissure is also explained by venous stasis, but the migration of definite pigment cells (chromatophores) to this region has been described (Dor, I899), and in many cases there is no doubt as to the presence of pigment in the skin (p. I25).

The main folds or wrinkles of the skin in and around the eyelids are of topographical interest. They may be folds of movement or structure. In each eyelid there is a horizontally curved "palpebral" or orbito-palpebral infold, distinguished as superior and inferior according to the lid, and dividing it into tarsal and orbital areas: the tarsal region overlies the tarsal plate and the globe, the orbital is related to the space between the latter and the bony margin. Sappey states that these folds exactly superimpose the lines of reflection of the conjunctiva on to the globe (the "fornices"), but according to Charpy they indicate the limit between the smooth and folded parts of that membrane. The superior palpebral fold is the deeper; it lies 2 or $3 \mathrm{~mm}$. above the highest point of 
the cutaneous insertion of the levator palpebrae superioris, the contraction of which muscle causes it to become deeply recessed; the orbicularis oculi muscle is thinnest along this line. The skin of the orbital region of the lid above it often sags forward in middle age when it has lost its elasticity, and forms a fold ("Deckfalte" or " pli de recouvrement" of foreign authors) which covers the tarsal region even as

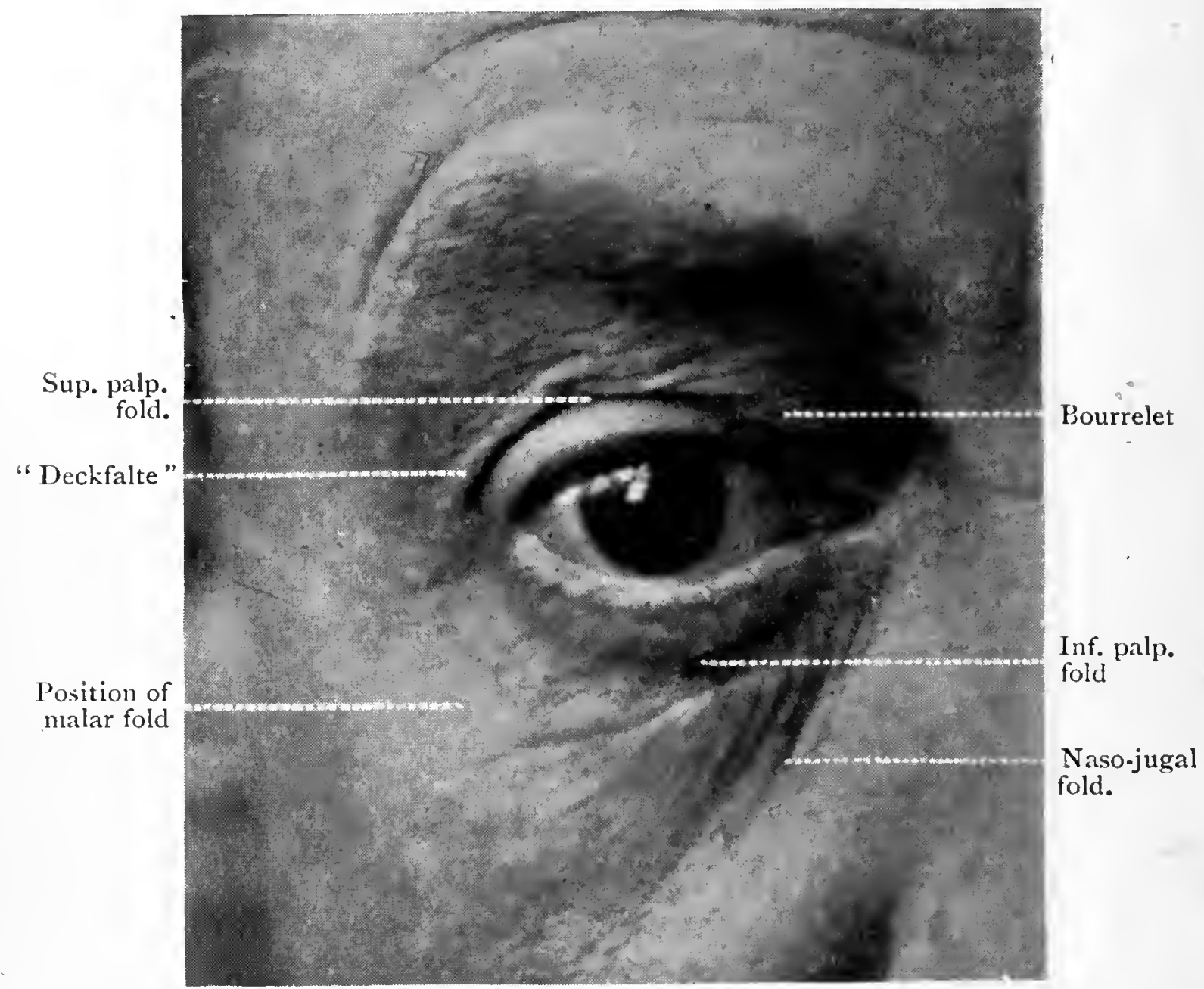

FIG. 48.- The Eyelids and Palpebral Folds. Natural size.

low down as the eyelashes; it is most marked on the lateral side, and may even form a kind of lateral epicanthus in old subjects (ptosis adiposa) ; the fold is obliterated on drawing the eyebrow up. In the medial corner of this region, particularly in the aged when the orbicularis muscle has become weak, there commonly presents a small swelling, the " bourrelet sénile," or " palpebral hernia," caused by a hernial protrusion of the orbital fat; such a swelling can be produced or accentuated by pressing the eyeball backwards and towards the nose; the lobe of fat which forms it is 
shown in Fig. 49. The writer has seen cases where the skin over the lump appeared yellow from the underlying fat.

The volume of the lacrimal gland has no influence on the configuration of the lateral part of the upper eyelid, behind which it lies. The inferior palpebral fold is well marked in infancy, but may be slight or absent in the adult.

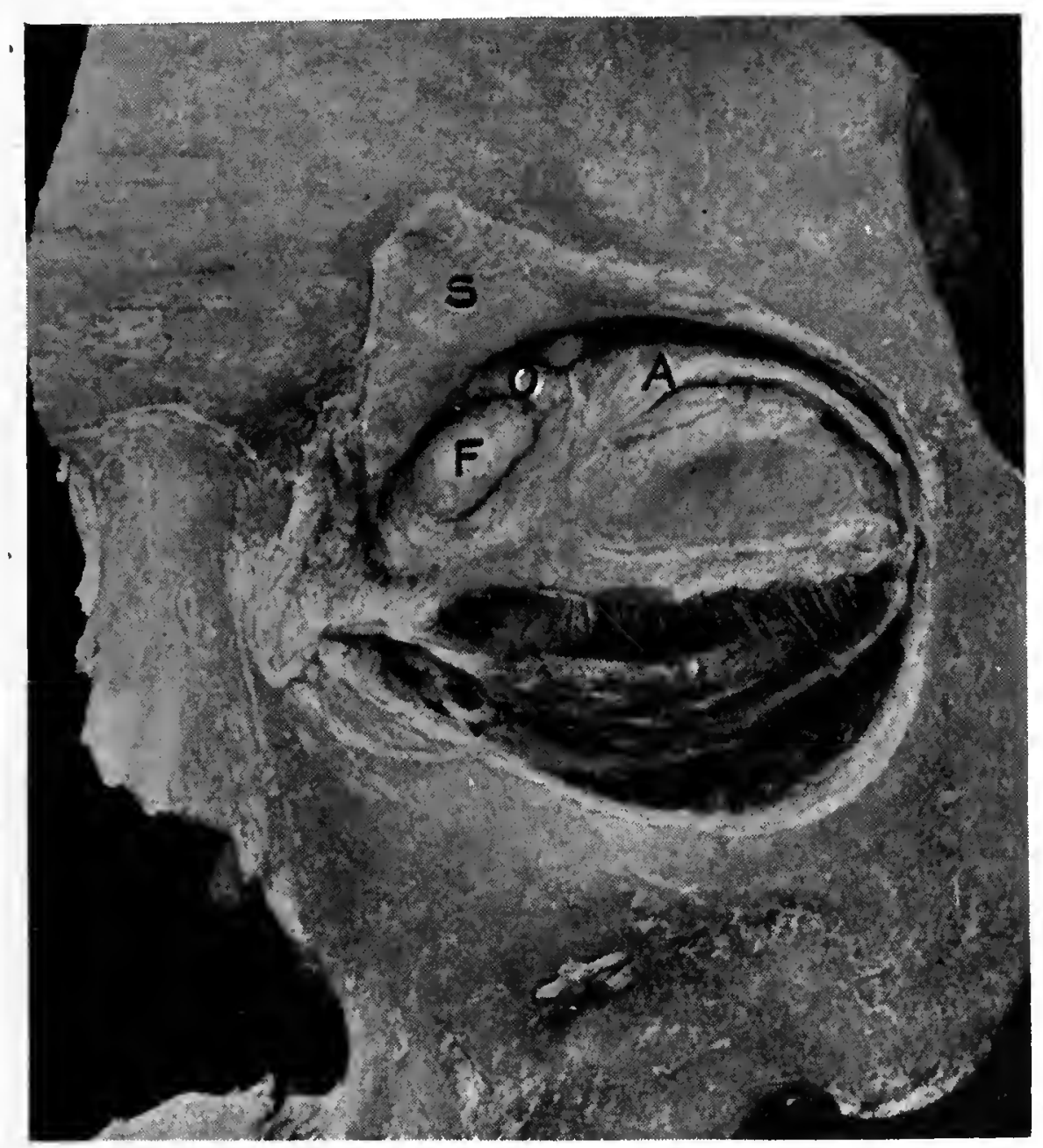

FIG. 49. - The palpebral hernia adiposa. Dissection of Left Orbit, seen from in front after removal of the skin, orbicularis oculi muscle, and septum orbitale; a part of the septum (S.) has been left and turned up. 'The orbital fat (F.) is seen protruding below the reflected tendon of the superior oblique muscle (O.), and between the orbital margin and the medial extremity of the aponcurosis (A.) of the levator palpebrae superioris muscle. The swelling caused in life by this hernial protrusion of the orbital fat is shown in Fig. 48. Above the tendon of the superior oblique is seen a loop formed by the terminal part of the ophthalmic artery. Natural size.

Below the lower lid two wrinkles are usually present on the cheek: a "naso-jugal" fold running downwards and laterally from the medial commissure, and always well marked, and a " malar" descending from the opposite side, but often ill-defined; these tend to become continuous with one another, forming the "Wangenlidfurche" of Arlt 
or " sillon palpebro-genien " of Charpy. They are not only folds of movement, seen when the cheek is raised, for example as in laughter, but are structural in that they indicate the differentiation between the loose tissue beneath the skin of the lower lid region and the denser fatty superficial fascia of the cheek; hence they are accentuated by effusion into the lower lid. They also become more marked in ocular fatigue or in the aged when the orbicularis oculi muscle has lost its tone. The naso-jugal fold, moreover, corresponds to a fascial interstice fixed to the bone between

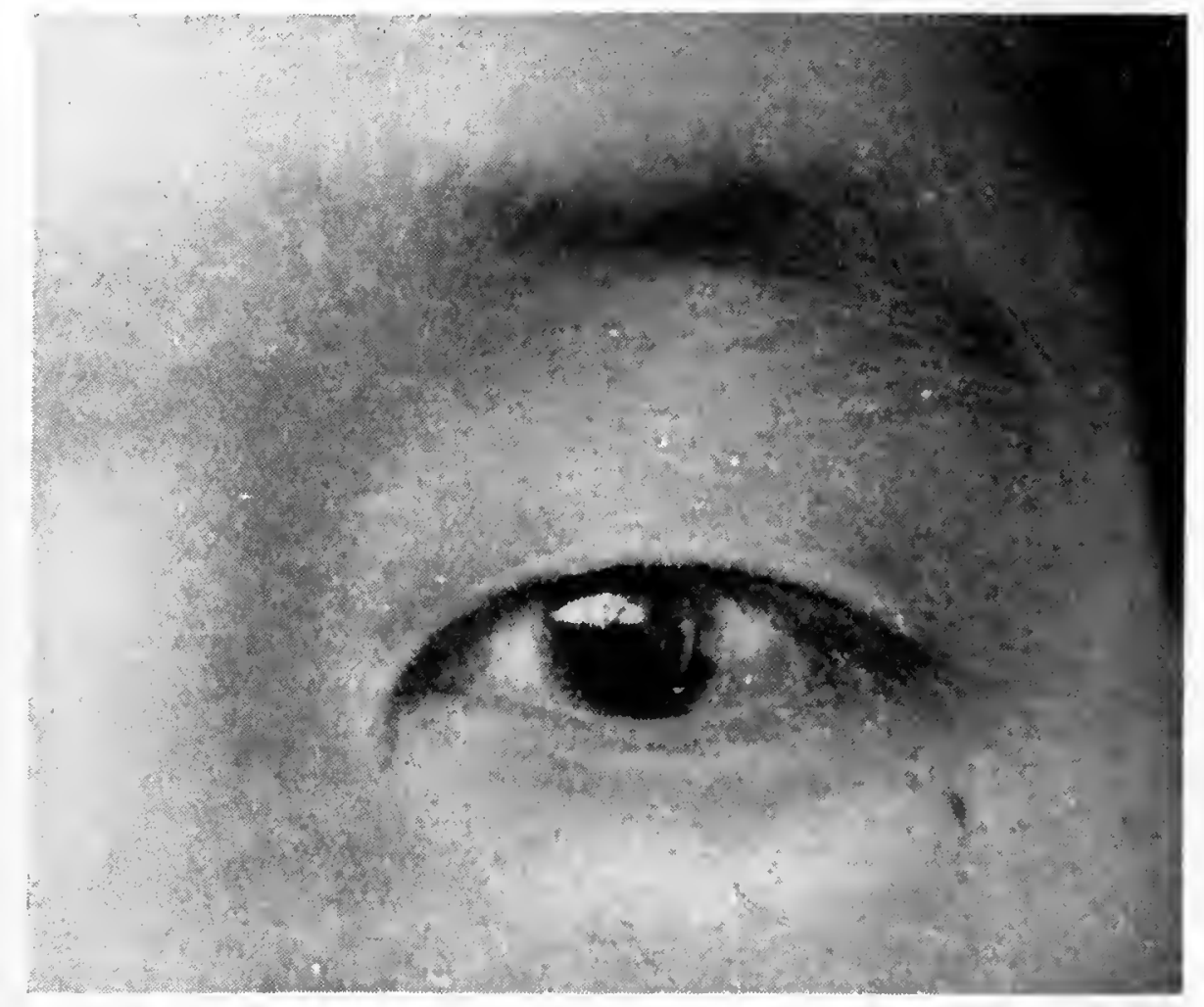

FIG. 50. - Mongolian Eye, showing the epicanthus or Mongolian skin-fold.

the orbicularis oculi and angular head of the quadratus labii superioris (levator labii superioris alaeque nasi) muscles, and along its line there run the facial vein and artery, while its upper part overlies the lacrimal sac (Fig. 52).

Another fold is known as the "epicanthus" or Mongolian fold ; it is formed by the skin alone of the medial part of the orbital region of the upper lid, and it curves downwards, covering the medial canthus (as the name implies) and joining the naso-jugal furrow of the lower lid. It is characteristic of the Mongolian eye, in which it has been recently studied by Bloch (I9II), but is present in all races in foetal life (Keith), and is seen in infants, disappearing 
gradually as the bridge of the nose develops: Metchnikoff (I874), indeed, considered it to be only the persistence of a character peculiar to this period of life and dependent upon the flatness of the nasal bones. Duckworth (I904), however, notes its absence in negroid races, where these bones are even flatter and smaller than those of Mongols, though Testut states that it occurs sporadically in Hottentots. It has been found associated with congenital ptosis (Pockley, I9I9).

Other minor skin-folds will be found described in a special article by Charpy (I9IO).

The palpebral opening (rima or fissura palpebrarum, rictus oculi) is limited above and below by the free margins of the lids, and at its ex-. tremities by their commissures. When the eye is wide open the curves of the margins are not symmetrical, that of the upper lid being more pronounced than the lower, and its greatest height being nearer the medial angle, whereas that of the lower lid is nearer the lateral

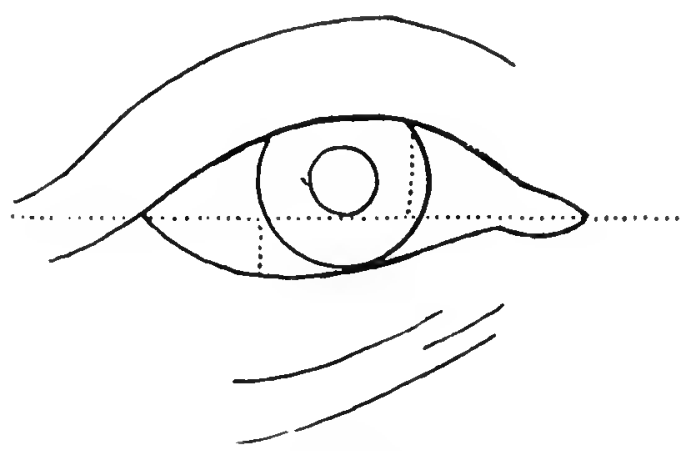

FIG. 5I. - The Palpebral Opening, showing the curves of the lid margins. Compare with Fig. 48. angle, as shown in Fig. 5I; the radius of the upper curve is $16.5 \mathrm{~mm}$., that of the lower $22 \mathrm{~mm}$. (Merkel). The long axis of the opening is not quite horizontal, the lateral extremity being about $4 \mathrm{~mm}$. higher than the medial; in some of the Mongoloid races an obliquity of $50^{\circ}$ from the horizontal may be found, the slope being emphasised by the presence of the epicanthus. The opening is about $30 \mathrm{~mm}$. long and $15 \mathrm{~mm}$. high in normal regard, but is smaller in females and rounder in children, and larger in equatorial than in northern races. It exposes nearly one-fifth of the surface of the globe, and, it should be noted, represents the mouth of the conjunctival sac. The lateral angle is more acute than the medial, and lies in close contact with the globe ; the medial angle extends towards the nose, and is from 5 to $7 \mathrm{~mm}$. away from the globe, being separated by the caruncula and the plica 
semilunaris. When the lids are closed the opening becomes a fissure, which is not quite straight but forms an elongated and flattened S-shaped curve; in relation to the globe it corresponds to the inferior border of the cornea, which is therefore entirely covered by the upper lid when the eye is shut.

The free margin of each lid is divided at about the junction of the medial $\frac{1}{6}$ th with the lateral $\frac{5}{6}$ ths of its length into two definite parts by a small tubercle, the papilla lacrimalis, which presents on its summit an orifice, the punctum lacrimale. The papilla of the lower lid is directed upwards and slightly inwards, and appears more prominent, whereas that of the upper lid is hidden and the contour of the whole upper margin is uninterrupted when viewed from the front, as seen in Fig. 48 ; the papillae are best seen by eversion of the lid edge. The medial or "lacrimal "part of the lid margin (so called from its containing buried in its thickness the lacrimal canaliculus) extends from the papilla to the medial canthus, and is about 5 to $8 \mathrm{~mm}$. long ; its surface is rounded, smooth, and devoid of eyelashes; it unites with its fellow of the opposite lid in a semi-elliptical curve, so describing an area, the lacus lacrimalis, the floor of which presents a small reddish mass, the caruncula lacrimalis, partly lying on a thin fold of mucous membrane, the plica semilunaris conjunctivae. The lateral, bulbar, or " ciliary" part of the lid margin extends from the papilla to the lateral canthus; it is thicker, sharper cut, and more closely related to the globe than the lacrimal part, and it bears the eyelashes. Further details are given on p. I54.

The deep relations of the lids: the lateral angle of the palpebral opening.lies Io $\mathrm{mm}$. below the line of the zygomatico-frontal suture and from 5 to $7 \mathrm{~mm}$. distant from the orbital margin; the medial angle lies a little below the fronto-maxillary suture (Fig. 8, p. 20). On dissecting away the superficial parts of the lids (orbicularis oculi muscle and septum orbitale), a view of the contents of the orbit is obtained such as is shown in Fig. 54. The anterior surface of the globe is covered above by the levator muscle expansion and superior tarsal plate, and below by the inferior tarsal 
plate and orbital fascia; the space between the globe and orbital walls is occupied by masses of fat, removed in the above-mentioned figure to show the position of more important structures. The expansion of the levator extends behind the whole width of the upper lid, the lacrimal gland lies behind its lateral quarter, the pulley and reflected tendon of the superior oblique muscle behind its medial part; the origin of the inferior oblique is situated behind the medial quarter of the lower lid, but the muscle itself recedes deeply away from its mid-point; the lacrimal sac lies in its fossa behind the medial commissure. If the lids, therefore, be divided into quadrants by a vertical line crossing the centre of a horizontal one drawn between the commissures, important structures are found to lie behind each quadrant, except the infero-lateral one, where there is only a mass of orbital fat, and where the finger can be pushed deepest into the orbit between its bony margin and the eyeball.

(2) The Structure of the Eyelids.-There may be distinguished in each lid four layers or planes of tissue, which are from before backwards:

(i.) Cutaneous.

(ii.) Muscular (the orbicularis oculi muscle).

(iii.) Fibrous (the tarsal plate and the septum orbitale).

(iv.) Conjunctival (the membrane lining the lids and reflected from them on to the globe).

The tarsal plates form the skeleton of the lids; at each commissure their extremities unite to form medial and lateral palpebral or tarsal ligaments, by means of which they are attached to the corresponding orbital margins at about their mid-points, the medial ligament splitting to embrace the lacrimal fossa. The septum orbitale is a thin fibrous membrane arising from the whole circumference of the orbital margin and extending inwards towards the peripheral margins of the tarsal plates, thus completing the plane of this central fibrous layer. The orbicularis oculi sheet of muscle fibres is laid over these two structures. The conjunctival membrane lines the deep surface of the tarsal plates and is reflected from them on to the anterior surface 
of the globe, so uniting the eyelids to it, and the fold of reflection is called the fornix conjunctivae. Further, the upper eyelid contains and is rendered more complex by the insertions of a special muscle, the levator palpebrae superioris, which forms an additional structural layer intervening between the orbicularis and the tarsal plate; it passes from within the orbit over the summit of the tarsal plate, carrying before it and uniting with the lower dependent edge of the septum orbitale, and its terminal fibres reach the skin by traversing the orbicularis muscle; hence the levator cuts the fibrous layer of the upper lid in two, whereas in the lower lid the septum orbitale reaches the tarsal plate. Finally, in each lid there is a thin sheet of smooth muscle fibres, the palpebral involuntary muscle, attached to the margin of the tarsal plate, and passing thence to the levator muscle in the upper lid and towards the inferior rectus muscle of the globe in the lower lid.

This complex architecture of the lids will best be understood by following the steps of a dissection from the superficial aspect, as represented in Figs. 52, 53, 54, 55. The skin can easily be raised from the underlying orbicularis, and in doing so it will be seen that on the lateral side and below the orbit the only attachment between the two is by means of connective-tissue septa which pass between the fasciculi of the muscle, whilst in the eyebrow region and below the medial angle of the lower lid, cutaneous insertions of actual muscle fibres have to be cut; moreover, the skin may be found adherent to the exposed lower edge of the medial palpebral ligament at the medial commissure. The marked difference between its thinness and delicacy over the eyelids and its thickness and coarseness in the eyebrow and cheek regions is appreciated on carrying the dissection beyond the limits of the lids.

The orbicularis can next be removed with care in one sheet, commencing the reflection on the lateral side (see Fig. 58); in the circum-orbital region the muscle will be found connected to the underlying bony surface by a dense felting of connective tissue; over the septal region the reflection is difficult owing to the close adherence of the 
underlying septum orbitale, which is so closely attached to the deep surface of the muscle as to resemble a fascial lining ; in the tarsal part of the lid the muscle is more easily lifted. Near the head of the eyebrow the corrugator supercilii muscle will require severing at its origin from the bone, and from this point onwards towards and below the medial

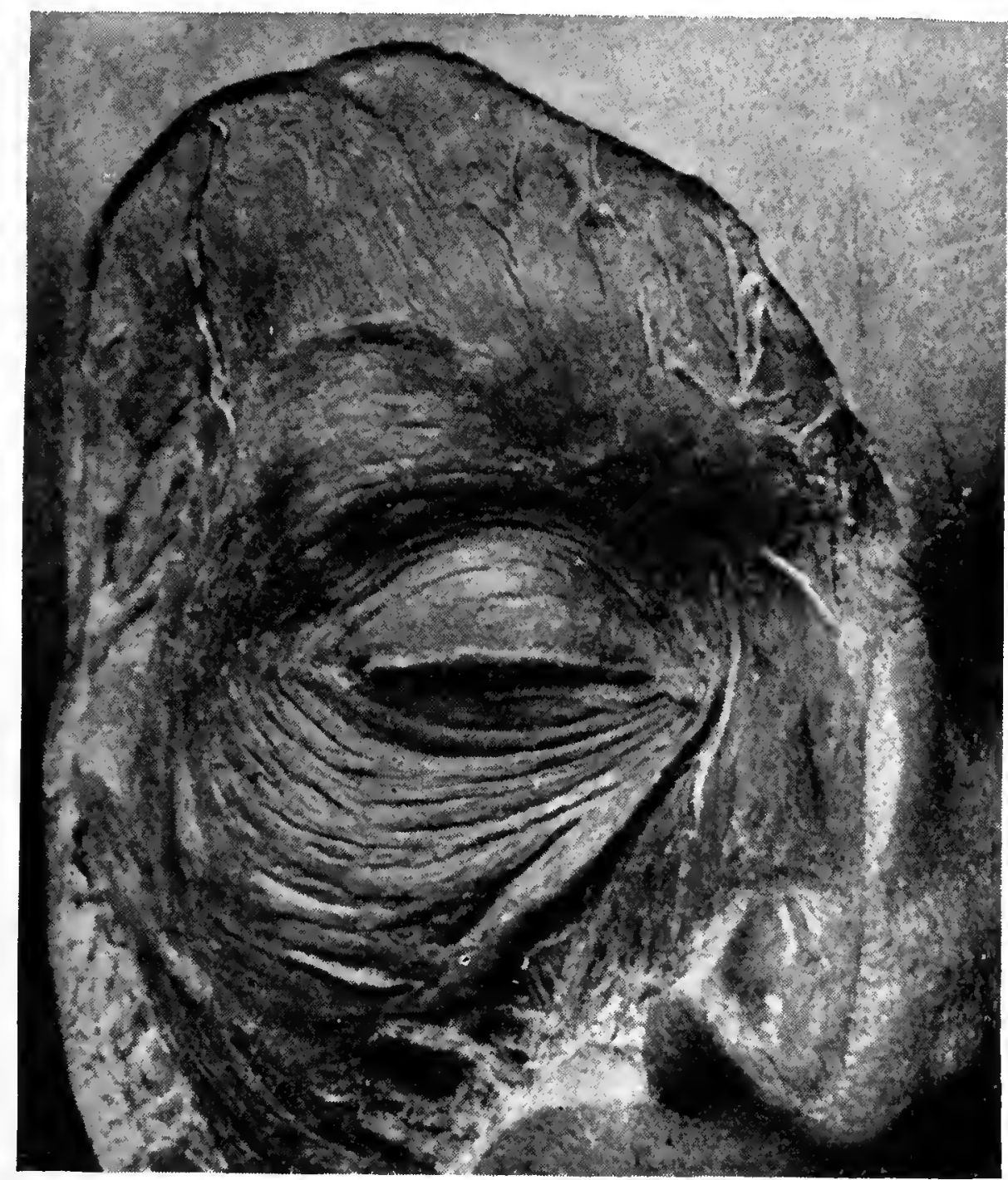

FIG. 52,-Dissection of the Eyelids : first stage. The skin only has been removed, showing the orbicularis oculi sheet of muscle fibres, which should be compared with Fig. 56 for names of constitnent parts. Note the angular vein, running in the cleft between the orbicularis oculi and the levator labii superioris alaeque nasi or caput angulare of the quadratus muscle, along the line of the naso-jugal skin-fold. Natural size.

angle of the eye a broad line of muscle fibres has to be cut, marking the osseous attachment of the orbicularis itself. In reflecting the muscle sheet downwards from the superior tarsal plate a succession of fine connective-tissue strands are severed; these are the terminal fibres of insertion of the levator, which radiate forwards and traverse the fasciculi of the orbicularis to reach the skin. When cut, they collapse 
to form a definite layer of fascia, which has to be raised to expose the superficial surface of the tarsal plate (Fig. 66, p. I40). On lifting this fascial layer further upwards, it will be seen to carry the septum orbitale with and in front of it, and the fact is thus demonstrated that the cutaneous

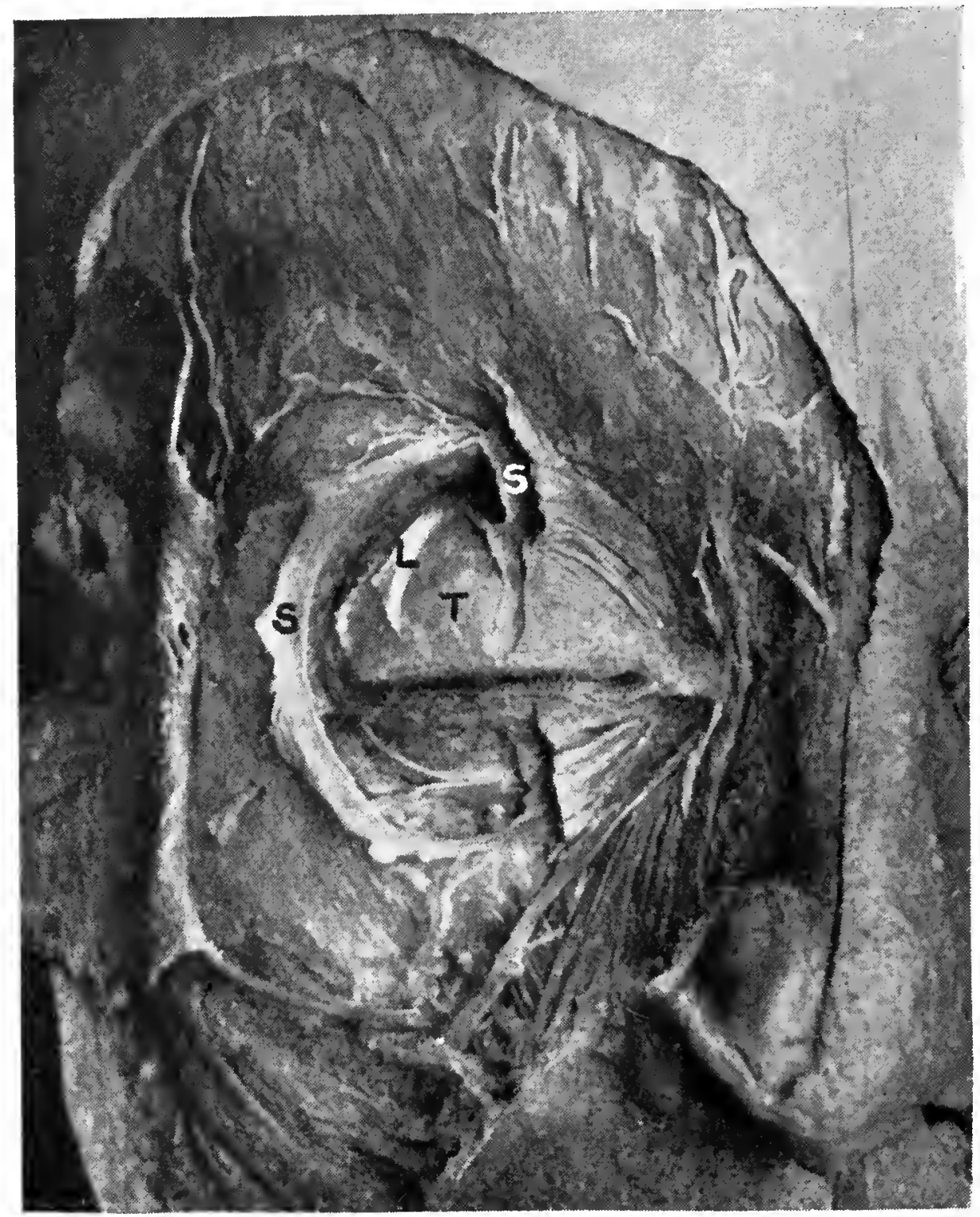

Fig. 53.-Dissection of the Eyelids: second stage. The lateral half of the orbicularis oculi has been turned back over the temple, the medial half is left in situ; the lateral half of the underlying septum orbitale (S.) has likewise been turned aside, and the part left shows its connection with the aponeurosis of the levator palpebrae superioris (L.); the latter has been split and uplifted to expose the tarsal plate ('T.).

insertion of the levator intervenes between the septum and the upper margin of the tarsal plate, and therefore the two last are not continuous with one another, as is sometimes stated. After severing and lifting the septum orbitale, the fascial layer of collapsed levator fibres can be traced backwards, and seen to arise from the front edge of a glistening 
fibrous band transversely arched across the orbit over the globe ; this is the expansion or " aponeurosis" of the levator, in the posterior edge of which the muscle fibres end (Fig. 65).

It will further be noticed that the upper border of the

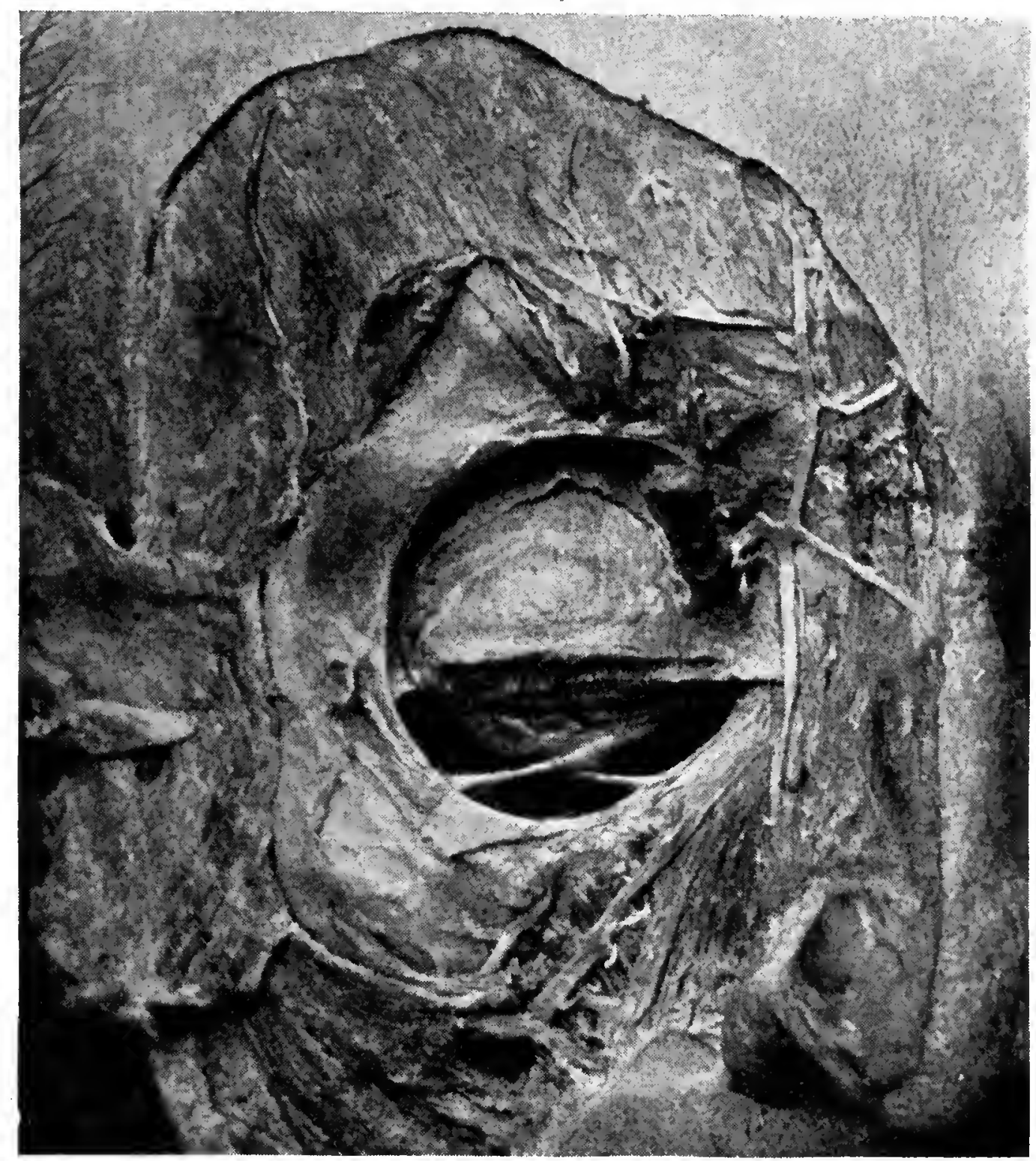

FIG. 54.-Dissection of the Eyelids: third stage. 'The orlicularis oculi and the septum orbitale have been completely removed, and the forc edge of the aponeurosis of the levator cut away to expose the tarsal plate; the orbital fat has been cleared away. 'The preparation shows the supra-orbital and supratrochlear nerves (compare Fig. 8), the pulley of the superior oblique musele, the anastomosis between the ophthalmic and angular veins, the inferior oblique muscle with its so-called "check ligament" (the only instance of this structure the writer has met), and the lacrimal gland subdivided into its two parts by the lateral horn of the aponeurosis of the levator.

exposed tarsal plate is not free, but is continued upwards and backwards into the recesses of the orbit above the globe by means of a pale reddish membrane; this is the superior palpebral involuntary muscle, and in a vertical section it can be seen to be attached posteriorly to the muscular belly 
of the levator and to lie immediately beneath its aponeurosis (Fig. 6r, p. I34).

No further demonstration of layers is possible; the tarsal plate cannot be dissected away from the underlying conjunctiva, though the superior palpebral muscle may with difficulty be isolated from it. After removal of the

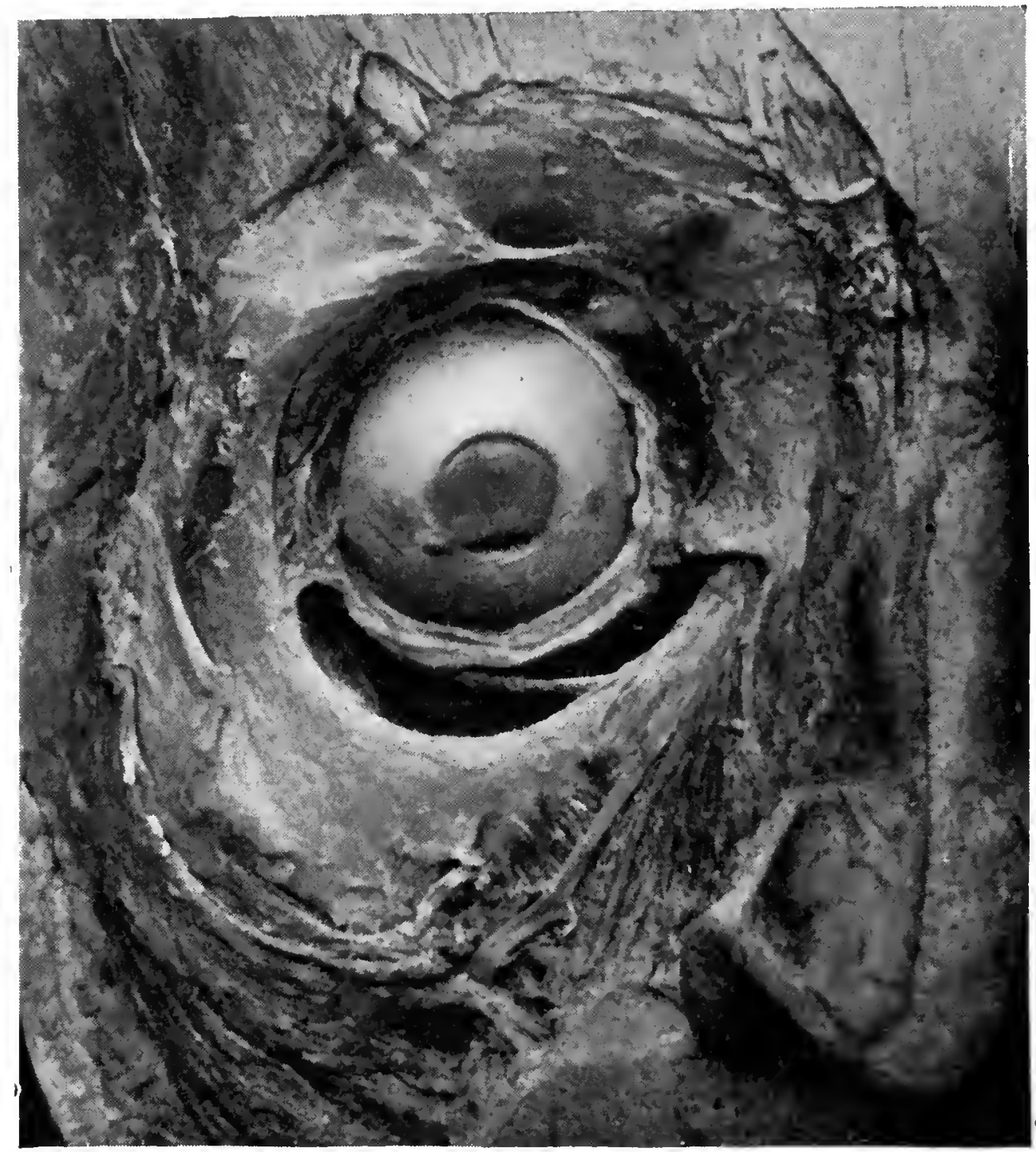

FIG. 55.-Dissection of the Eyelids: fourth stage. The globe is exposed by removal of the tarsal plates, and shows below it "the suspensory ligament" of Lockwood. 'The medial palpebral ligament is well displayed; also the two parts of the lacrimal gland, and below it the retinaculum oculi laterale of Hesser.

septum orbitale, the orbital fat surrounding the globe is exposed behind both lids; the lacrimal gland is seen to lie behind the lateral corner of the upper lid, and the pulley and reflected tendon of the superior oblique muscle behind its medial corner; the origin of the inferior oblique muscle is seen to lie behind the medial half of the lower lid, just wide of and below the lacrimal sac. 
These various structures will now be considered in detail. The skin is extremely thin, and contains no fat in its deeper layer; the hairs are represented by fine down, or may be so short as not to project beyond the follicles; the dermal papillae, sweat and sebaceous glands are also less developed than elsewhere; pigment cells are found in the stratum

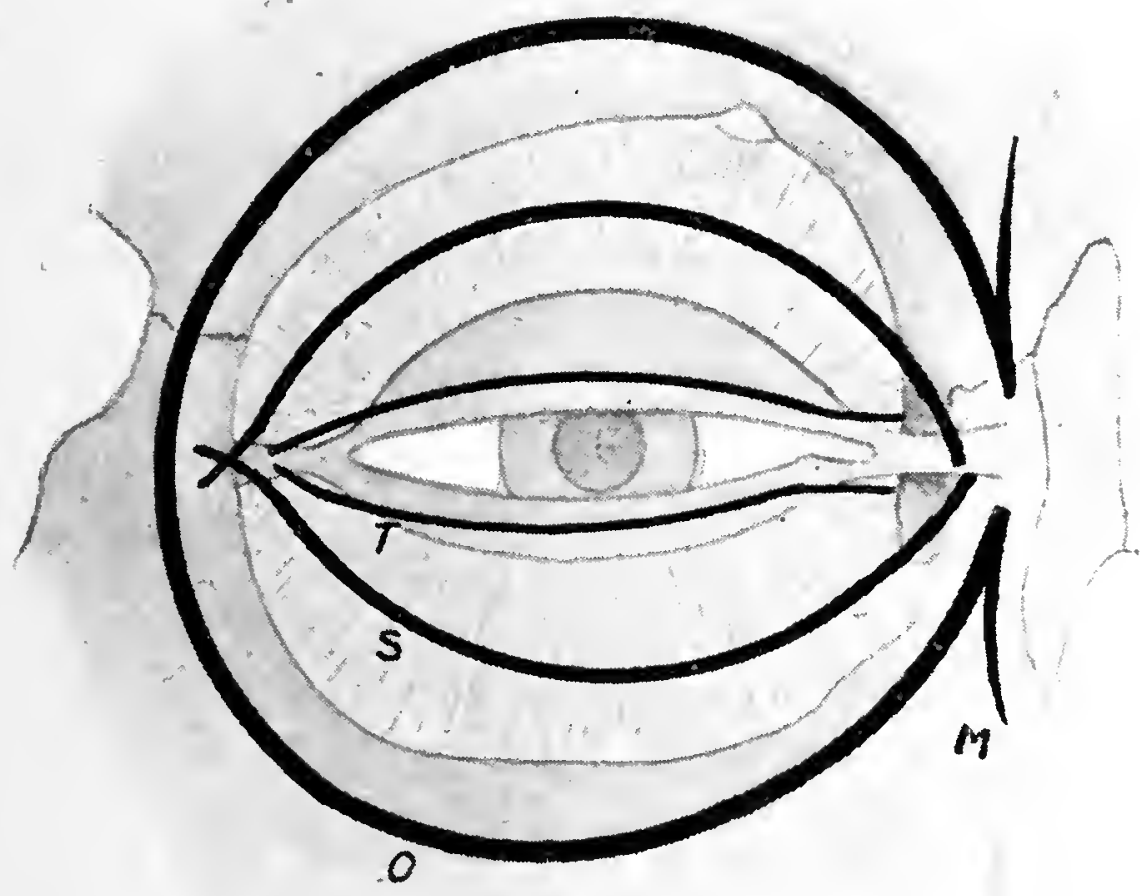

FIG. 56.-Diagram of 'Right Orbicularis Oculi Muscle to show the direction and insertion of its main fasciculi. Compare with Fig. 52.

$\mathrm{O} .=$ The pars orbitalis, covering the orbital margin.

$\mathrm{S} .=$ Pre-septal portion $\}$ of the pars palpebralis covering the eyelids.

M. = Pars malaris.

Note the medial ends of the pre-tarsal fibres passing behincl the fossa for the lacrimal sac and constituting the pars lacrimalis or Horner's muscle, and the lateral ends of the pre-septal portion which interlace over the oprbital margin to form the "lateral palpebral raphe." After Merkel, modified.

mucosum, especially near the medial commissure, and mast and plasma cells are normally present (Parsons, I905). The skin is highly elastic, and but loosely attached by fine connective-tissue strands to the underlying muscle; this tissue represents the "superficial fascia" of other parts of the body, but differs in being devoid of fat (though Adachi has found it normally present in the eyelids of the 
Japanese); its laxity readily allows the skin to be pinched up or raised by effusions, but it is absent at the commissures where the skin is more adherent, so that effusions in the one lid will not readily pass into the other.

The orbicularis oculi (orbicularis palpebrarum) muscle is a thin sheet of fibres covering the eyelids and circumorbital region (Fig. 52); it is somewhat oval in shape, the long axis being horizontally disposed and corresponding to the palpebral opening, round which its well-marked fasciculi are concentrically arranged. Although it forms one continuous sheet, it can arbitrarily be divided into two main portions, an orbital overlying the orbital margins, and a palpebral lying in the eyelids. This distinction, whilst not absolute as regards the attachment of the fibres or their course, is, on the whole, justifiable on both anatomical and physiological grounds, since the two portions show important differences in their relations, attachments, structure, and action.

(i.) The orbital portion (pars orbitalis) is much broader than the palpebral ; it extends on to the regions of the eyebrow, of which it has already been described as a constituent, the temple, where it lies over the anterior part of the temporal muscle, and the cheek, where it covers the origin of the lip elevator muscles and the infra-orbital and zygomatico-facial nerves. The bony attachment of the fibres is to the medial orbital margin and side of the nose, along a curved line extending from the supra-orbital notch to the infra-orbital foramen; fibres also arise from the medial palpebral ligament, which interrupts this line of bony origin (Fig. 59).

The fasciculi arising from the line above the ligament sweep uninterruptedly round the orbital circumference, and are fixed again to the bone below the ligament, so forming complete ellipses on the lateral side ; in their course they give off cutaneous fibres only to the eyebrow and medial part of the lower lid, elsewhere being connected to the skin merely by connective tissue. In the new-born child the superficial orbicular fibres are not interrupted even on the medial side, but pass continuously over the ligament, and it is usual to find one broad fasciculus passing 
right over the ligament in the adult, as seen in Fig. 52. The most outlying fibres above and below the orbit have been named the musculus superciliaris (Merkel, I887) and the pars malaris (Henle) respectively (Fig. 56); a few of the latter fibres pass into the skin below the medial commissure, and can cause a fine wrinkling in this region, but as a whole these aberrant fasciculi are of no special importance. The pars orbitalis is composed of thicker and coarser fibres than the pars palpebralis, and is redder in colour ; its action is auxiliary to that of the latter, and it enters into play in forced closure of the lids, in bright light, and in excessive accommodation of the lens of the eye (p. I8I).

(ii.) The palpebral portion (pars palpebralis) is the essential part of the muscle, and forms an integral part of the eyelids; it is composed of two half ellipses, one in each lid; the fibres arise from the middle of the medial orbital margin and sweep across the lids, to terminate at the lateral commissure, where they cross to form the "lateral palpebral raphe" (raphe palpebrarum lateralis). This raphe is formed purely by the interlacing of the muscle fibres, strengthened on its deep aspect by the septum orbitale, and it does not represent the lateral termination of the tarsal plates, as is erroneously described (see p. I49). The pars palpebralis covers both the tarsal plate and the septum orbitale, and consequently " pre-tarsal " and " pre-septal " fasciculi may be distinguished; the junction of these two divisions is the thinnest part of the muscle sheet, and it coincides with the superior and inferior palpebral skin-folds.

(a) The " pre-septal " or more peripheral fasciculi arise from the medial palpebral ligament, chiefly from its superficial and partly from its deep aspect, but the lower edge of the ligament is usually left uncovered by muscle fibres (Figs. 52, 53) ; they also arise from a short line on the bone above and below it; some of the fibres which arise from the deep surface of the ligament have been described by Gerlach (I880) as forming an " anterior lacrimal muscle," from their close relation to the lacrimal sac, and in microscopical sections their connection with the lacrimal fascia covering the sac is well seen (Fig. I2I, p. 23I). 
(b) The "pre-tarsal" fibres (pars tarsalis), on the other hand, as can well be seen in a dissection of the posterior aspect of the lids (Fig. 57, and Fig. I20, p. 230), form at the medial end of the latter a thick muscular mass about $7 \mathrm{~mm}$. broad by $4 \mathrm{~mm}$. thick, which passes with the deeper part of the medial palpebral ligament behind the lacrimal fossa to be attached to the upper part of the posterior lacrimal crest; the importance and strength of the attachment is shown by the frequent presence in the skull of a thickening

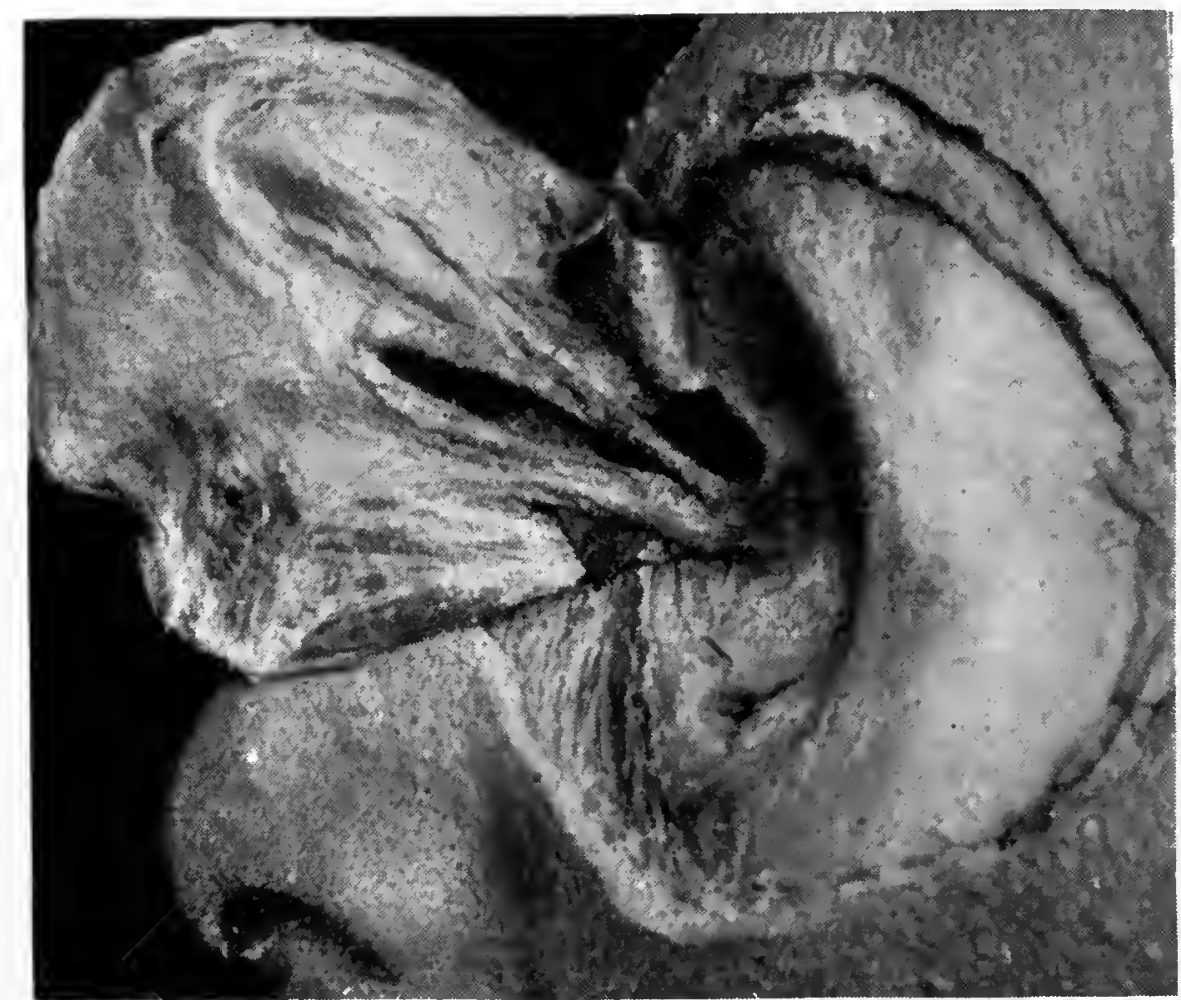

FIG. 57.-The Orbicularis Oculi Muscle dissected away from the lateral side and swung medially, to show the direct continuity of its pars lacrimalis with the pretarsal or pars tarsalis fibres which run along the lid margins. The relation to the upper part of the lacrimal sac, which has been exposed by cutting through the lacrimal fascia, is shown, but is better displayed in Fig. I20, p. $230 . \times \frac{2}{3}$.

or tubercle at this point (Fig. 59), but the area of attachment often encroaches forwards on to the lacrimal fascia or periosteal roof of the fossa in which lies the lacrimal sac. It is this well-defined mass of fibres that has been described as a separate muscle under various names, most commonly as the "muscle of Horner" (I824), but chronologically more correctly as that of Duverney (I749), who well named it the tensor tarsi muscle, or as the posterior lacrimal muscle, or pars lacrimalis of the orbicularis. A few fibres are short and end upon the tarsal plate, others are related to the 
lacrimal canaliculi, which are buried in its mass, as seen in Fig. I2I, p. 23I, but it should be realised that the above names merely denote the conjoint medial ends of two continuous muscular bands which sweep across the lids from one side to the other and are placed over the tarsal plates, hence being more properly called the pre-tarsal portion or pars tarsalis of the orbicularis. Some fibres of the upper bundle cross over to join those of the lower, which latter in their entirety form a specially robust bundle. The practical importance of this part of the muscle lies in its relation to the lacrimal sac, which is considered on p. 23I, and on this account the name " pars lacrimalis" is retained.

Thus the pars palpebralis has a superficial origin from the medial palpebral ligament and adjacent bone, from which fibres sweep over the septal region of the lid, and a deep origin from the posterior lacrimal crest, the fibres from which pass across the tarsal plates. Both sets of fibres interlace in the lateral palpebral raphe:

There is a further subdivision of the orbicularis which calls for mention; this is a small fasciculus of extremely fine fibres, amongst the finest, indeed, of the whole body, which runs close along the free margin of each lid, separated off from the rest of the muscle by and lying behind the follicles of the eyelashes; it is known as the marginal part of the orbicularis (pars marginalis or pars ciliaris) or ciliary bundle of Riolan, and a few of its fibres, seen in microscopical section to lie behind the openings of the ducts of the tarsal glands, are called the "subtarsal portion" (Klodt, I893, and Virchow, I908) ; they are seen in Figs. 77, 78, 80, and 93.

The fibres of the pars palpebralis are paler and finer than those of the pars orbitalis ; in action they are not only voluntary, but also involuntary (see p. I77).

To sum up, the orbicularis oculi muscle sheet may be subdivided into :

(i.) Pars orbitalis, covering the orbital margin ;

(ii.) Pars palpebralis, covering the eyelids; and in the latter these can be differentiated :

Pars septalis, covering the septum orbitale:

Pars tarsalis, covering the tarsal plates, and including: 
Pars lacrimalis (of Horner), behind the lacrimal fossa;

Pars ciliaris, along the lid margins ;

Pars subtarsalis, behind the tarsal glands.

Considerable variations are found in the thickness and development of this muscle. It is thicker in the negroid and yellow races than in white (Chudzinski). Accessory fasciculi may be present, forming connections with neigh-

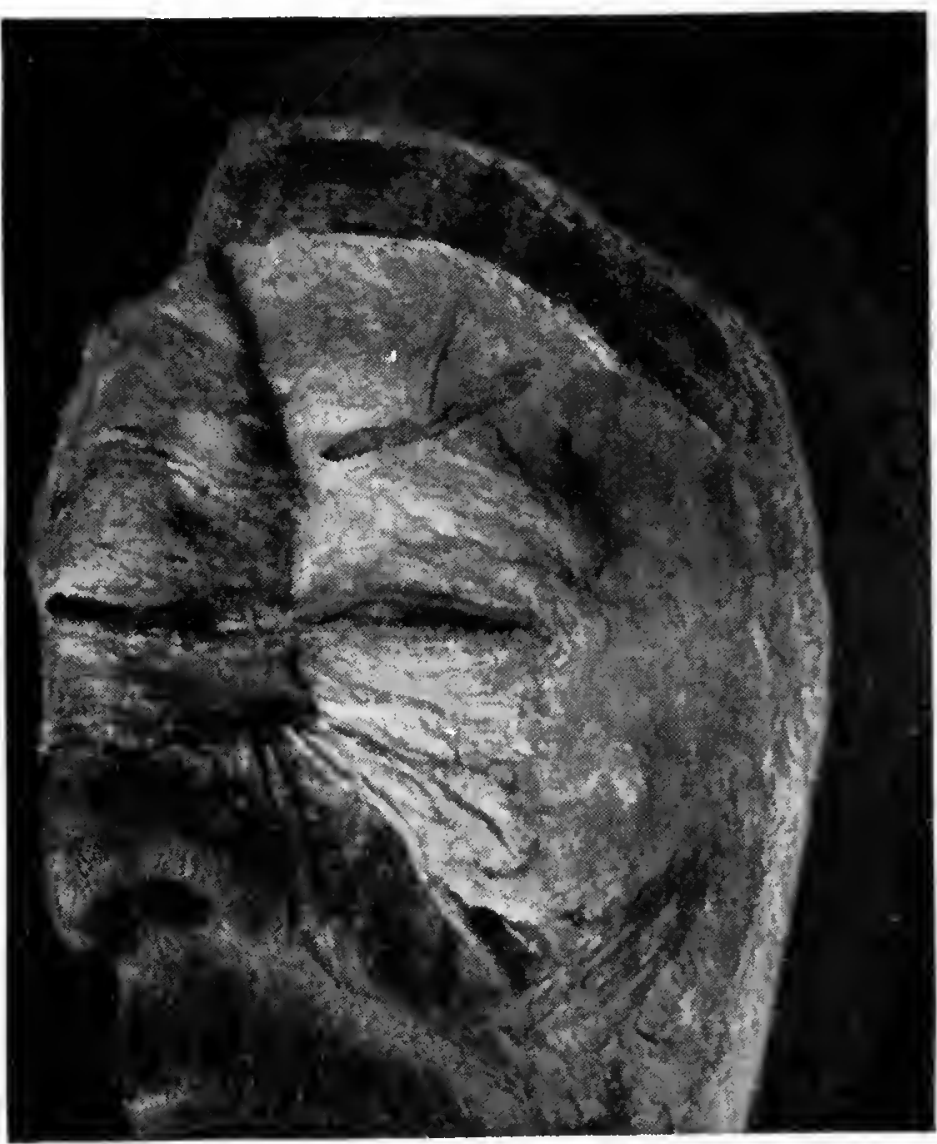

FIG. 58. - The Septum Orbitale exposed by reflection of the orbicularis oculi, which has been turned medially. "Note the supra-orbital nerve piercing it above, and the terminal cutaneous fibres of the lacrimal nerve emerging just below the lateral angular process of the frontal bone. bouring muscles of the face, and it may even be united to its fellow of the opposite side by a transversus glabellae muscle (Ruge). There may be a complete separation of the palpebral from "the orbital portion. - On the other hand, cases of absence of the orbital or a rudimentary condition of the palpebral portion have been noted. Such variations are of interest, since they indicate the developmental origin of the orbicularis as a specialised part of a continuous platysmal sheet of muscle fibres; they are recorded in detail in the works of Macalister (I875) and Ledouble (I897).

The septum orbitale (the broad ligaments of the eyelids or of the tarsal plates; superior and inferior palpebral ligaments; palpebral fascia or tarsal membrane; fascia tarso-orbitalis or orbito-palpebralis) is a thin membrane of connective and elastic tissue, which extends from the entire orbital margin towards, but not reaching, the palpebral opening. Whilst the septum lies in the same morphological 
plane as the tarsal plates, it is essential to consider it not as a fixed vertically disposed diaphragm, but as a supple floating membrane, changing its contours with the movements of the lids. In fact, its disposition and relations are best realised by regarding it as the deep fascia lining the palpebral part of the orbicularis oculi muscle, and in gross dissection

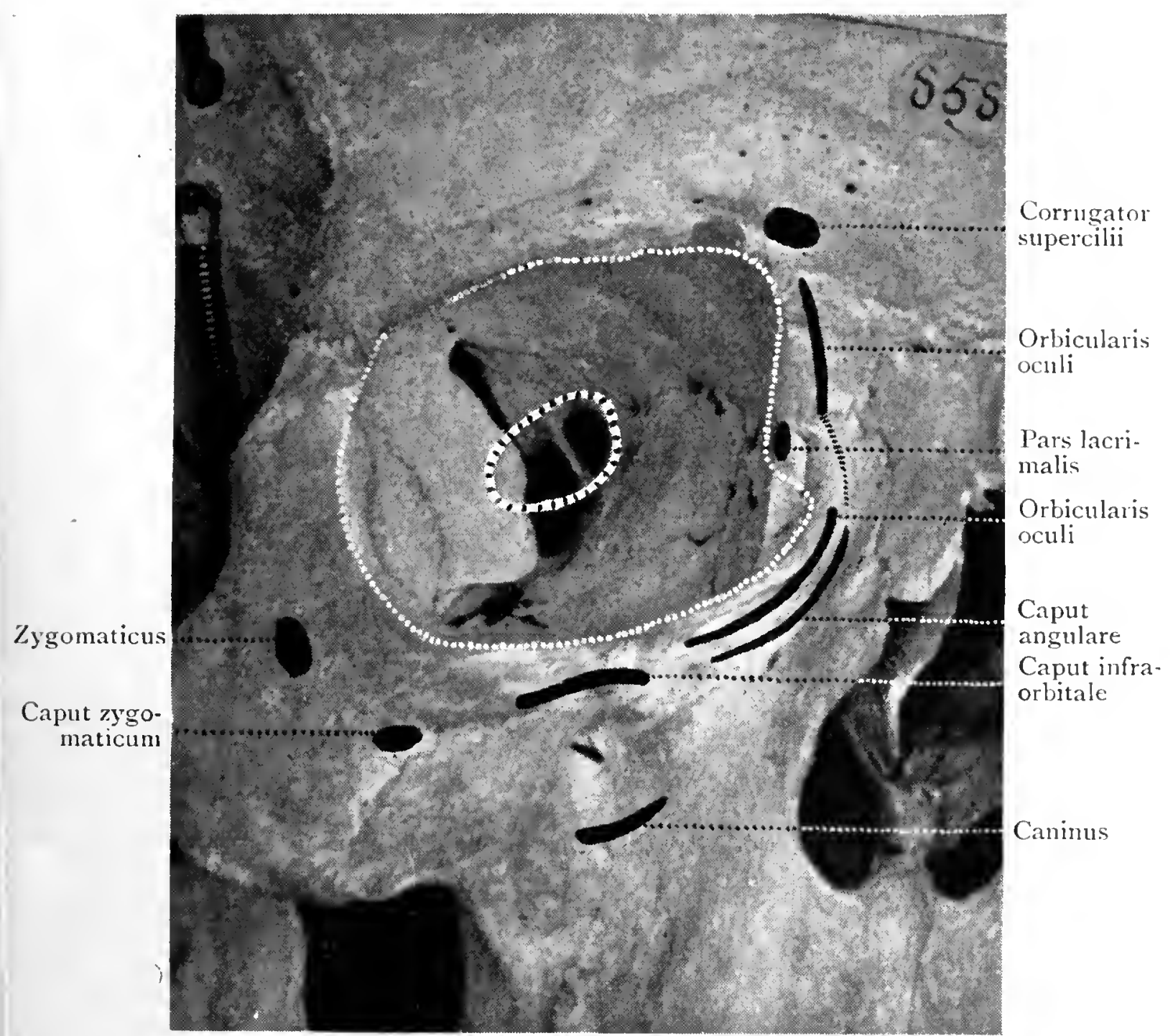

FIG. 59.-Markings of Muscle Attachments in and around the Orbit. The centre ring represents the fixation of the Annulus of / $/$ inn ; the white dotted nuarginal line indicates the origin of the septum orbitale.

of the lids, the skin, muscle, and septum together form one combined layer covering the space between the globe and the orbital margin; it is recognisable as a definite structural layer only by careful dissection, but merits its name in that it can form a barrier to the passage of extravasations from the orbit to the lids and vice versa (see pp. I38, 299).

Along its line of origin from the bone it is directly continuous with the periosteum, which is here thickened and 
raised along the exact line of the orbital margin to form a sharply defined ridge, the "marginal arcade" or arcus marginale (Charpy, I9II). In tracing the septum round the orbital margin, as may be followed in Fig. 59, it is found specially well marked on the lateral side; it then springs from a periosteal bridge across the supra-orbital notch (Fig. 55), and passes in front of the fovea trochlearis along a rough line on the bone to descend behind the area of attachment of the pars lacrimalis muscle (for which the septum acts as a thin posterior covering); it then skirts the lower border of the muscle and crosses the middle of the lacrimal fossa to gain the anterior lacrimal crest and pass on to the inferior orbital margin. The pars lacrimalis muscle and the lacrimal sac thus lie outside the orbit, of which the septum exactly defines the anterior boundary.

In the direction of the palpebral opening the attachrinents differ in each lid; in the lower lid the septum blends with the anterior face of the tarsal plate; in the upper it has been seen to be separated from the plate by the terminal fibres of the levator muscle and carried forwards with them to the skin; at its junction with the levator, however, some of its fibres are reflected backwards towards the aponeurosis of that muscle, so that a smooth concave barrier is presented to the orbital contents above the globe, the concavity containing a well-defined long roll of fat with tapering ends (Figs. 62, 67). Its dependence as a curtain from the supra-orbital margin and its connection with the levator can be demonstrated by such a preparation as is shown in the following figure, where the bony margin has been sawn and turned aside, carrying the septum with it.

The septum presents local differences in thickness; it is best developed, like the marginal arcade from which it springs, on the lateral side, where it stretches between the orbital margin and the angle of the eye and reinforces the raphe formed by the overlying muscle fibres of the orbicularis; it is thin in the lower lid and most delicate in the medial region; it is perforated by the vessels and nerves passing to or from the orbital cavity, the orifices lying along its peripheral margin (Fig: 54). 
The levator palpebrae superioris, the special elevator
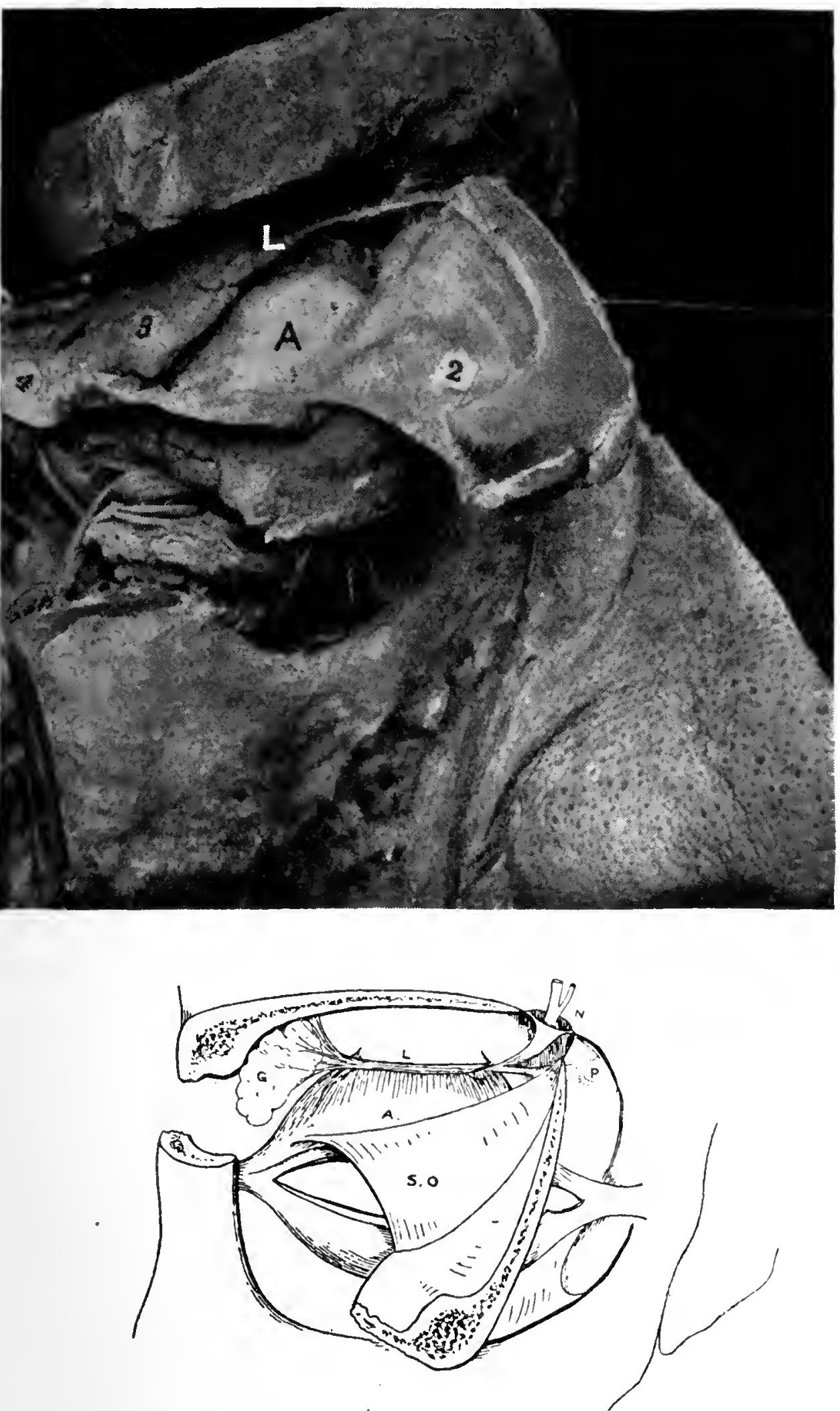

FIG. 60.-Dissection to show Septum Orbitale. After removal of the skin and orbicularis oculi muscle, the bone forming the supra-orbital margin has been cut and turned down with the attached septum orbitale (S.O. and 2), exposing the palpebral aponeurosis (A.) of the levator. Above this is seen lying the transversely placed ligament (L.) formed by its sheath; its expansion which passes forward to bridge the supra-orbital notch (N.), and attachment to the pulley (P.) of the superior oblique on the medial side, and comections with the lacrimal gland (G. and 3 ) on the lateral side, are shown. Compare with Fig. 68, p. r 43.

muscle of the upper eyelid, lies within the orbit, and only 
its terminal part enters into the lid. It is thin, flat, and of

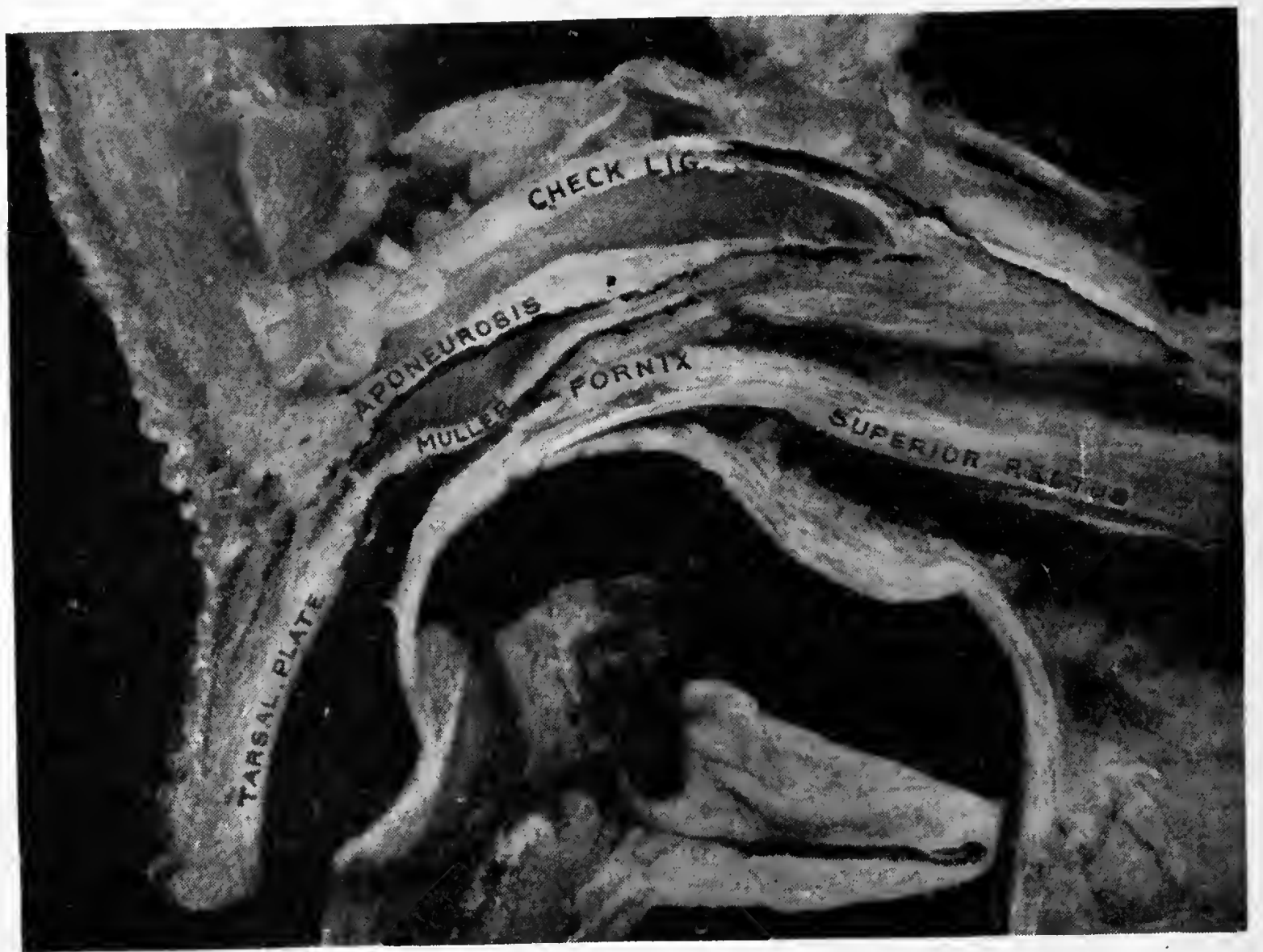

FIG. 6I. - Sagittal section through Upper Eyelid and Globe, to show connections of Levator Palpebrae Superioris muscle. $\quad \times 2$.

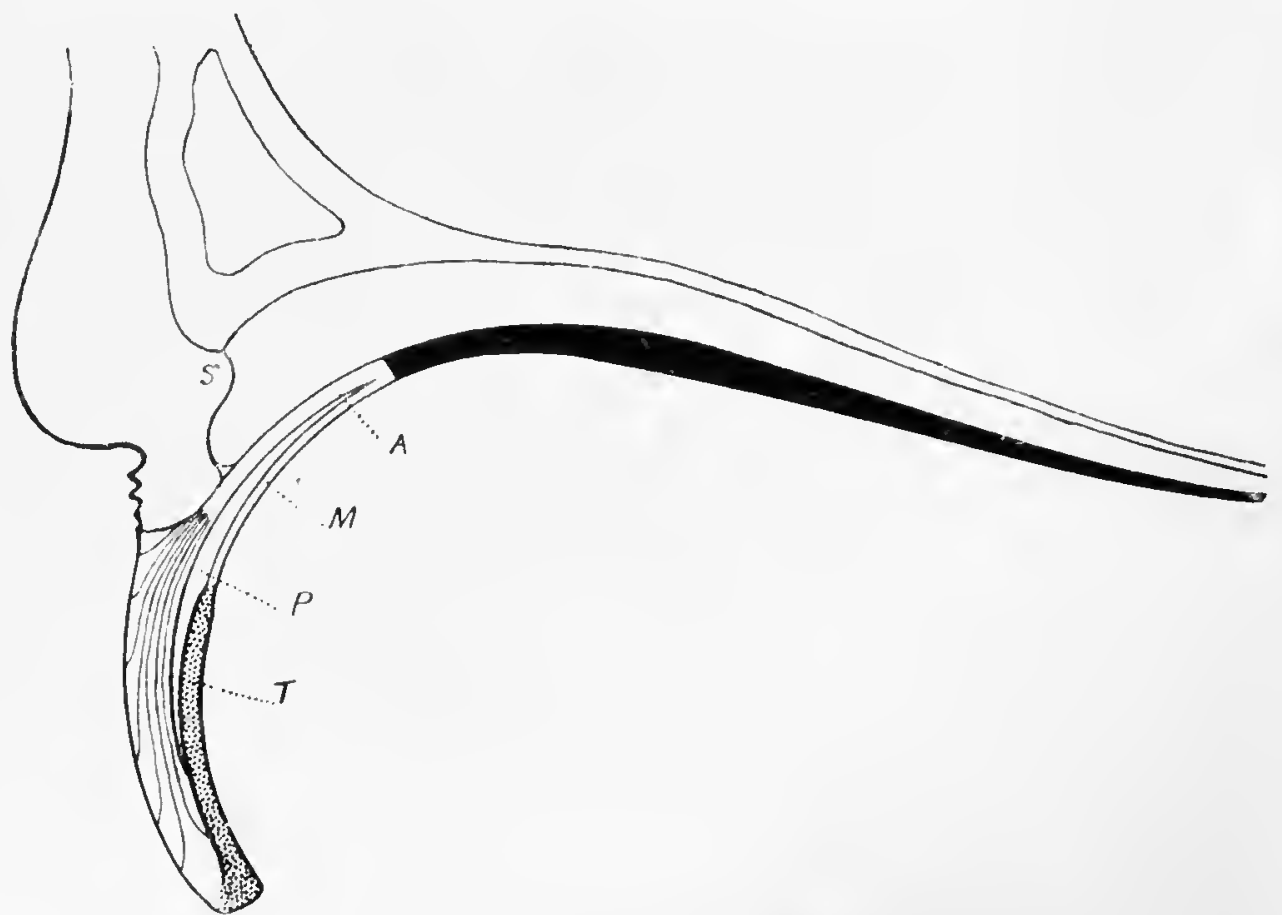

FiG. 62.-Diagran of the Levator Palpebrae Superioris, showing its connections.

A. $=$ Aponeurosis.

M. = Superior palpebral involuntary muscle of Müller.
$\mathrm{T} .=$ Tarsal plate.

P. = Pre-tarsal space.

$\mathrm{S} .=$ Septum orbitale.

the shape of an isosceles triangle. It arises at the apex 
of the orbit and passes forwards towards the base, where its fleshy belly terminates in an expanded tendon called the "aponeurosis" ; the fore-edge of this band breaks up into fine connective tissue fibres, which radiate upwards and downwards and are inserted into the skin of the lid. There are therefore three parts of the muscle to be seen in a longitudinal section: the fleshy belly, the aponeurosis, and the terminal fibres (Fig. 62). Moreover, lying in close contact be-

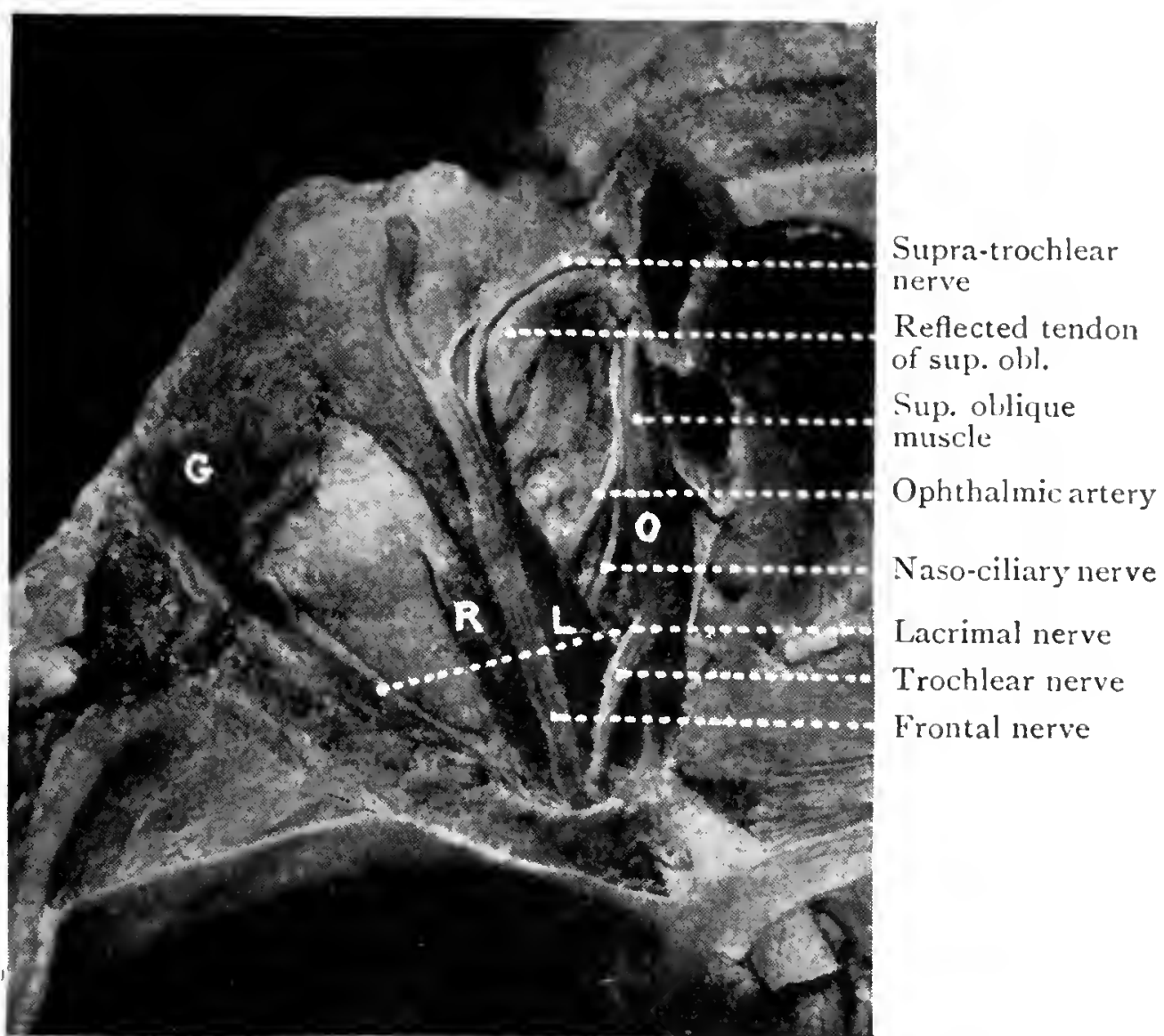

FIG. 63. - Dissection of Left Orbit from above, to show the levator palpebrae superioris (L.) and superior oblique (O.) muscles, and the nerves in relation. R. =Superior rectus muscle: G. =lacrimal gland. Compare with Fig. 65 . Natural size.

neath the aponeurosis is a thin lamella of pale smooth muscle fibres, which appears to be a direct continuation of the fleshy belly, but is inserted directly into the upper margin of the tarsal plate. This is the superior palpebral involuntary muscle, and constitutes a secondary insertion of the levator.

The levator arises by a short narrow tendon from the lesser wing of the sphenoidal bone, just above and a little lateral to the optic foramen; it is separated from this orifice by the origin of the superior rectus muscle, with the tendon of which it is blended (Fig. I35, p. 260); shortly 
after its origin it is crossed obliquely by the trochlear or fourth nerve, which passes medially to enter the adjacent superior oblique muscle. As it passes forwards the levator inclines laterally and lies close beneath the roof of the orbit, the frontal nerve and the supra-orbital artery lying upon it; the nerve crosses it diagonally from the lateral border behind to the medial border in front, and then divides into supra-orbital and supra-trochlear nerves, and the vessel lies over its anterior half only. In its whole course the levator lies upon the superior rectus muscle, but does not cover it entirely, the lateral border of the latter being exposed and free; the medial borders of the two muscles coincide and are adherent by their thin fascial sheaths; they are also connected by means of the nerve to the superior rectus, which after piercing that muscle supplies also the-levator. These relations are shown in Fig. 63. A bursa is said by Motais to exist between the two muscles, but none could be found in the writer's series of preparations.

The palpebral aponeurosis or expanded tendon of the levator is a glistening white band of dense connective tissue about $7 \mathrm{~mm}$. in depth from before backwards and $0.5 \mathrm{~mm}$. thick; it lies just behind the supra-orbital margin, and arches over the globe from side to side. Its anterior edge splits up into terminal cutaneous fibres; its extremities become narrower but remain tendinous, and are attached to the medial and lateral orbital margins at their mid-points, and may be distinguished as lateral and medial "horns." The attachments of the aponeurosis, which, it will be realised, are the actual insertions of the levator, are therefore both cutaneous and osseous. The former are best studied in vertical section, the latter by dissection from above; both are seen in a dissection of the lids from the front.

The cutaneous insertion is into the skin of the whole of the pre-tarsal part of the lid by means of vertically radiating fibres of delicate connective tissue; this is the primary and essential attachment of the muscle. In reaching the skin the fibres necessarily traverse the horizontally disposed fasciculi of the orbicularis oculi muscle, as can clearly be seen in microscopical sections (Fig. 73, p. I5I). They sweep 
over the bare superficial face of the tarsal plate in their downward course, and it is the condensation of these fibres that forms the definite layer of tissue which has to be lifted before the plate is exposed in a methodical dissection of the lid, as shown in Figs. 65, 66 ; they are also raised in splitting the lid along the midline of its edge. The importance of realising this arrangement in operations on the lids for ptosis; trichiasis, or plastic operations is emphasised by Wolff (I905). The uppermost fibres meet and fuse with the septum orbitale; the lowermost fibres are inserted into the lower third of the face of the tarsal plate and mingle with the bulbs of the cilia (Fig. 62). It will be seen that the centrally situated aponeurosis of the levator with its radiating fibres divides the lid into two potential spaces, a "pre-tarsal" confined between the tarsal plate behind and the levator in front, and a "pre-septal" which lies in front of the levator and the septum orbitale, and is limited above by the fatty cushion of the eyebrow (Fig. 62). The pre-tarsal space contains between its boundaries laterally the palpebral lobe of the lacrimal gland and terminal branches (lateral palpebral) of its artery, and within its upper part there runs from side to side the superior tarsal arterial arcade.

These spaces can be injected in the fresh subject by means of coloured gelatine (Clermont, I909; Charpy, IgII); by using a small amount of fluid the two spaces can be injected separately, but on pushing the injection further the masses become confluent and the fluid will next pass between the fasciculi of the orbicularis and lie beneath the skin, so that the whole lid becomes infiltrated, the muscle lying in the 
middle of the mass, as shown in Fig. 64. The injection will not readily pass backwards into the cavity of the orbit, owing to the barrier afforded by the septum orbitale, which is rarely penetrated even by abundant injections, though a gradual transudation and percolation through the nervous and vascular orifices of that membrane can take place;

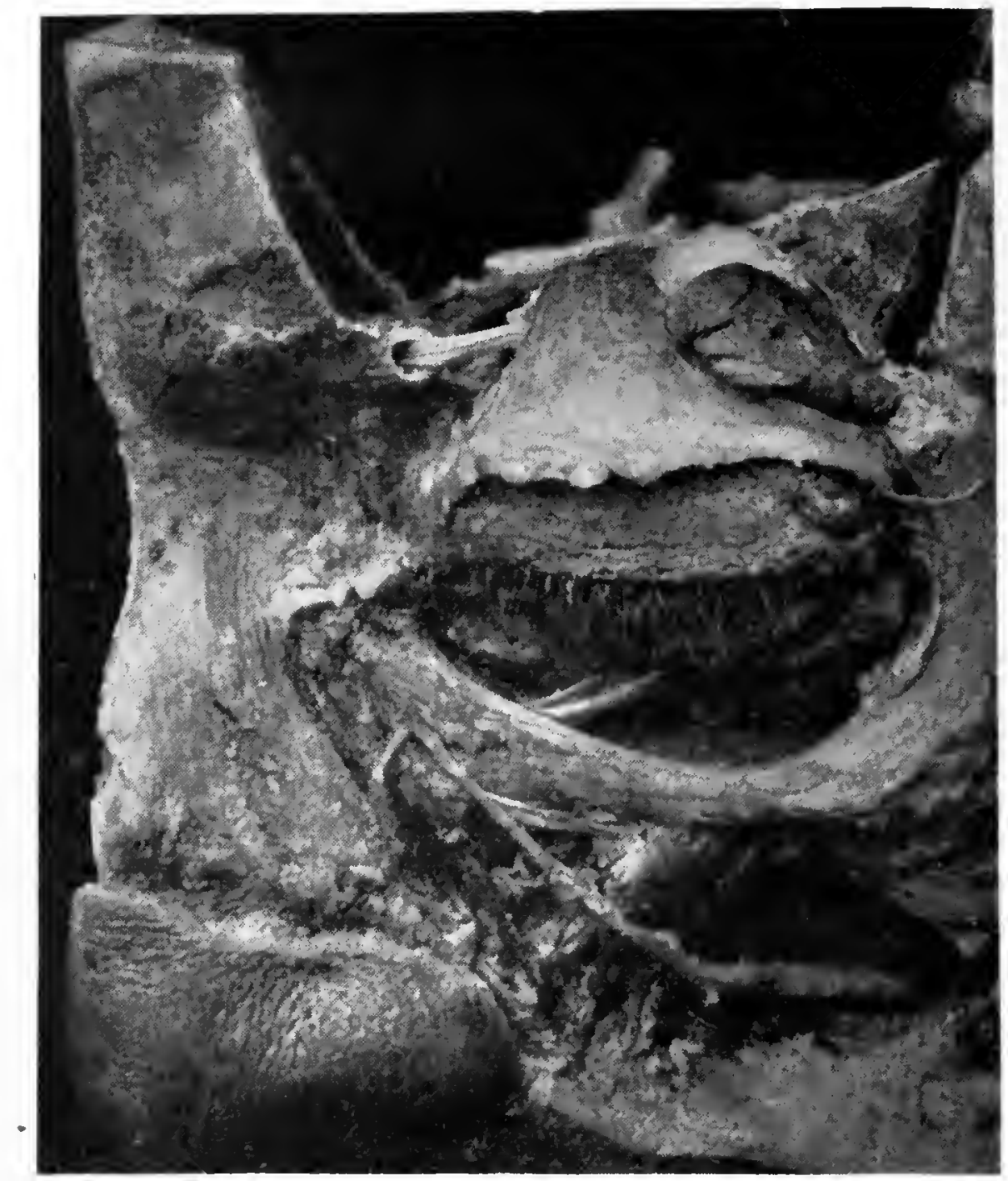

FIG. 65. - Dissection of the Left Orbit from above and also in front (the same preparation as Fig. 63, viewed from in front) to show the aponeurosis of the levator palpebrae superioris muscle and the lacrimal gland. 'The pulley of the superior oblique muscle and its tendon are also seen. Natural size.

extension on to the temple or cheek is prevented by the felting of connective tissue which lies between the orbicularis oculi and the bone in these regions. (See also p. 299.)

The osseous or orbital attachments of the aponeurosis are effected by means of its medial and lateral horns to the corresponding orbital margins at their mid-points and opposite the commissures of the eyelids. The lateral horn is much more strongly developed, and in the form of a 
conspicuous ligament, 3-4 mm. broad, cuts into and subdivides the lacrimal gland, which is, as it were, folded round it (Fig. 65, and see also Fig. I05, p. 206); below the gland the lateral horn is inserted into the orbital tubercle of the zygomatic bone, covering the insertion of the true lateral ligament of the tarsal plates (just as the terminal cutaneous fibres of the aponeurosis pass in front of the plate) ; it is not uncommon to find a slender continuation of the horn passing downwards round the lateral angle of the eye into the lower lid, where it joins the fascia beneath the globe, as is seen in Figs. 49 and 53. On the medial side the aponeurosis loses abruptly its tendinous nature as it passes over and comes into close contact with the reflected tendon of the superior oblique muscle; the opposed surfaces are smooth and shining, but no definite bursal formation can be identified. From this point the medial horn turns downwards at an angle, and can be traced only with difficulty in the form of loose strands to fuse with the medial palpebral ligament, and so reach the bone. Thus from the greater strength and more definite development of the lateral horn, and the fact that the fleshy fibres of the muscle curve laterally and extend further forwards in that direction, whilst the medial border of the muscle is parallel to the mesial plane, the main osseous insertion is the lateral one into the orbital tubercle. Retraction of the muscle is consequently more restrained on this side, and the greater excursion of movement on the other may explain the accentuation of the marginal curve of the upper lid in its medial part noted above on p. II7.

The Palpebral Involuntary Muscles or " tarsal muscles" (musculi palpebrales, or tarsales of Merkel, or orbitopalpebral muscles of Sappey) were first described by Müller in 1859 ; they are not to be confused with the involuntary " orbitalis" muscle, also described by him, which lies in the inferior orbital fissure and is connected with the periorbita (p. 82). They are found in each lid in varying degrees of development, but usually form a thin sheet of pale red fibres connected with the peripheral margin of the tarsal plate and lying in front of the conjunctiva. That in the upper lid, namely the superior palpebral involuntary muscle, 
is the better developed structure, and is about I cm. broad antero-posteriorly; its position immediately beneath the aponeurosis of the levator has already been noted; but so closely does it lie in contact with it that it may not be recognised as a distinct structure, though the two can be easily separated and their several connections made out in a vertical section. The fibres arise from between the striated fibres of the levator by elastic tendons, and have a similar mode of fixation to the upper margin of the tarsal plate,

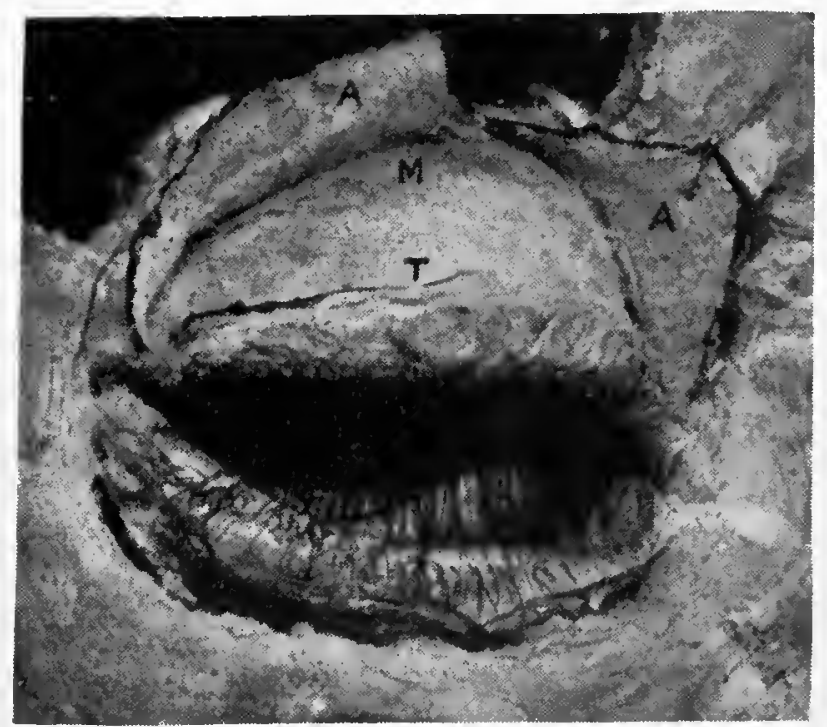

FIG. 66.-Dissection of Right Orbit and Eyelid from in front. 'The aponeurosis of the levator palpebrae superioris muscle (A.) has been reflected upwards from the face of the tarsal plate ('T.), to the upper margin of which is attached the superior palpebral involuntary muscle (M.). $\times$ about $x \frac{1}{4}$. a connection to be seen in such a microscopical section as is shown in Fig. 74, p. I5I, and well displayed in Fig. 66, in which the levator fibres have been turned upwards to expose the tarsal plate. For the most part the fibres run in a vertical direction, and are mingled with connective tissue and fat cells; groups of nerve cells analogous to ganglion cells are also present (Groyer, I905). In the lower lid the inferior palpebral muscle is a much less definite structure and is not easily recognisable by dissection, though the fibres can be traced microscopically. It arises from the fascial sheath of the inferior rectus muscle or from the expansion which this sheath gives to the inferior oblique, and it divides into two lamellae at the conjunctival fornix, one of which ends in the bulbar conjunctiva, whilst the other enters the lower lid, where it does not reach as far as the tarsal plate (Virchow, I908), as represented in Fig. 67.

The morphology of this palpebral involuntary musculature is interesting. In the aquatic mammals, as for example the seal and the dolphin, each of the four recti muscles of the globe divides anteriorly into two lamellae, 
one of which, the inner, is inserted onto the globe and so constitutes the rectus proper, whilst the other, the outer, enters the eyelid as a "palpebral muscle" ; both lamellae are composed of striated muscle fibres, and both receive the same innervation. In other mammals the outer palpebral lamellae derived from the inferior medial and lateral recti are wholly replaced by smooth muscle fibres, and in man are represented by the inferior palpebral muscle and also by the scattered involuntary fibres found in the lateral expansions of the sheaths of the last two muscles, fibres which are sometimes dignified by the name of medial and lateral " palpebral muscles." In consequence of the greater development of the upper lid, the outer or palpebral lamella derived from the superior rectus remains almost entirely striated and forms the levator muscle, but a deeper part of the same palpebral lamella, in harmony with the fate of the whole of the corresponding layer of the other muscles, becomes converted into smooth fibres, and is called the "superior palpebral muscle" (Groyer, I903, I905, and I906). This involuntary musculature as a whole forms an incomplete ring around the globe, and is intimately connected with the fascia around that organ (Tenon's capsule), under which heading further reference will be made to it (p. 296). It is innervated by the sympathetic nervous system, possibly by fibres from the cavernous plexus, which enter the third nerve and run along its branch of supply to the levator. Here the superior palpebral muscle calls for notice, since it indirectly forms one of the insertions of the levator palpebrae superioris.

There remains a last so-called insertion of the muscle to be described; this is the conjunctival attachment, which is effected not by the muscle itself, but through the agency of its FASCIAL SHEATH. As is the case in all the ocular muscles, the fascial sheath of the levator is so thin and transparent around its posterior half that the fleshy fibres appear quite devoid of covering ; anteriorly, however, the fascia becomes gradually thicker, until just behind the aponeurosis the whole muscle is enveloped in a thick opaque sheath, which fuses underneath with the similar covering of 
the superior rectus; the common mass of tissue so formed fills up the angle of divergence between the two muscles as they pass to their several insertions, the one on to the lid,

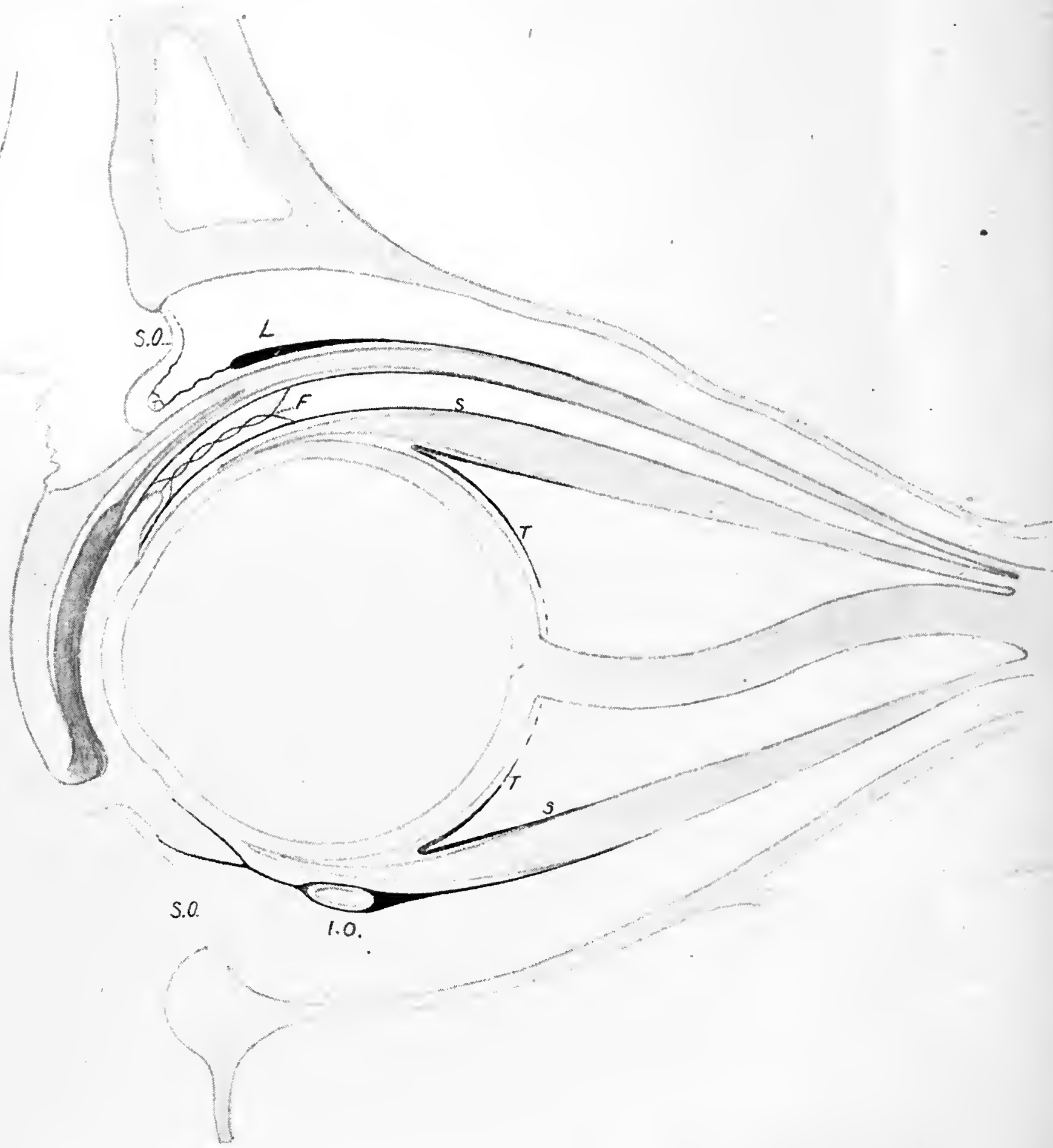

FIG. 67.--Scheme of the Fascia of the Orbit (the muscle sheaths and Tenon's capsule) in sagittal section. Compare with dissection shown in Fig. $6 \mathbf{I}$.

S.O. = Septum orbitale.

$F_{.}=$Conjoint fascial sheaths of the levator and superior rectus.

S. = Muscle sheaths.

T. ='Tenon's capsule.

L. = Thickened part of sheath of levator.

I.O. = Inferior oblique with the "suspensory ligament" of Lockwood posterior to it.

the other to the globe. Anteriorly this mass abuts on and is fixed to the conjunctiva along the line of its reflection from the lid on to the globe (superior fornix), and though no. 
muscle fibres pass into it from the levator, the attachment is described as an additional insertion of that muscle (see Figs. 6r, 67). It is by means of this fascial attachment that the conjunctiva is drawn up and backwards when the globe is rotated upwards, and harmoniously with it the upper eyelid is elevated; it would be as accurate to describe it as being an additional "insertion" of the superior rectus muscle.

A further point of interest is presented by this sheath of the levator: its superficial part covering the upper aspect of the muscle just behind the aponeurosis is condensed to form a definite ligamentous band, which stretches transversely across to reach the orbital walls on either side, parallel with but attached at a higher level than the aponeurosis; medially the main connection is to the trochlea, but behind this some fibres pass to the bone, and a well-marked slip passes forwards to bridge the supra-orbital notch ; laterally the band is connected with the stroma of the lacrimal gland and cuts into it, as does the lateral horn of the aponeurosis, and beyond the gland it reaches the lateral orbital margin; it lies for the most part free above the aponeurosis, but stout strands of connective tissue may unite them (Figs. 6o, 6r, 68). In front of this ligamentous condensation the sheath becomes abruptly so thin that it appears to end in a free border, but it can be traced forwards as a very delicate layer up to the supra-orbital 
border (see Fig. 67). The band is well developed in the foetus. When traction is made upon the levator from behind this band becomes tense and thus forms a "check ligament" to the muscle, preventing its overaction, a function it can perform by reason of its situation and attachments better than the aponeurosis (the horns of which are fixed at a lower level), which is generally considered to act in that capacity (Whitnall, Igro). The action of the levator is therefore checked by the attachments of its fascial sheath, as is the case in all the other ocular muscles.

To recapitulate: the anterior attachments of the levator palpebrae superioris muscle are as follows:

Primary.-(i.) Cutaneous, by means of its terminal fibres to the skin of the eyelid; (ii.) Tarsal, by means of (a) some of its terminal fibres, (b) the superior palpebral involuntary muscle.

Secondary.-(iii.) Osseous, by the horns of its aponeurosis; (iv.) Conjunctival, by means of its fascial sheath fused with that of the superior rectus muscle.

Three ABNORMALITIES of the levator have been described:

(i.) The commonest variation is a slip passing from the medial border of the muscle to the pulley of the superior oblique muscle. Such a fasciculus was described by Budge (I859) as the musculus tensor trochleae, and appears to be identical with muscles described by Vesalius, Molinette, Kolmus, and Sandifort, the comes oblique superioris of Albinus, and the gracillimus orbitis of Bochdalek. Budge found it present in fifteen out of twenty cases examined, but Virchow considers it much more rare, as indeed it was in the writer's observations; an instance is recorded by Rush and Schaeffer (I9I7). (See also p. 284.)

(ii.) Similar fasciculi may be present passing from the lateral border of the muscle to the lacrimal gland. Such strands could have a much more effective action as a retractor. glandulae lacrimalis than could the medial offshoots as atensor trochleae, since the gland is movable, whilst the trochlea is fixed. In the example figured below traction on the levator pulled the gland backwards, owing to the 
attachment of these fasciculi. Both these offshoots replace normal connections of the fascial sheath of the levator to the trochlea and lacrimal gland.

(iii.) As a musculus transversus orbitis Bochdalek (1868) described certain fleshy bundles passing from the fore and upper part of the os planum of the ethmoidal bone across the orbit to the lateral wall, passing over but closely con-

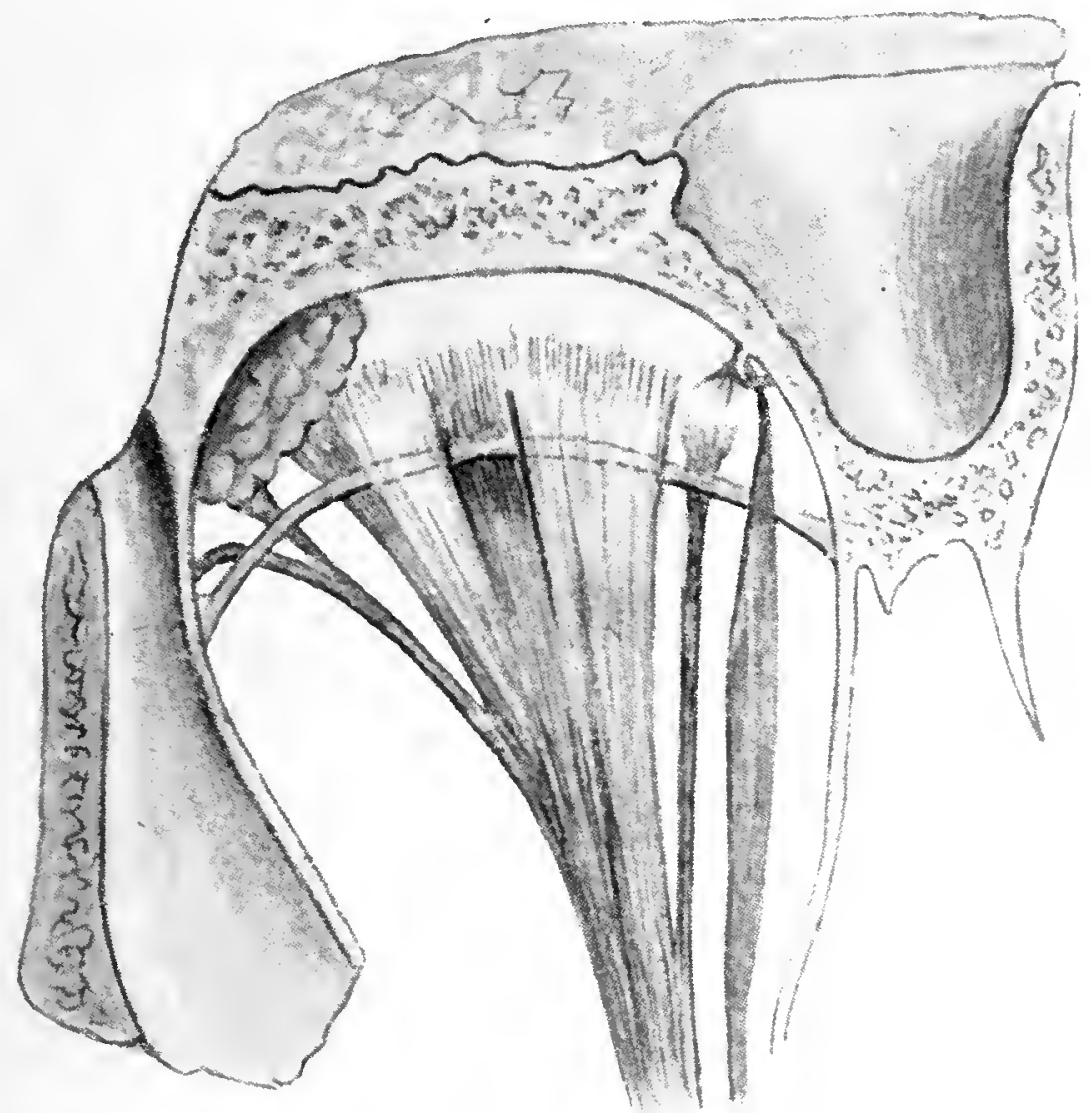

FıG. 69. - Sketch of abnormal Levator Palpebrae Superioris Muscle, seen in left orbit after removal of roof. A transiersus or-bitis muscle is represented by the transversely disposed fasciculus; a tensor trochleae muscle is seen passing to the trochlea from the medial edge of the levator, and on the lateral side fasciculi pass to the lacrimal gland.

nected to the levator, and giving off various attachments to neighbouring fascia. Macalister and Ledouble consider it to be a backwardly displaced slip of the orbicularis oculi. Perna (I905) also described such a muscle slip, and considered it, like the levator itself, to represent the remains of the primitive muscular membrane which surrounds the globe in lower vertebrates. An instance of its occurrence in both orbits has recently been reported by Hueber (I9I8).

The specimen drawn in Fig. 69 is interesting, in that it presents all three of the above abnormalities. It is 
suggested that the musculus transversus orbitis is normally represented by the transversely disposed check ligament formed by the superficial part of the levator muscle sheath, which was not present in this case (Whitnall, I92I). From the occasional persistence of such variations the levator is evidently derived from a much more extensive muscle sheet, and probably corresponds to the palpebralis muscle of lower animals (see above, p. I4I). Wiedersheim (I895) places it in the list of organs showing retrogressive char-

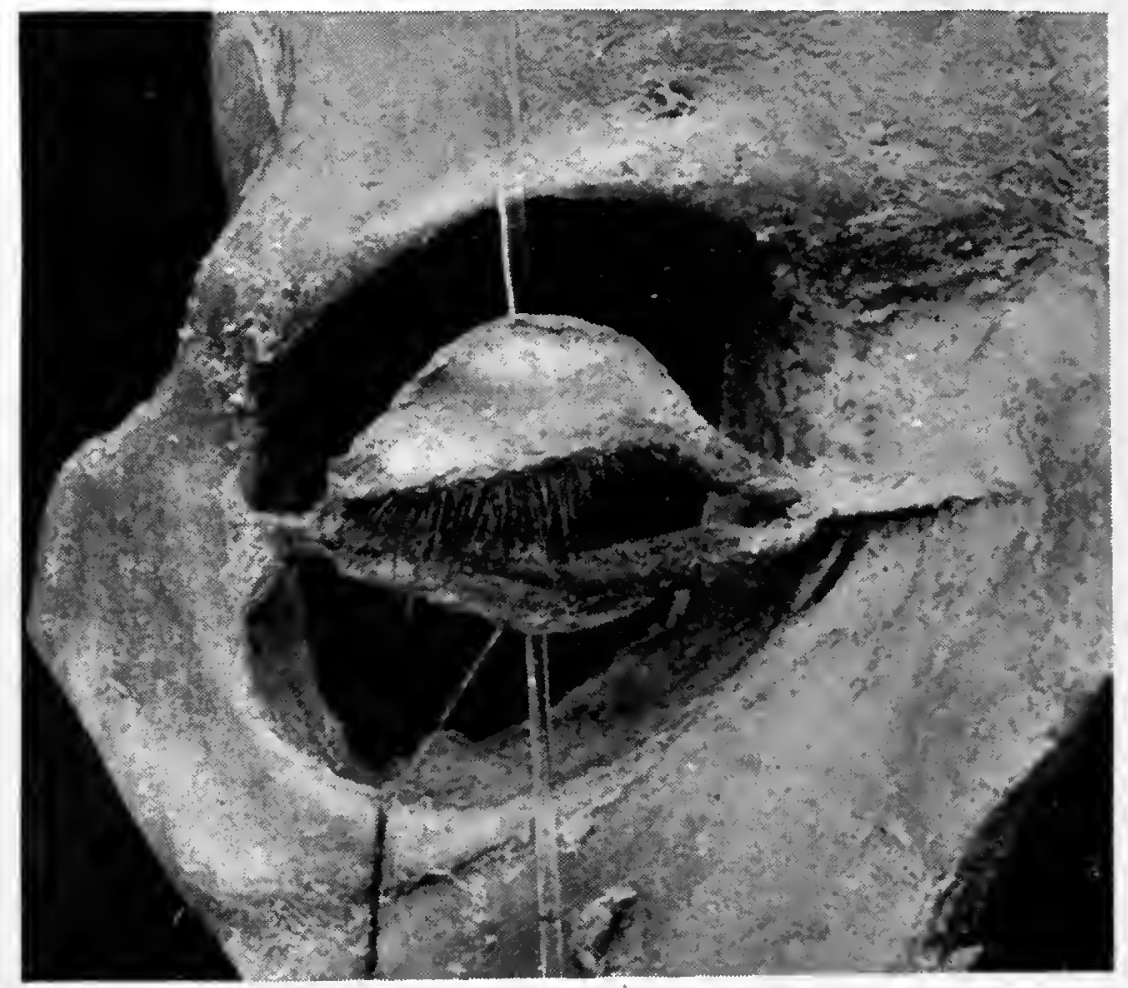

FIG. 70.-Dissection of the Tarsal Plates and the Lateral and Medial Palpebral or Tarsal Ligaments. Note the relation of the latter to the upper part of the lacrimal sac. Natural size.

acters, modified, but still performing clearly recognisable functions.

The tarsal plates (tarsus superior vel inferior, tarsal

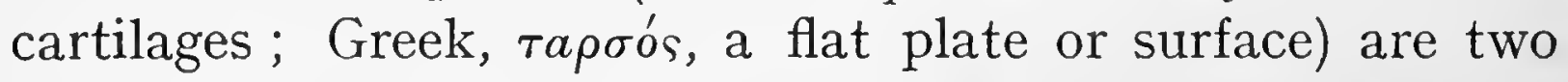
plates of dense connective tissue situated one in each eyelid, and hence distinguished as superior and inferior. Their shape, the upper that of a D laid on its side, the lower an elongated band, is shown in Fig. 70.

They have two borders : a horizontally straight or free border, coextensive with the ciliary margin of the eyelid up to the punctum lacrimale and about 25 to $30 \mathrm{~mm}$. long, and 
an attached or orbital border, which describes a well-marked curve in the superior plate but is nearly straight in the inferior. The upper plate is from ro to $\mathrm{I} 2 \mathrm{~mm}$. broad in its middle, the lower one is about $5 \mathrm{~mm}$. wide throughout; they are thickest at the ciliary border and I mm. thick in the centre, but taper off towards the attached borders. The curved upper outline of the superior plate can be felt and sometimes seen through the skin; to it is attached the superior palpebral involuntary muscle; it is covered in front by the terminal fibres of the levator, some of which are fixed to its lower third. The inferior plate is covered by the orbicularis ; its lower edge is united to the septum orbitale and to an expansion from the fascial sheath of the inferior rectus muscle, in which lies the inferior palpebral involuntary muscle. The plates are moulded upon the anterior surface of the globe, from which they are separated by the closely adherent conjunctiva; hence they are bent with a posterior concavity, a configuration shared by their free margins, and the closeness with which they fit the globe can be appreciated on making them spring from its surface by approximating the thumb and fingertips placed at opposite ends of the lids. Their extremities meet at each end in the commissures of the lids, and are prolonged to form the medial and lateral palpebral (or tarsal) ligaments, by means of which they are attached to the middle of the corresponding margins of the orbit.

The medial palpebral or tarsal ligament (ligamentum palpebrale mediale, tendo oculi, internal palpebral ligament, or orbicular tendon) is formed by the junction of direct continuations of the two tarsal plates on the medial side. It is a broad band of connective tissue which is attached to the upper half of the anterior lacrimal crest, but it also spreads out over the frontal process of the maxilla in front of this ridge, as is seen in Fig. 70 ; it therefore lies in front of the lacrimal fossa, but is partly adherent to the lacrimal fascia which roofs over that space. Superiorly it is thin and blends with the periosteum, so that definition of an upper margin is artificial; it is the weakness of this upper part that allows the fundus of the underlying lacrimal sac to 
bulge forwards in pathological conditions; inferiorly it is thicker and terminates in a free margin, which is prominent owing to the generally oblique position of the ligament from above downwards and forwards, and is conspicuous since it is. bare of muscle fibres (Figs. 53, I42). This edge shows through the skin as a prominent white band on drawing the lids laterally. Fibres of the orbicularis oculi muscle arise from nearly the whole of the anterior surface of the ligament, with the exception of the free edge, and from the deep surface where it is not adherent to the bone or the lacrimal fascia. The relations to the lacrimal fossa are illustrated by Fig. 70, whence it will be realised that the ligament is placed in front of the upper half only of the lacrimal sac, but covers it over completely, so that there is no formation of a narrow band crossing its middle as is sometimes figured. Superficially the ligament is crossed by the angular artery and vein, the latter lying nearer to the angle of the eye and about $8 \mathrm{~mm}$. from it (Fig. 84). Almost immediately after the ligament arises from the tarsal plates it divides into two lamellae, an anterior or direct portion, described above as passing in front of the lacrimal fossa, and a posterior or reflected portion, which passes behind it to be attached to the posterior lacrimal crest ; this posterior lamella is very thin and of quite secondary importance; it appears in dissections merely as a fascia clothing the front of the pars lacrimalis muscle, being intimately connected with it as it passes behind the fossa (Fig. I5o, p. 287). In one full-term foetus, however, this posterior lamella was found better developed than the anterior; possibly the latter becomes the stronger as the orbicularis comes into use.

The lateral palpebral or tarsal ligament (ligamentum palpebrale laterale, tendo oculi externus, ligamentum canthi externum, external palpebral ligament, or lateral palpebral raphe): the lateral extremities of the tarsal plates form a conjoint tendon as in the case of the medial ends, but this consists only of a single and direct lamella; it is fixed to the orbital tubercle of the zygomatic bone just within the lateral orbital margin at about its mid-point. There 
is some confusion in the interpretation of this attachment and in the terms applied to it. Commonly the fixation is stated to be by means of the lateral palpebral raphe, which is formed by the interlacing ends of the palpebral fibres of the orbicularis, strengthened by the underlying septum orbitale, and passing superficial to the orbital rim ; on cutting

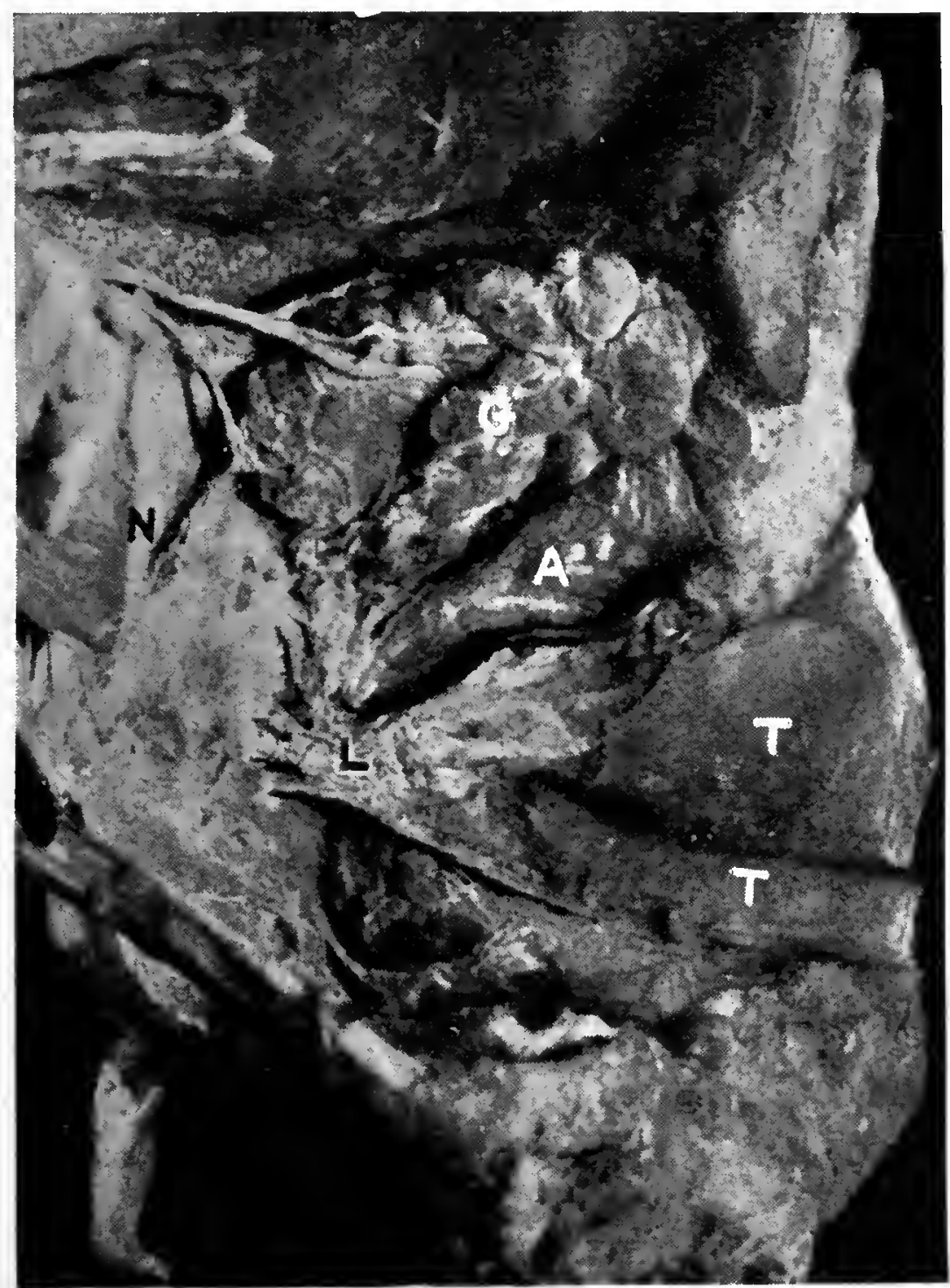

Fic. 7ı.-Dissection of Antero-Lateral Region of Left Orbit, seen from the medial aspect, to show the lateral halves of the tarsal plates ( $Г$.) and the true lateral palpebral or tarsal ligament (I..), attached to the orbital tubercle of the zygomatic bone. The preparation also shows the lacrimal gland (G.) and the anastomosis between its nerve and the zygomatico-temporal nerve $(N$.$) ; \lambda$. is the aponeurosis of the levator. 'I'he orifices of the tarsal glands can be seen along the lower margin of the superior tarsal plate. $\times 2$.

through the raphe, however, the plates, though a little slackened, will be found still attached to the bone by a deeper-lying and stronger band of fibres, well displayed from the inner surface, as in the above figure. This is the true lateral palpebral ligament, and it is separated from the more superficially placed raphe by a narrow but definite cleft, in 
which there may be found a few lobules prolonged downwards from the lacrimal gland. The ligament is also closely covered at its attachment to the tubercle by the lateral horn of the aponeurosis of the levator, just as the tarsal plate itself above is covered by the same muscle expansion. Hence in this lateral region the order of structures from the surface backwards is skin, orbicularis and septum orbitale in one layer (the raphe), lateral horn of aponeurosis, and the lateral palpebral ligament (Fig. I50, p. 287).

It will be seen that by the recessed position of the lateral palpebral ligament on the one side and the deeply placed attachment of the pars tarsalis muscle on the other, the

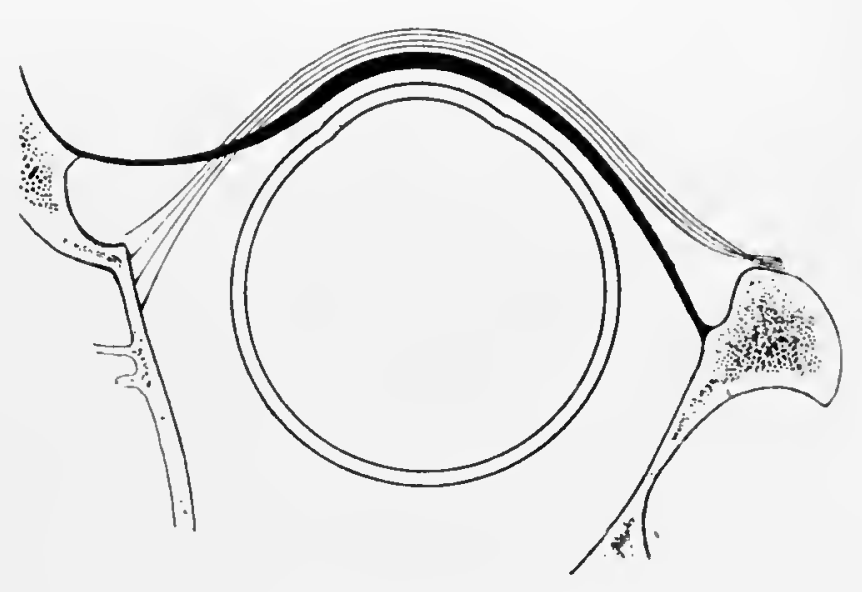

FIG. 72. - Scheme to show relation of attachments of tarsal plate (in solid black) and of pre-tarsal fibres of orbicularis oculi muscle (the lines) to the medial and lateral orbital margins. tarsal plates are kept closely curved against the globe in their whole length, an approximation that could not be effected to so complete a degree were they fixed to the bone merely by the raphe laterally and the medial palpebral ligament medially. Moreover, the contractile muscle fibres ensure this contact between tarsal plates and globe under all conditions, whether the latter is recessed or protruded or whether its scleral or more prominent corneal region be presented to the lids in its various movements (Fig. 72).

- In structure the tarsal plates are formed by dense connective tissue with a certain amount of elastic fibres; they are so firm as to account for their being sometimes called " tarsal cartilages," though C. Krause pointed out in I842 that there are no cartilaginous cells present. They are not solid plates, but are hollowed out by a series of large glands (tarsal or meibomian) ; indeed the most striking feature seen in microscopical sections is the enormous size of these structures, which excavate the plates to such an extent that there is more glandular formation than connective tissue substance (Figs. 74 and 75). 


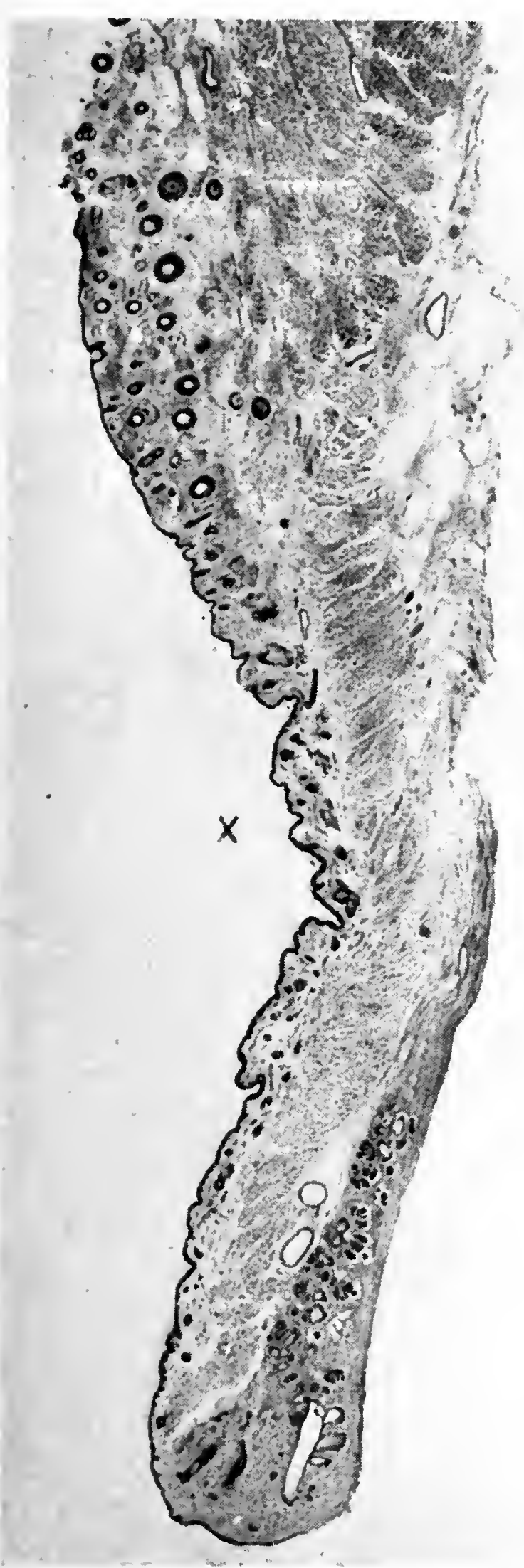

FiG. 73 .

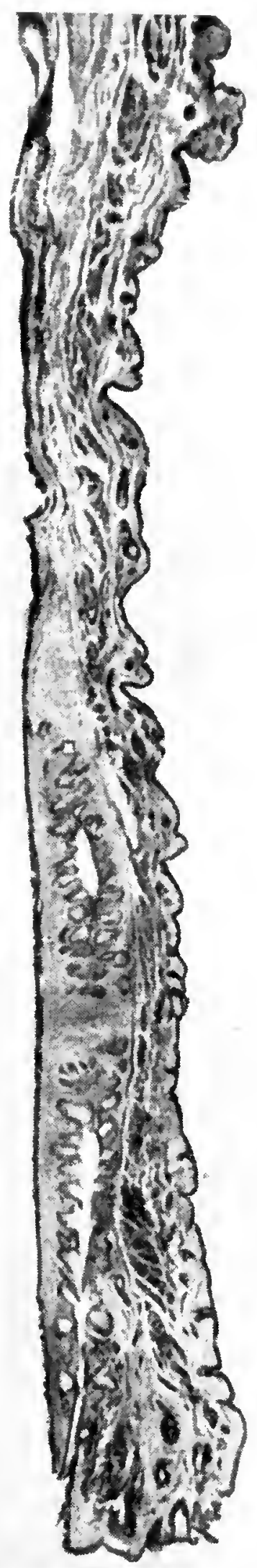

FiG. 74 .

Fig. 73. - Longitudinal section of Upper Eyelid and Eyebrow, showing various parts and degrees of coarseness of orbicularis oculi muscle, and the terminal fibres of the aponeurosis of the levator traversing its fasciculi to reach the skin. The tarsal plate is small. $\mathrm{X}$. is the region of the superior palpebral skin-fold.

FIG. 74. -Longitudinal section of Upper Eyelid, showing a large tarsal plate and an almost complete section along a tarsal gland. The superior palpebral involuntary muscle appears as a dark line leading upwards from the upper margin of the tarsal plate. 
The connective tissue appears simply as a dense supporting stroma or " peri-meibomian capsule," concentrated in front and behind the glands and connected by transverse strands passing between their acini. This stroma is, moreover, permeated by a system of lacunae and lymph spaces (Parsons, I904), and is transversed by a network of nerves and blood-vessels supplying the glands. The elastic tissue is concentrated round the glandular acini, where smooth muscle fibres are also stated to be present, and some fat cells are found in the borders of the plates, especially in the lateral convex border of the upper one (Virchow, I905). Towards the free borders of the lids the connective tissue

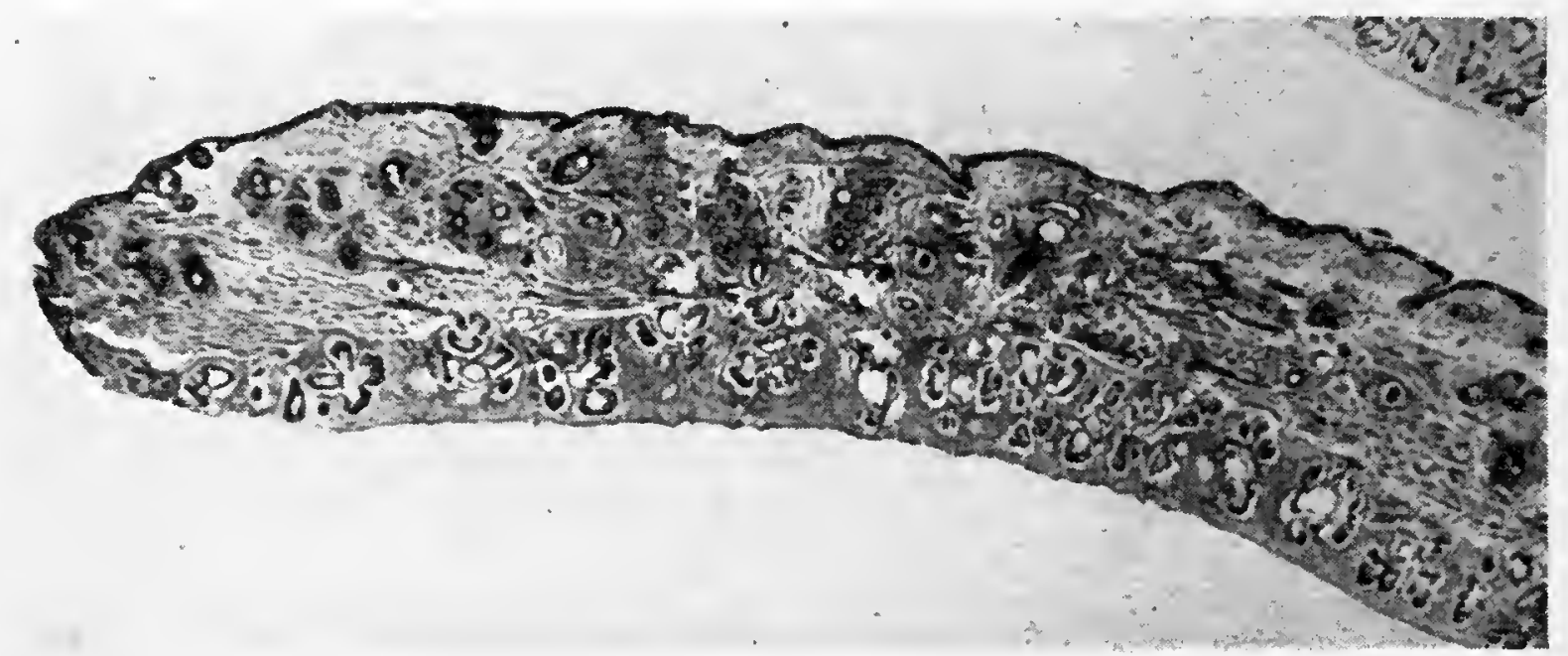

FIg. 75.-Transverse section of the Upper lyelid to show the tarsal glands, and the extent to which they excavate the tarsil plate (on the concave aspect of the section).

of the plates spreads out anteriorly to intermingle with and support the bulbs of the cilia, so forming a thick marginal basis or " ciliary mass" (Figs. 73, 80). Their deep surfaces are lined by the closely adherent palpebral part of the conjunctiva.

The tarsal plates stiffen the lids, and by their density render them more efficient organs of protection to the eye from injury or strong light. Phylogenetically they are dermic tissue condensed under the influence of the constant tension of the lids against the globe. By some authors they are considered to be correlated with the presence of the tarsal glands and the greater mobility of the eyelids (Eggeling, I904), since they do not develop clearly until the secretory activity of the glands is manifest, but 
others disagree with these conclusions on morphological grounds, finding that their presence is not conditioned by that of the glands, since the eyelids of mammals contain either both glands and plates or only one of these according to species (Argaud, I9I3).

The tarsal or meibomian glands (glandulae tarsales) were described by Meibomius in I666, but had already been figured by Casserius in I6og. They are long, narrow, and nearly straight, but the upper ends may be bent over, and are regularly arranged in a single row perpendicularly to the free margin of the lid; they occupy almost the entire thickness of the tarsal plate, as seen in Fig. 75 , sometimes forming a series of ridges on its anterior surface, and extend its whole height, consequently being tallest in its middle and shortest at the extremities. They are relatively longer in the infant.

Each gland is composed of a single central canal closed at its upper end and

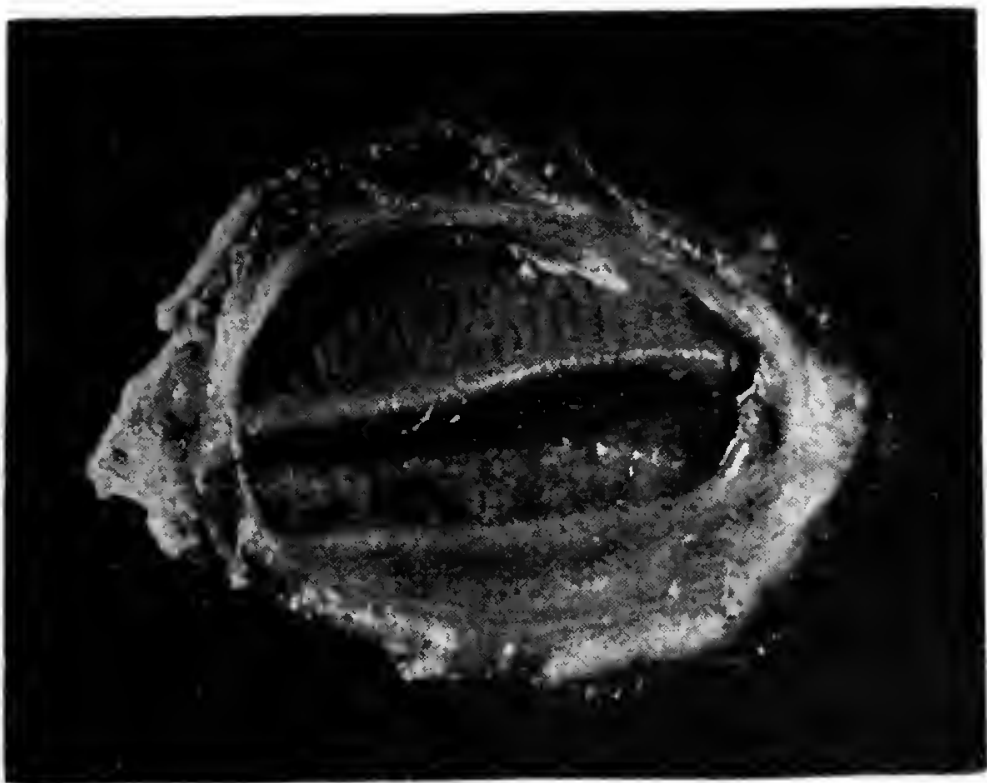

FIG. 76.-.'The Eyelids seen from behind after treatment with a saturated solution of urea, showing the tarsal glands of the tarsal plates. The puncta lacrimalia are also well displayed.

opening below on to the palpebral margin immediately in front of its posterior edge, where the regularly arranged row of minute orifices of these glands can be seen (Fig. $7 \mathrm{~T}$ ). Around nearly the whole length of the canal are grouped the acini, which open into it by lateral ducts; the acini are single or composite, and number thirty to forty in the longest glands (Sappey, I867) ; they are surrounded by a lymph space lined by endothelium (Parsons, I904), and the ducts are lined by cubical epithelial cells which become stratified at their mouths. They are sebaceous glands, and the secretion is composed of finely granular fat, the "sebum palpebrale," which by its greasy nature 
lubricates the edges of the lids and prevents them from sticking together and also the tears from overflowing; they are absent from the lacrimal part of the lid margin where the tears, unless unusually abundant, first begin to trickle down. Owing to the yellow colour of the secretion the glands can be seen through the conjunctiva on everting the lid. The relation of the horizontally disposed fibres of the pars ciliaris of the orbicularis oculi muscle to the terminal canals of these glands is seen in Figs. 78, 80 ; they lie chiefly in front, partly behind them, and some minute bundles are even interlobular, interciliary, and fetrotarsal in position.

The tarsal glands represent the ordinary sebaceous glands of a primitive secondary range of eyelashes which have disappeared in man, a fact that explains the presence of an extra row of cilia and the absence. of these glands in cases of congenital districhiasis (Brailey, I906), and the aberrant lashes found in a cyst on the posterior face of the tarsal plate (Szili).

The free palpebral margin is a region of such practical importance as to merit a special description. It is divided along its length into two regions, ciliary and lacrimal, the point of division being marked by the lacrimal papilla. The lacrimal region is devoid of eyelashes, and is smooth and rounded; in the substance of the lid beneath it runs the lacrimal canaliculus. The ciliary region bears the eyelashes, and is flat and about $2 \mathrm{~mm}$. broad; it therefore presents two lips or limbi, an anterior, slightly rounded and continuous with the skin, and a posterior, sharply cut and continuous with the conjunctiva and lying against the globe ; in the aged the edges lose their sharpness owing to the loss of elasticity of the fibres composing them. When the lids are closed a longitudinal capillary space (rivus lacrimalis) is said by some authors to exist between their posterior lips and the globe. The flat surface of the margin between the lips is termed the "intermarginal region " (Terson, I903), and it may present along its middle a slight groove or a fine grey or pigmented line, called the " intermarginal groove," 
or sulcus intermarginalis of Graefe, best seen in the upper eyelid. This line subdivides the flat margin along its length into a ciliary part in front, from the surface of which spring the cilia, and a tarsal part behind, marked by the range of orifices of the tarsal glands; by a reference to the structure of the lids it will be seen that the lid can be split along this line into an anterior layer containing the orbicularis and ciliary follicles, and a posterior containing the tarsal plate (Fig. 77). The conjunctival membrane extends as

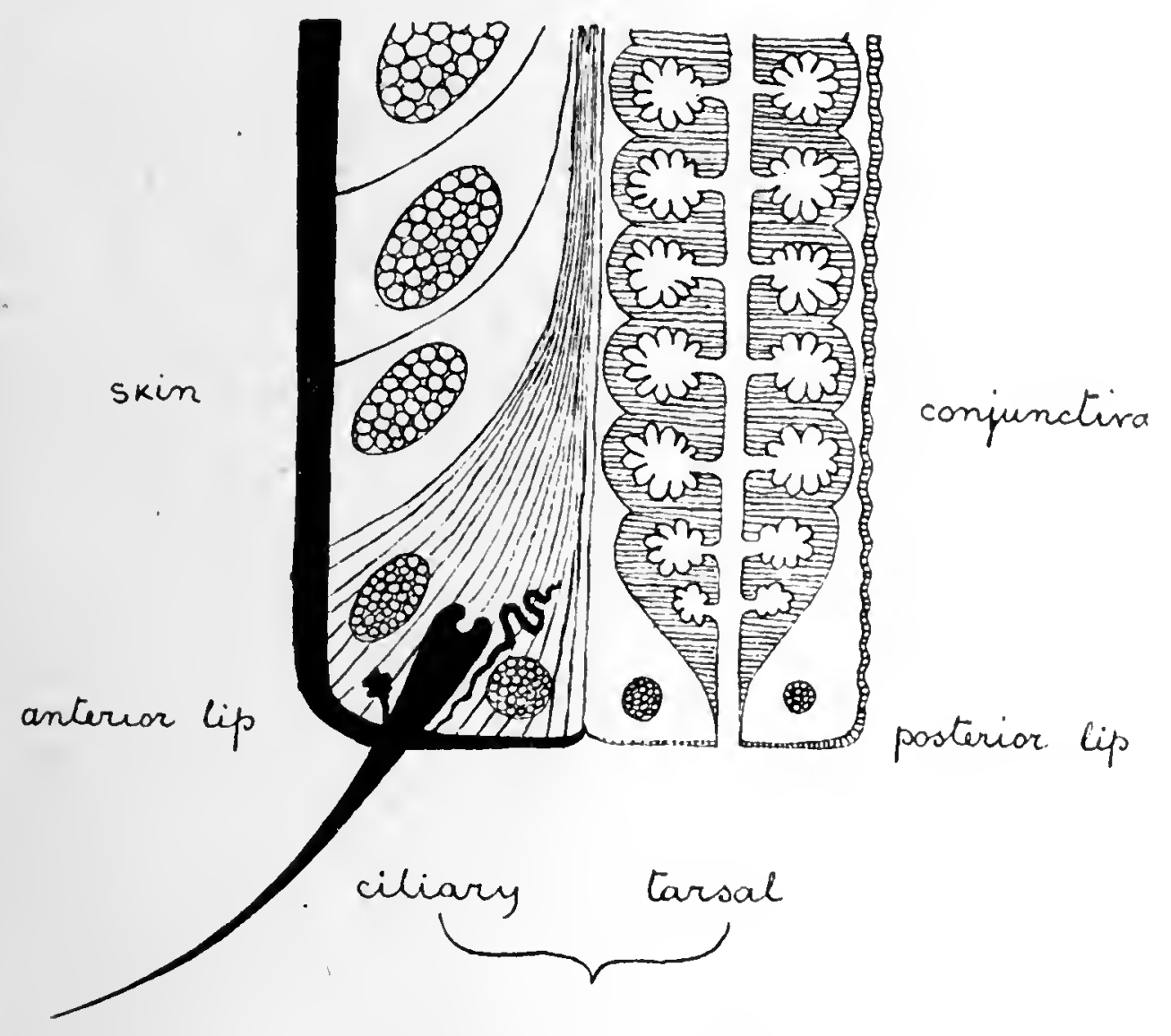

INTER MARGINAL REGION.

Fig. 77.-Scheme to illustrate the Structure of the Free Palpebral Margin.

far as the glandular orifices, the rest of the border anteriorly being cutaneous. Structurally, the lid margin consists largely of dense connective tissue directly derived from the splayed-out free border of the tarsal plate : this ciliary mass appears triangular in vertical section, and embedded in it are the follicles of the eyelashes, together with their particular glands, and the fasciculi of the pars ciliaris muscle (Fig. 78).

The cilia, or eyelashes, are short, thick, curved hairs, usually deeper in colour than those of the head, but not 
blanching with age; they are relatively longer and finer in the female and infant, and are but-little developed in mongoloid races (Branca, I9I2). They are arranged in two or three irregularly placed rows. Those in the upper lid are larger, longer, more closely set, and more numerous than in the lower; in the upper lid they number from Ioo to I20 or even I5O (Donders, I858), and are from 8 to I2 $\mathrm{mm}$. long; in the lower lid they are from 50 to 75 in number, and 6 to $8 \mathrm{~mm}$. long; they are larger in the middle

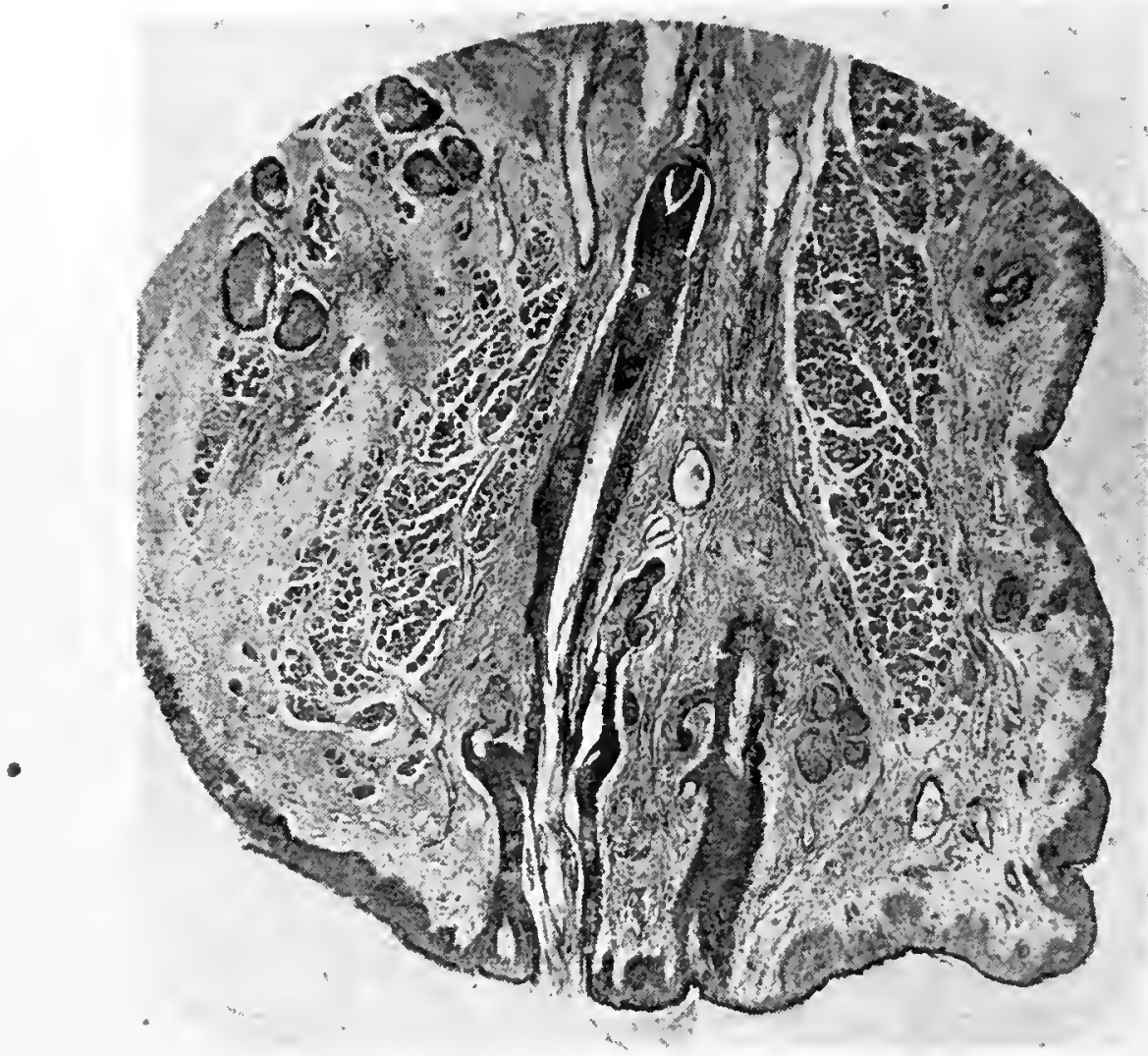

Fig. 78. - The Upper Palpebral Margin, showing ciliary follicle with glands of Zeis ; sagittal section, enlarged; skin surface to right, conjunctiva on left. The pars marginalis of the orbicularis oculi muscle and its relation to the cilia, as clescribed on p. ז29, are well shown.

region of either lid. In the lower lid they are implanted more on its anterior face than on the actual margin; they curve upwards in the upper lid, downwards in the lower, and their convexities meet in occlusion of the lids; cylindrical in early life, they become a little flattened in the adult, a change in shape attributed to the compression exerted upon them by the marginal muscle fibres. The follicles are from $\mathrm{I} \cdot 5$ to $2.5 \mathrm{~mm}$. deep, and are obliquely planted in a dense fibrous tissue derived from the tarsal plate, up to which they extend; they are surrounded by a rich plexus of sensory 
nerve fibres (the "marginal plexus" of von Mises, I882), and their relation to the ciliary muscle bundles is seen in Fig. 78 . From the sides of the primary follicles secondary ones develop and give origin to new lashes, which replace the older ones after their life of about three months; certain follicles date from foetal life, and may develop late and out of their proper order.

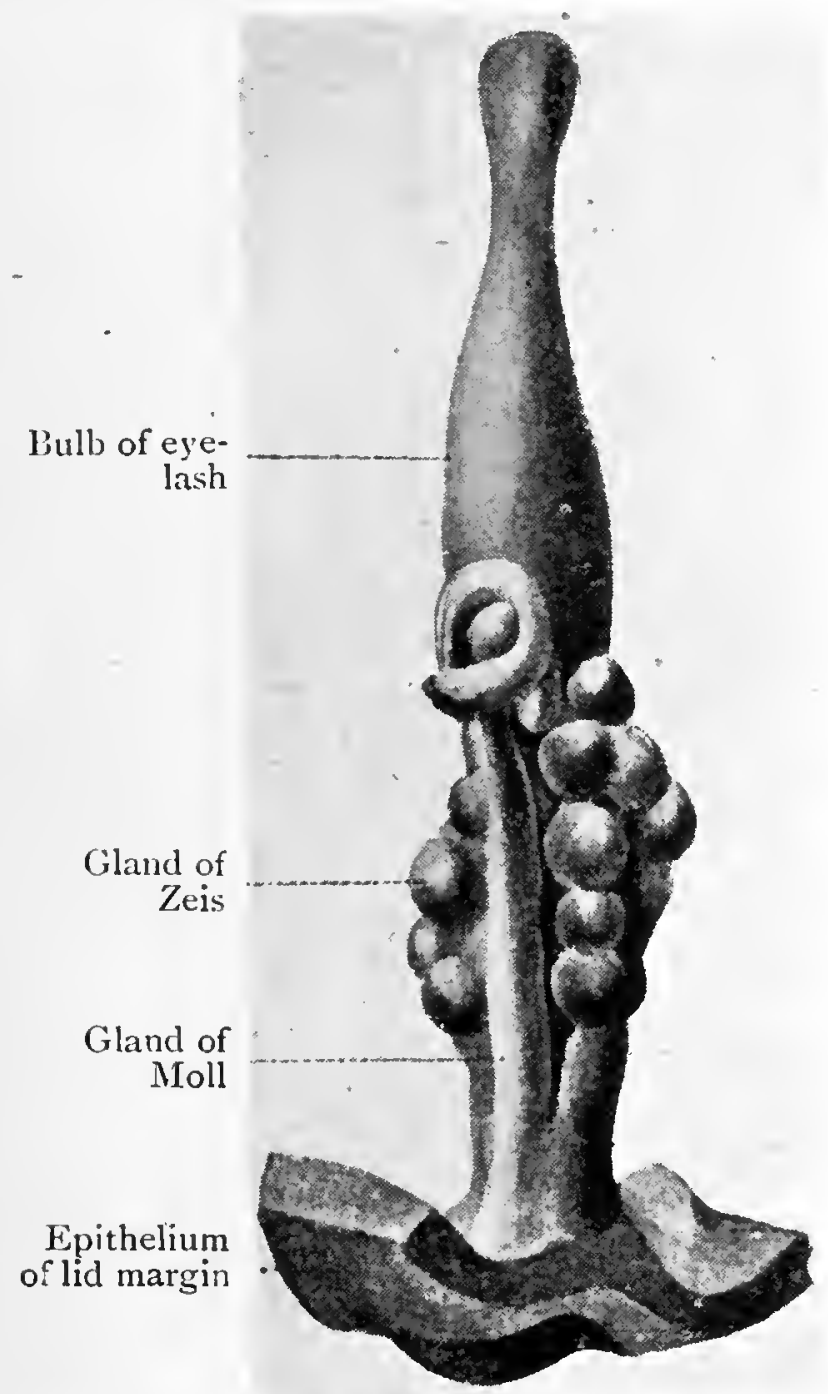

FIG. 79.- Reconstruction of the Bulb of an Eyelash, with its appendages.

(Contino, ig07.)

It has already been pointed out that the tarsal glands are to be regarded as greatly modified glands of primitive cilia which have aborted; similarly it is found that the other skin glands at the lid margin differ from the normal.

The sebaceous glands are larger than those of the hairs elsewhere, and are known as the glands of Zeis (I835; generally spelt incorrectly as Zeiss); there are usually two for each follicle, developed by outgrowths of its epithelial wall, and they pour their secretion round the base of the 
hair. They consist of a short wide duct leading from a cluster of small rounded saccules (Fig. 79), which are lined with cells loaded with particles of fatty substance, and are supported by a basement membrane reinforced with a covering of connective tissue (Schäfer, I9I2) ; they are better developed in the infant than in the adult.

The sweat glands are distinguished as the glands of Moll (I857) or "ciliary glands" (a name which- should

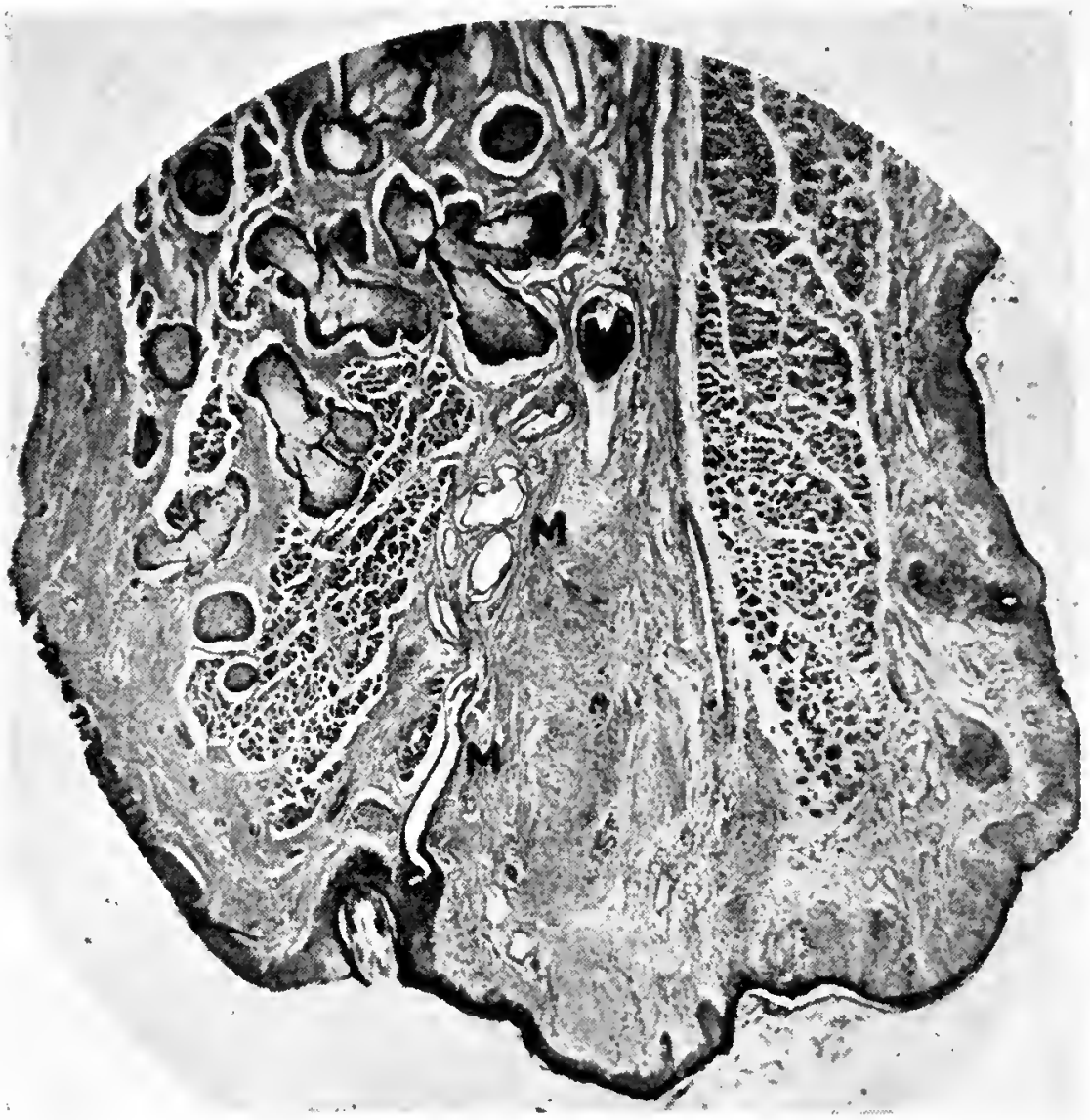

FIG. 80.

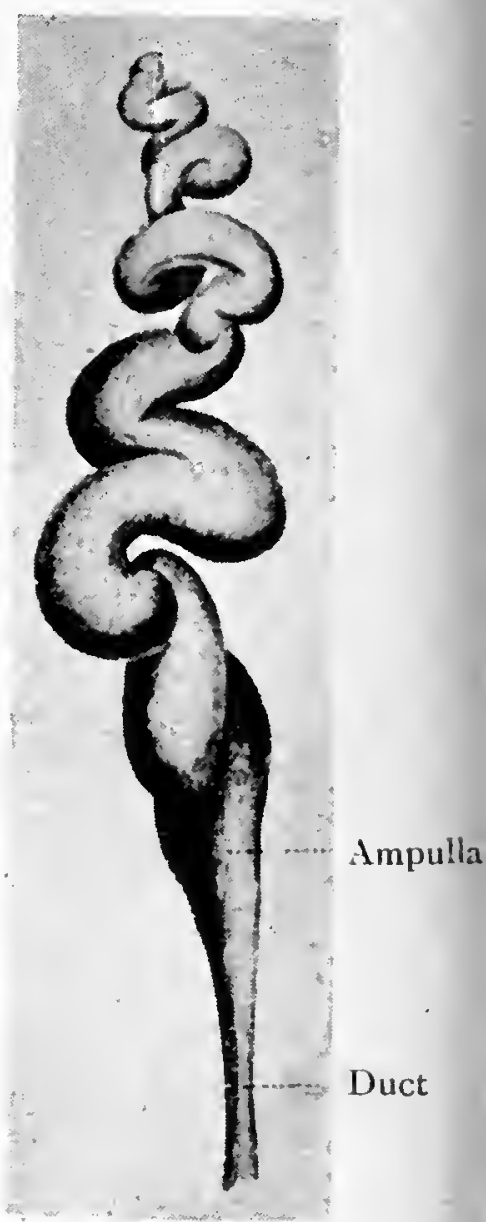

FIG. 8I.

FIG. 80. - The Upper Palpebral Margin, sagittal section enlarged, showing a Gland of Moll (M., M.), and the various parts of the pars marginalis of the orbicularis oculi muscle (see p. I29 for description). Skin surface on right, conjunctiva and tarsal plate on left.

FIG. 8I. - Reconstruction of a Gland of Moll (Contino, 1907).

be applied more appropriately to the glands of Zeis) ; they are ordinary sweat glands retarded in development, the secretory tubule forming a spiral instead of a glomerulus, but having a wider duct, and they open either into some (I4-26) of the ciliary follicles close to the surface (Virchow, Contino, Sättler, Parsons) or into the duct of a Zeis gland, or on to the surface of the lid margin between the eyelashes 
(Waldeyer, Picou); usually there is one placed between each two cilia (Sättler, I877). They extend deep into the lid even as far as the tarsal plate (Fig. 8o), and are better developed in the lower eyelid. The secretory part of the gland is lined by an epithelium formed by a single layer of cuboid columnar cells which increase to two or three layers in the duct, becoming keratinised and continuous with the epidermis at the orifice; it is supported by a basement membrane, and surrounded by smooth muscle fibres and a felting of elastic tissue (Carlini, I907).

Blocking of the duct of either of these glands causes a "stye," and the fact that they open into a follicle explains the relief obtained by pulling out the eyelash.

For the development of the lid margin see p. I84.

The difference between the two eyelids may here be summarised: the lower lid is thicker, shorter, and less mobile, having no special levator muscle; the size of its tarsal plate and glands and the length of its cilia are less; the orbicularis muscle is thicker over the lower lid and its marginal fasciculi are less differentiated; the inferior palpebral involuntary muscle is shorter and less developed than that of the upper lid. A section is shown on p. I94.

The Lacrimal Caruncle (caruncula lacrimalis, from the Latin diminutive of caro, flesh) is a small reddish oedematous-looking body about $5 \mathrm{~mm}$. high by $3 \mathrm{~mm}$. broad, lying in the lacus lacrimalis at the medial angle of the eye ; from its mucoid appearance and position it looks as if it were a formation of the conjunctiva, but developmentally and structurally it is a detached and modified part of the lower lid margin (see p. I86). It is more prominent when the eyeball is directed laterally, being dragged forwards by the plica semilunaris of the conjunctiva, with which it is connected on its under surface, and is deeply recessed when the eye is turned medially; this recessed position is normal in infancy, but may follow as. a disfigurement after operation on the medial rectus muscle, probably owing to the fact that fibres of the fascial sheath of the muscle enter 
its base, and so can draw it backwards (Fig. I50, p. 287). Its prominence serves to stem the flow of tears into the groove between it and the more laterally placed plica semilunaris, into which groove the lacrimal papillae are directed.

In structure the caruncle presents many traces of its developmental origin; it consists of a modified epithelium, hairs with sebaceous glands, an accessory lacrimal gland (ectodermal structures), and connective tissue and fat (mesodermal structures). The surface epithelium resembles

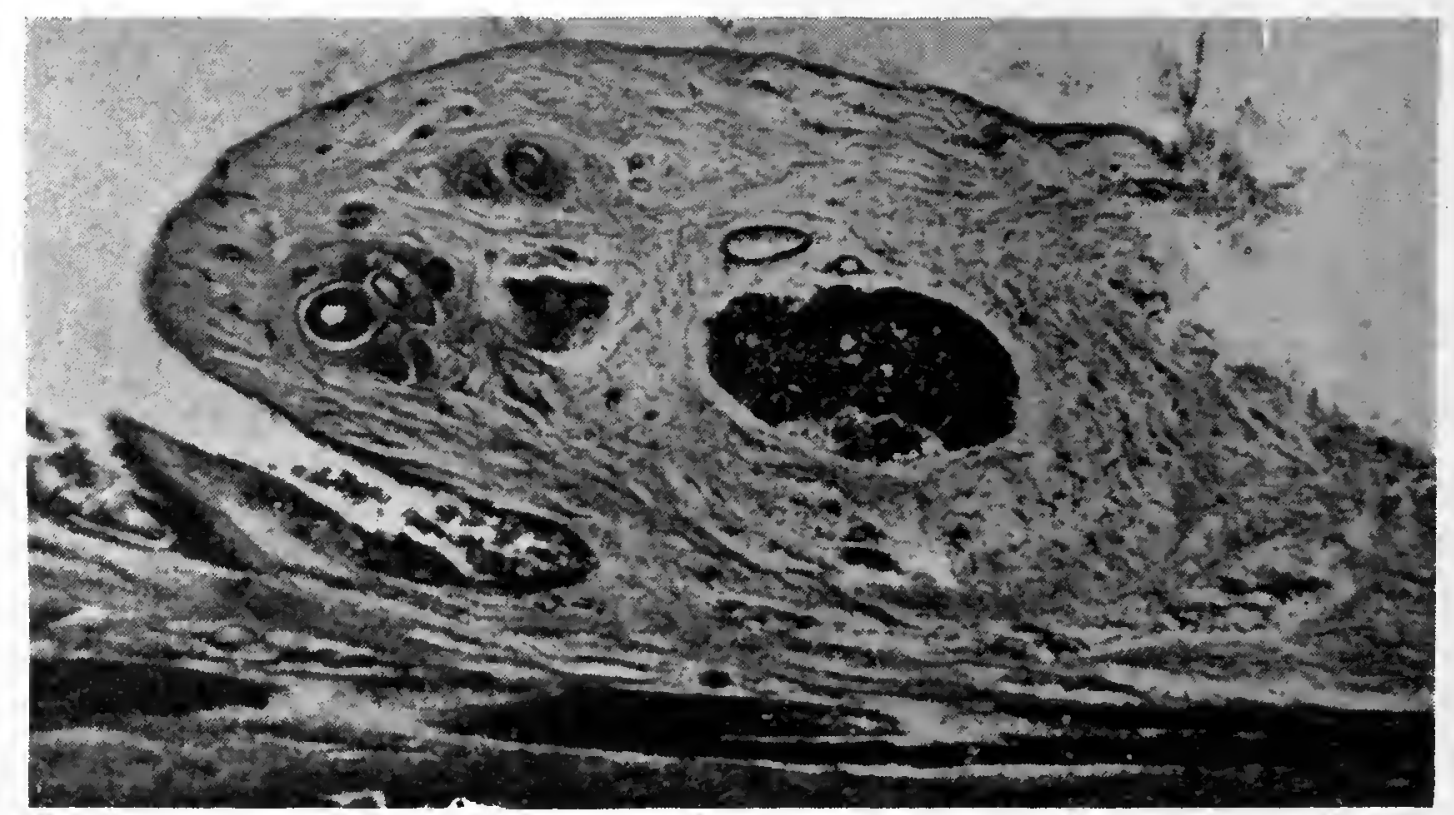

Fig. 82. - Section through the Lacrimal Caruncle, greatly enlarged, showing an accessory lacrimal gland in the centre, and sebaceous glands resembling the meibomian glands of the tarsal plate towards the left. 'The plica semilunaris just appears on the left side of the preparation.

that of the ciliary border, being of stratified pavement type but not keratinised, though Peters (I9I9) reports twentysix cases of cornification of the caruncle by thickening of its epithelium. At the margins it shows a transition to the conjunctiva by a cylindrical formation and the presence of goblet cells (Terson); " intra-epithelial glands "of simple mucoid alveolar type are described by Enslin (I905), but their existence under normal conditions is denied by Stieda (I890). The surface presents some fifteen colourless hairs which are directed towards the nose (Merkel); they may be developed to such an extent as to become a nuisance and require removal, and may even resemble aberrant eyelashes in size (Berger, I893); into the hair follicles there 
open glands like those of Zeis, and other sebaceous glands are seen, larger and resembling the acini of tarsal glands. These glands are the source of the white secretion that collects in this region of the eye, and are usually largely developed. Sweat glands were found by Waldeyer, but have not been observed by Stieda and Terson, and could not be found in the writer's serial sections; they occur, however, in the embryo (Contino), and when persistent in the adult are perfectly formed (Virchow). The most conspicuous object in microscopical sections is the large centrally-placed " acino-tubular gland " ; structurally this resembles the accessory lacrimal glands of the conjunctiva described by Krause; it varies greatly in volume, and there may be from one to four present, though sometimes none can be found; in the writer's sections there was one large central gland with two smaller ones above it; it opens upon the surface by a large tortuous excretory canal. It is not a relic of Harder's gland, which is absent in man (vide plica semilunaris, p. I98), though both are present in the calf. A few smooth but no striated muscle fibres are present (Müller).

The blood-vessels are derived from the superior palpebral artery, and on account of the connective tissue through which they run they gape on section, and free haemorrhage occurs; the lymphatics drain into the submaxillary nodes. The sensory nerve supply is provided by the infratrochlear branch of the ophthalmic division of the trigeminal nerve.

The Vessels of the Eyelids. - The blood-vessels are derived from two sources, those of the face and those of the orbit, but there are free anastomoses between the two systems at the orbital margin, and since the facial vessels are extra-cranial whilst the orbital are intra-cranial in origin, these junctions, especially of the veins, are of considerable importance. The facial group of arteries are all derived from the external carotid; the orbital (ophthalmic) is derived from the internal carotid. The facial system of veins drains into the internal and external jugular veins, 
the ophthalmic veins into the cavernous sinus of the intracranial dural system (see Fig. I62, p. 3I3).

It will simplify description to retain here the older and more commonly known names of facial artery. and facial vein, instead of the newer external maxillary artery and

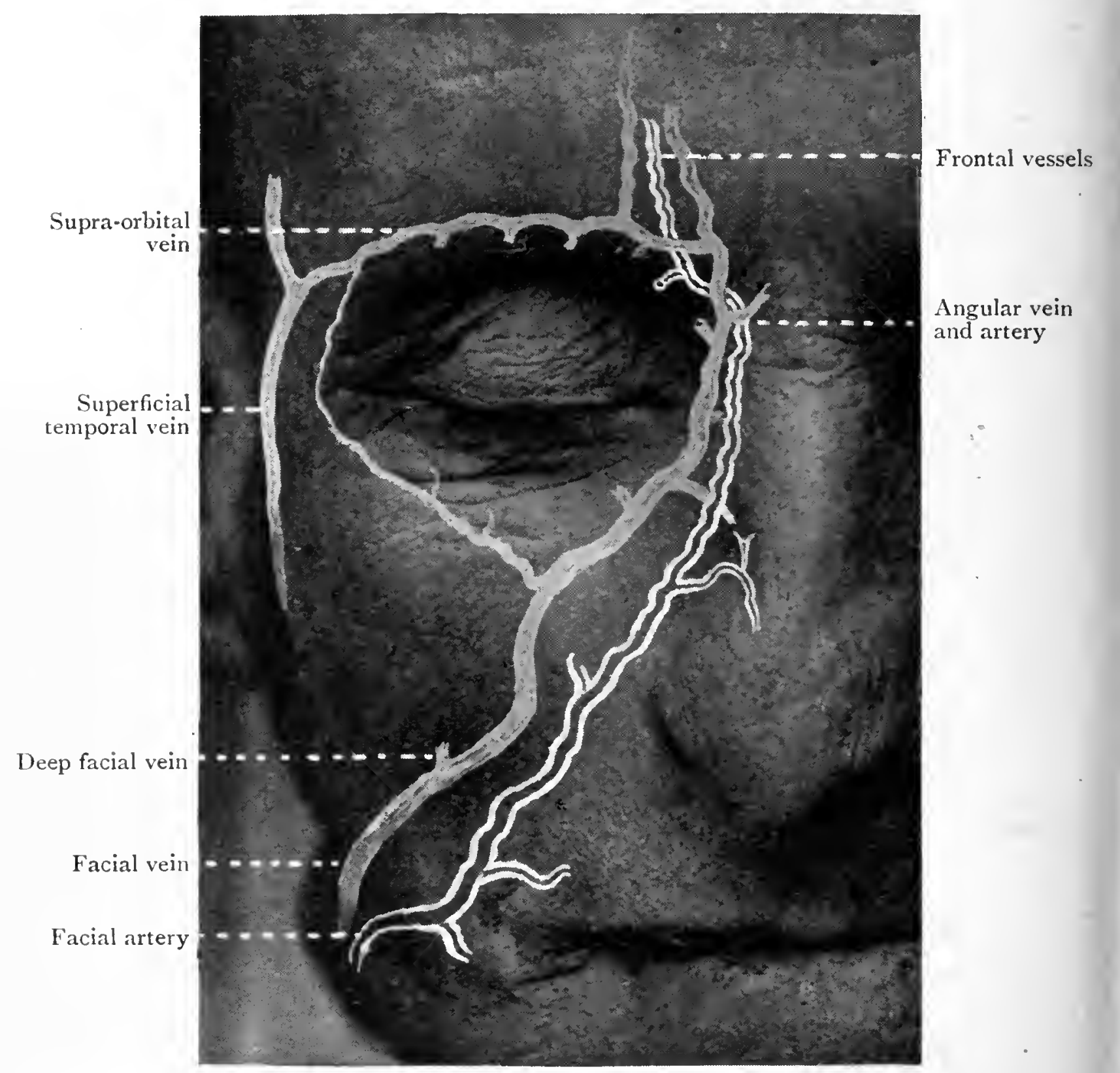

FIG. 83. -Approximate positions of main vessels of the face.

anterior facial vein, for these two collaterally placed vessels.

I. The facial vessels are the facial and superficial temporal arteries and veins, and their branches supply most of the circum-orbital region and the superficial parts of the lids, as shown in the above figure.

The facial artery (arteria maxillaris externa) arises from the external carotid in the upper part of the neck, and reaches 
the face by passing over the ramus of the mandible just in front of the anterior border of the masseter muscle, where its pulsations can be felt in life, and where it may be compressed against the bone. It pursues a sinuous course obliquely upwards and medially towards the naso-labial angle, being crossed by the risorius and zygomaticus muscles ; it then passes vertically upwards, beneath or rarely over the levator muscles of the upper lip (quadratus labii superioris) and below the infra-orbital foramen and nerve, to the medial angle of the eye, where it is termed the "angular artery" ; in this part of its course it runs along the line of the naso-jugal skin-fold, and passes superficial to the medial palpebral ligament with the facial vein on its lateral side. Both vessels lie about $5 \mathrm{~mm}$. anterior to the lacrimal fossa and are covered by skin, and also in most cases by fibres of the orbicularis muscle. The facial artery anastomoses with the transverse facial and infra-orbital arteries and with its fellow of the opposite side, and on piercing the septum orbitale between the trochlea and the medial palpebral ligament, it terminates by becoming directly continuous with the nasal branch of the ophthalmic artery. It supplies in the ocular region the orbicularis oculi muscle, the lower eyelid, and the lacrimal sac. In the upper part of its course it may be very small, the blood-supply to the lower eyelid being then derived from the vessels with which it anastomoses.

The facial vein (vena facialis anterior) is formed at the medial end of the eyebrow by the junction of the frontal and supra-orbital veins; it runs obliquely downwards and backwards across the face, along the anterior border of and then over the lower part of the masseter muscle, to cross the mandible and end in the internal jugular vein in the neck. In the first part of its course between the eye and the nose it is called the " angular vein," and presents relations already described in connection with its companion artery (see Fig. 54, p. I23) ; as it passes over the medial palpebral ligament it is distant about $8 \mathrm{~mm}$. from the angle of the lids, and in this position often forms a prominence in the living; its position may also be indicated by the dark colour of the contained blood showing through the skin. 
The facial vein lies lateral and posterior to the artery throughout, but takes a straighter line down the face and is more superficial, being covered only by the zygomaticus muscle. It is less flaccid than most of the superficial veins, and therefore tends to remain patent after section; it is a fairly large channel and may exhibit varicosities, as illustrated in the figure below. Such a condition would explain

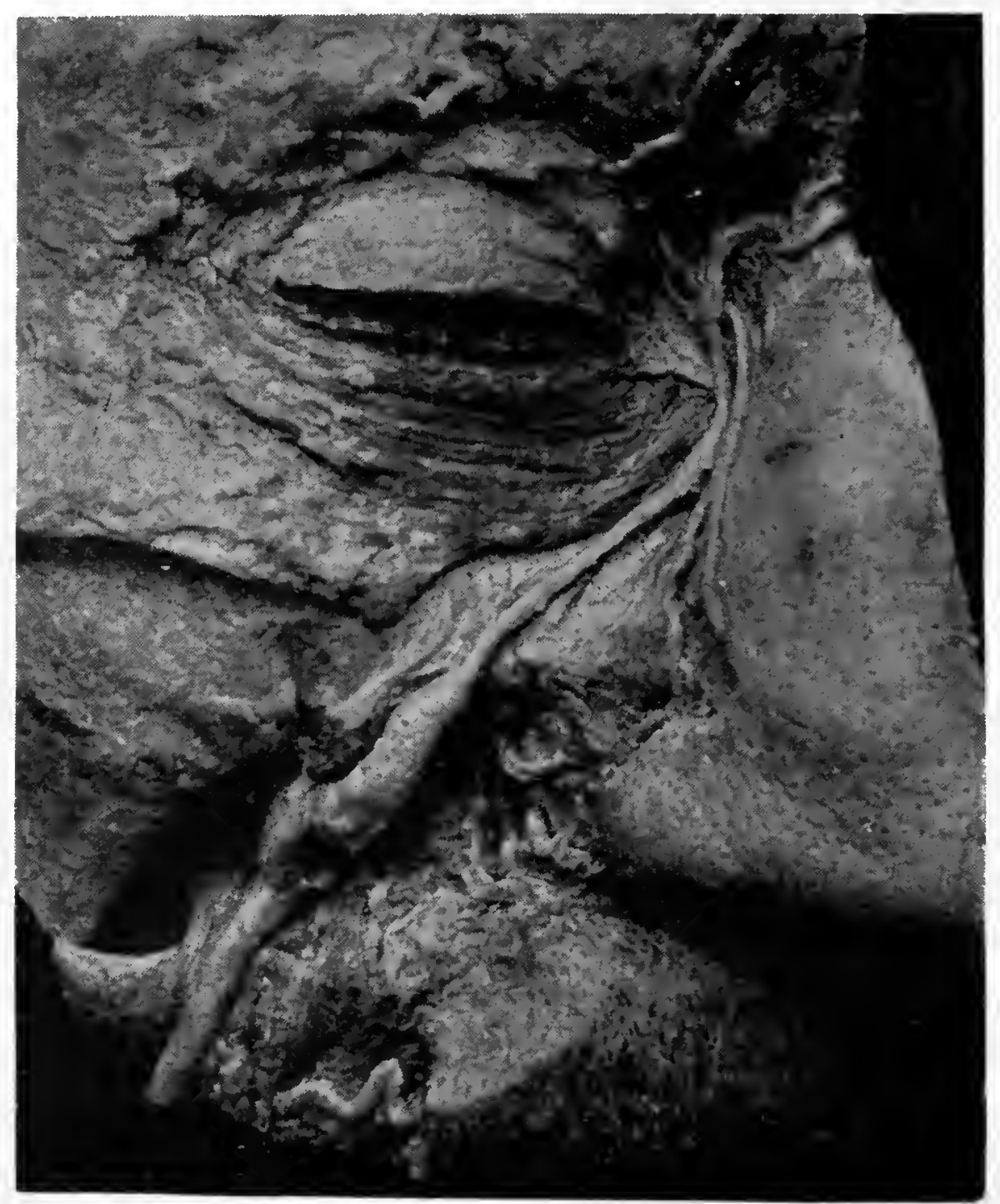

FIG. 84. -The Facial Vein (vena facialis anterior), showing varicosities. Its angular portion is seen crossing the medial palpebral ligament, with the corresponding angular artery lying to its nasal side.

the swelling sometimes seen in the living along the line of the upper part of its course when the vessel is compressed and the blood-flow retarded by the contraction of the overlying muscle, as in laughing. Among its tributaries the following are important :

(i.) The supra-orbital vein does not accompany the artery of the same name down the forehead, but runs horizontally 
along the margin (hence the name marginal vein or arcade sometimes applied to it) from the temporal side, where an anastomosis is formed with the middle temporal branch of the superficial temporal vein; it lies beneath the orbicularis oculi muscle, which it pierces below the head of the eyebrow to join the frontal and so form the angular vein (Figs. 83, 85). In its course it sends a large branch through the supraorbital notch to join the superior ophthalmic vein within the orbit, and as this branch passes through the notch it receives the frontal diplöic vein, which drains the mucous membrane lining the frontal air-cell and establishes a communication with the superior longitudinal blood sinus.

(ii.) The frontal vein (or fronto-nasal, represented as separate vessels by Merkel in Fig. 85) runs vertically down the forehead immediately beneath the skin, in company with the frontal or supra-orbital artery and nerve; it communicates with the superior longitudinal sinus through small foramina in the frontal bone (Piersol), and at the root of the nose is connected with its fellow of the opposite side by a short transverse " nasal arch" (seen in Fig. 84).

(iii.) The superior and inferior superficial palpebral veins are small veins which drain the skin of the eyelids and surrounding parts and enter the angular vein; the lower one is sometimes seen to reach a considerable size.

(iv.) The angular vein, like its tributary the supraorbital, communicates freely with the superior ophthalmic vein by a branch passing into the orbit below the trochlea; it is chiefly by the junction within the orbit of these two communications from the angular and supra-orbital veins that the superior ophthalmic vein is formed.

(v.) At about the middle of its course, where it passes beneath the zygomaticus muscle, the facial vein forms another important connection by receiving the deep facial (anterior internal maxillary) vein, as seen in Fig. 84. This vessel passes backwards between the masseter and buccinator muscles into the infra-temporal fossa to join the pterygoid plexus, a venous network which communicates with the cavernous sinus and with the inferior ophthalmic vein by 
branches passing through the inferior orbital fissure (see Fig. I62, p. 3I3).

Thus the facial vein has two chief connections with the cavernous sinus of the intra-cranial venous system : by its free union with the superior ophthalmic vein, and by a pathway through the pterygoid plexus. It is said to be devoid of efficient valves, though Merkel, Festal, and others state that they exist at its junction with the ophthalmic vein so disposed as to ensure a flow in a forward or facial direction (such valves could not be found in the specimen photographed in Fig. 84). Usually, however, there appears to be no hindrance to the passage of at least part of the blood-flow from the frontal region into the orbit, and this is no doubt the usual course, since when the ophthalmic system is congested during laughter or expiratory or straining efforts it is common to see the frontal veins become prominent, and in cases of orbital or intercranial tumour the ophthalmic, angular, and facial veins become congested and tortuous from pressure interference with the blood-current. Moreover, the angular vein is frequently found very small on both sides, and obviously insufficient to carry the whole flow from the frontal region into the facial vein. As a consequence of the valvular deficiency, septic diseases such as malignant pustule, furuncle, carbuncle, or cancrum oris, involving the face or forehead, are exceptionally dangerous, since the infection may spread by way of the ophthalmic veins or pterygoid plexus to the cavernous sinus (Piersol, rgr9). See also p. 3I4.

The superficial temporal vessels call for but brief description. The artery (arteria temporalis superficialis) is one of the terminal branches of the external carotid, and runs up behind the angle of the jaw and in front of the ear ; among other branches it gives off (i.) a transverse facial branch, which runs forwards below the zygoma to terminate near the lateral margin of the lower eyelid by anastomosing with the infra-orbital and lacrimal arteries.; (ii.) a zygomatico-orbital branch, which passes along the upper border of the zygoma to supply the lateral parts of the lids; it also anastomoses with the lacrimal artery, and sends branches 
into the cavity of the orbit; (iii.) a frontal branch, large and tortuous, which passes upwards and forwards across the temple to supply the frontalis and orbicularis oculi muscles, and anastomoses with the lacrimal and supraorbital branches of the ophthalmic artery.

The vein (vena temporalis superficialis) communicates with the frontal and supra-orbital veins, draining the blood from the lateral region of the upper lid; it runs across the temple, then downwards in front of the ear, joins the internal maxillary vein to form the temporo-maxillary or posterior facial vein, and enters the external jugular vein; it receives a middle temporal vein, which communicates with the lacrimal and facial veins at the outer angle of the eye, and runs along the upper border of the zygoma beneath the temporal fascia.

(2) The intra-orbital system of vessels are the supraorbital, frontal, nasal, and lacrimal branches of the ophthalmic artery, which supply the eyelids and the supra-orbital region in its medial half, and the general arrangement of which is shown in Fig. 158, p. 302. (i.) The supra-orbital artery leaves the orbit by passing through the supra-orbital notch, or, like the nerve of the same name which it accompanies, it may emerge in two branches, frontal and supra-orbital proper, as in Fig. 85; it supplies the skin of the forehead and upper eyelid, anastomosing with the superficial temporal artery and with its fellow of the opposite side. (ii.) The frontal artery has a similar but more medial distribution than the supra-orbital. (iii.) The nasal artery (arteria dorsalis nasi) leaves the orbit by passing over the medial angle of the margin between the trochlea and medial palpebral ligament; it is the direct continuation of the main ophthalmic vessel, and becomes continuous with the angular artery on the face. (iv.) The lacrimal artery, after supplying the lacrimal gland, gives off terminal twigs to the lateral ends of the eyelids. The ophthalmic veins have a similar distribution. The intraorbital origin and course of the vessels are described later (p. 300); and an account of the infra-orbital artery, which, though extra-cranial in origin, is intra-orbital in 
position and supplies part of the orbital contents and the lower eyelid, will be found on p. 307.

(3) The skin of the lids and the orbicularis oculi muscles are supplied by freely-anastomosing branches of all the above-mentioned vessels; the deeper parts of the lids are supplied by the specially denoted palpebral branches of the nasal and lacrimal vessels, which are medial and lateral respectively.
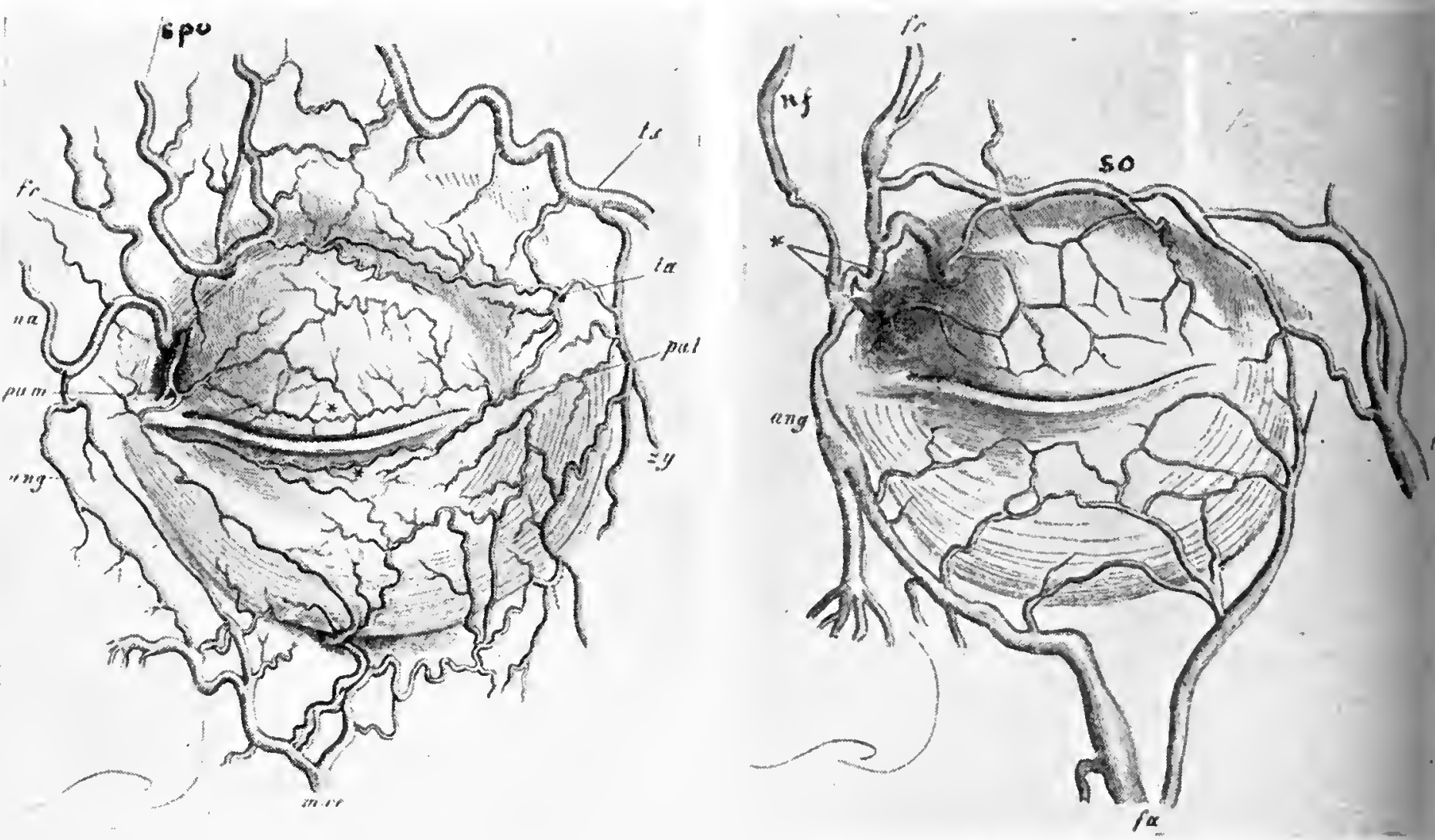

FIG. 85. - The Arteries and Veins of the Eyelids (Merkel, I gor).

In the first figure of the arteries : ang.=angular, na. = clorsalis nasi, $f_{0} .=$ frontal, spo. = supraorbital, $t s_{.}=$superficial temporal, $l a .=$ lacrimal, $z y .=$ zygomatic, $m x e$. = facial or external maxillary with the infra-orbital just above, pam. and fal.= medial and lateral palpebral, and the asterisks mark the superior and inferior tarsal arcades.

In the second figure of the veins : ang. =angular, $f r_{0}=$ frontal, $t_{0}=$ temporal, $f a .=$ facial, $n f_{0}=$ naso-frontal, so. = supra-orbital or marginal arcade, and the asterisk points to the anastomoses with the ophthalmic vein.

The medial palpebral arteries (arteriae palpebrales mediales) are two vessels which arise, usually by separate origins, from the nasal branch of the ophthalmic just before it leaves the orbit below the trochlea; they descend behind the pars lacrimalis muscle and lacrimal sac, and one enters the medial end of each lid. Before doing so the superior vessel supplies the caruncle, lacrimal canaliculi, lacrimal sac, and pars lacrimalis muscle; the inferior is joined by an orbital branch from the infra-orbital artery, and supplies 
the naso-lacrimal duct as far as its entrance into the inferior meatus of the nose. On entering the lids each palpebral artery bifurcates into a larger marginal and a smaller peripheral branch, which run along in relation to the borders of the tarsal plates towards the lateral extremities of the lids, where they unite and anastomose with two corresponding lateral palpebral branches (arteriae palpebrales laterales) provided by the lacrimal artery, and make connection also with the zygomatic-orbitalis branch of the superficial temporal artery; in this way two arterial arcades (arcus tarseus superior vel inferior) are formed in each lid, an inferior or marginal and a superior or peripheral, a distinction much better marked in the upper and broader eyelid. The marginal tarsal arcade with its accompanying vein forms a conspicuous feature in sections of the eyelids (Fig. 73, p. I5I); it lies about $3 \mathrm{~mm}$. from the free border of the lid, at the base of the ciliary region, directly on the tarsal plate and beneath the orbicularis muscle; it supplies the ciliary follicles and glands. The peripheral tarsal arcade pursues a sinuous course along the convex border of the tarsal plate, and in the upper lid is found in the pre-tarsal space between the aponeurosis of the levator and the superior palpebral involuntary muscle. Most of its twigs pass as " posterior conjunctival arteries" through the latter muscle to supply the conjunctiva of the fornix and bulbar region (Fig. I03, p. 200). The peripheral arcade of the lower lid is inconstant, being replaced by branches of the superficial vessels, and it does not supply the conjunctiva. Minute twigs pass from one arcade to the other, forming plexuses in front and behind the tarsal plates; the pretarsal plexus supplies all the structures in front of the plate and ramifies between the tarsal glands, whilst the posttarsal plexus (retro-tarsal or sub-conjunctival) supplies chiefly the conjunctiva, as described on p. I99 (Fuchs, I878; Langer, I878). The circulation at the margins of the lids is terminal, so favouring stagnation and causing redness.

The veins of the lids are larger and more superficially placed than the arteries, of which they run independently; in the deeper parts of the lids they form less regular arcades 
and plexuses, which present similar relations to the tarsal plates. The arcades drain medially into the superior ophthalmic vein, and laterally into the superficial temporal and lacrimal veins. The pre-tarsal plexus drains blood from around the tarsal glands, ciliary follicles, and free border of the lid; the superficial venules of the skin pass backwards between the fasciculi of the orbicularis oculi muscle to enter this plexus, and consequently are occluded in severe blepharospasm, so causing transudation and subcutaneous oedema. The retro-tarsal or sub-conjunctival plexus in the upper lid drains into the superior arcade, and thence into the superior ophthalmic vein; it communicates with the veins of the muscles of the globe, but also with the cutaneous veins; in the lower lid the sub-conjunctival veins pass entirely into the inferior ophthalmic vein, and do not communicate with the cutaneous veins.

The lymphatic vessels of the eyelids, like the bloodvessels, are divided into superficial and deep groups, the former draining the skin and orbicularis oculi, the latter forming plexuses in relation to the tarsal plates and the conjunctiva; but from a practical point of view they are better divided into medial and lateral groups, which collect into larger trunks and pass by separate paths to different lymph nodes.

The medial group of vessels (which are connected with those of the opposite side of the face) drains the superficial parts of the medial half of the lower lid, the medial commissure and medial end of the upper lid, and by deeper branches the conjunctiva of the medial two-thirds of the lower lid, and the lacrimal caruncle; from this field two superficial and two deep trunks follow the course of the facial vein to enter the submaxillary lymph nodes close beneath the lower border of the mandible. The superficial vessels enter a node lying in front of the vein, the deep ones into a node just behind it. Interruptions in the course of the vessels by either facial, infra-orbital, lateral nasal, or buccal nodes appear to be rare, according to most observers; the writer has not found any present in his series of dissections. 
The lateral group of vessels drains a larger field; the superficial ones come from the skin of nearly the whole of the upper and the lateral half of the lower lid, the deeper ones from the conjunctiva of the whole of the upper

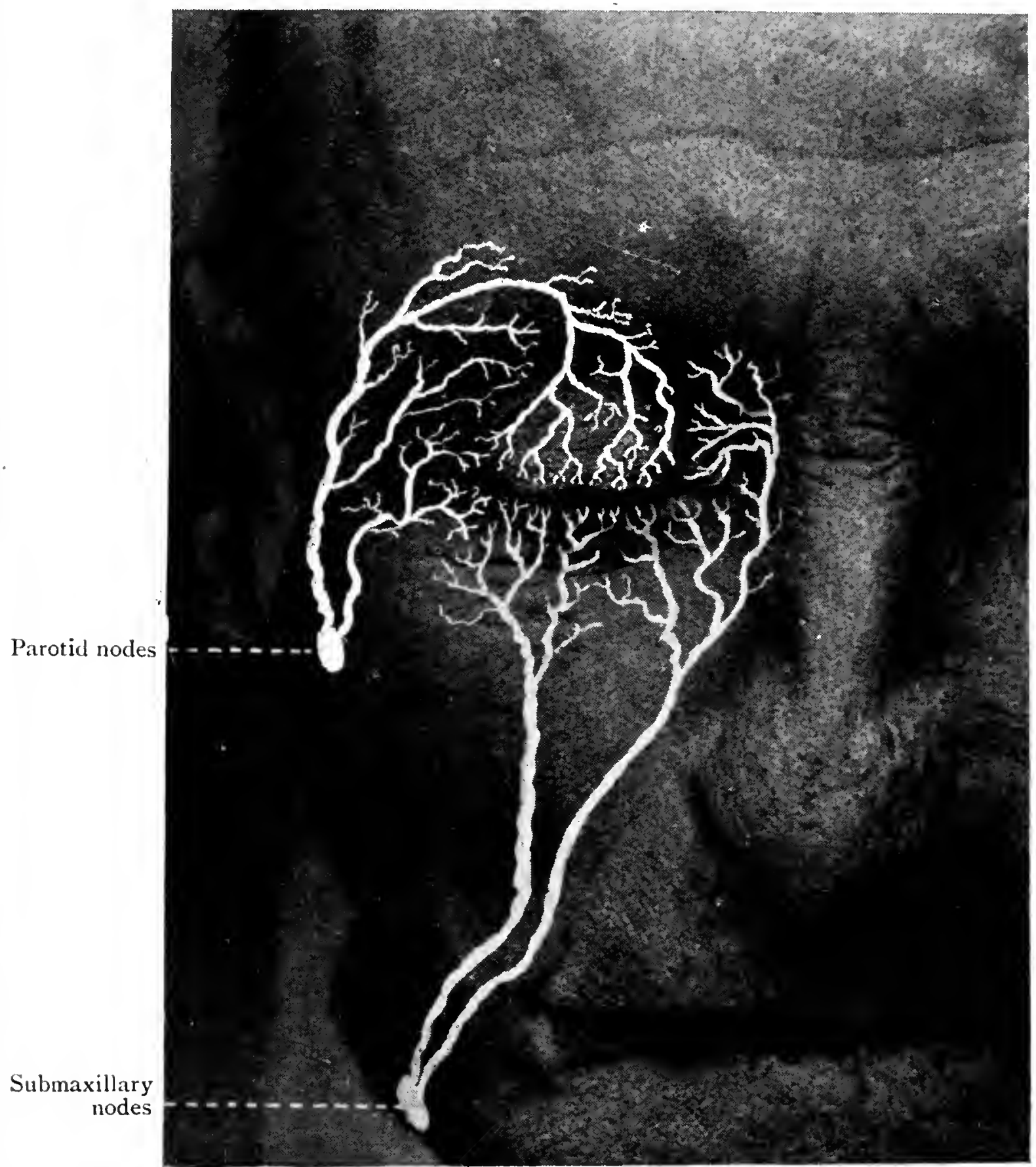

FIG. 86. - To illustrate the areas of the Skin and Conjunctiva of the Eyelids, which drain by lateral and medial groups of lymph vessels into the parotid and submaxillary lymph glands.

and lateral third of the lower lid; they pass as three or four trunks across the upper part of the cheek to enter the upper superficial and deep parotid nodes in front of the ear, the first and chief regional gland being situated superficially in the parotid gland at the level of the external acoustic 
meatus. Both these and the submaxillary nodes are connected with the deep cervical glands near the junction of the common facial with the internal jugular vein (Most, I905; Bartels, I909; Dewey, I920).

The deeper lymphatic vessels of the lids form pre-tarsal and retro-tarsal or sub-conjunctival plexuses which communicate with one another through the thickness of the tarsal plate (Grunert, I9OI) ; there is also a rich plexus along the convex border of the plate, the presence of which explains why the conjunctiva in this region is more readily invaded by pathological processes. The lymph round the tarsal glands passes into the sub-conjunctival plexus, the vessels of which are provided with valves, whereas the pre-tarsal vessels are devoid of them, so. that the deep lymph-flow is towards the conjunctiva and is in the opposite direction to the blood-flow (Fuchs, I878). See also p. 201.

(4) The Nerves of the Eyelids. - The nerves are motor, sensory, and sympathetic; the motor nerve is the facial, and it innervates the orbicularis oculi, corrugator supercilii, and frontalis muscles in this region; the sensory nerves to the skin are provided by numerous branches of the trigeminal nerve; the sympathetic nervous system supplies the involuntary palpebral muscles, the skin glands, and the vessels, etc.

The facial or seventh nerve (nervus facialis) arises from the facial nucleus in the lower part of the pons, forms a curved loop over the nucleus of the abducent or sixth nerve, and emerges on the surface of the brain at the lower border of the pons (Fig. I93, p. 387) ; it then passes into the opening of the internal acoustic meatus of the temporal bone in company with its sensory part, the nervus intermedius, and the acoustic nerve ; it traverses the facial canal or aqueduct of Fallopius and leaves the bone by the stylo-mastoid foramen; it passes on to the face below the external ear and behind the ramus of the mandible, and divides in the substance of the parotid gland into numerous radiating terminal branches, which are distributed to all the muscles of the face and the platysma of the neck. These branches 
are grouped into two main divisions of the nerve, an upper temporo-facial and a lower cervico-facial division.

Of the upper division, the temporal branches pass forwards and upwards over the zygoma, to be distributed to the upper part of the orbicularis oculi, the corrugator supercilii, and the frontalis muscles, which they enter from the lateral side and upon their deep surfaces; the branch to the upper part of the orbicularis (superior palpebral branch) runs a little above the lateral angle of the eye and parallel to the supra-orbital margin as far as its mid-line. The zygomatic branches (malar and infra-orbital) pass across the cheek to supply the lower part of the orbicularis oculi and lower facial muscles, the chief branch running along the lower border of the zygomatic bone (Friteau). The most important innervation of the muscles round the eye is therefore seen to be provided by the temporal branch of the facial

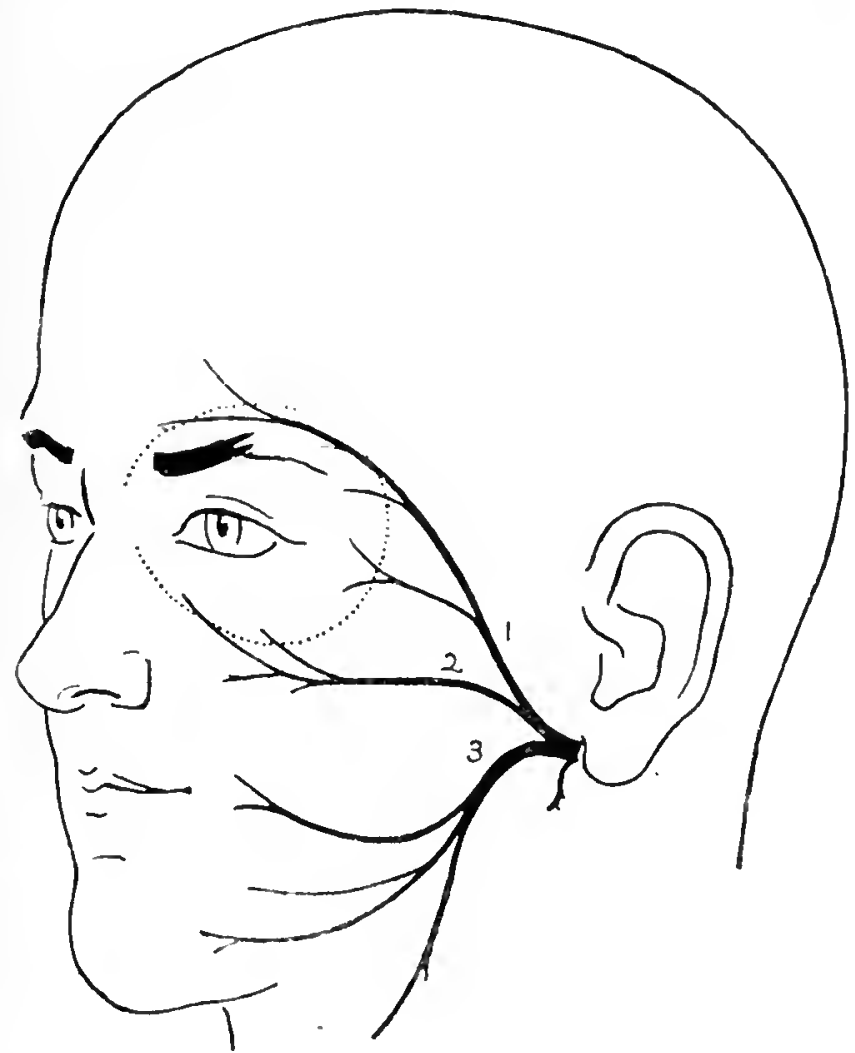

FIG. 87.-Diagram of the main branches of the Facial Nerve (from a dissection by G. Farmer, in the Oxford Anatomical Museum).

I and 2 are the iemporal and zygomatic branches respectively of the temporo-facial division of the nerve ; 3 is the cervico-facial uivision with its thrce branches, buccal, mandibular, and cervical. The orticularis oculi muscle is outlined by a dotted line. nerve.

It may be added that the levator palpebrae superioris is innervated by the upper division of the oculomotor or third nerve, in common with the superior rectus muscle of the eyeball.

All the motor branches of the facial nerve communicate with sensory branches of the trigeminal; the temporal branch with the auriculo-temporal, zygomatico-temporal, and supra-orbital nerves, the zygomatic branch with the zygomatico-facial, infra-orbital, infra-trochlear and nasal 
nerves; by the union of the zygomatic branch and infra. orbital nerve an extensive sensori-motor plexus is formed below the infra-orbital foramen, which is the most important anastomosis between the facial and trigeminal nerves.

The cortex of the brain is connected with the motor facial nucleus by fibres proceeding from cells situated in the lower third of the praecentral convolution (Fig. I90, p. 377) ; they traverse the genu of the internal capsule and the cerebral peduncle in reaching the pons. The facial nucleus also receives fibres from the spinal tract or descending root of the trigeminal nerve by means of which the orbicular reflex path is formed ( $\mathrm{p} .39 \mathrm{I}$ ) ; the nucleus is connected with the auditory nerve, and also with the visual and auditory centres, probably by paths through the medial (posterior) longitudinal fasciculus, by means of which the facial nerve cells respond to impulses of sight and hearing, as showriby the automatic closure of the lids to a threatened blow or loud sound.

The trigeminal or fifth nerve (nervus trigeminus) is the great sensory nerve of the face and forehead, and is also the motor nerve to the muscles of mastication ; its sensory part consists of three divisions-ophthalmic, maxillary; and mandibular (the last of which contains also the motor part of the nerve). The ophthalmic nerve supplies the skin of the eyelids and surrounding region by its supra-orbital, supra-trochlear, infra-trochlear, lateral nasal, and lacrimal branches; the maxillary nerve contributes infra-orbital, zygomatico-facial, and zygomatico-temporal branches; the mandibular nerve contributes no branches to the palpebral region, and is not related to the orbit, being destined for the muscles of mastication, the teeth, and other parts in connection with the lower jaw. A description of the origin and course of the trigeminal nerve and its divisions will be given in connection with the nerves of the orbit (p. 337), but here it may be noted that all the above-mentioned terminal cutaneous branches of the ophthalmic and maxillary divisions have passed through the cavernous sinus and the orbital cavity before reaching the face; the first group (ophthalmic) enter the orbit through the superior orbital 
fissure, are mostly related to its roof, and pierce the septum orbitale at the orbital margin ; the second group (maxillary) enter by the inferior orbital fissure, are related to the floor and lateral wall of the orbit, and emerge on to the face through bony canals.

The cutaneous areas supplied by the ophthalmic and maxillary divisions of the trigeminal nerve, as given by Zander (I897), are shown in Fig. 88, which demonstrates the areas of loss of touch and of pain after section of the nerves, but he states that the cutaneous distribution is so inconstant that he never found two identical fields, even on
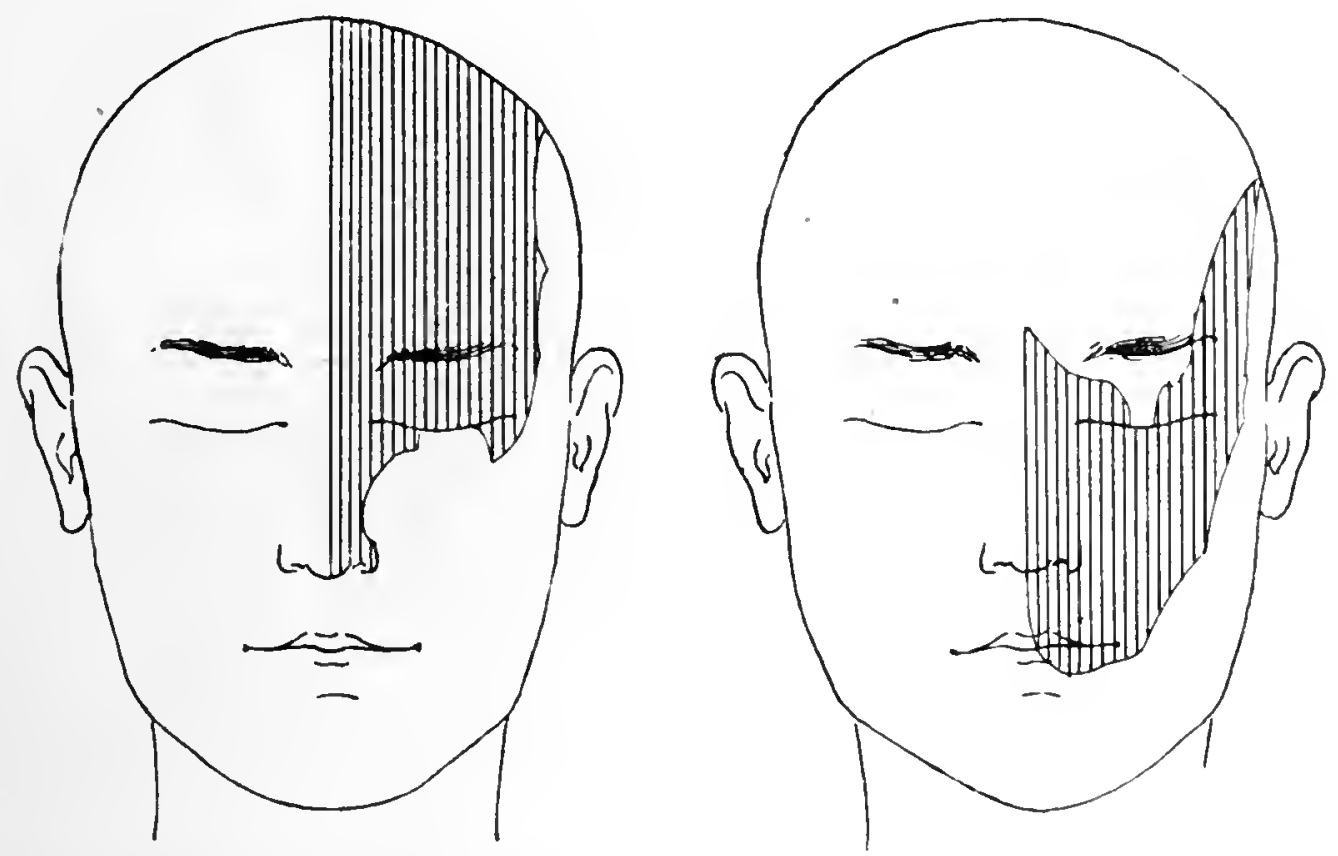

FIG. 88.-Cutaneous distribution of the Ophthalnıic (left-hand figure) and Maxillary (right-hand figure) Divisions of the Trigeminal Nerve, after Zander (I 897). The shaded areas show loss of touch and pain after section of the respective nerves. Note that there is considerable overlapping of the areas.

the two sides of the same head; the areas supplied by the two nerves largely overlap on the nasal side.

The skin of the upper eyelid is mainly supplied by palpebral branches derived from the supra-orbital branch of the frontal (ophthalmic) nerve, the skin of the lower lid by the palpebral branches of the infra-orbital branch of the maxillary nerve; the medial extremities of both lids are supplied by the supra- and infra-trochlear nerves, and the lateral extremities by the lacrimal nerve, all three of which are branches of 'the ophthalmic (Fig. I76, p. 342). The lacrimal nerve is stated by Frohse not to supply the skin (Quain), but 
the writer has several times traced its cutaneous branches, as in Figs. 58, 65. The nerves run at first beneath the orbicularis, then traverse it to reach the skin; in each lid a plexus is formed on the face of the tarsal plate, from which branches pass backwards through it to supply the tarsal glands and conjunctiva (Bach, I895). The nerve supply is especially abundant in the free margins of the lids, forming a " marginal plexus". (von Mises, I882), which is a special sensory apparatus connected with the cilia; terminal nerve corpuscles analogous to those of

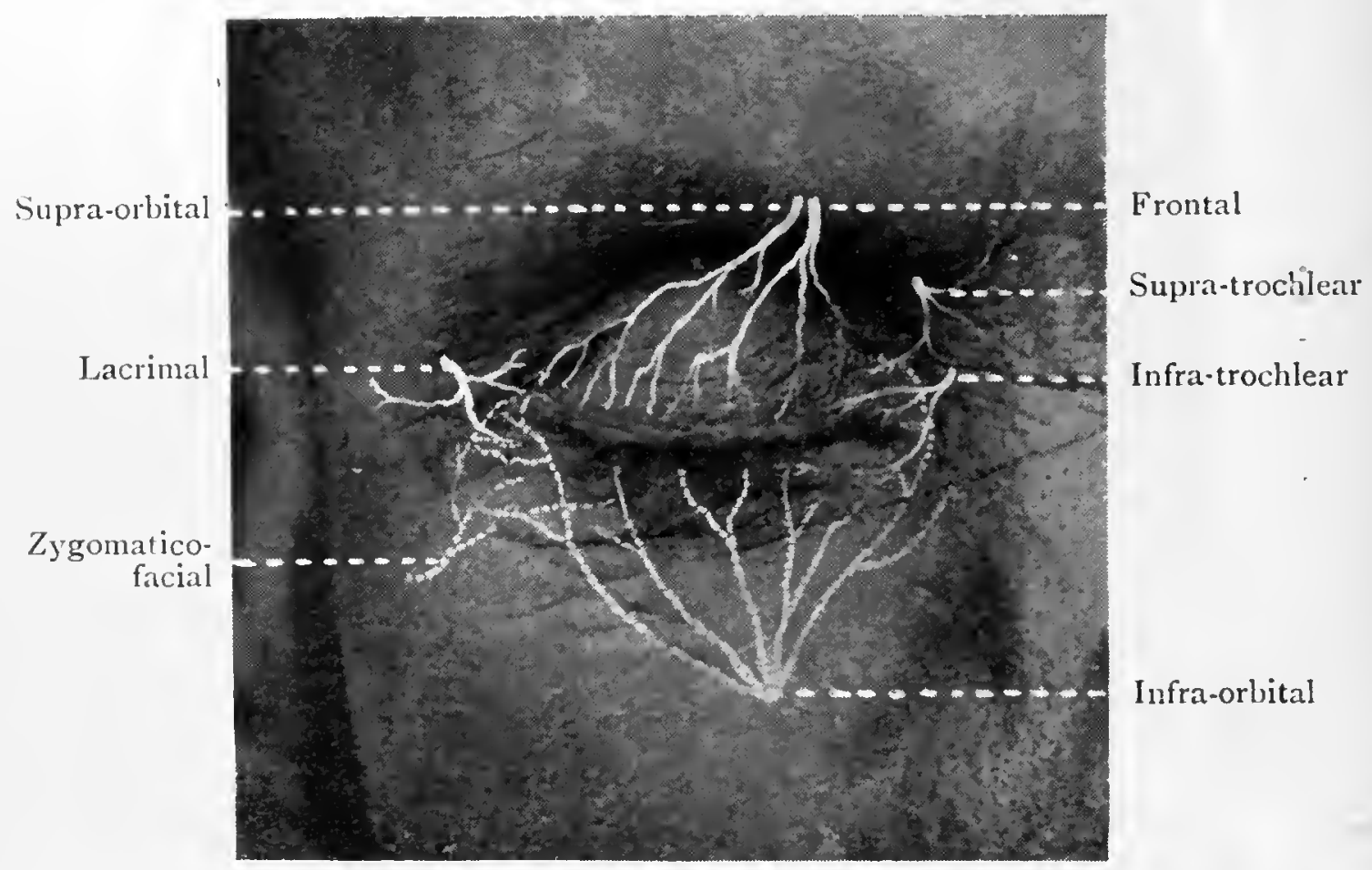

FIG. 89.- The Palpebral Branches of the Ophthalmic Nerve (solid lines) and of the Maxillary Nerve (dotted lines), right eye.

Meissner and Krause have been found present between the posterior edge of the lid margin and the orifices of the tarsal glands (Dogiel, I895).

Irritation or excitation of the sympathetic system in the neck causes widening of the palpebral opening by its stimulatory action upon the involuntary palpebral muscles, and section of the nerve causes a drooping of the upper eyelid and narrowing of the fissure by relaxation of the muscles. The nerve fibres to these muscles may either accompany the arterial twigs or be incorporated with the nerves to the levator and inferior oblique muscles. 
(5) The Action of the Muscles and movements of the eyelids.-The muscles which affect the movements of the eyelids are the orbicularis oculi, corrugator ${ }^{*}$ supercilii, frontalis, levator palpebrae superioris, and the involuntary palpebral muscles. Their individual actions are as follows :

The orbicularis oculi is a sphincter muscle, and is seen to act as a whole in forced occlusion of the lids, as in the presence of strong light or in protecting the eye from a blow : the palpebral margins are closely approximated, the skin is pursed up all round the fissure, the eyebrow is drawn downwards, and even the cheek is raised, the zygomaticus and levator muscles of the upper lip participating in the exaggerated movement. (It is also stated that in strong contraction of the orbicularis the eyeball is rolled upwards, and hence the superior rectus and inferior oblique muscles are also thrown into action.) The muscle also supports the orbital contents and prevents congestion of the globe in such forced actions as accompany vomiting, straining, etc., in which the intra- and extra-ocular pressure is raised. In partial closure of the lids, as occasioned by a glaring light, the lacrimal portions of the palpebral margins come in contact over the lacus lacrimalis, so that the whole palpebral opening is much reduced in horizontal width as well as in height, as represented by Fig. 90; the effect, however, is not seen in childhood, where the curve of the lacus lacrimalis is relatively wider.

As regards the various subdivisions of the muscle, a distinction in their actions is to be noted. A difference between the orbital and palpebral parts of the muscle is illustrated by the act of holding an eyeglass : it is kept in place by the voluntary contraction of the pars orbitalis, whilst the pars palpebralis retains its independent function of moistening the surface of the globe by involuntary periodic contraction; moreover, paralysis of the one part may occur without the other being affected, illustrating the fact that a voluntary muscle may be paralysed for a voluntary action and yet be perfectly free to act in an involuntary action (Wood Jones). In gentle voluntary closure the movement can be confined to the palpebral 
part, and in the involuntary act of winking the tarsal subdivision alone contracts. The pars tarsalis keeps the tarsal plate in close contact with the globe (see p. I50), whilst its lacrimal fibres (Horner's muscle) more especially direct the puncta of the lacrimal papillae backwards towards the lacus lacrimalis in which the tears collect, so that when

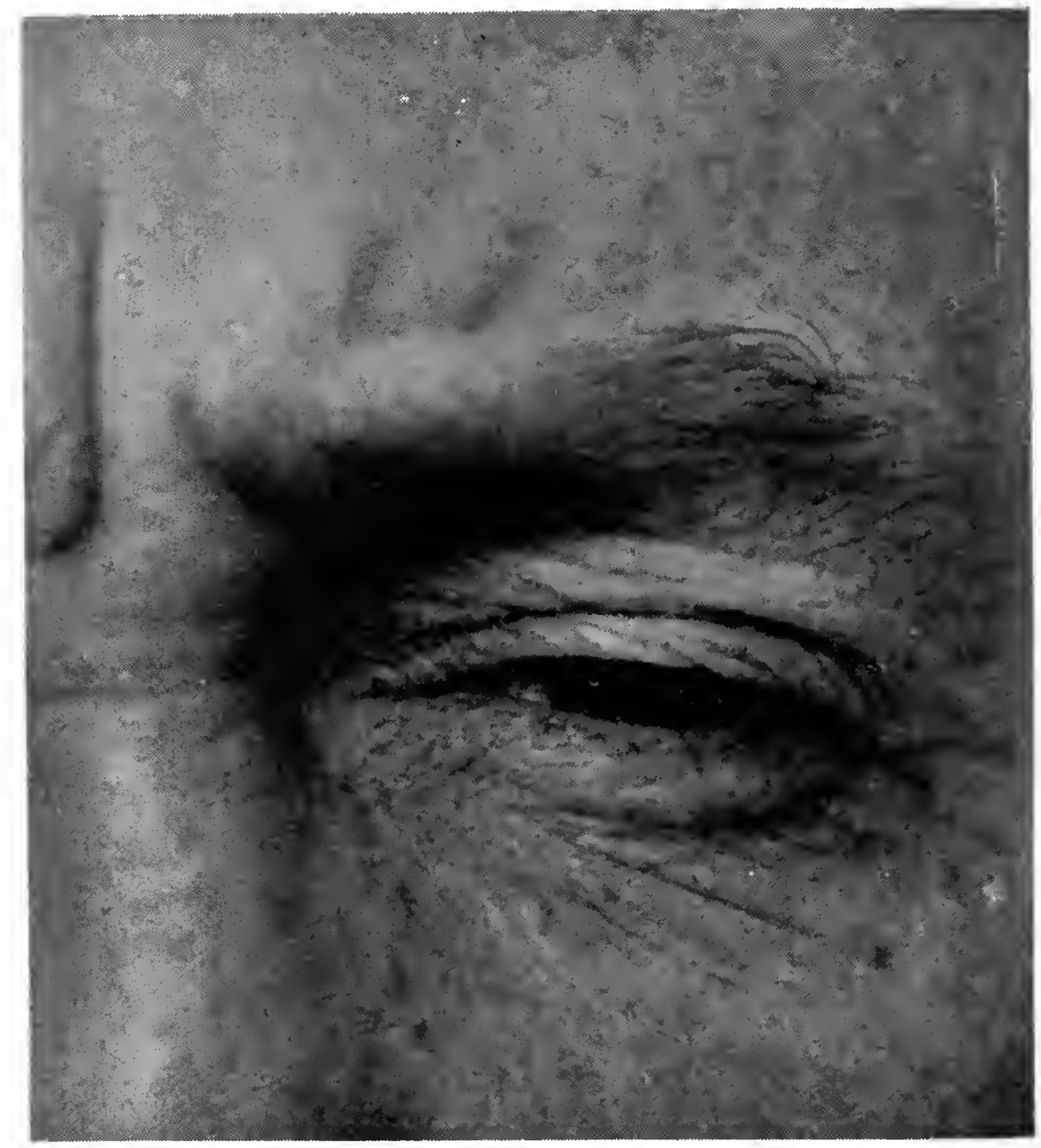

FIG. 90. - Photograph showing the action of the Orbicularis Oculi Z. Iuscle on exposing the eye to glaring light; the lacrimal portions of the lid margins are closely approximated by contraction of the pre-tarsal fibres, so that the palpebral fissure is narrowed in the horizontal direction as well as in the vertical. Note the wrinkling of the skin above the head of the eyebrow, caused by the corrugator supercilii muscle, and compare with Fig. 47, p. Iog.

this part of the muscle is inactive the lower lid tends to fall away from the surface of the globe, and the condition known as ectropion is produced. The effect of action of this part of the muscle on the canaliculi is also referred to on p. 224. The fibres of the muscle nearest the palpebral border (pars ciliaris) have the important function of keeping the lid edges in close approximation to the globe, so con- 
tracting the mouth of the conjunctival sac and preventing the entrance of foreign bodies; these fibres, from their close relation to the terminal portion of the tarsal glands, may be concerned with the secretion of the latter, and those immediately overlying the bulbs of the cilia may, by their pressure in contraction, determine the curve of the outgrowing eyelashes. The orbicularis is antagonised in its palpebral part by the levator palpebrae superioris, and in its orbital part by the frontalis in the upper eyelid, and by the inferior rectus and by gravity in the lower lid.

The corrugator supercilii muscle acts in harmony with the orbicularis, drawing the eyebrow downwards, as in shielding the eye from strong light; it also draws the brow towards the root of the nose, and so contracts the skin of the forehead into vertical folds; it is the special muscle of frowning, an essentially human gesture to be noted even in the infant, though the muscle is not peculiar to man (Darwin, I872). Sir Charles Bell, indeed, ranks it as the most remarkable muscle of the human face, since frowning irresistibly conveys the idea of mind and gives the countenance an aspect of intellectual energy.

The frontalis muscle raises the eyebrow and throws the skin of the forehead into horizontal folds, a movement that may become fixed in the aged; as regards facial expression, it is the muscle of attention and astonishment. It is antagonised locally and moderately by the orbicularis oculi when it takes its action from the galea aponeurotica as a fixed point; distally and more strongly it is counteracted by the occipitalis, the simultaneous contraction of which upon exaggerated raising of the eyebrows can be felt by placing the hand on the back of the head.

The levator palpebrae superioris muscle is the special elevator of the upper eyelid; by the contraction of its muscle belly the transversely-disposed aponeurosis is swung backwards over the globe like the vizor of a helmet, pulling with it the skin of the lid into which its terminal fibres are inserted, and so deepening the superior palpebral fold; at the same time the tarsal plate is raised both by the terminal fibres attached to the lower part of its face and by the con- 
joint action of the involuntary palpebral muscle inserted into its margin, and the loose conjunctiva of the superior fornix is pulled up by the fascial attachment of the levator muscle sheath. The levator acts in harmony with the superior rectus muscle of the eyeball, with which it shares the same nerve supply, though it also acts independently of it, as in winking ; it can further act in unison with the frontalis, as in expressing astonishment, and all three muscles act together in looking upwards in extreme excursion of the globe. The light degree of synergy which exists between the actions of these muscles is sometimes used to remedy cases of ptosis by connecting the eyebrow and eyelid more closely together, and so bringing the lid under direct influence of the frontalis. The levator is antagonised by the palpebral part of the orbicularis oculi muscle and by gravity. The action of the palpebral involuntary muscles is to widen the palpebral fissure in the vertical direction.

The movements of the eyelids. - The lids are closed by the action of the orbicularis oculi, the upper lid being. also lowered by gravity and slightly by relaxation of the palpebral invo'untary muscle; they are separated by contraction of the levator and relaxation of the orbicularis. Since the tone of the orbicularis is stronger than that of the levator, the lid is lowered in sleep. Neither lid can move quite independently of the other, since they are connected at the commissures. The movements of the globe are to some extent transmitted to the eyelids, the lower lid appearing to be pushed forwards and slightly raised in turning the eye upwards, though this movement may be caused by its commissural attachments to the upper lid. The association of these muscle movements is tabulated by Gowers (I879) as follows (with additions) :

\begin{tabular}{|c|c|c|c|c|}
\hline & Looking down. & Looking up. & Closing gently. & Closing forcibly \\
\hline $\begin{array}{l}\text { Orbicularis } \\
\text { Levator } \\
\text { Superior rectus } \\
\text { Inferior rectus }\end{array}$ & $\begin{array}{l}\text { inaction } \\
\text { relaxation } \\
\text { relaxation } \\
\text { contraction }\end{array}$ & $\begin{array}{l}\text { relaxation } \\
\text { contraction } \\
\text { contraction } \\
\text { relaxation }\end{array}$ & $\begin{array}{l}\text { contraction } \\
\text { relaxation } \\
\text { inaction } \\
\text { inaction }\end{array}$ & $\begin{array}{l}\text { contraction } \\
\text { relaxation } \\
\text { contraction } \\
\text { relaxation }\end{array}$ \\
\hline
\end{tabular}


As regards types of movement there are three : voluntary (as in occlusion with effort), automatic (winking), and reflex (blinking). The reflex path for automatic involuntary winking is from the cornea (naso-ciliary nerve) and conjunctiva (infra-trochlear and lacrimal nerves) by afferent sensory branches of the trigeminal nerve to the cerebral centres, and thence by efferent motor branches of the facial nerve to the orbicularis. There are two reflex arcs in spontaneous winking :

(i.) Trigemino-facial, the reflex excitable stimulus being cooling and dryness of the cornea or touching of sensory parts.

(ii.) Optico-facial, the excitable stimulus being strong light or proximity to danger (Lans, I9oI)

Certain facts concerning the relation of these muscles to vision are of clinical interest. The frontalis is affected in certain forms of imbalance of the muscles of the globe, and a horizontal wrinkling of the forehead is a characteristic symptom in hyperphoria (the name given to the condition when one pupil is higher than the other); moreover, the connection of the muscle by means of the galea aponeurotica and occipital bellies with the deep fascia at the back of the head and neck (Fig. 46, p. Io6) would explain the occurrence of an occipital headache, which in some cases is relieved by the use of suitable glasses, and the relief of severe headaches associated with efforts of accommodation of the lens which is said to follow complete severance of the muscle. The corrugator contracts in the presence of too strong a light, in most myopic subjects in far vision, and in nearly all subjects in close work; indeed one of the symptoms of actual excessive accommodation of the lens is vertical wrinkling of the forehead. On such grounds the frontalis, orbicularis, and corrugator have been grouped together under the name of " the accessory muscles of accommodation," and they become of clinical importance in connection with certain forms of ocular headache (Howe, 1907). These muscles (which are supplied by the facial nerve) are called into action by the efforts of the ciliary muscle of the lens (supplied by the oculomotor nerve) which accompany exces- 
sive accommodation, and corroborative evidence of connection between the two sets of muscles supplied by the two nerves is further shown by the occurrence of cases of total ophthalmoplegia (paralysis of the muscles of the globe, both extrinsic and intrinsic) accompanied by paralysis of the frontalis and orbicularis (Aldren Turner, I893). Moreover, a differentiation between these muscles (the upper facial group) and the rest of the facial muscles (the lower group, comprising the muscles of the nose, cheek, and mouth) is indicated by the fact that the former commonly escape in facial nerve paralysis of cortical origin, though the peripheral part of the nerve supplies both groups of muscles and all are affected in paralyses of extra-cerebral origin.

Various explanations of the above conditions are given. An explanation of the headache is that the ophthalmic division of the trigeminal nerve, which supplies the skin of the forehead, represents the sensory somatic nerve of the same primitive segment of the neural canal from which the oculomotor nerve is derived, and frontal headache in disorders of accommodation is caused by reflex pain (Keith, I9I3). An afferent path from the ciliary region of the eyeball to the brain is afforded either by the naso-ciliary nerve or by the communicating branch which the oculomotor nerve receives from the ophthalmic nerve in the cavernous sinus, or by both; in each case the brain may refer the pain not to the site of origin but to some other sensory field of the trigeminal (of which the naso-ciliary and ophthalmic are branches), just as it does in a neuralgia of dental cause, for example.

As regards the synergy of action of the upper facial with the ciliary muscles, whilst no actual branch of the oculomotor nerve has been traced to enter the upper facial. group, it is suggested that nerve fibres destined for their innervation, although passing out of the brain in the substance of the facial nerve, either (i.) actually arise from cells of the lower part of the oculomotor nucleus and then pass via the medial longitudinal bundle as a "superior facial nerve," to emerge as part of the facial nerve itself (Mendel, 
I887), or (ii.) arise from a more dorsally-placed sub-group of facial nucleus cells, which is distinguished as a " superior facial nucleus," and is presumably associated by the same bundle with the oculomotor nucleus (Bishop Harman, I903; Bruce and Pirie, I9o8; van Gehuchten; Quain; Villiger), as illustrated in Fig. I93, p. 387; as regards the escape of the upper group of facial muscles in a paralysis, apart from the possible non-implication of these cells in a nuclear lesion, it is generally considered that the superior facial nucleus receives a bilateral innervation from the motor centres of both cerebral hemispheres, and the muscles it supplies will therefore be unaffected by a unilateral cortical lesion.

The phenomenon of "jaw-winking," when there are associated movements of the jaw and eyelids, may be due to some alteration of the supra-nuclear connections of the nerves (Lutz, I9I9; Posey, Darrel, and Natale also report instances).

(6) The Development of the eyelids.-The face begins to be developed towards the end of the third week of foetal life; about this time five processes arise from the base of the primitive cerebral capsule, and by the end of the second month they have completely united to form the facial part of the head and surround the stomodaeum or primitive mouth. Of these five processes one, the fronto-nasal, is median in position, and is composed of symmetrical right and left halves, the others are placed laterally, two on each side, and are called the maxillary and mandibular processes (Fig. 9I). The fronto-nasal process becomes subdivided by the appearance of two olfactory pits into a median and a lateral nasal process on each side. The cleft between each lateral nasal and the adjacent maxillary process leads upwards to the already developed eyelids, and is called the naso-optic groove; in its lower part the naso-lacrimal duct is developed, and its upper part is continuous with the future orbit; it is obliterated by fusion of the adjacent processes in the second month of foetal life. The parts of the adult face derived from each process are shown in Fig. 92. 
The eyelids are formed in the earlier weeks of the third month of foetal life by folds of epiblast, which project above and below the front part of the eyeball and enclose mesoblastic tissue in their thickness. The lower eyelid is derived from the primitive maxillary process; the upper eyelid is developed from the fronto-nasal process in two parts, medial and lateral, and occasionally in the adult a notch on the margin may mark their point of union. The epiblast on the outer surface of the folds develops into the skin of

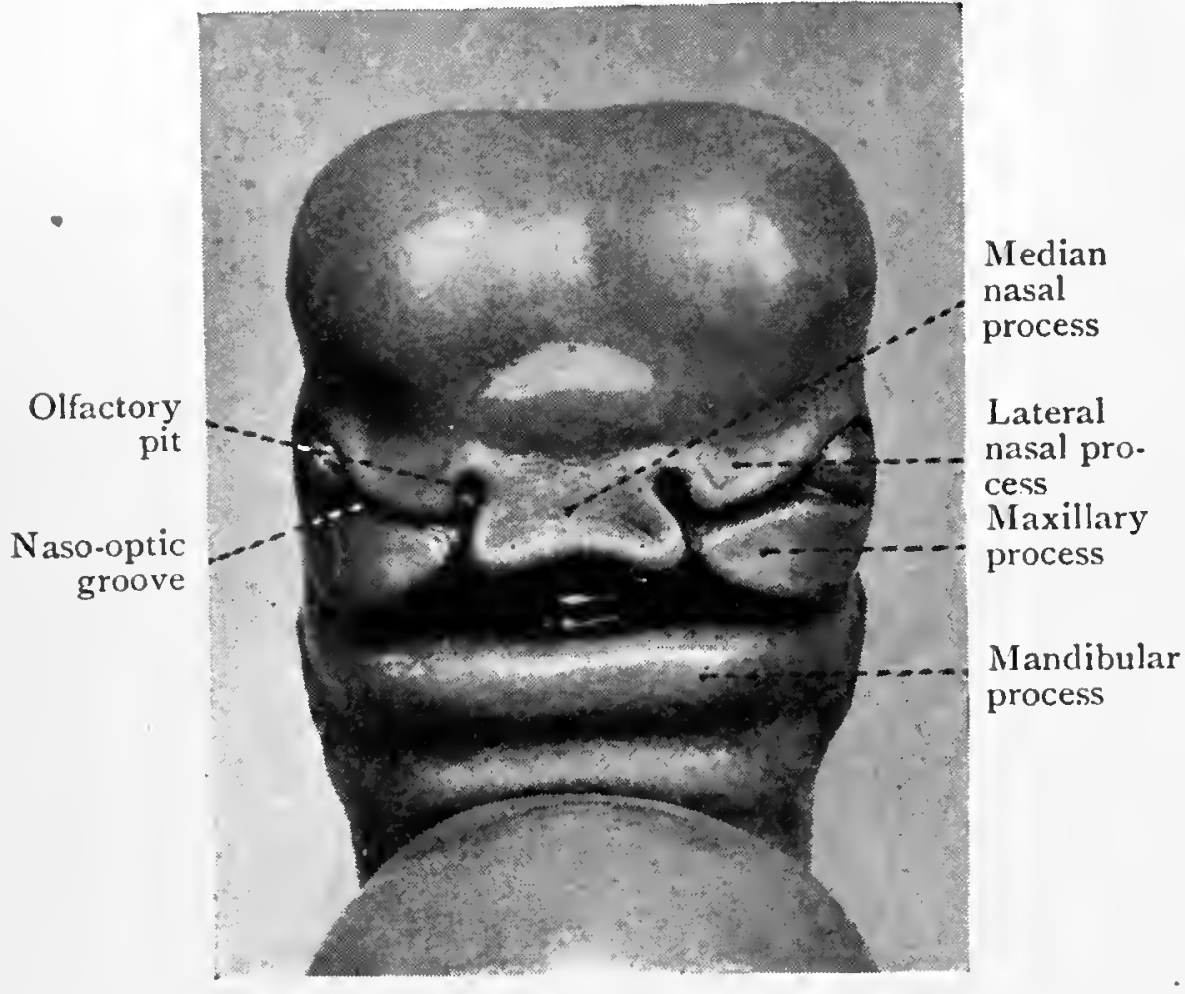

FIG. 9 I.

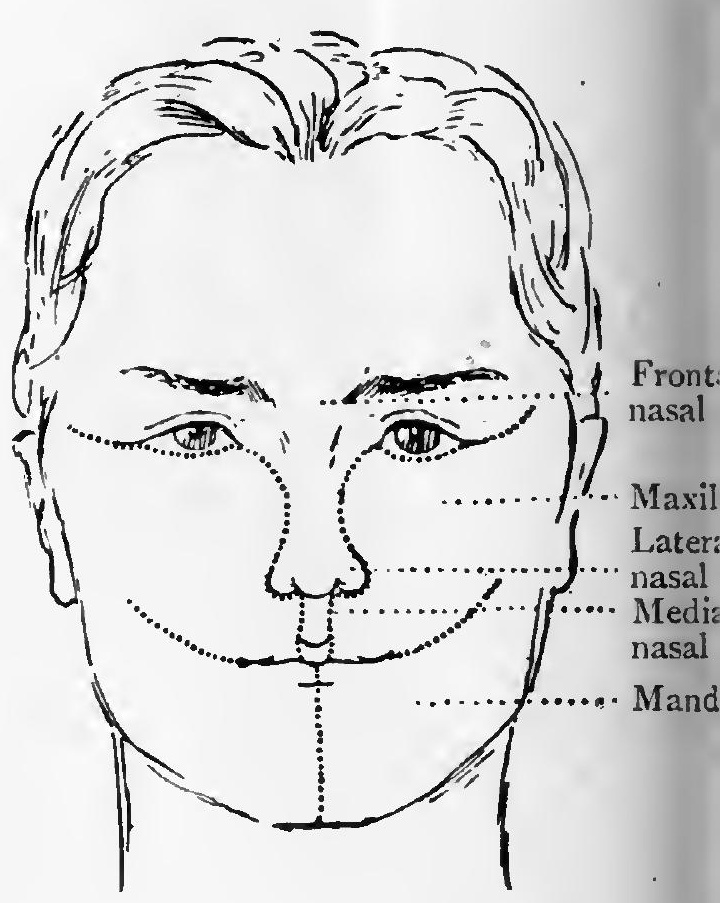

FIG. 92.

FIG. 9I.-Model of the Face of an Embryo (reconstruction by His) to show the processes which form it. The two mandibular processes have fused at this stage.

FIG. 92. - Showing the corresponding parts of the Adult Face.

the lids, whilst that on the ocular surface becomes modified into the conjunctival membrane, which comes into contact with the anterior surface of the globe and is thus continuous with the epiblast of the cornea. The edges of the folds become the palpebral margins, and ingrowths from the epiblast in this region into the substance of the lids form the ciliary follicles and the tarsal and other glands, which are all therefore ectodermal in origin. From the mesodermal tissue enclosed between the skinfolds there are developed the orbicularis oculi muscle, 
tarsal plates, vessels, and connective, elastic, and fatty tissues.

About the beginning or middle of the third month (that is, as observed in an embryo of $3 \mathrm{I} \mathrm{mm}$. by Ask, Igo8) the edges of the lids meet, adhere, and do not separate until the end of the sixth month; the fusion begins at the two extremities of the palpebral fissure, and was seen quite complete in an embryo of $37 \mathrm{~mm}$. (Contino, I907) ; it is after the fusion of the lids that the epiblast on their deep

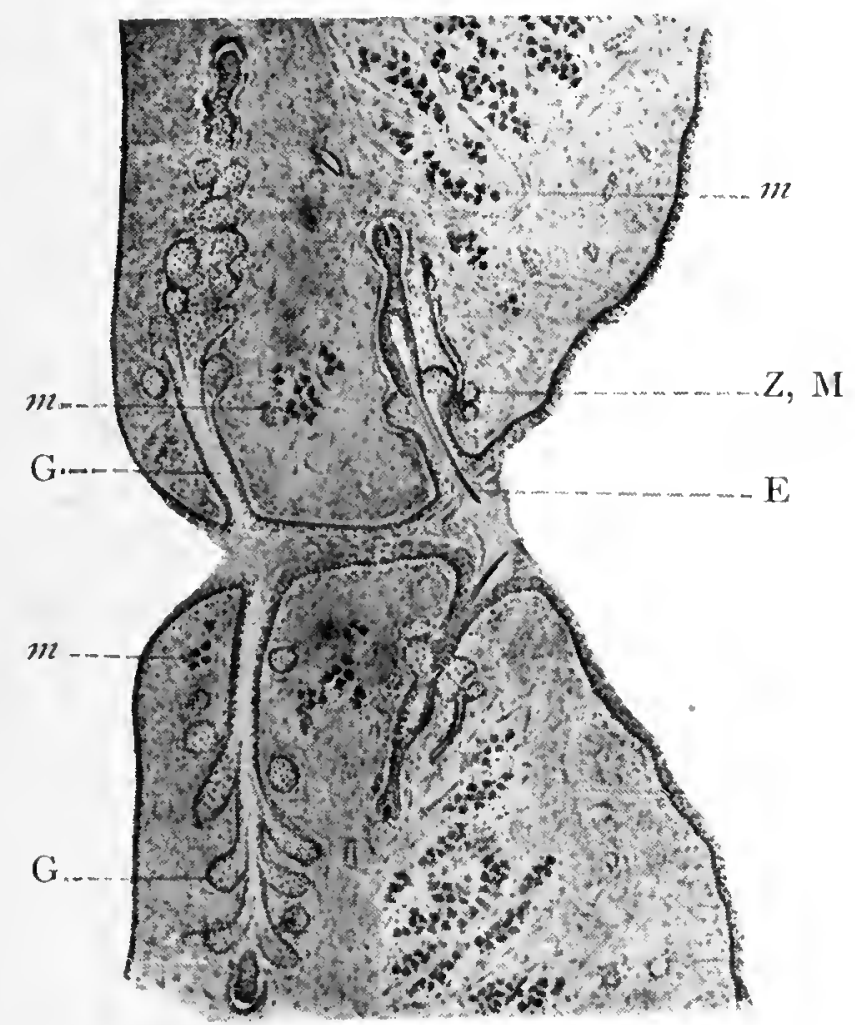

FIG. 93.-Developing lid margins at the time of separation in a foetus of $225 \mathrm{~mm}$. A tarsal gland (G.) and an eyelash (E.) with glands of Zeis (Z.) and Moll (M.) are seen in each lid, and the subdivisions of the pars marginalis of the orbicularis oculi muscle ( $m$.) are shown. (Contino, 1907.)

surfaces becomes modified into the conjunctiva. The separation of the eyelids begins at the end of the fifth month on the nasal side, and is affected by a process of cornification of the fused edges, aided by the pressure of the growing eyelashes and secretion of the glands (Fig. 93); the actual date of separation presents individual variations, and Oppenheimer records a case where it was not complete at term, a condition which is normal in kittens, rabbits, and mice. The details of the development of the lid margin will be found in articles by the authors quoted above, 
and the comparative anatomy of the lids has been specially studied by Eggeling (I904).

The tarsal or meibomian glands begin to develop in the embryo at about eleven to twelve weeks, arising as inward buddings from the epithelium of the posterior edges of the fused margins of the lids; those of the upper lid exceed the lower ones in length only after the middle of embryonic life. The glands of Zeis and Moll arise as pouchings out of the basal cells of the Anlage of the cilia at about three months. The tarsal plates appear as a condensation of mesoderm at about ten weeks, but develop more clearly

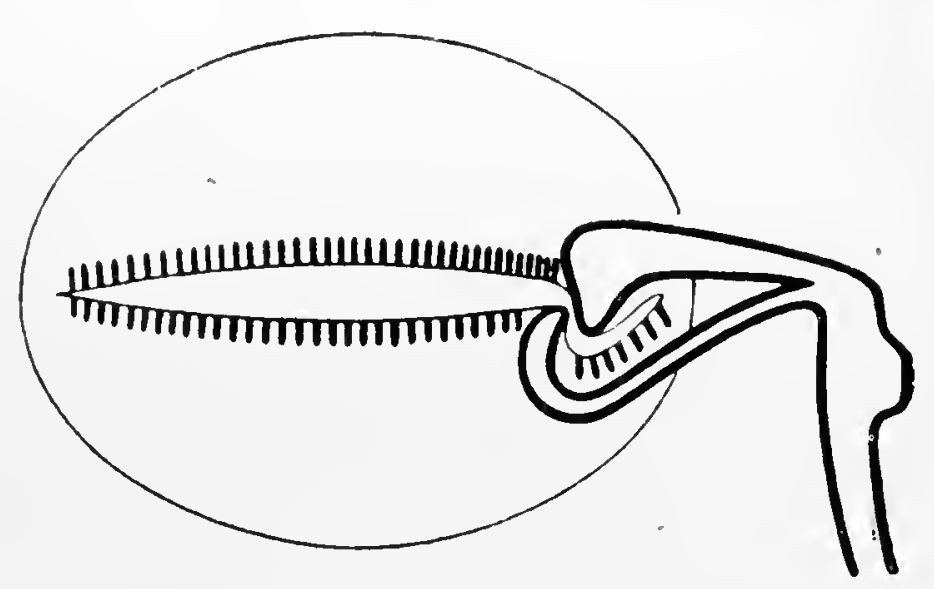

FIg. 94. - Development of the Lacrimal

Caruncle. Scheme of the modification produced in the medial part of the Eyelids by the growth of the Lacrimal Canaliculi; the lower one cuts off a part of the lid margin which becomes the lacrimal caruncle. 'The lids are really fused at this stage (embryo of $170 \mathrm{~mm}$.) but are shown separated for the sake of more clearly representing the meibomian glands (the vertical lines). (From Ask, 1908.) when the secretory activity of the tarsal glands is established. The septum orbitaleand aponeurosis of the levator palpebrae superioris begin to be differentiated from mesoblastic tissue at about six months. The orbicularis appears at ten weeks, and its ciliary bundle is separated off by the ingrowing bulbs of the eyelashes at twelve weeks. The development of the vessels of the lids has been studied by Engel, of whose work a résumé is given by Picou (I9I2).

The lacrimal caruncle appears immediately after the fusion of the lids, and is formed from the medial end of the lower lid margin by a portion being cut off in the development of the inferior lacrimal canaliculus (Fig. 94). Hence the caruncle is not developed in animals, such as the pinnipedes, which have no lacrimal passages (Broman and Ask, I9IO). It is definitely isolated in about five days, when its ectodermal covering becomes modified, as does its mesodermal body. In many animals, as, for example, in the 
calf, it retains a cutaneous formation and is still connected with the lid margin by a bridge of skin, a primitive condition that has been seen to persist in man (Peschel, I903). Its cutaneous origin is also shown by the occasional presence of small papillomata.

(7) The congenital abnormalities of the eyelids are explained by reference to the above short account of the development. Only chief examples are here noted; and for further details the reader should consult the articles by Lang and Treacher Collins (IgOo), and Hardy (I9I4).

(i.) The eyelids may be absent, when the conjunctiva fails to develop and the front of the globe is covered by skin; all the lid appendages, including the lacrimal gland and passages, are absent in the condition known as cryptophthalmia; part of either lid may be absent, but the deformity usually affects the medial part of the upper lid.

(ii.) The margin of either lid may be notched or fissured, sometimes up to the orbital margin, and more commonly on the nasal side of the upper lid; the deformity may be caused by imperfect descent or union of the embryonic processes, and is called a coloboma of the eyelid. Associated with it there is frequently found a small nodule of skin on the surface of the globe, forming a congenital mole or dermoid of the bulbar conjunctiva; this nodule does not represent a detached part of the skin which should have filled the gap in the eyelid, but is an islet of the embryonic skin-fold over the eyeball which has not become metamorphosed into conjunctiva owing to its exposed position (Fisher).

(iii.) The palpebral fissure may be wider than usual (lagophthalmos), a condition that has been found to be a congenital malformation in four generations by Peters; on the other hand, the fissure has been seen narrowed by incomplete separation of the margins (Hoeve), or the lids may even be completely united by vascularised tissue, the prenatal separation not having taken place, causing the condition known as ankyloblepharon.

(iv.) Dermoid cysts may occur at the inner angle of the eye or even within the orbit, but more commonly develop near the tail of the eyebrow (one of the favourite sites chosen, 
curiously enough, for the affixation of a "beauty spot"); they lie beneath the orbicularis oculi muscle, and may hollow out the frontal bone, or even be connected with the dura mater lining of the skull by an opening through it; they are caused by an inclusion of epiblast between the growing bones; occasionally they are present in the middle of the upper eyelid, where the two parts from which it is formed fuse, but here they have no deep connections.

(v.) The muscles of the lids may fail to develop, and congenital ptosis results. The superior rectus is not uncommonly defective as well as the levator. The condition may be hereditary, and Briggs (I9I9) reports sixty-four such cases.

(vi.) A reduplication of the conjunctival membrane lining the upper tarsal plate, known as the "epitarsus" or " double tarsus," is described on p. 203.

Abnormalities of the orbicularis oculi muscle are given on p. I30, and of the levator palpebrae superioris on p. I44.

A reduplication of the caruncle has been recorded by Stephenson (I8g6); a case of a "supernumerary eyelid," formed by an outgrowth of the caruncle and containing both palpebral and caruncular elements, is reported by Shoemaker (I9I4).

\section{THE CONJUNCTIVA}

The conjunctiva (tunica conjunctiva) is the mucous membrane lining the posterior surface of the eyelids and reflected from them on to the anterior face of the eyeball, thus forming a union between the lids and globe. In its entirety the membrane forms the "conjunctival sac," the mouth of which is bounded by the free margins of the lids and so corresponds to the palpebral fissure, but the walls are in the closest apposition throughout, and the sac is only a potential one. At the lid margin it is continuous with the skin through a transitional zone common to both layers. It lines the ocular surfaces of the tarsal plates and extends some short distance beyond their-peripheral limits into the supra- and infra-tarsal regions before being reflected 
on to the globe; the fold of reflection forms a circular cul-de-sac round the globe known as the conjunctival fornix. The membrane becomes smoother as it passes over the front of the eyeball, and over the cornea it is structurally continuous with and is represented by its epithelium. On the medial side of the globe it forms a fold called the plica semilunaris conjunctivae. The ducts of the lacrimal glands open into the upper lateral region of the conjunctival sac, and on the medial side the puncta lacrimalia lead from it into the lacrimal passages, through which the tears are drained into the nose.

The conjunctiva is a continuous membrane throughout its whole extent, but since it presents special characters and relations in different regions, it is divided topographically into :

(i.) The palpebral conjunctiva, lining the eyelids ;

(ii.) The conjunctiva of the fornix, in its fold of reflection;

(iii.) The bulbar conjunctiva, covering the front of the eyeball ;

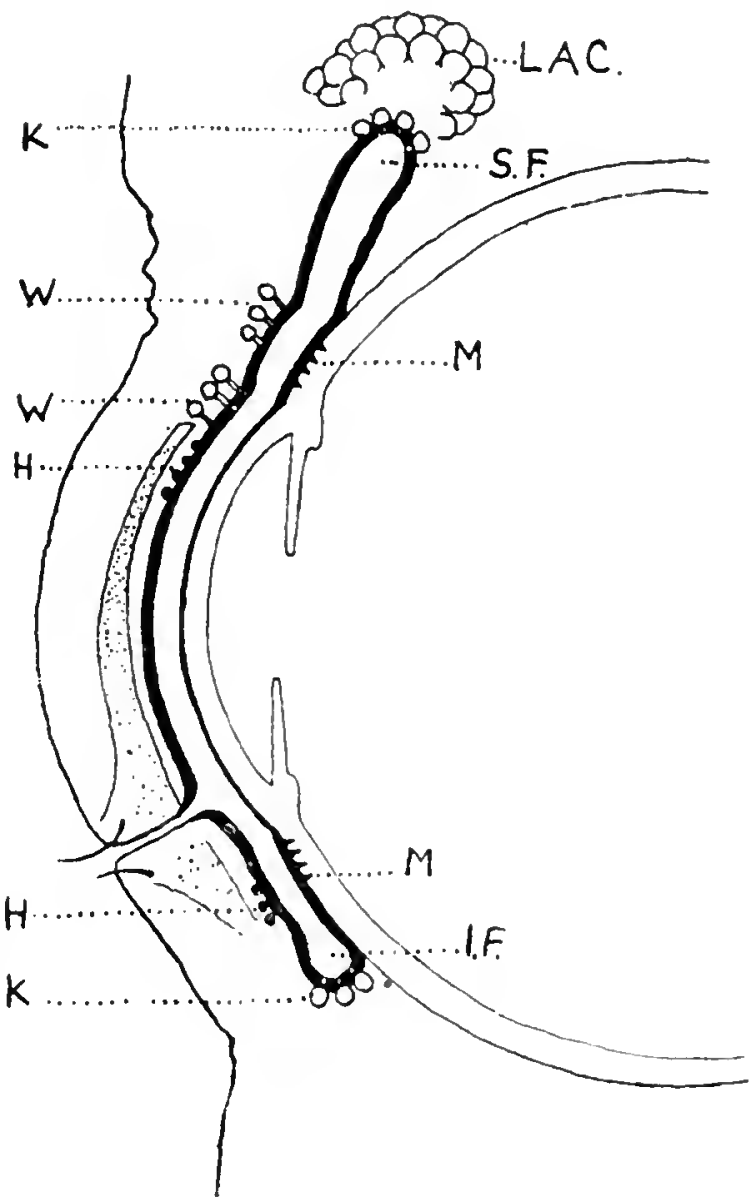

FIG. 95. - Scheme of a sagittal section through the Eyelids and Eyeball to show the Conjunctival Sac and the position of its Glands (after Dubretil, rgo8).

S.F., I.F. are the superior and inferior fornices respectively ; LAC. $=$ the lacrimal gland proper ; $\mathrm{K} .=$ the accessory lacrimal glands of Krause, and $\mathrm{W}_{\text {. }}=$ those of Wolfring $; H_{.}=$the crypts of Henle; M. = the "glands" of Manz.

(iv.) The plica semilunaris.

The palpebral and bulbar portions of the membrane are fixed, but where it forms the plica and in the region of the fornix it is mobile.

(i.) The palpebral conjunctiva (tunica conjunativa palpebrarum) is more vascular than the rest of the membrane, and its condition is referred to as a sign in anaemia; it is smooth and intimately adherent to the posterior faces of the 
tarsal plates, the meibomian glands of which can be seen through it on everting the lids; a slight groove, " the subtarsal sulcus," in which foreign bodies commonly lodge, may exist a short distance from the lid margin, and minute folds can be seen on this surface by the aid of a lens (Quain). At the puncta lacrimalia the membrane is directly continuous with the lining of the lacrimal passages, and through them with the nasal mucous membrane, an important continuity of structure in view of the spread of infection from one region to the other. Near the convex border of the tarsal plate the surface presents a network of grooves limiting minute islets, the zone of plateaux and grooves described by Virchow (I905); the same author states that the surface of the membrane over the plates is covered by papillae, but their presence is denied by others. Beyond the borders of the tarsal plates the palpebral conjunctiva of the surpraand infra-tarsal regions is looser, being thrown into horizontal folds, which are folds of movement since they only appear after birth and become effaced in occlusion of the lids; the membrane is here related on its deep surface to the involuntary palpebral muscles. In the lateral region in the upper lid there rests upon it the palpebral lobe of the lacrimal gland, the ducts of which pierce it.

(ii.) The conjunctival fornix (Latin, fornix, a vault or arch) is the circular cul-de-sac formed by the fold of reflection of the membrane from the eyelids on to the globe; its exact limits are somewhat indefinite, but in the different regions it is referred to as the superior, inferior, lateral, or medial fornix. The distances from the orbital margin, corneal limbus, and lid edges are given in Fig. 96, though great individual differences occur.

The superior conjunctival fornix corresponds nearly with the superior palpebral skin-fold, but may lie at a higher level; the centre of the inferior fornix is about $6 \mathrm{~mm}$. above the bend of the orbital margin; on the medial side the plica semilunaris and caruncle lie in the fornix between the palpebral and bulbar conjunctivae. The membrane in the region of the fornix is thicker and more loosely disposed than elsewhere, being thrown into folds when the eye is opened. 
and allowing the globe to move freely independent of the lids; its looseness also allows it to be easily raised by subjacent infiltrations. It is surrounded by connective tissue or " retinacula," formed by expansions of the fascial sheaths of the recti muscles of the eyeball and containing smooth muscle fibres (Charpy, I9r2), so that its folds are drawn back and its recess is deepened by the action of these muscles in turning the globe. If an instrument be thrust through the summit of the superior fornix it will lie in the plane

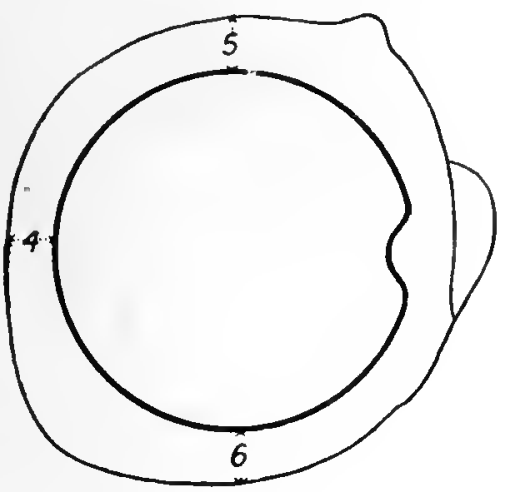

A

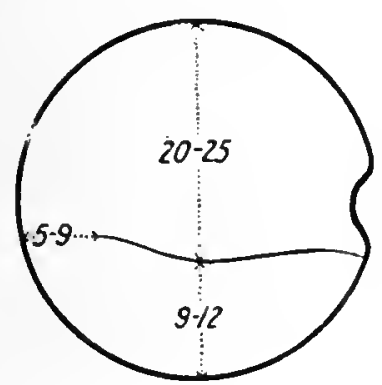

C

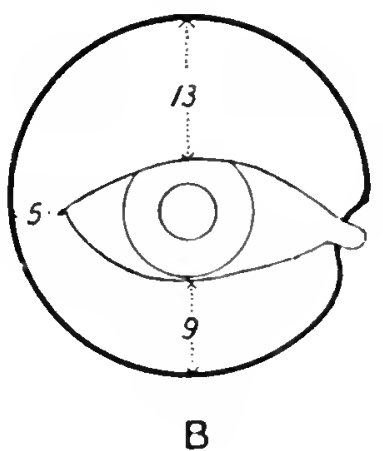

B

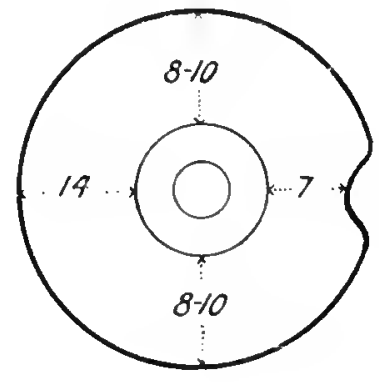

D

Figs. $6 \mathrm{I}$ or 67 . Through the inferior fornix the instrument would strike the inferior oblique muscle covered by a fascial expansion prolonged from the inferior

FIG. 96.-Diagrams to show the distance in millimetres of the Conjunctival Fornix :-

A. From the orbital margin (figures according to Gerlach).

B. From the palpebral margins with the eye open (figures as given by Baker).

C. From the palpebral fissure (eye closed) (after Henle, Dwight, Charpy, Richet).

D. From the corneal margin (as given by Testut and Merkel).

The conjunctiva is indicated in each case by the heavier outline.

rectus muscle. In the diagonal regions of the fornix, that is, in the intervals between the muscle sheath expansions, the connective tissue behind the membrane abuts on the orbital fat, and it is through these areas of less obstruction that, according to Charpy, artificial injections within the orbital cavity reach the fornix, and can then extend beneath the bulbar conjunctiva nearly up to the corneal 
margin; it is along these paths that the large subconjunctival ecchymoses due to haemorrhage into the orbit following a fractured skull travel (see also p. 298).

(iii.) The bulbar conjunctiva (tunica conjunctiva bulbi) is that part of the membrane which is reflected on to the anterior face of the globe, of which it covers approximately one-third; it consequently presents a scleral and a corneal portion. The conjunctiva covering the sclera is thinner and less vascular than in either the palpebral or fornical regions, so thin, indeed, that the white of the eye shows through it, and sub-conjunctival haemorrhages in this region retain their red colour, the oxygen in the air enabling the blood to maintain its arterial character (Treves). It is separated from the globe by the anterior part of the fascia bulbi (capsule of Tenon), with which it blends as it approaches the corneal margin, the junction of the two membranes taking place about $3 \mathrm{~mm}$. away, and being sometimes marked by a slight ridge, which is more apparent in certain ophthalmic catarrhs. In consequence of their union these two layers, bulbar conjunctiva and anterior part of the fascia bulbi, are raised together in exposing the scleral surface of the globe and the recti muscle tendons, and the capsule, filled with delicate episcleral tissue, is opened into (Fig. I42, p. 265, and Fig. 97, below). At the sclero-corneal junction the fascia bulbi ceases, but the conjunctiva, now reduced to its epithelial layer only, passes on to become directly continuous with the epithelium covering the face of the cornea, which thus forms the corneal portion of the bulbar conjunctiva. This direct continuity of the epithelium of the bulbar conjunctiva with that of the cornea explains the spread of disease from one part to the other.

(iv.) The plica semilunaris conjunctivae is a narrow fold of the bulbar conjunctiva, situated in the medial angle of the eye on the lateral side of and deep to the caruncle, together with which it intervenes between the bulbar and palpebral conjunctivae, and forms the floor of the lacus lacrimalis. It is vertically elongated and crescentic in form, with the concave and free border directed towards and projecting a little over the globe ; its lower horn reaches 
the middle of the inferior fornix, the upper is not quite so long ; its extent and connection with the bulbar conjunctiva is made manifest by turning the eye laterally. It first appears in the upper lid in embryos of $18 \mathrm{~mm}$. long (Popoff, I9I2), is relatively large in the foetus, and is well developed in infancy; usually it is about $2 \mathrm{~mm}$. broad, but in one Malay tribe it is found $5 \mathrm{~mm}$. broad (Wiedersheim). In structure it resembles the rest of the conjunctiva, but in its base or adherent border is embedded a fatty lobule, and some smooth muscle fibres are present, vestiges of the lateral palpebral muscle detached from the medial rectus; these fibres are striated in marine mammals (Groyer). In ruminants, anthropoids, and most mammals it contains a plate of hyaline cartilage, and a small nodule has been found in Negroes (in I2 out of I6 by Giacomini), in Japanese (25 per cent by Adachi), in Caucasians (3 out of 548 by Giacomini), and very rarely in Europeans (5 out of rog6 by Testut). The vestiges of glands which may be found in the plica semilunaris are described on p. I98.

The Structure of the conjunctiva:-Like other mucous membranes, it consists of two main layers, a surface epithelium, with a connective tissue basis or tunica propria; its special features are the scantiness of the mucous secretion and the presence of large serous lacrimal glands. The epithelium is composed of several layers of cells limited by a basement membrane; the cells are stratified squamous in character at the lid borders, like the epidermis with which they are continuous; in the palpebral region and fornix they are columnar or cylindrical in two or three layers, with islands of cuboid cells among them over the tarsal plates; in the bulbar region they gradually assume a squamous formation as the cornea is approached, and they then form the stratified pavement epithelium of its surface, some 5 cells deep. Clear oval mucous secretory or goblet cells are normally present (Leedham Green, I894), especially in the bulbar region and fornix, where they may be aggregated to resemble glandular crypts, but they are rarer in the palpebral conjunctiva and absent towards the lid margins. Pigment granules occur in the deeper cell layers of the bulbar 
conjunctiva, especially near the corneal margin in the triangular space of the bulbar surface exposed to light by the opening of the palpebral fissure; here they may even form pigment spots (Pergens, I898; Coppez, I905; Fischer, r905; Kusel, I907), as found in Negroes, Indians, Chinese,

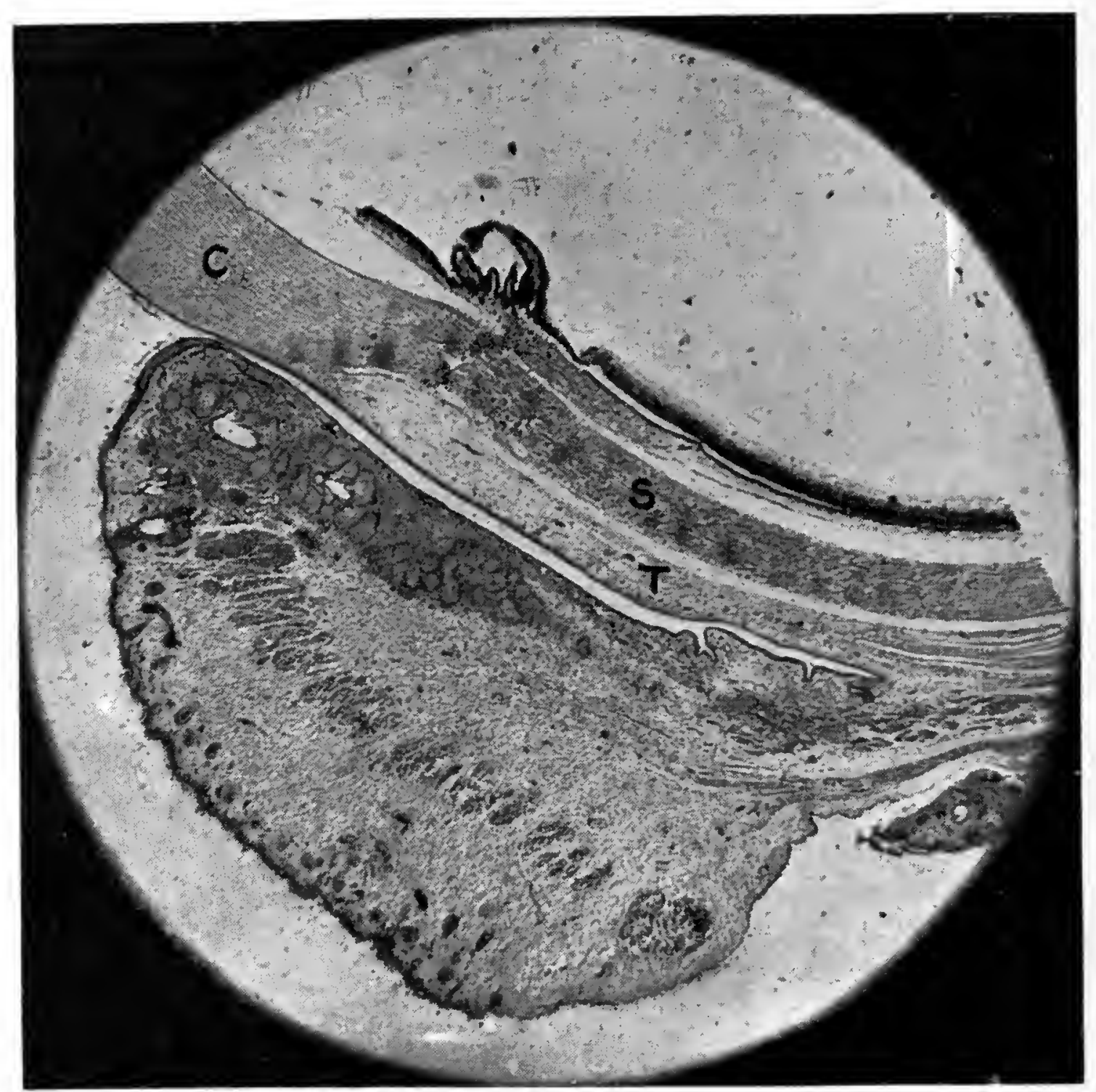

FIG. 97.- Section through the Lower Eyelid and part of Globe. In the inferior conjunctival fornix there are seen the folds or crypts described as "glands" by Henle (see Fig. too for enlarged view).

C. $=$ cornea $; \mathrm{S} .=$ sclera $; \mathrm{T} .=$ the anterior part of the fascia bulbi or capsule of Tenon.

and Japanese ; they are also present in the palpebral conjunctiva of trachomatous subjects (Steiner, Ig06). Hiwatari (I92I) finds pigment also in the corneal margin itself. The tunica propria corresponds to the dermis or chorion of all the integuments; it is reduced over the tarsal plates, well developed in the fornix, and absent over the cornea, and consists of a thin adenoid layer beneath the epithelium and a thick fibrous deeper layer with an abundance of elastic 
tissue. The adenoid layer is loose in texture, and is invaded throughout by white corpuscles, though less freely in the bulbar part; this lymphatic infiltration is especially abundant in the region between the convex border of the tarsal plates and the fornix, and principally towards the commissures of the lids, where it is concentrated to form lymph nodules (noduli lymphatici conjunctivales), termed " trachoma glands" by Henle. Papillae of the dermis occur in a circum-corneal zone of about $2 \mathrm{~mm}$. broad (Villard, I896; Nakagawa, I903; Virchow, I905; but denied by Hiwatari, I92I), and near the margins of the lids, but the appearance of papillae described elsewhere, as by Virchow in the tarsal region, may only be due to circumscribed islets of mucous membrane. The deeper fibrous layer of the dermis is not a true constituent of the mucous membrane, being really formed by sub-conjunctival connective tissue, reinforced in the palpebral region and fornix by fascial expansions of the sheaths of the levator and recti muscles, and in the bulbar region by the anterior part of the fascia bulbi; it does not exist in the tarsal region. Near the corneal margin the adenoid layer of the bulbar conjunctiva is absent, that membrane being represented by its epithelium only, supported by the fascia bulbi. The comparative histology of the conjunctiva is given by Zeitschmann (I904).

The glands of the conjunctiva are either lacrimal glands of various degrees of development or simple epithelial formations; the true glands comprise :

(i.) The lacrimal gland proper (glandula lacrimalis), the large mass in relation to the upper lateral region of the fornix, which will be described in accordance with custom as part of the lacrimal apparatus (p. 204).

(ii.) The accessory lacrimal glands (glandulae lacrimales accessoriae), which are smaller and are found in the fornix elsewhere; they are sometimes referred to as the glands of Krause, Wolfring, and Ciaccio.

The glands of Krause (I854) are microscopic acinotubular glands or single acini scattered along the conjunctival fornix, especially laterally, where they are more numerous in the upper eyelid ( 8 to 20 , or even 42 according 


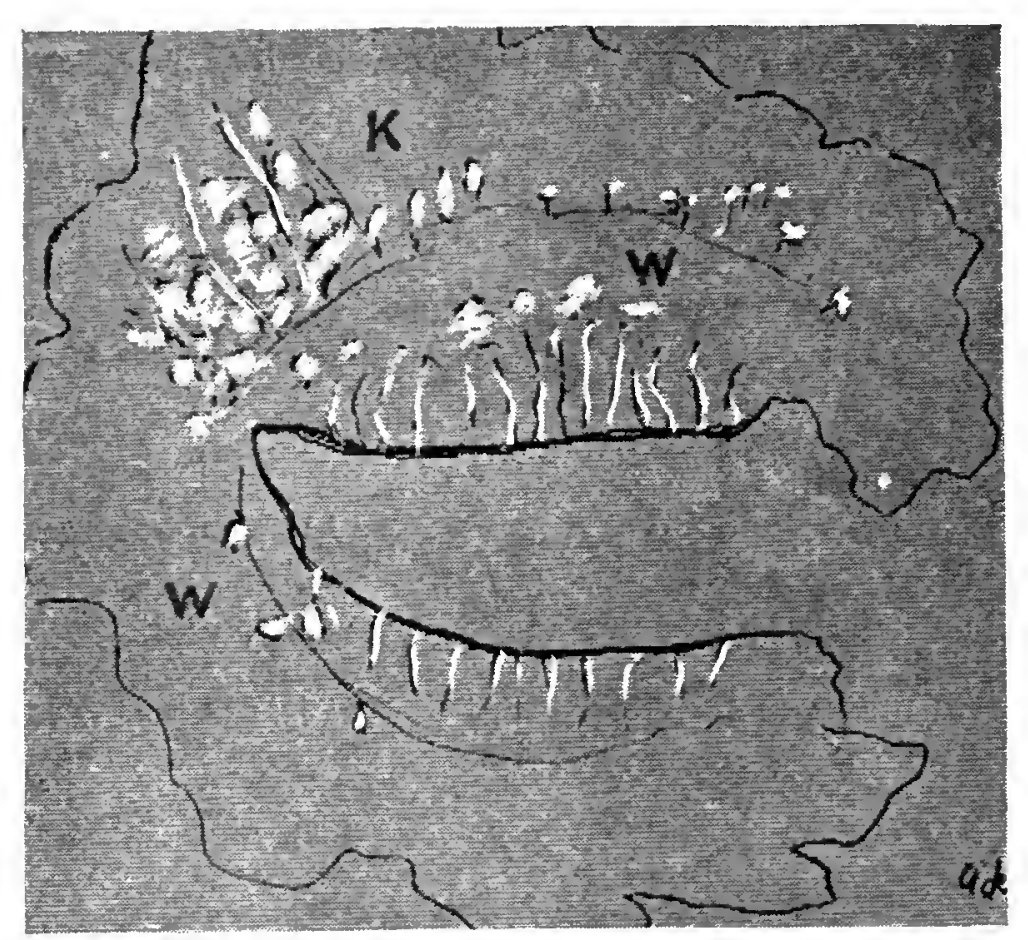

FIG, 98. - The Accessory Lacrimal Glands of Krause (K.) and of Wolfring (W.) as figured by Terson (I903).

plates close to, and even between, the upper extremities of the tarsal glands; they are larger, but fewer in number, than those of Krause, being from 2 to 5 in the upper lid, with rarely a single one in the lower (Terson). All these accessory lacrimal glands are structurally identical with the lacrimal gland proper, and differ only in possessing large sinuous excretory canals. Hocevar (Igoo) described "sterile lacrimal glands" without ducts in the same region.

The "glands" described by Henle (I853) in the palpebral conjunctiva of both lids between the edges of the tarsal plates and the fornix to Parsons) than in the lower ( 2 to 8 ). These are doubtless what is meant by the glandulae mucosae Krausei of the B.N.A. terminology, though they are not mucous glands. Those described by Wolfring (I872) and Ciaccio (I874) are similar acino-tubular glands situated along the middle of the convex border of the tarsal

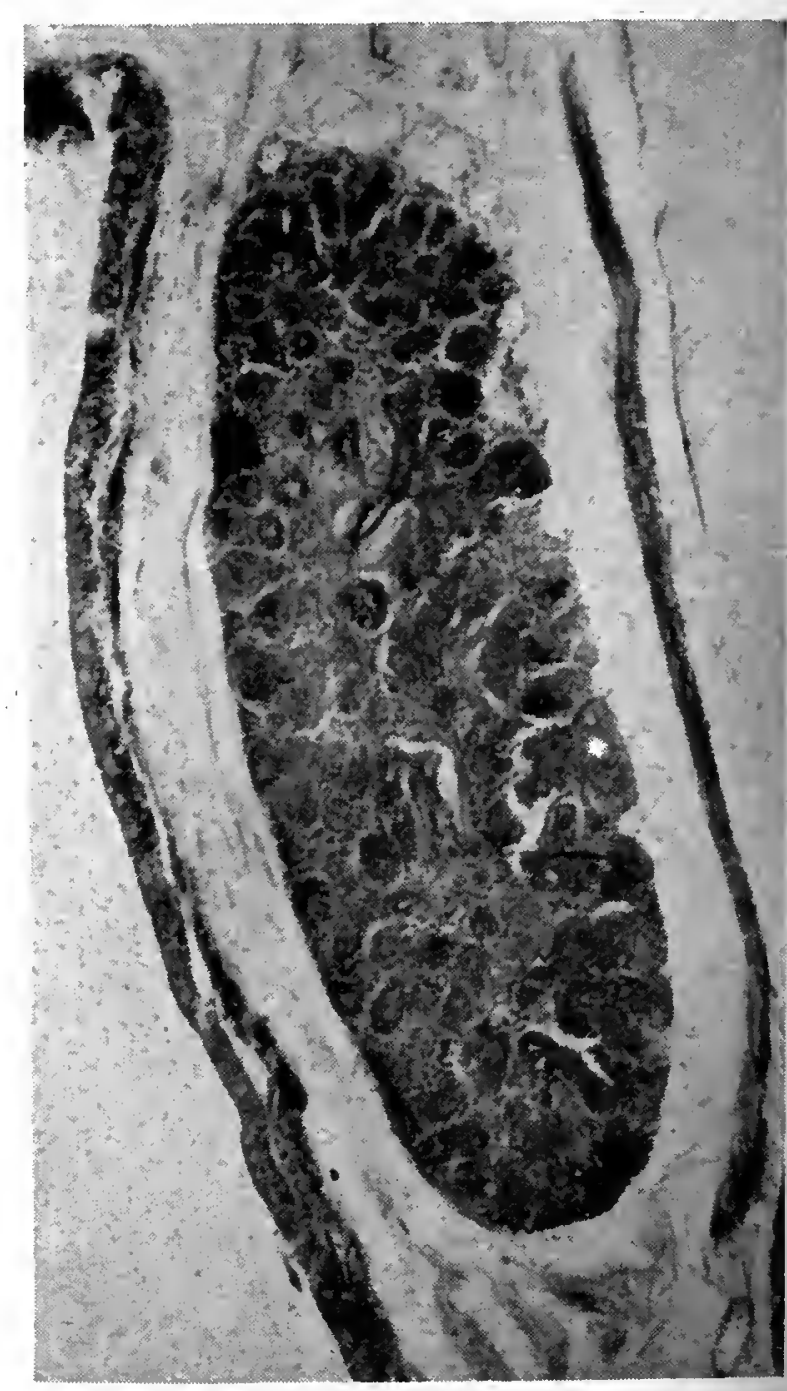

Fig. 99.- An Accessory Lacrimal Gland (gland of Krause), from the conjunctival fornix of the. upper eyelid. $\times 60$. 
appear to be simple epithelial invaginations; Zaluskowski (I887), Ciaccio, and Reich (I875) consider them "mucous glands " formed of goblet cells, but many authors regard them not as true glands but as folds of the conjunctiva seen in transverse section (Stieda, Waldeyer, Krause, Sättler, Terson), and they are well named " conjunctival crypts" by Dubreuil (Igo8).

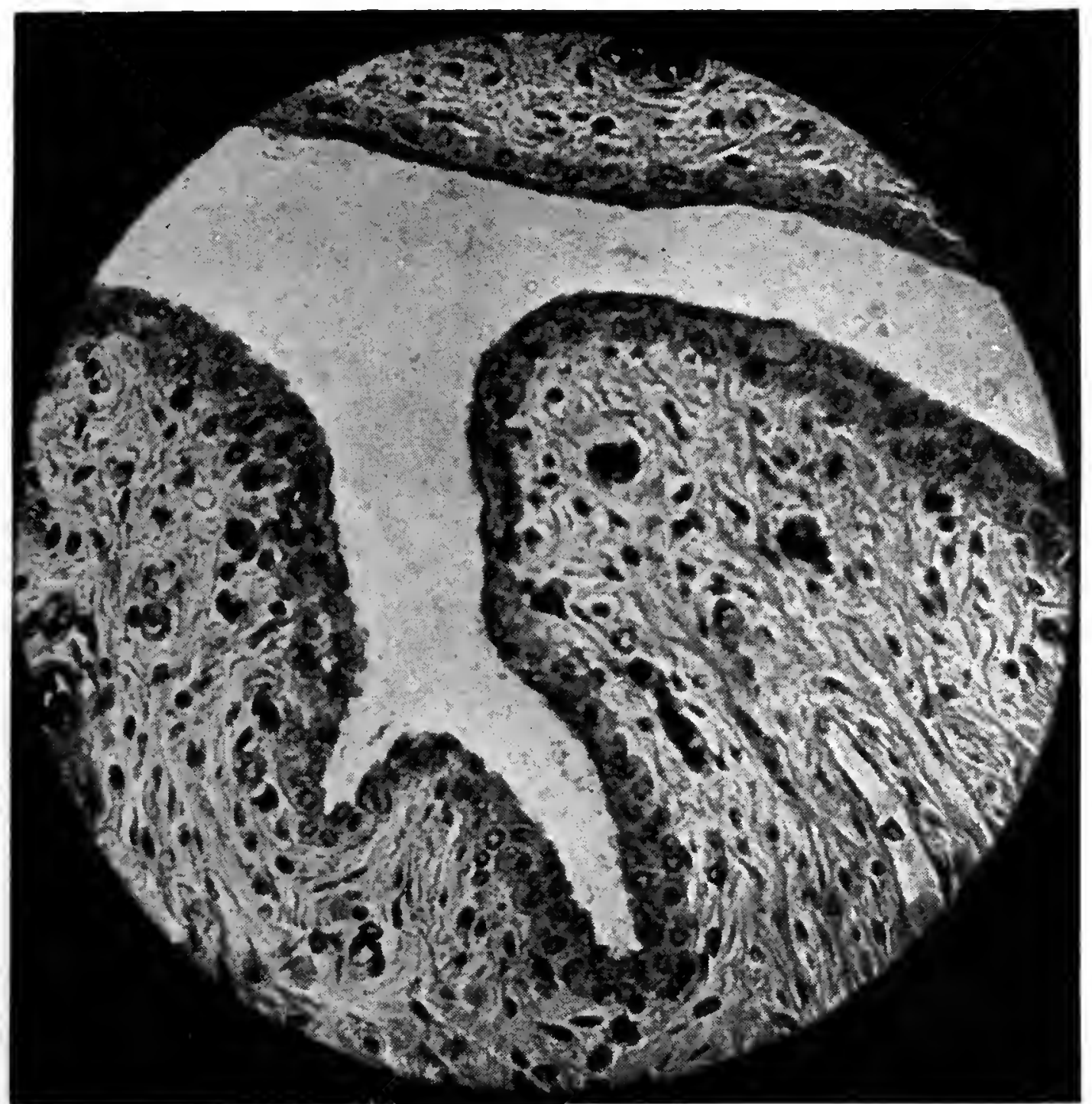

Fig. roo. - Conjunctival Crypt, sometimes described as a "gland of Henle, or "mucous gland of Reich." Magnified from Fig. 97.

Similarly the "glands of Manz" (I859) are utricular formations in the circum-corneal region of the bulbar conjunctiva, described by him in the pig, and since found in other animals; they are not hollow diverticula, but resemble crypts filled with epithelial cells (" epithelial nests ") in the dermis; they are said to exist in man by Stromeyer, Henle, and Ciaccio, and more recently by Bartels (I908), and they have been thought to be con- 
nected with trachoma, but their presence and function is uncertain.

The position of these various glands in the conjunctiva is shown in Fig. 95, as given by Dubreuil (I907-8), who has made a special study of the lacrimal glands in man and animals.

(iv.) In addition there have been described in the plica semilunaris of the human foetus, and as persisting in extremely rare cases in the adult, vestiges of glands found normally in lower animals. The plica semilunaris corresponds to the third eyelid of lower vertebrates and the nictitating membrane of birds and reptiles, and in all cases where the membrane is well developed, as in birds, ruminants

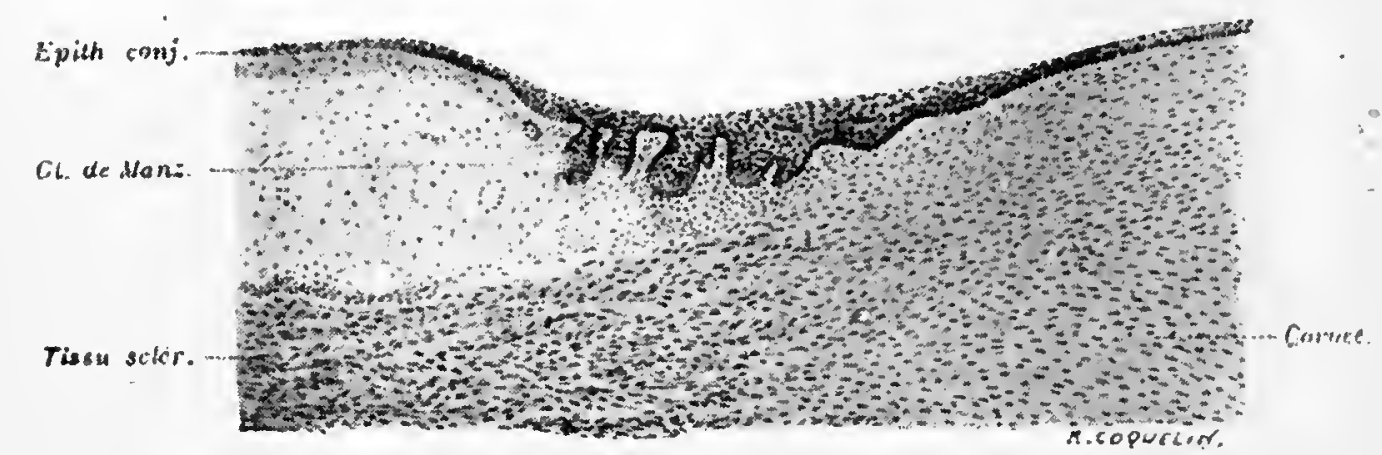

Fig. roi, - Epithelial Nests at the Sclero-Corneal Junction, sometines described as the "glands of Manz," as figured by Druault (I9r2), from a seven months' foetus. $\times$ about 55 .

large and small, pigs, carnivores, and rabbits (Law, I905), there is found a special acino-tubular gland which secretes a fatty substance ; this gland was first discovered by Harder in 1694 , and has recently been investigated by Löwenthal (I892); a rudiment of this gland was found in a case of malformation in man (Fleischer, I907), and also in a Bushman (Giacomini), and both Ask and Contino consider that a glandular organ which appears in the plica semilunaris of the human embryo towards the eleventh week but usually disappears about the eighth month is a transitory form of Harder's gland, and that in rare cases it may persist in the adult.

Finally, the so-called "infra-orbital gland" of lower animals such as the sheep and the rabbit, which is an acinotubular gland of the lacrimal type but phylogenetically 
more ancient, is said to be represented in the infero-lateral fornix of the human foetus of I7 cms. (Löwenthal, I892, I894, I9I0). Probably this "infra-orbital gland" is only one of the outlying accessory lacrimal glands of Krause, as, indeed, Popoff (I9I2) considers any rudiments of glands occurring in the human plica to be, and in regard to their various positions the diagram shown in Fig. IO2 of the phylogenetic migration of the lacrimal gland proper, as given by Wiedersheim, is of interest (see also p. 219).

The vessels : The arterial supply for the greater portion of the conjunctiva is derived from the two tarsal arcades formed by the palpebral branches of the nasal and lacrimal arteries in each eyelid; the marginal arcade and the posterior tarsal plexus afford a rich supply to the conjunctiva lining the tarsal plates, hence the red colour in this region; the peripheral arcade by its perforating "posterior conjunctival" branches sup-

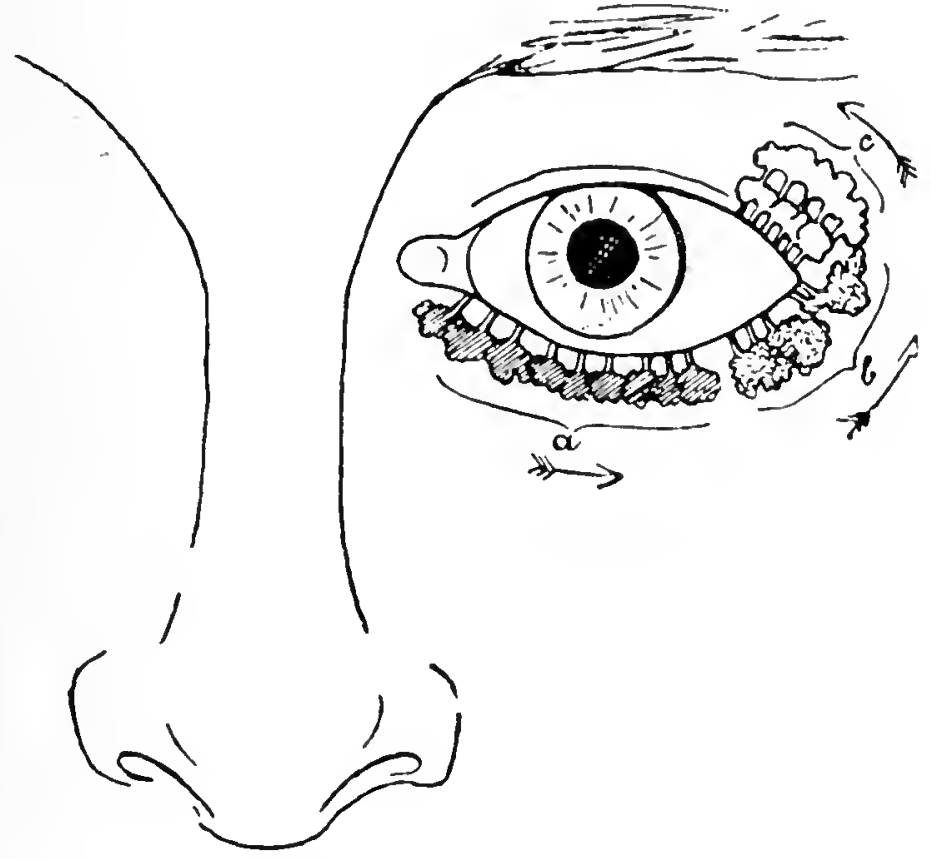

FIG. I02. - Diagram to illustrate the shifting of the Lacrimal Gland in the course of Phylogeny. (From Wiedershein, r895.)

$a_{.}=$position in amphibians; $b_{0}=$ position in reptiles and birds; $c_{.}=$the normal position in man.

plies both the fornix and the bulbar portion, and consequently is the more important; its branches become finer and therefore the membrane paler towards and over the globe, where they are usually only visible in hyperaemic conditions. The details of the vascular supply in the region of the sclero-corneal junction are of considerable clinical interest. About $3 \mathrm{~mm}$. from the edge of the cornea the posterior conjunctival branches anastomose with " anterior conjunctival" branches derived from the anterior ciliary arteries, which come from the ophthalmic artery and accompany the recti muscles in their course from the 
back of the orbit. The two sets of vessels are in close relation, but the posterior conjunctival are more superficial and more movable than the more deeply placed immovable anterior conjunctival branches. The latter, after giving off their anastomotic twigs, form a "peri-corneal plexus" about $4 \mathrm{~mm}$. broad, which is disposed in two planes, a superficial or conjunctival and a deep or episcleral, and sends loops of minute vessels into the edge of the cornea.

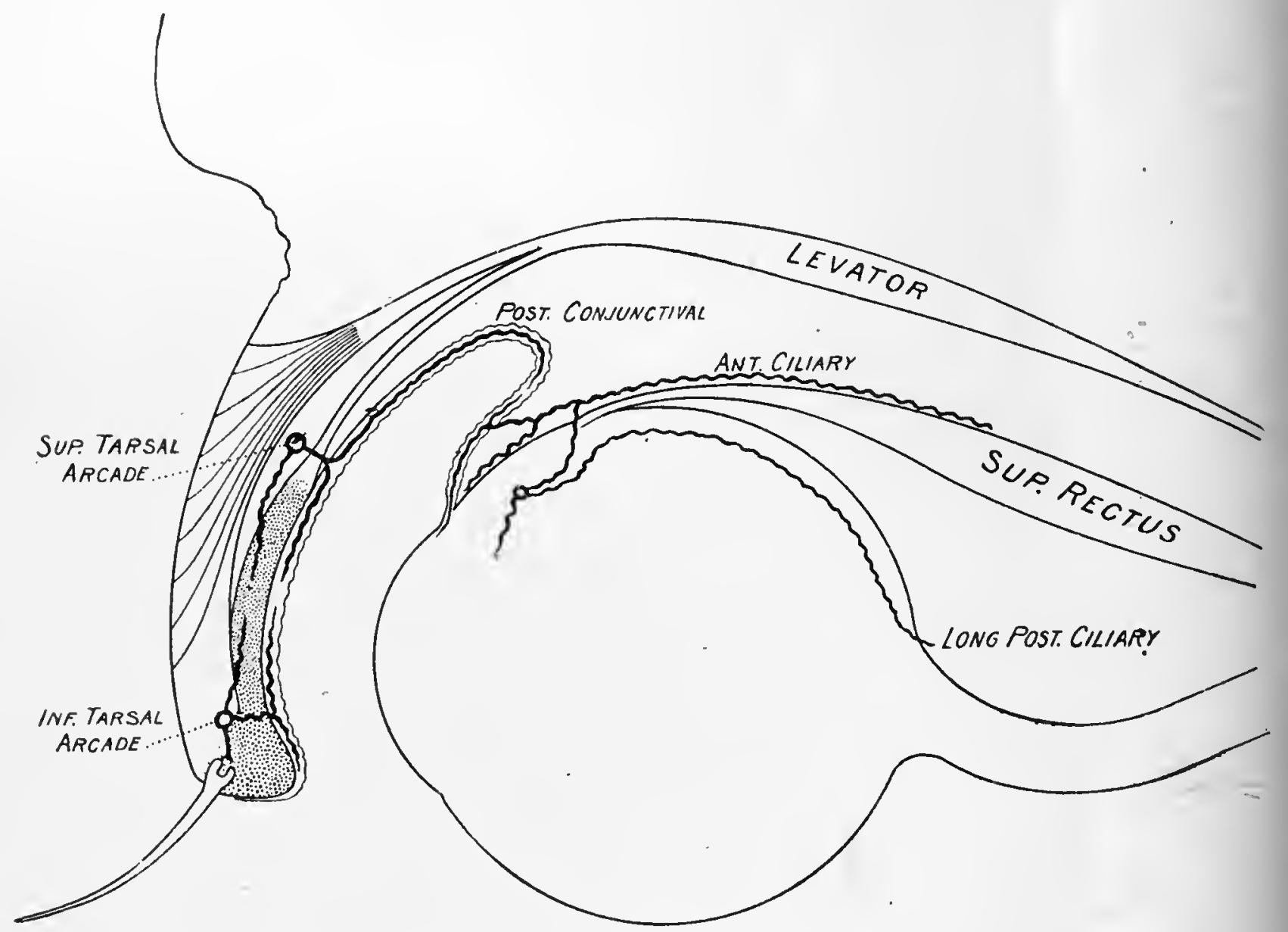

Fig. ro3.-Diagram to show the deep Arteries of the Upper Eyelid and Conjunctiva and their relation to those of the forepart of the Eyeball.

It is further to be noted that the anterior ciliary arteries, after giving off their conjunctival branches, turn abruptly to pierce the sclera just in front of the muscle insertions (the point of flexion being marked by the presence of pigment granules) and enter the base of the iris to form the circulus arteriosus major iridis (indicated by the unmarked circle in Fig. I03; see also Fig. I59, p. 304). The above arrangement of the vessels explains the significance of inflammation in this region: 
(i.) In inflammation of the conjunctiva the posterior conjunctival vessels, normally scarcely visible, appear as a close network over the bulb, fading away towards the corneal margin; they move freely with the conjunctiva and do not disappear under pressure.

(ii.) In superficial inflammation of the cornea the corresponding plane of the peri-corneal plexus formed by the anterior conjunctival vessels is engorged, and spreads in a freely branching manner into the corneal margin.

(iii.) In affections of the iris and deeper parts of the cornea the episcleral plane of the peri-corneal plexus, normally invisible, appears as a pink band of fine straight vessels round the cornea; the vessels do not move with the conjunctiva and disappear on pressure.

The veins of the conjunctiva generally accompany the arteries, one or two to each. The greater number drain into the palpebral vessels through the retro-tarsal venous plexus; near the corneal margin, where they form an episcleral network apparent in hyperaemia, the venules accompany the anterior conjunctival arteries, draining backwards by the veins of the recti muscles into the ophthalmic vein and cavernous sinus.

The lymphatic vessels form two communicating networks in the dermis of the conjunctiva; a smaller, superficial, and a larger, more deeply placed in the fibrous layer (Leber, I876). Most (r905) found the lymphatic vessels of the lower eyelid easier to inject than those of the upper; they are minute in the tarsal region, and at both margins of the plates they anastomose with those of the skin of the lids. Near the corneal margin an extremely minute plexus of vessels was stated by Recklinghausen to communicate directly with the lymph spaces of the cornea, but Koeppe (I9I8), who has made clinical studies of the conjunctival vessels with the corneal microscope, denies this; he finds, however, the blood-vessels of the bulbar conjunctiva and sclera near the limbus to be surrounded by lymph spaces. All the vessels pass into the deeper layer of the conjunctiva and drain towards the palpebral commissures, where they join those of the eyelids, as described above on p. I70. 
The nerve supply of the conjunctiva is provided medially by the infra-trochlear branch of the naso-ciliary nerve, and laterally by the lacrimal nerve, which supplies a larger area of the conjunctiva than of the skin of the lids; the epithelium covering the cornea and a narrow zone of the bulbar conjunctiva round it are supplied by the ciliary nerves, which have followed an intra-ocular path before piercing the sclera to gain this region (p. 353). The details of the distribution to the cornea are given by Boucheron (I890). The nerves terminate in the conjunctiva either by free ends or in tactile corpuscles of Krause. In the former case, after losing their nerve sheaths, they form a " sub-epithelial plexus" (Dogiel, I895), especially well marked at the free margins of the lids and near the convex borders of the tarsal plates (Bach, I895), and containing formations resembling ganglion cells. The corpuscles of W. Krause (I867) (which occur in the skin and mucous membrane elsewhere) are round or oval bodies of a granular substance, with a nucleated membrane of connective tissue; one or more nerve fibres approach the body, lose their sheaths, and end as delicate fibrils with enlarged heads. They lie close beneath the epidermis, especially in the supero-lateral region of the conjunctival sac supplied by the lacrimal nerve. They are also found in the corneal limbus on the ends of the ciliary nerves (Dogiel), and at the margins of the eyelids (Piersol) ; they have again been investigated by Ciaccio (I874) and Poncet (1875), and the latter points out that the presence of the tactile corpuscles in the lateral part of the conjunctiva of the upper lid can be realised by touching the skin over it with a blunt point such as that of a pencil; in this area the sensation is more poignant than elsewhere, and it feels as though sand were beneath the lid. Other types of sensory nerve terminations, such as tufts and ribbons, have been found by Crevatin (rgo3).

The development of the conjunctiva has been referred to in connection with that of the eyelids (p. I84); the ectoderm lining the primitive palpebral folds begins to differentiate into a mucous membrane after the eyelids have 
met and fused at about the third month of foetal life. All the glands of the conjunctiva, including the lacrimal, are formed by invaginations of the ectoderm; the mucous cells appear after the seventh month; the glands of Henle first appear as simple depressions of the surface, and Ask found them in the bulbar as well as the palpebral regions in an embryo of $39 \mathrm{cms}$. Dubreuil saw glands of Manz clearly defined in a seven months' old foetus. The plica semilunaris has been seen in an embryo of ten weeks by Contino, earlier than the caruncle and quite independent of it. The development of the lacrimal gland follows on p. 217 .

Abnormalities of the conjunctiva.-The so-called "epitarsus " or " double tarsus" is a congenital malformation in the upper lid formed by a reduplication of the lining conjunctival membrane. First described by Schapringer (I905), numerous instances of its occurrence have since been recorded by Radinski (" double tarsus "), Mathewson (" accessory eyelid"), Robertson (" third eyelid "), Krankow, Adamueck, Polack, Contela; it most commonly takes the form of a triangular fold arising by its base from the superior fornix and attached at its apex to the palpebral margin, and is regarded either as a developmental malformation or as the result of an amniotic adhesion or as a cicatricial result of an ophthalmia neonatorum (Fuchs).

Congenital dermoids (see p. I87), naevi and fatty growths may be found.

On the bulbar conjunctiva, along the line between the medial, and less often the lateral, side of the cornea and the corresponding angle of the lids, there may be formed a horizontally elongated yellow mass known as a " pinguecula " (Latin, pinguis, fat); such bodies occur most commonly in adults subject to conditions which irritate the conjunctiva, the site being in that triangular part of the sclera exposed by the opening of the palpebral fissure; they are pigmented in tropical races. The mass, accoreling to Parsons, is not of a fatty nature, but is composed of vascular conjunctiva thickened by fibrous and yellow elastic tissue; Fuchs states that besides an increase in the 
number and size of elastic fibres there is an additional formation of numerous concretions of a yellowish hyaline substance. It may develop into a " pterygium," which is a larger wing-like growth extending over the cornea towards the pupil. Pingueculae are present on both sides of the cornea in the subject photographed in Fig. 55, p. I24, where they appear as dark areas in the position described above.

\section{THE LACRIMAL APPARATUS}

The lacrimal apparatus consists of a gland, which secretes the tears into the conjunctival sac, and a system of canals, the lacrimal passages, through which they are drained away into the nasal cavity.

I. The lacrimal gland (glandula lacrimalis) is, as previously noted, the chief mass of conjunctival glands, there being also present "accessory lacrimal glands" which, though microscopic in size, are of similar nature. It forms a small flattened and lobulated mass, yellowish pink in colour and a little darker than the orbital fat, from which it is not easily distinguishable at first sight. There are to be described its situation and relations, form, attachments, structure, vessels and nerves, development and variations.

It lies deep beneath the lateral part of the upper eyelid, covered in front by the septum orbitale, orbicularis oculi muscle, and skin, and is placed just within the orbital margin, wedged in between the lateral angular process of the frontal bone and the globe (Fig. 55, p. I24); the bone is slightly hollowed out for its reception, so forming the fossa glandulae lacrimalis, in the lower and fore part of which it mainly lies; posteriorly the gland is adherent to a mass of orbital fat which fills the hinder part of the fossa, termed the loge accessoive by Rochon Duvigneaud (I903), and sometimes a slight undulation can be felt across the surface of the bone demarking the regions occupied by the two masses. The gland extends downwards as low as the zygomatico-frontal suture, which, as illustrated in Fig. Io, 
p. 25, may be raised in a low ridge limiting the fossa below, and it rests upon, and is moulded by, the eyeball.

The gland is crossed in front and deeply grooved by the lateral horn of the aponeurosis of the levator palpebrae superioris muscle, round which it is folded; so deep is the

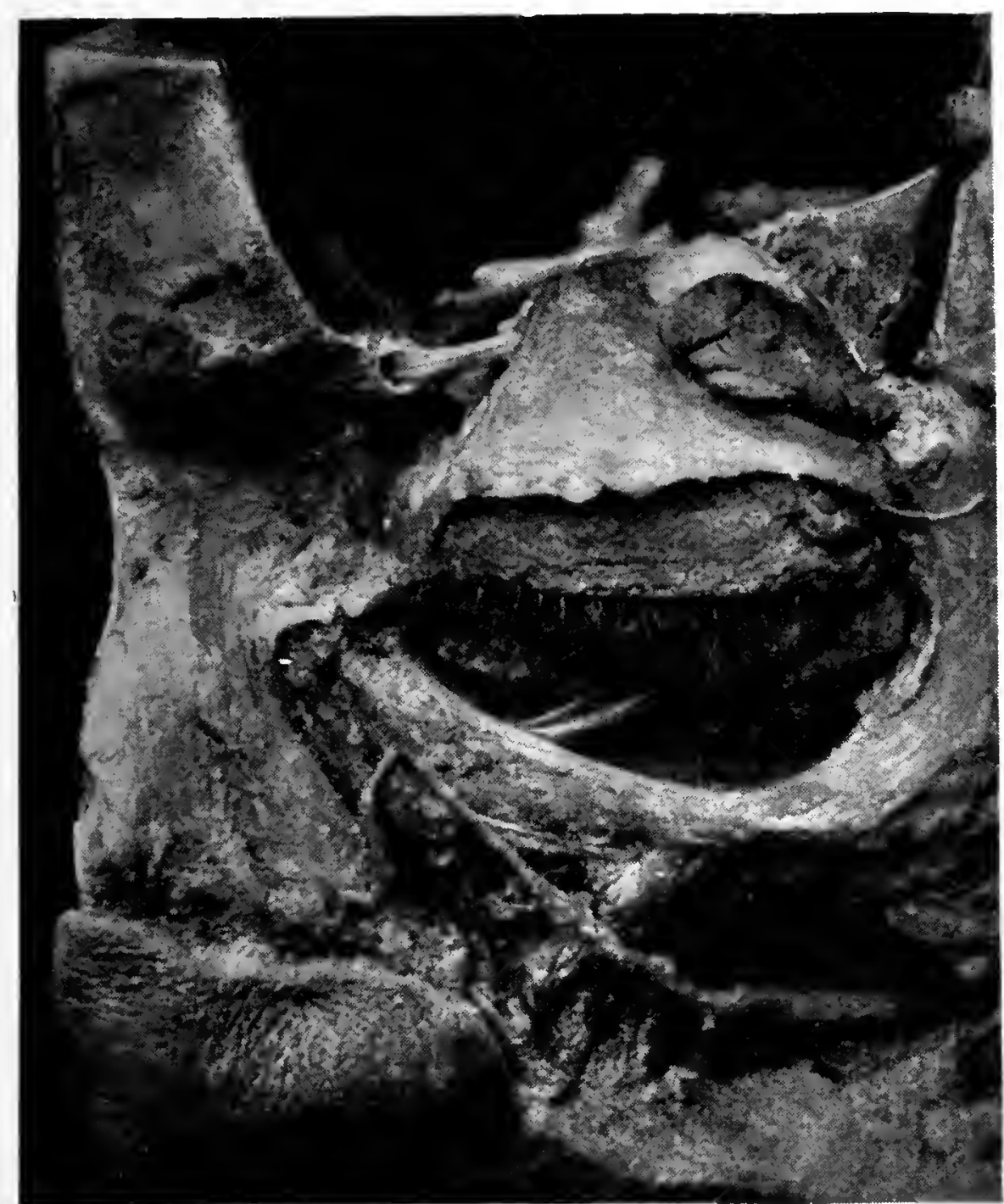

Fig. 104.-The relations of the Lacrimal Gland. Dissection of the Left Orbit from above and also in front (the same preparation as Fig. 63 , viewed from in front) to show the aponeurosis of the levator palpubrae superioris muscie and the lacrimal gland. The pulley of the superior oblique and its tendon are also seen. Natural size.

groove that the gland is almost divided into two parts or lobes, an upper and a lower (Fig. Io4). They are distinguished as the glandula lacrimalis superior or orbital lobe, and the glandula lacrimalis inferior or secondary or palpebral lobe, but the writer has met with no instance of entire separation of the two parts of the gland, and the term "lobe" is preferable to gland, since they are not even as 
completely separated as are the lobes of the lungs for cxample; they differ in size, position, and relations.

The superior or orbital lobe (glandula lacrimalis superior) is the larger, and its lobules, owing to its confined position. between the globe and the bone, are more closely pressed together; it forms an oval mass rather like a haricot bean, about $20 \mathrm{~mm}$. long by I2 $\mathrm{mm}$. wide and $5 \mathrm{~mm}$ thick, but the

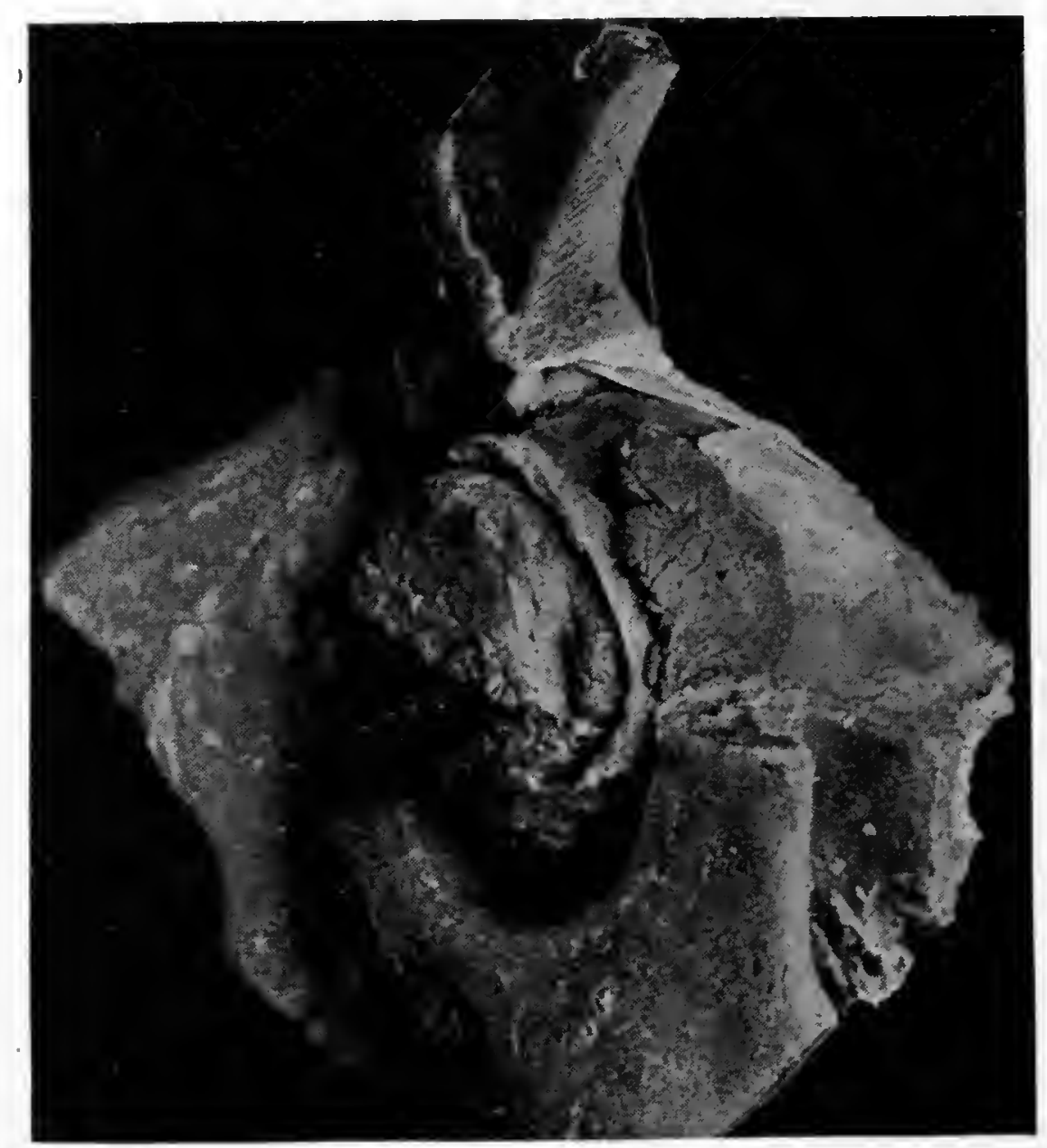

Fig. I05.-Dissection of Left Orbit viewed from the side to show the lacrimal gland subdivided by the aponeurosis of the levator, the moulding of its upper lobe by the frontal bone (removed), and its close connection behind with the orbital fat. Natural size.

size and shape, like the weight, which is given as on an average $0.78 \mathrm{gr}$. by Göz (I908), are very variable (Fig. Io6). Its orbital or lateral surface is smooth and convex, in harmony with the contour of the bony fossa in which it is closely lodged and from which it is separated only by the periorbita; the forepart of the ocular or medial surface lies on the aponeurosis of the levator muscle, but the hinder part, which is confluent with the palpebral lobe, lies on the fascial expansion which passes from the sheath of the 
superior rectus to that of the lateral rectus muscle, whereby it is separated from the fascia bulbi covering the eyeball. The long axis of this upper lobe is curved, and reaches from the lateral margin of the muscular part of the levator above to the zygomatico-frontal suture below; its upper extremity or pole is usually free, but the deep surface is often connected to the fascial sheath of the levator; the lower pole is fixed by its stroma to the periorbita in the angle between the lateral expansion (check ligament) of the sheath of the lateral rectus muscle and the zygomatico-frontal suture, a fixation often strengthened by the entrance at this point of the anastomotic branch from the zygomatic (temporo-malar) nerve to the lacrimal (Fig. 7I). The anterior edge of the lobe is thin and free, and runs parallel to the orbital margin, just behind which it normally lies, and is separated by a slender roll of fat from the septum orbitale; the posterior edge, thick and ill-defined, extends to a variable distance backwards into the orbit, and is not free but is connected with the mass of orbital fat lying between the superior and lateral recti muscles, as is seen in Fig. 105, and at about its middle there enter the lacrimal nerve and blood-vessels (Fig. 63, p. I35, and Fig. 7I, p. I49).

The inferior, palpebral, or accessory lobe (glandula lacrimalis inferior) is from one-half to one-third the size of the superior lobe; it forms a thin plate with diffuse limits, but is usually about $15 \mathrm{~mm}$. wide, and its lobules are more loosely connected; they number from I5 to 40 (Sappey), and the writer has never found only one or two present, as Fuchs states to be the normal number. Behind, it is confluent with the superior lobe, and presents the same relations; in front, it extends well beyond the orbital margin, beneath the aponeurosis of the levator and on to the lateral part of the upper eyelid, where it lies partly on the superior palpebral muscle, but chiefly on the palpebral conjunctiva, to which it is firmly adherent and through which its ducts open; they can be seen by raising its fore edge, which extends a few millimetres over the convex border of the tarsal plate. It is not uncommon to find a few lobules of this part of the gland extending downwards 
between the lateral raphe of the orbicularis muscle and the lateral palpebral ligament.

The gland (presumably its orbital lobe) is described as being enclosed in a delicate but definite capsule (Picou, I9I2), which separates it almost completely from the surrounding parts and most definitely behind from the orbital fat, but the writer has never found anything comparable to such a structure : the lobules of the gland appear bound together by a connective tissue stroma, which is

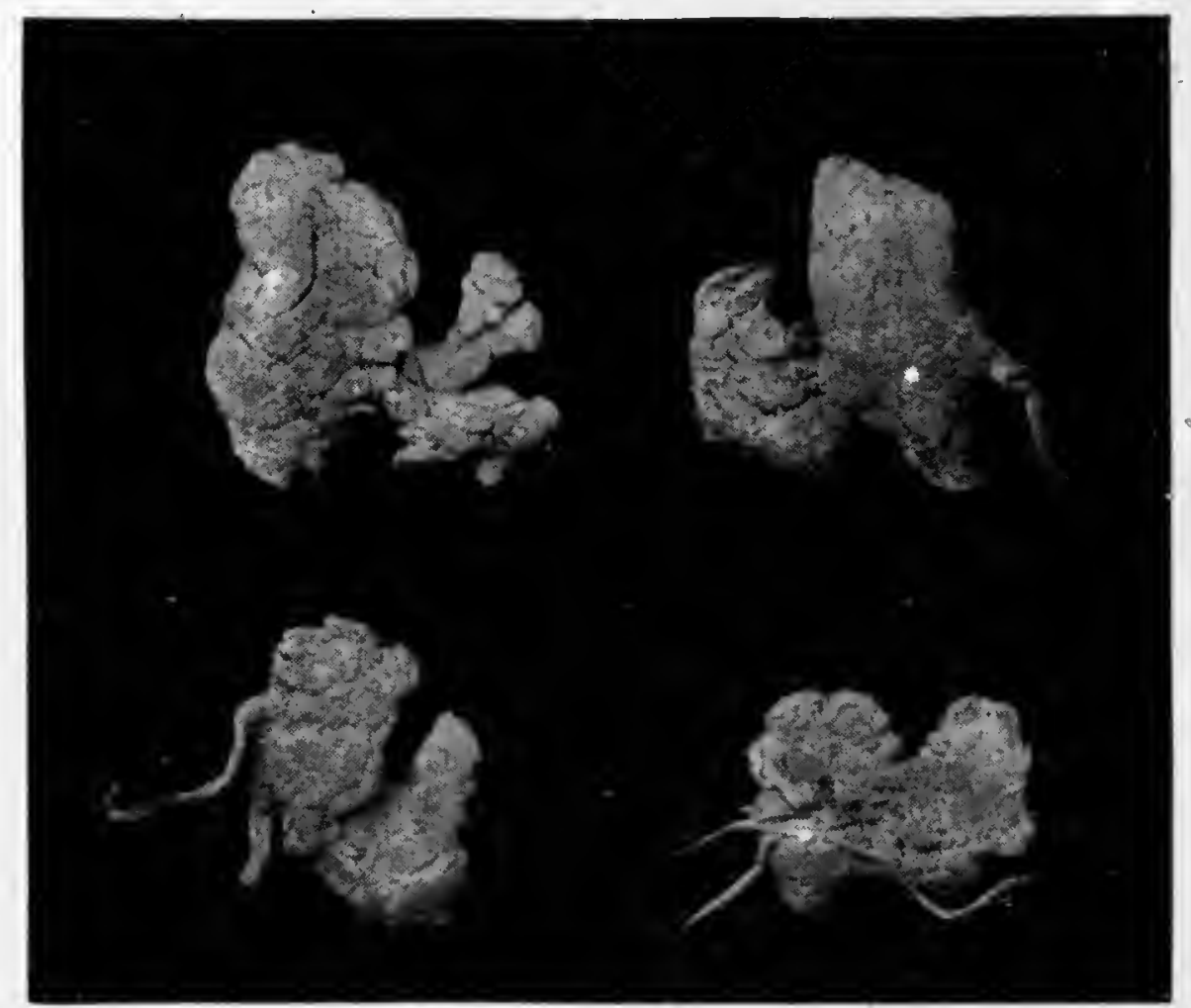

FIG. Io6. - Four Lacrimal Glands dissected out entirely ; the two left-hand ones are from right orbits and are viewed from the lateral aspect; the upper right-hand gland is from a left orbit; the lower right-hand gland is viewed from its ocular or medial aspect. All show clearly the subdivision into superior and inferior lobes. Natural size.

slightly more abundant on the surface and is certainly continuous behind with the tissue between the adipose lobules of the orbital fatty mass, to separate the gland from which a careful dissection is needed.

Similarly, various ligaments have been described, none of which, however, present any special degree of development, and to which the application of the term "ligament" is pretentious and misleading. These are as follows:

(i.). A suspensory " ligament of Soemmering," fixing the upper surface of the gland to the orbital wall. This consists 
merely of loose, delicate strands of connective tissue passing from the stroma of the gland to the periorbita, and they can easily be destroyed with a blunt instrument.

(ii.) An inferior "ligament of Schwalbe;" fixing the lower part of the gland in the angle between the expansion of the sheath of the superior rectus muscle and the lateral orbital wall at the zygomatico-frontal suture. This is the most definite attachment of the gland.

(iii.) A "posterior ligament" passing to the periorbita at the point of entrance of the lacrimal nerve into the gland (Holmes).

(iv.) An " internal ligament" accompanying the excretory ducts of the gland. This, like the last, is neither a true ligament nor a means of fixation of the gland.

In dissecting out the whole gland, as in the preparations made for Fig. Io6, the first structure to be severed is the lateral horn of the aponeurosis of the levator palpebrae superioris, which is often fixed to the gland where the latter is folded round its hinder edge ; then the upper part of the gland requires freeing from the fascial expansion of the sheath of the same muscle, and further back from the orbital fat, where its nerve and blood-vessels must be cut; the gland can then be pulled forwards and freed at its lower angle, the most fixed point, from the so-called inferior ligament; the deep surface of the palpebral lobe of the gland is strongly adherent to the conjunctiva, upon which it rests, and is separated only with difficulty from it.

The gland is kept in position between the globe and frontal bone not by any such ligaments, but by being folded round the lateral horn of the aponeurosis of the levator which lies in front of it, and by the support afforded to it by the mass of orbital fat behind; as just noted, it is most firmly fixed at its lower pole, but elsewhere a certain amount of movement is possible, and probably does take place when the aponeurosis is swung back by contraction of the levator in raising the lid. In the specimen referred to above, where muscle offshoots passed from the levator to the gland (Fig. 69), traction on the muscle pulled the gland backwards even though the preparation had been hardened in spirit. The gland regains its more forward position upon 
relaxation of the muscle owing to the resiliency of the orbital fat against which it has been withdrawn. The movement is slight, but may be a means of stimulating or aiding the expulsion of the tear fluid.

Structure and Ducts. - The lacrimal gland consists of a mass of lobules, each about the size of a pin's head; they are more loosely aggregated in the palpebral portion, but present the same detailed structure in each lobe. It is a tubular-racemose gland-that is, each lobule consists

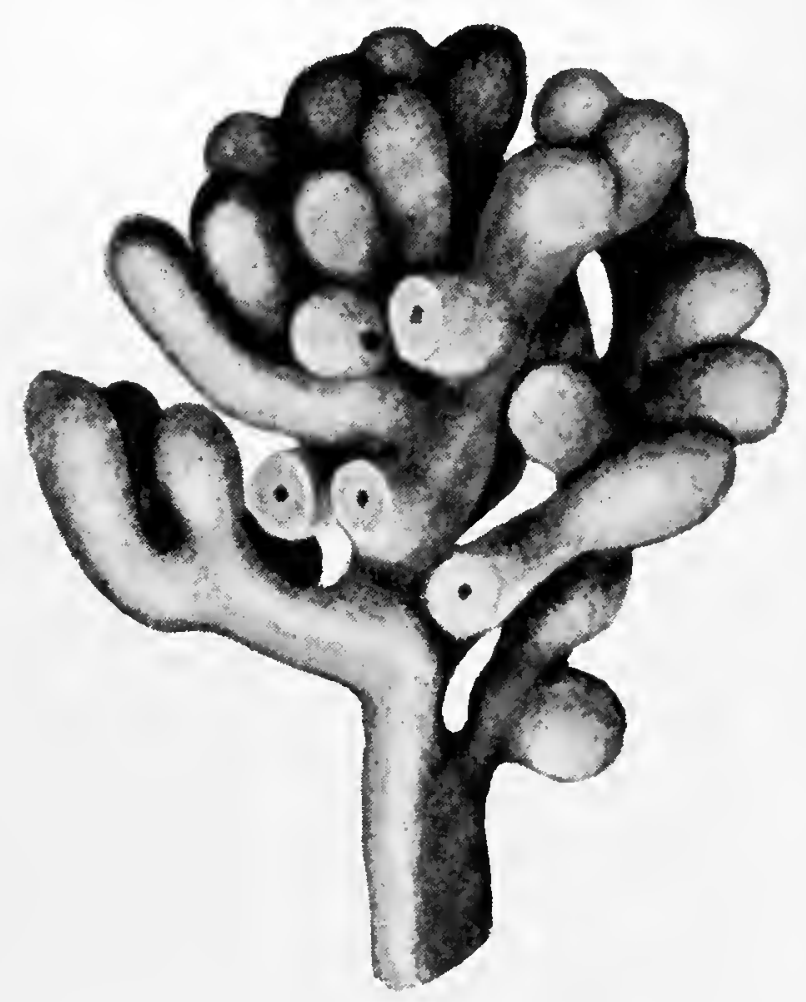

FIG, 107.-Reconstruction of a Lobule of the human Lacrimal Gland (Maziarski, I902). $\times$ I70. of a small mass of ramifying tubes, and each tube branches further into tubules or acini of secreting cells, as illustrated in Fig. I07.

The acinus has a basement membrane supporting two kinds of cells arranged round a central canal (Fig. Io9) ; one kind of cell is flat basal and contractile (myoepithelial), the other is cylindrical and secretory, and the protoplasm appears under three different aspects according to the stage of secretory activity; thus the cell in charging contains refringent granules and is vacuolated, and in resting shows obscure details (Noll, Igor; Fleischer, I904; Dubreuil, I908). Fat granules have also been observed in the secretory cells of normal glands (Axenfeld, I898).

By contraction of, the protoplasmic network of the cell, the secretion is expressed into the central canal of the acinus, and thence passes into the excretory canals. These resemble the acini in structure, but soon become lined by two rows of epithelial cells, the deeper of which are contractile. At first they are intra-lobular in position (Fig. I08), but join with others to form larger extra-lobular or collecting canals, which eventually become the ducts of the gland (ductuli 
cxcretorii glandulae lacrimalis). These are constructed by an adventitia outside a basement membrane which encloses an epithelium of two rows of flat cells lined by cylindrical cells. The chief characteristic of all the ducts is their relatively large lumen; they are white, delicate-walled vessels, not easily distinguishable at sight from the blood-vessels or nerves. In number they seldom exceed a total of I2, of which 2 (Gosselin, I843) or from 3 to 5 (Sappey, I867) drain the orbital lobe, and from 6 to 8 (Gosselin) or 2 to 5 (Sappey) the palpebral portion. Some of the upper may

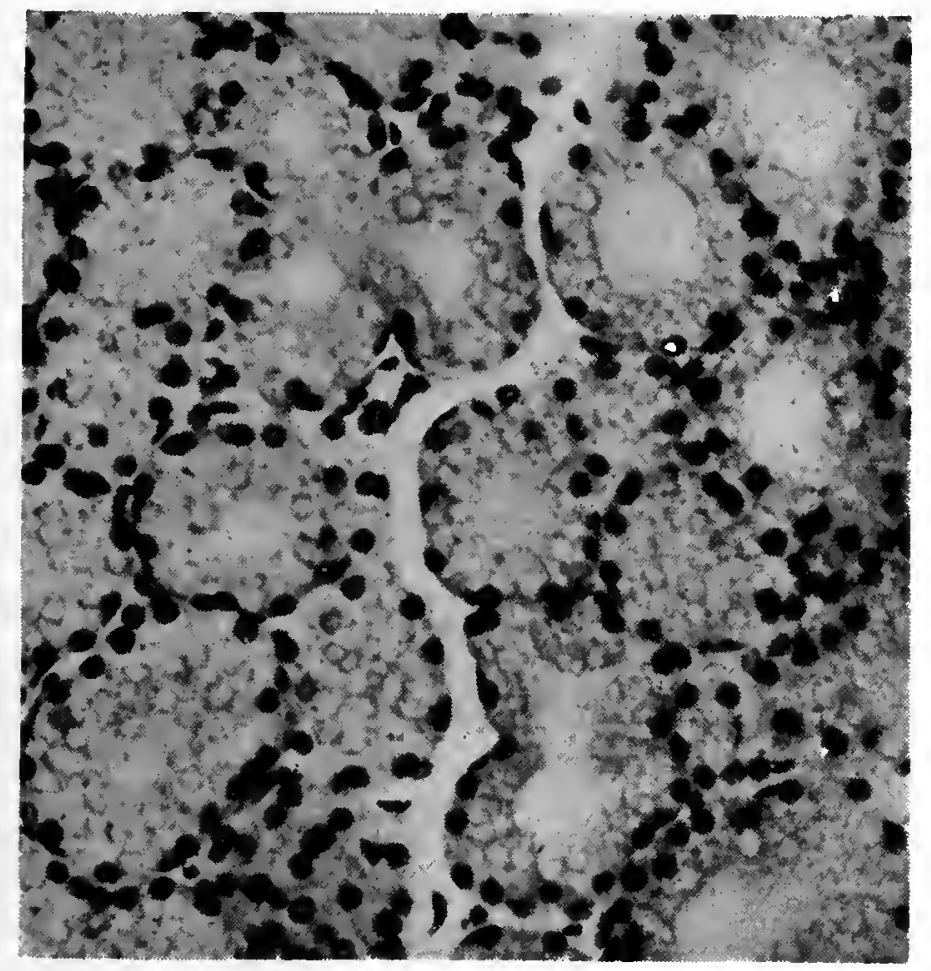

FIG. 108. - Section of human Lacrimal Gland, showing Acini ; running down the middle of the section is an intra-lobular secretory canal. $\times 200$.

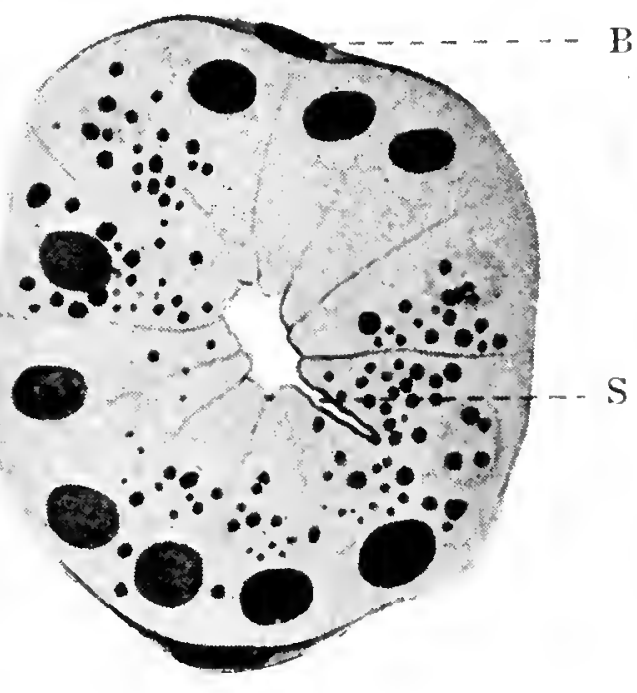

FIG. I09. - Microscopical section of an Acinus of the human Lacrimal Gland (Schirmer, I904).

$\mathrm{B}=$ basal cell $; \mathrm{S}=$ secretory capillary.

join those of the lower group, but nearly all open separately into the supero-external region of the conjunctival sac along a line just in front of the superior fornix and about 4 to 5 $\mathrm{mm}$. above the convex border of the tarsal plate. One duct, the largest, opens behind the lateral commissure, and one or two from the palpebral lobe may open as low down as the corner of the inferior fornix below the level of the commissure. From a practical point of view, it is important to note that the ducts of the upper or orbital lobe traverse or pass in close contact with the lower or palpebral lobe before they reach 
the fornix, so that removal of the palpebral lobe through the conjunctiva of the everted eyelid severs the ducts of both lobes, and is equivalent to extirpation of the whole gland as far as its secretory function is concerned (Schirmer, I904); it will be realised, however, that such an operation does not entail dryness of the conjunctiva, owing to the presence of the accessory lacrimal glands and its own mucous cells, though the condition may temporarily occur.

The stroma or supporting tissue between the lobules (less developed than in the salivary glands, with which the lacrimal gland is often compared, but from which it differs in the shape of its secretory cells and by the absence of crescent cells of Gianuzzi) is mesodermal tissue derived developmentally from the dermis of the conjunctiva, and like it consists of loose connective, elastic, and lymphoid tissues; the lymphoid tissue is absent in the new-born, sparsely present at puberty, but well developed in the adult, especially in the female; in old age it may hypertrophy; Stanculeanu (rgoo) found it present around the ducts and vessels, and chiefly concentrated towards the lower pole of the gland. Plasma cells have been found present by Hannes (I9II).

The lacrimal secretion is a clear fluid, salty, and slightly alkaline; the exact composition varies according to the histological condition of the epithelium which secretes it, and, it is worthy of note, according as the fluid analysed is the secretion of the gland itself collected from its ducts (as in Magaard's analysis which follows), or the "tears," which are the product of all the secretory cells including the mucous cells of the conjunctival sac. The details of the chemical composition are given by Schirmer (I904), from whose tables the following are quoted : 


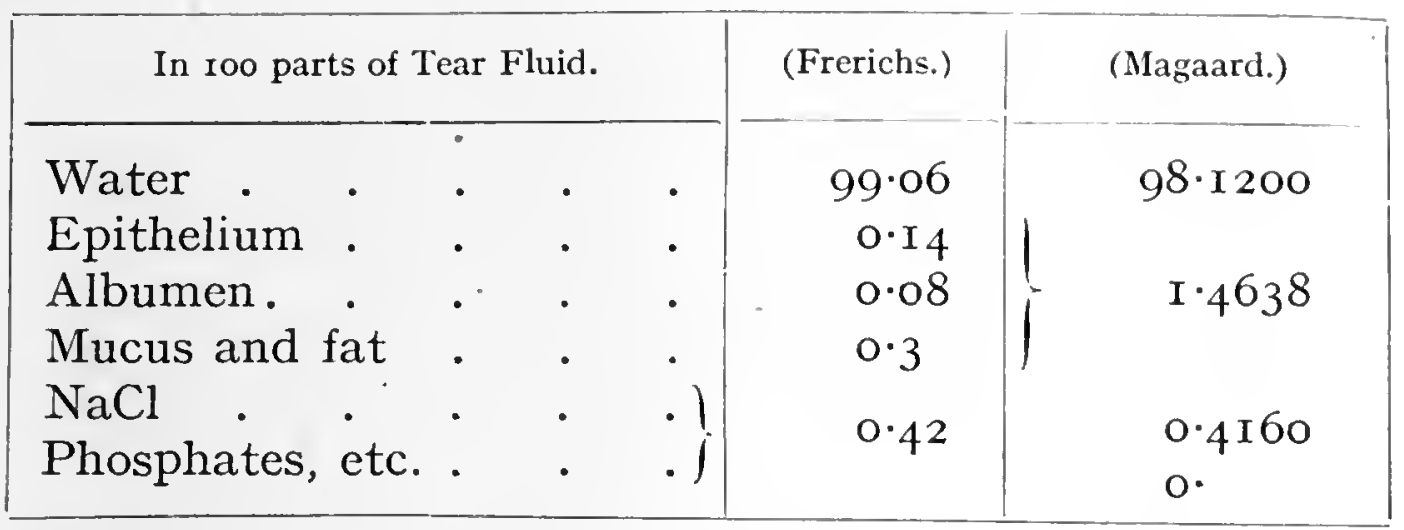

The normal quantity excreted in twenty-four hours is $6 \cdot 4 \mathrm{gr}$. (Magaard), of a specific gravity of $\mathrm{I} \cdot 0086$ at $20^{\circ} \mathrm{C}$. (Arlt).

The possession by the fluid of any bactericidal action is uncertain. It remains sterile indefinitely if collected under aseptic conditions, and is a bad culture medium; some authors consider it to have a bactericidal action lost on boiling (Kalt, Starling), others deny this property (De Bono and Frisco, I90I; Nedden, I907). Schirmer considers it to have an insignificant antiseptic action against the majority of bacteria, but none on the tubercle bacillus or bacillus coli. Lindahl (I907) studied its effect on various bacteria, and found a potency against some owing to the presence of an albuminoid substance of the nature of an enzyme, which is secreted by the lacrimal gland; the action is feeble on the staphylococci, and is lost on heating. The conjunctival sac is never free from organisms, save in the new-born, the most common being the bacillus Xerosis, which was found present by Lawson (I899) in II 8 out of I59 cases examined, and in over one-half of these unaccompanied by other bacteria, and designated by him " bacillus conjunctivae communis" in consequence; a staphylococcus albus of low virulence is also found (Parsons).

Vessels.-The arterial supply is derived from the lacrimal (p. 305), a branch of the ophthalmic artery, which enters the gland at its posterior margin and passes superficially on to the eyelids; an additional supply is often afforded by the infra-orbital branch of the internal maxillary artery. The venules form a lacrimal vein (p. 3II), running backwards as a tributary of the ophthalmic vein to enter the cavernous sinus. The lymphatics are probably limited to the surface 
of the gland, and enter the conjunctival and palpebral systems, whence they will drain into the pre-auricular nodes. In malignant tumours of the gland the facial and preauricular lymph nodes have been found enlarged.

Nerves. - The gland is supplied by the lacrimal nerve, by the facial nerve, and by the sympathetic nervous system. The lacrimal nerve is one of the three branches of the ophthalmic division of the trigeminal, and its course and relations within the orbit are described on p. 339; it anastomoses with the zygomatic branch of the maxillary division of the trigeminal nerve, and enters the gland with the blood-vessels ; part of its fibres end in the gland substance as fine non-medullated ramifications between the secretory cells of the acini and round the ducts and vessels (Dogiel, I893), and on some of the fibrils small ganglion cells are present (Puglisi-Allegra, I903); the bulk of the fibres traverse the gland and supply the conjunctiva and skin of the eyelids (p. 202).

The fibres from the facial nerve to the gland follow a long and complicated path; they arise from cells of the geniculate ganglion of that nerve in the facial canal (aqueductus Fallopii), but may have a central connection with the glosso-pharyngeal nucleus in the brain (Parsons, I902); under the name of the greater superficial petrosal nerve, theyjoin the deep petrosal nerve (sympathetic) to form the nerve of the pterygoid canal or Vidian nerve, and so enter the spheno-palatine or Meckel's ganglion; thence they are said to pass into the zygomatic (temporo-malar) branch of the maxillary nerve, and reach the gland through the anastomosis between the zygomatico-temporal division of this branch and the lacrimal nerve. That this anastomosis is of importance is indicated by the fact that in cases where the lacrimal nerve is absent a branch from the zygomatic nerve takes its place. On the other hand, it is stated that the minute orbital rami passing directly from the ganglion as its ascending branches into the orbit supply the lacrimal gland (Gray, I920). It should be realised that the greater superficial petrosal nerve probably contains facial motor fibres, as well as sensory from the geniculate 
ganglion. The direct sympathetic nerve supply is derived from the carotid plexus of the cervical part of this system, and accompanies the lacrimal artery to the gland; sympathetic fibres from the same source may also pass via the spheno-palatine ganglion and reach the gland by being incorporated with the zygomatic nerve.

As regards the unsettled problem of the actual source and path of the fibres conveying secretory impulses to the gland, it is first to be noted that the secretion is provoked :

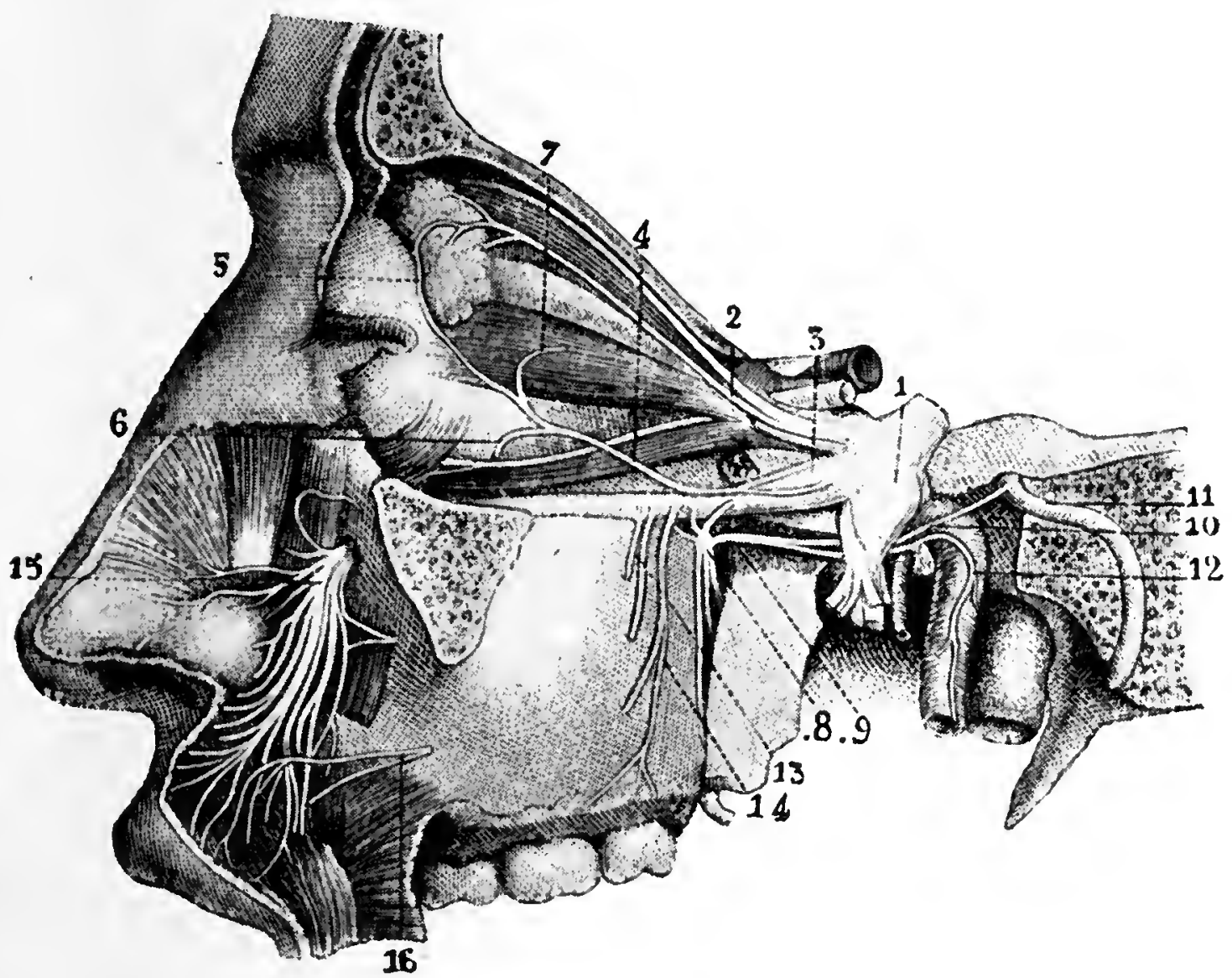

FIG. Iro.-The Maxillary Division of the Trigeminal Nerve, its branches and their connections. (From Sappey, after Hirschfeld and Leveillé.)

$\mathbf{I}=$ semilunar or Gasserian ganglion $3=$ the maxillary nerve $; 4=$ the zygomatic branch with its anastomosis (5) with the lacrimal nerve (2); II = the facial nerve; 10 is the greater and 12 the deep petrosal nerves which join to form the Vidian nerve (9) and enter the spheno-palatine ganglion (8). The other branches can be followed from the text.

(i.) as a normal physiological act, in the slight amount necessary to prevent drying of the conjunctiva ;

(ii.) by stimulation of a supposed tear secretory " centre," whereby psychical weeping, which is a human peculiarity, occurs ;

(iii.) as a reflex act following stimulation of the terminal nerve-endings of branches of the trigeminal nerve to the conjunctiva or nasal mucous membrane ; 
(iv.) by impulses passing through the retina and optic nerve, as when the eye is exposed to bright light.

It may be added that the tears which are forced in yawning, coughing, laughing, etc., more probably result from stimulation by co-innervation than, as is sometimes stated, from mechanical pressure of the orbicularis oculi muscle on the gland.

Further facts bearing upon the question are, first, that there is a secretion of tears on stimulation of either the lacrimal nerve itself, or of the facial nerve, or of the cervical sympathetic nerves (Starling, I920). Next, that the trigeminal nerve represents the reflex centripetal path, since there is a secretion of tears upon stimulation of its trunk or of almost any of its branches (Czermak quoted by Tepliachine, I894) ; indeed, the secretion following stimulation of the lacrimal nerve may be but a reflex action, since irritation of the area of conjunctiva supplied by this nerve produces the same effect. Then it is to be noticed that the muscles innervated by the facial nerve are usually thrown into action during weeping; and finally, that a connection with the sympathetic system is apparent since its stimulation produces tears, even after section of the lacrimal nerve (Kalt, I903), and the lids become swollen and the face red in crying. Most authors consider the facial nerve as the source (Parson, Lagrange, Schirmer, Quain, Dubreuil, Gaskell, Blum), but most probably both this nerve and the sympathetic system provide the secretory fibres, the latter acting in normal conditions, the facial in the superabundant secretion of weeping. Merkel, following Campos (I897), supposes that the impulse may travel by all the three nerves. Arloing considers that the sympathetic fibres convey the excito-secretory impulses. Kalt objects to the complicated facial route on the grounds that (i.) the zygomatic nerve leaves the maxillary nerve just after the latter has emerged from the foramen rotundum and before it is connected with the spheno-palatine ganglion-that is, before the Vidian fibres enter the main nerve; (ii.) the anastomosis between the zygomatic nerve and the lacrimal is not constant; (iii.) total paralysis of the facial nerve causes loss of the action 
ofjweeping though not of the lacrimal secretion, and Campos found that section of the great superficial petrosal nerve in monkeys did not cause loss of lacrimal secretion on the same side.

The most probable conclusion is that the lacrimal nerve merely contains centripetal fibres to the brain, as do other sensory branches of the trigeminal, conveying impulses called forth by the movement of the gland in the involuntary act of winking or by drying or irritation of the conjunctiva; that the sympathetic system provides fibres responsible for normal secretion; and that the facial nerve supplies fibres following the above outlined route and terminating in the lacrimal nerve path, which occasion the superabundant secretion of weeping.

It may be of interest to note that an article has been written by Rutherford (IgI3) on the behaviour of the lacrimal gland during the various, stages of surgical anaesthesia, wherein the amount of the secretion is shown to bear a definite relation to the depth of the narcosis.

Development. - The lacrimal gland is formed by the ramification and agglomeration of simple tubular buddings from the epithelium lining the conjunctival fornix in its supero-lateral region; they push their way along the path of least resistance between the globe and the orbital wall. The outgrowths appear in two successive tides ; the earliest were seen as knobs in embryos of $22-26 \mathrm{~mm}$. long by Keibel and Mall (I9I2) and in embryos of $32 \mathrm{~mm}$. (ten weeks) by Speciale-Cirincione (I908), and by increase in length they ultimately form the more deeply placed orbital lobe of the gland ; the secondary buds appear a week later (in embryos of $40-60 \mathrm{~mm}$.), are shorter, and form the palpebral lobe ; the tubules become hollow in embryos of 50-55 mm. long. In the mesodermal tissue between the two masses of outgrowths, the aponeurosis of the levator muscle begins to be developed as an interglandular septum in embryos of 38 $\mathrm{mm}$., and was found complete in one of $60 \mathrm{~mm}$. The accessory lacrimal glands appear làter, and have been first seen in a foetus of $17 \mathrm{cms}$. (Ask, I9Io). In the new-born the gland is only a quarter to two-thirds the adult size, 
and differs also in the appearance of its cells; the early growth is rapid, but the full structural development is not complete until the age of three to four years (Kirschstein, I894). After the tenth year there is a gradual change in the histological structure, the glandular epithelium becoming decreased in height, the lumen of the acini widening, and the interstitial and adenoid tissue gradually increasing,

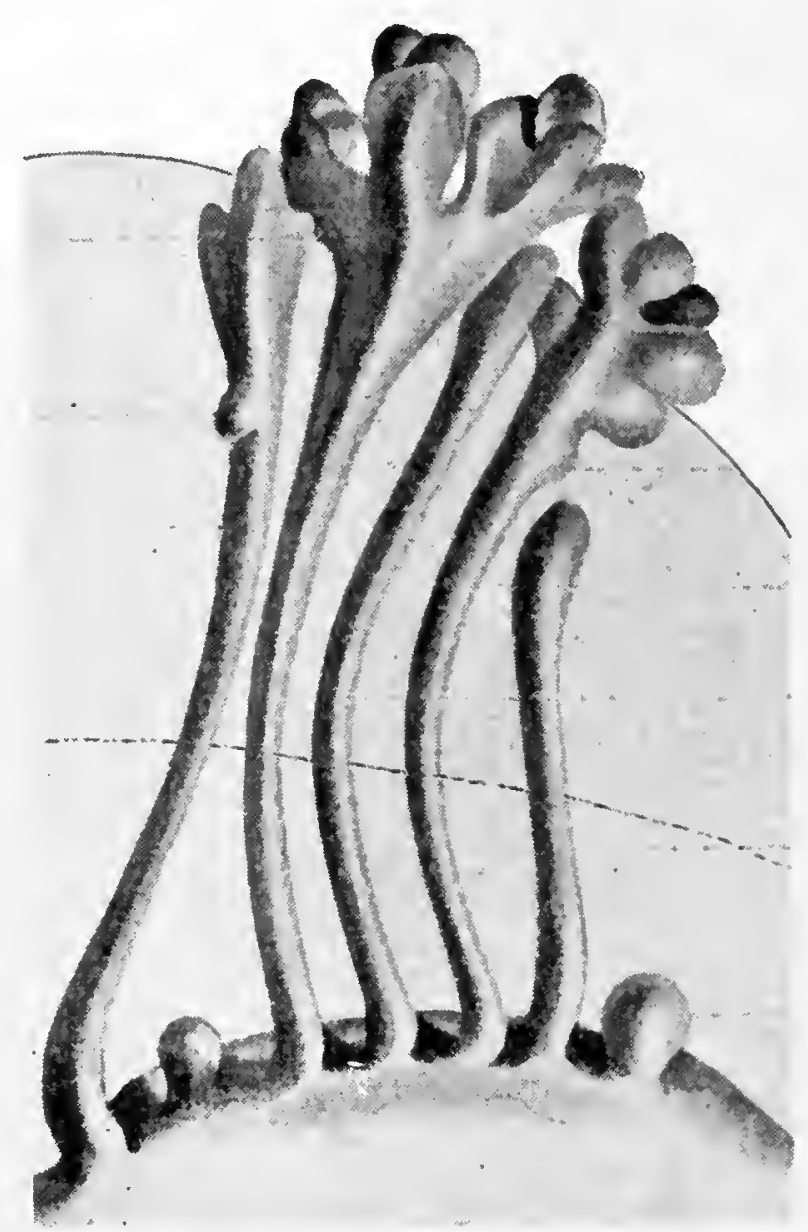

FIG. III. - Reconstruction of the developing Lacrimal Gland in an Embryo of to $\mathrm{mm}$. The transverse curved line indicates the position of the aponeurosis of the levator, developed between the two successive tides of outgrowths from the conjunctival fornix ; the upper larger ones form the orbital lobe, the lower knobs form the palpebral lobe of the gland. (Speciale-Cirincione, r909.)

though less rapidly, after the age of forty (Göz, I908) ; in old age the glandular substance atrophies and the interstitial tissue increases.

Considerable variation is found in the size and form of the gland, as is to be expected from the mode of development. Bock (I896) found it as usually described only in 9 out of 20 cases; the orbital lobe extended to a very variable distance backwards into the orbit, and varied from II to $22 \mathrm{~mm}$. in length and from 5 to $\mathrm{I} 2 \mathrm{~mm}$. in breadth; the 
palpebral lobe was undeveloped in seven cases. The gland is relatively larger in the child, as in the female, where the upper eyelid, of which the gland is but an annexe, is also relatively larger and more mobile than in the male. Terlinck and Gallemaerts describe cases of congenital lacrimal fistula in which the normal secretion was discharged by an opening on to the face of the upper eyelid.

The lacrimal gland is found first in the amphibia, and in air-breathing vertebrates it appears first in the lower lid, as in the triton, and the higher the series examined the further the gland is found to have migrated towards and round the lateral commissure, until the outer part reaches the upper eyelid and there predominates (Fig. I02). The phylogeny of the gland in mammals has been studied by Broman and Ask (IgIO).

II. The Membranous Lacrimal Passages.-The tears excreted by the lacrimal gland into the supero-lateral region of the conjunctival sac are spread over the surface of the globe by the movement of the eyelids aided by gravity, and collect in the lacus lacrimalis at the medial angle of the eye; they then pass by capillarity through the lacrimal puncta, the minute openings on the summit of the lacrimal papillae, into the lacrimal canaliculi, which run horizontally through the thickness of the lacrimal portions of the lid margins and conduct the fluid into the lacrimal sac lying in the lacrimal fossa; the membranous passage formed by the sac is continued directly downwards as the naso-lacrimal duct, which traverses the bony naso-lacrimal canal, to open by its lower extremity into the inferior meatus of the nasal cavity (Fig. II2). The lacrimal passages thus lead from the conjunctival sac to the nasal cavity, and may conveniently be described in three parts:

(i.) The lacrimal canaliculi.

(ii.) The lacrimal sac.

(iii.) The naso-lacrimal duct.

(i.) The lacrimal papillae (papillae lacrimales) are placed one on each lid margin at the junction of its ciliary and 
lacrimal portions, and on the posterior edge. They are pale, being but little vascularised ; their prominence is increased in the aged owing to atrophy of the muscle fibres which

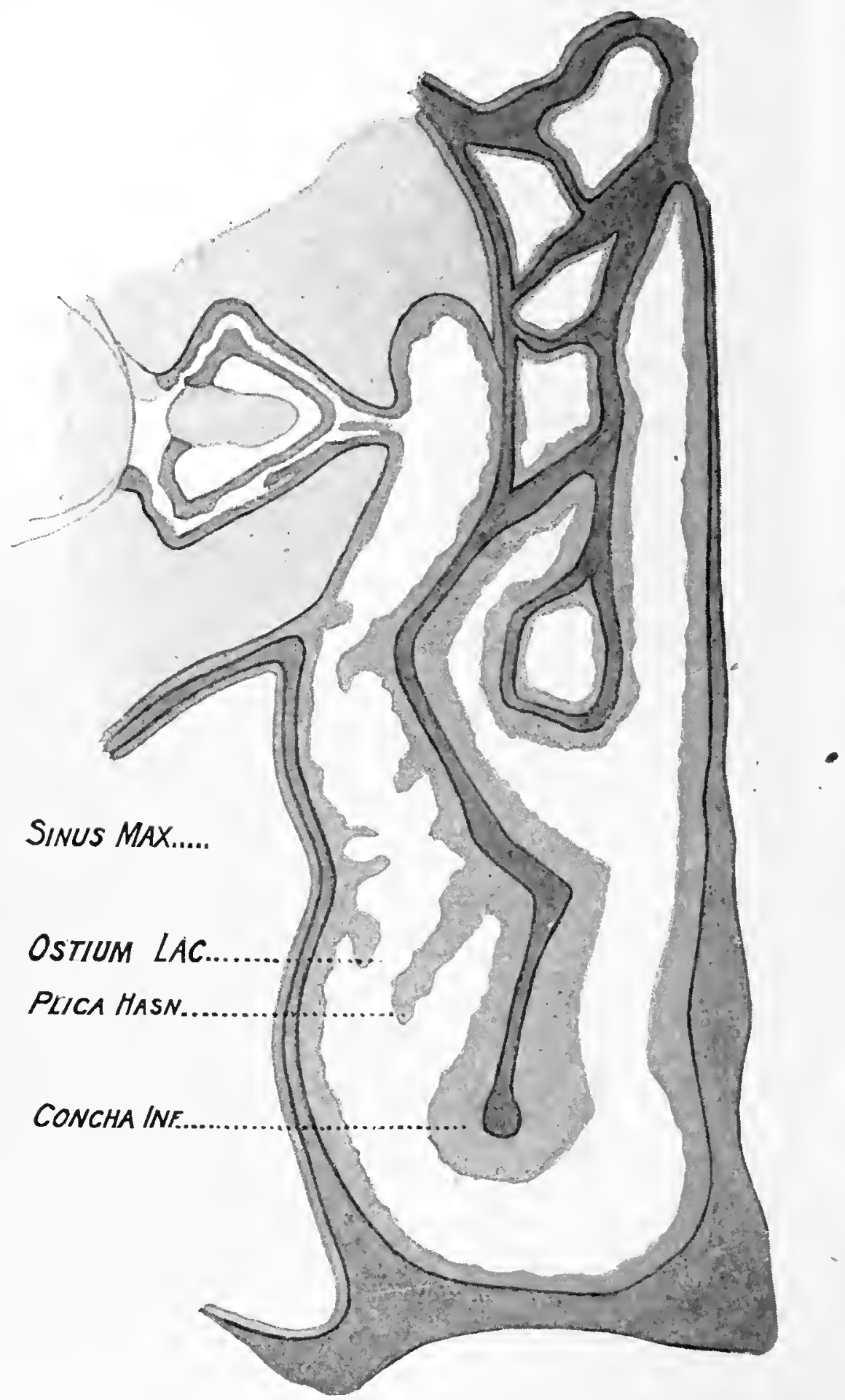

FIG. I I2. - Sketch from a frontal section through the Right Nasal Cavity viewed from in front, to show the relation of the lacrimal passages to the ethmoidal cells, the inferior concha, and the maxillary sinus. The lining mucous membrane is coloured to emphasise its continuity. The lacrimal sac is represented as usual in an abnormal dilated condition. (After Gérard, 1907.) $\times$ about 2.

encircle them at their base. The lacrimal point or punctum (punctum lacrimale) is the minute circular or oval opening of the canaliculus which appears on the summit of the 
papilla, and since the papillae are directed backwards the lids must be slightly everted to see the openings. They are well displayed in Fig. 76, p. I53, and in Fig. I20; they lie in the same plane as the orifices of the tarsal glands, the nearest of which is only $\frac{1}{2}$ to $I \mathrm{~mm}$. distant. The inferior punctum lies $6.5 \mathrm{~mm}$. and the superior $6 \mathrm{~mm}$. lateral to the medial canthus, so that when the lids are shut the points are not superimposed, but the inferior lies just to the lateral
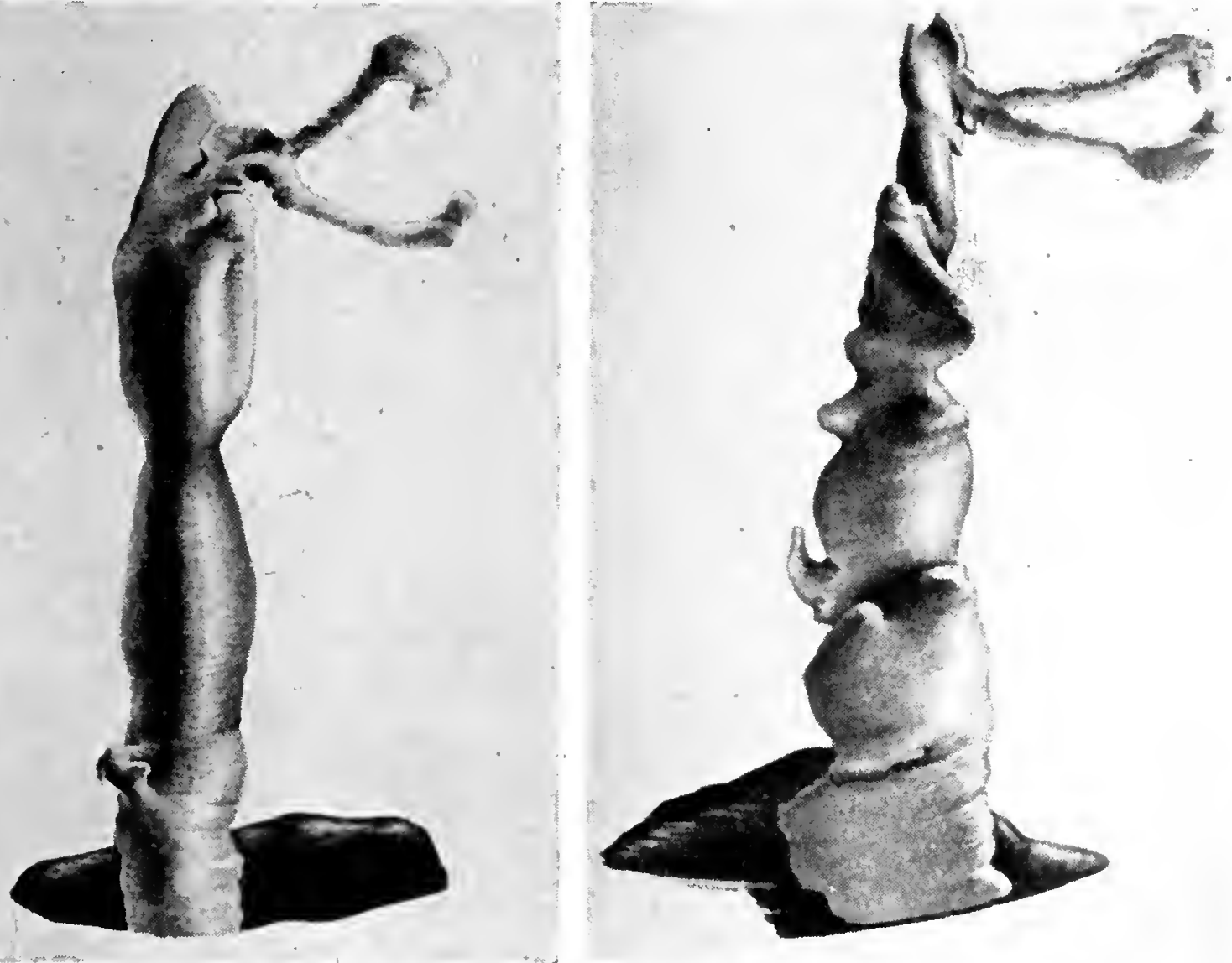

Fig. II3. - Reconstructions of two types of the Naso-Lacrimal Passages. The left one is from an adult aged 60 years, and shows a regular type. The right one is from a man aged 65 years, and shows the marked irregularity these passages may present. (From Schaeffer, I9I2.) $\times 2$.

side of the superior ; the reverse arrangement has, however, been seen to occur (Dieulafé, I905); the diameter of the lower one is $3 \mathrm{~mm}$., that of the upper $2.5 \mathrm{~mm}$. They are plunged into the lacus lacrimalis, the inferior orifice, like the papilla on which it is placed, being directed backwards into the groove between the globe and the plica semilunaris, whilst the superior papilla with its punctum is directed downwards as well as backwards into the groove between the plica and the caruncle; both puncta touch the cornea when the eye is turned strongly nasalwards. The orifices 
are encircled by a ring of dense connective tissue, so that they always remain patent and capillarity is ensured.

The lacrimal canaliculi or ducts (ductus lacrimales), into which the puncta lead, are two minute canals, each running in the thickness of the lacrimal part of the lid margin behind the medial commissure to enter the lacrimal sac. They therefore constitute the palpebral part of the lacrimal passages.

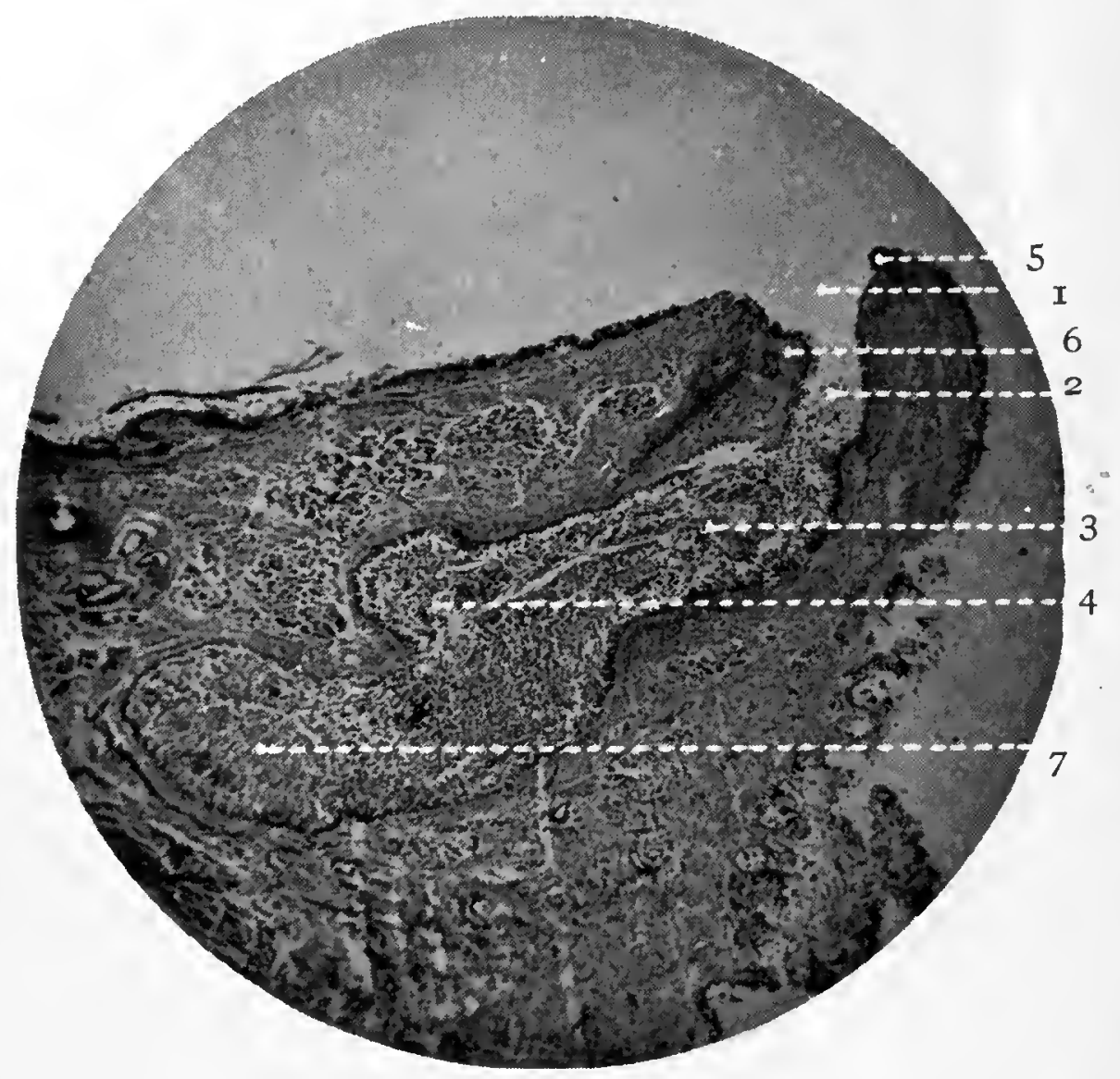

FIG. I I 4. - Magnified section of the Punctum and vertical portion of the Lacrimal Canaliculus of the Lower Eyelid.

$\mathrm{I}=$ infundilulum; $2=$ angustia; $3=$ piriform dilatation, and $4=$ sacculiform dilatation, the two forming the ampulla; $5=$ "valve" of Bochdaleck; $6=$ "valve" of Foltz; $7=$ commencement of horizontal part of canaliculus.

The total length is about Io $\mathrm{mm}$. (Gerlach, I880), the lower one being a little longer than the upper, in harmony with the more lateral position of its punctum. Each is bent nearly at right angles into two unequal portions, a shorter vertical part lying in the papilla, united by a widened angle to a longer horizontal part which runs along the lid margin.

The vertical part (pars verticalis) begins with an inverted funnel-shaped lumen, the infundibulum of Foltz, of which the base corresponds to the punctum and the neck is called the angustia of Gerlach; it leads into the dilata- 
tion of the angle or ampulla, which is subdivided into two secondary piriform and sacciform recesses, and then narrows slightly as it turns into the horizontal part; there may be further recesses of the ampulla directed in the opposite direction towards the ciliary part of the lid margin, as found in a model reconstructed by the writer. The total height of the vertical part is from $\mathrm{x} \cdot 5 \mathrm{~mm}$. to $2 \mathrm{~mm}$., of which $0.5 \mathrm{~mm}$. is formed by the infundibulum (Heinlein, I875); the diameter of the punctum is from 0.2 to 0.3 $\mathrm{mm}$., that of the ampulla is I mm. (Merkel).

The horizontal portion (pars horizontalis) runs from the base of the vertical portion medially along the lid margin to the lacrimal sac; this part is not strictly horizontal, but inclines towards its fellow of the opposite lid as it courses medially; the lower one is also slightly curved. The length is from 6 to $7 \mathrm{~mm}$. (Gerlach) or from 7 to $9 \mathrm{~mm}$. (Henle), and the diameter is from $0 \cdot 3$ to $0 \cdot 6$, but can be dilated to $\mathrm{x} \cdot 5 \mathrm{~mm}$., the inferior being $.5 \mathrm{~mm}$. longer and a little wider than the superior; though the lumen is small the elasticity of the walls allows of a dilatation to three times the normal calibre, and for the same reason a probe will easily straighten out the angle between the vertical and horizontal portions, but the sclerous nature of the punctum does not favour its regaining the normal size after passage of an instrument, and the capillarity of the opening may thereby be interfered with.

The base of the papilla lies buried in the fibres of the 
orbicularis oculi muscle (pars lacrimalis), which cross one another and are disposed in a more or less sphincter-like manner (Fig. I26, p. 242), forming the musculus quadrangularis constrictor puncti lacrimalis (Halben, I903; Gerlach, Merkel), which is considered to have a definite sphincter action, but owing to the density of the walls the lumen is probably not reduced in normal winking, though it may be in blepharospasm. The horizontal portion of the canaliculus is likewise surrounded on all sides by the same muscle fibres (as seen in Fig. I2I), which some authors describe as being spirally arranged round the tube and even attached to its walls by elastic fibres (Klodt, I893; Krehbiel, I878; Heinlein, I875), though this arrangement was not found by Halben and could not be confirmed by the present writer, who found the fibres running parallel with the canaliculus on all sides, though less abundant posteriorly; contraction of these fibres draws the papillae medially and directs them into the lasus lacrimalis, a fact that can be verified by everting the medial parts of the lids so as to expose the points, and then instructing the patient to try and blink; the action may also increase the calibre of the canaliculi.

The canaliculi usually pierce the lacrimal fascia separately, as shown in Fig. II8, and then unite to form a common. canal of union about $.8 \mathrm{~mm}$. long, which narrows slightly before it enters the lacrimal sac; a conjoint termination of the canaliculi into the sac is described as the normal by some writers (Sappey, Foltz, Hyrtl), and has been found to occur in Iog cases out of II 2 by Lesshaft (I868), who has made a special study of the question; on the other hand, Huschka (quoted by both Merkel and Schirmer) found a common opening only in I4 per cent. The different views may be accounted for by a distinction not having been made between the lacrimal fascia and the wall of the sac itself. The canal of union is really a diverticulum of the sac, as shown by its structure, and it is often enlarged to form a so-called "sinus of Maier," as found in I4 per cent by Huschke; the position is shown in Fig. I25 and an example in Fig. II3. The point of entrance into the sac is situated a little above the middle of its lateral wall about 
$2 \frac{1}{2} \mathrm{~mm}$. from the upper end; it lies nearly opposite the mid-point of the medial palpebral ligament, but $2-3 \mathrm{~mm}$. deep to its surface; the ligament covers the medial third of the canaliculi and their common canal of union, and behind this part of their course lies the pars lacrimalis or Horner's muscle (Fig. I2I, p. 23I).

The lacrimal sac and the naso-lacrimal duct form one continuous membranous tube, and a distinction between them is hardly justified on developmental, structural, or physiological grounds, though one must be drawn for practical purposes on their anatomical relations. It is really an "orbito-nasal tube," of which the upper part lies beneath the periorbita or lacrimal fascia in a groove (lacrimal fossa) on the medial margin of the orbit, and the middle part is contained in an osseous channel (nasolacrimal canal) in the maxilla; moreover, beyond the lower end of this part the tube often runs downwards some distance beneath the lining mucous membrane of the lateral wall of the inferior meatus of the nose before it opens into that space. Thus there may be demarked three parts of the tube, orbital, maxillary, meatal, which are respectively subperiorbital, inter-osseous, and submucous as regards their positions; the first part is called the lacrimal

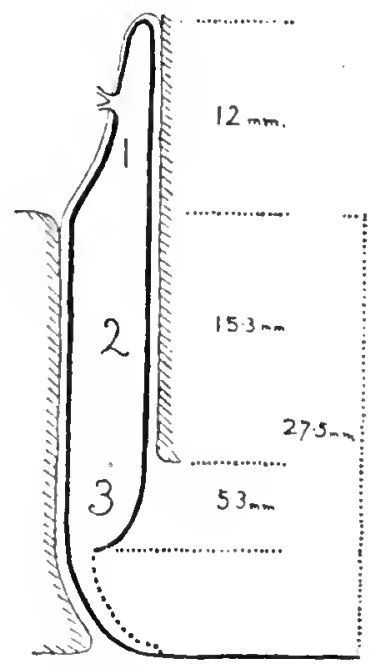

FIG. I 66.-Diagram to show the Orbito-Nasal Tube subdivided into three parts according to its external relations. 'The figure is drawn to scale, natural size, after the measurements of Power and Aubaret.

I $x$ the lacrimal sac, covered by the lacrimal fascia.

$2=$ the inter-osseous part of the naso-lacrimal duct.

$3=$ the meatal part of the duct the rare condition of a prolongation of the passage below the opening is indicated by a dotted line. sac, the second and third together form the naso-lacrimal duct. The whole tube can be dissected out from the surrounding parts, when it will be realised that the differentiation is largely extrinsic and relational (Figs. II6, II7).

In the new-born infant the whole channel is regularly cylindrical in shape (Rochon-Duvigneaud, I900 ; Aubaret, I9Io; Lepage), but marked secondary modifications of 
the form, both internal and external, are usually found in the adult.

At this period of full development two main types of tube are found :

(a) Regularly cylindrical in outward form, with no differentiation into sac and duct. This retention of the primitive shape was found by Aubaret to occur in 32 out of 1 I6 specimens. The external diameter was about $5 \mathrm{~mm}$.

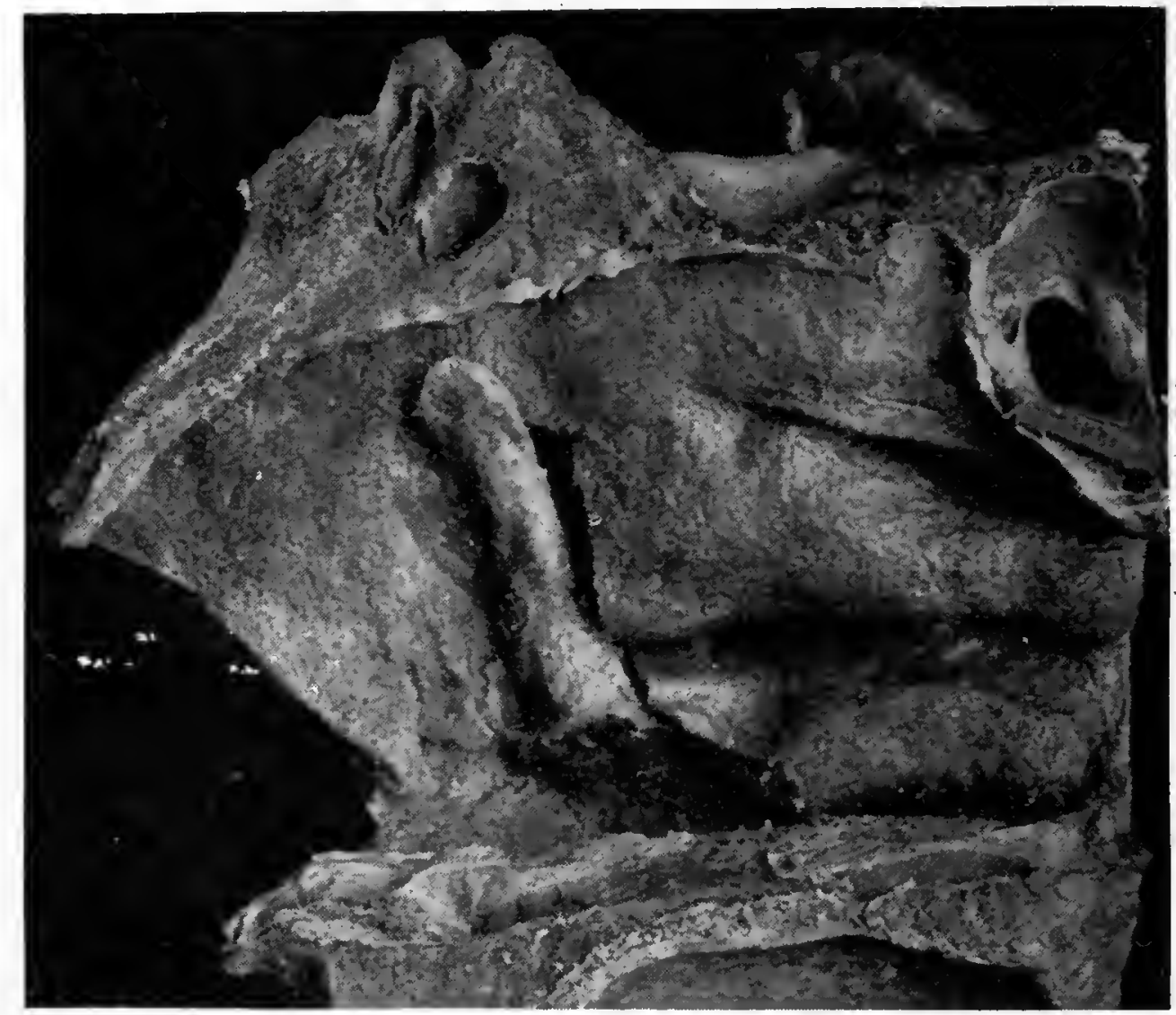

FiG. I 7. - Right Lateral Nasal Wall viewed from inside, showing the lacrimal sac and naso-lacrimal duct lifted out of their osseous channels. Natural size.

(b) Irregular types; in some (28 out of II6) the upper end of the tube is enlarged, so forming a sac as usually described, though it is to be noted the increase in size is in an antero-posterior direction only; in others (26) the lower part of the tube presents a second dilatation, giving the classical hour-glass shape, but the constriction between the two parts does not coincide with the bony junction of fossa and canal, being situated below this point; in some (6) the whole tube is uniformly enlarged. In these irregular types the external diameter varies from $2 \mathrm{~mm}$. in the narrowest to $9 \mathrm{~mm}$. in the widest part. 
It will be realised that the tube necessarily conforms in shape to the bony fossa and canal in which it is lodged, and the casts of these bony passages taken by Zabel (I90o), like the reconstructions of Schaeffer, therefore serve to illustrate the outward form of the soft contents (Fig. 34, p. 74, and Fig. II3).

Since the sac and duct into which the tube is conveniently divided present marked differences in their anatomical relations, they must now be described separately, the internal configuration and structure being subsequently considered as a whole.

(ii.) The lacrimal sac (saccus lacrimalis) is lodged in the fossa sacci lacrimalis on the medial margin of the orbit. On its lateral or ocular aspect it is covered over by the periorbita, which splits at the posterior lacrimal crest, one layer following the bone and lining the fossa, the other bridging straight across it to reach the anterior lacrimal crest and being attached below to the incisura lacrimalis or upper edge of the bony naso-lacrimal canal. The lacrimal sac is roofed over completely by the periorbita, and so smoothly does this membrane maintain the contour of the medial orbital wall that after removal of the eyelids and the entire contents of the orbit the only indication of the position of the lacrimal sac is afforded by the orifices of the severed canaliculi (Fig. II8). This layer of periorbita which roofs the lacrimal fossa is termed the "lacrimal fascia" (fascia lacrimalis), and although it forms a strong definite layer of fibrous tissue readily demonstrable by dissection and conspicuous in cross-sections (Figs. II8, I2O, and I2I), it is not figured, and but rarely mentioned, in accounts of the anatomy of this region, though sometimes it is referred to by such misleading terms as "deep" or even "palpebral fascia." Failure to realise its existence in passing a probe through an opening in the supposed wall of the lacrimal sac down the duct leads the student to force the instrument between the sac itself and the periosteal lining of the naso-lacrimal canal, and naturally a difficulty is experienced in reaching the nasal cavity. Usually there exists a narrow space between the lacrimal fascia and the sac, filled by a minute venous 
plexus continuous with that around the duct and draining into the angular or supra-orbital vein, and a separation between them is easily effected; occasionally, however, they are adherent, as indeed the sac generally is to the periosteum lining the medial wall of the fossa and always at its summit (fundus). The fascia is pierced separately by the two canaliculi, at which points it sends reflected

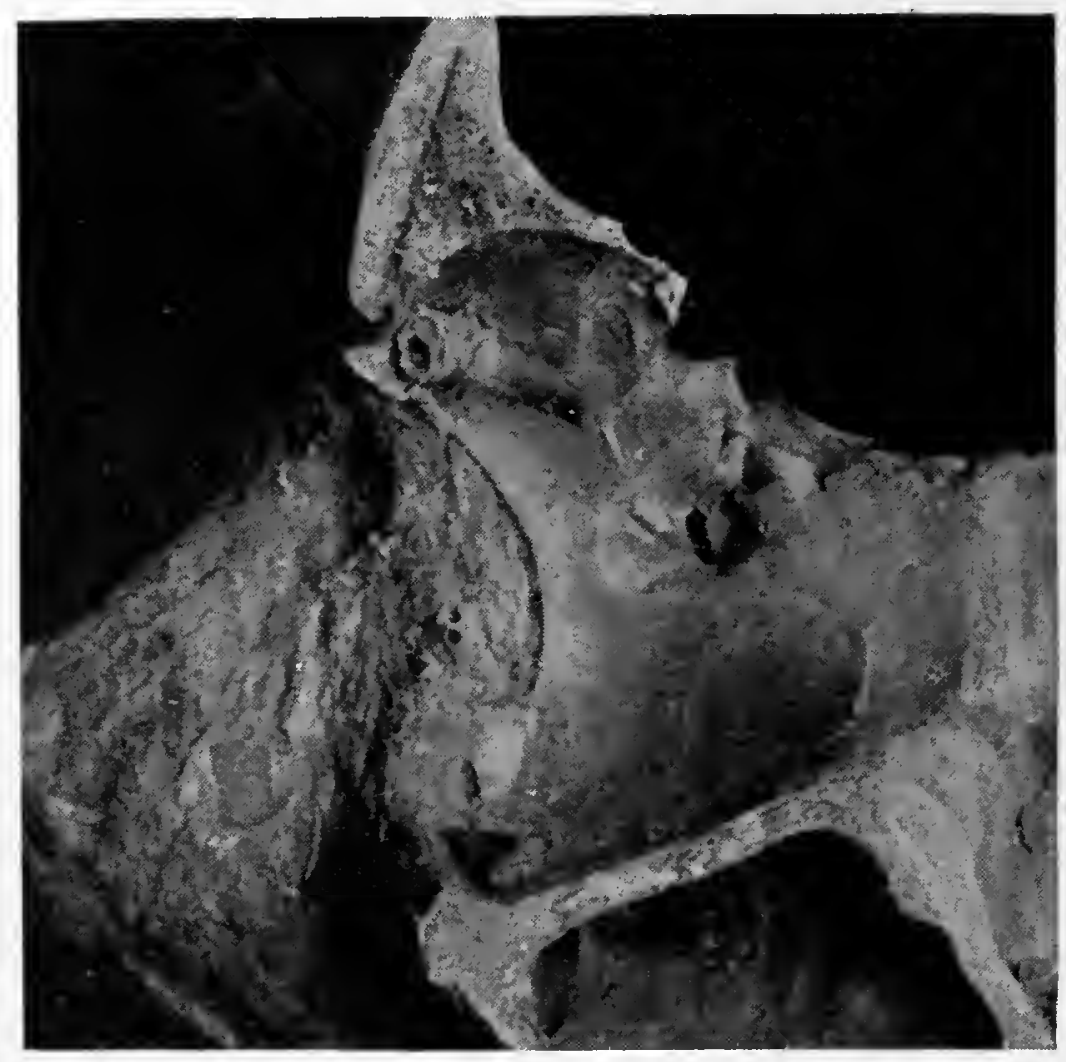

FIG. II8. - Dissection to show the lacrimal fascia (fascia lacrimalis). Medial Wall of Left Orbit viewed from inside. The lining periorbita is left in situ. The cut orifices of the canaliculi are seen in the roughened part of the periorbita which covers the lacrimal fossa and so forms the lacrimal fascia; the position of the lower part of the lacrimal sac is indicated by a slit in the lacrimal fascia, immediately below which is the cut end of the inferior oblique muscle. There was an ethmoidocoele in this wall. The trochlea is seen above. The ostium maxillare can just be seen. Natural size.

sheaths along them nearly as far as the lacrimal papillae; it is also pierced in its lower part by a twig passing from the infra-orbital artery to the naso-lacrimal duct, and by filaments of the infra-trochlear nerve.

Thus, confined by the tense lacrimal fascia, as is well shown in Fig. I2I, the lacrimal sac naturally conforms to the fossa within which it lies; it is flattened from side to side in the shallow upper part, becoming more rounded below where it joins the cylindrical naso-lacrimal duct. 
It therefore does not represent the upper dilated end of the duct, as commonly described (e.g. in Figs. II2, I25), but the two together may be likened to the end of a quill pen seen sideways, as is well illustrated in the next figure and by Fig. 34, p. 74. Just as deep or shallow forms of lacrimal fossae are found in the skull, so variations in the size of the sac may be expected. The average dimensions are given on p. 237.

The flattened upper part of the sac above the entrance of the canaliculi is termed the fundus (fornix sacci lacrimalis) ;
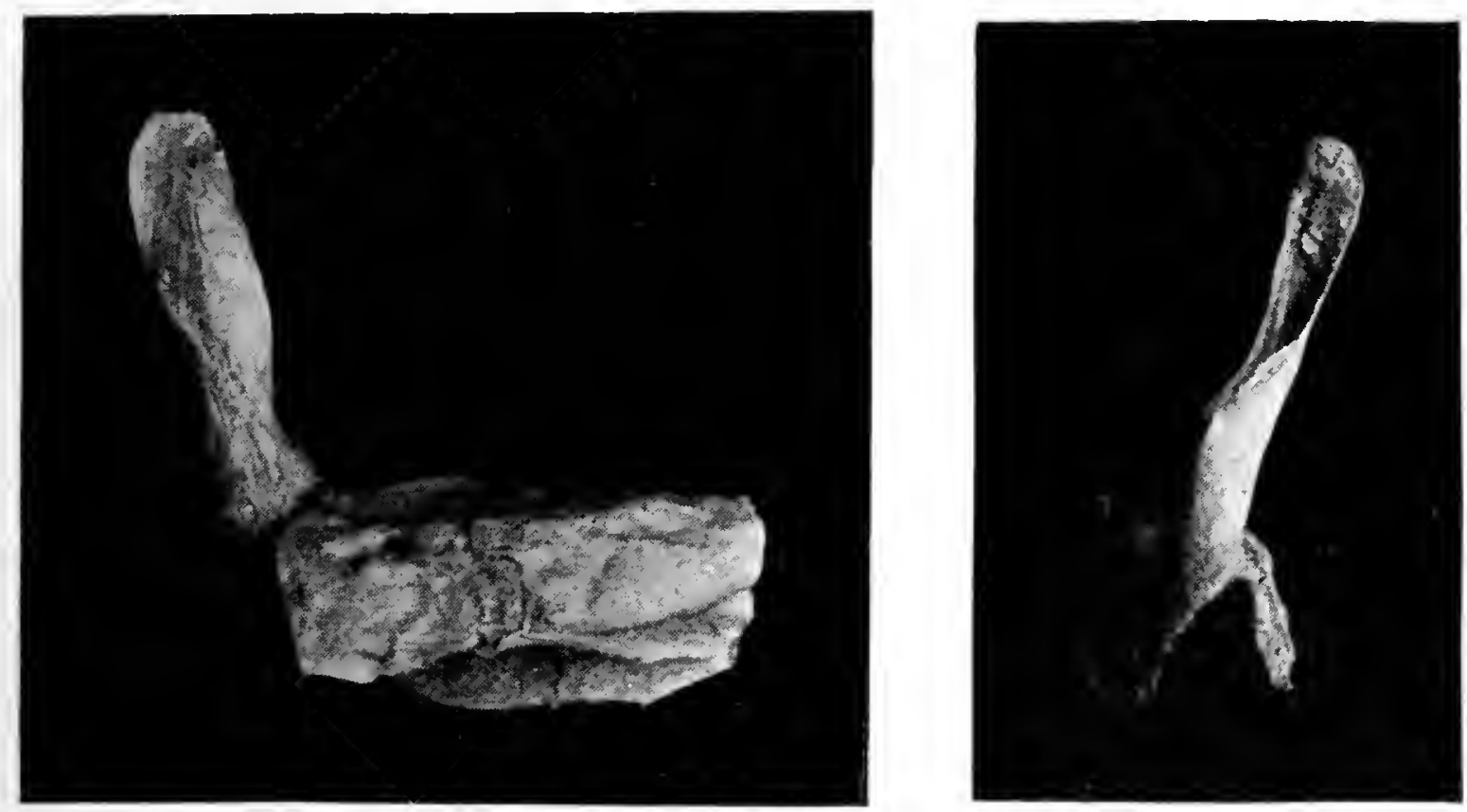

FIG. I I9. - The Right Lacrimal Sac, the Naso-Lacrimal Duct, and the Mucosa of the Inferior Meatus of the Nose, dissected out entire and viewed from the medial side (left figure) and from in front (right figure). 'The mucous membrane is atrophied, the specimen having been taken from an aged subject, but the normal flattened shape of the lacrimal sac and the inclination of the whole tube are well illustrated. Natural size.

its summit lies from 3 to $5 \mathrm{~mm}$. above the line of the medial canthus and from Io to $\mathrm{I}_{5} \mathrm{~mm}$. below the trochlea of the superior oblique muscle, in the interval there being situated the anastomoses between the angular and ophthalmic vessels and the branches of the infra-trochlear nerve.

The relations of the lacrimal sac on its medial or nasal aspect are those of the corresponding wall of the bony fossa, which have been described above on p. 66. On its lateral or ocular side it is first covered over entirely by lacrimal fascia, a primary relation to be accepted throughout the whole of the following description. Outside this the sac 
presents different relations in approximately its upper and lower halves, and both in front and behind. In front of the upper half lies the anterior limb of the medial palpebral ligament (tendo oculi internus); this does not form a narrow band crossing the sac about its middle and allowing it to bulge out above as is sometimes depicted, but covers over the whole of this half of the sac, and is first attached to the upper part of the anterior lacrimal crest and then spreads out to blend with the periosteum of the bone beyond,

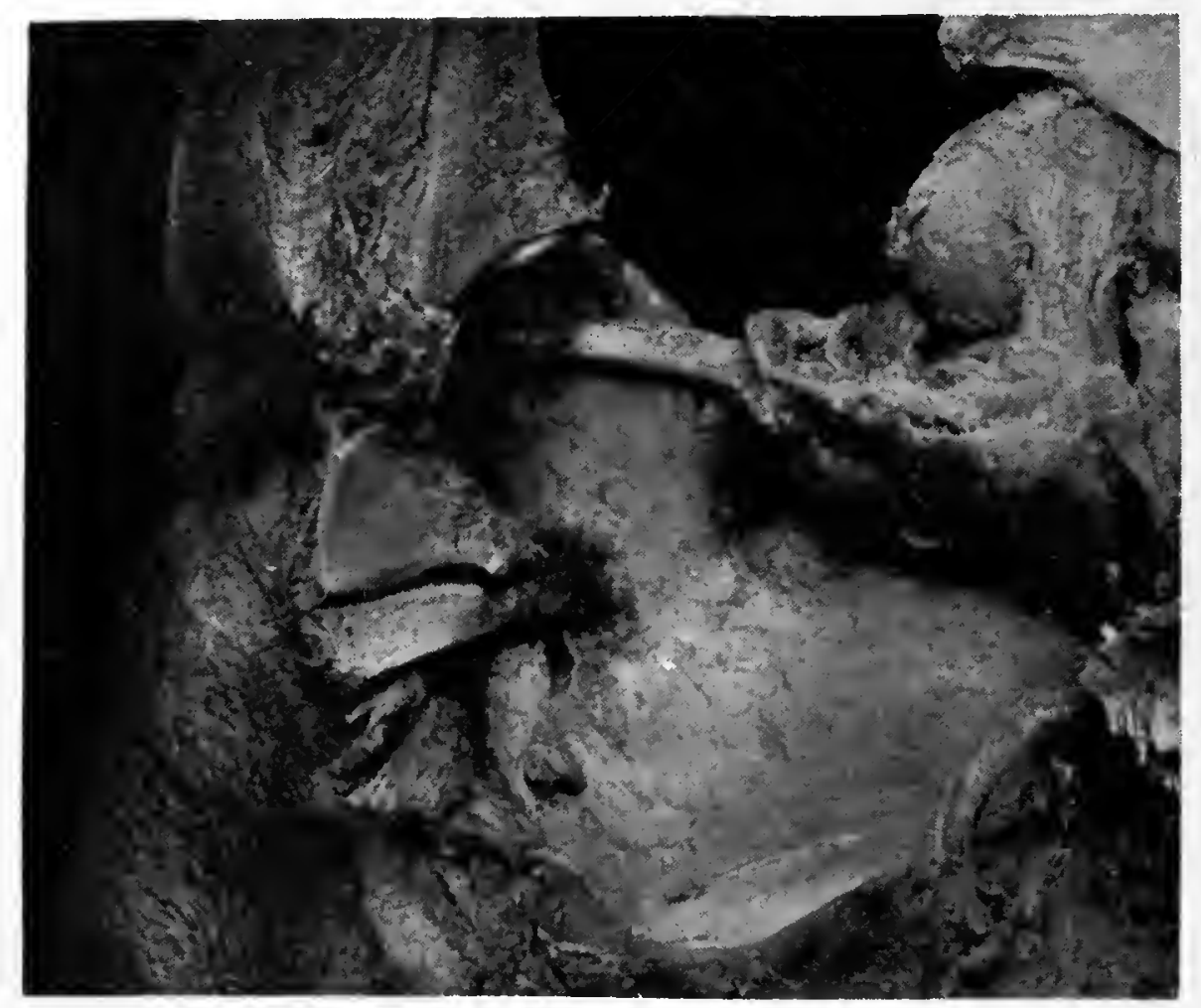

Fir. I 20. - Dissection of the Medial Wall of the Left Orbit to show the relation of the pars lacrimalis of the orbicularis oculi or Horner's muscle to the upper half of the lacrimal sac; a window has been cut through the lacrimal fascia to show the position of the lower half of the sac. The relative positions of the two lacrimal puncta are clearly shown. Natural size.

as is well displayed in Figs. 55, 70, I42. Its lower border is thick and free, and is separated from the sac by fibres of origin of the orbicularis, as noted on p. I48; its upper part becomes thinner, and blends with the lacrimal fascia covering the fundus of the sac. This intimate connection is noteworthy as affording a possible explanation of cases where the lids swell up on blowing the nose after a comparatively light blow on the eye, as in boxing: a sudden strain is thrown upon the ligament and the sac is torn (Fisher, I904). Superficial to the ligament lie fibres of 
origin of the orbicularis oculi muscle, the angular vessels, and the skin.

Behind the upper half of the lacrimal sac there passes the posterior or reflected limb of the medial palpebral ligament, a thin sheet of fibres which is difficult to identify and must be of little importance. Deep to it lies the pars lacrimalis (Horner's muscle), of which, indeed, the reflected part of the ligament seems to form but a fascial covering. The muscle arises from the upper part of the posterior lacrimal crest (Fig. 59, P. I3I, and Fig. I2I), frequently

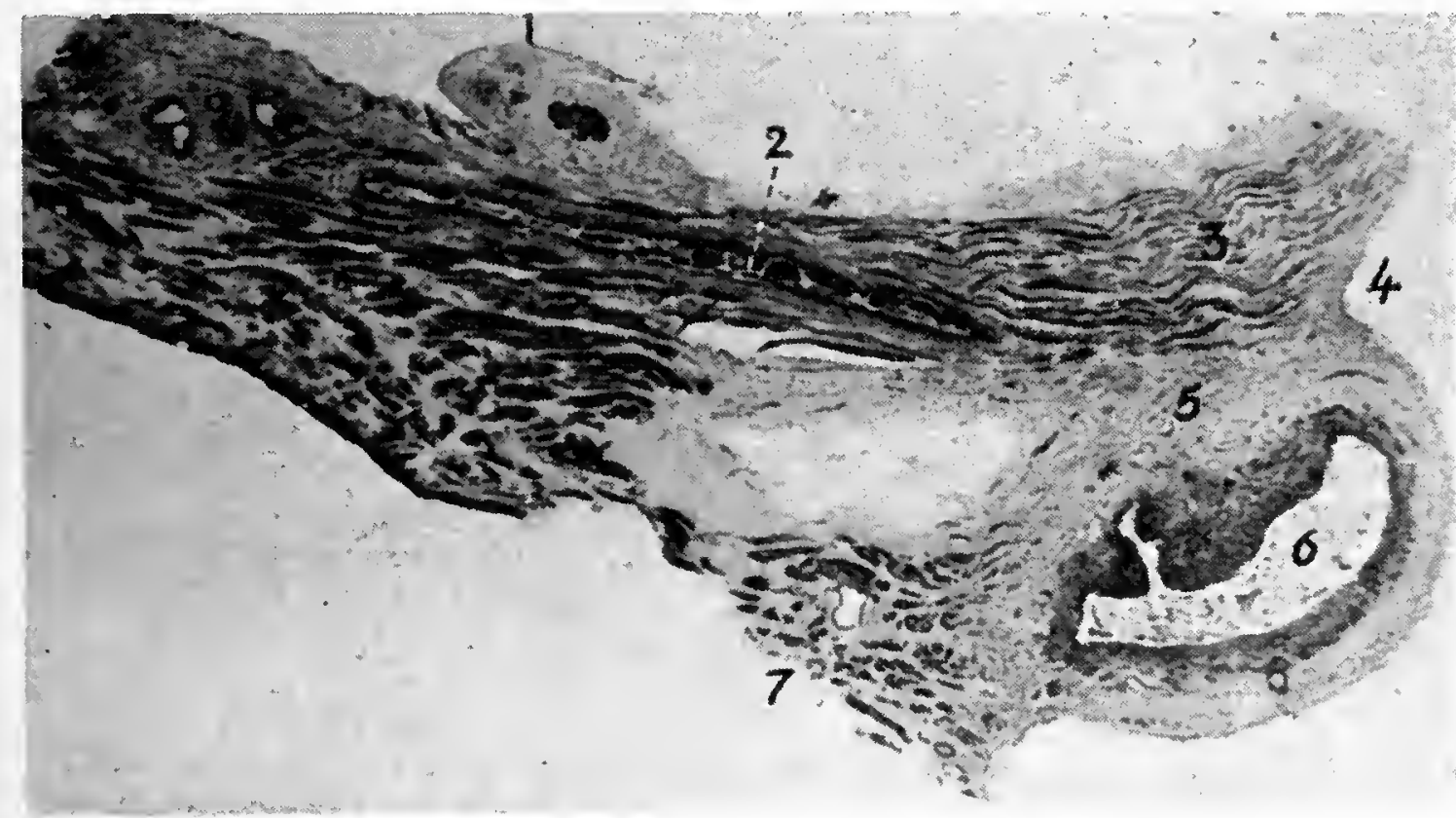

FIG. I2I.- Relations of the lacrimal sac. Horizontal section through the Medial End of the Lower Eyelid, showing-

I, the lacrimal caruncle ; 2 , the lacrimal canaliculus; 3 , the pars lacrinalis muscle attached to the posterior lacrimal crest (4); 5 , the lacrimal fascia covering the lacrimal sac (6); and 7 , the anterior lacrimal muscle fibres. Magnified.

encroaching forwards upon the lacrimal fascia, and passes forwards upon and laterally across the upper part of the sac from behind, as seen in Fig. I20, to divide and run along each lid margin as the pars tarsalis of the orbicularis oculi muscle. Deeper still behind the muscle origin lies the septum orbitale, separating it from the fascial expansion or check ligament of the medial rectus muscle of the eyeball and the orbital fat, as is shown diagrammatically in Fig. I5o, p. 287. The chief structures, then, in relation to the upper half of the lacrimal sac are the medial palpebral ligament in front, and the pars lacrimalis muscle behind. As previously noted, when traced from the lateral side the muscle fibres 
in the lids lie in front of the tarsal plates, and hence are distinguished as the pars tarsalis, but at the medial angle of the eye they pass deep behind the sac and form the pars lacrimalis muscle, whilst the plates are continued on as the medial palpebral ligament which passes in front of the sac, as shown in Fig. 72, p. I50. The more posterior attachment of the muscle ensures the plates being kept in close contact with the curved anterior surface of the globe, as noted above on p. I5o, and it will be understood why, when the ligament is severed in operations giving access to the lacrimal sac, an ectropion or falling away of the lower lid margin from the globe will not result if the pars lacrimalis muscle behind the sac be not interfered with (Whitnall, IgI3).

The lower half of the sac presents simpler relations, since it lies altogether below both medial palpebral ligament and pars lacrimalis muscle, as seen in Fig. 70, p. I46, and Fig. I20 above. In front lie the skin, orbicularis muscle, septum orbitale, and lacrimal fascia; and access to the sac can readily be obtained from this aspect by exposing and separating (spanning) the muscle fibres along its line, and, if need be, by upraising the ligament. Pathological swellings or diverticula of the sac (Fig. I29, p. 247) are most commonly present in this region, that is, below the medial palpebral ligament. On the lateral side the inferior oblique muscle may arise partly from the fascia covering the sac, and in this region there enter the latter branches from the inferior palpebral and infra-orbital arteries; otherwise on this side and posteriorly it is in contact with the orbital fat.

The guides to the position of the lacrimal sac are the medial palpebral ligament, made conspicuous by drawing the lids laterally, and the anterior lacrimal crest, which can be felt with the finger-tip along the line of the upper part of the naso-jugal fold ; there is sometimes a tubercle present at the base of the crest (Fig. 28, p. 65). It is often easy to identify a prominent posterior lacrimal crest as well as an anterior by placing the pulp of the finger flat against the side of the nose in this region, or the finger nail can be pressed into the hollow of the fossa. It is important to 
relations to the bony naso-lacrimal canal in which it is lodged, and these characters have been described on p. 74 . The form is also illustrated by Figs. 3I, 34, II7, II9, I22. The direction taken by the duct in its downward passage is described on p. 77 .

The lower or meatal part of the duct lies buried in the mucous membrane of the lateral wall of the inferior meatus,

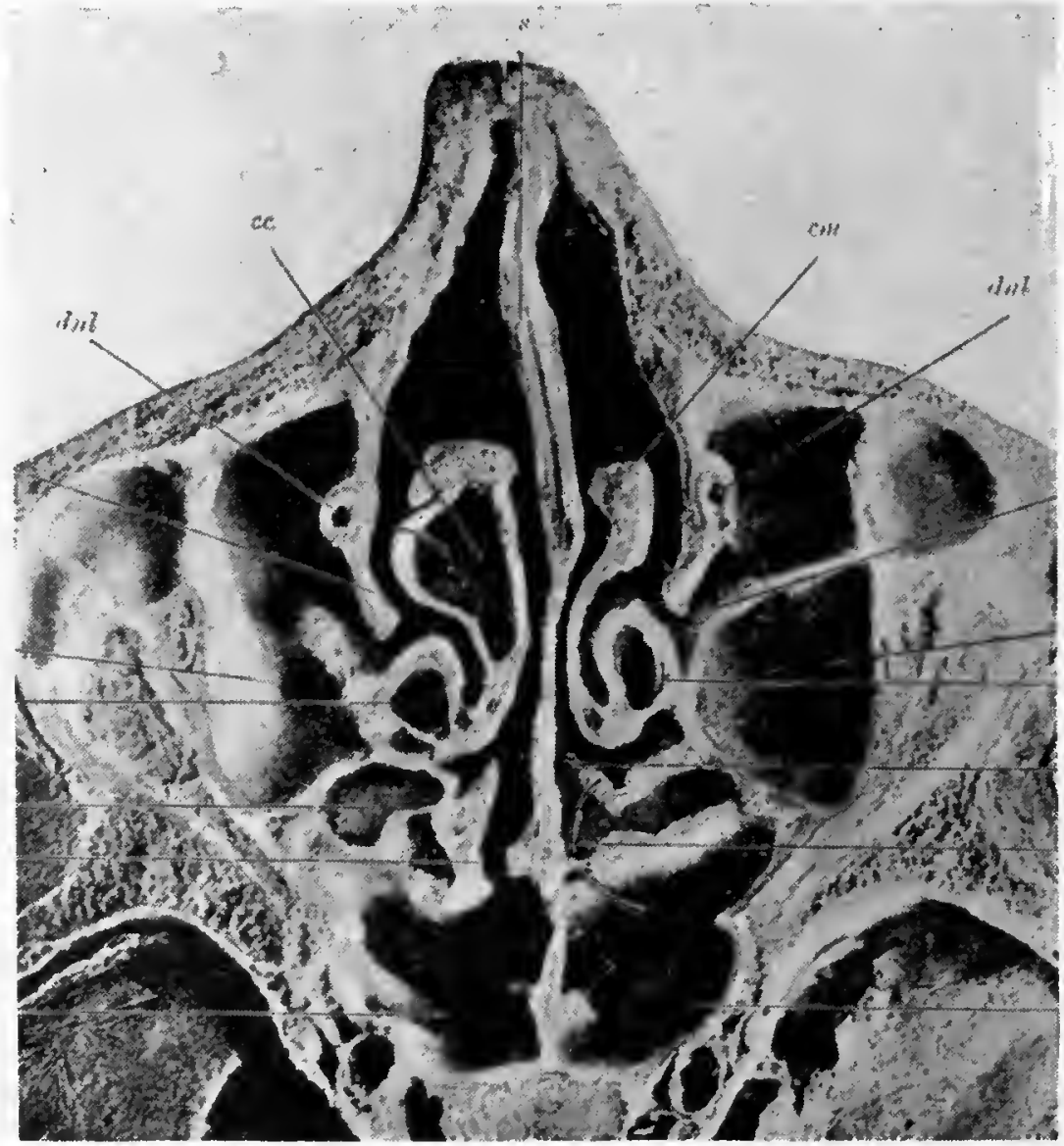

FIG. I23.-Horizontal section through the Nasal Cavities of an Adult to show the bulging (lacrimal protuberance) of the naso-lacrimal canal $\left(d n l_{0}\right)$ into the maxillary air sinus. (From Onodi, r913.)

$c c .=$ the bulla ethmoidalis; $c m .=$ the middle concha.

and is therefore related on the one side to the maxillary sinus, into which it may bulge, and on the other to the meatus itself. It is the extent of this part that chiefly determines the variation in length of the whole tube.

The inferior opening, meatal aperture, or ostium lacrimale of the naso-lacrimal duct is most variable in shape and position, and is often difficult to discover by inspection. In the foetus up to eight months, often up to birth, and sometimes even in the new-born child, the duct is closed below by. a thin partition formed by the approximation 
of the lining membrane of the nose to that of the duct, the ostium being subsequently formed by its dehiscence. The resulting opening, which is rarely as large as the lumen of the duct, may be round or linear, vertical or transverse, punctiform or even duplicate. Holmes found it nearly always slit-like and very fine; it may be guarded by a valve, a flap, or a diaphragm.

It may be followed by a gutter or groove continuing the line of the duct downwards (the "lacrimal sulcus" of Verga); sometimes this gutter is bridged across by threads of mucous membrane (Henle), or even completely closed over, so that the duct is continued down beyond the ostium, and the blind end may reach as far as the incisive foramen on the nasal floor (Bochdalek) ; in such cases a probe might be passed along this lower continuation of the duct without opening up the ostium (Fig. II6).

Aubaret (IgIO) emphasises the importance of the degree of permeability of the opening and its influence upon both the shape and the lining membrane of the duct and sac; out of I39 specimens examined by him, 8o presented visibly open orifices and 59 appeared closed; but he concludes that whatever be the shape, dimension, or degree of opening, the ostium is permeable to the air of the nose, and if valves are present they are ineffectual. A widely patent opening would allow of a raising of the air pressure within the duct and sac each time the nose is blown, with a consequent expansion in the direction of least resistance during development and possibly a resulting evolution of the lining mucosa into an atrophic type; the greater the degree of permeability, the more will the air pressure affect the calibre of the duct. He found the regularly cylindrical types of tube correlated with a small inferior meatal orifice, whilst the irregular types all had permeable openings, and in those cases where the whole duct was uniformly enlarged there was a wide ostium. The writer's cases corroborate these observations, though a fine slit-like opening was more commonly found. It would therefore appear possible to ascertain to some extent the probable condition and size of the duct by inspection of the opening. 
The position of the ostium is variable. Swerschewsky (IgIo) described three positions :

(i.) In the roof of the inferior meatus of the nose, coinciding with the aperture of the bony naso-lacrimal canal, and found in 45 per cent. There was a fold guarding the opening in 27 per cent of these, a diaphragm in I2 per cent, a circular orifice in 6 per cent. Rochon-Duvigneaud, how-

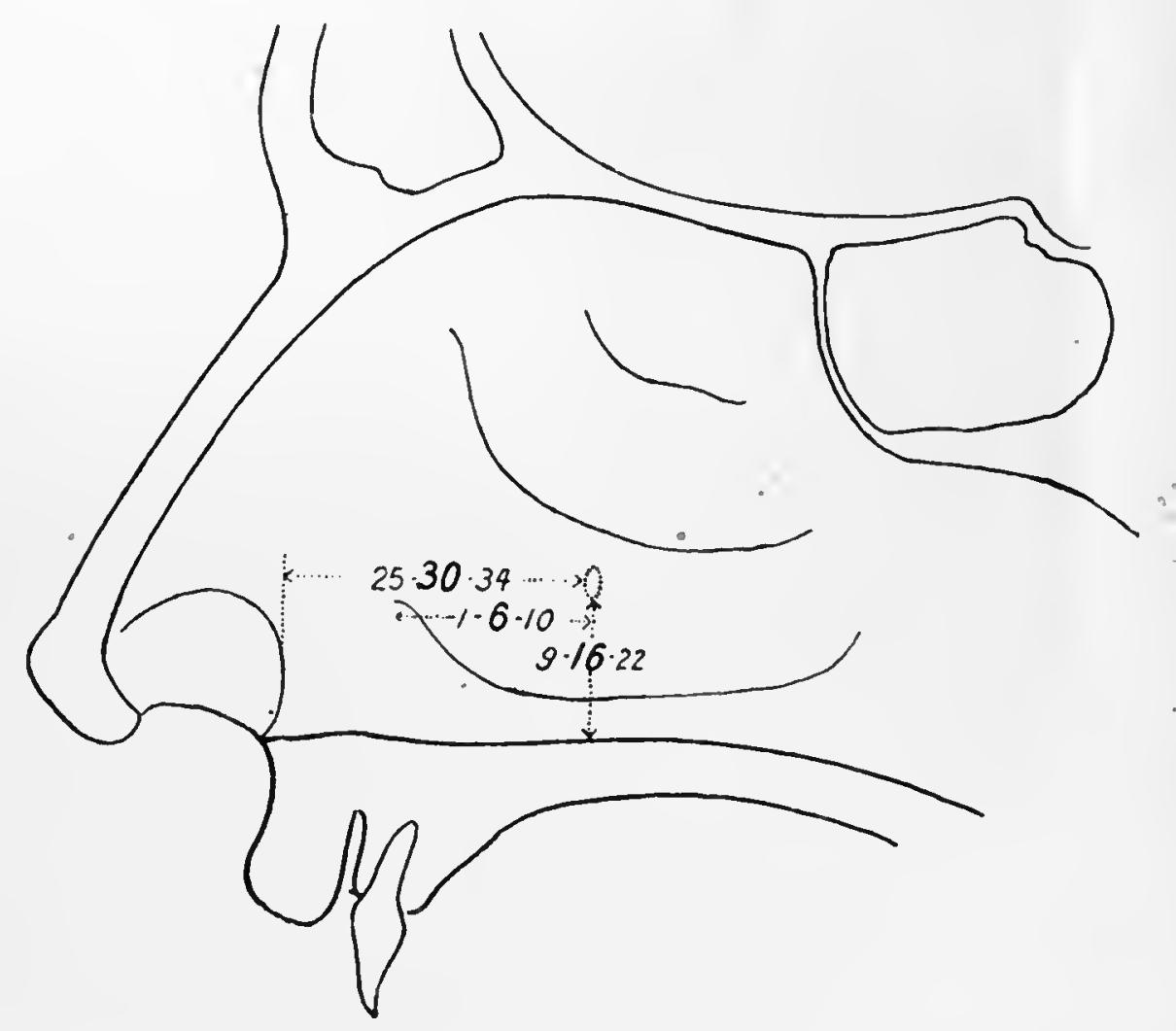

FIG. I 24. - Diagram to illustrate the position of the opening of the Naso-Lacrimal

Duct (ostium lacrimale) on the lateral wall of the inferior meatus as if seen through the overhanging inferior concha. The figures (taken from the measurements of Holmes, 1919) show in millimetres the average of 50 specimens in heavy type, with the extremes on cither side in lighter type.

ever, found this position to occur only in 5 per cent of cases.

(ii.) Opening below this point on the side wall of the interior meatus in 49 per cent, the generally described position. The aperture was a wide one in I8 per cent, a half-open canal in 16 per cent, and a small groove in 15 per cent of these. This last was the condition considered by him to be most favourable to disease.

(iii.) Opening far in front or behind as a small canal in 3 per cent.

The generally described position is at about the middle of the side wall of the meatus, $30 \mathrm{~mm}$. or $\mathrm{I}_{4}^{\frac{1}{4}}$ inch behind 
the lateral margin of the anterior nares, and opposite the junction of the first and second quarters of the attached border of the inferior concha which overshadows it. The detailed measurements of its position from various points are shown in the above diagram made from the figures given by Holmes (I9I9) after an examination of fifty specimens.

It would appear that Winslow's location of the position by intersecting lines, one drawn horizontally from the inferior border of the ala of the nose, the other vertical and passing through the second molar tooth or between the second and third molars, can give only approximate results.

The dimensions of the lacrimal sac and naso-lacrimal duct correspond with those of the bony fossa and canal given on p. 73 ; the dimensions of the sac are a little less than those of the bony fossa, being on an average $\mathrm{I} 2 \mathrm{~mm}$. ( $\frac{1}{2}$ inch) long and from 4 to $8 \mathrm{~mm}$. ( $\left.\frac{1}{4} \mathrm{inch}\right)$ broad; the lateral width differs according to the part of the sac measured, but is about 2 or $3 \mathrm{~mm}$. in the mid-region. The inter-osseous part of the duct measured in length on an average $12.4 \mathrm{~mm}$. $\left(\frac{1}{2}\right.$ inch), the meatal portion $5.32 \mathrm{~mm}$. ( $\frac{1}{4}$ inch), with extremes of from I to I2 mm. in sixty observations made by Aubaret. Onodi gives a total length of the tube as about $30 \mathrm{~mm}$. ( $\mathrm{I}_{4} \frac{1}{\mathrm{inch}}$ ), comprised by the sac of $\mathrm{I} 2-\mathrm{I} 4 \mathrm{~mm}$., intra-osseous part of duct Io $\mathrm{mm}$., meatal portion $5 \mathrm{~mm}$., as illustrated in Fig. II6, p. 225. A further measurement of practical value is the distance that the upper or orbital opening of the duct lies above the nasal floor, since the figure includes the extreme extent of any meatal part of the duct. Power (I886) found an average distance of $27.4 \mathrm{~mm}$. in 292 European skulls; Gérard (I907) an average of $23.3 \mathrm{~mm}$., with extremes of $25 \mathrm{~mm}$. and $40 \mathrm{~mm}$. in 69 skulls, and he emphasises the importance of retaining this highest extreme of $40 \mathrm{~mm}$. ( $\mathrm{I}_{2}$ inch) as the least length that should be given to that part of the catheter destined to traverse completely the whole duct.

The external calibre of the duct will be in harmony with, and vary according to, that of the canal, as described on 
p. 75 , but the lumen presents greater variations, since not only is the lining mucous membrane commonly thrown into folds and ridges (as illustrated by the second type in Fig. II3), but, like the nasal mucosa, may vary from the hypertrophic condition found in chronic catarrh to the atrophic form of old age. Normally the lumen of both sac and duct is a mere cleft, but will take a probe of $3.5 \mathrm{~mm}$. diameter (Treves). The normal capacity of the sac is given by Hyrtl as 20 c.mm., but Arlt found that it could be injected until it measured $4 \mathrm{~mm}$. in transverse width, with a capacity of 120 c.mm. Aubaret, again insisting upon the effects of a permeable lower opening, describes narrow ducts of from $\mathrm{I}$ to $3 \mathrm{~mm}$. in internal diameter and dilated ones of from 3 to $7 \mathrm{~mm}$., 4I out of 60 specimens being of the latter class; in the former the mucous membrane is thick (as in Fig. I27), in the latter thin (as in Fig. I2I). His fusible metal casts of the sac and duct illustrate well the variable morphology of the passages, but a slightly artificial dilatation of the sac is to be expected by this means. Theobald obtained an average transverse width of from 4 to $4.7 \mathrm{~mm}$. in ten ducts measured on the cadaver, slightly greater than the canals in the skull, and explained by the elasticity and yielding of the bones in the fresh condition. Onodi (I9I3) gives the following diameters (presumably internal) of the nasal duct in children: in the new-born infant between $\mathrm{I} \cdot 5$ and $2 \mathrm{~mm}$.; in one of 2 months, $2 \mathrm{~mm}$.; at 5 months, between 2 and $3.5 \mathrm{~mm}$.; at 8 months between 3 and $8 \mathrm{~mm}$.; in a child of II months it was $2 \mathrm{~mm}$., in a child of I 2 months, $3 \mathrm{~mm}$. ; in a child of 14 months, $\mathrm{I} .5 \mathrm{~mm}$. ; in one of 3 years, $2.5 \mathrm{~mm}$. The average diameter, therefore, in young children is about $2 \mathrm{~mm}$.

Internal Configuration of the lacrimal passages.-In the new-born the lining mucous membrane of the passages is utricular, irregularities being left in the walls after disintegration and absorption of the solid epithelial cord from which they are developed. In the adult most of the crypts and folds disappear, but some persist, and if correlated with a patent lower meatal orifice may develop under 
the influence of a raised air-pressure into so-called valves and sinuses; on the other hand, they may be occasioned by a gradual distension due to accumulation of tear fluid and mucus, with an insufficiently clear lower passage. The folds are of no special structure or anatomical regularity, but occasionally some are sufficiently well developed to be termed "valves," though their rôle as such is doubtful.

They have been specially studied by Aubaret (I908), and the positions they occupy and the names by which they have been dignified are shown in Fig. I25; they appear, however, most inconstant, as for example the valve in the middle of the naso-lacrimal duct (Taillefer), which is said to occur only in 6 per cent of cases. The most important and most frequently developed fold is one situated at the meatal opening of the naso-lacrimal duct, the plica lacrimalis or valve of Hasner, though previously described by others; it is the remains of the foetal membrane closing the lower end of the duct, and a blocking and distension of the tube may be caused by accumulation of epithelial débris above it ; when present in the adult it is rarely efficient as a valve, since incompetency was found experimentally by Aubaret in 90 per cent of cases. On the other hand, Bert (quoted by Aubaret) found that coloured fluids injected into the nose escaped from the lacrimal puncta only three times in eighteen experiments, whereas direct injections into the duct invariably appeared at these points, showing that the valves above Hasner's were always incompetent. - Passages of the enlarged atrophied type associated with widely open ostia would explain the instances recorded of smokers who could cause fumes to escape from the puncta. From a clinical point of view, a paper by Tartuferi (I902) on catarrhal conditions of the passages, well illustrated by microscopical sections showing various folds, is here worthy of reference.

The folds are considered to be the usual cause of obstruction to the course of the tears or the passage of a sound; and in connection with this, it may be of interest to summarise the possible natural obstacles to the passage of a probe along the lacrimal passages (Gérard, I907): 
(a) Osseous.-Accentuation of the angle between the long axes of the lacrimal fossa and naso-lacrimal canal, such as occurs when the anterior nares is narrow; the development of a spur at the base of either the anterior or posterior lacrimal crest may narrow the upper aperture of the canal, or the presence of a well-developed hamular process may

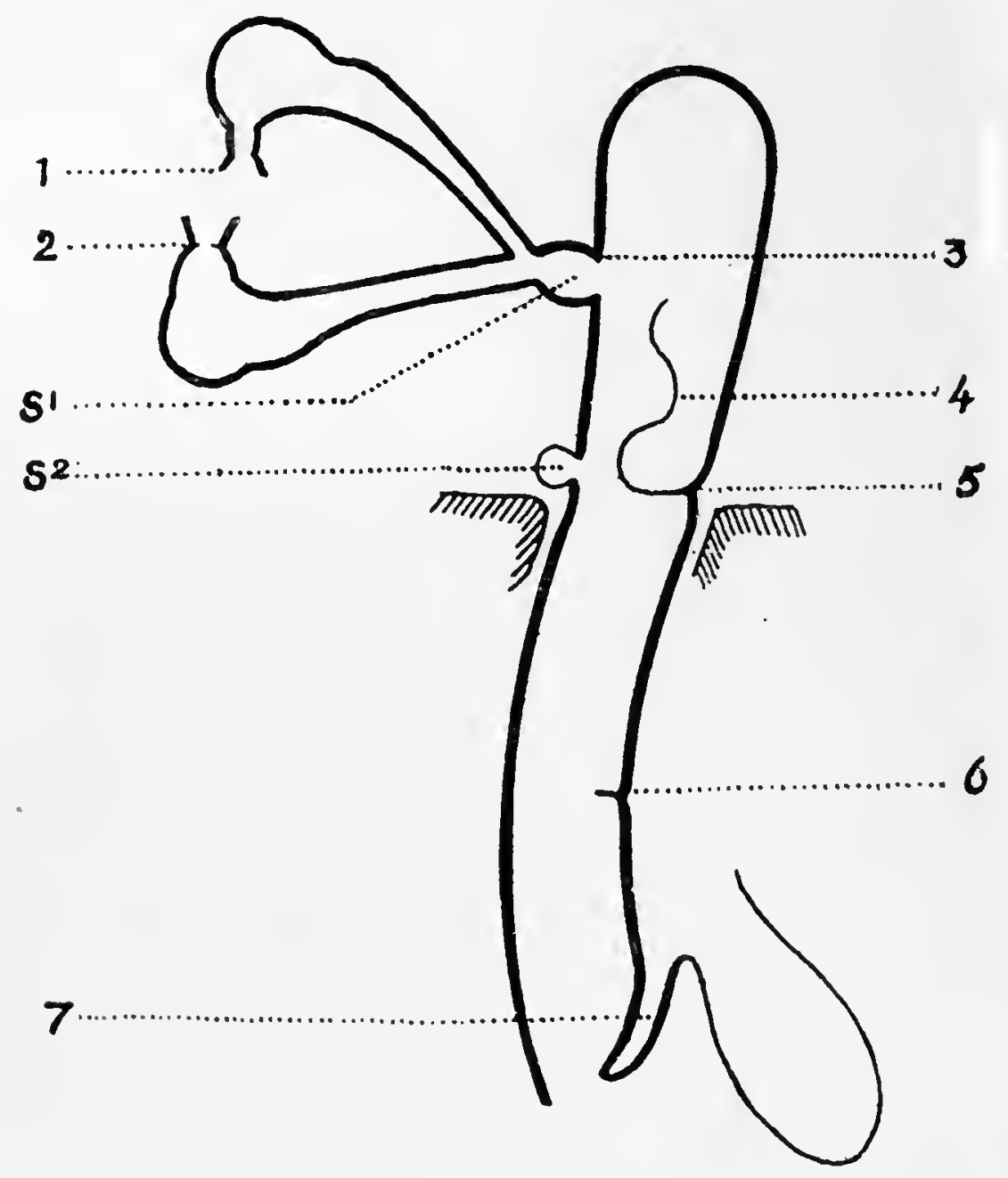

FIG. I25.-Diagram to show the position of the so-ealled Valves of the NasoLacrimal Passages (after Aubaret). The valves are numbered as follows, with the names by which they are known:

$\mathrm{I}=$ Bochdaleck ; $2=$ Foltz; $3=$ Rosenmuller or Huschka ; $4=$ spiral valve of Hyrtl (which by an error is wrongly placed in the diagram: it should lie in the duct, not the sac, and:end below in valve 6 , not 5 ) ; 5 , the valve of Krause or Béraud ; $6=$ Taillefer ; $7=$ Horner, Hasner, Bianchi, or Cruveilhier, the plica lacrimalis; $\mathrm{S}^{1}=$ the sinus of Maier; $\mathrm{S}^{2}=$ the sinus of Arlt.

The lacrimal sac is shown in the conventional dilated condition.

raise its level ; the canal may be constricted about its middle (see p. 76) ; a prominent supra-orbital ridge may throw the upper end of the probe too far forwards.

(b) Membranous.-Mucous folds in the canaliculi, dilatations or sinuses of the sac, or valves in the duct, may be sources of difficulty; the ostium may be guarded by a diaphragm; the point of a probe may pass beyond 
the meatal opening and lie in a blind continuation of the duct.

The Structure of the Lacrimal Passages.-They are essentially formed throughout by a mucous membrane continuous with the conjunctiva at the lacrimal puncta, and with the nasal mucosa at the inferior orifice of the nasolacrimal duct (Fig. II2, p. 220). This continuity of the membrane is of considerable clinical importance, since in Kuhnt's experience disease of the lacrimal passages has a nasal origin in $93^{\circ} 7$ per cent of cases, though micro-organisms of conjunctival origin may cause inflammation of the lacrimal sac (Onodi, I9I3). This lining membrane is reinforced by elastic, connective, and muscle fibres in the canaliculi, by fibro-elastic tissue in the sac, and by cavernous erectile tissue in the duct. It differs from the conjunctiva in that its mucous tissue is rich in elastic fibres, is thicker, and more vascular ; it gradually assumes the characters of the nasal mucosa as it approaches the nasal cavity, and it shares the degree of atrophy or hypertrophy of that lining membrane.

The canaliculi are lined by an epithelium which is thicker here than elsewhere in the passages, stratified six to twelve layers deep on the surface, but cylindrical below; the presence of a basal membrane is disputed. Outside the epithelium of the canaliculi is a fibrous layer derived from the lacrimal fascia, which is reflected along them from the points where they pierce it; it is characterised by a rich development of elastic fibres, which spread out amongst the muscle fibres of the pars lacrimalis in which these channels are buried (Halben, I903; Fig. I26). Round the vertical part of the canaliculus and papilla the elastic tissue is denser and the connective tissue of these portions is continuous with that of the tarsal plates; round the puncta the tissue is almost sclerous. The muscle fibres surround the base of the papilla in sphincter-like form, as described on p. 224, and as figured below.

The lacrimal sac and naso-lacrimal duct are lined by two or more layers of columnar epithelium resting upon a basement membrane; cilia are said to be present by some 
authors (Schäfer, Quain, Tourneux, Santos Fernandos), but their presence is denied by others (Werncke, Halben, RochonDuvigneaud); goblet cells are present. There is a delicate dermis or tunica propria, infiltrated in the adult, though not in the new-born, by lymphocytes, which are often, though possibly abnormally (Parsons, Werncke), aggregated to form follicles. Krehbiel has several times seen a small lymph nodule in the wall of the sac immediately behind the point of entrance of the canaliculi. The outermost coat is

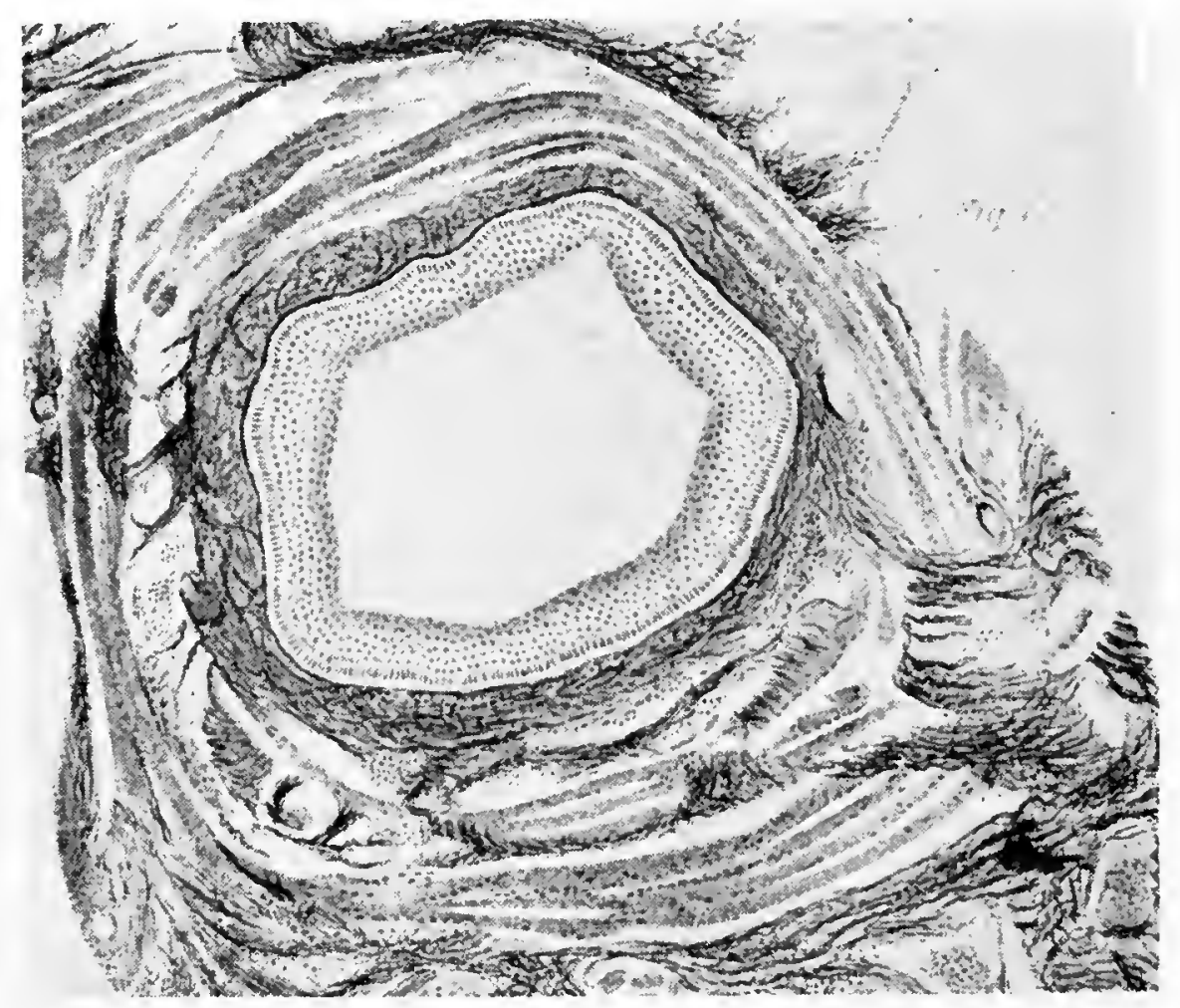

FIG. I 26. - Cross-section of base of vertical part of Lacrimal Canaliculus of threeyear-old child, showing the sphincter-like arrangement of muscle fibres constituting the musculus quadrangularis constrictor puncti lacrimalis, and also the large amount of fine elastic tissue surrounding its wall. (From Hallen, 1903.) Magnified.

of fibro-elastic tissue, containing a venous plexus especially well developed towards the meatal end of the tube, where it resembles the erectile cavernous tissue characteristic of the nasal mucosa with which it becomes continuous. On the whole, the elastic tissue is found to decrease from the puncta downwards, whereas the venous plexus and thickness of the walls increase. The presence of glands in the walls of the sac and duct is disputed; they have not been found either by Stanculeanu in the embryo or by Rochon-Duvigneaud in the adult, and such as have been described may be 
pathological formations or simple diverticula of the mucous membrane. On the other hand, definite mucous glands have been noted in normal sacs by Kuhnt, Arlt, Halben; and Werncke (I905), in a special investigation, found both composite and single tubular glands present in 8 out of I4 lacrimal sacs, whereas in I7 pathological sacs none were found; they were situated in the antero-medial wall near the summit, and contained mucous cells and crescents of Giannuzzi. Joerss (I905) found small serous glands, resembling those of Krause of the conjunctiva, in 8 per cent of cases in the submucosa of the fundus of the sac. The writer has found one instance of such in the lacrimal sac. Whilst the duct wall at its upper end can be separated from the lining periosteum of the canal in which it lies, as it passes downwards the two become more intimately blended to form a muco-periosteum, a close association which explains the

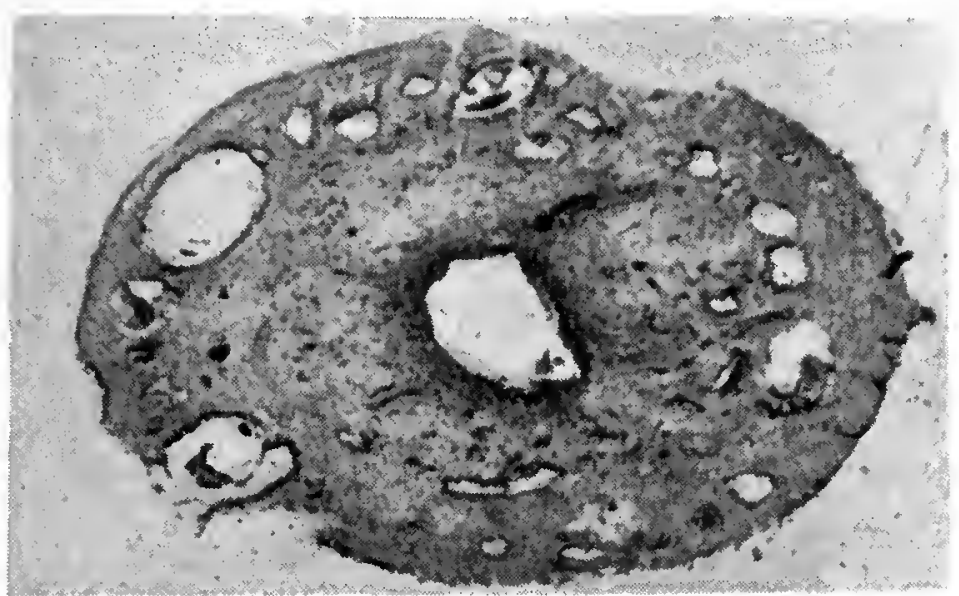

FIG. 127. - Transverse section through the middle of the Naso-Lacrimal Duct of an adult ; hypertrophied type of mucous membrane. The actual diameters of this specimen were: outside transverse, $4 \mathrm{~mm}$; ; antero-posterior, $6 \mathrm{~mm}$. ; inside transverse, $\mathrm{I} \cdot \mathrm{I} \mathrm{mm}$. $\times$ about 9. extension of chronic inflammatory diseases of the duct to the surrounding bone.

The Vessels and Nerves of the naso-lacrimal passages: The arteries are derived (i.) from the ophthalmic, the superior palpebral branch supplying the sac, the inferior palpebral the duct; (ii.) from the angular artery of the facial, which supplies both parts ; (iii.) from the infra-orbital artery, which in the forepart of the infra-orbital sulcus gives off a branch which sends twigs to the lower eyelid and inferior oblique muscle, then grooves or pierces the lateral margin of the upper orifice of the naso-lacrimal canal and supplies the sac and duct. The lower part of the duct receives a supply from the nasal branch of the spheno- 
palatine artery, which is a derivative, like the infra-orbital, of the internal maxillary artery.

The rich venous plexus which surrounds the duct, and to a lesser extent the sac, is connected above with the angular and inferior orbital veins and below with those of the nasal cavity, which latter drain through the spheno-palatine veins into the pterygoid plexus and internal maxillary vein ; venules pass from the plexus round the lacrimal sac through the lacrimal bone or its sutural lines into the mucous membrane of the anterior ethmoidal cells.

The lymphatic vessels from the sac accompany the facial vein in their course and drain into the submaxillary nodes (Fig. 86, p. I7I) ; those of the lower part of the duct join the lymphatic vessels of the inferior nasal meatus, which drain

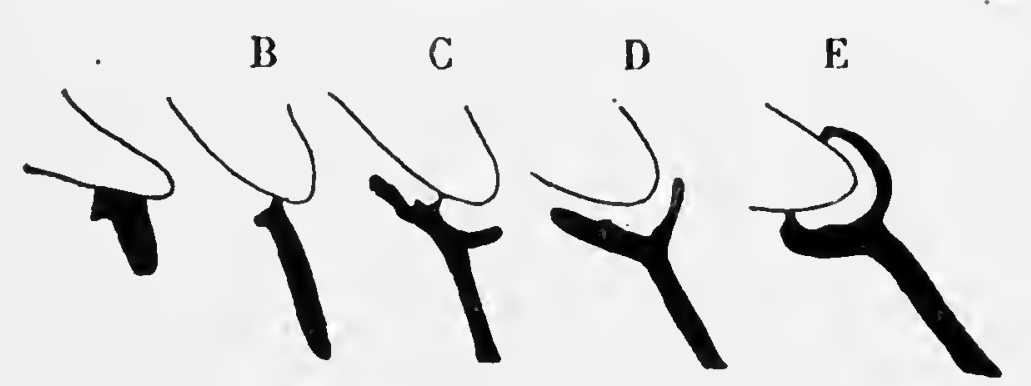

FIG. I28. - Schematic representation of the successive stages of development of the Lacrimal Passages to form the canaliculi. (From Matys, 1905.) both anteriorly towards the skin of the anterior nares and thence to the submaxillary nodes, and posteriorly to the retro-pharyngeal and deep cervical nodes (Most, I905).

The nerves of the sac are derived from the infra-trochlear branch of the ophthalmic division of the fifth nerve; the lower part of the duct receives a twig from the anterior superior alveolar branch of the maxillary division of the same nerve. There would appear to be some physiological relation, probably through the branches of the nerve, between the innervation of the lacrimal gland and that of the lacrimal sac, explanatory of the known clinical fact that destruction of the sac leads to suppression of the tear secretion; the epiphora of dacryocystitis is most likely caused by reflex irritation from the diseased sac.

Development and Abnormalities.-The lacrimal passages are developed along the line of the cleft between the lateral nasal and maxillary processes of the embryonic face (p. I83) ; 
they are formed, not by the cleft being primarily converted into a tube by the approximation and fusion of its boundaries, as was supposed until the observations of Born in 1876 , but by canalisation of a solid rod of ectoderm cells formed beneath the surface along this line. In later years the development has been an object of special study by many (Stanculeanu, Igoo; Rochon-Duvigneaud, Igoo; Monesi, I904; Matys, I905; Fleischer, I906; Cosmettatos, I906; Contino, I907; Lang, I9II; Schaeffer, I9I2). It may be described in two stages: a first, consisting in the formation of the solid epithelial rod; a second, the hollowing out of the rod to form the lacrimal passages.

The rod appears, after the cleft has become obliterated by the fusion of the processes which bound it, as a thickening of the deeper. layers of the epithelium along the line of junction, the first appearance being at a point corresponding to the medial part of the conjunctiva of the lower lid; the lower end elongates and sinks beneath the surface, the whole rod following and becoming surrounded by mesenchymal tissue, but retaining its contact with the skin longest at the point of origin. The first appearance has been seen in an embryo of $9.5 \mathrm{~mm}$. in length, and the rod was found completely separated from the surface in one of $15 \mathrm{~mm}$. The canaliculi are formed secondarily by buddings from the upper end of the rod, and reach the surface of the lid margins about the same time that the lower end of the rod reaches the nasal cavity, as has been seen in embryos of from 18 to $24 \mathrm{~mm}$. The inferior canaliculus appears to be the direct continuation of the main stem, the upper one being a secondary outgrowth. The inferior canaliculus in its growth upwards cuts off a part of the lid margin which is destined to form the lacrimal caruncle (Fig. 94, p. I86).

The process of canalisation begins by disintegration of the central cells, first in the middle of the lower canaliculus, as has been seen in an embryo of $35 \mathrm{~mm}$. long or at three months, then in the upper, and then in the lacrimal sac. In embryos of $6 \mathrm{cms}$. in length a lumen is established throughout the whole system, but is closed at the puncta and the inferior orifice of the duct; the former open in embryos 
of $I 3 \mathrm{cms}$. just before the eyelids separate towards the seventh month; the latter remains closed until the end of the eighth month, or even after birth. According to Monesi, after the sixth month the lower end of the duct is swollen by an accumulation of the debris of disintegrated cells, which eventually causes the perforation of the septum between it and the nasal fossa, and the remains of the septum form the plica lacrimalis.

The abnormalities are readily explained by irregularities in this mode of development. The puncta may be supernumerary, two (Steffan, Greeves), three (Chase), or even four (Majewsky) having been noted. Duplication of either canaliculus, but more often of the lower, has been observed (Cosmettatos, Coats, Merkel, Schirmer, Nielson, Michel, Schaeffer, Nance, etc.), but such developments must be rare, since Wicherkiewicz found only I case of double punctum and canaliculus in 60,000 patients examined, and according to Schoute, not more than 22 cases had been recorded in the literature up to Igor. On the other hand, cases are recorded of congenital imperforation of the puncta (Hawley), of non-development of the canaliculi (Onodi, Kusel), and of atresia of the whole duct or part of it (Geddes, Aubaret). The lacrimal sac may present a congenital fistula (Cosmettatos, Elschnig, Beer, Agnew, Hartridge, Casey Wood).

Diverticula or sinuses of the passages are also found. The usual site is on the lateral wall of the lacrimal sac, where the resistance to expansion is least. A small dilatation at the point of entrance of the canaliculi is common (Fig. II3, p. 22I), and is known as the "sinus of Maier." Another, called the sinus or " recessus of Arlt," bulges outwards from the sac just beneath the medial tarsal ligament; Aubaret (Ig09) states that he found the latter present in 24 out of 50 specimens (a proportion quite at variance with the writer's experience, though it is not clear in Aubaret's paper whether or no he includes dilated sacs in this category); an example is photographed in Fig. I29. Such diverticula or folds, if largely developed, may cause the appearance of a bilocular or double lacrimal sac (Bochdalek, 
Luschka, Vlacovich, Tartuferi, Aubaret, etc.). The nasolacrimal duct itself may be double throughout (Monesi), or it may present two inferior apertures. The various folds or valves of the passages have been described above.

For accounts of the lacrimal apparatus in domestic animals, papers by Lical (I9I9) and Rochat (I9I5) may be referred to, and an admirable study of the histological

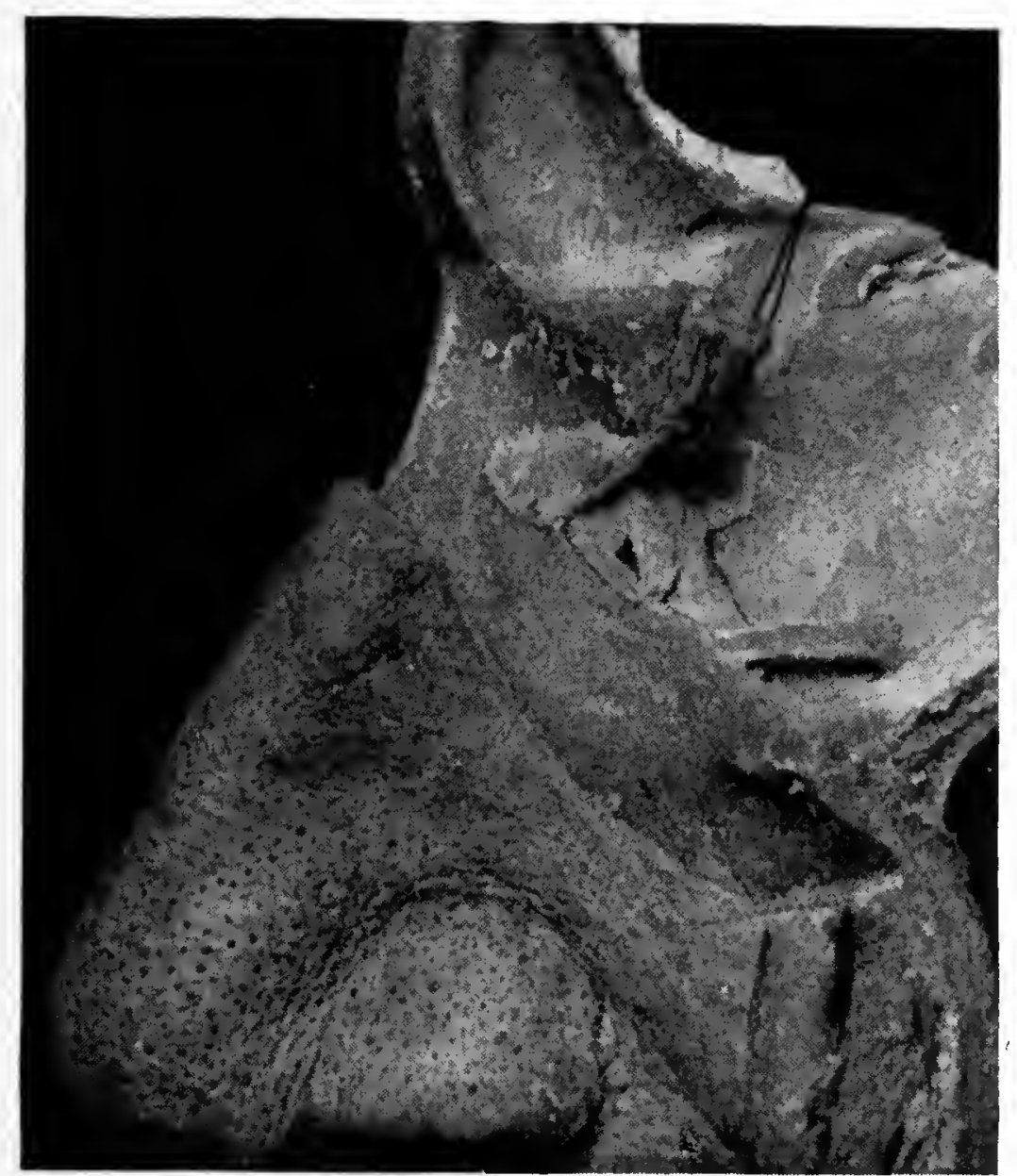

FIG. I29. - Dissection of forepart of Medial Wall of Left Orbit, to show relation of medial palpebral ligament (in front) and pars lacrimalis muscle (behind), both of which have been lifted upwards, to the lacrimal sac, which shows a wellmarked anterior dilatation or sinus of Arlt (opened). Natural size.

structure of these organs in the ox will be found in a paper by Sundwall (rgr6).

The Mechanism of the drainage of the tears from the conjunctival sac through the lacrimal passages has been the subject of much discussion. Normally the lacrimal gland secretes fluid in just sufficient quantity to replace that which is lost by evaporation; the fluid is prevented from running over the edge of the lower eyelid by the greasy lubrication of the tarsal glands, and is supposed to be 
induced to flow towards the medial canthus and collect in the lacus lacrimalis by a slight "spiral movement" of the lids in closure; more probably the direction of the flow is determined by the general contraction of the orbicularis muscle from its mobile lateral to its fixed medial side. For the tears to enter the lacrimal sac two conditions are essential : first, the puncta must be directed towards the floor of the lacus lacrimalis, that is, there must be no paresis of the marginal fibres of the orbicularis, or epiphora will result ; second, the puncta must retain their capillary action. The canaliculi are elastic-walled tubes, and in the act of winking they are shortened and widened by the pull of such fibres of the pars lacrimalis muscle as are connected to the walls of their horizontal portions (Halben, I903), and consequently, the fluid may be sucked into them. The vertical portion of the canaliculus is too dense to be compressed by the action of the quadratus muscle surrounding its base in light closure of the lids, and the lumen always remains open in normal physiological conditions, but in blepharospasm or forced closure the muscle does act as a sphincter; the lumen is then effaced, and the tears are seen to accumulate in the lacus lacrimalis (Halben, RochonDuvigneaud). Frieberg (IgI8) and West (I9I8) both consider that the canaliculi play the leading part, the tears being sucked in by capillarity and then expelled into the sac by their occlusion in closure of the lids.

It is as regards the action of the lacrimal sac, and especially of the pars lacrimalis or Horner's muscle in relation to it, that the most widely divergent views are expressed. The muscle is said either to compress the sac or to dilate it. It will be realised that, according to the above description of the sac and its relations, normally it is neither in a position nor in a condition to be compressed; its position is one of lying flattened in the lacrimal fossa covered over by the lacrimal fascia, and no part of the orbicularis muscle is actually inserted into it; the arc described by the pars lacrimalis muscle may be slightly flattened in contraction, but by so much less would the muscle press upon the sac since it lies behind the latter; the medial palpebral ligament, 
bound to the fore and upper part of the lacrimal fascia, will be straightened and pulled away from the sac on contraction of the orbicular fibres which arise from it (Schirmer, Fuchs). The normal condition of the sac, flattened from side to side so that its lumen is a mere cleft, also allows of no further compression. On the other hand, the sac is both in a position and in a condition that readily admits of dilatation, as is readily seen in pathological conditions and by experimental injection. It has been pointed out above that the area of attachment of the pars lacrimalis muscle frequently encroaches upon the fascia covering the sac, and if the two be adherent, contraction of the muscle will dilate the sac; indeed, in one preparation of a horizontal section through the parts where the muscle fibres were so disposed, the slightest traction upon them made the cleft of the sac visible, and, moreover, in cases of epiphora due to paralysis of the orbicularis it has been observed that tears can be made to enter the sac by drawing the lids laterally. The importance of the pars lacrimalis muscle is further shown by the fact that the first symptoms of a facial paralysis may be a complaint of epiphora even before the puncta become everted (Kalt, I903). Scimemi (I892) found experimentally that the capacity of the sac is increased by 2 c.mm. in each physiological closure of the lids, by Io c.mm. with moderate effort, and by as much as 30 c.mm. by forced effort, and that an upward and outward traction of the upper eyelid will increase the capacity much more than the most energetic closure of the lids will do; further, clinical observations have shown that a drop of fluid at the entrance to a fistula of the sac is aspirated in winking (Kalt). On such grounds, therefore, the lacrimal sac must be considered to be dilated and not compressed in the act of winking. It then regains its normal and reduced capacity owing to the elasticity of the walls, and will drive its contents onwards into the wider channel, the naso-lacrimal duct, rather than backwards along the narrower canaliculi. That the sac must have some action is shown by the fact that in atony of this structure the proper conduction of tears is arrested (Fuchs). 
Finally, as regards the passage of the tear-fluid down the duct into the nasal cavity, the flow is favoured by gravity, by the passage from narrow to wide channels, and perhaps by inspiration of air through the nose, since one sniffs in the act of weeping. Permeability of the duct is not a necessary condition for the absorption of tears, since the sac will fill in spite of complete obliteration of the passage below it. The so-called valves of the lacrimal passages play no part in occasioning the downward flow.

To sum up, then, it is probable that the capillarity of the puncta primarily suffices to draw off from the conjunctival sac any slight surplus of fluid left over from evaporation; that the canaliculi, shortened and dilated in the act of winking, can assist the drainage; and that the lacrimal sac participates in the removal of excess fluid by aspiration, being slightly dilated in the act of winking, and markedly so in forced closure of the lids; the elastic rebound of the sac to its resting dimensions drives the fluid into the nasal duct, whence the flow into the nasal cavity is directed by gravity. 


\title{
PART III
}

\section{THE CONTENTS OF THE ORBIT}

\author{
I. The Eyeball. \\ 2. The Muscles. \\ 3. The Fascia and Fat. \\ 4. The Vessels. \\ 5. The Nerves.
}

General Arrangement. - The eyeball is situated in the anterior and roomiest part of the orbit, of which it occupies about one-fifth the volume; from its posterior surface the optic nerve emerges and passes backwards to leave the apex of the space by the optic foramen; the nerve lies in the midst of the four recti muscles, which, arising close around the foramen, diverge as they pass forwards to be inserted on to the eyeball. Within the "cone" so formed by the muscles lie, as well as the optic nerve, their own nerves (third and sixth), the naso-ciliary nerve and ciliary ganglion, the ophthalmic artery and vein, together with many of their branches. Outside the cone of muscles lie the superior oblique and levator palpebrae superioris muscles and the fourth, frontal, and lacrimal nerves. Beneath the eyeball lies the inferior oblique muscle, above it is the reflected tendon of the superior oblique muscle, whilst on its upper and lateral, side is placed the lacrimal gland. The rest of the orbital space, both within and without the cone of muscles, is completely and compactly filled with the orbital fat, from which the eyeball is separated by a fascial envelope, the fascia bulbi or capsule of Tenon.

I. The Eyeball (bulb or globe of the eye, bulbus oculi).- 
The eyeball is here considered only as regards its external

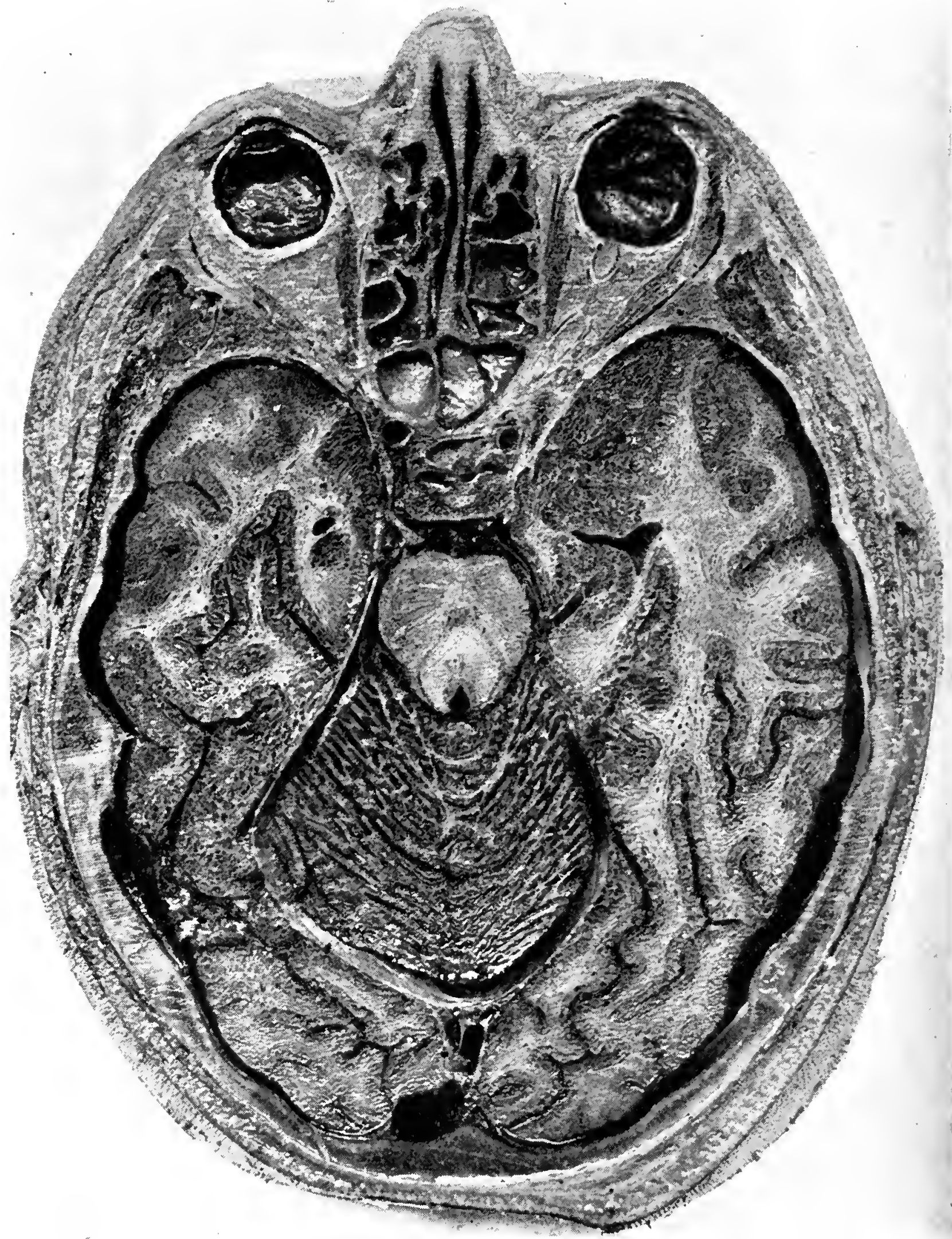

FIG. I30. - Horizontal section of Adult Male Head to show the relative position and relations of the orbits and their contents. Compare with Figs. 3 and 15. From the McGill Anatomical Museum. $\times \frac{2}{3}$.

configuration and relations, that is, the organ as a whole is described; for the gross structure the reader is recom- 
mended to consult The Anatomy of the Human Eye, as illustrated by Enlarged Stereoscopic Photographs (Thomson, Clarendon Press, I9I2) ; the development and histological structure will be found fully described in the "Organe de la Vision" (Druault, in Poirier's Traité d'anatomie humaine, Paris, IgI2) ; or in the articles by Merkel and Kallius and Pütter in the Graefe-Saemisch Handbuch der Augenheilkunde, or by Piersol in Norris and Oliver's System of Diseases of the Eye.

The eyeball has the form of a sphere, but not a strictly symmetrical one, being slightly flattened from above downwards (forming an "oblate spheroid"), with the contour interrupted by the bulging of the cornea in front. It may be described as composed of segments of two spheres, an anterior corneal segment with a radius of $8 \mathrm{~mm}$., and a

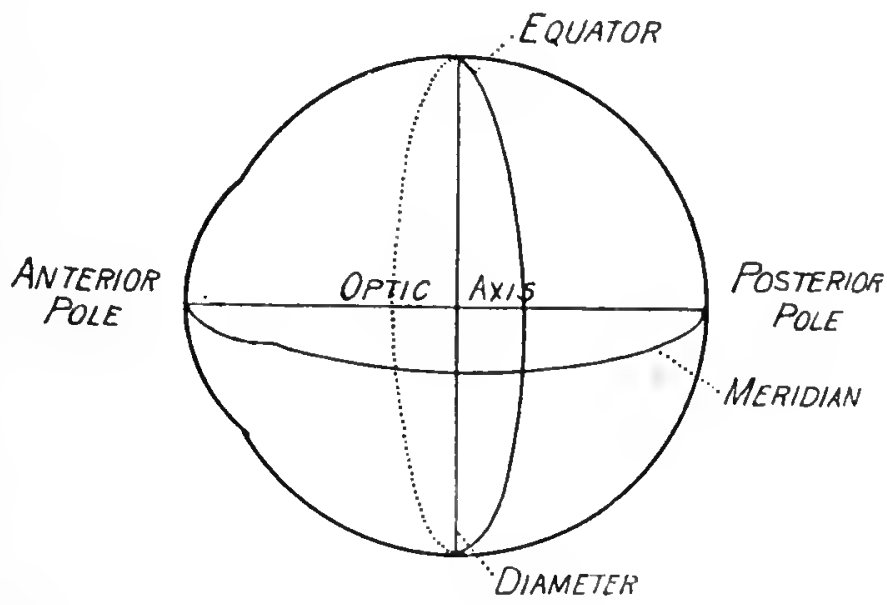
posterior scleral segment of $12 \mathrm{~mm}$. radius; the corneal area extends

over about one-sixth of the whole bulbar surface. Where the two areas join, that is, at the sclero-corneal junction or limbus corneae, is a shallow groove, the sulcus sclerae. The central points of the curvatures of the corneal and scleral spheres are called the anterior and posterior poles (polus anterior vel posterior) of the globe; and a straight line joining them represents its axis (axis oculi externa vel interna); a line encircling the globe midway between the poles is the anatomical equator (aequator), and it divides the surface into two hemispheres, anterior and posterior; a line surrounding the surface of the globe and passing through both poles is a meridian, and crosses the equator at right angles; the antero-posterior length, height, and width of the globe are expressed by sagittal, vertical, and transverse or horizontal diameters respectively. 
The "visual axis" (axis optica) passes from the fovea centralis of the retina through the "point of rotation" of the globe, and cuts the cornea rarely at its mid-point but usually slightly to its nasal side; it therefore does not exactly coincide with the axis of the globe. When the visual axis is directed straight forwards, the axis of the cornea presents a deviation from this line of $5^{\circ}$ laterally and $2^{\circ}$ below; the long axis of the orbital cavity deviates still further. from the visual axis, being from $23^{\circ}$ to $25^{\circ}$ lateral and $I 5^{\circ}$ to $20^{\circ}$ below it (Druault, I9I2); the visual axes are roughly parallel to one another, at most forming an angle of $10^{\circ}$ (Testut), whereas the orbital axes enclose an angle of about $45^{\circ}$, as already noted on p. 93.

Most eyeballs vary slightly from the schematic form; the most important variations are those where the length of the optic axis is either less (hypermetropic globe) or greater (myopic) than the normal (emmetropic), since in such eyes, when accommodation of the lens is completely relaxed, the image of a distant object falls respectively either behind or in front of the retina instead of exactly upon it.

The dimensions of the normal globe vary (but not beyond one millimetre) according to the findings of different authorities. The figures in millimetres given by Sappey (and quoted by Dwight, Druault, and Baker) from 26 adult eyes (I4 male, I2 female) measured from 24 to 36 hours after death are as follows:

$\begin{array}{lccc}\text { Diameter. } & \text { Antero-Posterior. } & \text { Transverse. } & \text { Vertical. } \\ \text { Maximum } & 26 \cdot 4 & 27^{\cdot 1} & 25^{\circ} \\ \text { Mean } & 24^{\circ} \cdot 2 & 23^{\circ} & 23 \cdot 2 \\ \text { Minimum } & 22 \cdot 9 & 22 \cdot 2 & 22 \cdot 2\end{array}$

Each diameter is on an average five to six-tenths of a millimetre greater in the male than the female. Merkel (IgOI) gives

$$
\begin{aligned}
& 24 \cdot 3 \text { (external axis) } 23 \cdot 6 \\
& 22 \cdot 5 \text { (internal axis) }
\end{aligned}
$$$$
23 \cdot 3
$$

The last-named author gives, in addition, numerous other dimensions of the parts of the globe as found by himself, and 
also by Quain, Rauber, Schwalbe, and Weiss; the mean of the combined above measurements of all these authors is

Antero-Posterior.

$24 \cdot 15$
Transverse.

$24 \cdot 13$
Vertical.

$23 \cdot 48$

The average diameters of the eyeball in the new-born, as found by Weiss (I897) in I4 observations, are

$$
\text { I6.40 } \quad 16.00
$$

I $5 * 40$

and he found the eyeball at this period to be hypermetropic in 92 per cent. Weiss gives the comparative diameters and also the circumferences at various ages from birth to adult ; and Seefelder (I908) has made a very complete series of measurements of the embryo and infant eyeball.

The cornea has an average horizontal diameter of I2 $\mathrm{mm}$. and a vertical one of II mm. (Druault).

The weight of the eyeball varies in individuals, but may, be considered to be about 7 grammes; Sappey estimated it at 7 to 8 grs.; Testut at $7 \cdot 14$ grs. on a mean of ten eyes taken from the cadaver; Weiss at $7 \cdot 45$ grs. on a mean of 5 adult emmetropic eyes, with weights of $2 \cdot 29$ grs. at birth, 4.05 grs. at one year, and from 5.87 grs. to $6.50 \mathrm{grs}$. at from I3 to I5 years; the volume is about $6.5 \mathrm{c} . \mathrm{mm}$., with a specific gravity of I·077 (Schäfer).

The Structures attached to the Eyeball. - The optic nerve emerges not exactly from the posterior pole, but a little medial to and below it, the central point of the nerve lying $3 \mathrm{~mm}$. medial to the vertical meridian and I $\mathrm{mm}$. below the horizontal meridian (Fig. I32). Round the nerve exit in an irregularly scattered ring are the points of entrance of the minute ciliary

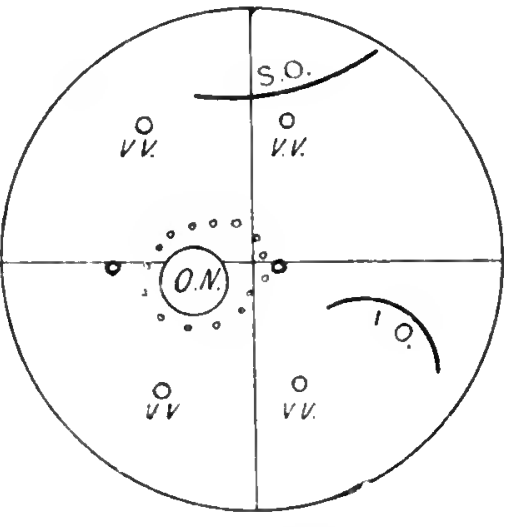

FIG. I32.- Scheme of the Eyeball seen from behind, to show the position of the various structures attached to it. (After 'Testut.) $\times 1 \frac{1}{2}$.

O. N. = the optic nerve, surrounded by a ring of ciliary nerves and vessels, with a long posterior ciliary artery on the horizontal meridian on either side of it; V.V. = the four zenae z'orticosae; S.O. and $\mathrm{I.O}=$ the lines of insertion of the superior and inferior oblique muscles respectively.

nerves and short posterior ciliary arteries; the long posterior ciliary arteries pierce the globe one on each side of the horizontal meridian and a little distant from the optic 
nerve; four venae vorticosae emerge on or a little behind the equator, and the anterior ciliary arteries enter just behind the sulcus sclerae. The four recti muscles are inserted by tendons into the globe in front of the equator, and the two oblique muscles behind this line and on. the lateral side. The fascia bulbi or capsule of Tenon is attached to the globe in front immediately behind the corneal margin, and at this point is fused with the bulbar conjunctiva, the two being closely applied to the anterior third of the bulb; the capsule closely envelops the entire scleral part of the globe, being united to it by a very delicate loose connective tissue, and terminates behind round the site of emergence of the optic nerve ; it must consequently be pierced by all the above-named structures which are attached to the globe, and it becomes fused with the fascial sheaths of the muscles where they enter it.

The relation of the eyeball to the orbit is best studied in horizontal and vertical views, as shown in Figs. I30, I55, and Fig. 55, p. I24. As already noted, it occupies about one-fifth of the cavity, lying in its roomiest forepart and slightly nearer the upper and lateral sides, being nowhere in contact with the walls.

The summit of the cornea is normally just touched by a ruler held vertically against the superior and inferior orbital margins, but the prominence varies in individuals and according to age and state of health; it thus may vary from I2 $\mathrm{mm}$. in front to Io $\mathrm{mm}$. behind this plane, and in exophthalmic goitre may even lie $24 \mathrm{~mm}$. in front (Cohn); indeed, Ambialet (I905) finds that in 85 per cent of living cases the protrusion is positive, that is, in front of the vertical axis; it is more pronounced in infants. The lateral orbital margin, on the other hand, is so recessed that half of the eyeball is here exposed, as is well shown in Fig. I33, and a straight line passing from this margin to the anterior lacrimal crest would traverse the globe behind the ora serrata of the retina on the one side and emerge at the junction of the ciliary body and iris on the other. A strong medial rotation of the globe will almost bring the macula lutea in line with this margin (Henke). The distances 
which separate the equator of the globe from the orbital walls are: $4 \mathrm{~mm}$. from the roof, $4.5 \mathrm{~mm}$. from the lateral wall, $6.8 \mathrm{~mm}$. from the floor, and $6.5 \mathrm{~mm}$. from the medial wall. The mid points of the two pupils lie from 58 to $60 \mathrm{~mm}$. apart. The posterior pole lies about I $8 \mathrm{~mm}$., varying from I4 to 24 (Weiss), or $\frac{3}{4}$ inch in front of the apex of the orbit.

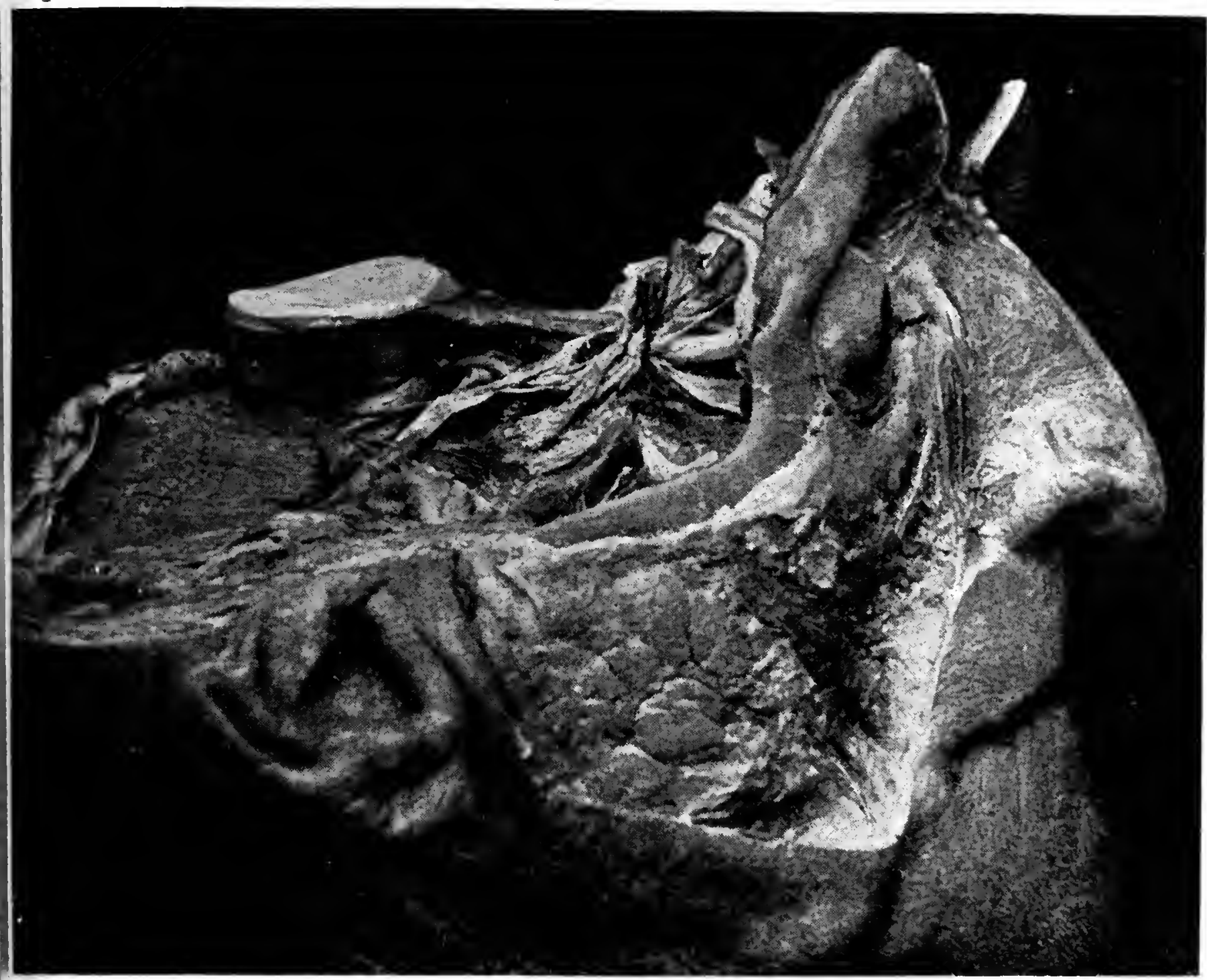

FIG. I33. - Dissection of the Head, to show the relation of the eyeball to the orbital margin, the course of the optic nerve, the position of the optic chiasma, the trochlear nerve in its whole course, the cavernous sinus, and the semilunar or Gasserian ganglion. The same preparation is viewed from above in Fig. I 84. $\times \frac{2}{3}$.

2. The Muscles (musculi oculi). - The muscles which move the eyeball (sometimes referred to as extrinsic, in contradistinction to the intrinsic muscles which are connected with the mechanism of the lens and iris within the globe) are six in number. There are four recti, distinguished as superior, inferior, medial, and lateral, and two obliques, the superior and inferior, all named from their position 
relative to the eyeball. Each rectus muscle presents a narrow, tendinous origin from the apex of the orbit, an elongated, band-like, fleshy belly, and a thin tendon of insertion on to the eyeball, all four being attached in front of the equator. The superior oblique arises with the recti from the apex of the orbit, runs forwards above the medial

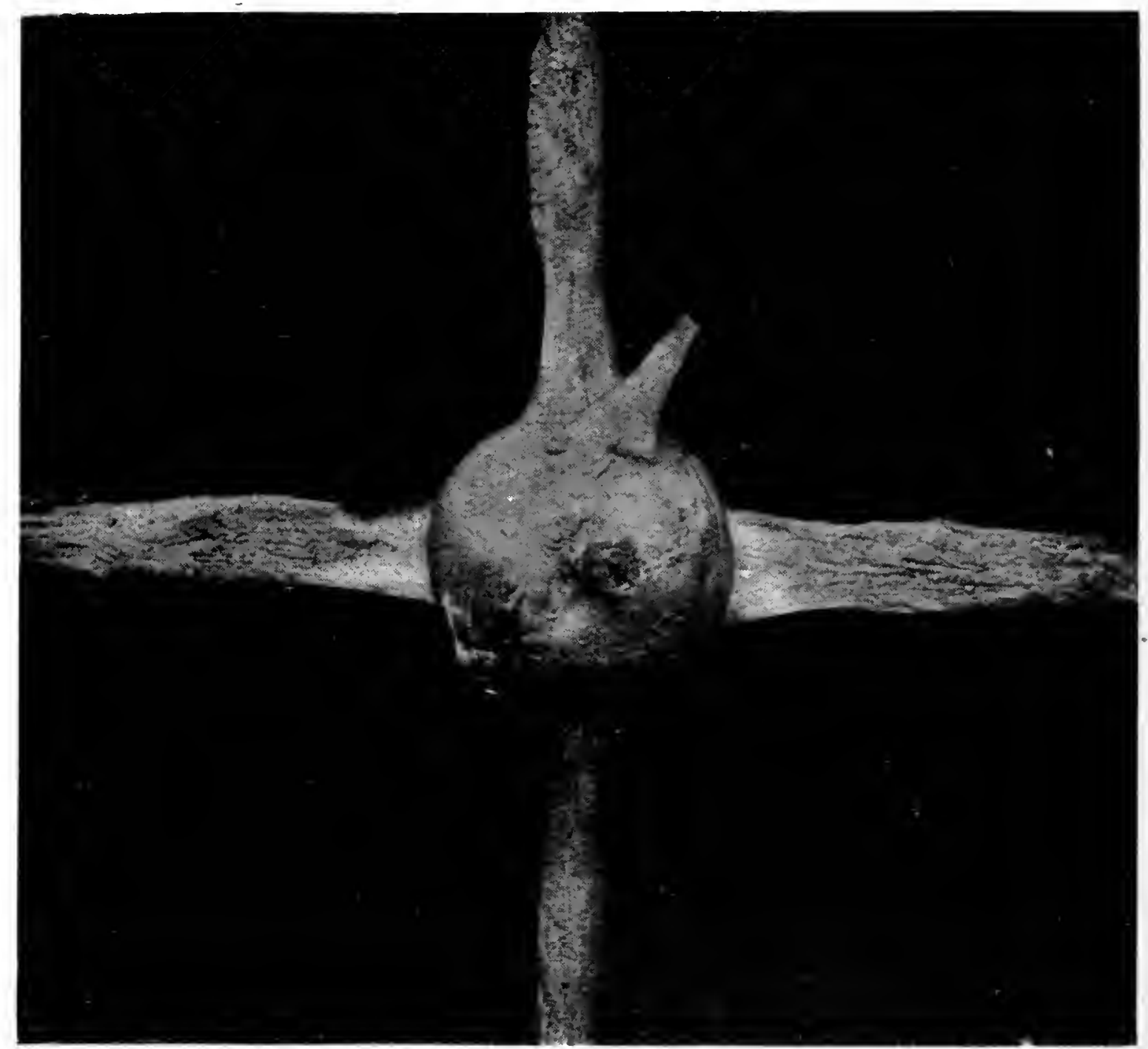

FIG. I34.--The Left Eyeball viewed from bchind to show the four recti muscles, spread out, and the oblique muscles in their respective positions. Natural size. Note the curved edge of the tendon of the lateral rectus on the left.

(An enucleated eyeball can always be relegated to the side to which it belongs if viewed from this aspect and if sufficient of the oblique muscles be left to distinguish the superior (tendinous) from the inferior (muscular); these cut ends point towards their origins on the nasal or medial sicle of the orbit, or, it may be said, away from the side to which the eye belongs.)

rectus to the trochlea, where it becomes tendinous and is reflected backwards on to the globe. The inferior oblique is a much shorter muscle, arising near the orbital margin at its inferior medial angle, and passing laterally and posteriorly across the forepart of the floor of the orbit to reach the globe behind the equator, being attached, like the superior 
oblique, to the postero-lateral quadrant of the eyeball. Each muscle is enveloped in a fascial sheath, which is so thin as to be practically invisible posteriorly, but becomes thick and opaque where the muscle pierces the fascia bulbi (capsule of Tenon), at which point the two fascial structures blend.

The lateral rectus is innervated by the abducent or sixth nerve, the superior oblique by the trochlear or fourth nerve, and the superior, medial, and inferior recti, together with the inferior oblique, by the oculomotor or third nerve. Their blood-supply is derived from the ophthalmic artery, and is drained away by the ophthalmic veins into the cavernous sinus.

The structure of the ocular muscles has been specially studied by Schiefferdecker (I905), who found that the parallel fasciculi of muscle fibres of which they consist are remarkable for the richness of their nerve-supply and the large amount of elastic fibres in the perimysium and intermuscular septa. The latter character is responsible for a passive contraction of the muscle after extension by its antagoniser, the action of which is, moreover, moderated by the same means; the provision ensures a delicate regulation of the eye movements. Muscle spindles, the sensory nerveend organs, are present, but are few in number.

The Recti Muscles (recti oculi) are approximately equal in length, each being about $40 \mathrm{~mm}$. or $\mathrm{I}_{2} \frac{1}{2}$ inch long including the tendon of insertion, but there is a difference in the length of the tendons and also in the size of the muscle belly. The medial rectus is thickest, the lateral rectus is thicker than the inferior, and the superior is thinnest of all. The weights as given by Volkmann are:

Medial rectus, 747 gramme; inferior, $\cdot 67 \mathrm{I}$ gramme.

Lateral ". 7 I 5 , ; superior, $5{ }^{\mathrm{r}} 4$,

and the exact lengths in millimetres are :

Medial rectus, $40^{\circ} 8$; inferior, $40^{\circ} \mathrm{O}$.

Lateral ", 40.6; superior, $4 \mathrm{I} \cdot 8$.

The origin or posterior attachment of the recti at the apex of the orbit is complicated by the configuration of this region and by the presence of the many important structures 
which here enter the space. Looked at from the outside, after they have been stripped from the bone as in Fig. I39, the muscles are seen to have a common origin from a short tendinous tube, the annulus of Zinn (I755) (annulus tendineus communis). This tube is oval in cross-section, and is most simply considered as being implanted by its base on to the apex of the orbit in such a way as to embrace both the optic foramen and the medial extremity of the superior

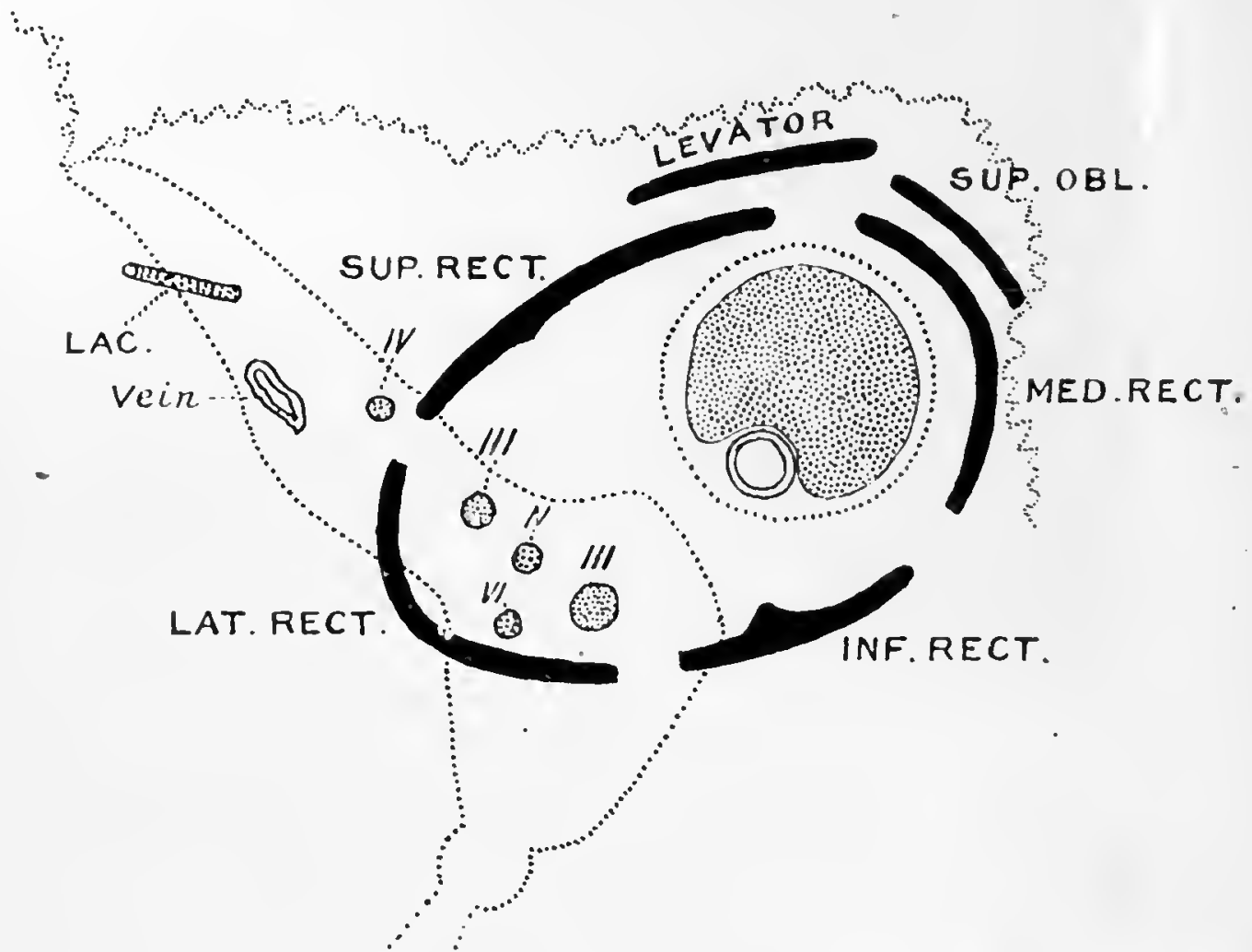

FIG. 135. - Scheme to show the position of the annulus of $\boldsymbol{Z}$ inn, implanted on the apex of the orbit in such a way as to embrace both the optic foramen (in which is seen the optic nerve and oplithalmic artery) and the broad end of the superior orbital fissure. See Fig. 59, p. I3I.

The annulus is resolved into its component muscle origins, and the relative positions of the nerves which enter the orbit through the superior orbital fissure both within and without the annulus are shown; the frontal nerve should be shown lying above the lacrimal; N.=the naso-ciliary nerve. (After Poirier, modified.)

orbital (sphenoidal) fissure (Figs. 59, I35); that sector of the annulus which embraces the end of the fissure is fixed to the bone forming its anterior boundary, that is, to the tip of the lateral orbital wall, a site frequently marked by a spur (the spina recti lateralis, p. 34); in the specimen photographed in Fig. I36 the spur is crescentic in outline, indicative of the contour of this portion of the ring.

At its base the annulus is intimately related to the dura mater lining the middle cranial fossa of the skull, 
The muscles arise from the fore end of the annulus in their respective positions; the superior and medial recti are most closely related to the optic nerve, being, indeed, adherent to its sheăth, a fact that would explain painful movements of the eyeball in inflammation of the nerve, and owing to the great obliquity of the optic foramen their origins are on a plane anterior to the rest ; just outside and above them lie the origins of the levator palpebrae superioris

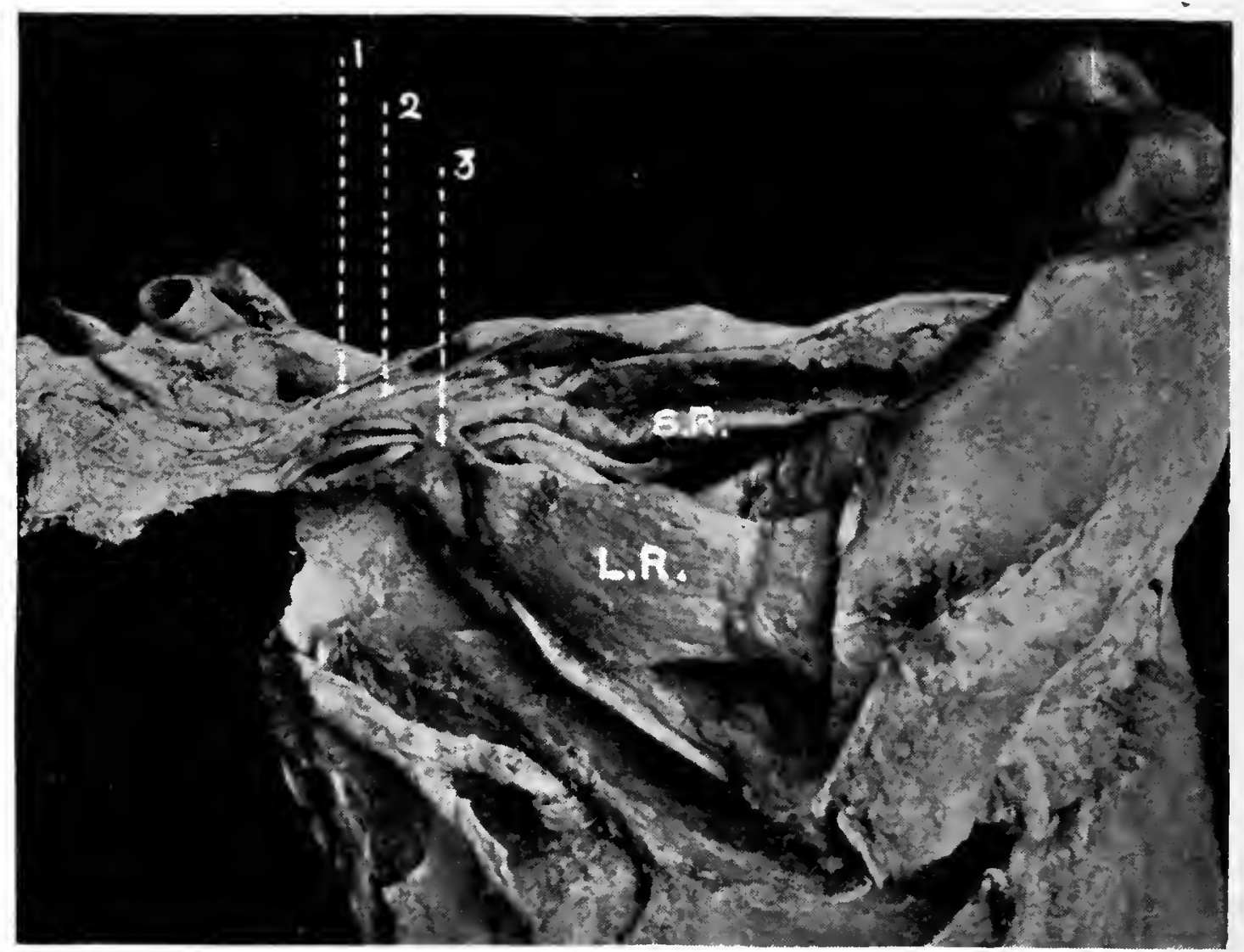

FIG. I39. - Dissection of Right Orbit in which all the hony walls have been removed and the margin alone left, viewed from the lateral aspect. The preparation shows the trochlear ( $\mathrm{r}$ ) and frontal (2) nerves as they enter the orbit outside the annulus of $Z$ inn (3); entering within the annulus are seen the oculomotor, niaso-ciliary, and abducent nerve, in that order from above downwards. The continuity of origin of the lateral rectus (L.R.) with that of the superior rectus (S. R.) is shown. Natural size.

and superior oblique muscles; the inferior rectus arises below and a short distance away from the nerve.

The lateral rectus arises from that part of the annulus which spans the superior orbital fissure, a position that has led to the statement that it arises by "two heads," one from each margin of the gap ; but such description, like so many drawings of the parts, gives an exaggerated idea of the actual facts, for there is no break in the annular origin of the muscle belly, as can clearly be seen in Fig. I39; 
its upper edge is fused at this point with the lower edge of the superior rectus, and similarly below it blends with the inferior rectus. Merkel (I9OI), indeed, described and figured "two heads" of origin of the lateral rectus, one from the spina recti lateralis and the other from the annulus next the optic nerve sheath, but they do not span the fissure, no structures enter the orbit through them, and they are not the same " heads" as are commonly described in later works.

If the annulus be now examined from the interior by splitting it open between two adjacent muscle origins, as

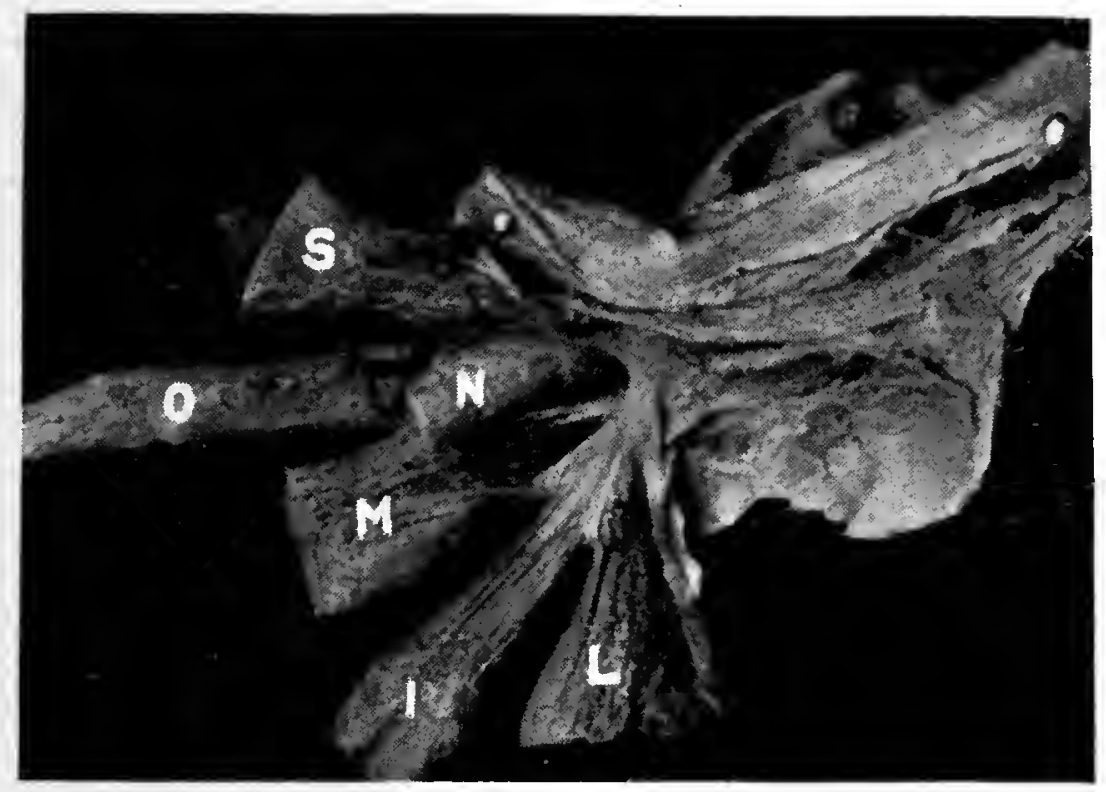

FIG. I40.-Dissection of the parts in relation to the Apex of the Left Orbit, lateral view. 'The annulus of Zinn has been split open between the superior (S.) and lateral (L.) recti to expose the tendon of Zinn (T.). The inferior (I.) and medial (M.) recti and superior oblique (O.) are spread out, and in the centre of the muscles lies the optic nerve (N.). 'The right-hand part of the preparation shows the lateral wall of the cavernous sinus torn open. Natural size.

has been done between the superior and lateral recti in Fig. I40, further complications of the muscle origins are seen. The ring on this inner aspect is not regularly smooth as on its outer side, but presents below a well-marked tendinous ridge, which gives origin to fibres of three of the muscles, namely, to part of the lateral, all of the inferior, and part of the medial rectus; this tendon is called the tendon of Zinn, or tendo orbitalis inferior, and its attachment is sometimes marked either by a minute "infra-optic tubercle," as seen in Fig. 9, or by a small fossa (Sappey) situated on the lower part of the bridge of bone which separates the optic foramen from the superior orbital fissure. On the 
upper half of the interior of the annulus a similar but much less definite thickening, the tendo orbitalis superior, was described by Lockwood (I886) as giving origin to part of the lateral, all of the superior, and part of the medial recti. Rouvière (IgI4), in a special study of this region, describes a single tendon, that of Zinn, arising from the bone as above noted but dividing into six parts from which the muscles arise.

Since the annulus of Zinn surrounds the optic foramen and the medial end of the superior orbital fissure, it follows that all the structures which enter the orbit through the foramen and this part of the fissure will lie at first within

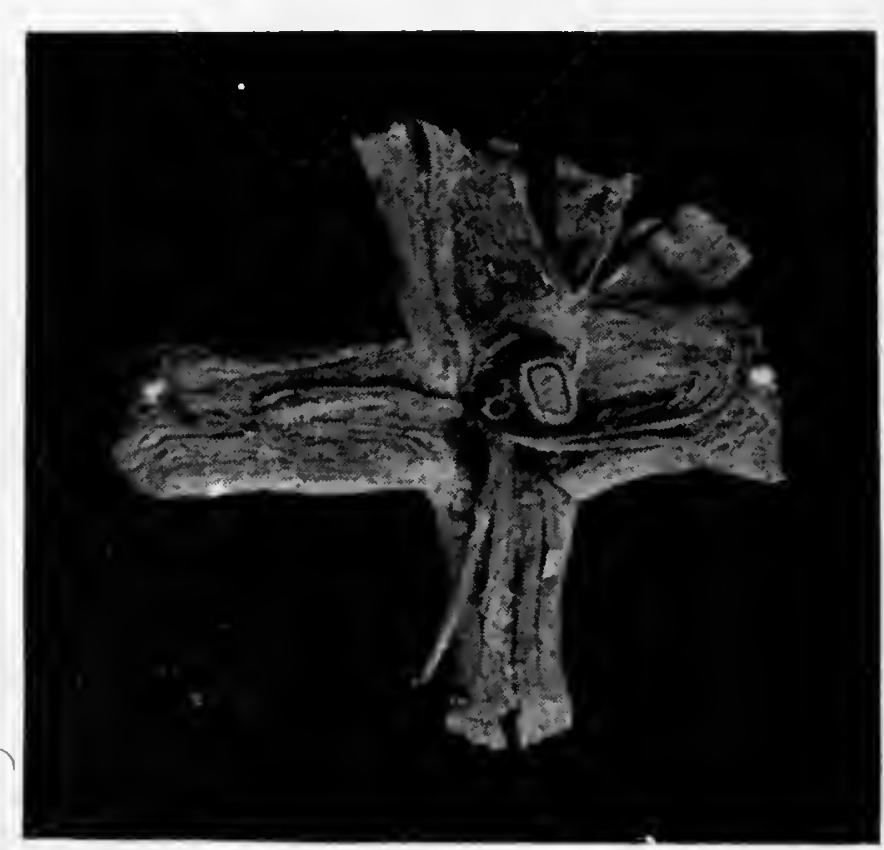

FIG. I4I.-Dissection of the Structures in the Apex of the Right Orbit seen from in front. The four recti muscles have been spread out and occupy their respective. positions, and the nerves are seen entering the orbit within the cone of muscles. In the centre is the optic nerve in its sheath with the ophthalmic artery on its inferolateral side. Below the artery is the inferior division of the third nerve, the upper division of which lies above the vessel. The sixth nerve lies lateral to the artery. The cut ends of the levator (left) and superior oblique (right) appear between the superior and medial recti. Natural size. the cone of muscles. Through the optic foramen there enter the optic nerve, enveloped in its dural sheath, and the ophthalmic artery, embedded in the sheath and accompanied by branches from the carotid plexus of the sympathetic nervous system; through the medial end of the fissure there pass the oculomotor or third nerve, the abducent or sixth nerve, the naso-ciliary branch of the trigeminal or fifth nerve, the sensory root of the ciliary ganglion, and usually the superior ophthalmic vein (p. 3Io).

These latter structures are all closely packed together in a narrow space, as is seen in Fig. I4I, and also in the fourth section in Fig. I85, p. 366, and their relative positions are shown in Fig. I35. The space through. which they pass is formed into a smooth and glistening tendinous fora- 
men bounded medially by the optic nerve sheath and laterally by the loop of the annulus bridging the fissure; it is termed the "oculomotor foramen" (foramen oculomotorium), and its contained structures are better described as entering the orbit through this foramen, or " within the cone of

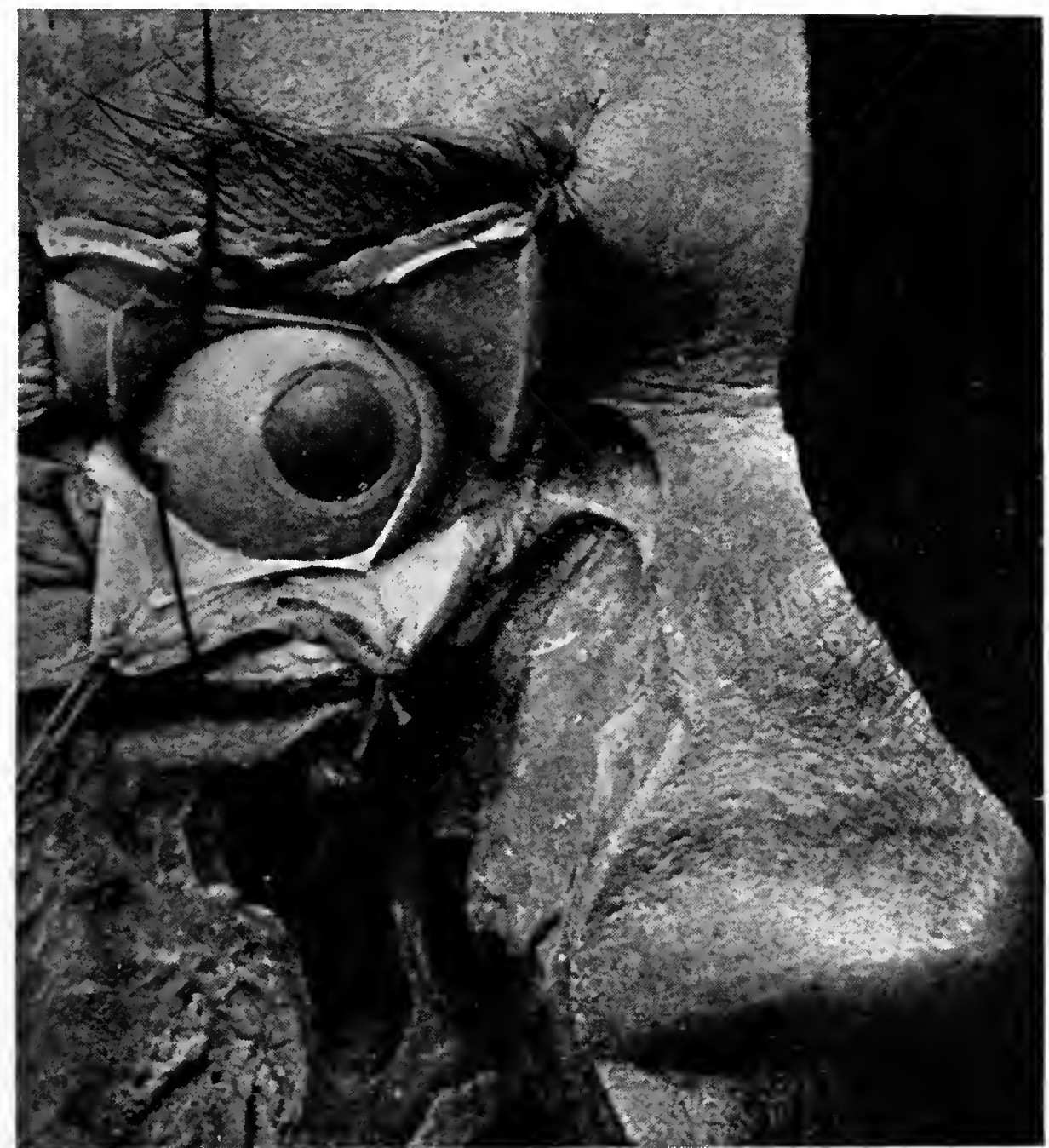

Fig. I42. - Dissection of the Right Eyeball from in front. The eyelids as a whole have been crucially incised and the flaps pulled aside. The bulbar conjunctiva and anterior part of the fascia bulbi or capsule of Tenon have been cut just wide of the cornea, and pulled away from the globe on the lateral side to expose the tendon of the lateral rectus muscle, beneath which a bristle has been placed.

The lacrimal sac and naso-lacrimal duct have been exposed from in front in their entire extent. Natural size.

muscles," than "between the two heads of the lateral rectus muscle."

The insertions or anterior attachments of the recti muscles on to the globe are effected by means of thin, flat, glistening tendons, formed by parallel rectilinear fasciculi of connective tissue, without anastomoses but weakly united by transversely placed and easily separated fibres. The fasciculi are several layers deep, those of the medial 
rectus being thickest, but form a single layer at the edges, which are recurved to form a little.cul-de-sac (Fig. I34). Some of the fasciculi leave the main layer to be inserted separately as far back as from I to $5 \mathrm{~mm}$. behind the remainder, and consequently, like the recurved edges, may escape the tenotomy hook in operations. The tendons penetrate the sclera to unite most firmly with it, causing a slight thickening of the surface as they spread out within it in fan-like manner. They are accompanied by the anterior ciliary arteries, and lie beneath the anterior part of the fascia bulbi, which, with the overlying bulbar conjunctiva, must be removed to expose them from the front, as shown in Fig. I42; so closely are they applied to the sclera and so much do they resemble it in colour, that the student may not easily recognise them.

The antero-posterior length of the tendons in millimetres is given by Merkel (IgOI) as follows:

$\begin{array}{cccc}\text { Rectus Medialis. } & \text { Rectus Inferior. } & \text { Rectus Lateralis. } & \text { Rectus Superior. } \\ 3 \cdot 7 & 5^{\circ} 5 & 8 \cdot 8 & 5 \cdot 8\end{array}$

The breadth of the actual line of insertion is given by Fuchs as
$10 \cdot 3$
$9 \cdot 8$
$9 \cdot 2$
$10 \cdot 6$

By Weiss (in adult) :
$10 \cdot 76$
$10 \cdot 35$
$9 \cdot 67$
$10 \cdot 75$

By Weiss (in new-born) :
$7 \cdot 35$
$6 \cdot 25$
$5 \cdot 85$
$6 \cdot 95$

The distance of the mid-point of this line from the corneal margin varies slightly according to the observations of different authors. The measurements made by Fuchs (I884) were taken from the largest series of eyes, and are most widely accepted ; they are
$5 \cdot 5$
$6 \cdot 5$
$6 \cdot 9$
$7 \cdot 7$

and a mean of the figures given by Howe, Adachi, Weiss, Fuchs, Testut, Gerlach, Sappey, Merkel, Motais, Tillaux, Macalister, and Krause is 
ORBIT

Rectus Medialis.

Rectus Inferior.

$5 \cdot 84$

$6 \cdot 65$

Rectus Lateralis.

Rectus Superior.

$7 \cdot 18$

$7 \cdot 88$

The corresponding distances in the new-born are given by Weiss as
$3 \cdot 6$
$5^{\circ} \mathrm{O}$
$4 \cdot 9$
$5 \cdot 8$

The above measurements of the length, breadth, and distances from the corneal margin are shown diagrammatically

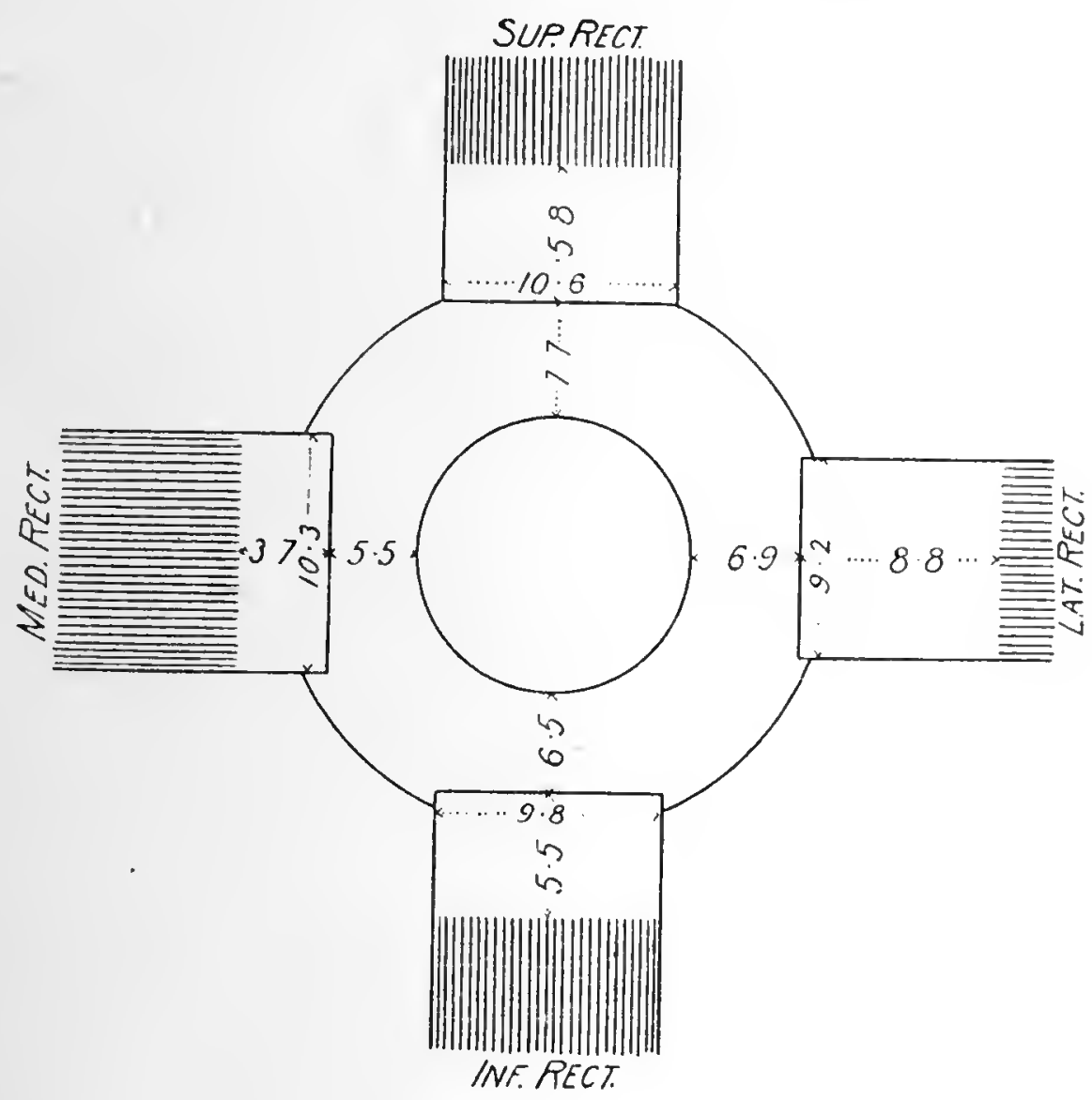

Fig. 143.- Scheme to show the anterior attachments of the Recti Muscles to the Eyeball, with the various measurements given in the text. $\times 1 \frac{1}{2}$.

in Fig. I43, and the more exact positions of the lines on the globe in Fig. I44.

It is to be noted, however, that these lines of insertion are not straight, but are curved or sometimes even wavy; they lie neither parallel to the corneal margin nor at right angles to the respective meridian either of the muscles or of the cornea, though those of the medial and lateral recti are approximately so placed (Fig. I45). The line of insertion of the medial rectus is straight or feebly convex forwards, and may either cut the horizontal meridian symmetrically or cross it obliquely and unequally, with the greater length lying above; in the case of the inferior rectus, the line is 
oblique and markedly convex forwards, and in two-thirds of cases the greater moiety lies lateral to the vertical meridian; the line of the lateral rectus is usually straight and lies perpendicular to the horizontal meridian, though in abnormal cases it presents an asymmetry the reverse of that of the medial rectus; the line of the superior rectus is convex forwards and is most often asymmetrically placed
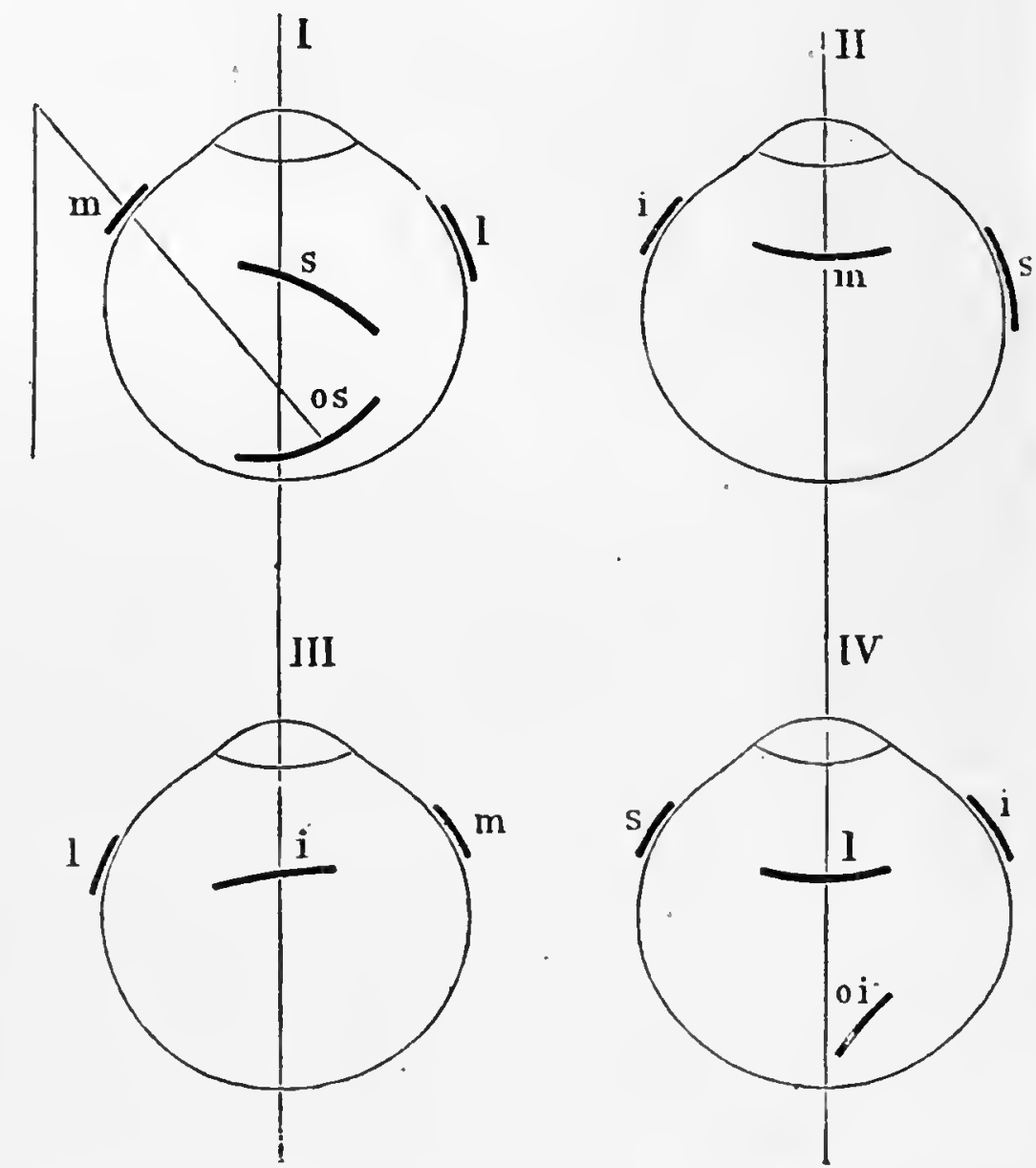

FIG. I44. - Schematic representation of the lines of the Muscle insertions on to the Eyeball (from Merkel, I901). Natural size.

$I_{s}=$ seen from above, II. from the medial side, III. from below, IV. from the lateral side. $s .=$ superior rectus, $i_{0}=$ inferior rectus, $m$. =medial rectus, $l_{0}=$ Jateral rectus; os. = superior oblique, $o i_{0}=$ inferior oblique.

relative to its meridian, sloping obliquely upwards and lateralwards with the greater length on the lateral side.

It follows, therefore, that the above distances, measured from the mid-points of the lines of insertion, are not the shortest nor the mean distances from the cornea, and Motais suggests taking the nearest and farthest points, which he finds are respectively as follows, in millimetres:

Rectus Medialis.

\section{$5 \cdot 5$}

$7^{\circ} 0$
Rectus Inferior.

5.5
Rectus Lateralis.

Rectus Superior. 
and he maps out the lines as in Fig. I45, from which it is seen that the so-called spiral line on which the insertions lie is really a very irregular one.

Finally, Weiss gives the distance of the insertions from the optic nerve as follows:

$$
\text { I6.95 } 18.80 \quad 20 \cdot 60 \quad \text { I } 8 \cdot 5 \mathrm{I}
$$

That there is much individual variation in the position, length, and form of these lines of insertion is seen from Fig. I46, as found by Holmes, and is illustrated also by Fuchs (I884).

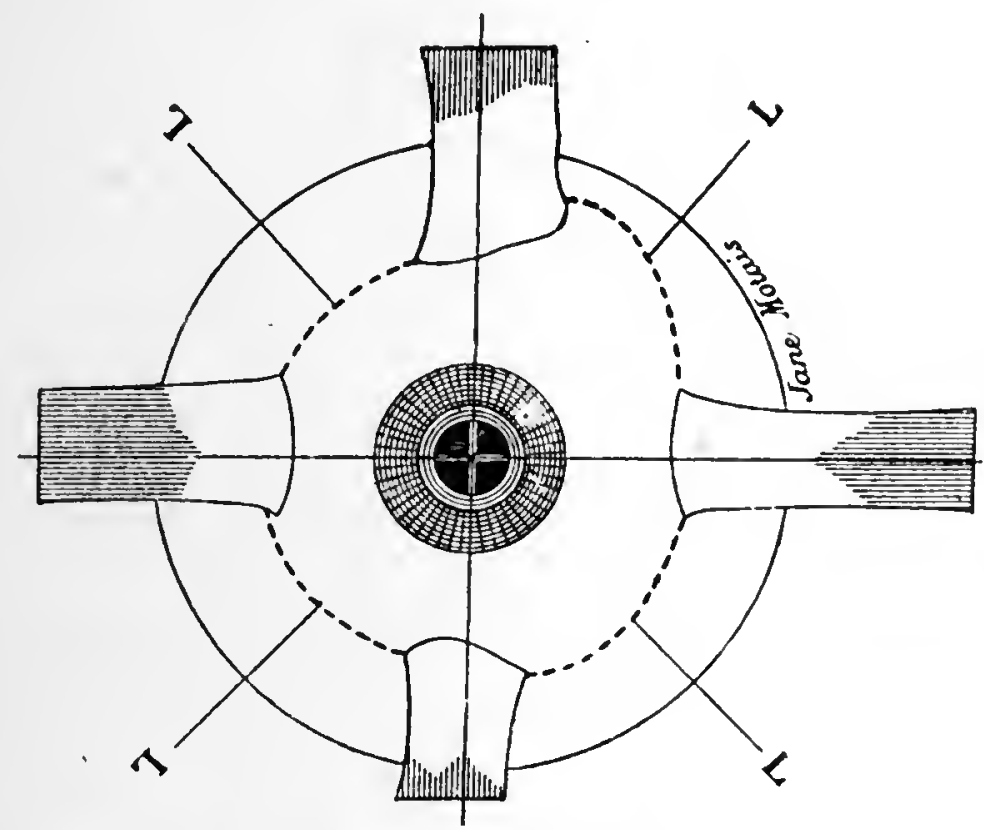

FIG. I 45. - The insertions of the Recti Muscles on to the Left Eyeball according to Motais (in Poirier, I9I2). The line joining them (L.) describes a very irregular spiral. 'The medial rectus lies to the reader's left.

On the whole, the medial rectus is the largest and heaviest muscle, and is most advantageously placed nearest the corneal margin, whilst the superior rectus is the weakest and is farthest away, especially at the lateral edge of its insertion.

The above described tendon is sometimes termed the "primary or principal insertion " of the muscle, the following being then considered as "secondary insertions" :

(i.) The thin recurved strands at the edges of the tendons.

(ii.) The few fasciculi which are inserted on to the globe separate from and behind the remainder.

(iii.) Certain attachments of the muscle sheaths to the walls of the orbit, which form the so-called "check ligaments," described on p. 293. 
The "arc of contact" which the muscle tendon makes with the globe prior to its insertion has been studied by Weiss (r894), especially as regards the lateral and medial recti, and he points out the increase of the arc of the lateral muscle and decrease of that of the medial consequent to the greater divergence of the orbits during growth ; the changes

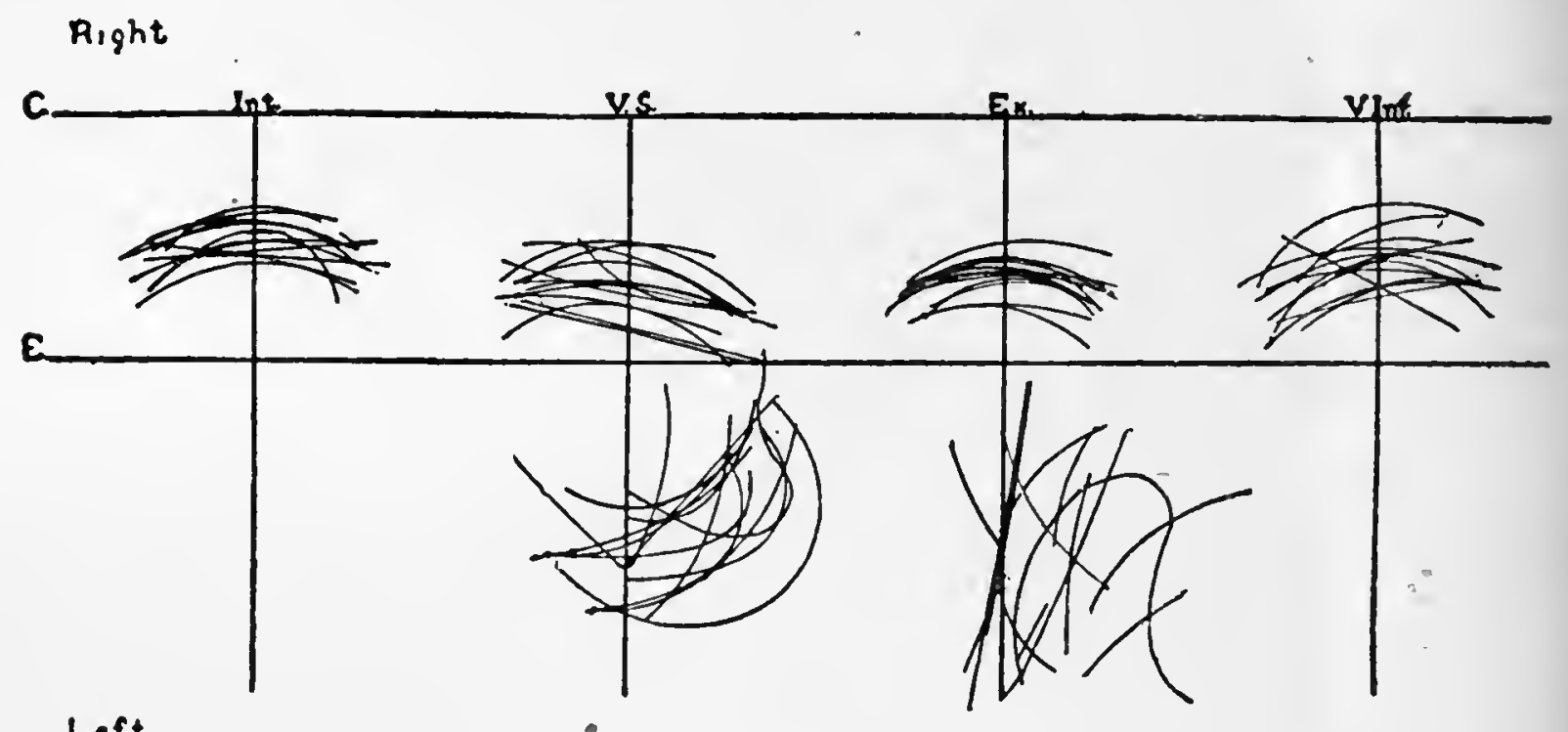

Left.

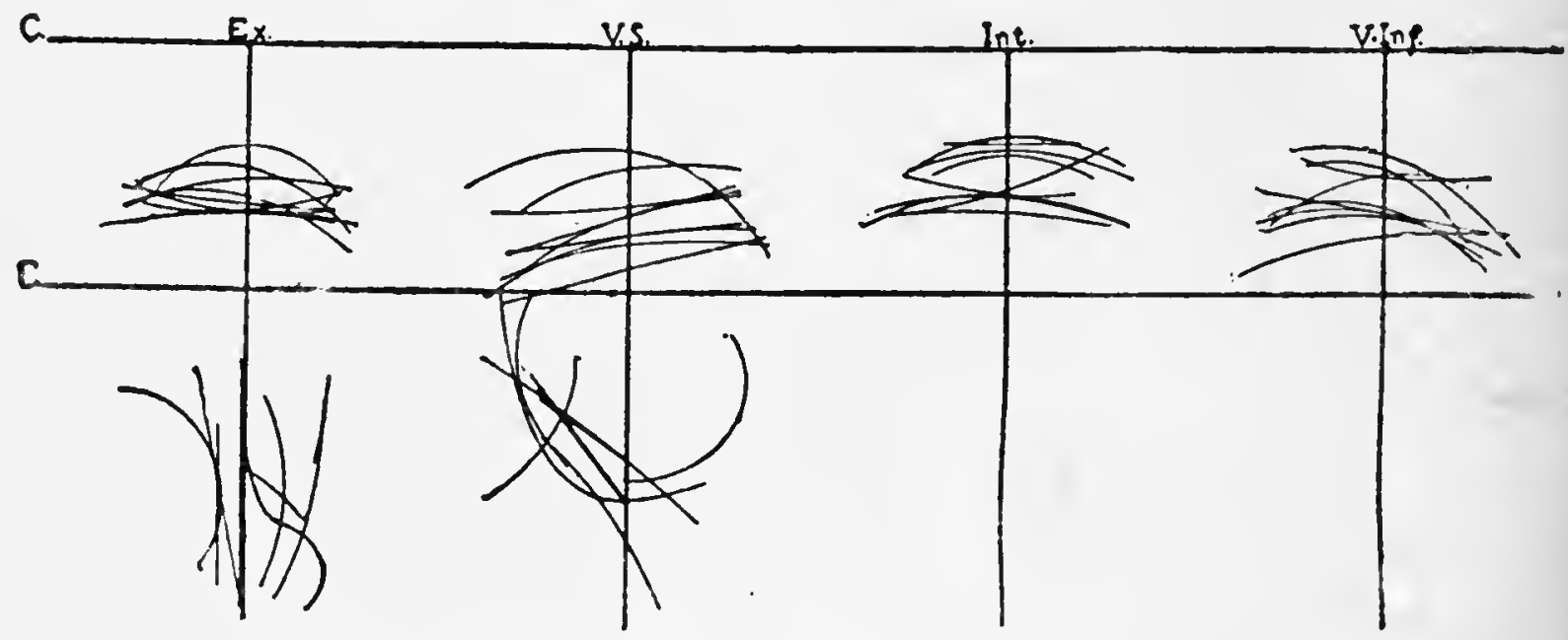

FIG. I 46. - Lines of insertion of the Muscles on several Eyeballs plotted together in order to show the difference in the form and place of the insertion. All the lines of the right eyes are grouped together in the upper portion of the figure, and all of the left eyes in the lower portion. In both, C. represents the line of the cornea, E. the equator of the globe. (From Howe, 1907.)

may explain cases of the spontaneous cure of convergent strabismus which existed in childhood (see also p. 283).

Having considered the recti muscles together as regards their origin and insertion, the relations and certain other points of interest remain to be described for each muscle.

The medial rectus ( $m$. rectus medialis) from its origin, 
where it is closely applied to the medial side of the optic nerve sheath, runs forwards and inclines a little upwards and medialwards; at first it lies in contact with the corresponding wall of the orbit, separated by the thin os planum from the ethmoidal air-cells, but in its anterior third it inclines from the orbit wall to reach the globe, the intervening space being filled by a compact mass of fat (Fig. I30). Above it lies the superior oblique muscle, closely applied posteriorly but separated by the naso-ciliary nerve, ethmoidal branch and terminal part of the ophthalmic artery anteriorly. A branch from the oculomotor nerve enters its substance in several strands on the ocular side about $15 \mathrm{~mm}$. from its origin, and its blood-supply is provided by the inferior muscular branch of the ophthalmic artery.

The inferior rectus (m.rectus inferior) runs along the floor of the orbit closely related to the palatinal air-cell near its origin, but separated by a mass of orbital fat from the roof of the sinus maxillaris or antrum of Highmore in front (Fig. I70, p. 326) ; its infero-lateral margin lies above the infraorbital nerve in the hinder part of its course. Anteriorly and a little in front of the equator of the globe the inferior oblique muscle passes laterally and obliquely beneath the inferior rectus, the fascial sheaths of the two being fused at this point and forming the suspensory ligament of Lockwood. The lower division of the oculomotor nerve runs along its ocular or superior aspect, and the branches of supply enter about the junction of its posterior third and anterior two-thirds; the nerve to the inferior oblique is closely bound to its lateral margin (Fig. I39). The blood-supply is derived from the inferior muscular branch of the ophthalmic artery.

The lateral rectus ( $m$. rectus lateralis) is attached at its origin to the apex of the lateral orbital wall, being thereby separated from the temporal pole of the brain; between the muscle origin and the optic nerve is the fibrous oculomotor foramen through which there enter the orbit the oculomotor, the abducent, and the naso-ciliary branch of the trigeminal nerves, and the ophthalmic vein, these structures 
being consequently here closely related to the muscle (Figs. I39, I4I). A little in front of this point $(5 \mathrm{~mm}$. or about $\frac{1}{4}$ inch) lies the ciliary ganglion (ganglion ciliare), and further forwards the ophthalmic artery and the naso-ciliary nerve lie likewise between the muscle and the optic nerve (Fig. I78, p. 349). In its forepart it is separated laterally by orbital fat from the wall of the temporal fossa (Fig. I30); above it is in contact with the lacrimal gland, the nerve and artery to which run along its upper border; whilst between its lower border and the inferior rectus lies the nerve to the inferior oblique; at the globe it crosses over the inferior oblique muscle. The sixth nerve enters it on the medial aspect, about $\mathrm{I}_{5} \mathrm{~mm}$. beyond the oculomotor foramen, and its blood-supply is derived from the $\underset{z}{\operatorname{lacrimal}}$ artery.

The superior rectus ( $m$. rectus superior) lies beneath and in intimate contact with the levator palpebrae superioris, separated by it from the frontal nerve and roof of the orbit (Fig. 63, p. I35) ; it is united to the levator along its medial border by the adherence of their fascial sheaths, and also by the passage through the latter of the superior division of the oculomotor nerve, which supplies both muscles. The ophthalmic artery and the naso-ciliary nerve lie beneath it posteriorly, between it and the optic nerve; in front it passes over the tendon of the superior oblique muscle. The direction of its long axis from the apex of the orbit to the globe inclines laterally, and so does not coincide with the sagittal axis of the latter, but forms an angle of about $25^{\circ}$ with it. It does not lie directly "above the inferior rectus, but is placed slightly more lateral.

The superior oblique muscle ( $m$. obliquus superior) lies along the angle between the medial wall and roof of the orbit (Fig. 63, p. I35). It arises by a short, slender tendon antërior and medial to the optic foramen, from which it is separated by the medial rectus, in the angle between the annulus of Zinn and the periorbita. It may further be attached to the medial orbital wall for a few millimetres from its origin. The belly of the muscle, more rounded than that of the recti, runs forwards, parallel and close to the 
nasal wall above the medial rectus, but separated from the latter by the naso-ciliary nerve and ophthalmic artery, and crossed by their ethmoidal branches; a few millimetres behind the orbital margin at its supero-medial angle the muscle becomes tendinous and passes through a cartilaginous ring, the pulley or trochlea; it then bends abruptly backwards, slightly down, and lateralwards at a " trochlear angle" of about $54^{\circ}$, and the round tendon broadens out, pierces the fascia bulbi, and passes beneath the superior rectus, to be inserted into the back and lateral side of the eyeball. The first part of the muscle from its origin to the trochlea is referred to as the direct portion, and the tendinous part between the trochlea and the globe as the reflected portion. The tendon measures $20 \mathrm{~mm}$. or $\frac{3}{4}$ inch in length from pulley to globe, and is enveloped by a prolongation of the fascia bulbi as far as the pulley.

In most mammals this reflected portion is also fleshy, and indeed in lower vertebrates the whole muscle is represented by the reflected portion only, which arises from the medial angle of the orbit near the inferior oblique. The insertions of the tendons of the oblique muscles differ from these of the recti in being placed on the posterior instead of the anterior hemisphere of the eyeball; they also show much more variation in position, as is shown in Fig. I46.

The line of insertion of the superior oblique tendon crosses the vertical meridian obliquely and is curved with a backwardly directed convexity. The breadth as measured by Fuchs on 3 I emmetropic globes was on an average I0.7 mm., with extremes of from 7.5 to $12.7 \mathrm{~mm}$., but was found narrower in myopic globes, in 20 of which it averaged $9.6 \mathrm{~mm}$. with extremes of from 6.8 to $\mathrm{I}_{4} \mathrm{~mm}$. The distance from the corneal margin to where the line crosses the meridian is from I6 to $17.9 \mathrm{~mm}$. (Fuchs), but Weiss in 5 adult emmetropic eyes found the anterior edge of the obliquely placed line to lie $13.85 \mathrm{~mm}$. from the cornea and $14.66 \mathrm{~mm}$. from the optic nerve, whilst the posterior end was $18.8 \mathrm{~mm}$. from the cornea and $7.56 \mathrm{~mm}$. from the nerve; the anterior end also lies on the same meridian as the lateral end of the line of attachment of the superior rectus, but 
distant $4.6 \mathrm{~mm}$. (with extremes of from 0.5 to $8 \mathrm{~mm}$.) from it, though the two tendons are often connected by connective tissue strands. The line of the superior oblique is similarly connected with that of the inferior oblique, but is separated from it by $I 5 \mathrm{~mm}$. at the anterior end and II.9 $\mathrm{mm}$. at the posterior end. Fuchs (I884) described two types of insertion : one in which the line is broad with a strongly marked anterior concavity, and lies in an equatorial direction across the vertical meridian with the greater part on its lateral side (Fig. I44), as more commonly found in emmetropic and hypermetropic eyes ; the other narrower and flattened, and lying entirely in the lateral quadrant almost parallel to the vertical meridian, as found in myopic (though occasionally in normal) globes.

The trochlear or fourth nerve crosses over the muscle from the medial side to enter its substance at about $\mathrm{I} 2 \mathrm{~mm}$. from its origin; the blood-supply is derived from the superior muscular branch of the ophthalmic artery.

The trochlea or pulley of the superior oblique muscle consists of a finely grooved and curved plate of hyaline cartilage, $4 \mathrm{~mm}$. long by $6 \mathrm{~mm}$. broad; its edges are attached to the fovea trochlearis of the frontal bone (p. 24) by fibrous retinacula, so completing the tube through which the muscle tendon slides, as shown in Fig. I04. The tendon here, as it actually passes through the pulley, is invested by very lax areolar tissue with an imperfect endothelial lining in its clefts, but there. is scarcely a true synovial membrane lining the trochlea (Macalister, I889). The fascial sheath, which envelops the tendon from its point of entry into the capsule of Tenon, extends up to the pulley, and is fixed to and terminates at the latter. The trochlea was primitively a specialised part of the sheath of the muscle at its origin, which was situated near the orbital margin, but the need for a longer muscle belly in mammals has led to the development of the pulley and the retrogression of the origin backwards towards the apex of the orbit. Maddox (I907) suggests that the reason why the superior oblique has a pulley is that since the speed with which a muscle's point of insertion moves is proportional to its length, it is necessary for the superior 
oblique, the virtual origin of which as regards its action is from the pulley, to have a long, fleshy, reflected portion extending to the back of the orbit if it is to keep pace with the movements of the recti ; and as regards this advantage over the inferior oblique, the reason may be that prolonged looking downwards is more important for daily work than looking upward, and therefore the former excursions of the eye are more amply provided for than the latter. It may be pointed out, however, that the disparity between the lengths of the contractile parts of the two muscles is not great, since the muscle belly of the superior is approximately $40 \mathrm{~mm}$. long, and the total length of the inferior, which is fleshy throughout nearly its whole extent, is about $37 \mathrm{~mm}$.

The inferior oblique ( $m$. obliquns inferior) muscle is the only one of the ocular muscles which does not arise from the apex of the orbit. The origin is from a small shallow depression on the orbital floor, just within the inferior orbital margin lateral and usually close to (see p. 284) the opening of the naso-lacrimal canal (Fig. I6); some fibres frequently arise from the lower part of the fascia covering the lacrimal fossa, and so are closely related to the lower part of the lacrimal sac (Fig. I29). The muscle passes laterally and backwards as a flat band beneath the inferior rectus, to which it is closely connected by the fusion of their fascial sheaths (Fig. 67), is separated from the floor of the orbit by a compact mass of fat, and is inserted, like the superior oblique, on to the posterior hemisphere of the eyeball; its course intersects the axis of the orbit at an angle of $75^{\circ}$; it is fleshy in nearly its whole extent, the tendon being only $2.6 \mathrm{~mm}$. long or, indeed, often non-existent, so that the muscle fibres pierce the sclera (Fig. 134). The line of insertion lies obliquely in the lateral and inferior quadrant of the posterior hemisphere, farther back than that of the superior oblique, with which it is not parallel but makes an angle of $25^{\circ}$, and nearer the optic nerve, from which it is distant about $5.2 \mathrm{~mm}$. in normal but $7 . \mathrm{I} \mathrm{mm}$. in myopic globes; it lies $9.5 \mathrm{~mm}$. away from the insertion of the lateral rectus and almost on the same meridian, though at right angles to 
the line of the latter, and is only $2 \cdot 2 \mathrm{~mm}$. from a spot corresponding to the fovea of the retina. The distance from the corneal margin is from $I 7 \cdot 3 \mathrm{~mm}$. to I9.I mm. (Fuchs). The variations in its position are seen in Fig. I46. Salzmann (I9I2) notes that the line of its insertion often shows gross irregularities, angular serrations, or even dehiscences. The branch from the inferior division of the oculomotor nerve enters the muscle belly about the middle of its hinder border, and its blood-supply is derived from the infra-orbital artery as well 'as the inferior muscular branch of the ophthalmic artery.

For the comparative anatomy of the oblique and recti muscles the reader interested should consult the work of Motais (I887), and papers by Struthers (I849), Ottley (I879), and Corning (I902); Poole. (I905) discusses the variation in the trochlear angle in the domestic animals.

The Action of the Muscles.-The movements of the eyeball have been resolved by Fuchs (I9I7) into components which correspond to three primary axes, perpendicular to each other and intersecting at its "centre of movement" or "point of rotation" ; this point in a emmetropic eye is situated not at the mathematical centre of the globe, but. a little behind the mid-point of its antero-posterior axis, or I4 $\mathrm{mm}$. behind the summit of the cornea and Io $\mathrm{mm}$. in front of the posterior pole; in myopic globes the distance is $14.52 \mathrm{~mm}$. and in hypermetropic $13.22 \mathrm{~mm}$. behind the anterior surface of the cornea (Donders). The three primary axes are:

The vertical axis, around which lateral movements occur to the right and left, that is, abduction and adduction or divergence and convergence of the cornea on the horizontal plane ;

The frontal axis, running from right to left, and related to movements of elevation and depression of the cornea ;

The sagittal axis, passing from before backwards, corresponding to the visual axis, and correlated with medial and lateral rotation, or intorsion and extorsion of the cornea.

As regards the actions of the muscles, it is first to be 
emphasised that the eyeball is delicately poised in the fascia and fat of the orbit; the capsule of Tenon forms its primary socket, and the two, globe and fascia, move together in any but the smallest excursions upon the bed of fat which completely fills the surrounding space up to the bony orbital wall. The globe undergoes no change of place as a whole, but simply rotates about the centre of movement; since, however, this point is not the mathematical centre of the eyeball, it is more correct to describe the movement as oscillation rather than rotation. A state of equilibrium is maintained by the tonicity of all the ocular muscles, and individually it is impossible for any one muscle to act without the others participating both in the movement and in the maintenance of the new position of the globe; in every motion from three to five muscles participate, one or two acting directly and the others helping by steadying the course.

Incidentally there is to be noted the extent to which the head itself participates in all except the most narrow ranging of the visual regard, a co-ordination which serves to relieve the ocular mechanism of wide excursions and favours concentration of effort upon the finer movements; the prevalence of this unconscious act is well realised when the observer is suffering from a stiff neck, for example, and is a noticeable feature in the muscular rigidity of paralysis agitans.

As a whole, the four recti may be regarded as retractors of the eyeball; they are antagonised by the two obliques, which from their origins and directions are protractors (apart from their pull nasal-wards on the globe), and this is probably the active element in maintaining the equilibrium of the globe (Theobald, I9I8); the medial and lateral recti are nearly perfect antagonists in movements of the cornea upon a horizontal plane; the superior and inferior recti are opposed in producing elevation and depression and in torsion, but both act as adductors; the two oblique muscles rotate the eyeball in opposite directions in elevation and depression of the cornea and in torsion, but both produce abduction. Thus the equilibrium of the globe is assured by the anatomical dispositions of the muscles; 
in movements, the action of a contracting muscle, which shortens in full extent about a quarter of its length, is moderated by its antagoniser, which elongates to the requisite degree. A rise of intra-ocular pressure in consequence of the muscle action, especially in convergence of vision, has been assumed, but Levinsohn (IgIo) considers the action to be too slight to affect the pressure; Lederer (IgI2), on the other hand, found that a rise of pressure equal to $5 \mathrm{~mm}$. of mercury occurs in marked lateral motions of the eye, due, however, to the compression of the globe against the orbital fat. It may be noted that Ochi (IgI9) ascribes juvenile progressive myopia to the pressure of the muscles on eyeballs with weak sclerotic coats.

The individual action of each muscle can be described as determined by its anatomical disposition as follows, but it is to be noted that, except for the medial and lateral recti, each has a main and a subsidiary action.

The medial rectus is the adductor of the eye, turning the cornea medialwards, and the movement is sensibly direct, with a possible excursion of $45^{\circ}$; the simultaneous contraction of two muscles produces convergence of vision.

The lateral rectus has a directly opposite action; it is the abductor of the eye and directs the cornea lateralwards; the motion is direct and has an excursion of nearly the same extent as that of the medial rectus.

The superior rectus is an elevator of the cornea, but since its direction does not coincide with the sagittal axis of the eyeball but forms an angle of $23^{\circ}$ with it, and since its insertion falls on the anterior hemisphere in front of the centre of movement of the globe, it will not only elevate but adduct, and can, moreover, incline the upper end of the vertical axis medialwards, that is, rotate the globe in this direction. The action then is to elevate, adduct, and intort the cornea.

The inferior rectus is a depressor of the eye, but since, like the superior rectus, its long axis inclines laterally forwards and so makes an angle with the visual axis of the globe, it also possesses a slight action of adduction, directing the cornea medially; it can also cause a small 
degree of extorsion, and its action, therefore, is to depress, adduct, and extort the cornea.

The superior oblique must be considered to act relative to the globe from its physiological origin at the pulley. Primarily it will raise the posterior hemisphere of the globe, to the upper aspect of which it is fixed, and so depress the cornea, but since it is also inserted behind the centre of movement of the globe at an angle of $39^{\circ}$ to the optic axis

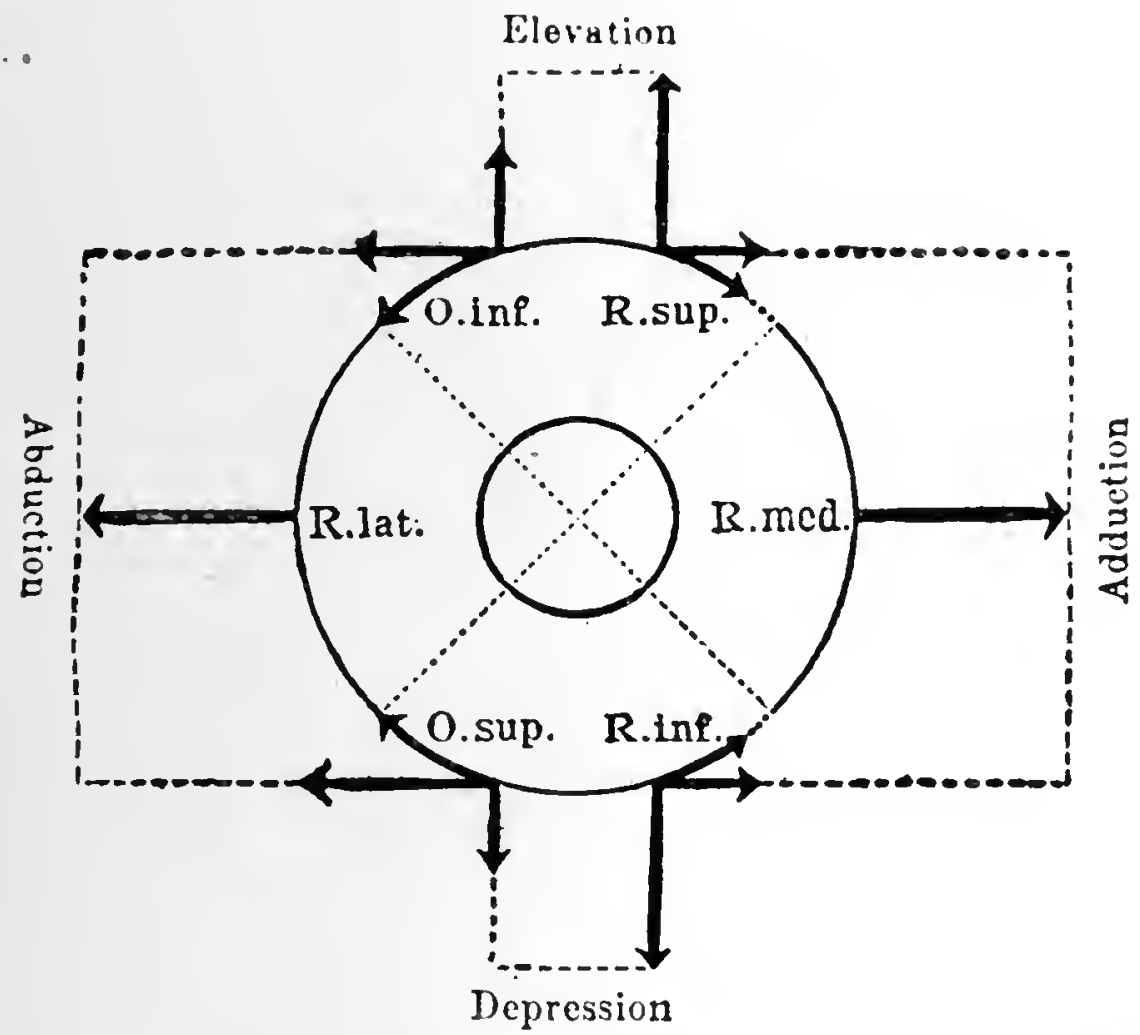

Fig. 147. - Schematic plan to illustrate the action of the Ocular Muscles.

(After Marquez, from Fuchs, I9I7.)

The action of each muscle is shown by arrows, which represent this action as decomposed into its components, according to the parallelogram of forces. The components representing torsion are drawn on the outer circle and indicate with their apices the position of the extremity of the vertical meridian, when the upper end of the latter has been tilted by the muscle inward or outward, as the two dotted lines crossing in the centre of the figure represent. The curved arm, in other words, shows the direction in which the vertical meridian is tilted, and the amount of tilting. The inferior oblique, in accordance with its elevating action, is drawn on the upper rim of the outer circle, and the superior oblique, because of its depressing action, is drawn o 1 the lower rim. There are shown by this plan-

(I) That the action of the medial rectus and lateral rectus alone is simple, tnat o all the other muscles being made up of the two or three components.

(2) That for all movements, except the purely lateral ones, two or three muscles act in concert.

(3) That when this occurs, two out of the three components of each muscle are in large part nullified, because opposed by those of other muscles, so that in general only the third set of components, which have the same direction and hence act as if added together, come into play.

(Fig. I44, I.), it will at the same time draw the posterior pole medially, so abducting the cornea ; finally, from its insertion lateral to the sagittal meridian, it can rotate the eyeball slightly medialwards. Its action, therefore, is to depress, abduct, and intort the cornea. 
The inferior oblique similarly can be understood from its position beneath the globe and its insertion on the posterior hemisphere to the lateral side of the sagittal meridian, primarily to elevate, secondarily to abduct and extort the cornea. It would appear that were it not for the forward divergence of the orbital axes and the consequent angular pull of the superior and inferior recti, the

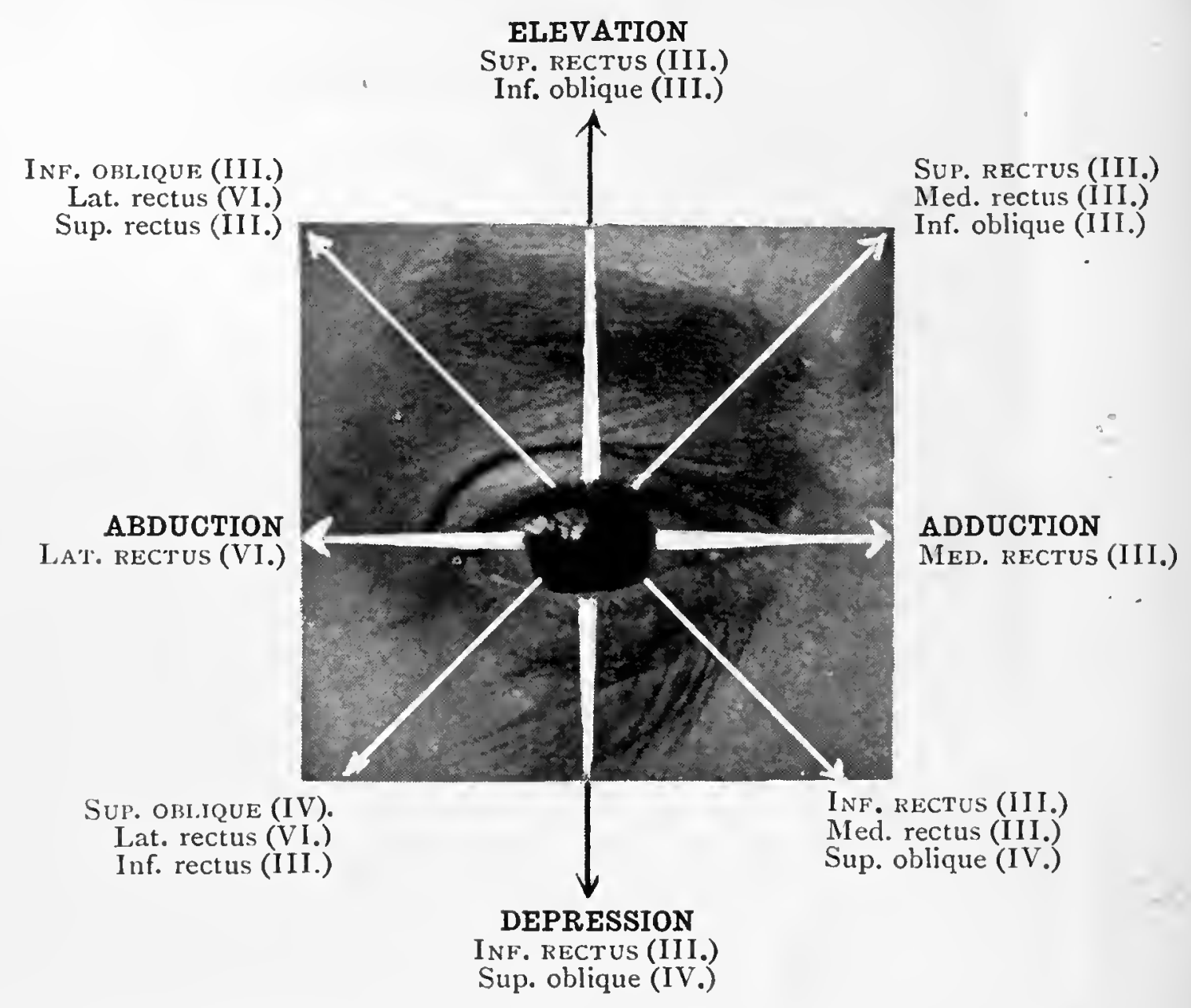

FIG. I48. - Scheme of movements of the Cornea with the muscles that affect them. The predominant muscles are in capital letters; the roman numerals after the names indicate the nerve supply. (Adapted from Testut, r9I4.)

need for post-equatorial insertion of the oblique muscles to counteract this secondary action of the recti would disappear.

The movements of the cornea are caused by combinations of these individual muscular actions; thus direct

Elevation results from the combined action of the superior rectus and inferior oblique (both supplied by the third nerve) ;

Depression is effected by the inferior rectus (third nerve) and superior oblique (fourth nerve); 
Abduction by the lateral rectus (sixth nerve), assisted by the two obliques (third and fourth nerves), especially towards the end of the movement ;

Adduction by the medial rectus assisted towards the end of its excursion by the superior and inferior recti (all supplied by the third nerve) ;

\section{Further movements}

$\mathrm{Up}$ and lateralwards are effected by the superior and lateral recti and inferior oblique muscles;

Up and medialwards by the superior and medial recti and inferior oblique ;

Down and lateralwards by the inferior and lateral recti and superior oblique ;

Down and medialwards by the inferior and medial recti and superior oblique ;

Intorsion by the superior oblique and superior rectus;

Extorsion by the inferior oblique and inferior rectus.

The actions of the muscles are well illustrated by the schematic plan of Marquez (Fig. I47), as reproduced by Fuchs, from whose classical text-book the above description is largely taken, and the movements of the cornea are shown in Fig. I48. More detailed descriptions of the action of these muscles and the movements of the eyeballs, of which the above is but a brief summary of the main facts, will be found in the works of Stevens (I887), Maddox (I907), Howe (I907), and in any of the current text-books of ophthalmology. See also pp. 389 and 39r.

From a clinical point of view, the position of the insertion and action of the muscles in the myopic or elongated eyeball are of interest. Fuchs states that the distances of the recti from the corneal margin are not increased, the elongation only affecting the posterior hemisphere as regards axial length. The oblique muscles, however, are thereby affected, passing more transversely and less antero-posteriorly to their insertion, so that the angles which they make with the medial plane are increased; their abducting power is greater, and the adducting power of the superior and inferior recti diminished, conditions favouring the condition of divergent strabismus (Fisher, I904). As regards the range 
of movement of the insertions, Landolt finds an equal excursion of $47^{\circ}$ from the position of rest in all directions, and Charpy gives ranges of $45^{\circ}$ (medial rectus), $50^{\circ}$ (inferior), $46^{\circ}$ (lateral), $45^{\circ}$ (superior).

As quite subsidiary actions of the muscles, it may be added that by means of the connections of their facial sheaths the superior rectus pulls up the superior conjunctival fornix and also assists the levator in raising the upper lid, the inferior rectus slightly depresses the lower lid, the lateral rectus draws the corresponding commissure slightly backwards, and the medial rectus causes marked recession of the lacrimal caruncle.

Development. - It is commonly assumed on morphological grounds that the orbital muscles are derived from the anterior head or cephalic mesoblastic somites, though the latter have never been observed in man or mammals. Their mode of development in lower forms is described in some detail by Ryder (I900). The first somite gives rise to the levator, superior, medial, and inferior recti and inferior oblique, supplied by the third nerve; the second somite gives origin to the superior oblique, supplied by the fourth nerve; and the third somite to the lateral rectus, supplied by the sixth nerve. Neal (I9I8), however, produces evidence from comparative anatomy to support the contention of Dohn that the lateral rectus is derived in part also from the second somite ; in his paper will be found the literature upon the subject. Lewis (in Keibel and Mall, rgro) found that in man all the eye muscles arise from a common pre-muscle mass, first recognisable in an embryo of $7 \mathrm{~mm}$. in length, and connected with the third nerve only; the fourth and sixth nerves entered at the $9 \mathrm{~mm}$. stage, wherein the muscle mass began to show signs of cleavage ; at $\mathrm{I}_{4} \mathrm{~mm}$. all the muscles are to be distinguished, and have nearly the same position relative to the eyeball as in the adult, though the inferior oblique does not completely separate off from the inferior rectus until later. In a reconstruction by Ask (I9IO) of the muscles of the eye in an embryo of $55 \mathrm{~mm}$. long, the levator is only just begin- 
ning to separate off from a well-developed superior rectus. Poyales (I9I7) has also studied the muscles in the human embryo and finds that the lateral rectus shows inferiority as compared with the medial rectus in age, length, and number of fibres; he considers that the consequent greater power of the medial rectus would be the cause of the congenital strabismus of the new-born (see also p. 270).

Abnormalities. - It is probable, to judge from the writer's individual experience in finding quite a number of gross abnormalities of the ocular muscles in his series of dissections, that such are by no means as excessively rare as would appear from the number recorded in the literature ; dissecting-room conditions do not favour their identification, and in life some may be unrecognisable through compensatory action of the other muscles. They are explained by errors in development by cleavage from the common pre-muscular mesoblastic mass. See also Hardy (I9I4).

The superior rectus has been found to give off a muscular slip I5 $\mathrm{mm}$. long, which arose from the same origin from the annulus of Zinn and passed downwards and forwards across the lateral face of the optic nerve to join the inferior rectus about its mid-point; the nerve-supply came from the inferior division of the third nerve (Aubaret). See also p. I88.

The medial rectus has been found absent in some cases of divergent strabismus (Ledouble, Krause). Its posterior third may be fused with the inferior rectus. A bifid sclerotic insertion by two tendons, I6 $\mathrm{mm}$. in length, is recorded (Wicherkiewicz).

The lateral rectus has likewise been found undeveloped in some cases of convergent strabismus (Ledouble, Krause), and a case of atrophy of the muscle has been noted on operating for strabismus in the living (Bourgeois). A fasciculus may pass from it to the inferior rectus, as is normal in certain ruminants, or to the lateral wall of the orbit. A lateral rectus with two extra fasciculi which passed forwards to end on the inferior tarsal plate and lateral wall is recorded (Curnow). In the specimen photographed in Fig. 37 there was a well-marked, fleshy bundle $7 \mathrm{~mm}$. long and $2 \mathrm{~mm}$. in diameter, passing from the lateral rectus across 
the posterior third of the orbit beneath the optic nerve to fuse with the belly of the medial rectus; no nerve could be traced to it.

The inferior rectus in the preparation photographed in Fig.' 55 can be seen giving off a large muscular bundle which passed lateral to the optic nerve and joined the superior rectus; it was innervated by the lower division of the third nerve.

The superior oblique may be closely accompanied by an offshoot from the levator palpebrae superioris, sometimes called the comes obliqui superioris (see p. I44). In the preparation shown in Fig. I84 there were present two long muscle bundles, arising in common with the levator and ending anteriorly, the one upon the fascia bulbi between the superior oblique and the globe, the other on the orbital margin beneath the pulley; the nerve supply came from the fourth nerve; the superior oblique was broader than usual. Ledouble has found supernumerary fasciculi accompanying the reflected tendon; and has further recorded a case where the direct or normal fleshy part of this muscle was absent, the reflected or usually tendinous part being muscular and arising from the site of the pulley, recalling the type normally found in non-mammalian vertebrates.

The inferior oblique has been found by the writer to have an abnormally placed origin in several instances. Out of Ioo orbits examined, the origin was in the usually described position immediately adjacent to the incisura lacrimalis of the maxilla in 45 instances; it lay from 2 to $5 \mathrm{~mm}$. distant in 47 cases (most often in the left orbit); and was situated from 6 to $7 \mathrm{~mm}$. lateral to the normal position in 8 cases. Of the latter the muscles were examined in three eyeballs and found to be inserted higher up than usual, and their total length was normal ; the position resembles that found in certain fishes (Whitnall, I92I).

An abnormal muscle bundle (musculus obliquus accessorius inferior) has been found by Rex passing from the apex of the orbit to the inferior oblique, but also sending a slip to join the inferior rectus; it was found in both orbits, and was supplied by the third nerve. 
Vestiges of the musculus retractor bulbi (retractor oculi, superior oculi, rectus posterior, choanoid) muscle have on rare occasions been recorded in man, and what would appear to be a well-marked example is illustrated in Fig. I49. The muscle is first met with in amphibians and certain reptiles, and exists in the greater number of mammals, reaching its highest development in the ruminants; vestiges occur in some monkeys (e.g. macacus rhesus). The muscle arises from the apex of the orbit, lies wholly within the cone of muscles formed by the recti, and is inserted on to the posterior hemisphere of the globe ; it may exist either as a cone-shaped mass cleft only

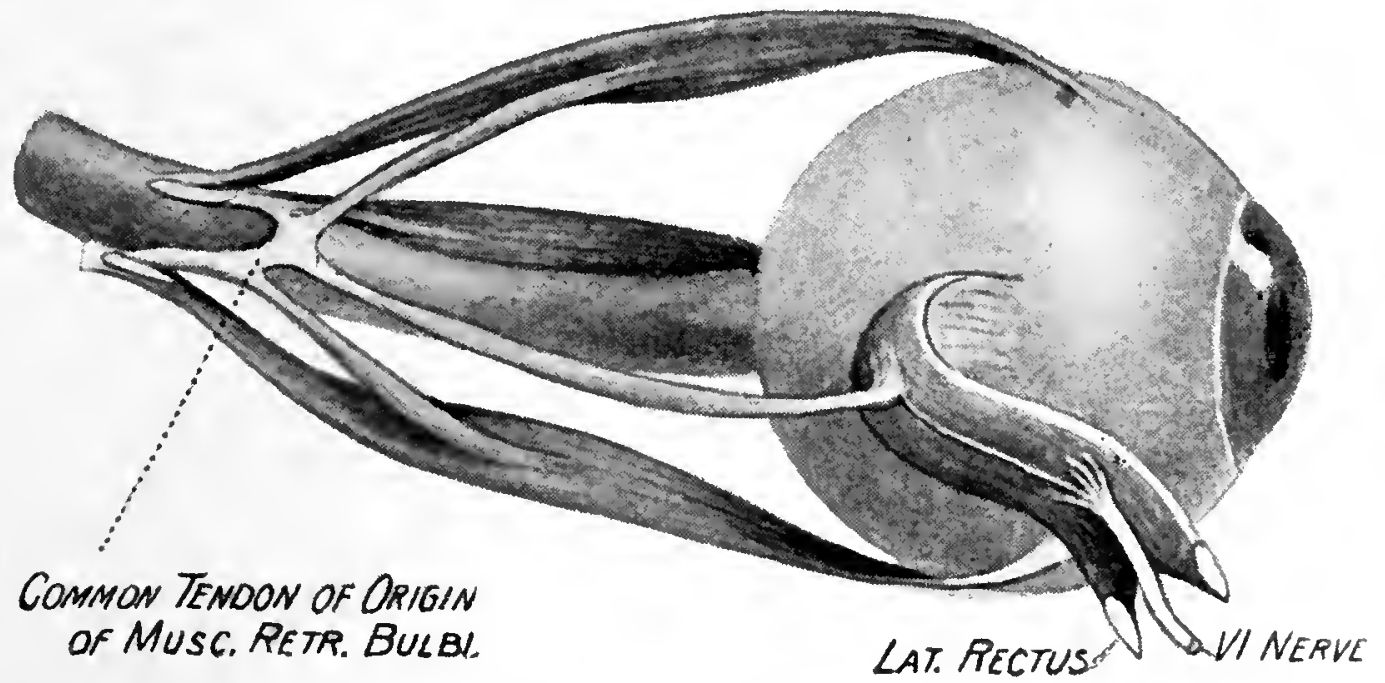

FiG. 149.--Sketch of an instance of the Retractor Bulbi Muscle in Man. There were four muscular bundles passing forwards towards the globe, but each fused with a rectus muscle before reaching it; one bundle was innervated by the sixth nerve as shown in the sketch, but the others were supplied by the third nerve. For description see the paper by the writer, I9r.

by a passage for the optic nerve, as in the pig, or be subdivided into two or more parts; in the cat, for example, the muscle is divided about $2 \mathrm{~mm}$. from its origin into four slips, which diverge and pass forwards to be inserted on to the globe behind the attachments of the recti and opposite the intervals between them. It is to be regarded as a derivative of the lateral rectus (Corning, I902; Johnson, I90I), and is innervated in the domestic animals by the sixth nerve (Hopkins, I9I6), though in the writer's example in man the third nerve shared. Its action is antagonised by the membrana orbitalis musculosa or protractor of the globe. In the macaque monkey it is found reduced to a single bundle placed between the superior and lateral recti, in 
which position Ledouble found delicate muscle bundles in two cases in man. Other instances have been noted by Nussbaum, and Fleischer (I907), and according to Lewitsky (IgIO) it is always represented in man (thirty specimens examined) by a well-marked strand of connective tissue (fascia retro-bulbaris) lying between the lateral rectus and the optic nerve, and attached to the back of the fascia bulbi anteriorly; it does not appear, however, to be readily identifiable. The literature will be found in a paper by the writer (Whitnall, IgII).

3. The Fascia of the Orbit (fasciae orbitales). - This comprises :

(i.) A condensation of connective tissue containing both collaginous and elastic fibres round the eyeball, which forms a primary socket for it called the fascia bulbi or Tenon's capsule.

(ii.) The fascial sheaths of the muscles, with extensions to the orbital walls known as " check ligaments."

(iii.) The connective tissue supporting the orbital fat.

The muscle sheaths blend with the fascia surrounding the globe, and both are connected to the stroma of the orbital fat, but for descriptive purposes the three parts of the fascia are best considered apart.

(i.) The fascia bulbi (Tenon's capsule, Bonnet's capsule, aponeurosis orbito-ocularis, tunica vaginalis oculi, etc.), from the great attention that has been paid to it and the conflicting views regarding its exact constitution, deserves a brief historical note. Mention is vaguely made of it under the name of " tunica adnata " by Galen, Colombo, Casserius, Riolon, and others, but Tenon, of Paris, in I8o6 gave the first good description of the structure, which bears his name. It then appears to have been forgotten until about I840, when Stromeyer's discovery of strabotomy raised the question of the more exact relations of the muscles of the eye and the orbital fascia, and investigations by Malaigne again brought the capsule into notice under the name of the " orbital aponeurosis." Then Bonnet in I84I pointed out the surgical importance of the relation of its 
posterior part in connection with enucleation of the eyeball, and Ferrall gave a very clear description of the "tunica vaginalis oculi" about the same time. Sappey in I867

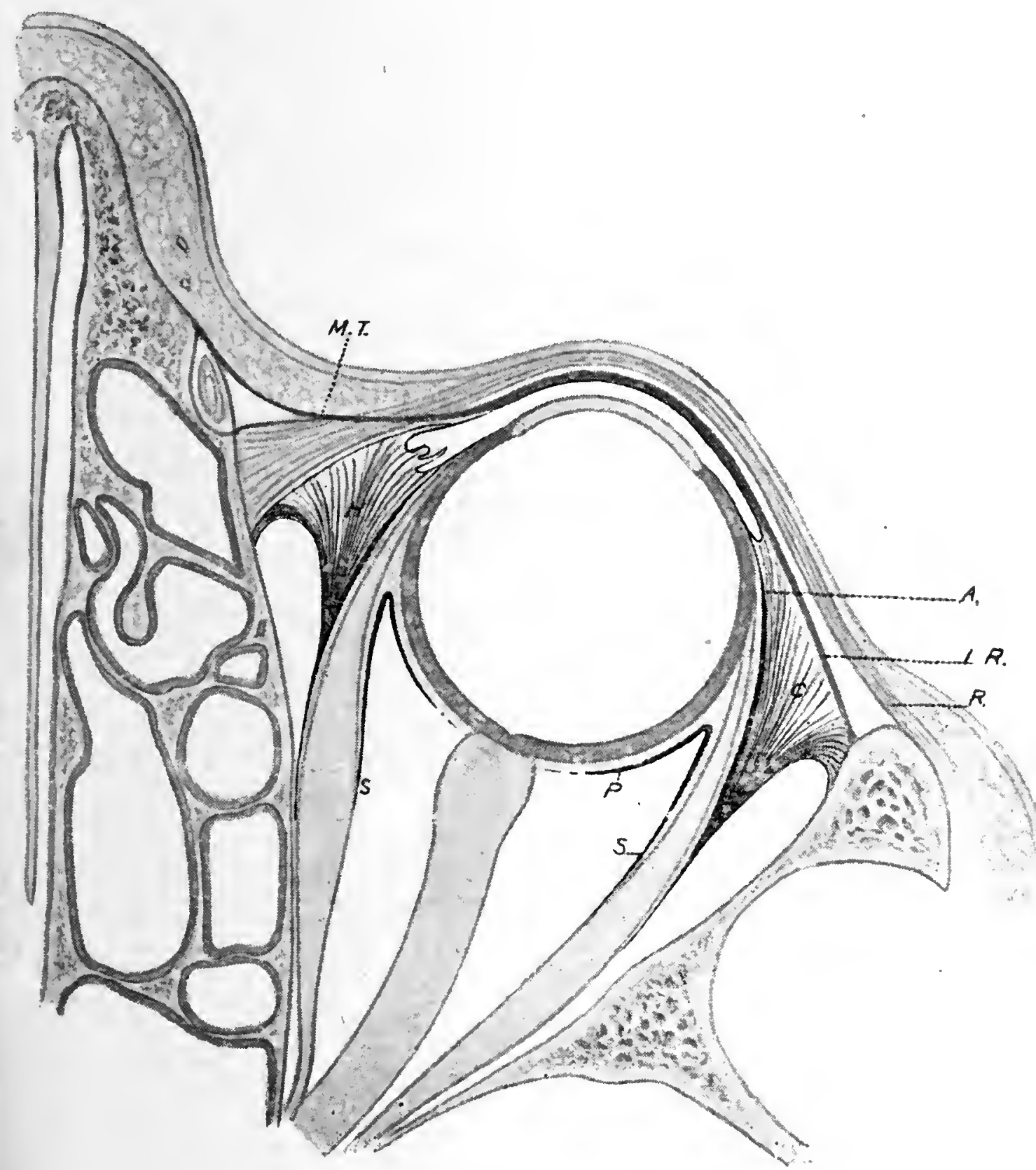

FIG. I50.-Schematic view of a horizontal section through the Right Orbit to illustrate the Fascia of the Orbit. Compare with Fig. 155. Fig. 67, p. 142, shows the arrangement in vertical section.

The fascia bulbi or capsule of Tenon is shown by A., its anterior part, and P., its posterior part. The fascial sheaths of the muscles are marked by S., and their offshoots form C., the lateral, and H., the medial "check ligaments."

The drawing also illustrates certain points in the anatomy of the eyelids, M.T. being the medial palpebral ligament with its two limbs passing in front and behind the fossa for the lacrimal sac, I.R. being the lateral palpebral ligament, and $R$. the lateral palpebral raphe.

first described the presence of smooth muscle fibres in the check ligaments of the lateral and medial recti, and showed that these ligaments were not formed by the muscles themselves but by their fascial sheaths. As regards later 
accounts, like fascial dispositions elsewhere in the body such as the pelvic fascia, the capsule has received most elaborate attention, and is still to this date the subject of reinvestigation and laboured description. Dwight, indeed, remarks that " the complications of this membrane are limited only by the perverted ingenuity of those who describe it." Motais, for example, devotes nearly fifty pages to its description in Poirier's Anatomy, and deals with it from the standpoint of comparative anatomy in his book on the motor apparatus of the eye (I887) ; his method of demonstrating sheets of fascia by pressing the connective tissue between sheets of hot blotting-paper to absorb the fat within its meshes appears liable to form artificial conceptions of its disposition. Schwalbe (I887), Lockwood (I886), Howe (I907), Lewitsky (I9I0), Gallemaerts (I899), Virchow (I902), and Charpy (I9I0), the latter of whom has studied the effects and extent of experimental chemosis by gelatine injections into the capsule and beneath the bulbar conjunctiva, have also written papers on it. Hesser (I9I3) has compiled what is practically a monograph upon the subject; the latest description is by Sutton (I920), who has, however, little to add to previous descriptions.

The writer has studied the capsule and muscle sheaths. not only by repeated dissections of material preserved in the usual way, but by examining practically fresh preparations in the post-mortem room, and feels convinced that connective tissue strands, hardened by alcohol or formalin and isolated by dissection from fat and neighbouring structures, assume in many regions an unwarrantable distinction as membranes, ligaments, or capsules. It should be realised that the fascia of a muscle is the remains of a mesoblastic tissue mass undifferentiated into muscle fibre; also that if a firm body such as the eyeball revolves against a cushion of fatty tissue, the fat will be pressed away and the connective tissue mesh-work supporting it condensed into a membrane-like surface. Such appears to be the case in the formation of the capsule of Tenon: the surface applied to the globe is shown definitely membranous after enucleation of the latter (hence meriting the term capsule, which for clearness of 
description will be used in this section), but the posterior surface is connected with the mesh-work of the orbital fat, and the most careful dissection from this aspect leaves it ragged and perforate.

The following account of the capsule is such as is demonstrable by ordinary dissection.

The capsule of Tenon is a thin envelope of connective and elastic tissue completely surrounding the eyeball from the circumference of the cornea in front to the entrance of the optic nerve behind; it is so closely applied to the globe that in sections of the orbital contents it is not readily identified, but by pulling the structures apart posteriorly a space between it and the globe, the "episcleral space" or spatium interfaciale, can be formed. Careful scrutiny will show, however, that the interval is really occupied by a felting of extremely fine connective tissue, the tissue of Tenon, episcleral tissue or tunica adventitia, comparable to the arachnoidea of the brain, though finer and denser. There is no sign of an endothelial lining, and the second or visceral layer of the capsule, as sometimes described (Schwalbe, Parsons), is merely that part of the adventitia which remains adherent to the sclera after separation of the capsule (Fig. I54); the space, according to Charpy and Hesser, is neither a true serous nor a formed lymph cavity, as described by some authors (Schwalbe); Leber (I876) also denies that it communicates with the perichorioidal space of the eyeball or contains lymph. The eyeball can move within the capsule, the adventitia being lax enough to allow of slight excursions, but in wider movements both globe and capsule move together as a whole upon the bed of orbital fat which is loosely connected to the capsule behind. It is obviously impossible for the eyeball to rotate freely within a socket that is fixed to and terminates at the circumference of its anterior sixth, that is, the cornea, as well as attached to the muscles at their points of entrance.

Since the capsule envelops the globe from the cornea in front to the optic nerve behind, it follows that it is pierced by all the structures which are attached to the eyeball, namely, the ocular muscles, the venae vorticosae, and the 
ciliary arteries and nerves. The recti muscles enter the capsule in front of the equator of the globe and serve to demark a smaller anterior from a larger posterior part. The anterior part of the capsule, in extent about one-third of the whole and lying in front of the muscle attachments, is thinner and more closely applied to the globe, being fused

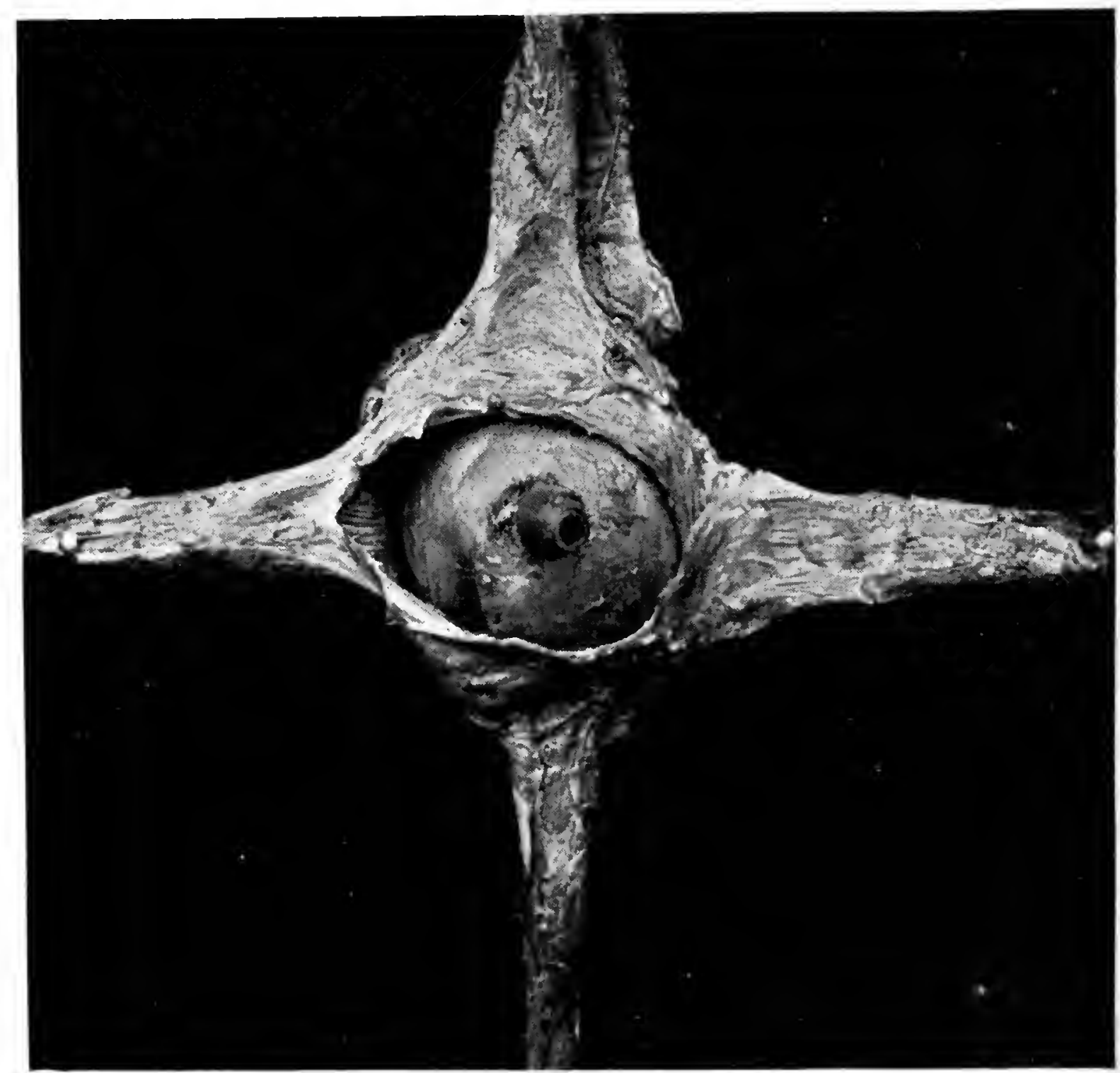

FIG. I5I. - Left Eyeball seen from behind. The four recti have been spread out to show the continuity of their fascial sheaths with the fascia bulbi, or capsule of Tenon, which has been cut away on the left side to expose the meclial rectus. Such a hardened and dissected-out preparation conveys an exaggerated iclea of the density of the capsule. Natural si\%e.

to the sclera for $\mathrm{I}$ to $2 \mathrm{~mm}$. wide of the corneal margin (Fig. 97, p. I94); on its external face lies the bulbar conjunctiva, and the two membranes are raised together in exposing the tendons of insertions of the recti, as is well illustrated in Fig. I42, p. 265 ; the episcleral space here is extremely narrow and the intervening tissue greatly reduced. 
The posterior part of the capsule is thicker and more yellow than the anterior, save just round the attachment of the optic nerve to the globe; here it is so broken up by the entrance of the posterior ciliary arteries and nerves that it is difficult to define, so much so that Merkel considers it to cease altogether a few millimetres from this point; in any case it is quite clear, as both Merkel and Virchow point out, that there is no continuation of the membrane backwards along the optic nerve to the apex of the orbit to form a so-called and often figured "supravaginal space" around it and continuous with the episcleral space (Schwalbe, Piersol); the optic nerve in its movements occasioned by the excursions of the globe has naturally formed a closer packing of the surrounding orbital fat.

The practical importance of the capsule lies in the fact that it forms a barrier between the globe and the other contents of the orbit, and after enucleation of the eyeball it forms a socket for an artificial eye, as is shown

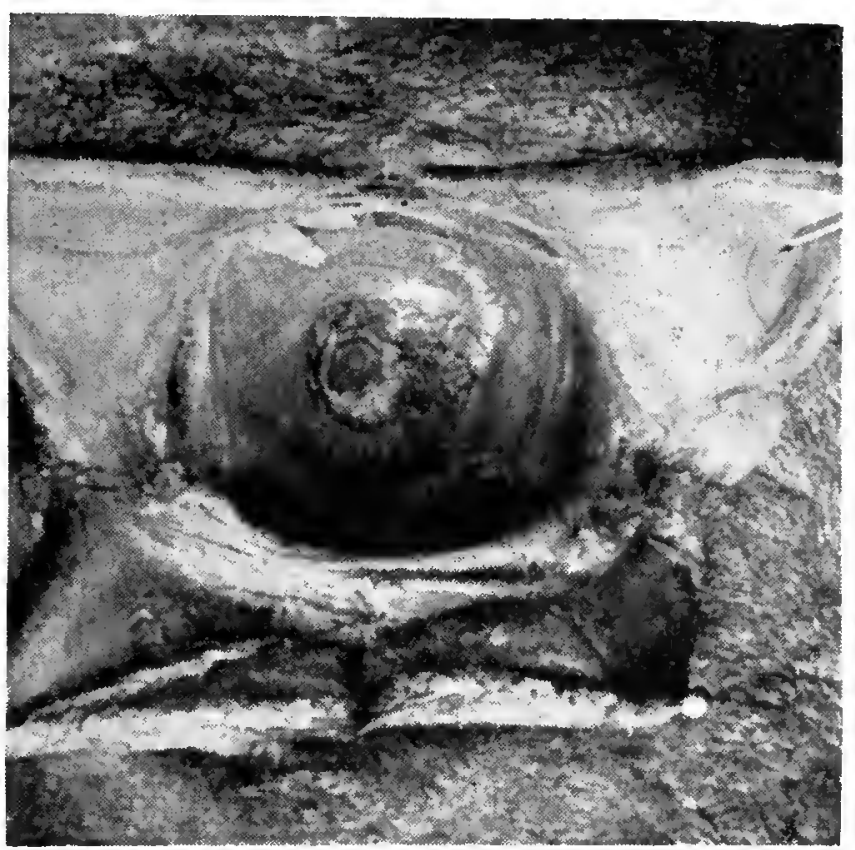

H'IG. 152.-.'The Left Eye-Socket. 'The eyelids have been crucially incised and pulled aside and the eyeball removed to show the fascia bulbi or capsule of Tenon forming a barrier to the orbital contents behind it. Natural size. in Fig. I52, where the slit-like openings of the muscle sheaths into the capsule are seen, and also in Fig. I54.

(ii.) The muscle sheaths (fasciae musculares) are so thin and transparent in their posterior third (and in the case of the fleshy part of the superior oblique in the whole extent) that they are practically invisible; traced forwards, however, they become thick and opaque, and at the points where the muscles pierce the capsule of Tenon, they blend and become directly continuous with the latter. So uniform is the connection that some authors consider the sheaths to be formed by backward diverticula from the capsule (Testut, 
Charpy, Virchow), and on the other hand the posterior part

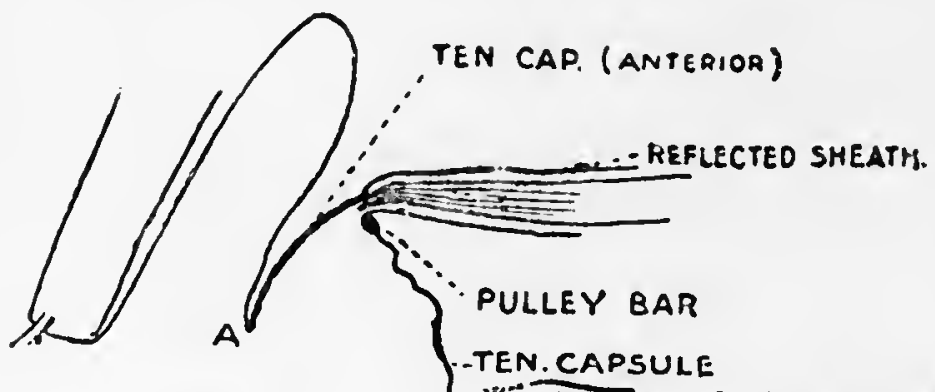
of the capsule has been considered to be formed by the. fusion of backward extensions of the sheaths (Motais) ; it is much clearer to describe, as Hesser does, the sheaths and their offshoots as separate formations which fuse with the capsule when they come into contact with it. Just before this union with the capsule, the adjacent margins of the sheaths are continuous with one another, with a wide sweep between them when laid out as in Fig. I5 $\mathrm{I}$, but in the natural position forming a backwardly directed " intermuscular membrane," which is especially well marked between the superior and lateral recti, where it passes beneath the lacrimal gland; the backward extension of the membrane is greatly exaggerated in many illustrations (e.g. those of Motais), and practically exists only a short distance behind the globe.

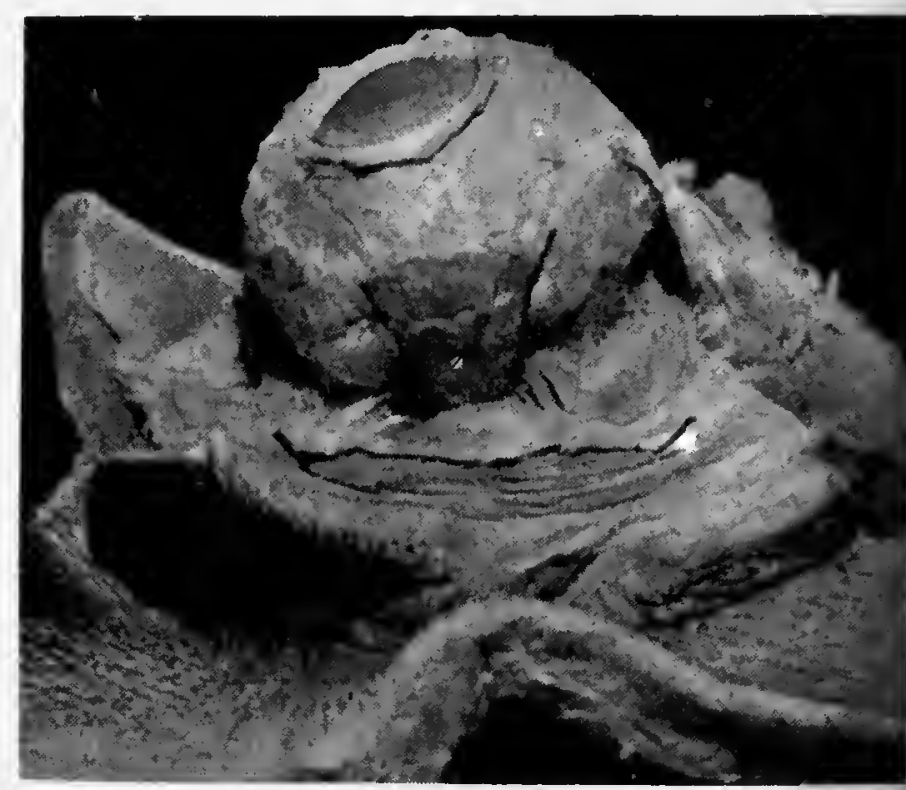

FIG. I 54.-Dissection of Eyeball to show the Muscles entering the fascia bulbi or capsule of Tenon, and the episcleral tissue adherent to the sclera. The eyelids were first cut and turned down, then the bulbar conjunctiva and anterior part of the capsule cut round the cornea and reflected from the forepart of the globe. Natural size.

Where the sheath is reflected backwards on the inner or 
ocular surface of a recti muscle to become continuous with the posterior part of the capsule it is thick and naturally forms a lip, but is not expressly developed into an " intracapsular ligament" (Lockwood) or a "falciform fold" (Charpy), and the appearance of such a thickening shown in Fig. ${ }^{54}$ is caused by a gathering together or rucking of the fascia, and disappears when the muscle sheath is outstretched. Nor does there appear to be specially developed any "pulley bar" (Fisher, I904) at this point, over which the muscle tendon turns; the muscle makes no angle at the beginning of its arc of contact with the globe such as would require such a contrivance, and the idea that there are so formed slings connected through the medium of the external part of the sheath or capsule with the orbital wall to prevent muscle pressure on the globe is also erroneous. The muscles are firmly connected with the margins of the openings through which they enter the capsule; moreover, their sheaths are adherent to their muscle bellies along the whole extent, a fact of considerable importance, since the attachment prevents their extensive retraction after severance in enucleation of the globe (Fig. I53).

The muscle sheaths give offshoots from their external surfaces to the orbital walls, known as " check ligaments," Fascienzipfel, or ailerons; they are best developed in the case of the medial and lateral recti (muscles whose excursions are greater and more exercised than are those of the others), and are therefore most clearly observed in horizontal sections through the orbit (Figs. I50, I55). That part of the sheath which clothes the lateral rectus on its external aspect becomes greatly thickened anteriorly and divides into two lamellae; one, the outer and larger, radiates forwards and laterally as a fairly dense triangular mass of tissue as broad as its muscle, and is mainly inserted into the periosteum over the zygomatic tubercle, just within the mid-point of the lateral orbital margin, but is also attached to the hinder face of the lateral palpebral ligament, which is attached to the same tubercle and to the lateral conjunctival fornix; this mass is the "check ligament" or lacertus of the lateral rectus. The 
inner lamella is thinner, and closely follows the tendon of the muscle forwards; it unites with the corresponding continuations of the other recti to form the anterior part of the capsule of Tenon, lying beneath the bulbar conjunctiva and extending up to the sclero-corneal junction. The check ligament of the medial rectus presents a similar formation, its anterior fixation being to the lacrimal bone

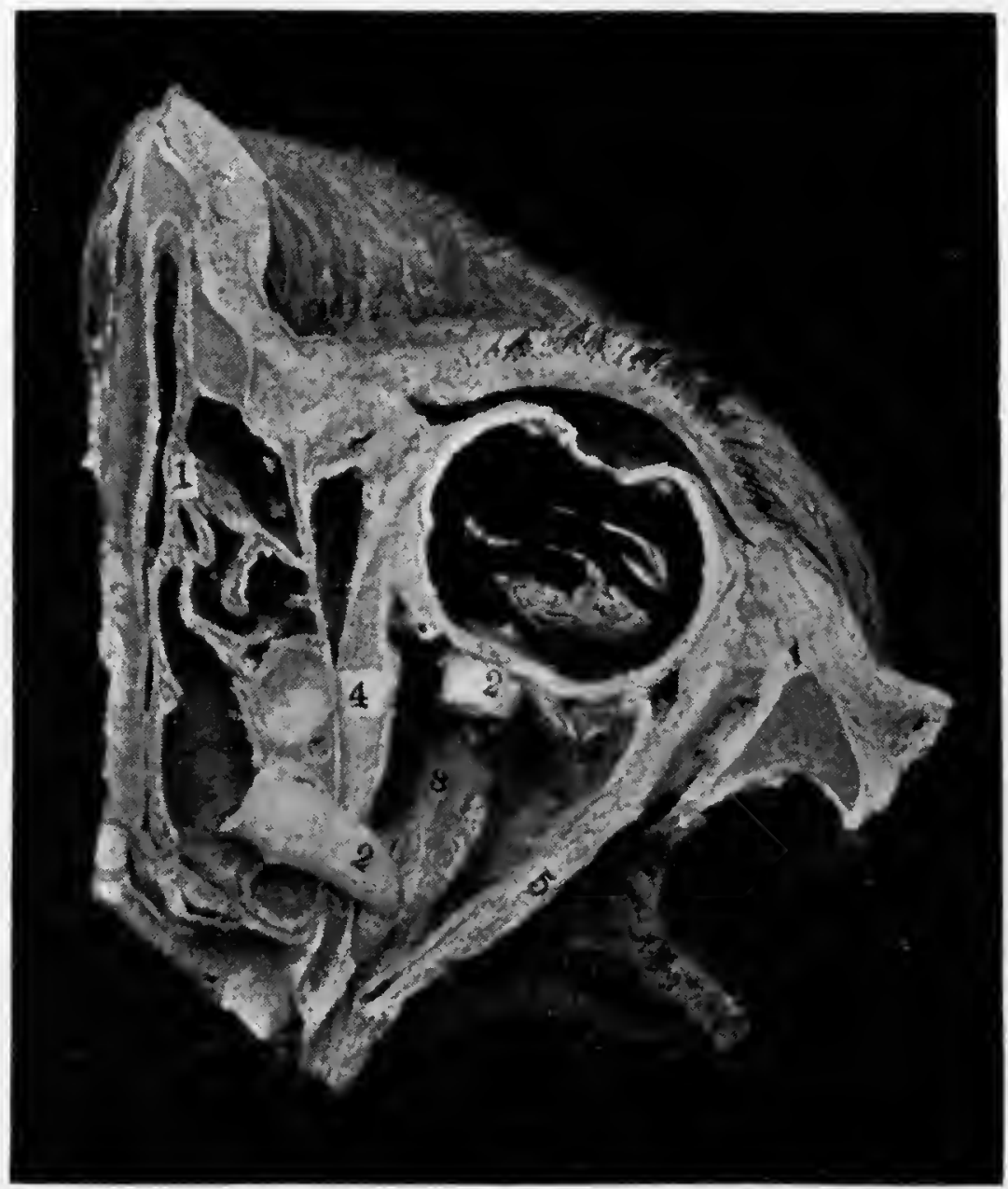

FIG. I 55.- Horizontal section through Right Orbit seen from above, to show the fascial sheaths of the medial $(4)$ and lateral (5) recti with their expansions which form the "check ligaments." 2 is placed on the optic nerve, which has been cut and turned aside to show 3, the inferior rectus, which in this instance presents a large abnormal bundle faintly marked 6 on its lateral aspect (see p. 284). Natural size. Compare with Fig. I50.

just behind the posterior crest, to the septum orbitale behind the pars lacrimalis muscle, and to the caruncle and medial fornix. Ledouble states that it is not rare to find in man a muscle fasciculus in either of these expansions, presumably an offshoot from the muscle itself, but Sappey first described smooth or involuntary muscle fibres in them, the presence of which has been verified by later authors (Motais, Groyer, Hesser). That these expansions do act 
as check ligaments to over-contraction of the muscles, can readily be verified by cutting one of them and noting the greatly increased range of excursion of the globe on pulling the appropriate muscle ; their attachments to the commissures explain the recession of the parts to be noticed on strong movements of the eyeball in the one direction or the other. The checking of the action of the superior or inferior rectus is not so apparent. The sheath of the former blends with that of the overlying levator palpebrae superioris, and the fixed mass between them is attached to the back of the superior conjunctival fornix ; the two muscles act harmoniously, and the superior rectus may be limited in range by the same fascial mechanism as has been described for the levator on p. I43. The fascial sheath of the reflected tendon of the superior oblique clothes it as far as, and is attached to, the pulley. A definite check ligament is described by Motais, Charpy, and Maddox for the inferior oblique, in the form of a fascial strand passing laterally and backwards from its sheath to the floor of the orbit, and called the "septal bridge" of the lower eyelid by Virchow; an example is seen in Fig. 54, p. I23, but it may be pointed out that this is the only instance the writer has found of a strand which could reasonably possess any such action. Distal to the point where the inferior oblique crosses the inferior rectus, their blended sheaths send forwards thin lamellae, one of which follows the tendon of the latter muscle to help form the anterior part of the capsule of Tenon, whilst the other can with difficulty be traced into the lower eyelid, as shown in Fig. 67, p. I42.

These expansions of the sheaths draw the fornices of the conjunctiva backwards, but a more important function is that by their fixation to the orbital walls they anchor the whole fascial apparatus of the globe in position. In this respect special note is to be made of the sheath of the inferior rectus which is thickened on the lower aspect and blended with the sheath of the inferior oblique. As previously noted, the sheaths of the muscles become confluent with each other by their adjacent margins immediately prior to the fusion with the capsule; the sheath of the 
inferior rectus is no exception to the rule, and the thickening of its sheath can be traced laterally and medially up to the sheaths of the lateral and medial recti, and, since these are fixed to the orbital walls by expansions, there is formed a continuous band about one-tenth of an inch thick beneath the globe, supporting it as in a hammock. The band was first described by Lockwood (I886) and named the ligamentum suspensorium oculi; it is well displayed in Figs. 55, 67 and I70; and its function as a suspensory ligament to the globe is manifest after operations in which the maxilla is removed for disease, since it then prevents the eyeball from sinking down. It may be added that the medial and lateral check ligaments, blended with the extremities of the suspensory ligament, meet the horns of the aponeurosis of the levator and are attached to the orbital margin at the same mid-points, and the conjoint masses of tissue so formed have been named the retinaculum oculi laterale and mediale respectively (Fig. 55, p. I24).

A final point of interest in regard to the capsule lies in the fact that there is found in close relation to it a scattered and ill-defined area of smooth muscle fibres. Hesser (I9I3) has recently investigated this peri-bulbar musculature in the foetus and the adult by means of serial microscopical sections, and finds it to extend round the anterior half of the globe in a nearly continuous but very thin layer, from 3 to $7 \mathrm{~mm}$. wide in antero-posterior extent, but broken on the lateral side, and he terms the whole the musculus capsulo-palpebralis. Anteriorly it extends into the eyelids, constituting the palpebral involuntary muscles (of Müller), and designated by Hesser pars superior and pars inferior respectively; these are the only parts of the musculature which form definite isolated lamellae, the pars medialis being an extension of the latter (Fig. I56). Posteriorly, behind the conjunctival fornix, it is quite indefinite. The whole sheet, which is innervated by the sympathetic system, is of interest in that its contraction was considered by Landström (Igo8) to be the cause of the exophthalmos of goitre (see p. 84). Groyer's morphological derivation 
of this involuntary musculature, as given above on p. I40 in connection with the levator palpebrae superioris, should be referred to.

(iii.) The orbital fat (corpus adiposum orbitae, capsula adiposa bulbi), which in life is no doubt semi-fluid, compactly fills all the space not occupied by the other structures, as is seen in Fig. I30; in formalin hardened specimens, indeed, it forms so firm a mass that excellent sections of the orbital

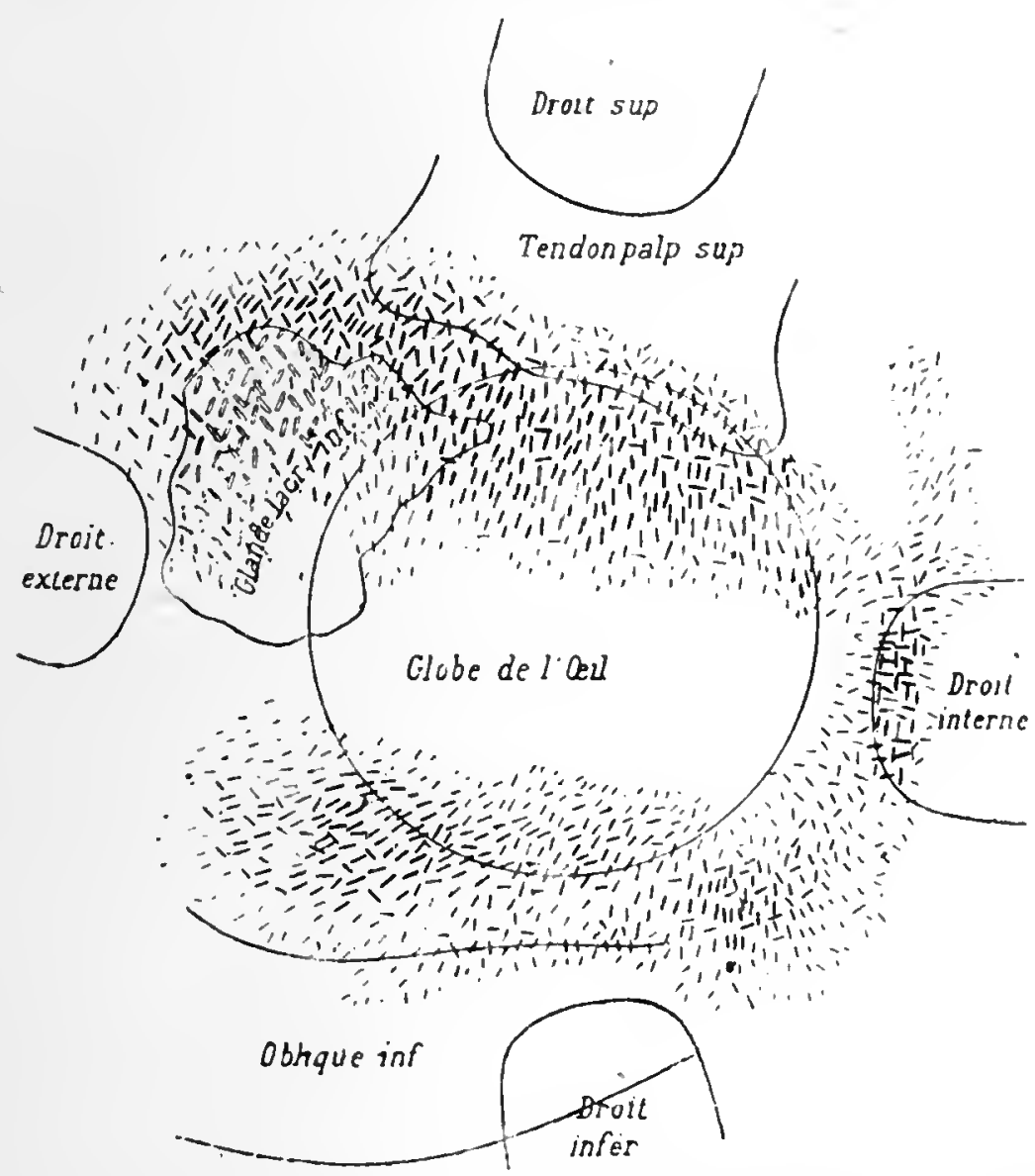

FIG. I56. - Diagram showing the position of smooth Muscle Fibres in the Fascia round the eyeball of an adult man, as described by Hesser (1913) and called by him the musculus capsulo-palpebralis. (From Hesser's figure, reproduced by Roussean, I9r6.)

contents can be cut with a razor by hand, as shown by Fig. I85, p. 366. It varies in consistency in different regions. Within the cone of muscles at the back of the globe and along its whole extent round the optic nerve (central part of the orbital fat) it appears as large and loosely connected lobules which can easily and cleanly be picked away with the forceps in dissections of the orbit; between them the ciliary nerves and arteries make their way forwards, and it is by the passage of these structures that the fat is broken up; the inter-lobular tissue is loose and can be made 
oedematous by artificial injections, and it is along these channels that blood extravasated in fractures reaches the deeper parts of the orbit; Birch-Hirschfeld (I909) thought that the spaces between the lobules represented the unknown orbital lymph channels. 'Outside the cone of muscles, especially beneath the globe, behind the lacrimal gland, and even between the muscles anteriorly, the fat is collected in larger and firmer masses, which are much more difficult to remove owing to the quantity of connective tissue they contain and their close connection thereby with the muscle sheaths; in this region it may be distinguished as the peripheral part of the orbital fat.

As regards the relations of the capsule and orbital fat to the conjunctiva and eyelids, it is first to be noted that if the eyelids, including the septum orbitale, be completely dissected away from the front of the orbit, the capsule and muscle sheath expansions form an interrupted barrier to the orbital fat between the globe and the walls of the orbit; the gaps through which the fat is visible are called the " orifices adipeux " of the base of the orbit by Charpy (I908), and are shown in Fig. 55, p. I24; it is through one such orifice that the lobule of fat (" palpebral hernia ") illustrated in Fig. 49, p. II5, protrudes.

Charpy (I9II) further studied the relations between the eyelids and the orbit by the injection of coloured gelatine between various muscle and fascial planes in the orbit, and his results may be summarised as follows:

(i.) Injections within the capsule of Tenon can distend and are confined to the whole episcleral space, from the corneal margin to the optic nerve, as shown in Fig. I57; they produce anteriorly a regularly disposed tumefaction round and limited by the cornea, and lying beneath the bulbar conjunctiva and the anterior part of the capsule.

(ii.) Injections into the central part of the orbital fat form a retro-ocular mass, which is confined anteriorly within the muscle cone by the conjoint anterior edges of the sheaths, the so-called intermuscular membrane, but in the posterior half of the orbit extends between the muscles into the peripheral region; there is no communication with the 
eyelids, since the injection is blocked by the globe and the muscle cone in front; hence moderate invasions of fluid into the central region neither invade the conjunctiva nor reach the lids, but produce an exophthalmos and immobility of the globe.

(iii.) Injections into the peripheral part of the orbital fat, outside the cone of muscles, are chiefly confined to the lower part of the orbit, and lie between the orbital fat and the periosteum lining the orbital walls. They can reach both the conjunctiva at the fornices and the eyelids behind the

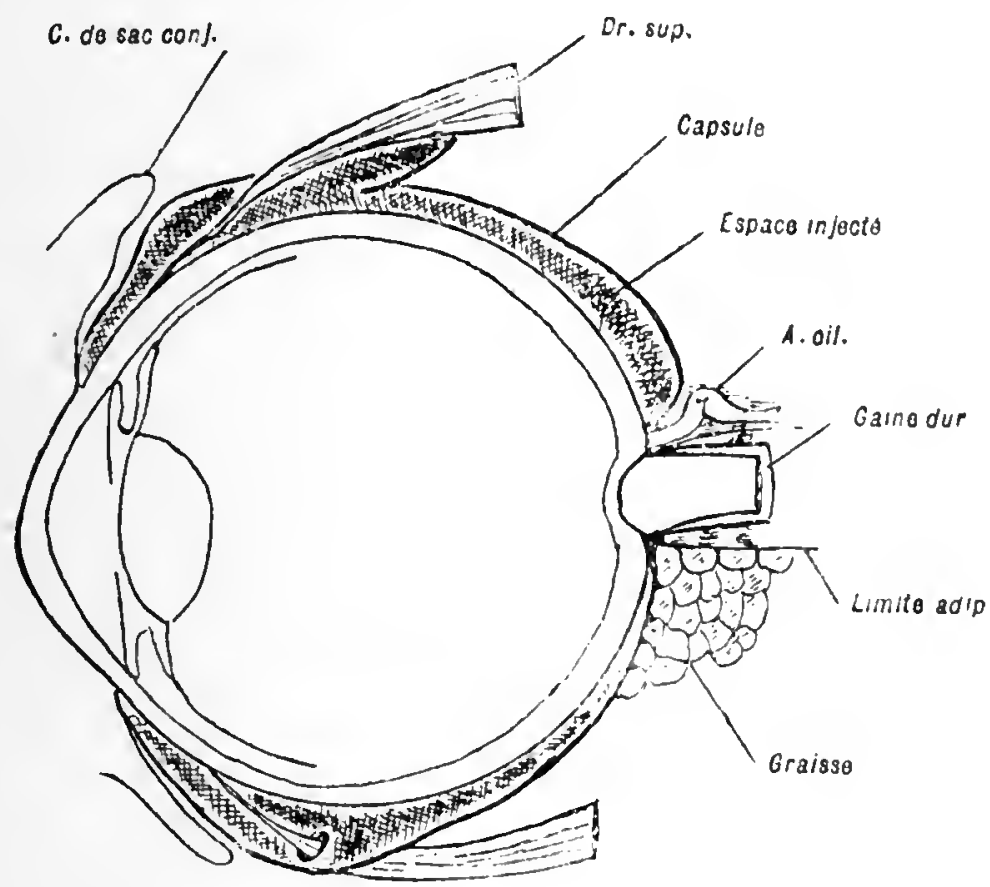

FIG. 157. - Diagrain of a sagittal section through the Eyeball, illustrating the confinements of various injection masses, as made by Charpy (I9I0).

septum orbitale by passing through any of the "adipose orifices" left between the attachment of the fascial sheaths of the muscles to the orbital walls, and this is the ordinary path along which fluids pass from the depths of the orbit to the eyelids. They may further transude through the venous or vascular apertures in the septum orbitale or through that membrane itself, and so reach the superficial parts of the lids, but Heerfordt (I904) has shown that the septum will stand a pressure of at least from 40 to $50 \mathrm{~mm}$. of mercurybefore allowing an intra-orbital injection to pass into the eyelids. The limits of injection masses in the superficial parts of the eyelids have been described above on p. I37. (See also p. 40.) 
Finally, sub-periosteal injections beneath the periorbita easily strip it off from the orbital walls and are confined to the space so formed, being arrested at the margin; they may rupture the membrane, especially if beneath the roof, and then behave like an invasion of the peripheral orbital fat.

Pathological observations support the results of such experimental injections (Friedberg, Magron, Girard, Königstein, Richet, Birch-Hirschfeld, etc.), and emphysemata, abscesses, and haemorrhage have been shown to follow the paths outlined above; the orbital course of haemorrhage in fractures of the base of the skull is determined by the same structures in reaching the eyelids (Liebrecht, I906).

4. The Vessels of the Orbit.-The arteries supplying blood to the orbital contents are chiefly the ophthalmic, with minor additions from the infra-orbital and middle meningeal branches of the internal maxillary.

The ophthalmic artery (arteria ophthalmica) arises from the internal carotid, being the last branch given off before it divides into its terminal branches, the anterior and middle cerebral arteries; it usually springs from the medial side of the fourth part of this vessel's course just as it ascends out of the cavernous sinus, and pierces the dura between the anterior clinoid process and the body of the sphenoidal bone, though sometimes it arises just within the sinus. The artery leaves the cranial cavity through the optic canal, embedded in the dural sheath of the optic nerve below and to its lateral side. Having entered the orbit, it pierces the nerve sheath, and lies at first within the apex of the cone of recti muscles, between the optic nerve on the one side, and the ciliary ganglion, third, fifth, and sixth nerves, and lateral rectus muscle on the other (Fig. I4I) ; it ascends and winds round the nerve, usually passing over it about halfway along its course beneath the superior rectus, and in company with the naso-ciliary nerve (Fig. I84); it then runs forward in a sinuous course towards the medial orbital wall between the superior oblique and medial rectus, and at the medial angle of the upper eyelid terminates by 
dividing into its terminal branches, the frontal and dorsal nasal arteries. These pierce the septum orbitale, and the latter vessel, passing between the trochlea and the medial palpebral ligament along with the infra-trochlear nerve, becomes directly continuous with the angular part of the facial artery (Fig. 85, p. I68).

As regards the origin of the vessel, the writer has often observed a marked reduction in the calibre of the internal carotid artery just after it has given off the ophthalmic branch, a narrowing out of proportion to the consequent diminution of its blood-stream as judged by measurement of cross-sections of the vessels (see Fig. I64, p. 3I7); on an average of ten specimens, the diameter of the internal carotid on entering the cavernous sinus was $5.4 \mathrm{~mm}$. (cross-section area $=22.89 \mathrm{sq} . \mathrm{mm}$.), but at a point $5 \mathrm{~mm}$. distal to the origin of the ophthalmic, the width was $3.8 \mathrm{~mm}$. (area $=$ Ir.33 sq. mm.), the corresponding dimension of the ophthalmic artery being $x .5 \mathrm{~mm}$. (area $=2.009 \mathrm{sq} . \mathrm{mm}$.). This conformation may be related to the intra-cranial bloodpressure, or be a means of reducing the shock of arterial pulsations to the brain (in conjunction with the curved course of the vessel as it traverses the carotid canal and cavernous sinus, a similar bending being found in the case of the vertebral arteries before they enter the cranial cavity), but it is also of interest to speculate whether the reduction in calibre of the main vessel, by forming a slight damming up of the blood-stream, does not favour the passage of the side current down this important ophthalmic branch. In connection with this suggestion it is to be noted that the branch not only comes off the main stem at right angles, but at once bends downwards to lie almost parallel with the latter at the commencement of its course, and it is not unreasonable to imagine that in such case the main stream would tend to draw blood out of the side branch rather than to guide fluid into it (analogous to the action of a spray producer, wherewith the air is blown across the end of the fluid-containing tube), were there not some such provision as apparently exists here.

The exact relation of the ophthalmic artery to the optic 
nerve in the intra-cranial part of its course appears subject to slight variation, the vessel lying more to the medial or lateral underside of the nerve according as it arises nearer or farther away from the optic foramen (Fawcett, I896).

The branches of the ophthalmic artery are small but very numerous, and, like the parent stem, are characterised by a tortuosity of course ; many anastomoses are formed with neighbouring extra-orbital vessels, though not as freely as is the case of the ophthalmic veins. They may be

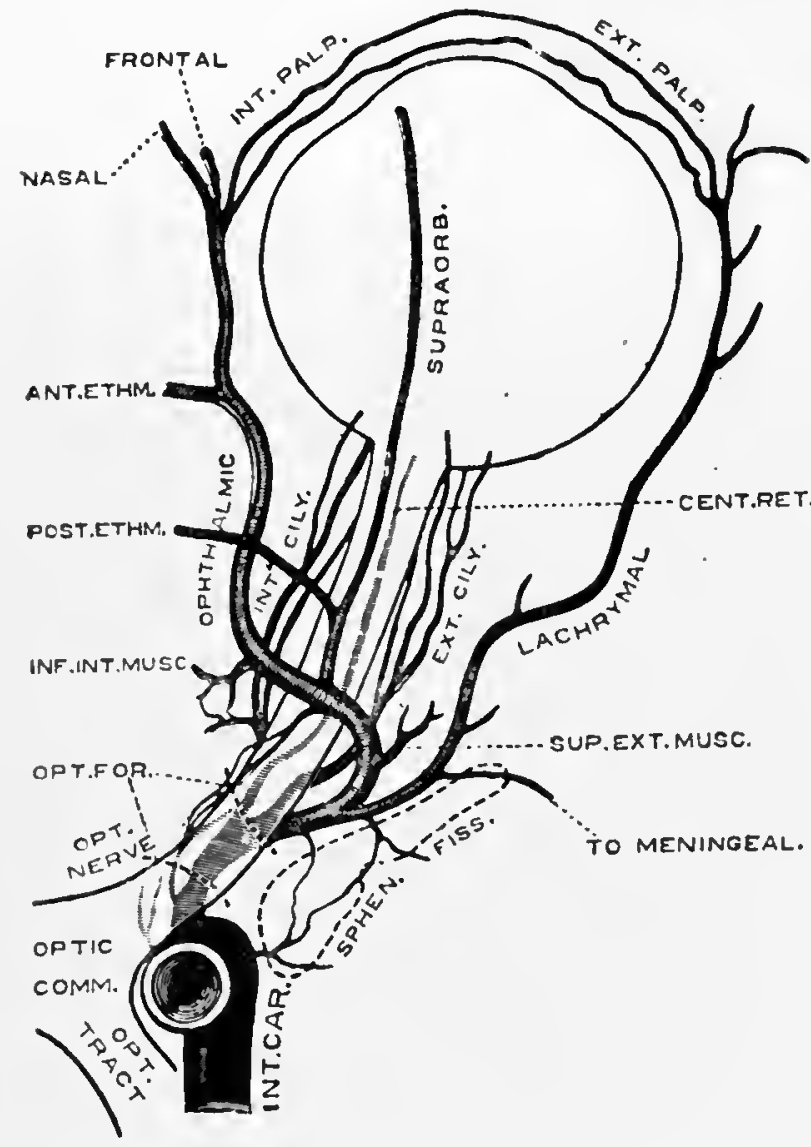

FIG. I58.-Diagram of the Cphthalmic Artery and its branches. (From Quain, I909, after Meyer.) classified in various ways, either :

(i.) Into groups supplying the eyeball, the other contents of the orbit, and extra-orbital regions; or

(ii.) From their sites of origin from the main vessel relative to the optic nerve, into groups arising lateral, superior, and medial to that structure, together with terminal branches; or

(iii.) According to their sequence of origin.

The eyeball is supplied by the central artery of the retina, two long posterior ciliary, and seven short anterior ciliary arteries; there are groups of branches to the muscles, and an artery to the lacrimal gland; and, passing on to supply parts beyond the orbit, are posterior and anterior ethmoidal arteries, the supra-orbital, and finally the terminal branches, the frontal, nasal, and palpebral vessels. Relative to the optic nerve, there lie laterally the central artery of the retina and the lacrimal, superiorly the supra-orbital, posterior ciliary, and muscular arteries, medially the ethmoidal and palpebral. The sequence in which the branches arise from the parent stem is, according to Meyer (I887), the retinal 
and medial posterior ciliary, the lateral ciliary, the lacrimal, the upper and lateral muscular twigs, the supra-orbital and posterior ethmoidal, the lower and medial muscular twigs, the anterior ethmoidal, and the terminal branches.

It is also to be noted (i.) that an artery lies in close relation to each of the walls of the orbit, namely, the supraorbital to the roof, the lacrimal to the lateral wall, the ethmoidal vessels and the terminal part of the ophthalmic artery itself to the forepart of the medial wall, and the infra-orbital (a branch of the internal maxillary) to the floor; (ii.) that each of the last-noted main branches is accompanied by a nerve; and (iii.) that they anastomose with vessels outside the orbit. The various branches are now to be described in detail.

The central artery of the retina (arteria centralis retinae), the first to be given off from the parent stem and the most important of its branches, arises from the latter whilst it still lies beneath the optic nerve, and runs forward to pierce the nerve sheath some Io to $I_{5} \mathrm{~mm}$. behind the posterior pole of the eyeball; the site of entry is variously given as either on the lower, the medial, or the lateral aspect of the nerve, but Deyl (I896), after a careful examination of twentyone specimens, found the position in each case to be inferomedial, and since this is the site of the optic fissure in the embryo where the mesenchyma invaginated the primary optic vesicle to form the vitreous, it appears that the vessel maintains its primitive position, and the supposition that the nerve and eyeball have rotated through an angle of $90^{\circ}$ during development (Vossius, in Keibal and Mall, I9I2) is without foundation. The artery is accompanied by a central vein and a sympathetic plexus derived from the ciliary nerves, the so-called Tiedemann's nerve, enveloped as it enters the nerve substance in a prolongation of the pial sheath, and it courses along the centre of the nerve to supply the inner layers of the retina; both vessels send recurrent branches backwards within the nerve almost up to the optic foramen (Quain). The finer branches of the network into which it ultimately breaks up in the retina make no connection with any other artery supplying the 
eyeball, though the main stem of the vessel may anastomose with a short posterior ciliary artery just after its entrance into the eyeball, such a cilio-retinal vessel being present in about I7 per cent of cases (Lang and Barrett). Sometimes the central artery may be replaced by a posterior ciliary artery, and indeed Meyer (I887) states that normally it arises in common with the medial long posterior ciliary, often within the sheath of the nerve.

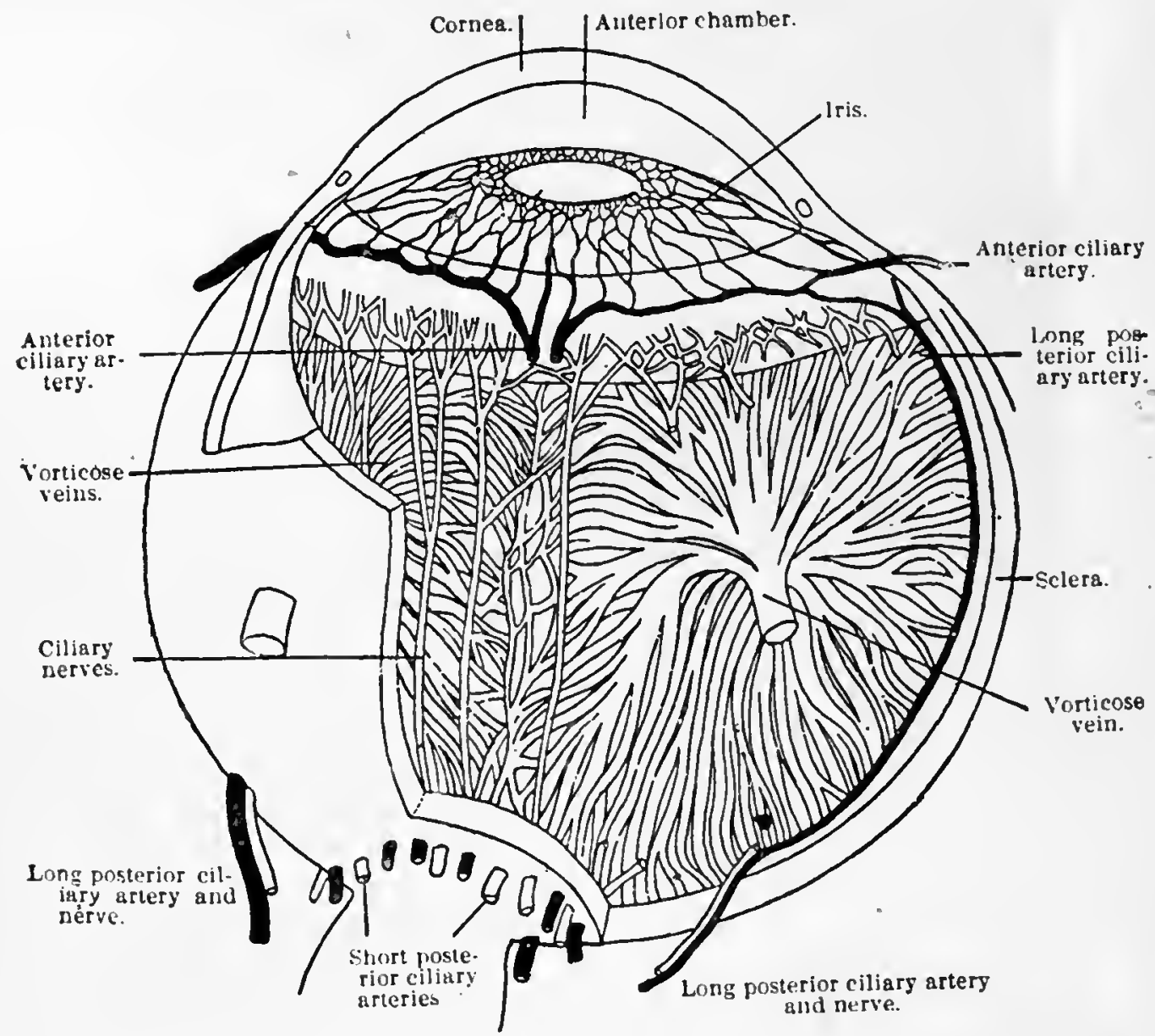

FIG. I 59. - The Vessels and Nerves of the Middle Coat of the Eyeball, to show the destination of the ciliary arteries and nerves. (From Baker, 1900, after Testut.)

The ciliary arteries, which are also destined to supply the eyeball (chorioid coat, ciliary processes, and iris), are variable in number and origin; they are distinguished as posterior and anterior groups. The posterior (arteriae ciliares posteriores longae et breves) arise from the ophthalmic artery as it crosses the optic nerve, and may appear as two single trunks or as six to eight vessels; in either case they subdivide near the globe into fifteen or twenty branches arranged round the optic nerve, and pierce the sclera to supply the chorioid. Two of them are larger than the 
others, and under the name of long posterior ciliary arteries enter the globe one on either side of the optic nerve (Fig. I32, p. 255); their ultimate destination is the ciliary muscle and the iris, where they anastomose with the anterior ciliary vessels to form the circulus arteriosus iridis. The anterior ciliary arteries (arteriae ciliares anteriores) arise usually from the branches of the ophthalmic artery which supply the muscles of the globe; they accompany the tendons of the recti (two to each, save the lateral rectus which has one), pierce the sclera just anterior to the line of tendon insertion, and anastomose with the long posterior ciliary vessels, as just noted; their main stems pass onwards toward the corneal margin to supply its periphery and the adjacent bulbar conjunctiva (see Fig. I03, p. 200) ; an anterior ciliary vessel may arise from the lacrimal artery.

The lacrimal artery (arteria lacrimalis) arises from the ophthalmic artery on the lateral side of the optic nerve, and in company with the lacrimal nerve passes forward along the upper margin of the lateral rectus muscle through the orbital fat to reach the gland. Early in its course it gives off a recurrent meningeal branch, which passes through the lateral end or pierces the bony margin of the superior orbital fissure and anastomoses with the middle meningeal artery in the middle cranial fossa. It then supplies the medial and superior recti muscles, and sometimes provides an anterior ciliary artery; it gives off temporal and malar branches which traverse the zygomatic bone in company with the zygomatic nerve and anastomose with the anterior deep temporal and transverse facial arteries in the temporal fossa. Finally, after passing outside or through the lacrimal gland, which it supplies, it bifurcates into the two lateral palpebral vessels and ends in the conjunctiva and skin of the eyelid. Since it also anastomoses with the infra-orbital artery, it forms an extensive series of unions with other vessels.

The muscular branches (rami musculares) of the ophthalmic artery are usually found as two main stems, with a variable number of smaller twigs; when distinct, one stem arises inferiorly close to the lacrimal artery and supplies the 
inferior and medial recti and inferior oblique muscles, the other stem, the upper, supplies the superior and lateral recti, the levator and superior oblique muscles. As noted above, the muscular arteries provide the anterior ciliary twigs.

The supra-orbital artery (arteria supraorbitalis) arises from the ophthalmic above the optic nerve, and runs forwards at first medial then superior to the levator, and between it and the roof of the orbit, in company with the corresponding nerve; it leaves the orbit through the supra-orbital notch, and ascends in the scalp over the forehead towards the vertex of the skull. Besides supplying the scalp, it sends twigs to the levator, the periorbita of the orbital roof, the diploë and periosteum of the frontal bone, and the upper eyelid, and it anastomoses with the superficial temporal artery (Fig. 85, p. I68).

The ethmoidal arteries, two in number, leave the ophthalmic as it passes along the medial wall of the orbit between the superior oblique and medial rectus. The posterior (arteria ethmoidalis posterior), smaller and less constant, leaves the orbit by passing through the foramen of the same name to end in the mucous membrane lining the neighbouring air-cells and upper part of the nasal fossa, anastomosing with the spheno-palatine branch of the internal maxillary. The anterior (arteria ethmoidalis anterior) is a constant and larger vessel; it leaves the orbit through the anterior ethmoidal canal in company with the nasal branch of the naso-ciliary nerve and a vein, enters the anterior cranial fossa, crosses the cribriform plate of the ethmoidal bone, and enters the nasal cavity by traversing a slit at the side of the crista galli; it then descends along a groove beneath the nasal bone to the tip of the nose. It supplies a meningeal branch to the dura of the anterior cranial fossa, twigs to the anterior ethmoidal cells, the frontal sinus, the nasal mucosa, and the skin of the dorsum of the nose.

The palpebral branches (arteria palpebrales mediales) arise from the ophthalmic just below the trochlea; one enters each eyelid to form the superior and inferior tarsal 
arcades, which anastomose laterally with the palpebral branches of the lacrimal artery and also with the supraorbital and infra-orbital arteries. The details of the palpebral distribution are given above on p. 168 .

The frontal terminal branch of the ophthalmic artery (arteria frontalis) leaves the orbit by piercing the septum orbitale in company with the supra-trochlear nerve, and ascends over the forehead to supply a small part of the eyelids, but chiefly the mesial region of the scalp; it anastomoses freely with the supra-orbital artery and with its fellow of the opposite side (Fig. 85, p. I68).

The nasal terminal branch (arteria dorsalis nasi) is the real termination of the ophthalmic artery, and, as already noted, it leaves the orbit by piercing the septum orbitale between the trochlea and the medial palpebral ligament, and becomes directly continuous with the angular part of the facial (external maxillary) artery; it supplies the lacrimal sac and the skin of the root of the nose, and anastomoses with its fellow of the opposite side and with the lateral nasal branch of the facial artery.

The infra-orbital artery (arteria infraorbitalis), although not a branch of the ophthalmic but a terminal part of the internal maxillary, itself a branch of the external carotid, deserves notice here from its relation to the floor of the orbit beneath the periosteum of which it lies. It originates in the pterygo-palatine fossa, enters the orbit through the posterior end of the inferior orbital fissure, and runs along the infra-orbital sulcus and canal to emerge on the face by the infra-orbital foramen; in the sulcus it gives branches to the inferior rectus and inferior oblique muscles, orbital fat, and lacrimal gland, besides the alveolar branches, and anteriorly it supplies the lower eyelid and lacrimal sac (Fig. 85, and see p. 243) ; on the face it divides into palpebral, nasal, and labial branches, and anastomoses with various branches of the facial artery, with the transverse facial branch of the superficial temporal, and with the dorsal nasal branch of the ophthalmic.

The anastomoses which the ophthalmic artery, a derivation of the internal carotid artery, forms with the internal 
maxillary and facial, which are branches of the external carotid, have been detailed in the above description; the chief communications are the supra-orbital with the transverse facial, the lacrimal with the middle meningeal, the nasal with the angular arteries Variations in the branches of the ophthalmic artery are common. In I $_{5}$ per cent of cases the main vessel passes beneath instead of over the optic nerve (Quain), and then may lie entirely to its medial side; it has been found buttonholed by the optic nerve (Poirier). The lacrimal artery may be reinforced by the anterior deep temporal; its communication with the middle meningeal may be unusually large, and, indeed, sometimes the latter vessel provides the lacrimal branch instead of the ophthalmic; as a further development, the whole of the ophthalmic arterial system has been found in very rare cases to be provided by the middle meningeal (a derivative of the internal maxillary branch of the external carotid), as in the specimen photographed in Fig. I78, p. 349, where there was no trace of an ophthalmic artery proper on either side. Conversely, the ophthalmic artery has been seen to give off the middle meningeal (Quain). The supra-orbital as well as the posterior ethmoidal is inconstant; the latter may be a branch of the former instead of coming directly from the ophthalmic. The nasal frequently supplies a deficiency of the upper part of the facial artery. Types of such abnormalities are figured in a paper by Meyer (I887). As Parsons (I903) states, the general tendency as we ascend the aninial scale is for the principal ophthalmic artery, which in the lower members is supplied by the external carotid, to be derived from the internal carotid; in intermediate forms there is usually one vessel derived from each source, commonly with an anastomosis between the two systems; hence individual variations may be expected. Henderson (I903) has studied the ophthalmic arteries in the rabbit and dog, and both papers will be found of value in a study of the intra-ocular circulation. An account of the development of the vessels is given by Dedekind (I909).

The Veins.-The blood is drained from the orbit by 
three routes ; firstly and in greater part, backwards by the ophthalmic veins into the cavernous sinus of the intracranial venous system; secondly, forwards by the free communications of the ophthalmic veins with the angular vein into the facial system ; thirdly, and in least extent, downwards through the inferior orbital fissure into the pterygoid venous plexus.

The ophthalmic veins are two in number, the superior and inferior. The superior (vena ophthalmica superior) is the chief vein of the orbit; it is formed behind the medial corner of the upper eyelid by the union of two branches from

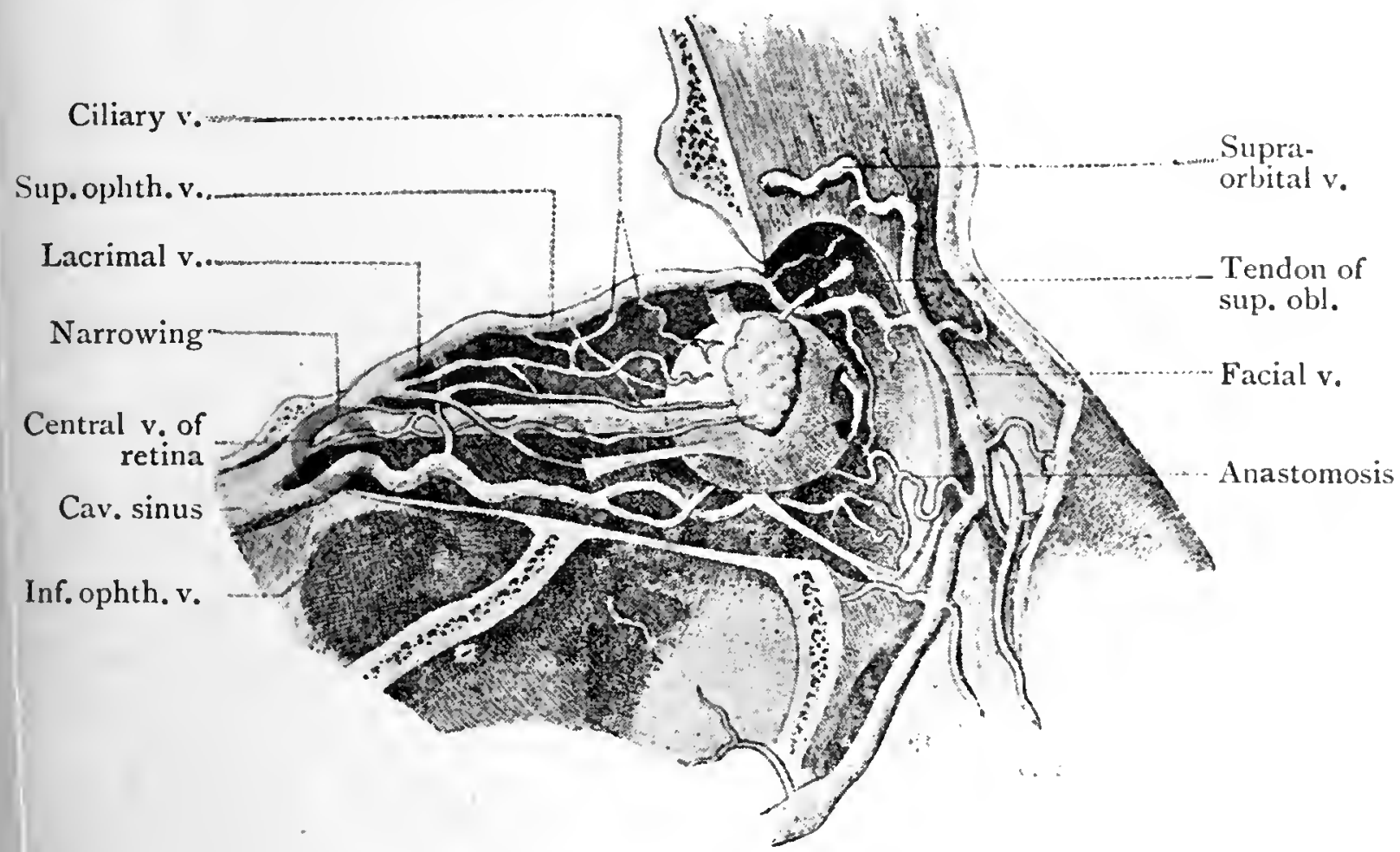

FIG. $160 .-$ The Veins of the Orbit and their connection with those of the Face. (From Poirier, after Sesemann.)

the supra-orbital and angular veins of the face, which enter the orbit by passing through the septum orbitale respectively above and below the trochlea; both these veins are seen in Fig. 85 , p. I68, and the communication with the angular vein is particularly well displayed in Fig. 54, p. I23.

The vessel so formed passes backwards in the orbit, closely though not so tortuously following the course of the ophthalmic artery across the optic nerve and beneath the superior rectus muscle, but having usually received the inferior ophthalmic vein it leaves the orbit by the superior orbital fissure, and not through the optic foramen ; it passes 
either above, or through, or below the oculomotor foramen (that is, that part of the annulus of Zinn which embraces the medial end of the fissure) according to the observations of different authors (Fig. I6I), and in the last mentioned situation may be compressed by the orbitalis muscle (Fründ, IgI2). It then bends acutely downwards (Hesser and Krauss) to enter the lower forepart of the cavernous sinus; before its entry it is stated to become markedly narrower according to

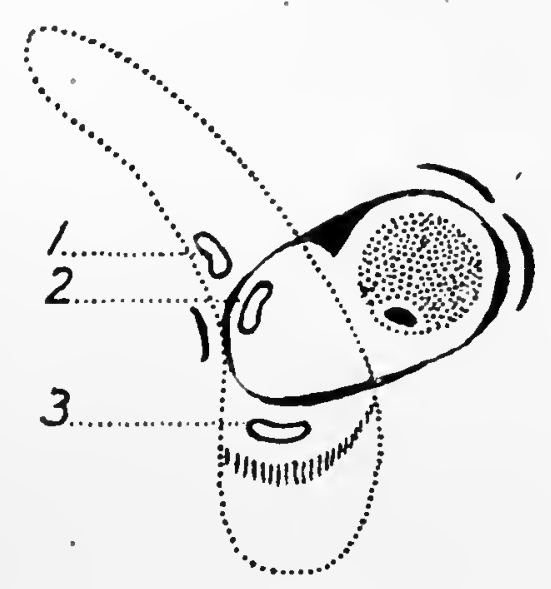

FIG. I6I. - Diagram to show the various positions of the Superior Ophthalmic Vein relative to the annulus of Zinn as it leaves the orbit through the superior orbital fissure, as described by different authors. (After Hesser, I9I3.)

I. According to Zinn, Festal, Merkel, Charpy, Hovelacque, and most others.

2. According to Schwalbe, Hesser.

3. According to Fründ. (This is the position of the inferior ophthalmic vein at this point when it enters the cavernous sinus separate from the superior vessel, accord. ing to Sesemann, Charpy, Hesser, and Hovelacque.)

The shading below 3 indicates the position of the orbitalis muscle of Müller.

ing mostly to the arterial occasionally receives the anterior frontal diploic vein; from the eyeball it receives the upper venae vorticosae, the lower ones joining the inferior ophthalmic vein. These veins, usually four in number and each draining a quadrant of the chorioid coat, ciliary body, and iris of the globe, emerge obliquely after a passage of from 2 to 4 $\mathrm{mm}$. through the sclera at nearly equidistant points relative to the vertical meridian, though the lateral ones are nearer, but from 6 to $8 \mathrm{~mm}$. behind, the equator (Figs. I32, 
I59); they are sometimes thought to be compressed by the muscle tendons, but whilst the movements of the recti have no action upon them, the ones passing beneath the oblique muscles, that is the upper and lower lateral pair, certainly appear to lie in such a position that they could be pressed upon, especially in convergence. Variations in number up to five or seven are not infrequent, generally on the nasal side, and they may empty into the veins of the muscles (Fuchs).

The vena centralis retinae accompanies the artery of the same name in its intra-neural course, though, according to Hovelacque (I920), it emerges from the optic nerve on either the lateral or inferior side and nearer (Io $\mathrm{mm}$. from) the globe ; it most frequently opens separately into the cavernous sinus, but sometimes joins the superior or even the inferior ophthalmic vein instead, and it has always at least one side connection, commonly with the former vein (Sesemann, I869), an important provision that ensures an escape for the blood of the retina in cases of thrombosis of the cavernous sinus. The ciliary veins accompany the corresponding arteries, the anterior ones joining the muscular veins; it is these anterior ciliary veins which become engorged and appear as dark serpentine vessels on the fore part of the sclera in cases of chronic glaucoma, the intraocular pressure occluding the obliquely running vorticose veins through which the bulk of the blood of the eyeball is normally drained (Fisher). The ethmoidal veins, anterior and posterior, drain part of the blood from the sphenoidal sinus, ethmoidal cells and superior meatus of the nose, where they communicate with the veins of that cavity. The veins from the upper and medial ocular muscles call for no special note.

The lacrimal vein (vena lacrimalis) is large enough to be considered as the third main vessel of the orbit; it originates in the lacrimal gland, but receives blood also from the neighbouring conjunctiva; it accompanies the artery in its course backwards, and usually opens into the superior ophthalmic vein. Gurwitsch found the lacrimal usually joined by veins from the superior and lateral recti 
and by a vorticose vein, the common trunk entering the superior ophthalmic vein farther back than any other tributary; in only Io per cent of cases did the vessel enter the cavernous sinus separately. It forms anastomoses with the temporal veins through the zygomatic canals.

The inferior ophthalmic vein (vena ophthalmica inferior) is the vein of the orbital floor; it originates in a plexus of small vessels in the orbital fat beneath the globe, and, indeed, may exist as a network throughout its whole course ; it receives venules from the lower and lateral ocular muscles, the two inferior vorticose veins, and also helps to drain the conjunctiva of the lower eyelid and the lacrimal sac. It runs backwards above the inferior rectus and either ends by joining the superior ophthalmic vein, as was always the case in Hesser's observations, generally so in those of Krauss, and found in two out of three by Gurwitsch, or enters the cavernous sinus separately by passing beneath the annulus of Zinn, where it lies in contact with the orbitalis muscle (Figs. I60, I6I). Anteriorly it anastomoses with the (anterior) facial vein by branches which pass over and sometimes groove the orbital margin; posteriorly it always communicates with the superior ophthalmic vein ; inferiorly it establishes communication (though very seldom, according to Krauss) through the inferior orbital fissure with the pterygoid plexus, in which it may terminate entirely; the connection, sometimes called the " ophthalmo-facial vein," may become of great importance. The pterygoid plexus, buried in the fatty tissue of the pterygoid fossa, communicates also with the cavernous sinus, and by the deep facial vein with the facial system; it receives blood from the field of distribution of the internal maxillary artery, and ultimately condenses to form the vein of the same name which in junction with the superficial temporal becomes the temporo-maxillary vein, a part origin of the external jugular. In its passage through the inferior orbital fissure the communication between the inferior ophthalmic vein and the plexus is subject to compression by the involuntary orbitalis muscle, which fills the fissure, and the vein itself is related to and may be affected by 
contraction of the same muscle where it passes beneath the annulus of Zinn to enter the cavernous sinus. By these relations Krauss and Fründ seek to account for the exophthalmos of Basedow's disease, a point that has already



FIG. I62. - Scheme to show the connections of the Vessels of the Orbit with those of the Face on the one hand and with those of the Intra-cranial System on the other. (The external jugular vein is not shown.)

C. $=$ the cavernous sinus, traversed by the internal carotid artery. $L .=$ the lateral or transverse sinus.

been referred to on p. 84, but the free anastomoses between all the ophthalmic veins, superior inferior, and lacrimal, and the absence of valves in them would negative such an explanation. 
The anastomoses of the orbital system of veins are of the greatest importance from a clinical point of view, and have been specially studied in this respect by Krauss (I9Io) and from the anatomical point of view by Sesemann (I869), Gurwitsch (I883), and Festal (I887). They communicate, as has been noted above on p. I66, with the superficial veins of the face, with the deep veins (pterygoid plexus), with the veins of the nasal cavity, and with the intracranial system of dural sinuses. The superior ophthalmic vein is the largest channel in the adult between the veins of the face and those inside the skull.

As regards the direction in which the blood of the orbit flows, if, as is generally stated, no valves exist in any of the branches of the ophthalmic veins, the blood can pass in either direction forwards or backwards and they form important emissary veins connecting the cavernous sinus with the facial system; Merkel, Gurwitsch, Dwight, Krauss, and Festal, however, state that valves do occur in these branches of junction with the facial veins just outside the orbit, and so disposed as to prevent the flow of injections from the face backwards into the orbit; but these valves, as others elsewhere in the body, tend to become ineffective in old age. Probably the orbital blood passes both forwards into the facial system along with the blood from the eyelids and backwards into the cavernous sinus, this last being the normal direction according to most authors, a conclusion supported by clinical and pathological observations, though the posture of the head may be a factor in determining its course. But it is to be noted that the ultimate channel formed by the ophthalmic vessels on reaching the sinus is small compared with the size of the numerous radicles they collect, and, moreover, may exhibit a marked narrowing at this point; also that the cavernous sinus is not only largely occupied by the internal carotid artery, but such space as is left is filled by trabecular tissue, so that the blood-flow through it must be sluggish. On the other hand, as noted on p. I66, the common appearance of a prominent frontal vein during laughter or coughing when the intra-cranial pressure is raised would point to a normal 
flow of at least part of the contents of this vessel into the orbital system and backwards to the sinus. It seems reasonable to conclude, therefore, that the orbital venous system consists of a network of vessels which can drain either forward and freely into the facial vein, or backwards into the cavernous sinus, or inferiorly and least freely into the pterygoid plexus, and through other minor channels, the object thus attained by the anastomoses between the vessels of the orbital system itself and by the multiplicity of paths along which the blood can drain away being to facilitate the ocular circulation.

The variations and anomalies of the orbital veins are numerous, and some are of clinical interest. The inferior may alone form the chief vein of the orbit and anastomose with the angular; the superior has been found to join the middle cerebral (Sylvian) vein of the brain (Hyrtl); the ethmoidal veins may enter the cavernous sinus (Sesemann), or communicate through the lamina cribrosa with the veins of the olfactory tract (Zuckerkandl). Stanculeanu states that he has sought in vain for the venule described by Gaillard as draining from the antrum through the orbital floor into the ophthalmic system.

The development has been studied by Dedekind (I909), from whose reconstruction of the embryonic network in the orbit which gives rise to the orbital veins it is not surprising to find such variations and anastomoses as do occur in the adult.

The cavernous sinus (sinus cavernosus), to which reference has been made, here calls for description not only from its connection with the ophthalmic veins, but from the fact that, apart from the optic nerve itself, all the nerves entering the orbit pass either through or in close proximity to it. It is generally considered to be formed, like the other intracranial blood sinuses, by a local separation of the two layers of which the dura mater lining of the cranium is composed, the outer endosteal layer closely following the contour of the bone, the inner meningeal layer forming the lateral wall of the sinus, whilst the space between them 
is lined by an endothelium continuous with that of the veins with which it communicates; Ferron (I9I3), however, has more particularly studied the constitution of the lateral wall of the sinus, and considers it to be formed by two layers, a thin deeper one, which is a continuation and enlargement of the carotid sheath, and a thicker superficial layer continuous with the dura lining the middle fossa ; between the two the nerves pass, carrying with them

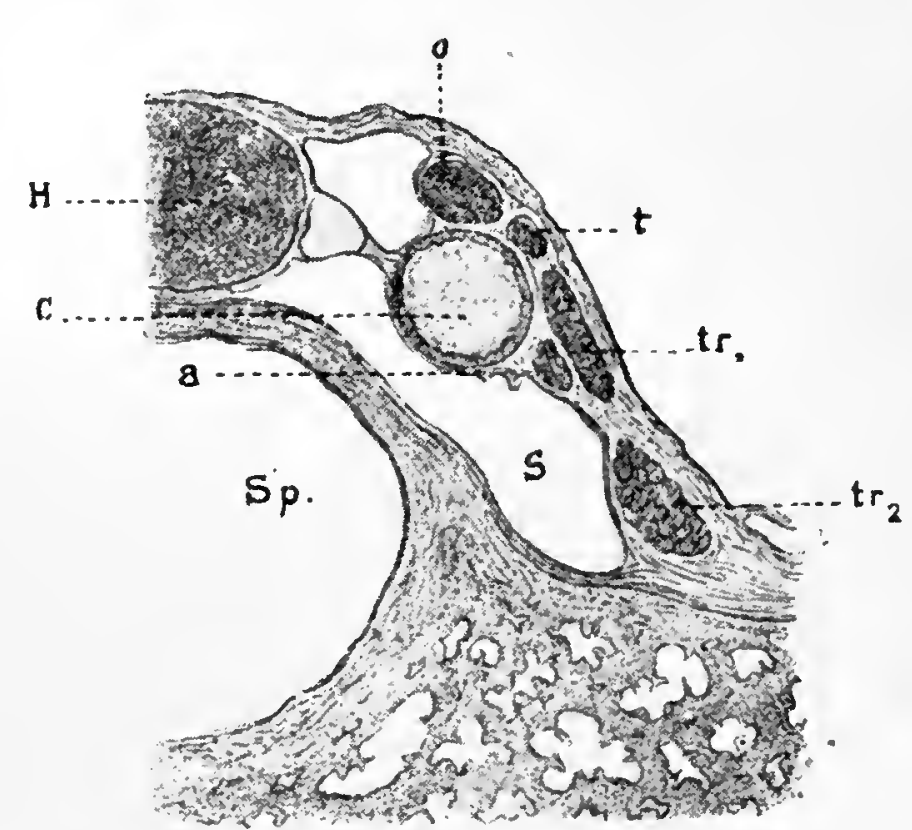

FIG. I63. - Transverse section through the Cavernous Sinus (S.), the Hypophysis Cerebri or Pituitary Body (H.), and the Sphenoidal Air Sinus (Sp.). (From Langer, I885.)

C. $=$ the internal carotid artery $; 0 .=$ the oculo-motor nerve; $t_{0}=$ the trochlear nerve; $t r_{0} 1$ and $t r_{.2}=$ the ophthalmic and maxillary divisions of the trigeminal nerve respectively; and $\alpha_{0}=$ the abducent nerve. sheaths formed by prolongations of the dura as they pierce it.

The cavernous sinus is situated on the side of the body of the sphenoidal bone, and is therefore related on the one hand to the air-sinus which hollows out that bone (with the hypophysis cerebri (pituitary body) resting above it, as seen in Fig. I63), and on the other to the middle cranial fossa (Fig. I69), in which is lodged the temporal lobe of the brain; it extends from the medial and widest part of the superior orbital fissure in front to the apex of the petrous portion of the temporal bone behind, where it is in close relation to the medial edge of the semilunar (Gasserian) ganglion of the fifth nerve, and has an average length of $20 \mathrm{~mm}$. or $\frac{3}{4}$ inch and a width of Io $\mathrm{mm}$.; it is wider in front than behind, and narrower below than above. Its lumen is traversed by numerous interlacing trabeculae and loose fringes of connective tissue derived from its walls, so that it appears in section filled with a spongy tissue, and it is still further reduced in size by the winding passage through it of the internal carotid artery and abducent nerve. 
The artery enters the sinus below, on emerging from the carotid canal; it first ascends towards the posterior clinoid process, passes forwards along the side of the body of the sphenoidal bone, and finally curves upwards and pierces the dura forming the roof of the cavernous sinus on the medial side of the anterior clinoid process; in this cavernous part of its course it is surrounded by a sympathetic nerve plexus and gives off minute branches to the walls of the sinus, to the hypophysis, and the semilunar ganglion. The abducent

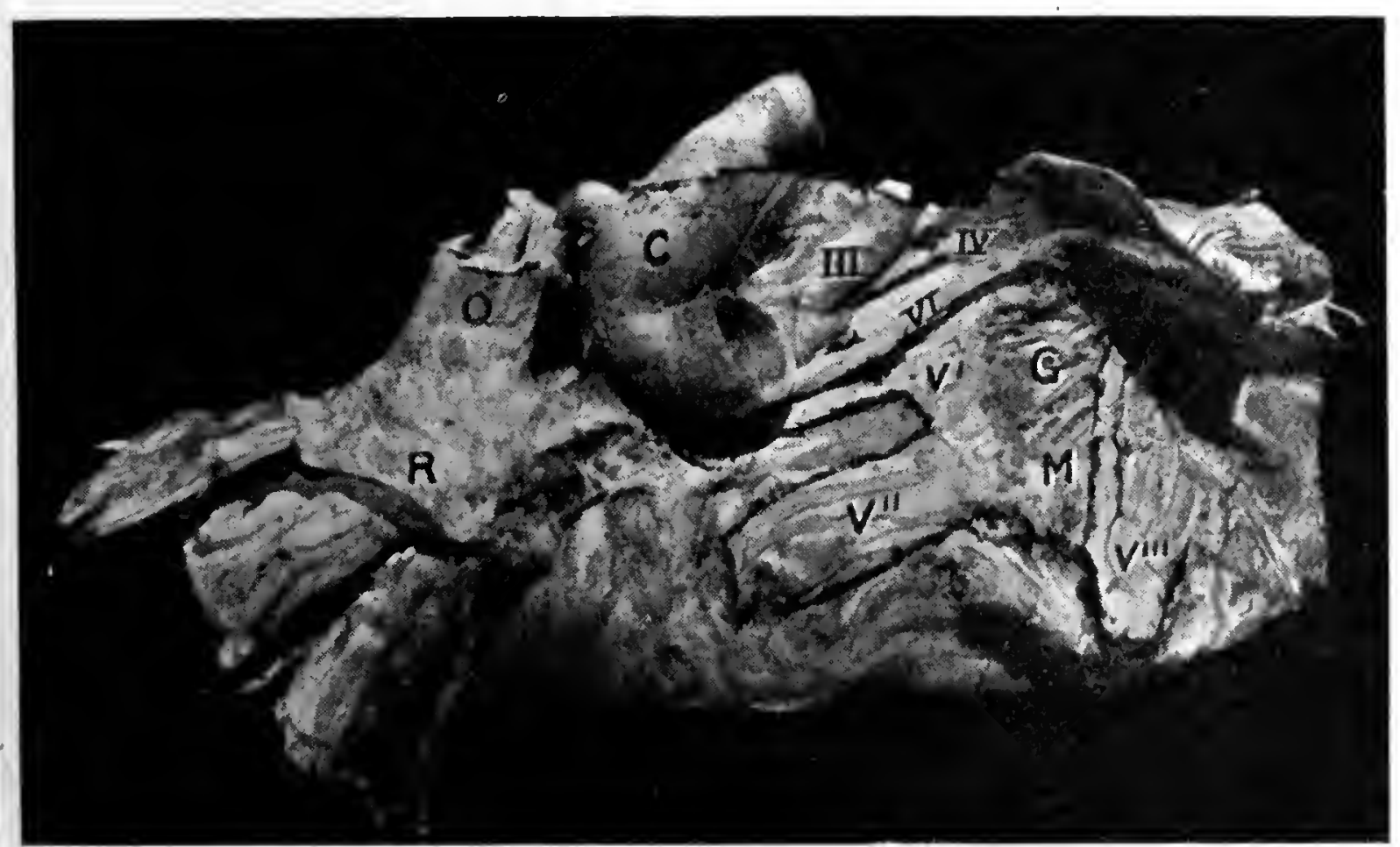

Fig. 164. - Dissection of the Lateral Wall of the Cavernous Sinus and its contents, viewed from the medial or inner side. The soft parts were stripped from the side of the body of the sphenoidal bone and the cavernous tissue cleared away. $\times \mathrm{x} \frac{1}{2}$.

$\mathrm{O} .=$ optic nerve $; \mathrm{R}_{0}=$ origin of the recti muscles $; \mathrm{C} .=$ the internal carotid artery, giving off the ophthalmic artery; I11., IV., V.', V.", V.'", VI. =the nerves; G. =the semilunar ganglion; M. lies just to the left of the motor root of $\mathrm{V}$. nerve.

nerve crosses the lateral side of the artery, lying free in the sinus in the first part of its course but being adherent to the lateral wall towards its exit; in a minority of cases it is united to the wall in its whole course by a short fibrous "mesentery."

Embedded in the lateral wall of the sinus are the oculomotor (third) and trochlear (fourth) nerves, and the ophthalmic and maxillary divisions of the trigeminal (fifth) nerve, in that order from above downwards (Figs. I63, I64, I70, and I74). Langer (I885), who has made a special 
study of the sinus, distinguishes two parts, a more reticular superficial one containing the third and fourth nerves, and a deeper, more spacious one nearer the bone.

The cavernous sinus is joined to its fellow on the opposite side of the sphenoidal bone by a pair of transverse intercavernous sinuses placed anterior and posterior to the sella turcica; the anterior is the larger, and the posterior is often absent (twenty-six times out of forty-four cases; Knott, I882); these, together with the portions of the cavernous sinus between their ends on either side, form a circular or

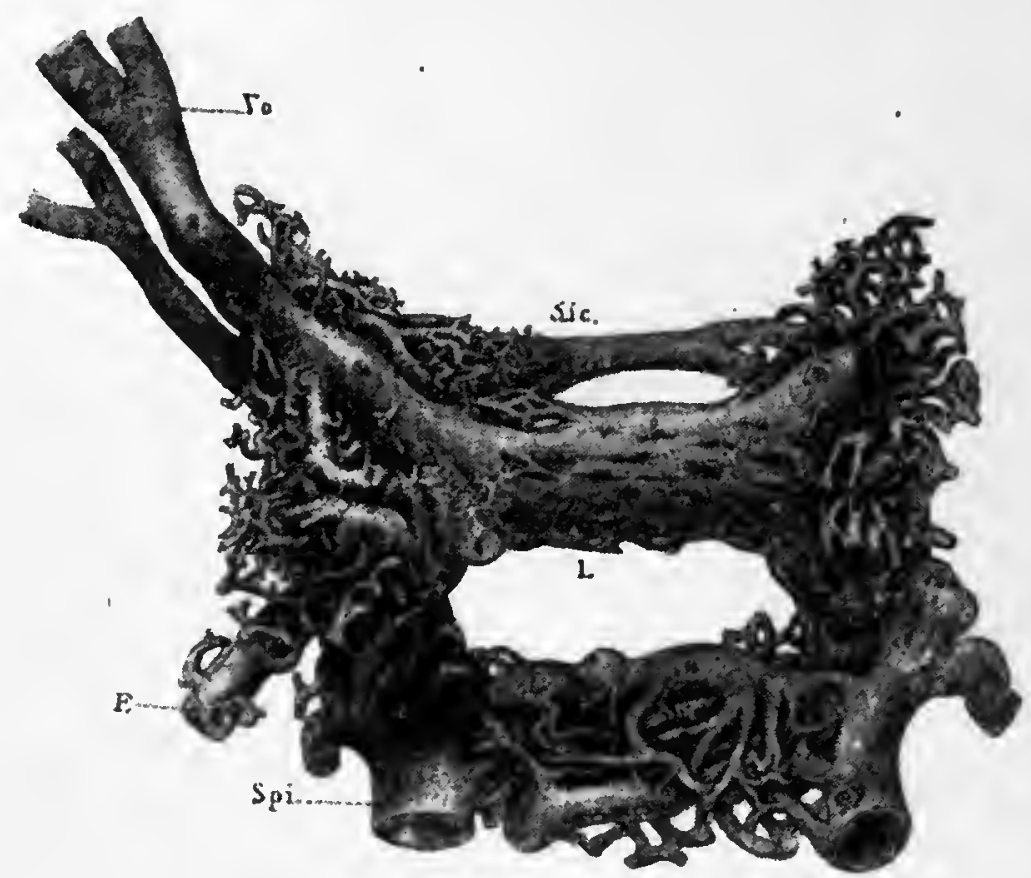

Fig. 165.-Corrosion preparation of the Cavernous Sinuses of a Child. (From Langer, r885. Note that the vessels are represented hollow, which would indicate a drawing and not a photograph of the actual injection nrass.)

Vo. = the superior ophthalmic vein, with the inferior vein below it; Sic.=the anterior intercavernous sinus; L. =the position of the hypophysis cerebri or pituitary body ; E.=emissary vein leading through the foramen oziale into the pterygoid plexus; $\mathrm{Spi}_{0}=$ the inferior petrosal sinus.

coronary sinus round the pituitary body, into which its veins pass. Farther back, the two cavernous sinuses are connected by the basilar or transverse occipital sinus or plexus.

The tributaries and emissaries of the cavernous sinus are numerous. Besides the ophthalmic veins, there enter it the spheno-parietal sinus, which runs along the margin of the lesser wing of the sphenoidal bone and sometimes joins the superior ophthalmic vein, veins from the neighbouring dura, and some of the veins from the muco-periosteal lining of the sphenoidal air-sinus which have pierced its 
bony wall. The middle cerebral vein or vein of the Sylvian fissure enters either the cavernous sinus or its tributary, the spheno-parietal sinus. The blood is drained away posteriorly by the two petrosal sinuses into which the cavernous sinus divides at the apex of the petrous bone;

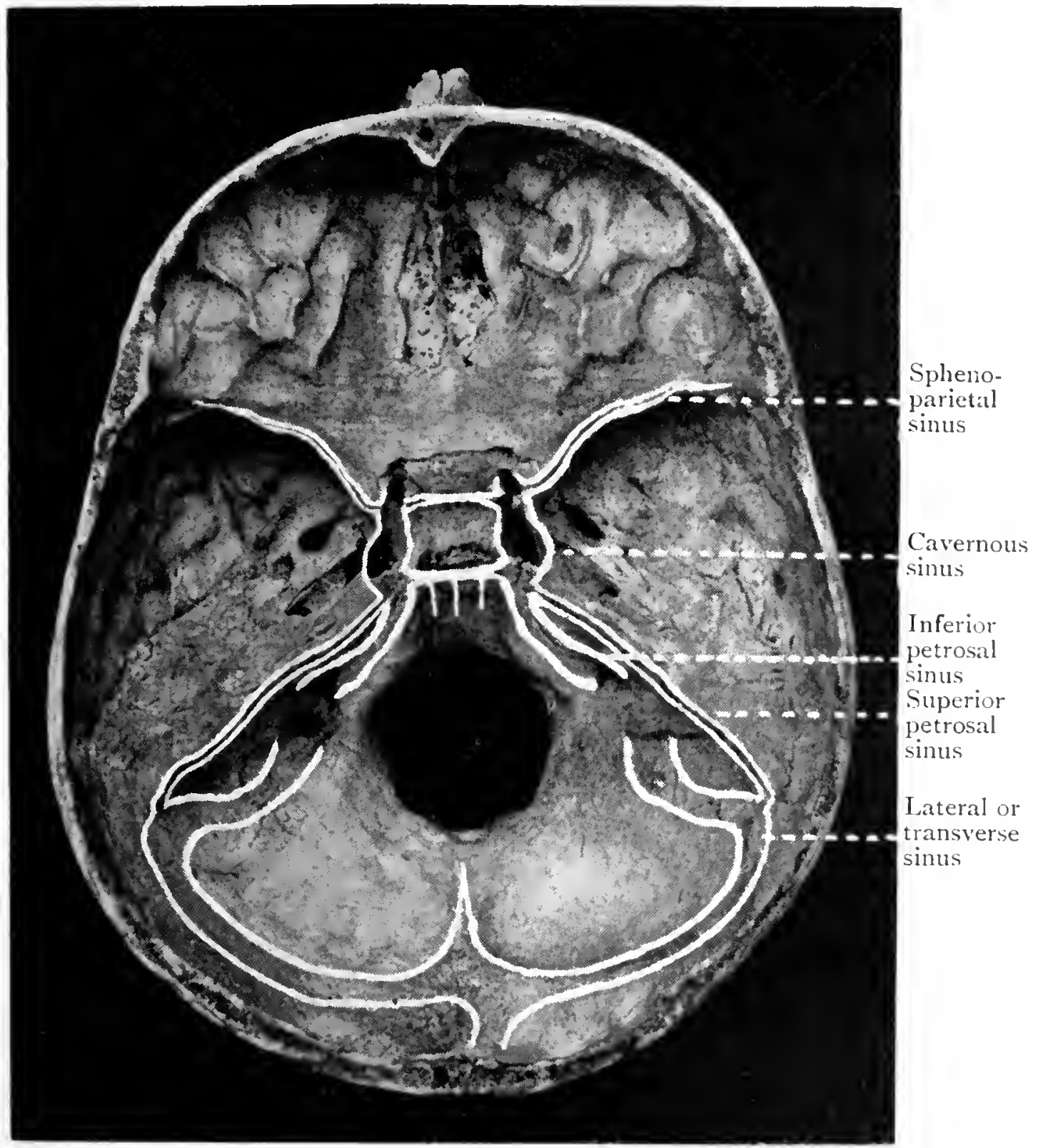

FIG. I66. - The Base of the Skull viewed from within, to show the position of the cavernous sinus and its connections. Compare with Fig. I69.

the superior petrosal sinus is the smaller, and runs along the upper border of that bone to enter the transverse (lateral) sinus, where it bends downwards to become the sigmoid sinus; the inferior petrosal sinus passes along the petrooccipital suture to the jugular foramen and enters the internal jugular vein; part of the blood also drains into the basilar sinus and thence into the anterior spinal plexus 
of veins. As emissary veins proper of the cavernous sinus are certain small veins leading from its lower part; they pass through (I) the foramen ovale, (2) the foramen lacerum (medium), (3) the foramen of Vesalius when this is present, (4) the foramen rotundum, though very rarely (Knott), all passing into the pterygoid plexus; and (5) a venous plexus (carotid plexus or sinus), which completely invests the carotid artery as it traverses the carotid canal, and is prolonged downwards to empty into the internal jugular vein.

The lymphatics of the orbital cavity are imperfectly known in man. As elsewhere in the body, the system no doubt consists of spaces which are peri-vascular in position (into which the fluid exudes from the blood capillaries), closed capillaries into which the lymph is then absorbed, and vessels. There are no lymphatic nodes, however, in the orbit. Definite lymph vessels can be demonstrated in the eyelids, conjunctiva, and lacrimal gland, to which reference has already been made, but as regards the orbit itself, although Schwalbe described Tenon's capsule as enclosing a definite lymph space lined with endothelium and continuous with a supra-vaginal space around the optic nerve (denied by subsequent observers; see p. 289), and others have considered the inter-lobular spaces of the orbital fat to be likewise lymph channels (Birch-Hirschfeld, Igog), such intervals do not show any special structural development, though, like similar spaces elsewhere in the body, they may contain plasma from the blood-vessels (Dewey, I920). Leboucq (I9I4) has studied the orbital lymphatics in the rabbit, and concludes that the sub-conjunctival space and that of Tenon's capsule are independent of the lymph circulation of the globe. The lymph from the latter passes out round the anterior ciliary and vorticose veins and the central vein of the retina, and eventually into the jugular lymph trunks (Bartels, I909). The lymph vessels of the orbit are supposed to pass through the inferior orbital fissure to the internal maxillary nodes, and thence to those of the upper deep cervical groups, and communications may 
also exist between the orbital system and that of the nasal cavity, or accompany vessels passing through the superior orbital fissure ; they have not been demonstrated in man.

5. The Nerves. - The nerves which enter the orbit are as follows :

The optic nerve, the special nerve of sight;

The oculomotor, trochlear, and abducent (third, fourth, and sixth) nerves, which are motor to the muscles of the eye and the levator of the upper eyelid;

The ophthalmic division of the trigeminal (fifth) nerve, which is the nerve of common sensation to the eyeball, and supplies also the lacrimal gland, conjunctiva, and eyelids, but which in greater part traverses the orbit to reach facial areas beyond.

The maxillary division of the trigeminal, which runs beneath the periorbita of the floor and gives off one branch into the orbit, but is likewise mainly distributed to extraorbital regions.

The sympathetic plexus of nerves.

As in the case of any other cerebral nerve, an orderly consideration must include these points :

(i.) The "nucleus of origin" ; that is, the mass of nerve cells from which the axons forming the nerve trunk arise, sometimes called its " deep origin." In the case of a motor nerve, the nucleus lies buried in the mid-brain, where it is connected with other nuclei and parts of the brain by fibre tracts. After a short intra-cerebral course, the nerve emerges at its

(ii.) "superficial origin" on the surface of the brain; in the case of a sensory nerve, such as the trigeminal, the nucleus of origin of its fibres (in this case the semilunar or Gasserian ganglion) lies outside the brain and is connected to it by centripetal fibres, which enter and terminate in a nucleus of reception. From its superficial origin in the one case, or point of entry in the other, the nerve has an

(iii.) intra-cranial course inside the skull between the brain and dura mater. In the case of the orbital nerves other than the optic nerve itself they then enter the 
(iv.) cavernous sinus, and pass through the superior orbital fissure (comparable to the point of emergence from the skull of other nerves) to enter upon the

(v.) orbital part of their course, and

(vi.) final distribution.

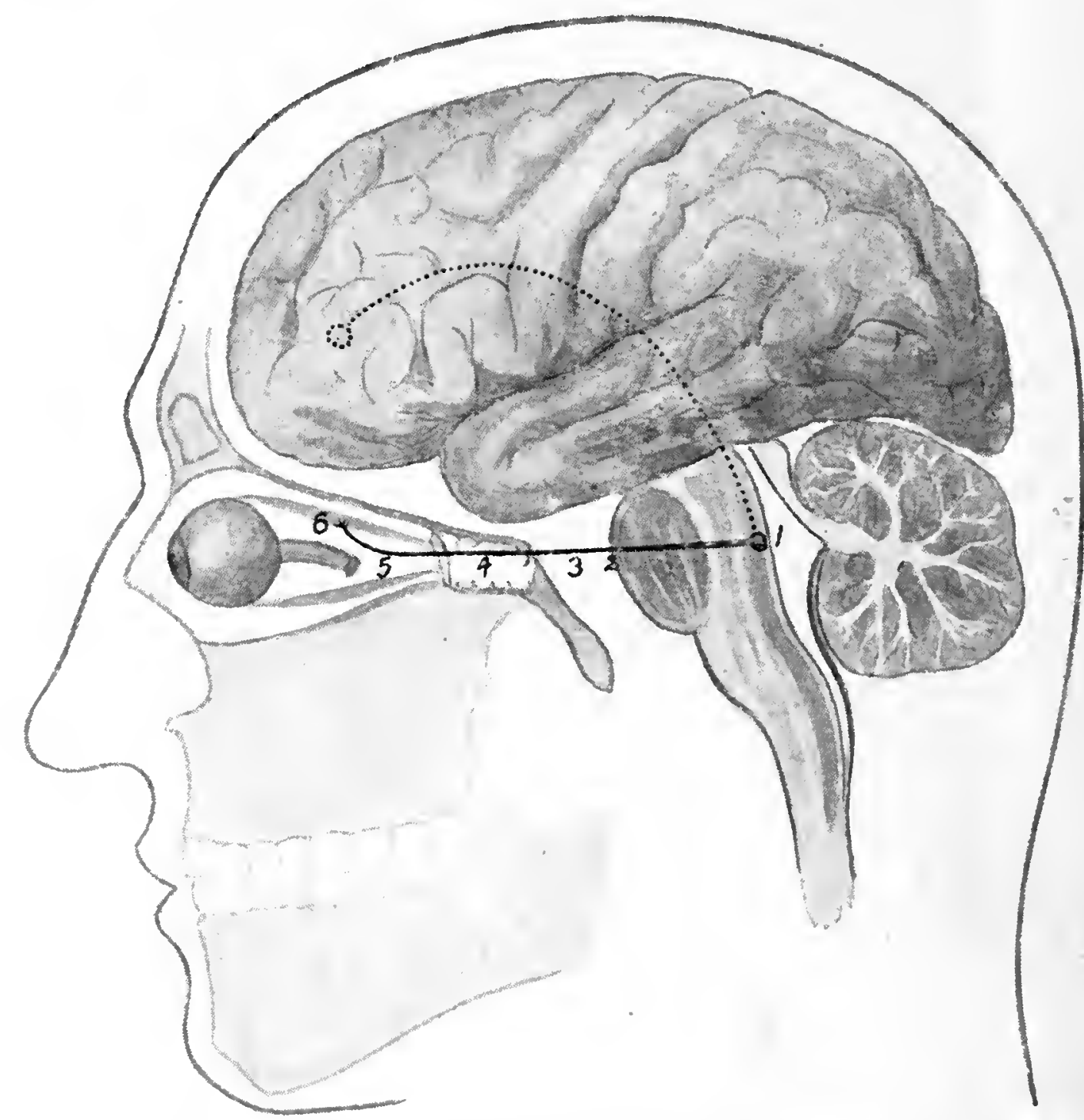

FIG. 167.- Scheme to show the course of a typical Orbital Motor Nerve. The fibres arise from a deeply-seated nucleus of origin ( 1 ), and after a short intracerebral course the nerve emerges from the surface of the brain stem at its superficial origin (2); it then traverses the cranial cavity $(3)$, passes through the cavernous sinus (4), and after entering the orbit (through the superior orbital fissure) passes along it (5) to reach its final distribution in a nuscle (6). The possible cortical connection of the nucleus of origin is indicated by the dotted line.

Fig. I67 represents schematically the course of a typical orbital nerve showing these points; and it will be found that in each part of the course so outlined a nerve is related to structures an alteration in the disposition of which can become of great clinical importance.

It will be convenient to describe the optic nerve last, so that its connections may be followed into the cerebral 
visual tracts to which the last section of this work is devoted. In that section there will also be found an account of the nuclei of origin and the intra-cerebral connections of the

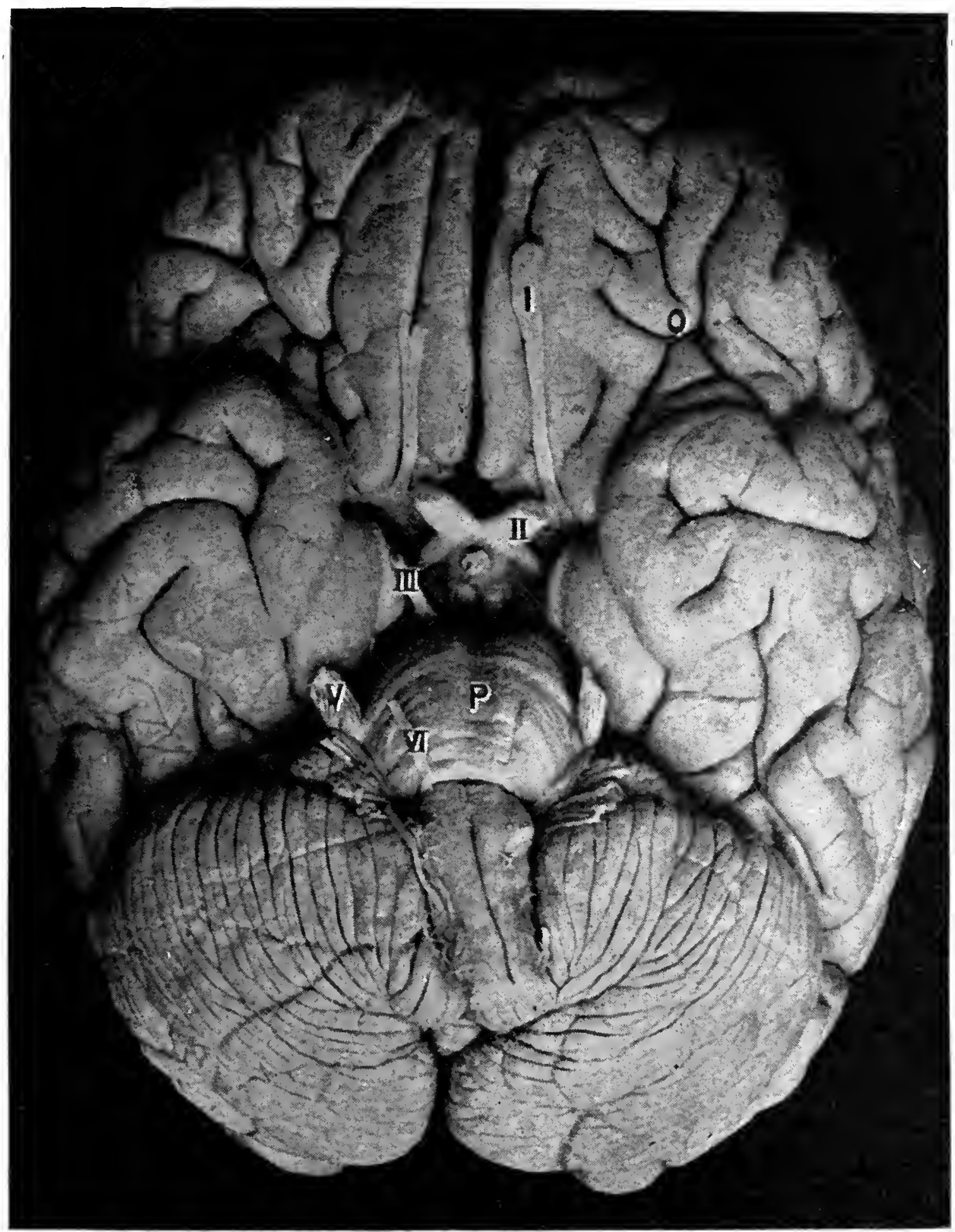

FIG. 168. - The Brain, inferior aspect, to show the superficial origins of the nerves (as numbered). 'The left (III.) nerve has been removed; the VII., VIII., and pars intermedia are seen just below $V$., followed caudally by the IX., X., XI. ; the fourth nerve is seen above V. on the reader's left-hand side.

$$
\mathrm{O} .=\text { orbital sulcus on base of frontal lohe; } \mathrm{P} \text {. }=\text { pons. }
$$

nerves. Here, in dealing particularly with the orbit, the nerves will be described from their superficial origin from the brain surface onwards. 
The 0culomotor or third nerve (nervus oculomotorius) supplies all the extra-ocular muscles with the exception of the lateral rectus and superior oblique; it also sends fibres through the ciliary ganglion to supply the ciliary muscle and sphincter pupillae within the eyeball.

The superficial origin on the surface of the brain is from the shallow oculomotor sulcus on the medial surface of the cerebral peduncle, in front of the pons, and in the interpeduncular space, on the floor of which is the posterior perforated substance or spot (Fig. I68) ; there are usually two groups of fibres at the origin, separated very often by an arteriole derived from the posterior cerebral artery.

In the intra-cranial part of its course, from this origin to where it pierces the dura to enter the cavernous sinus, the nerve lies in the posterior cranial fossa, and is about $25 \mathrm{~mm}$. long; it is closely invested by the pia mater, and traverses the sub-arachnoid space, which is enlarged in this region between the peduncles into a cisterna interpeduncularis, an imperfect subdivision of the larger cisterna basalis ; the nerve curves outwards and forwards, between and in close contact with two arteries, the posterior cerebral in front, the superior cerebellar behind; the posterior communicating artery then runs parallel with it on its medial side, and it lies against the uncus of the temporal lobe of the brain, as seen in Fig. I72; it pierces the arachnoid membrane forming the roof of the cisterna about $20 \mathrm{~mm}$. from its origin.

The nerve enters the cavernous part of its course by piercing the dura to the lateral side of the posterior clinoid process in the small triangular area formed by the free and attached borders of the tentorium (Fig. I69); it here for the first time comes in contact with the base of the skull, lying against the anterior clinoid process, and is now situated in the middle cranial fossa. Its position in the lateral wall of the cavernous sinus has already been referred to, and its relation to the other nerves is well displayed in the dissection photographed in Fig. I70, as also in Figs. I63 and I74.

It is to be noted, however, that the order of the nerves 
changes in the forepart of the sinus, the ophthalmic nerve becomes superior, the oculomotor nerve passes below and medial to the trochlear, and the abducent nerve is now in intimate contact with the lateral wall of the sinus (Fig. I70).

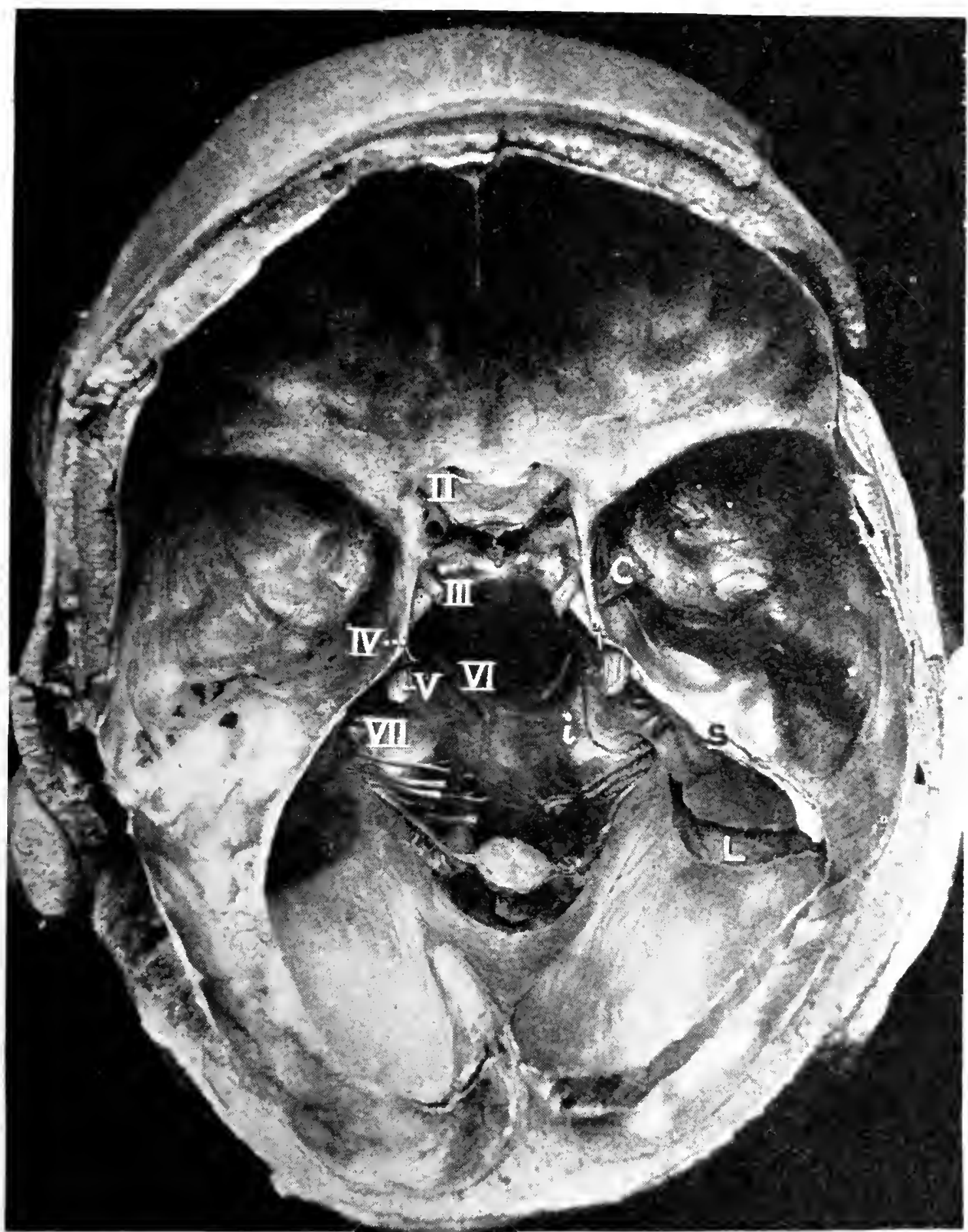

Fig. I69. - The Base of the Skull with the Dura in sitn after removal of the Brain, to show the nerve-exits and position of sinuses. On the right sicle the lateral wall of the cavernous sinus (C.) has been turned down as a thap. The superior ( $\mathrm{L}$.) and inferior $(i$.) petrosal and lateral or transverse (L.) sinuses have been opented up. Compare with Fig. I66. $\times \frac{2}{3}$.

The oculomotor nerve leaves the forepart of the sinus and enters the orbit through the superior orbital fissure. At or about this point it divides into two branches, superior 
and inferior, which enter the orbit through the oculomotor foramen, that is, inside the annulus of Zinn and within the cone of muscles, in close company with, but separated by, the naso-ciliary branch of the trigeminal, with the abducent nerve lying below. The position of these nerves relative to one another at this point, upon which considerable and unwarrantable stress is laid in the text-books, is illustrated

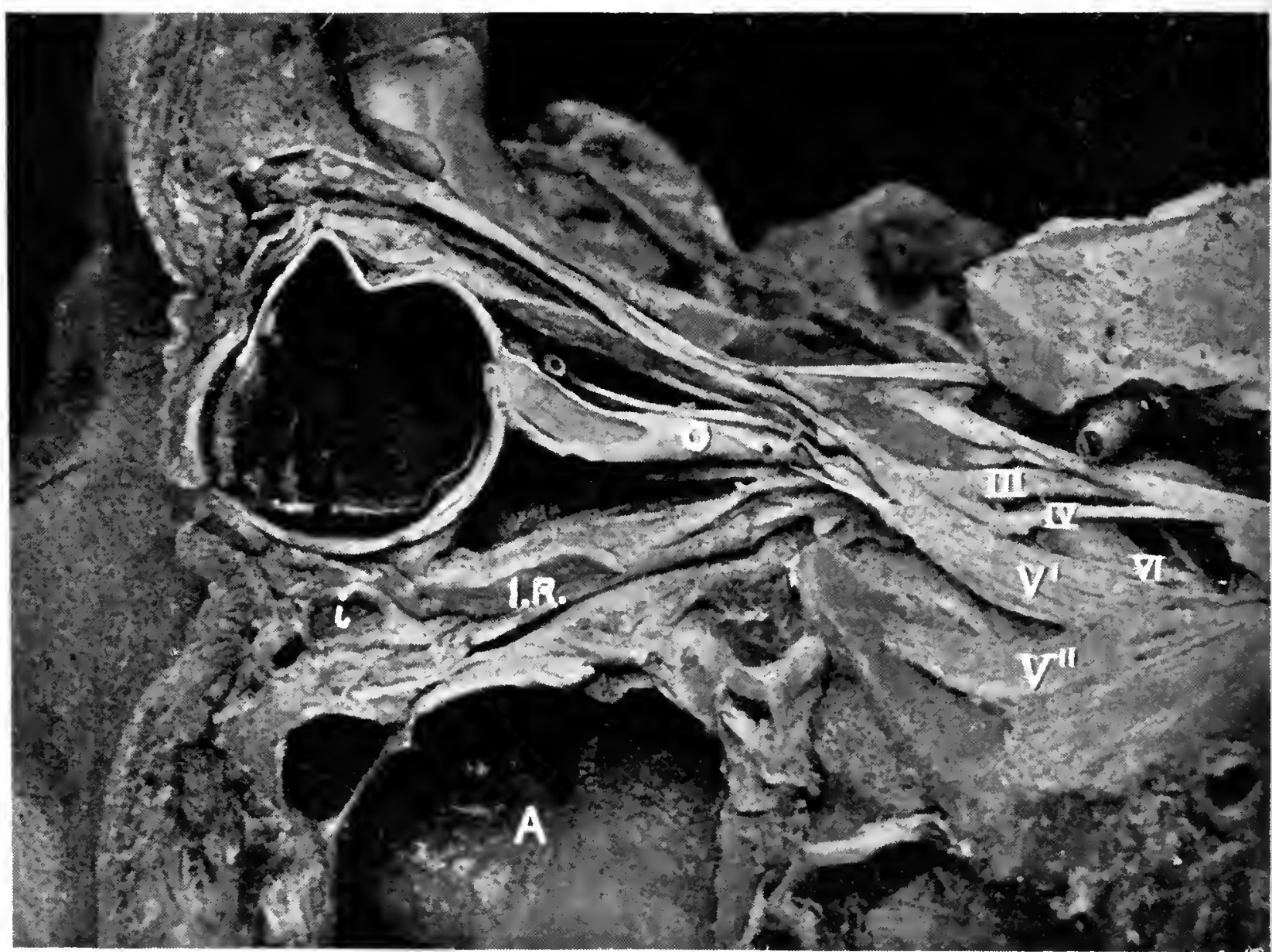

FIG. r7o.- Sagittal section through Left Orbit; the lateral wall of the cavernous sinus has been removed to expose the nerves. 'The maxillary sinus (A.) is cut open, and presents a well-marked pre-lacrimal recess in its fore and upper corner ; the ostium is also visible. Natural size. Compare with the scheme shown in Fig. 67, p. 142.

$\mathrm{O} .=$ optic nerve, cut to show its sheath; $1 . \mathrm{R} .=$ inferior rectus; $i_{0}=$ inferior oblique.

in Fig. I35, which should be compared with Figs. I39 and I4I, and the fourth section in Fig. I85, from which it will be realised that practically they are all crowded together here, compactly filling a narrow space certainly not more than $3 \mathrm{~mm}$. in diameter.

Within the orbit, the branches of the oculomotor nerve diverge; the superior and smaller of the two (ramus superior) passes upwards over the lateral aspect of the optic nerve 
to enter the ocular surface of the superior rectus, after supplying which it ends in the overlying levator palpebrae superioris muscle. The inferior and larger branch (ramus inferior), after supplying the medial and inferior recti, passes forwards beneath the optic nerve, between the inferior and lateral recti and closely bound to the former, to reach the posterior border of the inferior oblique, in which it ends. It contributes a short thick ganglionic branch, sometimes double, which forms the motor root of the ciliary ganglion (p. 35I), whereby fibres are conveyed to the intrinsic muscles of the eyeball. The course and branches of the nerve are illustrated in Fig. I $\mathrm{I}$.

As regards the ConstituTION and nature of the fibres of the oculomotor nerve, it is noteworthy that its size is remarkable in comparison with that of the muscles it supplies, a feature it shares with the other motor nerves of the eyeball; indeed, in this respect, the abducent nerve is relatively

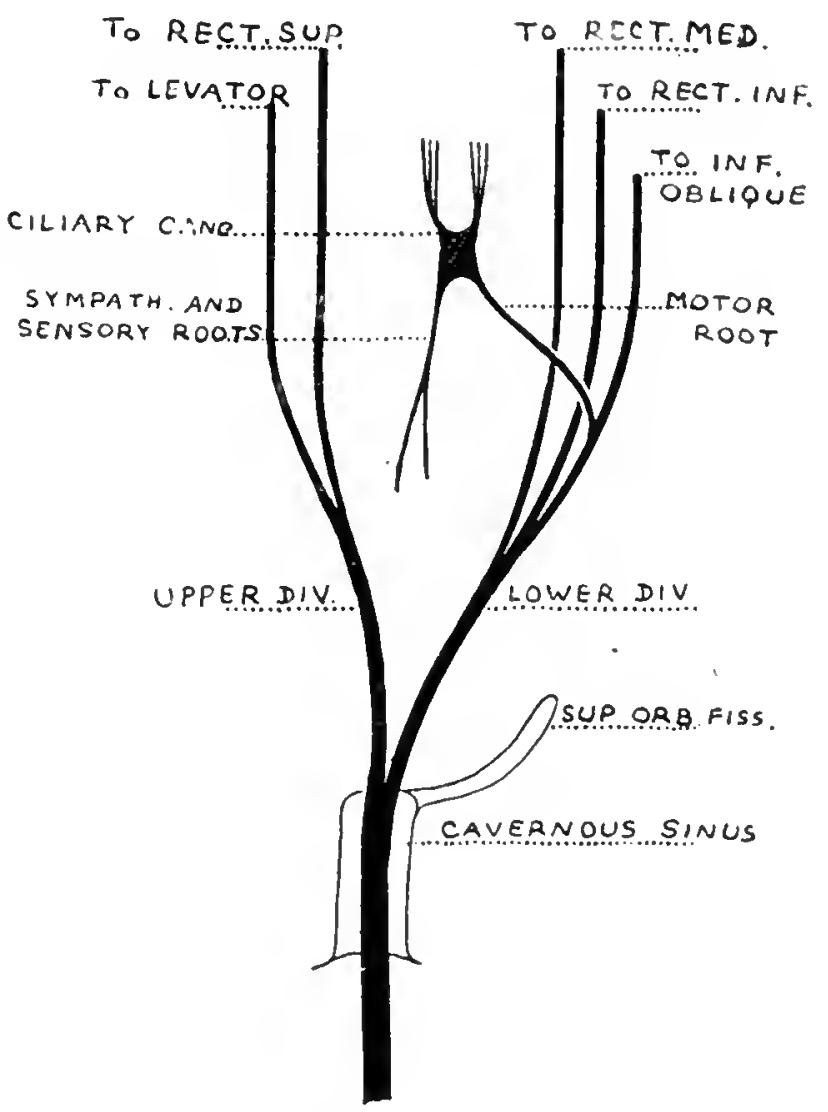

Fig. I7I.-Diagram of the Oculomotor or Third Nerve, with its branches. (Modified, after Quain.)

the largest in the body, and the only other motor nerve that may be compared with it in this respect is the hypoglossal. In all the muscles supplied by these nerves the function appears to be correlated not so much with rapidity as with a finely graded range of delicacy of movement; and, as Wood Jones points out, a muscle need not act: as a whole to perform a minimal action, but as more effort is demanded more fibres are brought into action; every fibre acts to its utmost and only sufficient fibres are called into action to perform the demanded task. The oculomotor contains I5,000 fibres 
distributed to about 40,000 muscle fibres (Macalister, r889); its fibres are generally large, but it also contains smaller ones destined for the ciliary ganglion. Whilst the great majority of its fibres are motor in function, it also contains a certain number of sensory fibres derived from the ophthalmic division of the trigeminal nerve, by an anastomosis usually considered to occur whilst the nerves are in the cavernous sinus, though Barratt (I9OI) in a study of transverse sections of the nerve failed to find the communication, and Bischoff (I865) denied its existence. Sherrington and Toser (I9IO), however, have shown experimentally that all three motor nerves of the eyeball contain some sensory fibres as well as the motor ones; and the former discusses the sensual rôle of the proprioceptive nerve supply of the extrinsic ocular muscles relative to the adjustment and maintenance of postures of the eyes in a later paper (I9I8). The presence of these afferent fibres explains the fact that after total desensitisation of the eyeball by means of cocaine, the ocular movements, the precision of which requires the co-operation of afferent impressions from the muscles, are carried out as accurately as in the normal animal (Starling, I920). In primitive animals these nerves are clearly mixed, containing both motor and sensory fibres; and Gaskell (I889), like Thomsen, has described the remains of a degenerate ganglion in the roots of the oculomotor as well as in the trochlear and the abducent nerves, which from a study of ancestral development he regards as representing an anterior root ganglion; Toser (IgI2) has made similar observations, as also has Nicholls (I9I5). The third nerve, like the fourth and sixth, is said to receive sympathetic fibres from the cavernous plexus whilst traversing the sinus; Bischoff (I865), who has studied microscopically the various anastomoses of the cranial nerves, figures such fibres as passing to the third and sixth, but not to the fourth nerve; Boeke (I9r3), however, has shown that in the cat the superior oblique muscle receives sympathetic fibres. A communication between the third and sixth nerves has been described, but its existence has been denied by recent 
observers. The nerve-endings of the motor nerves in these muscles, which differ by the richness of their ramifications from those of the skeletal muscles, have been studied by Levinsohn (I90I), and those of the sensory nerves by Dogiel (I906) ; Boeke (I9I5) found the sympathetic nerveendings to be the same in smooth as in striated fibres. The morphology of the nerves is the subject of a paper by Neal (I9I4).

A note upon the possible LEsions of the oculomotor nerve and their effects, may be added as emphasising its anatomical relations and distribution. A paralysis may result from injury to the nerve at its origin or in any part of its course, from cerebral disease, embolism, periarteritis, gumma or tumour, pressure of inflammatory exudate, periostitis, thrombosis of the cavernous sinus, and from fracture of the base of the skull or of the orbit. It may be affected by an aneurism or syphilitic periarteritis of the internal carotid, basilar, posterior cerebral, or superior cerebellar arteries, to all of which the nerve is closely related in its intra-cranial and cavernous course. In this respect the following notes may be of interest, taken from Stopford's paper (I9I7) on the arteries of the pons and medulla, in which an extensive bibliography will be found.

Aneurism of either the posterior cerebral or superior cerebellar arteries may easily cause oculomotor paralysis, since the nerve lies in the angle formed by these two vessels at their origin from the basilar, a relation, it is to be noted, shared, though not so intimately, by the fourth nerve. It is also possible, as Cushing has shown, for the posterior cerebral, which has a more constant anatomical disposition, to indent the third nerve in cases of cerebral tumour accompanied by any considerable alteration in the position of the brain stem. The basilar artery itself has caused a paralysis of the nerve. Beadles, in his collection of 555 cases of aneurism of the larger cerebral arteries, found that the oculomotor was the nerve most frequently compressed by aneurisms of the posterior communicating artery (the vessel which joins the posterior cerebral to the internal carotid and so completes laterally the arterial circle of Willis, as 
seen in the figure below), and that dilatation of this vessel clinically produced mechanical symptoms more frequently (47 per cent) than any other. Aneurism of the internal

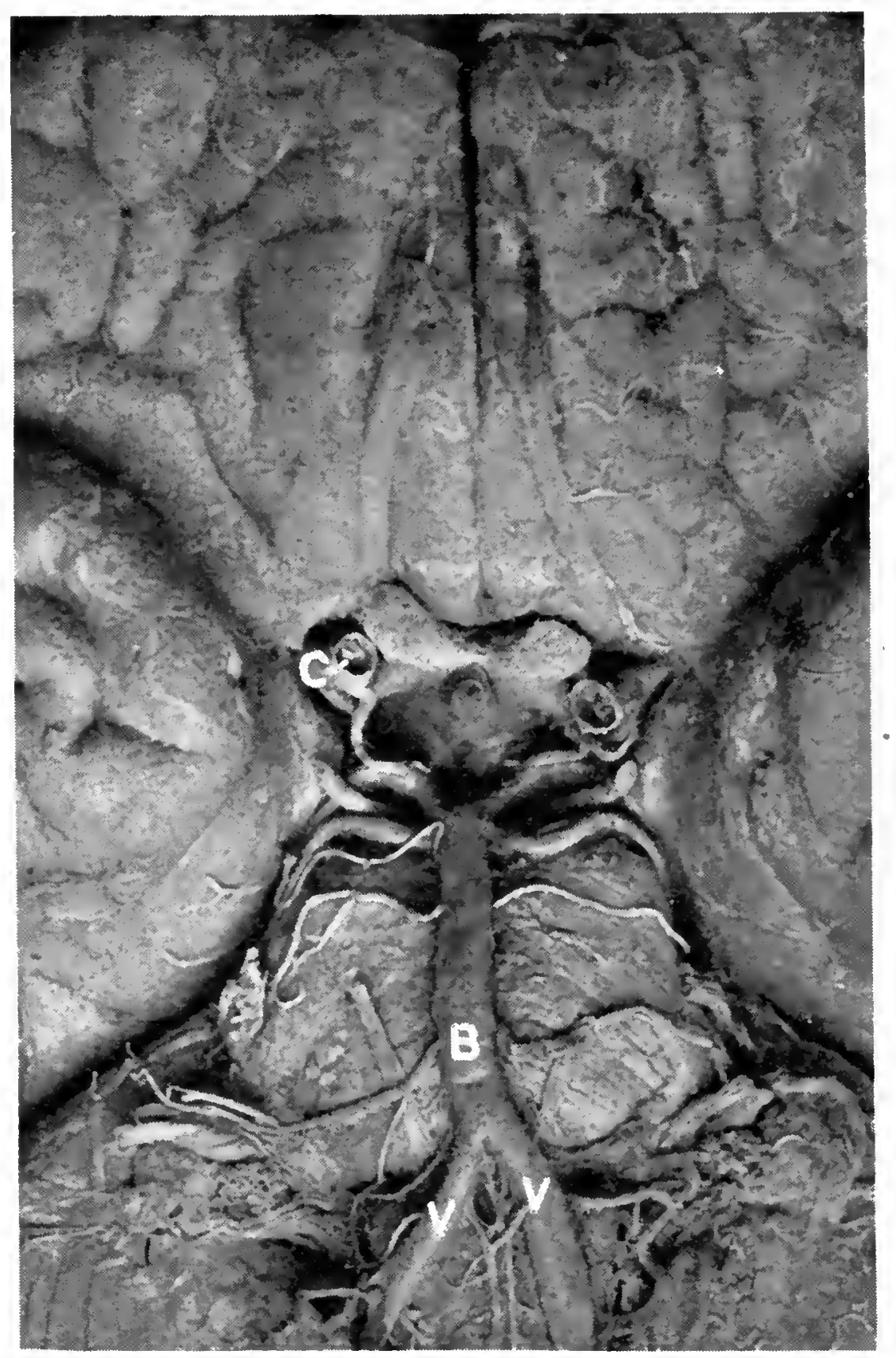

FIG. I72. - The base of the Brain, showing the relations of the arteries to the nerves. Natural size.

$V_{,}, V_{0}=$ the vertebral arteries. $B_{.}=$the basilar; this gives off on each side (opposite $B_{\text {.) }}$ an anterior inferior cerebellar branch which is seen crossing the sixth nerve, and, in front, the large superior cerebellar artery; immediately afterwards it clivides into the two posterior cerebral arteries, each of which is joined to the internal carotid artery (C.) of the same side by the posterior communicating artery. The relation of the third nerve to the superior cerebellar and posterior cerebral arteries is well displayed, and the fourth nerve on the reader's left can be seen to present a similar relation to these vessels.

carotid artery has frequently caused paralysis of this nerve, and an associated involvement of the trochlear, abducent, and ophthalmic division of the trigeminal would point to the localisation of a lesion in the cavernous sinus. Finally, 
it has been suggested that cases of recurrent oculomotor paralysis may be explained by vascular spasm or paralysis of the vaso-motor nerves of these vessels, causing their distension and consequent compression of the nerve.

As regards its relation to the base of the skull, the oculomotor nerve is in close contact with the bone at the anterior clinoid process, as seen in Figs. I69 and I70; it is related to it also in its passage through the superior orbital fissure, a position it shares with the abducent and the naso-ciliary nerves.

If complete, such a lesion of the oculomotor nerve will cause :

(i.) Ptosis, from paralysis of the levator palpebrae superioris. The skin of the eyelid appears abnormally smooth.

(ii.) Lateral strabismus, from non-opposition of the lateral rectus and superior oblique muscles. The eye is almost motionless, and cannot be turned upwards, directly downwards, or medial-wards, though it can be moved down and lateral-wards.

(iii.) Diplopia, the chief symptom complained of, is caused by deviation of the affected eye.

(iv.) Dilatation of the pupil, with no reaction to light, from paralysis of the sphincter papillae.

(v.) Loss of accommodation from paralysis of the ciliary muscle of the lens.

Variations of the Oculomotor Nerve.-These generally consist of abnormal branches, which replace one of the other orbital nerves; for example, the oculomotor may supply the lateral rectus, either in addition to, or even instead of, the sixth nerve (Generali, Harvey), or send a filament to the superior oblique; Martyn (I9I9) reports a clinical case in which the symptoms suggest an anomalous innervation of the levator by the same inferior branch of the third nerve which supplies the medial rectus; it has been seen to furnish a twig to the spheno-palatine ganglion (Poirier). It may anastomose with the sixth nerve, or the superior division may anastomose with the naso-ciliary. The branch to the inferior oblique may send a double supply to the 
inferior rectus, and has also been found traversing the latter, and even perforating the ciliary ganglion. It has been seen perforated near its origin by the posterior cerebral artery (Soemmering).

The Trochlear or fourth nerve.(nevvus trochlearis), the smallest of the cerebral nerves, supplies only the superior oblique muscle of the globe.

It emerges by separate rootlets at its superficial origin on the dorsal aspect of the mid-brain, just below the inferior colliculus at the side of the frenulum veli; it is the only motor nerve of the cerebro-spinal axis which arises from the dorsal aspect.

Its intra-cranial course is the longest of any of the nerves $(40 \mathrm{~mm}$.$) , and its total length from its superficial$ origin to the muscle is $85 \mathrm{~mm}$.; it crosses the brachium conjunctivum cerebelli and winds round the cerebral peduncle immediately above the pons, as is seen in Figs. I33 and I74. Towards the end of this part of its course it lies in the sub-arachnoid space between and parallel to the posterior cerebral and superior cerebellar arteries, and appears on the base of the brain in the interval between the temporal lobe of the brain laterally and the edge of the pons medially (Fig. I68). It then pierces the dura just beneath the free margin of the tentorium cerebelli, behind the third nerve and the posterior clinoid process, as shown in Fig. I69, and enters the cavernous sinus. Here it lies in the lateral wall, below the oculomotor nerve and above the ophthalmic nerve, but towards the fore end of the space it crosses and is bound to the third nerve on its lateral side, as seen in Figs. I70 and I74, then comes to lie above this nerve and on the same horizontal plane as the branches of the ophthalmic nerve ; in this part of its course it is considered to receive communications from the cavernous plexus of the sympathetic nervous system, and it is joined by a sensory twig from the ophthalmic division of the trigeminal. It enters the orbit through the lateral part of the superior orbital fissure, being the only motor nerve of the eye muscles that enters outside the annulus of Zinn and the cone of muscles. 
Its position and short course within the orbit is well displayed in the dissection photographed in Fig. I75, p. 34I, where it is seen on the medial side of the frontal nerve, crossing the superior rectus obliquely forwards and medialwards; it lies immediately beneath the periorbita of the orbital roof.

The trochlear nerve consists of about 2000 coarse fibres, distributed to about the same number of muscle fibres (Macalister, I889), and like the oculomotor shows close to its origin the remains of a degenerated ganglion (Gaskell, I889). Further details of its constitution have been given in connection with the oculomotor nerve on p. 328 above. Paralysis of the nerve, the causes of which have likewise been noted above in connection with the oculomotor, results in loss of function of the superior oblique muscle of the globe, and the patient cannot turn the affected eye down and lateral-wards, and diplopia occurs. Little change, however, may be noticed, as the loss of function of the superior oblique may be vicariously performed. The nerve is rarely affected alone.

As variations are recorded a branch to the orbicularis oculi, and communications with the supra-trochlear, infratrochlear, naso-ciliary, lacrimal, and frontal nerves, which are probably aberrant sensory branches of the trigeminal; it has been seen to pierce the levator on its way to the superior oblique (Shane).

The Abducent or sixth nerve (nervus abducens) supplies the lateral rectus muscle of the eyeball.

It emerges by numerous and sometimes separate rootlets at its superficial origin on the anterior surface of the hindbrain in the groove between the lower edge of the pons and the upper end of the medulla oblongata, just lateral to the pyramid (Fig. I68, p. 323).

In the first part of its intra-cranial course the nerve is flat and is closely applied to the surface of the pons for a distance of about $\mathrm{I}_{5} \mathrm{~mm}$., passing beneath and being bound down by the anterior inferior cerebellar artery (Fig. I72), and being contained in the cisterna pontis of the sub- 
arachnoid space ; it is invested by the arachnoid membrane at $15 \mathrm{~mm}$. from its origin, becomes rounded and pierces the dura opposite the dorsum sellae of the sphenoidal bone, medial and slightly behind the opening for the fifth nerve, and on the medial side of the inferior petrosal sinus (Fig. I69). It then bends slightly lateral-wards through the commencement of the sinus to reach its lateral side, passing forwards through the notch between the apex of the petrous bone and the posterior clinoid process, where it lies under the petro-sphenoidal ligament of Grüber (Fig. I73), and enters the cavernous sinus on the lateral side of the internal carotid artery. In the sinus its position relative to the artery and the other nerves has already been noted (p. 3I 7 , and Figs. I63 and I64); towards the end of its course within the sinus it lies medial to, and is intimately connected with, the ophthalmic nerve. Valentin described an anastomosis between the two nerves in this situation, a fact generally accepted by most authors, but not confirmed by Bischoff (I865); the nerve is also joined here by filaments from the internal carotid plexus of the sympathetic nervous system; some of these are supposed to supply the dilatator pupillae muscle, since a lesion of the nerve often entails a certain amount of contraction of the pupil (Gray); a branch ascending from the spheno-palatine ganglion to the sixth nerve whilst in the cavernous sinus has been described by Boch and Valentin (Quain).

The sixth nerve enters the orbit through the oculomotor foramen, within the cone of muscles, and between the optic nerve and the origin of the lateral rectus muscle (Figs. I35 and I4I) ; it enters the muscle on its ocular aspect at about the junction of its posterior third and anterior two-thirds, after a total course from its superficial origin of about $55 \mathrm{~mm}$. in length.

Its fibres, mostly large, have been estimated at from 2000 to 3600 in number, and are distributed to a muscle containing about 5000 fibres (Macalister, I889); the character of the fibres has been discussed above (p. 327).

Paralysis of the abducent nerve is particularly associated with fractures of the base of the skull, owing to its com- 
paratively long course beneath the dura covering the dorsum sellae and its close confinement beneath the petro-sphenoidal ligament. Panas, indeed, first recognised that a fractured base might be manifested by paralysis of the sixth nerve alone. As regards such injuries, Stephenson (I9I9) states that Hewett, in an analysis of sixty-four cases of fracture of the base, found that in upwards of 82 per cent the fissure passed through the middle cranial fossa, and that in about one-third of sixty-eight cases examined after death the

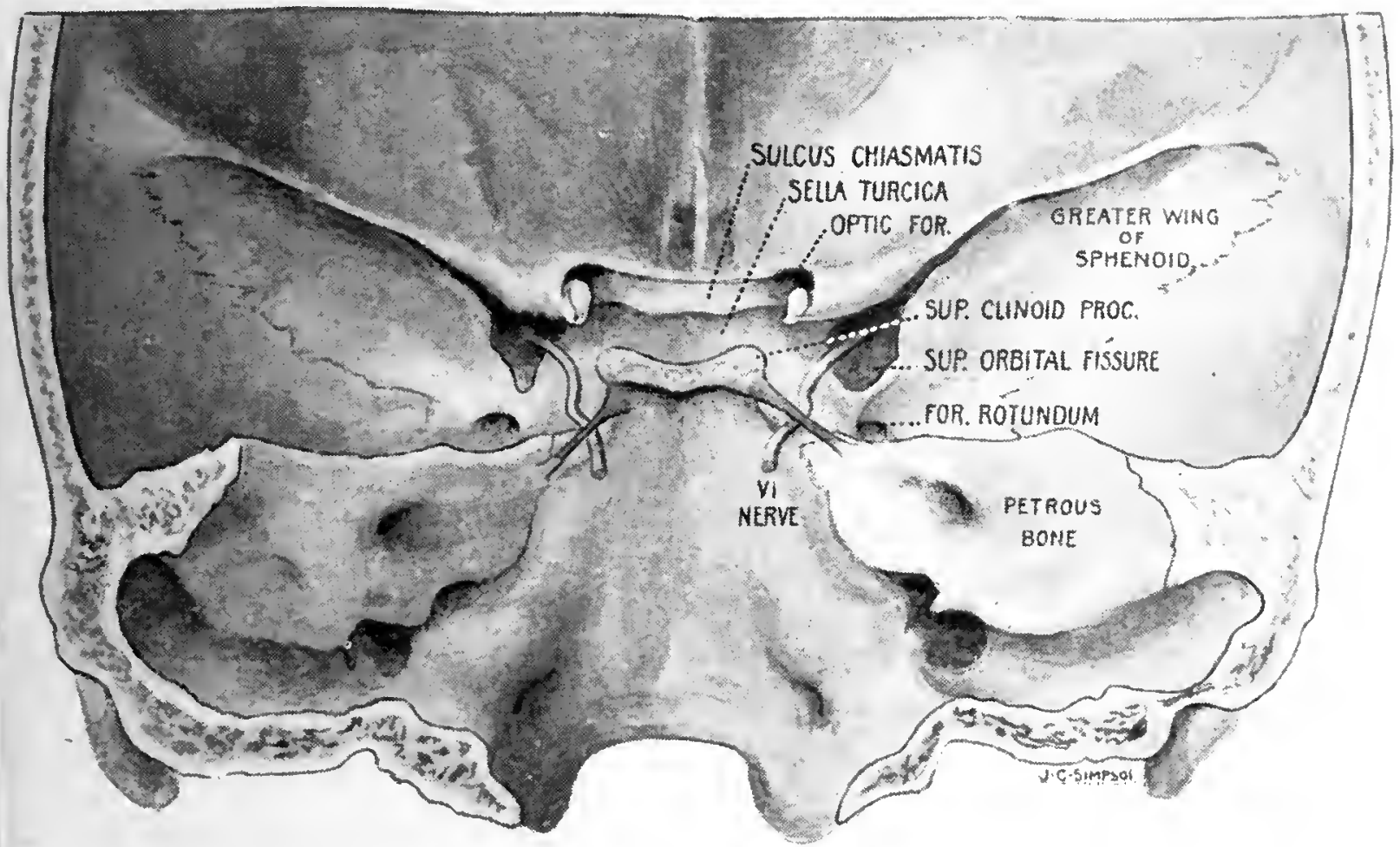

FIG. I73. - The petro-sphenoidal ligament. Sketch of the interior of the base of the skull viewed from behind to illustrate a dissection of the abducent or sixth nerve passing beneath the ligament before entering the cavernous sinus. 'The ligament, which is formed by the deeper or osteogenetic layer of the dura mater, presents differences in its extent and attachments, as is illustrated on the two sides.

orbital roof was also found broken; Liebrecht (I906) has specially studied the effect of such fractures on the eyes. Stopford (I9I7) notes the possible compression of the nerve by the anterior inferior cerebellar artery against the bone before it pierces the dura, and Cushing (IgIO) its strangulation by lateral branches of the basilar artery in cases of brain tumour. In its passage through the cavernous sinus it will be subject to the same affections as noted in the case of the third nerve (p. 329). The results of a paralysis are convergent strabismus and diplopia, and the cornea cannot 
be moved beyond the centre of the palpebral fissure; the oblique muscles may to some extent counteract the loss of abduction. It is to be noted that a paralysis of the lateral rectus on the one side accompanied by paralysis of the medial rectus on the other (conjugate deviation), would point to a lesion in the nucleus of origin of the sixth nerve (p. 3S9). The possibility of dilator pupillae fibres running in this nerve has already been referred to.

As variations there are recorded, a case of absence on one side, its place being taken by the third nerve (Generali); Cruveilhier (ISS6) has seen the origin to be from the olive, and has also described two separate roots of origin which may not unite until they reach the cavernous sinus and between which the artery may pass; this last anomaly has also been seen by Cushing, and is explained by the presence of an aberrant root of the nerve (Stopford, I9I6). Krause, on the other hand, has seen the origin placed at $S \mathrm{~mm}$. above the inferior border of the pons; Bremer (I90S, I92I) has written on the aberrant roots and branches of this nerve in the human embryo. Valentin states that it may receive a branch from the spheno-palatine ganglion instead of from the ophthalmic nerve; it may enter the orbit outside the annulus of Zinn (Poirier). It has been seen to furnish a branch to the superior rectus in addition to the usual supply from the third nerve (Magath, I9I9), or it may appear to give off the naso-ciliary nerve (Krause), or a root to the ciliary ganglion.

The Ophthalmic Nerve (nervus ophthalmicus) is the smallest of the three divisions of the trigeminal or fifth nerve, and is purely sensory. It supplies the eyeball and conjunctiva, the lacrimal gland, caruncle and sac, part of the nasal mucous membrane and the frontal air sinus, the upper eyelid, forehead, and anterior part of the scalp, the root and forepart of the nose (Fig. SS, p. I75).

It arises from the fore and medial part of the semilunar or Gasserian ganglion, the cells of which comprise the nucleus of origin of the sensory part of the trigeminal. The description of the ophthalmic nerve may therefore well 
be prefaced by a brief account of the main nerve from whis, h it is derived.

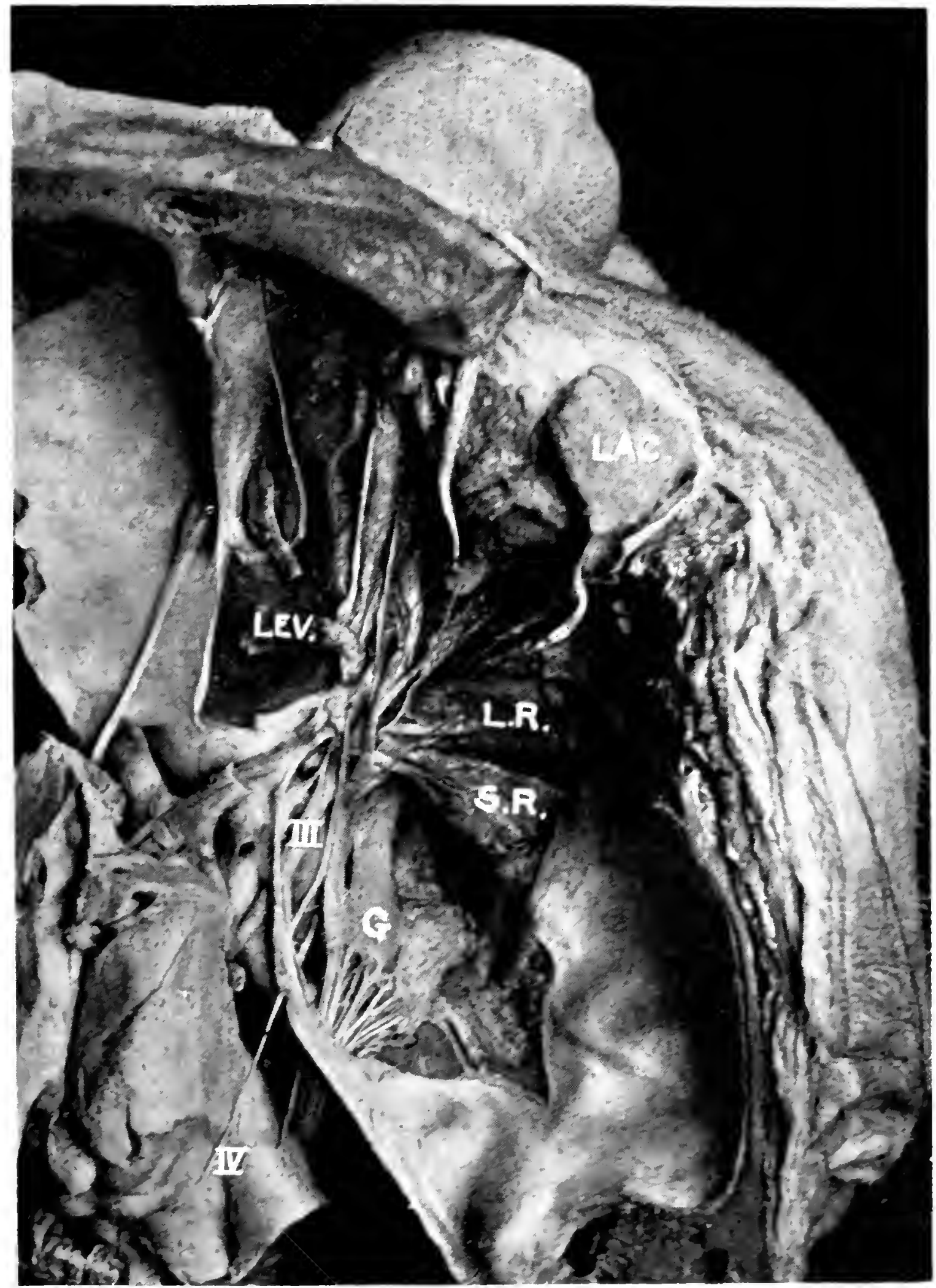

Fig. 174. -Dissection of Orlat, middle cranial Fossa to show the nerves. The preparation is risured frot to the left. Natural size. 
The trigeminal or fifth nerve (nervus trigeminus) is the great sensory nerve of the face and larger part of the scalp, and the motor nerve of the muscles of mastication. It is attached to the side of the pons by a large sensory and a small motor root, this point corresponding to the superficial origin of a motor cerebral nerve (Figs. I68, I74). Tracing the roots forward away from the brain, they are seen to enter an opening in the dura beneath the attached border of the tentorium, where the sensory fibres reach the semilunar ganglion from the cells of which they actually arise. The ganglion lies in a space (cave of Meckel) formed by a separation of the dura, and situated on an impression near the apex of the petrous part of the temporal bone in the floor of the middle cranial fossa (Fig. I69); it lies $2 \frac{1}{2}$ inches medial to, but a little higher than, the articular tubercle at the root of the zygoma (Figs. 'I33, I84). From the fore edge of the ganglion three large nerve trunks arise as the three divisions of the fifth nerve; the uppermost is the ophthalmic, the middle one is the maxillary (or superior maxillary), and the lowest and most lateral is the mandibular (or inferior maxillary) nerve. The motor root of the fifth nerve enters the cave of Meckel beneath the main nerve, and passes beneath the ganglion to become a component of the mandibular nerve, as is seen in Fig. I64, p. 3I7.

The ophthalmic nerve fibres arise as peripheral processes of cells of the semilunar ganglion. The nerve pierces the dura to enter the cavernous sinus almost immediately after its origin from the ganglion; it then passes upwards and forwards, embedded in the lateral wall of the sinus below the trochlear nerve and above the maxillary nerve, as is shown in Fig. I74. Whilst in the sinus the nerve first gives off a recurrent branch (nervus tentorii, or recurrent nerve of Arnold), which crosses and is adherent to the trochlear nerve, and is distributed to the tentorium cerebelli; it is then generally considered to give off three slender filaments, which join the oculomotor, trochlear, and abducent nerves and so provide sensory fibres for the muscles they supply, though Bischoff (I865), after microscopical examination, denied a junction with the third and sixth, and found it only 
for the fourth nerve, and Merkel regards the connections as inconstant; it also receives filaments from the cavernous sympathetic plexus.

After a total length from the ganglion of $25 \mathrm{~mm}$., or an inch, the nerve breaks up in the forepart of the sinus into three terminal branches, lacrimal, frontal, and naso-ciliary, though often the last alone is here separated off. All three enter the orbit through the superior orbital fissure, but the naso-ciliary nerve alone traverses the oculomotor foramen, inside the annulus of Zinn, and between the two divisions of the third nerve; the lacrimal and frontal nerves enter through the lateral part of the fissure and outside the cone of muscles (Figs. I35, I39). These nerves run forwards through the orbit, each accompanied in the greater part of its course by an artery, and all three end by finally supplying the skin in regions beyond the orbital margin. A complete lesion of the ophthalmic nerve occasions anaesthesia of the forehead, the upper but not the lower eyelid, the root and tip of the nose, the conjunctiva, cornea, and eyeball, and there is no reflex winking on irritation of the conjunctiva. Taste and smell are said to be impaired. The area of cutaneous anaesthesia is much less than might be expected from the distribution of the branches of the nerves, owing to overlapping by other adjacent branches of the trigeminal (Fig. 88, p. I7 ).

The branches of the ophthalmic, like the other sensory parts of the fifth nerve, anastomose on the face with branches of the facial nerve.

The lacrimal nerve (nervus lacrimalis), the smallest of the three, is closely bound to the fourth nerve at the fore end of the cavernous sinus, but leaves it to pass obliquely through the lateral extremity of the fissure, so intimately enveloped by the dura that it is difficult to dissect out. It then runs along the lateral orbital wall, embedded in the fat in company with the lacrimal artery and above the lateral rectus, toward the lacrimal gland. Just behind, or sometimes within its substance, the nerve divides into two branches. The uppermost one traverses the gland, to which it gives off most of its fibres, and after piercing the septum 
orbitale ends in the conjunctiva and skin of the upper eyelid in the lateral region (Fig. I04, p. 205); the inferior branch passes downward on the orbital wall behind the gland, to which it also sends fibres, and anastomoses with the zygomatico-temporal branch of the zygomatic nerve, which is derived from the maxillary division of the trigeminal (Fig. 7I, p. I49). The signification of this connection and the constitution of the fibres of this nerve have been referred to on p. $2 \mathrm{I} 4$; the cutaneous distribution is noted on Pp. I75, 202.

As variations, the lacrimal nerve may give off fibres which run for part of their course with the other orbital nerves; such junctions are recorded with the fourth nerve, with the frontal, and with the naso-ciliary (Laffey). It has been seen to give off a ciliary nerve, and to receive a filament from the long root of the ciliary ganglion or from the ganglion itself. A case has been observed where the lacrimal instead of the zygomatic (temporo-malar or orbital) nerve provided the usual zygomatico-temporal branch of the latter (Thane), and, on the other hand, the lacrimal has been found absent, its place being taken by the zygomatic nerve (Turner). The anastomosis between the two nerves has been found absent (Laffey). The writer has found two well-developed lacrimal nerves, both derived from the ophthalmic nerve; a similar instance is recorded by Delbet, though in his case one of the nerves was derived from the maxillary nerve, and so was apparently equivalent to an aberrant zygomatic branch.

The frontal nerve (nervus frontalis), the largest branch of the ophthalmic nerve, after entering the orbit above the muscles, runs forwards immediately beneath the periorbita of the roof, resting first upon the origin of the superior rectus, then upon the levator of the upper eyelid. At a variable point, but usually beyond the middle of the orbit, it gives off a supra-trochlear nerve, beyond which point it is continued forwards under the name of supra-orbital nerve (Figs. I75, I76). - The supra-trochlear nerve of Arnold (nervus supratrochlearis), as its name implies, passes inwards and forwards close above and sometimes bound to the pulley of the superior oblique muscle, pierces the septum 
orbitale, and runs up over the orbital margin through the frontalis and orbicularis muscles in company with the frontal artery, a finger's breadth from the mid-line, to supply by several branches the skin of the forehead above the head of the eyebrow (Figs. 8, p. 20, and 54, p. 123); near the pulley a branch joins the infra-trochlear branch of the nasociliary nerve, either before or after leaving the orbit, from which superior palpebral twigs supply the skin and con-

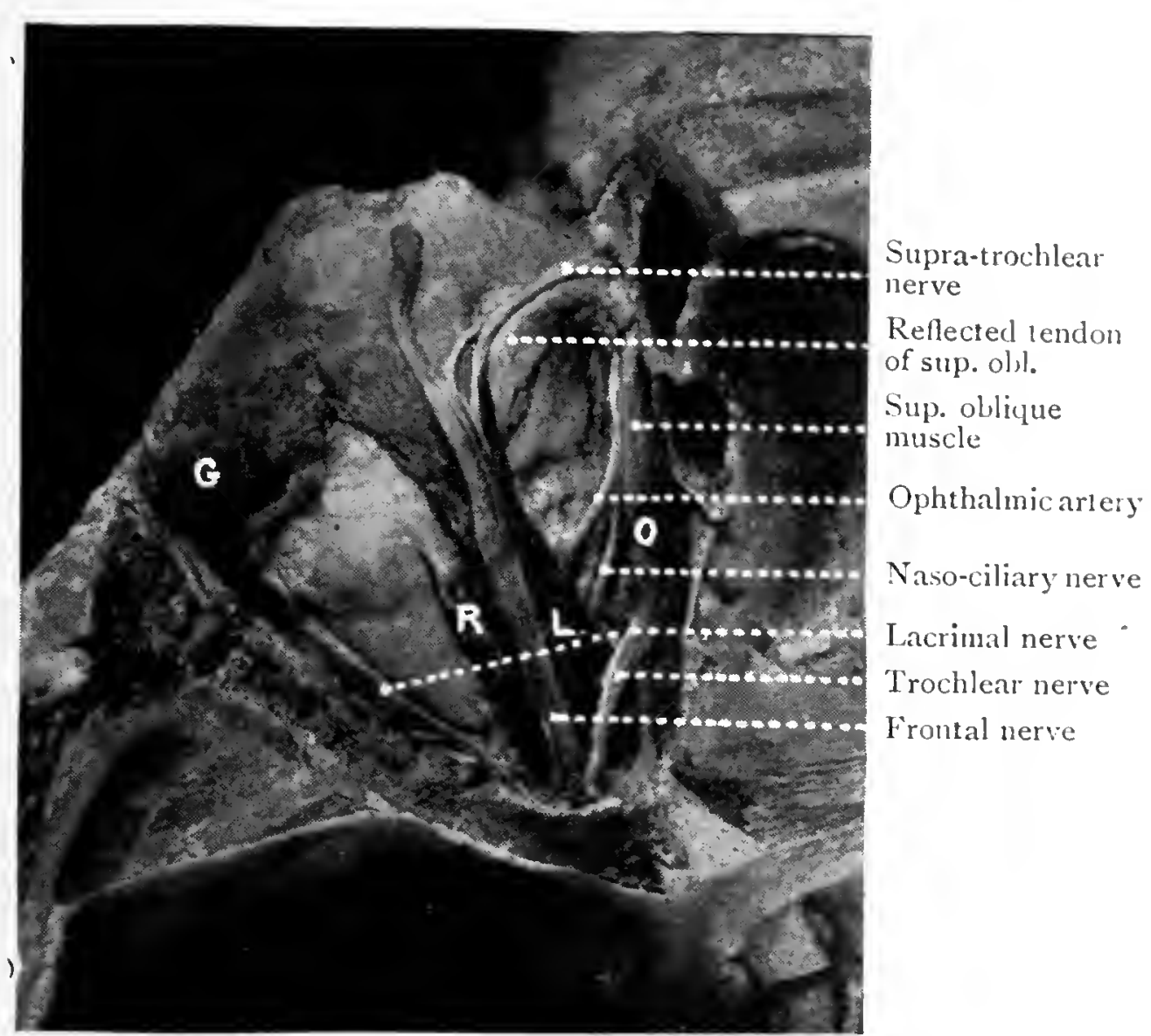

FIG. I75.-Dissection of Left Orbit from above, to show the levator palpebrac superioris (L.) and superior oblique (O.) muscles, and the nerves in relation. Natural size.

$$
\text { R.= superior rectus muscle; G. = lacrimal gland. }
$$

junctiva of the upper eyelid and side of the nose. The supraorbital nerve (nervus supra-orbitalis) is the direct continuation of the frontal nerve under a different name after it has given off the supra-trochlear nerve. It leaves the orbit by passing through the supra-orbital notch or foramen of the upper margin, and runs up over the forehead, sometimes grooving the bone deeply, in company with the artery of the same name as far as the occipital bone. It divides into a smaller medial and a larger lateral main stem, the former being sometimes called the internal frontal (which has been 
confused with the supra-trochlear) and the latter the external frontal or supra-orbital nerve proper; the division

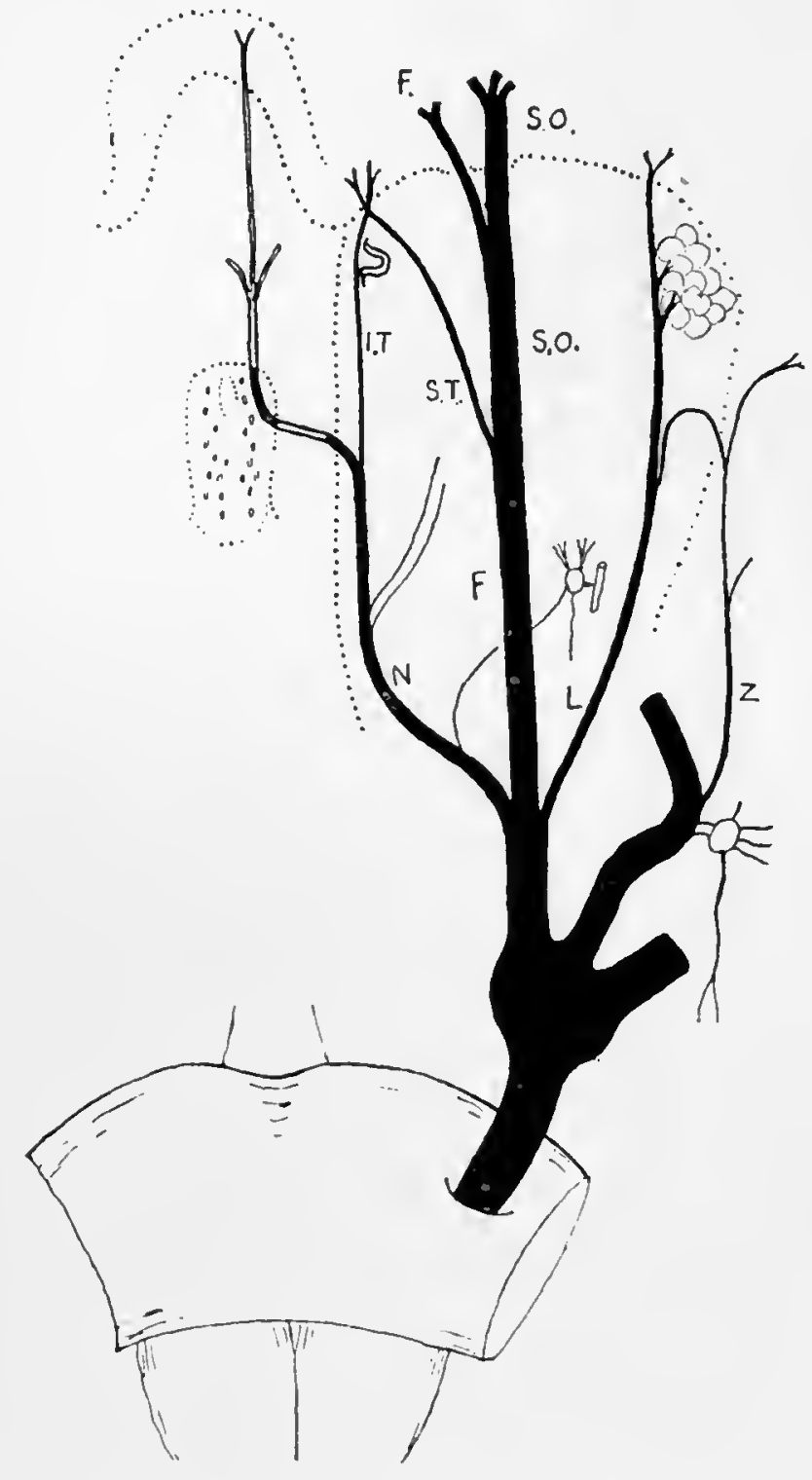

FIG. I76. - Scheme to show the distribution of the Ophthalmic Division of the Trigeminal Nerve.

$\mathrm{F}$. =frontal nerve, giving off the supra-trochlear branch (S.T.) and afterwards being known as the supra-orbital (S.O.), which may divide before leaving the orbit into a medial frontal branch (F.) and a lateral frontal or supra-orbital proper.

$\mathbf{N}$. = the naso-ciliary nerve; it is shown to give off the sensory root to the ciliary ganglion, the two long ciliary nerves to the eyeball, and, just before leaving the orbit through the anterior ethmoidal canal, the infra-trochlear nerve (I. T.) ; the rest of its course illustrates the description given in the text.

L. = the lacrimal nerve, the anastomosis of which with the zygomatico-temporal branch of the zygomatic nerve $(Z$.$) is represented.$

the orbit, in the cranial cavity, in the nasal cavity, and at the tip of the nose ; its parts and branches do not always receive the same names in the literature. After entering the orbit and the former branch then grooves the orbital margin in a frontal notch. Twigs are given to the eyelids, and whilst traversing the supraorbital notch to the diploë and frontal sinus. The nerve is illustrated in Figs. $8,54, \mathrm{I} 75$.

As variations of the frontal nerve, the writer has seen it provide a very fine infra - trochlear branch instead of the naso-ciliary; also a well-developed twig was found passing directly to the superior oblique muscle, which, when traced backwards, was found to be derived from the ophthalmic nerve, and so represents the sensory filaments usually accompanying the fourth nerve to the muscle. The supra-trochlear may abnormally arise in a branch of the supra-orbital. The writer has found it duplicated.

The naso-ciliary or nasal nerve (nerous nasociliaris) has a long and complicated course, since it is found in course, since it is found in

may occur within the orbit,

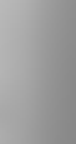


through the annulus of Zinn between the two divisions of the oculomotor nerve, it courses obliquely beneath the superior rectus muscle, to the tendon of which it may adhere, and over the optic nerve along with the ophthalmic artery towards the medial wall of the orbit, where it lies between the superior oblique and medial rectus, still in company with the artery; it leaves the orbit by traversing the anterior ethmoidal foramen (hence the name of anterior ethmoidal nerve sometimes applied to it here) and enters the cavity of the cranium, where it crosses the forepart of the cribriform plate of the ethmoidal bone beneath the dura of the anterior cranial fossa, running along the side of the olfactory lobe (Fig. I78); it then passes through a slit, the nasal fissure or anterior nasal canal, at the side of the crista, galli, enters the nasal cavity (being here related to the medial side of the infundibulum of the frontal sinus), and breaks up into three terminal branches. Two of these, the medial and lateral internal nasal branches (rami nasales mediales et laterales), are distributed to the forepart of the nasal cavity, but the third is continued forwards as the external nasal nerve to the end of the nose. In the orbit its branches are:

(i.) The long or sensory root (radix longa) of the ciliary ganglion (p. $\left.3^{2} \mathrm{I}\right)$, which leaves the nerve soon after or even before its entrance into the orbit, and lies lateral to the optic nerve (Fig. I78); it frequently anastomoses with the long ciliary nerves and with the short root of the ganglion (Valentin).

(ii.) The long ciliary nerves (nervi ciliares longi), two, or sometimes three in number, which lie also on the lateral side of the optic nerve, supplying its sheath; after joining some of the short ciliary nerves, they enter the eyeball round the point of attachment of the optic nerve and supply the iris, ciliary muscle, and cornea with sensory filaments, but in addition contain sympathetic fibres for the dilatator pupillae muscle (see pp. 353, 358).

(iii.) The infra-trochlear nerve (nervus infratrochlearis, sometimes referred to as the external nasal branch in contradistinction to the main trunk, which after this point 
of separation is then known as the internal nasal nerve) appears to be the direct though more slender continuation of the main nerve forward from the point where the latter turns to enter the anterior ethmoidal foramen; it runs beneath the superior oblique muscle and trochlea, forms a junction with the supra-trochlear nerve, and dividing into several. branches, usually before piercing the septum orbitale, is distributed to the parts of the medial canthus of the eyelid, namely to the skin and conjunctiva mainly of the upper eyelid and to a smaller extent of the lower, the root of the nose, lacrimal sac, canaliculi, and caruncle. Ledouble describes a filament passing through the lacrimal bone at the point dacryon into the nose.

(iv.) The three terminal branches in the nose are: (x) the medial internal nasal or septal branch (ramus nasalis medialis), which supplies the mucous membrane of the anterior part of the septum; (b) the lateral internal nasal branch (ramus nasalis lateralis, but sometimes confusedly called the external nasal branch, or again, sometimes described as a branch of the latter), which supplies the forepart of the middle and inferior conchae and lateral nasal wall; and $(c)$ the external nasal branch (anterior nasal, ramus nasalis externus or nasalis extremus), the continuation of the main nerve, which passes along a groove on the under surface of the nasal bone, emerges between its lower edge and the upper lateral nasal cartilage, and supplies the skin of the cartilaginous end of the nose.

As an inconstant branch within the orbit, there may be found in two out of six subjects (Delbet) the sphenoethmoidal nerve of Luschka, or posterior ethmoidal branch of Krause (ramus ethmoidalis posterior), which supplies the mucous membrane of the sphenoidal air sinus and posterior ethmoidal cells. Twigs are also said to be supplied by the naso-ciliary nerve to the frontal and anterior ethmoidal cells as it passes through the anterior ethmoidal canal (Merkel). From a clinical point of view it appears worth noting that, according to Ledouble, a neuralgia may recur after section of the supra-orbital nerve owing to the escape of the supra-trochlear nerve, and it is only after resection 
of the frontal nerve itself that Koenig has been able to cure a stubborn supra-orbital neuralgia.

As variations, the naso-ciliary nerve has been seen to send branches to the superior and internal recti and levator palpebrae superioris (Krause), doubtless representing the sensory twigs usually received by the motor nerves to these muscles in the cavernous sinus. An anastomosis with the lacrimal nerve has been found (Laffey). Instances are recorded of absence of the infra-trochlear nerve, the deficiency being provided for by the supra-trochlear (Testut). The internal nasal branches may be absent (Merkel), or they may enter the nose through the posterior ethmoidal canal. Other minor variations will be found recorded by Cruveilhier (I886).

The Maxillary Nerve (superior maxillary, nervus maxillaris) or second division of the trigeminal is, like the ophthalmic division, purely sensory. It supplies the skin of the cheek, front of the temporal region, lower eyelid, side of nose, and upper lip (Fig. 88, p. I75); the teeth of the upper jaw; and the mucous membrane of the nose, naso-pharynx, maxillary sinus, posterior ethmoidal cells, soft palate, tonsil, and roof of the mouth.

It arises from the middle of the semilunar ganglion and passes forwards in the lower part of the lateral wall of the cavernous sinus, the position being illustrated in Figs. I63, I64, I74.

It leaves the middle cranial fossa through the foramen rotundum, bends downwards and crosses the upper part of the pterygo-palatine or spheno-maxillary fossa, and curving laterally round the orbital process of the palatine bone as it forms the apex of the floor of the orbit, enters that space through the inferior orbital fissure at about its mid-point. It runs forwards along the orbital floor parallel to the plane of the medial wall, beneath the periorbita in the infra-orbital groove and canal, and emerges on the face through the infra-orbital foramen. In the orbital part of its course it is called the infra-orbital nerve.

Branches are given off from it in the cranium, in the pterygo-palatine fossa, in the infra-orbital canal, and on the face. 
(i.) The cranial branch is the recurrent or middle meningeal nerve to the dura mater.

(ii.) The branches given off in the fossa are the zygomatic, the spheno-palatine, and the posterior superior alveolar.

The zygomatic nerve (temporo-malar or orbital nerve; nervus zygomaticus; nervus subcutaneus malae) enters the orbit through the inferior orbital fissure, and running along

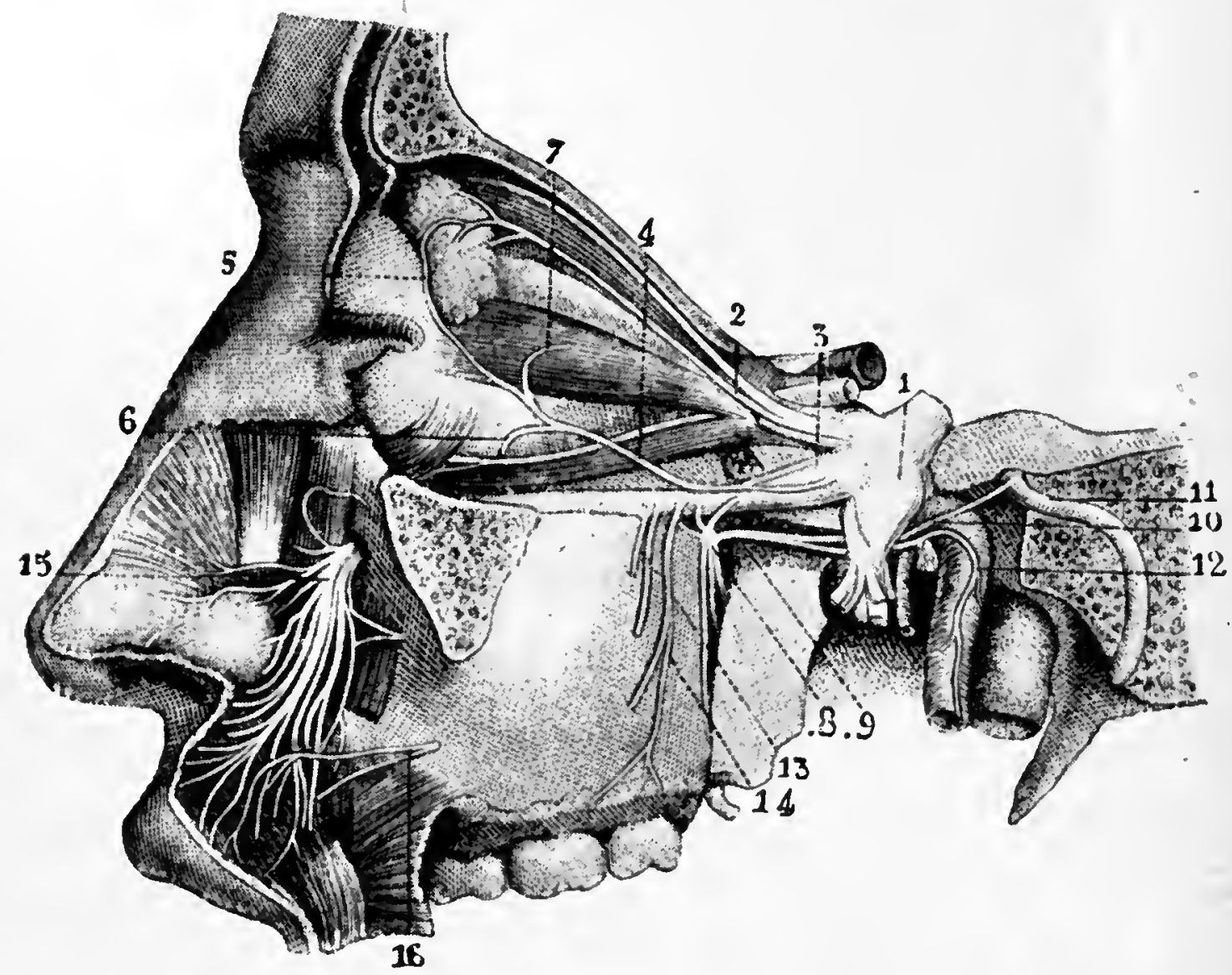

FIG. I77.- The Maxillary Division of the Trigeminal Nerve, its branches and their connections. (From Sappey, after Hirschfeld and Leveillé.)

$\mathbf{I}=$ semilunar or Gasserian ganglion; $3=$ the maxillary nerve $; 4=$ the zygomatic branch with its anastomoses (5) with the lacrimal nerve (2); II $=$ the facial nerve; Io is the greater and Iz the deep petrosal nerves which join to form the Vidian nerve (9) and enter the spheno-palatine ganglion (8). The other lranches can be followed from the text.

the lateral wall divides into two branches, temporal and malar. The temporal branch (nervus zygomaticotemporalis) sometimes grooves the orbital wall as it runs forwards in close contact with it ; it forms an important communication with the lacrimal nerve, as illustrated in Fig. 7I, p. I49; the significance of which has been noted on p. 2I4, and passing either through a canal in the zygomatic bone or through the spheno-zygomatic suture, enters the temporal 
fossa. After ascending and piercing the temporal fascia about $25 \mathrm{~mm}$. or an inch above the zygoma, it supplies the skin of the forepart of the forehead up to the lateral side of the orbit. The malar branch (nervus zygomaticofacialis) passes along the lower lateral angle of the orbit, and after traversing a canal of the same name in the zygomatic bone, perforates the orbicularis oculi muscle to supply the skin of the cheek; its emergence on the face is seen in Figs. 53, 89. Both branches of the nerve inosculate with twigs of the facial nerve. As variations, the whole nerve may pass through the bone before dividing, both branches may traverse separate canals, or the temporal branch may pass out by the fore-end of the inferior orbital fissure; either branch may be absent, its fellow supplying the deficiency; the infra-orbital nerve may replace the distribution of the malar, or a twig from the lacrimal may be substituted for the temporal, or vice versa, as previously noted on p. 340 .

The spheno-palatine branches (nervi sphenopalatini) are two or three short thick twigs which pass directly downwards into the spheno-palatine or Meckel's ganglion, only a small part of their fibres actually traverse the ganglion, the majority passing lateral to or in front of it to be continued directly on as its branches, but whilst in neither case are these trigeminal fibres interrupted in the ganglion, in both instances they receive sympathetic nerve fibres from it which accompany them to their destination (Piersol, I9I9). The ganglion lies in the upper part of the pterygo-palatine fossa, close to the spheno-palatine foramen; it receives three kinds of fibres, sensory by its branches from the maxillary nerve, motor (and probably also sensory) by the great superficial petrosal nerve from the geniculate ganglion of the facial nerve, and sympathetic by the great deep petrosal nerve from the carotid plexus, these two petrosal nerves running together in the latter part of their course to enter the ganglion as the Vidian nerve, or nerve of the pterygoid canal. Among the numerous branches of the ganglion, which chiefly supply the palate and the nasal cavity, there are to be noted here as of immediate interest the ascending 
or orbital branches (rami orbitales), two or three filaments which enter the orbit through the inferior fissure and supply the periorbita, including the involuntary orbitalis muscle, and after traversing the posterior ethmoidal canal or a minute special aperture, are also distributed to the sphenoidal sinus and posterior ethmoidal air-cells (Luschka); it is stated (Gray) that these minute filaments also supply the lacrimal gland (see p. 2I4), and others have been traced to the sixth nerve, ciliary ganglion, and optic nerve sheath (Quain).

The posterior superior alveolar or dental branches, usually two in number, arise just before the maxillary nerve enters the inferior orbital fissure; they descend on the zygomatic surface of the maxilla and enter minute canals to supply the molar teeth, gums, and mucous membrane of the maxillary sinus.

(iii.) The branches of the maxillary nerve given off in the infra-orbital canal are the middle and anterior superior alveolar or dental nerves (ramus alveolaris superior medius et anterior). The middle arises in the posterior part of the canal and runs downwards in the lateral wall of the antrum to supply the two premolar teeth. The anterior (usually double) leaves the lateral side of the main nerve in the forepart of the canal, curves medially beneath the infraorbital foramen in the anterior wall of the antrum, and then bends downwards to supply the incisor and canine teeth; its course is indicated in Fig. 5, p. 8, and further reference has been made to it on p. I6.

(iv.) On the face, after emerging by the infra-orbital foramen, buried in fat about Io $\mathrm{mm}$. from the skin surface, and under cover of the orbicularis oculi and the caput infraorbitale of the quadratus labii superioris muscles, the maxillary nerve terminates by dividing into three groups of branches: the inferior palpebral (rami palpebrales inferiores), distributed to the skin and conjunctiva of the lower eyelid; the lateral or external nasal (rami nasales extermi), which supply the skin of the side of the nose; and the superior labial (rami labiales superiores), which are the largest branches, and are distributed to the skin of the 
cheek, and the mucous membrane and skin of the upper lip.
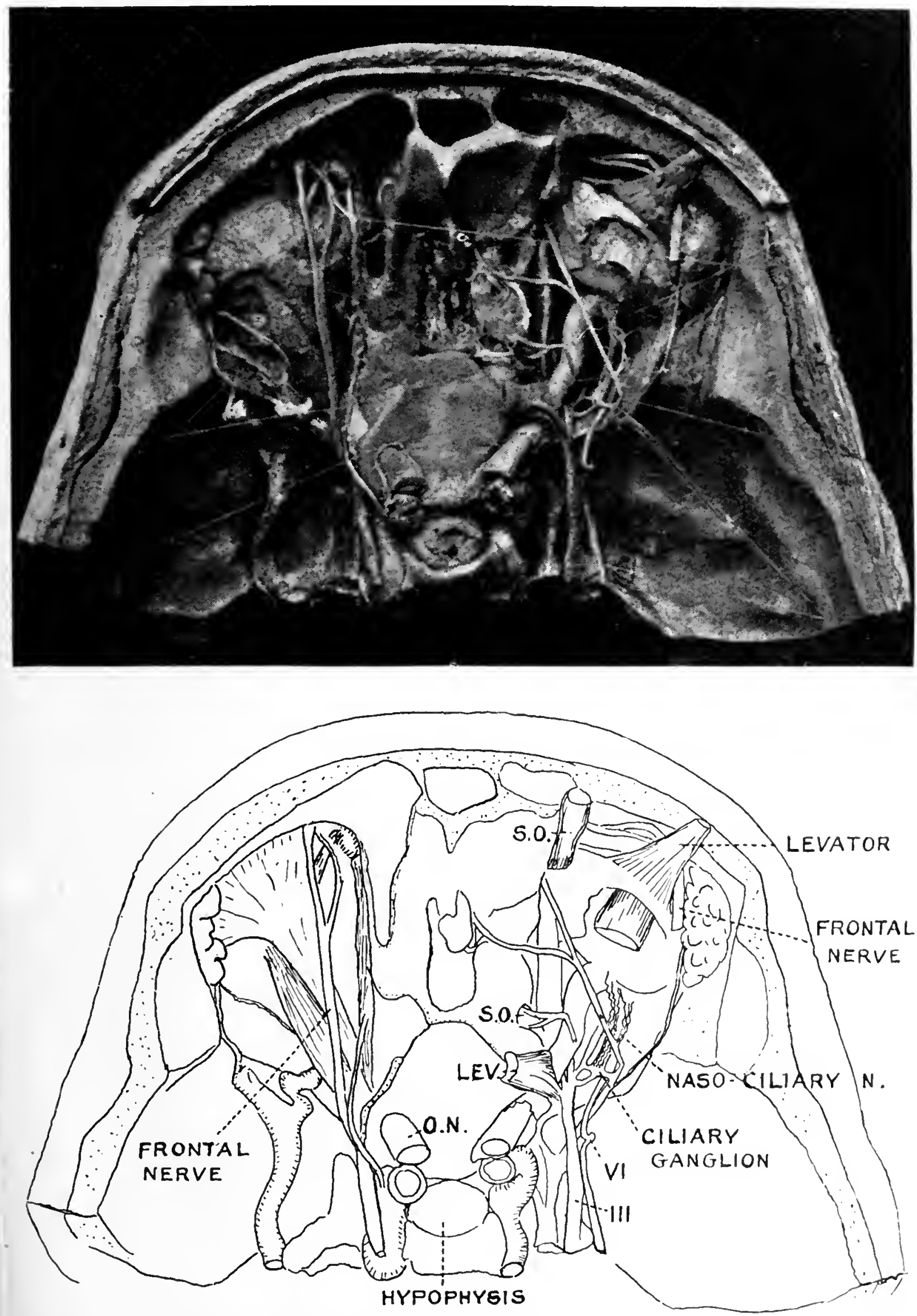

FIG. 178. -Dissection of both Orbits from above; superficial on the left and deep on the right, to show the nerves. Adult female. $\times \frac{3}{4}$.

$\mathrm{S} . \mathrm{O} .=$ superior oblique muscle $\mathrm{LEV} .=$ lezator palpebrae superioris; $\mathrm{O} . \mathrm{N} .=$ optic nerve.

All these branches inosculate freely with the zygomatic 
branch of the facial nerve, forming the important infraorbital sensori-motor plexus.

The Ciliary Ganglion and Sympathetic Nerve Supply of the Eyeball.-The ciliary ganglion (ganglion ciliare; lenticular or ophthalmic ganglion), which has been referred to above in connection with the oculomotor and naso-ciliary nerves, is a small mass of cells lying in the apex of the orbit. Like the spheno-palatine, otic, sublingual, and submaxillary ganglia connected with the other two divisions of the trigeminal nerve, it corresponds to the terminal ganglia of the sympathetic system, being chiefly composed of multipolar elements of three types, closely resembling stellate cells of undoubted sympathetic nature found elsewhere, though it contains also the bipolar cells of a cerebrospinal ganglion. Developmentally, in the human embryo its cells migrated from the semilunar ganglion along the ophthalmic nerve, and relatively few are contributed via the oculomotor (Kuntz, I920). It is essentially a relay station in which certain fibres, contributed by the various nerves which form its so-called roots, end in arborisations round the ganglion cells, the axons of which pass forwards as the short ciliary nerves to the globe; in other words, it serves as a centre for the supply of motor, sensory, and sympathetic nerves to the eyeball. It is noteworthy that since the ciliary nerves which leave the ganglion contain many more fibres than do the roots, probably each of the entering fibres is connected with several cells. (Langley and Anderson, I892; Retzius, I894; Marinesco, I908; Nicholls, I9I5. The earlier literature is given by Van Gehuchten, I906.)

It is a small pink mass about the size of a pin's head, $2 \mathrm{~mm}$. long, quadrilateral in outline and bilaterally compressed, though its shape is obscured by fat and connective tissue. It lies in the orbital fat near the apex of the orbit, between the lateral rectus muscle and the optic nerve, and close to the ophthalmic artery, a centimetre in front of the annulus of Zinn and about $15 \mathrm{~mm}$. behind the eyeball; it is most easily found by following backwards the nerve to the inferior oblique muscle, just above which it lies and to 
which it is connected by a short stout nerve twig, its motor root. This root, along with two others, the sensory and the sympathetic, enters its superior border, whilst from the corners of the anterior margin there arise two bundles of very delicate fibrils, at first sight not easily distinguishable from the connective tissue strands of the orbital fatty mass; these are the short 'ciliary nerves, and they can be traced forwards to the eyeball, which they enter round the optic nerve.

The roots convey to the ganglion :

(i.) Motor fibres from the oculomotor nerve.

(ii.) Sensory fibres from the naso-ciliary branch of the trigeminal nerve.

(iii.) Sympathetic fibres from the cavernous plexus on the internal carotid artery.

The motor or short root, or para-sympathetic root (radix brevis), is the thickest, and is derived from the branch of the oculomotor nerve which supplies the inferior oblique muscle. It enters the postero-inferior part of the ganglion and carries two orders of fibres:

(a) Contractile to the iris (the sphincter or constrictor pupillae fibres); these originate from cells situated in the anterior part of the nucleus of the third nerve in the midbrain, run along the main trunk of the nerve, and end in the ciliary ganglion, whence the impulses are carried by fresh fibres through the short ciliary nerves to the globe; degeneration does not occur in the motor fibre constituents of these nerves after section of the oculomotor proximal to the ganglion (Van Gehuchten) ; and the path is therefore interrupted in the ganglion. Contraction of the pupil has been experimentally produced in animals by excitation of the root of the third nerve, and by stimulation of the ciliary ganglion or of the short ciliary nerves (Starling, I920).

(b) Contractile to the ciliary muscle, the accommodation fibres; these have a similar origin and course, proved likewise by experiment, since stimulation of the semilunar ganglion or of the long ciliary nerves has no effect on the ciliary muscle.

The sensory, nasal, or long root (radix longa) arises from the naso-ciliary branch of the ophthalmic division of the 
trigeminal nerve; it leaves it in the oculomotor foramen, and runs along the lateral aspect of the optic nerve as a long slender filament which enters the upper posterior angle of the ganglion. Its fibres are chiefly sensory or centripetal from the whole eyeball, but the most important are those from the cornea, and they are not interrupted in the ganglion; this root is considered by some authors to contain also sympathetic fibres effecting dilatation of the pupil (vide infra), and other fibres which cause relaxation of the ciliary muscle (Poirier).

The sympathetic root (radix media vel sympathica), commonly multiple, is a minute filament which arises from the cavernous plexus and runs forwards to enter the upper posterior part of the ciliary ganglion. It conveys vasoconstrictor fibres to the whole eyeball, vaso-dilator to its anterior segment, and, it is somctimes stated, pupillo-dilator to the iris. The fibres are interrupted in the ganglion.

Variations in the number of roots are common. The motor root may be absent, double or multiple, or may bifurcate before entering the ganglion; it has been found arising from the trunk of the third nerve or from the sixth (Lagrange). The serisory root is commonly absent, the impulses passing backwards through the long ciliary nerves instead of partly through the ganglion; it is frequently fused with the sympathetic root; on the other hand, it may be multiple ; it may arise abnormally either from the semilunar ganglion, from the trunk of the ophthalmic nerve, or from the supra-orbital or lacrimal nerve. The sympathetic root frequently fuses with the sensory before entering the ganglion; it is so often multiple that some authors consider this to be the normal condition; some of these fibrils may accompany the third nerve. Additional roots of the ganglion may be provided by the superior division of the third, fourth, sixth, or lacrimal nerves, or by the spheno-palatine ganglion. As regards the ganglion itself, it may be very small or even absent, possibly due to the scattering of its constituent neurones among the nerves (Quain), or there may be two or more accessory ganglia; it has been seen perforated by a ciliary artery. 
The short ciliary nerves, or efferent branches (nervi ciliares breves), though very variable in number, usually consist of five or six fine filaments, which leave the anterior corners of the ganglion; they subdivide to form from twelve to twenty fibres, and pass forwards between the lobules of the orbital fat above and below the optic nerve, the lower group being the more numerous; they mix with, and a few are united to, the long ciliary nerves. They pierce the eyeball around the optic nerve entrance along with the short posterior ciliary arteries, run forwards between the sclera and the chorioid, to which they give filaments, and end in a rich plexus round the ciliary body (Fig. I59, p. 304). This plexus supplies the cornea, ciliary muscle and iris with sensory, motor and sympathetic fibres respectively, the origin and course of which are illustrated below in Fig. I79.

All the short ciliary nerves are mixed, and all, save two or three, contain pupillo-constrictor fibres. The motor fibres supply the intrinsic muscles of the globe, that is, the ciliary muscle or muscle of accommodation of the lens, and the sphincter pupillae or circular muscle of the iris; the fibres are the axons of cells situated in the ciliary ganglion, and transmit the impulses received from the oculomotor nerve, which are consequently interrupted here. The sensory fibres of the ganglion receive impulses from all parts of the globe, but chiefly from the anterior epithelium of the cornea; they are uninterrupted in the ganglion, and pass backwards by its sensory root into the naso-ciliary nerve. Since this nerve also receives sensory fibres from the globe through its long ciliary branches, it thus receives all the sensory impulses from the globe; there is consequently an intimate physiological relation between the skin and mucous membrane areas to which the rest of this nerve is distributed and the sensitive forepart of the eyeball. The sympathetic fibres end in the walls of the blood-vessels of the eyeball, but also provide pupillo-dilator fibres to the iris.

Besides these short ciliary nerves of the globe, the ganglion also supplies a few filaments to the sheath of the 
optic nerve, and others appear to be lost in the orbital fat near the globe.

The ciliary ganglion has been supposed to play a direct rôle in the formation of the aqueous humour, and to become of importance in glaucoma, but this view is now proved untenable. Landolt found that it contained no excitoglandular filaments, and it neither plays any part in the production of, nor does its ablation prevent the formation of the aqueous; Chimanowski showed also that the ganglion bears no relation to the intra-ocular tension. It may be added that Schweinitz (I9O3) states that experimental lesions of the ganglion do not cause neuro-paralytic keratitis, and its extirpation does not form trophic lesions, contrary to the results of resection of the cervical sympathetic. Finally, it is to be noted that the nerve plexus in the iris appears to be able to act independently of the ciliary ganglion, and is of the same nature as such post-ganglionic plexuses of the sympathetic system as those of Auerbach and Meissner in the intestines, since it has been found to persist after complete separation of the iris from the central nervous system by removal of the ciliary ganglion and superior sympathetic ganglion in the neck, a fact that explains the contraction of the pupil on exposure to light, as found by Brown-Séquard, and its dilatation by atrophine, as shown by de Rupter, in the excised eyeball (Pollock, rgI3).

The probable origin and course of the sympathetic nerve supply to the eyeball is illustrated in Fig. I79. Briefly, it is first to be noted that excitation of certain cortical regions on the brain surface of animals (for example, in the occipital region or in the sensori-motor area) cause dilatation of the pupil, accompanied by all the symptoms of excitation of the sympathetic cord in the neck, and Schäfer has found that constriction of the pupil can be obtained by stimulation of the quadrate lobe in monkeys, though these results may be associated effects, and do not necessarily imply cortical "centres" for pupillary movements (Parsons, I904). Then, the pupillo-dilator fibres are considered to arise from a supposed centre situated in the mid-brain, near the origin 
of the constrictor tract in the forepart of the oculomotor

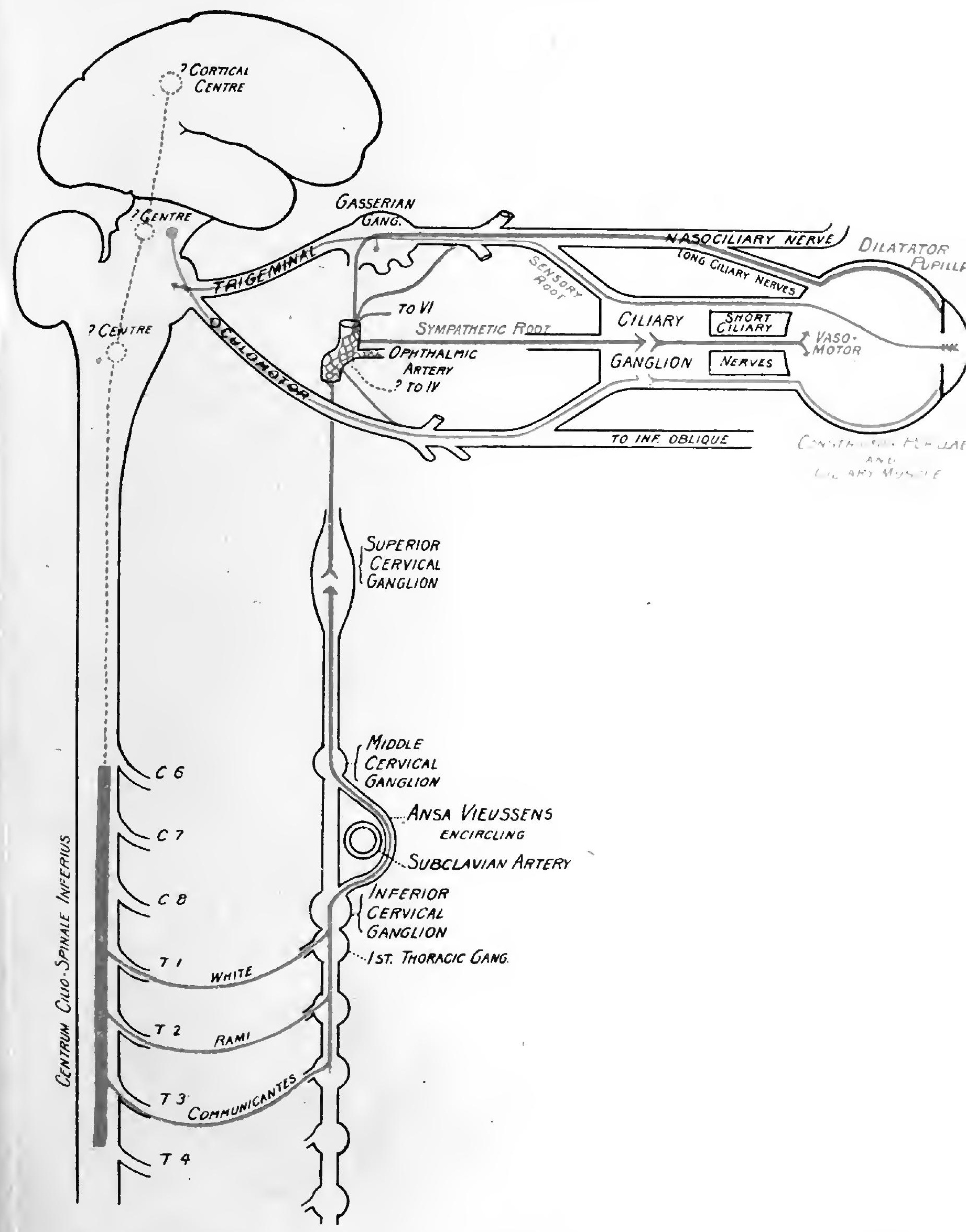

FIG. I79. - Diagram of the Ciliary Ganglion with its connections, and the origin and course of the pupillo-dilator sympathetic nerve fibres (in red). For simplification, sensory fibres, though present, are not drawn in the long ciliary nerves. For description see text.

nucleus, whence they pass through the medulla into the 
lateral columns of the spinal cord; and further, Dupuy has shown that puncture of the restiform body (inferior cerebellar peduncle) in the medulla produces exactly opposite effects to destruction of the sympathetic cord in the neck. There would therefore appear to be evidence of cerebral centres which may influence the sympathetic nerve supply to the orbit.

The first place, however, from which the course of the sympathetic fibres can more definitely be traced is the spinal cord at the root of the neck, there being strong clinical and experimental evidence for the assumption of a spinal centre (the centrum ciliospinale inferius of Budge) situated between the levels of exit of the sixth cervical and fourth thoracic nerves. The pupillo-dilator fibres have been traced in animals to leave the spinal cord by the ventral roots of the first three thoracic nerves (Langley), and to pass from them by the white rami communicantes into the first three thoracic ganglia (or first thoracic only) of the sympathetic cord. The fibres then pass up through the inferior and middle cervical ganglia to reach the superior ganglion, round the cells of which they terminate; this is their first interruption since leaving the spinal cord, and, according to Parsons, the only one in their passage to the eyeball. From the cells of the superior cervical ganglion fresh fibres carry the impulses by its superior, ascending, or carotid branch or branches (nervus caroticus internus) which accompany the artery through the carotid canal into the skull. Here the nerve breaks up into a plexus of fine nerve fibres, closely adherent to the vessel, and subdivided into a lower carotid plexus (plexus caroticus internus), which lies on the lateral side of the artery near the apex of the petrous bone, and the upper cavernous plexus (plexus cavernosus), lying medial and inferior to the artery as it lies in the cavernous sinus.

Numerous fine branches arise from these sympathetic plexuses, and their terminal filaments, continued along the branches of the artery, are connected around the anterior communicating artery with the corresponding system on the opposite side. The branches which enter the orbit 
nearly all arise from the cavernous plexus, and proceed as follows :

(i.) To the third nerve, at its point of division (recognisable).

(ii.) To the fourth nerve (doubtful).

(iii.) To the sixth nerve as it crosses the internal carotid artery in the cavernous sinus (from the carotid plexus).

(iv.) To the semilunar ganglion and also to the ophthalmic division of the fifth nerve (recognisable on its under surface).

(v.) To the ciliary ganglion, as its sympathetic root from the forepart of the cavernous plexus, entering the orbit through the superior orbital fissure.

(vi.) Vascular branches as a very fine plexus are continued along the ophthalmic artery and each of its twigs (derived from both plexuses).

These sympathetic fibres control in the orbit the dilatator pupillae muscle fibres of the iris, the vaso-constrictor mechanism of the blood-vessels of the orbit and eyeball, and the palpebral involuntary muscles, and provide trophic fibres to all the structures. The lacrimal gland, though affected by experimental excitation of the sympathetic, which causes secretion of tears, probably also receives secretory fibres from the seventh nerve (see p. 2I4). Accommodation of the lens is probably unaffected by the action of the sympathetic, and it has not been shown that the latter can provoke a negative accommodation.

As regards the pupillo-dilator fibres, it is first to be noted that the dilatation of the pupil is accompanied by contraction of the radial muscle fibres (the existence of which is now definitely proved) on the one hand, and by inhibition of the sphincter fibres on the other, the reverse of what happens when contraction of the pupil occurs; and its movements are independent of the state of its bloodvessels. There is a difference of opinion as to whether the dilator fibres reach their destination by passing (a) along the sympathetic root of the ciliary ganglion and into the short ciliary nerves (Merkel, Poirier, Piersol, Pegler), or (b) along the sympathetic branch to the semilunar ganglion, and thence into the naso-ciliary branch of the ophthalmic 
nerve, to reach the globe by the long ciliary nerves, a path which avoids the ciliary ganglion (Testut, Parsons, Schweinitz, Gray, Weeks, Halliburton). Since excitation of either the semilunar ganglion or of the long ciliary nerves causes a dilatation of the pupil, the latter path appears the chief one, a point that Testut is emphatic upon. Starling (I920) states that they travel by both routes; the short ciliary nerves contain both constrictor (oculomotor) and dilator (sympathetic) fibres, and when they are stimulated electrically both the sphincter and the radial muscles of the iris contract, but the sphincter, being the more powerful, will overcome the other, and therefore the pupil will contract. Cutting the sympathetic anywhere causes contraction, and section of the oculomotor nerve produces dilatation of the pupil. The mechanism of the "light-reflex" is described on p. 385 .

The Optic Nerve (nervus opticus), the second cranial nerve, and the special nerve of sight, extends from the eyeball to the optic chiasma, and is a part only of the visual tract. The great majority of its fibres originate as the axis cylinder processes of the ganglion cells which comprise the third or inner fundamental layer of the retina. Passing backwards from the eyeball through the orbit and optic canal to the optic chiasma (commissure), where part of them decussate, these fibres are carried on without interruption under the name of the optic tract to certain ganglionic masses of the mid-brain known as the primary or lower visual centres, namely, the lateral geniculate body, the pulvinar of the optic thalamus, and the superior colliculus or quadrigeminal body. Here the great majority of the retinal fibres terminate, but fresh fibres of cells in these bodies form a tract, the optic radiation, which transmits the visual impressions to the cortex of the occipital lobe of the brain, which constitutes the higher visual centre. The first half of the pathway lies outside the brain, the second part is deeply buried within its substance. These several parts of the whole visual tract from retina to cortex are represented diagrammatically in the figure opposite. 
The so-called optic nerve, therefore, is only a part of a continuous tract of fibres extending from the eyeball to the mid-brain. Moreover, since the retinal cells from which

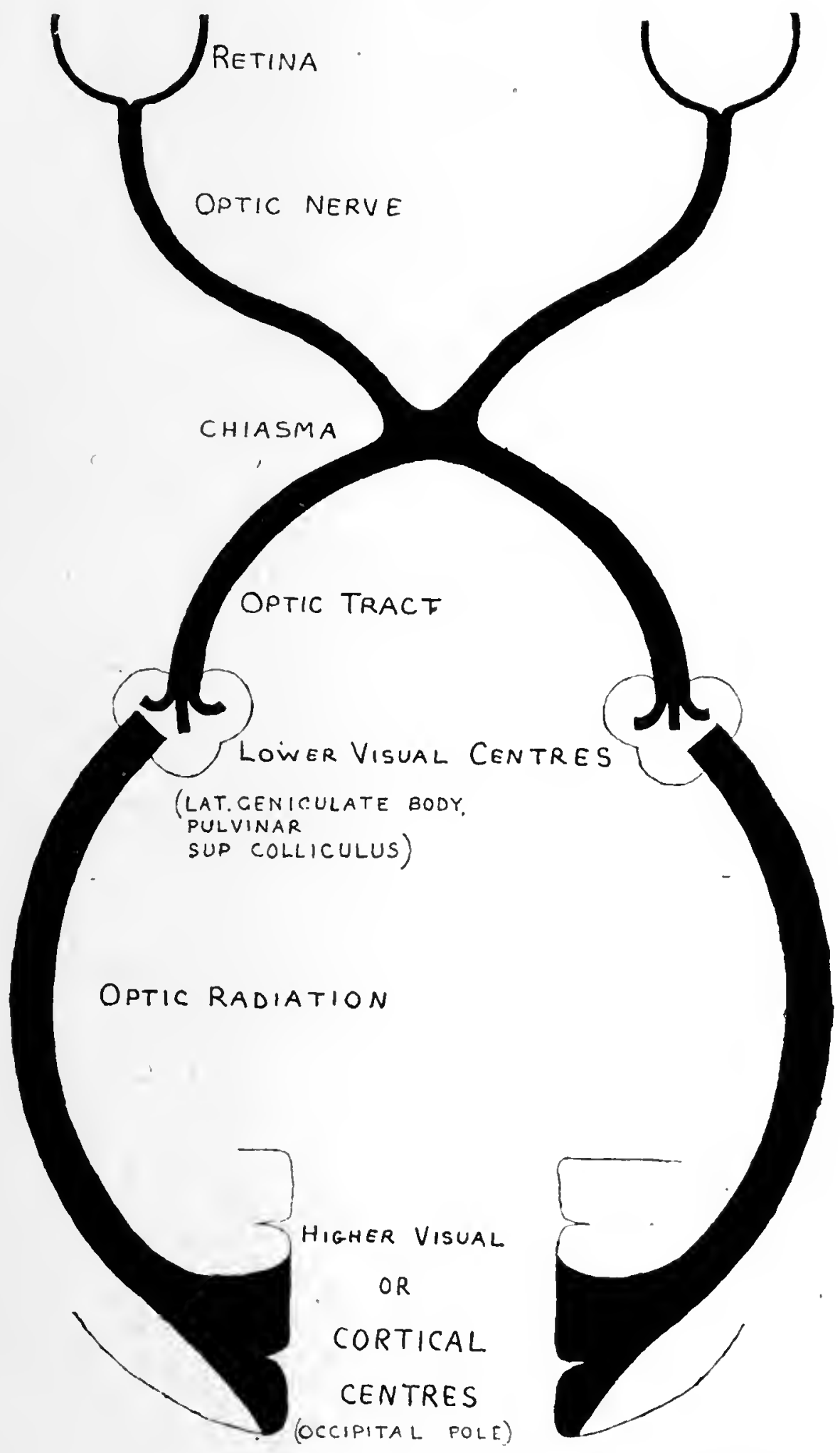

FIG. I80. - Scheme to represent the main parts of the visual pathways.

these fibres arise are not the primary but the tertiary elements of the visual tract (the visual impressions having previously traversed two sets of neurones, those of the rods and cones and those of the bipolar cell layer), and as is 
shown further by its development as an outgrowth from the brain, and by its structure and enveloping membranes, the optic nerve is not homologous with the other cerebrospinal nerves, but is really a part of the central nervous system, and corresponds to an outlying tract of brain fibres.

It is first formed by the development of the optic vesicle, which is an outgrowth from the ventral surface of the forebrain; the vesicle becomes collapsed by the invagination of its distal wall and forms the optic cup, into which the lens, an ectodermal derivative, is received, whilst the surrounding mesoderm gives rise to the coats of the eyeball. The narrow tube connecting the vesicle to the brain is

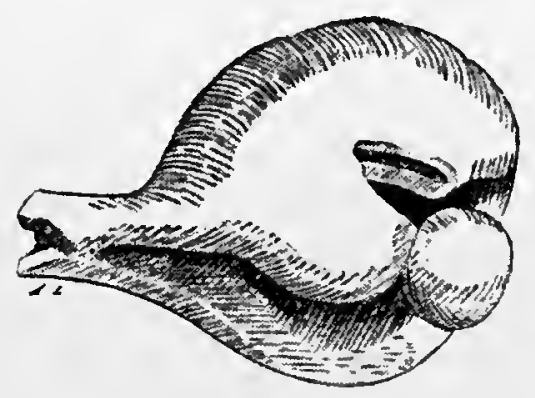

FIG. I81. - Model to illustrate the formation of the Second. ary Optic Vesicle and the fissure in the Optic Stalk. (From Druault, I912.) called the optic stalk, which later becomes solid. The outer layer of the cup forms the hexagonal pigment cells of the retina, and the inner layer develops into the retina proper. The central processes of the neuroblasts of the latter extend backwards along the optic stalk and form the optic nerve, chiasma, and tract. The invagination is on the infero-medial side of the vesicle rather than in front, and the infolding also affects the adjacent part of the stalk, so that the whole formation may be likened to a spoon with a deep bowl and a groove extending down the handle. In this groove anteriorly the central vessels of the retina become enclosed, and as a developmental error the optic disc, or the nerve and its sheath, may present a cleft or coloboma at the side of the infolding. The development of the nerve has been specially studied by Seefelder (I908-9-Io), and good accounts are also given by Druault (IgI2), Keibel and Mall (I9I2), and by Nussbaum (I9I2). The development of the medullary sheaths is given by Sättler (I9I5), who confirms Bernheimer's observation that they develop from the chiasma towards the eyeball, and at birth have almost reached it.

Structurally, the optic nerve is constituted by 800 to r200 fasciculi of myelinated fibres, of which there are nearly 
half a million, divisible into two classes, fine and coarse, the latter being much more numerous (Salzer, Gudden); they have been found to increase in number in growing animals as age advances (Boughton, I906). The fibres, unlike those of the cerebro-spinal nerves, and like those of the brain tracts, are denude of a sheath of Schwann, and also present similarity to the latter in being supported by neuroglia, the disposition of which has been specially studied by Jacoby (I905). It is also not uncommon to

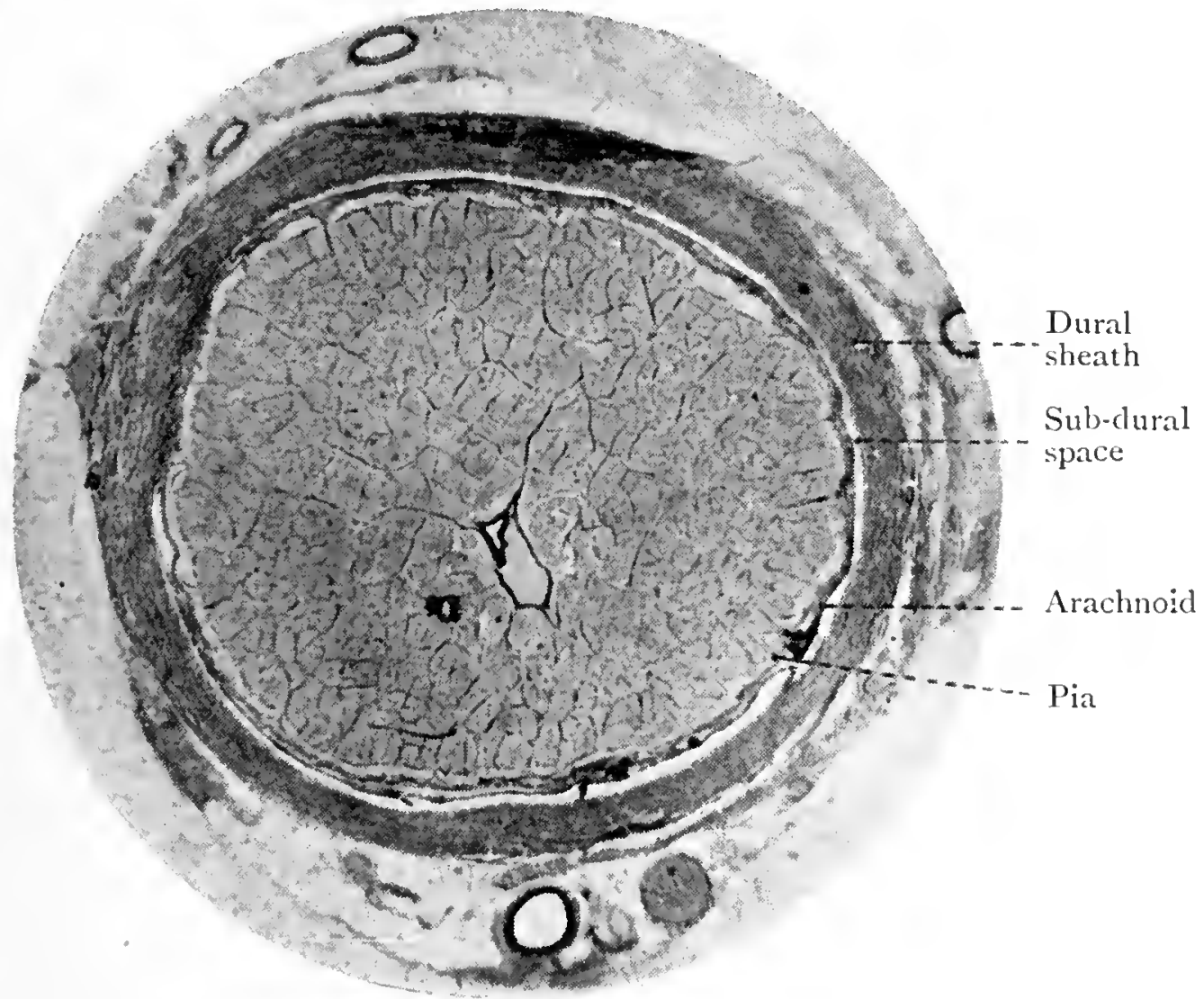

FIG. I82. - Cross section through the Optic Nerve and its Sheaths. The central vein is seen to the right and above the central artery of the retina. $\times$ ro.

find corpora amylacea present (Quain). The fasciculi into which the fibres are collected are separated by connective tissue containing many elastic fibres, and by lymph spaces. Ogawa. (I905-6) considers that the pigment cells found in the nerve in the region of the lamina cribrosa and sometimes seen with the ophthalmoscope on the optic papilla are hereditary anomalies, being correlated with the tint of the skin.

The nerve is pierced on its lower and medial aspect some Io or I $5 \mathrm{~mm}$. behind the eyeball by the central vessels of the retina ( $\mathrm{p}$. 303) accompanied by a fine sympathetic plexus, 
sometimes called the nerve of Tiedemann; the vein usually runs lateral to the artery, and they are enveloped by separate pial sheaths; their position as they run in the centre of the nerve is seen in the above figure. The central artery supplies both its anterior and posterior parts whilst in the orbit, where blood is also conveyed to it by ciliary twigs, and in the cranium it receives arterioles from the anterior cerebral artery. 'Its venules are tributaries of the central vein of the retina and the ciliary veins, and of the cranial veins posteriorly.

The nerve is enveloped by sheaths from each of the three meninges of the brain; the pia mater intimately clothes it throughout its whole extent (though separated from the nerve fibres at intervals by a layer of neuroglia), and sends prolongations into its substance which are continuous with the septa between the fasciculi; the arachnoid membrane comes into contact with it about half-way along its intra-cranial course as it emerges from the cisterna basilis; the dura mater, which is continued as a lining to the optic canal, splits, as previously described, at the orbital opening of this channel to become continuous with the periorbita on the one hand and to form a stout and somewhat loose sheath for the nerve in the orbital part of its course on the other (Figss. I38, I70, I85). All three membranes are continued along the nerve up to the eyeball, where they fuse with the sclera, though part of the pia becomes continuous with the chorioid coat of the nerve.

Similarly, the sub-dural and sub-arachnoid spaces of the brain between the membranes are continued along the nerve, and injections in them can be pushed up to the sclera, where they end, as seen in the next figure; they are lined by endothelium. It is stated that with pressure a subarachnoid injection will pass between the fasciculi and enter their lymph spaces, indicating the probable path of elimination of the lymph or tissue fluid in the reverse direction; the injection can even-be made to enter the peri-chorioidal space of the eyeball (Poirier), but, on the other hand, Behr (I9I2) found no elimination of fluids from the globe into the nerve. It is to be noted that since the 
arachnoidea round the nerve is very thin (and, indeed, said to be incomplete) and more closely united to the pia than elsewhere, the sub-arachnoid space is very much reduced and not easy to identify. The sub-dural space, on the contrary, is obvious in any section of the nerve with its sheaths (Figs. I82, I83, I85); it differs from that round the brain in being traversed at intervals by connectivetissue strands.

The nerve is divided, according to its anatomical relations,

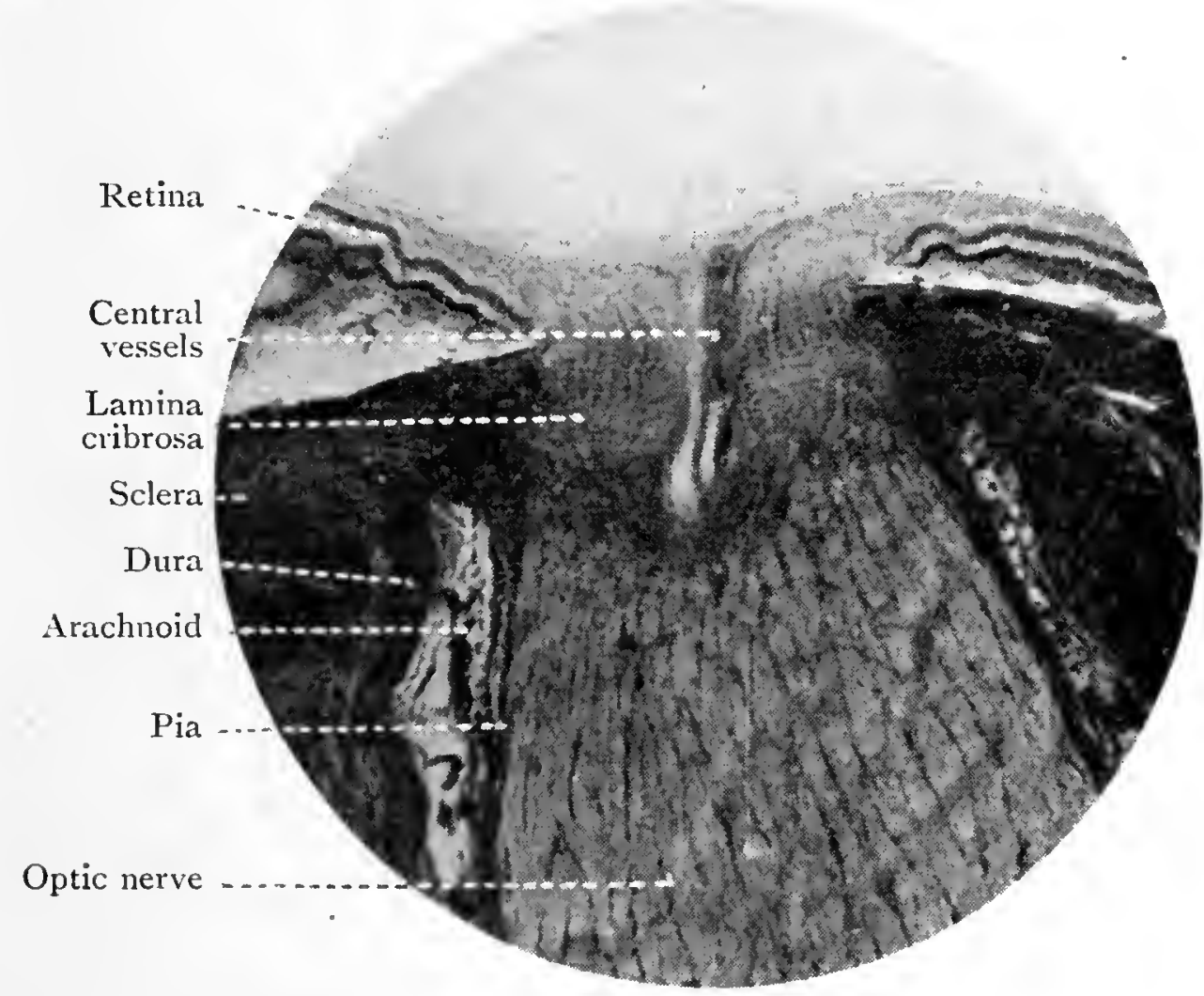

FIG. 183.-Longitudinal section through the Optic Nerve at its point of emergence from the Eyeball, and passing through the Optic Disc. $\times I_{3}$.

into four parts, since it traverses successively the sclera, the orbit, the optic canal, and the cranial cavity. The total length is about 45 to $50 \mathrm{~mm}$., or 2 inches, made up by a scleral portion of nearly I mm., an orbital part of 20 to $30 \mathrm{~mm}$., an intra-osseous length of 4 to Io $\mathrm{mm}$., and an intra-cranial part of Io $\mathrm{mm}$., varying from 4 to $\mathrm{I} 6 \mathrm{~mm}$., or, roughly, $\mathrm{I}$, 25. Io, Io $\mathrm{mm}$. respectively; the two nerves are seldom of equal length. Its diameter in the orbit is 3 or $4 \mathrm{~mm}$., and within the skull varies from 4 to $7 \mathrm{~mm}$., but is commonly $4.5 \mathrm{~mm}$. in cross diameter.

(i.) The scleral or intra-bulbar part is composed of the 
nerve fibres as they collect together at the optic disc and

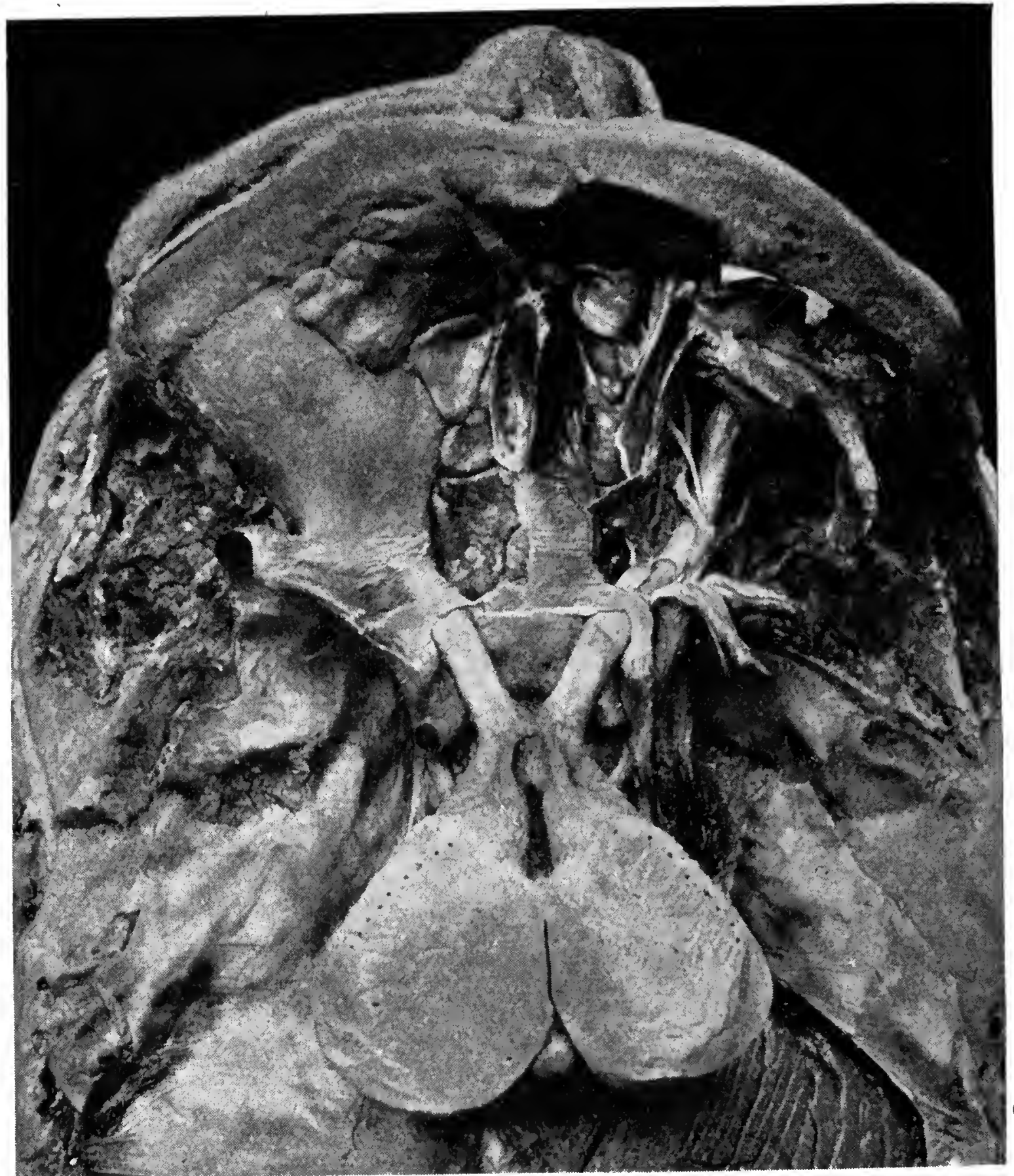

Fig. I84. - Dissection of Ilead of Adult Mlale Subject to show the Orbits from above. The bone has been removed from above thelleft orbit, leaving the periorbita intact and showing its connection with the dura mater lining the nidckle cranial fossa; the muco-periosteal lining of the frontal and ethmoidal air-cells has also been exposed. On the right side the orbit has been dissected to show the optic nerve crossed by the naso-ciliary nerve and ophthalnic artery. Note the position of the chiasma relative to the sphenoidal bone and sella turcica. The course of the optic tracts, indicated by the dots, can be traced round the cut crura cerebri to the grey masses of the lateral geniculate body and pulvinar. The distance between the optic nerve at its entrance into the eyeball and the lateral orbital margin is $30 \mathrm{~mm}$.; between the optic foramen and zygoma (the edge of which is bared on the left) is $55 \mathrm{~mm}$.; and between the centre of the chiasma and the zygoma is $70 \mathrm{~mm}$.

There is an abnormal clouble superior oblique muscle in this specimen. The same preparation is viewed from the side in Fig. I33. $\times \frac{4}{5}$.

pierce the chorioid and the lamina cribrosa of the sclera; 
the nerve here is only $1.5 \mathrm{~mm}$. in diameter, since the fibres do not become myelinated or supported by neuroglia until they have passed out of the globe (Fig. I83).

(ii.) The intra-orbital part of the nerve extends from the eyeball to the anterior optic foramen, and emerges from the former I $\mathrm{mm}$. below and $3 \mathrm{~mm}$. to the nasal side of its posterior pole (Fig. I32, p. 255) ; whilst the course of the nerve roughly corresponds to the long axis of the orbit, it is not straight, but describes two variable curves, usually bending downwards in its forepart and laterally in its hinder half, as seen in Fig. I84; the nerve is therefore a little longer than the distance between the eyeball and the optic foramen, and Weiss in sixty subjects found its length to average $24 \mathrm{~mm}$. (ranging between 20 and $30 \mathrm{~mm}$.), whilst the distance between the globe and the foramen was I8 $\mathrm{mm}$. (I4 to 24) ; this surplus or slack of 5 or $6 \mathrm{~mm}$. is associated with the mobility of the globe, being, like the curves, greater in animals with very mobile eyes, such as the chameleon, and less in exophthalmos. In extreme movements of the eyeball the nerve becomes nearly straight, and indeed it can be stretched enough in certain conditions to affect the optic disc and form a " conus."

The relations of the nerve in the orbit are as follows. It is surrounded by the fascia bulbi as it leaves the globe, though this is the weakest and most ill-defined part of the membrane. The posterior ciliary arteries and nerves surround its anterior half and pierce the globe round it (Fig. I32, p. 255). The central vessels of the retina enter on its infero-medial aspect about half-way along its course. It is crossed superficially by the naso-ciliary nerve and also by the ophthalmic artery (Fig. I84), though in I $_{5}$ per cent of cases the vessel passes beneath it ; further back the artery lies on its lateral aspect. On this side also is found the inferior division of the third nerve and the ciliary ganglion. At the apex of the orbit it is closely surrounded by the recti muscles at their origin from the annulus of Zinn; the superior and medial recti arise partly from the sheath of the nerve, a fact that probably accounts for painful movements of these muscles in inflammation of the nerve, but the lateral 
rectus is separated from it by the third, naso-ciliary, and the sixth nerves. Its relative position in regard to the muscles is well displayed in Fig. I4I, p. 264, and also in Fig. I85, from which it will also be realised how completely it is surrounded by the orbital fat; the latter, it may be noted, extends furthest into the apex of the orbit below the nerve.

Lagrange.(I903), quoting Lange, gives the following

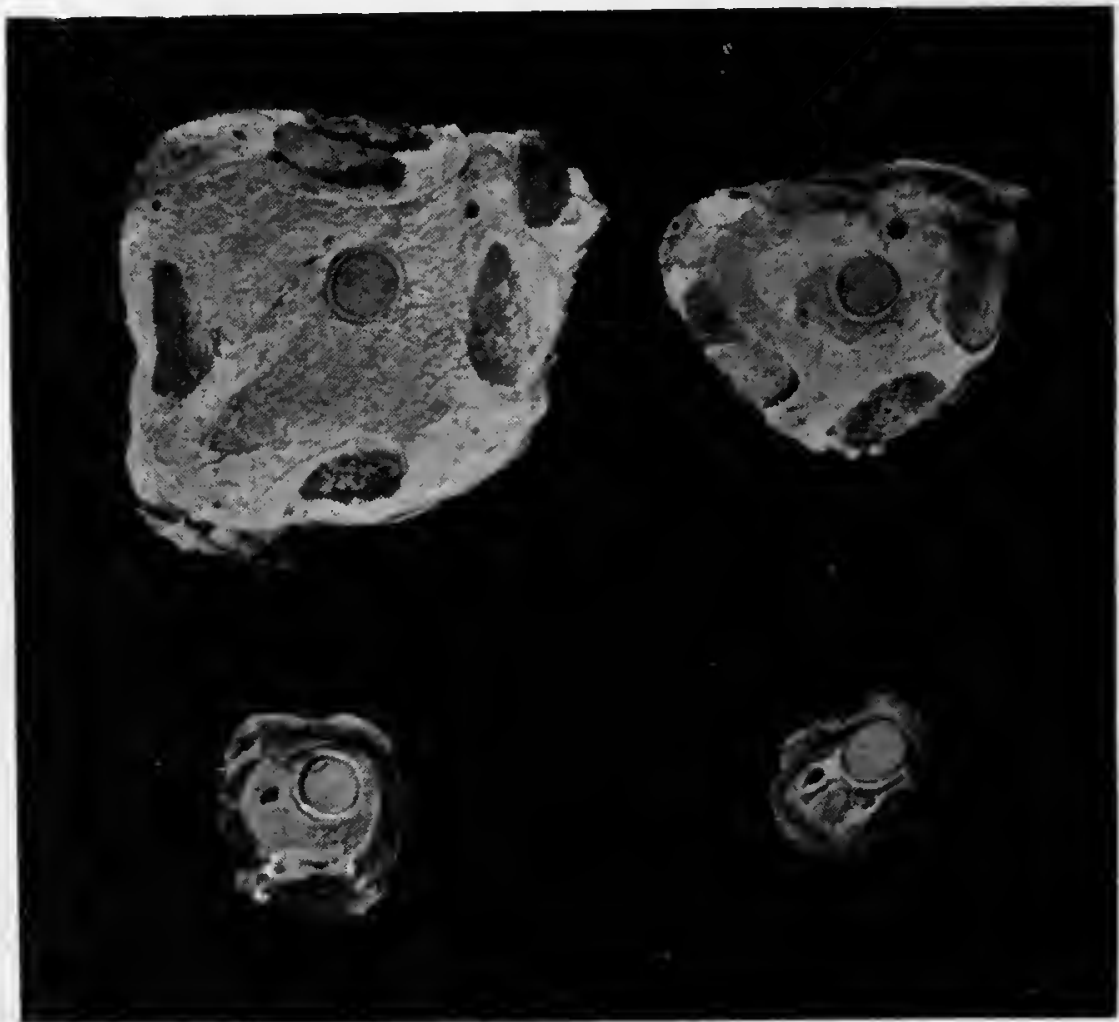

FIG. I 85. - Sections through the right orbital contents, seen from in front, after removal intact within the periorbita, to show the relations of the muscles to the optic nerve. 'The ophthalmic artery is also seen presenting different relations in its course forwards through the orbit. The sheath of the nerve is well shown.

The sections were made $5, x 5,20$, and $25 \mathrm{~mm}$. behind the eyeball. Natural size.

distances in millimetres which separate the nerve from the orbital walls at various points :

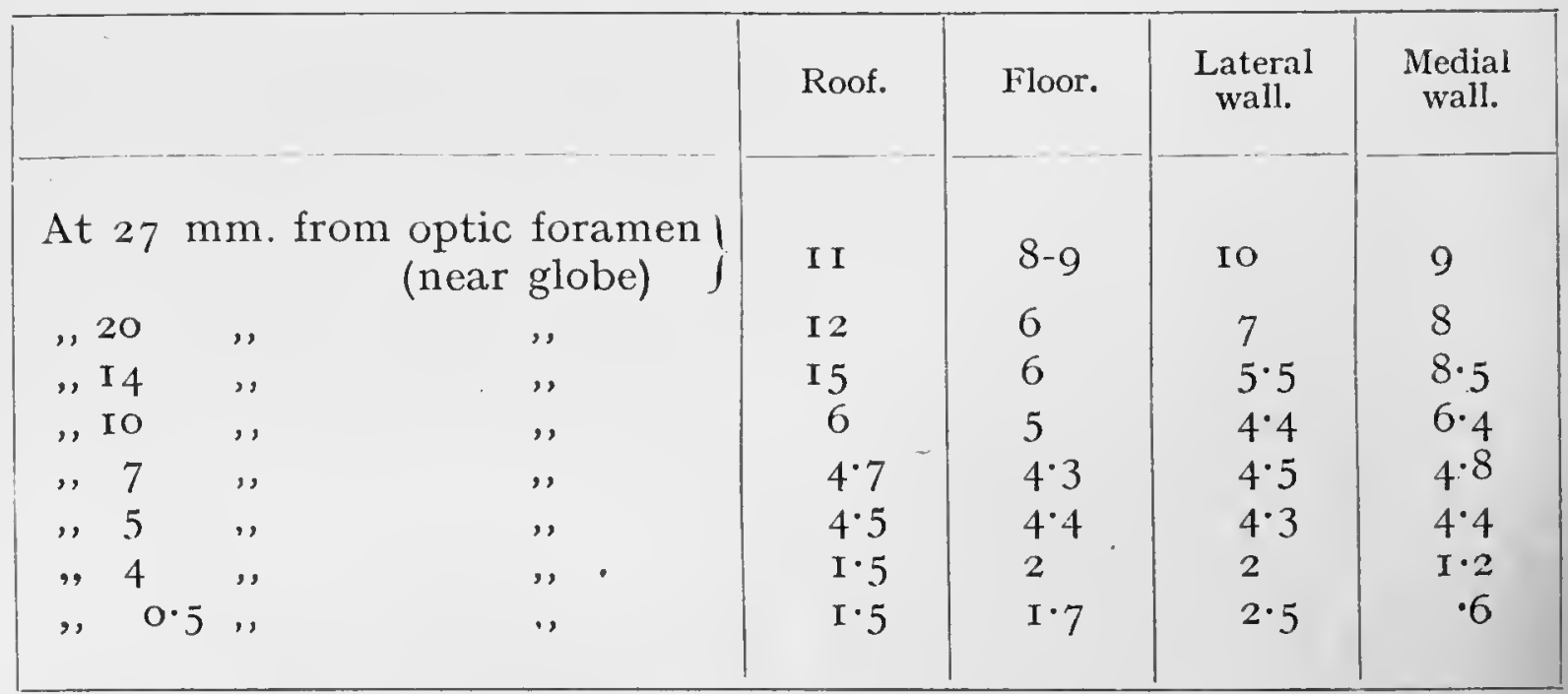


(iii.) The intra-osseous or canalicular part of the nerve, as it traverses the optic canal, has the ophthalmic artery embedded in its sheath first to the lateral side and then below it; by this sheath the nerve is firmly fixed to the walls of the canal, especially superiorly. 'The relations of the canal to the sphenoidal sinus have been described above on p. 27.

(iv.) The intra-cranial part of the nerve, from the posterior aperture of the optic canal to the chiasma, is flattened instead of being; as previously, round, measuring about $5 \mathrm{~mm}$. by $3 \mathrm{~mm}$. ; it is surrounded by pia mater and arachnoid, and above it lies a falciform fold of the dura mater prolonged a short distance behind the upper border of the canal. Superiorly, it is separated from the medial root of the olfactory tract and lateral part of the anterior perforated substance by the anterior cerebral artery; laterally lies the internal carotid artery (Figs. I72, I74), and infero-laterally the origin of the ophthalmic artery, the varying relations of which to the nerve have been mentioned above on p. $30 \mathrm{I}$; the sheath of the nerve is firmly connected to the internal carotid artery at the point of origin of the ophthalmic vessel, since the latter becomes embedded in the sheath (Fig. I64). Stopford (IgI7) notes the possible compression of the nerve by the internal carotid artery in disease. Beneath the nerve are the diaphragma sellae and the sphenoidal sinus in the body of the sphenoidal bone. The nerve does not lie exactly in the optic groove or sulcus chiasmatis on the upper aspect of this bone, but is placed lateral to it (vide infra).

The Optic Chiasma or commissure (chiasma opticum), formed by the junction of the two optic nerves, is transversely oblong in shape, but varies in size in different subjects. The transverse width in fifteen specimens has been found to average $12.8 \mathrm{~mm}$. (Gérard, I904), and in thirty-six specimens a mean of $13.29 \mathrm{~mm}$. was found, with extremes of from Io to $\mathrm{Ig} \mathrm{mm}$. ; the antero-posterior width in thirty-two cases varied from 4 to $\mathrm{I} 3 \mathrm{~mm}$., with an average of $8 \mathrm{~mm}$. (Zander, $\mathrm{I} 896$ ) ; it is from 3 to $5 \mathrm{~mm}$. thick. The dimensions 
in the specimen illustrated in Fig. I84 are: breadth, I3 mm.; antero-posterior width, II $\mathrm{mm}$.; thickness, $3 \mathrm{~mm}$.; that in Fig. I68 is $13 \mathrm{~mm}$. broad also. It is intimately clothed by the pia mater, and lies in the cisterna basalis of the subarachnoid space on the body of the sphenoidal bone. As regards the overlying brain, above, the chiasma is related to the third ventricle, in the anterior part of the floor of which it forms a prominence; behind it is the tuber cinereum, from which springs the infundibulum or stalk of the hypophysis cerebri (pituitary body); laterally is the anterior perforated substance (through which there pass the basal branches of the middle and anterior cerebral arteries) and, more anteriorly, the olfactory tubercle at the base of the olfactory tract. A more important relation is the internal carotid artery, which, ascending to the brain after piercing the roof of the cavernous sinus, is in close contact with the lateral side of the chiasma just before dividing into the anterior and middle cerebral vessels (Fig. I72); the two anterior cerebral arteries, united by the anterior communicating vessel, lie likewise in contact with the front of the chiasma, and aneurysms of these vessels could compress it against the cranial base (Mitchell and Bramwell). As regards its relations to the base of the skull, it is to be noted that the chiasma does not lie in the sulcus chiasmatis of the body of the sphenoidal bone, as is usually stated, but over the tuberculum sellae (olivary eminence) behind this groove, as in Fig. I69, or even further back still and over the diaphragma sellae, covering the hypophysis cerebri, as in Figs. I84, I86; the further back the chiasma lies the more acute will be the angle formed by the two optic nerves as they enter it. The exact position has been studied by Zander (I896), Lawrence (I894), who describes the development of the bony parts in this region, and Wallis (I9I7) from the clinical point of view. The last-named author examined eleven subjects, and in only one did the chiasma lie in the generally described position; he found it usually to lie over the diaphragma and partly on the dorsum sellae, as is seen in the figures last referred to; he concludes, in agreement with Lawrence and Zander, that 
whilst the chiasma does occasionally rest on the sulcus chiasmatis, it is nearly always completely posterior to this groove. Traquair (I9I6) considers it to lie above the posterior half of the fossa hypophyseos (sella turcica), and its posterior edge is on an average $\mathrm{I} \cdot 58 \mathrm{~mm}$. behind the dorsum sellae, and only exceptionally in front of this point

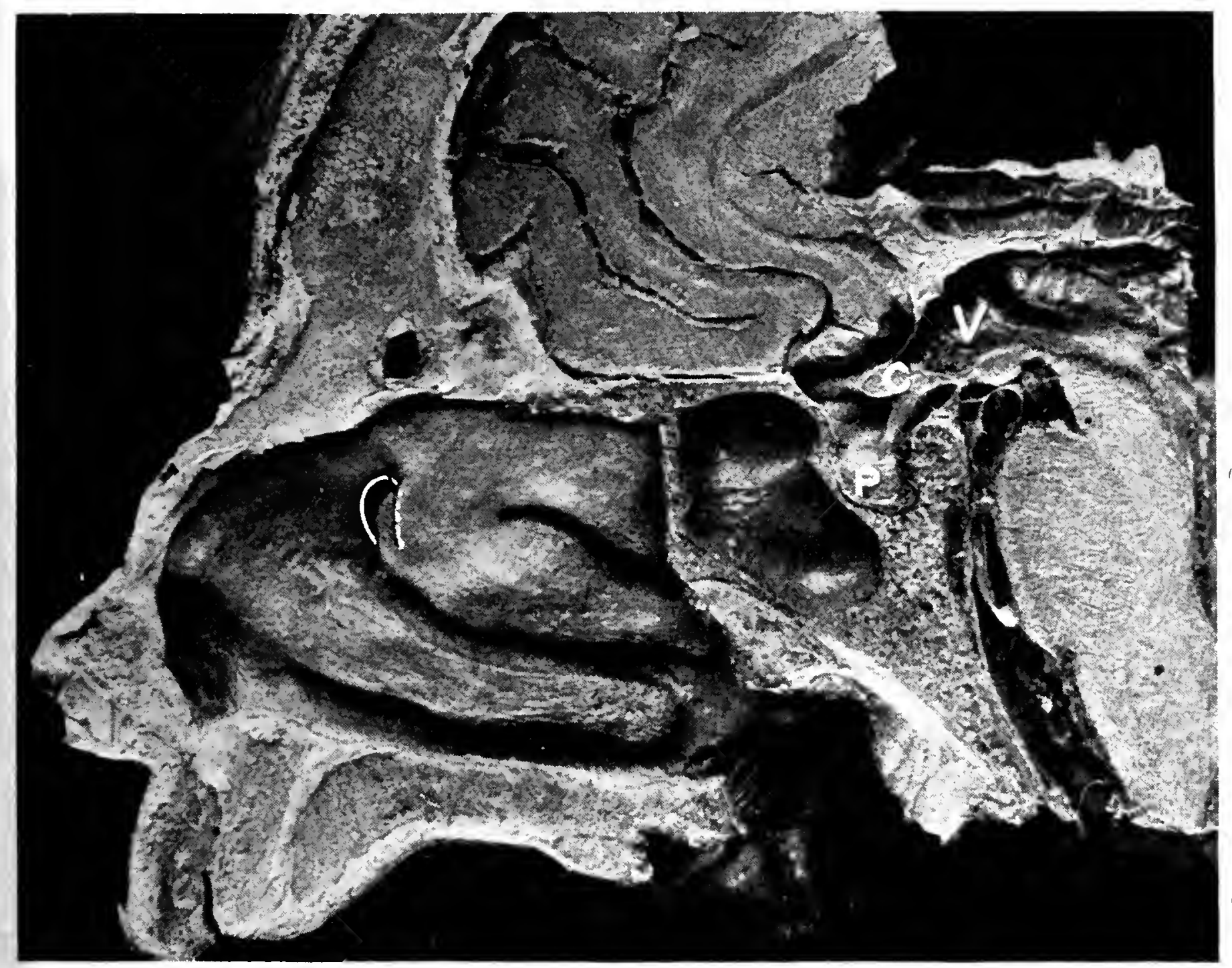

FIG. I86. - Half section of Head, adult male, showing right lateral nasal wall; the relative position of the fossa for the lacrimal sac is inclicated by the white outline. The position of the optic chiasma (C.), lying well above the hypophysis or pituitary body (P.) and bohind the sulcus chiasmatis, is seen, and the third ventricle (V.) is shown in its relation to both. $\times \frac{3}{4}$.

(Zander). Changes in the position of the sella turcica, it may be noted, have been found associated with hereditary optic atrophy (Fisher, I9I6; Taylor, I9I9). It is to be noted further that the chiasma does not lie in close contact with the diaphragma and the hypophysis, but is separated from them by a space, the cisterna basalis of the sub-arachnoidea; the interval is from $4 \mathrm{~mm}$. (Traquair) 
to $7.5 \mathrm{~mm}$. (Hirsch), or even Io $\mathrm{mm}$. (Cope, I9I6). In the specimen illustrated in Fig. I 86 the distance is $8 \mathrm{~mm}$.; in Fig. I33, p. 257, where the interval between the chiasma and the hypophysis is clearly seen, it measures $5 \mathrm{~mm}$.

The relation of the sphenoidal air-sinuses to the chiasma will naturally depend upon their degree of backward extension (see p. 62). They may extend only as far back as the posterior lip of the olivary eminence, and lie altogether in front of the fossa hypophyseos and the normally situated chiasma, but Cope (I9I7) found them more often to undermine the whole fossa, and they are often separated from it by only a thin plate of bone, as in Fig. I22, p. 233. Loeb (I909) found that the chiasma is nearly always related in front to both sphenoidal sinuses and never to the posterior ethmoidal cells. The relations of the chiasma to the third ventricle of the brain and to the pituitary body, of the optic nerve to the sulcus chiasmatis, and of the sphenoidal air sinus to all these structures are well shown in the above figure, in which preparation, it is to be noted, but little shrinkage and displacement of the parts have occurred.

The optic tract (tractus opticus), after leaving the chiasma, diverges from its fellow of the opposite side in front of the interpeduncular space, and sweeps .laterally and backwards between the tuber cinereum and the anterior perforated substance round the lateral surface of the crus cerebri, to which it is intimately applied, with the posterior cerebral artery, which supplies it, just below it ; it becomes flatter as it proceeds, and, reaching the postero-lateral aspect of the optic thalamus, becomes divided by a shallow furrow into two so-called roots. The medial root is the smaller, and appears to end in the medial geniculate body; as can be seen in Fig. I87, its fibres, though apparently forming part of the optic chiasma and tract, do not come from the optic nerve, but sweep across the back of the chiasma to the opposite side, forming Gudden's commissure (p. 383). The lateral root, containing all the fibres of retinal origin, can be traced to the lateral geniculate body, the pulvinar, 


\section{and the superior colliculus. The lateral geniculate body}

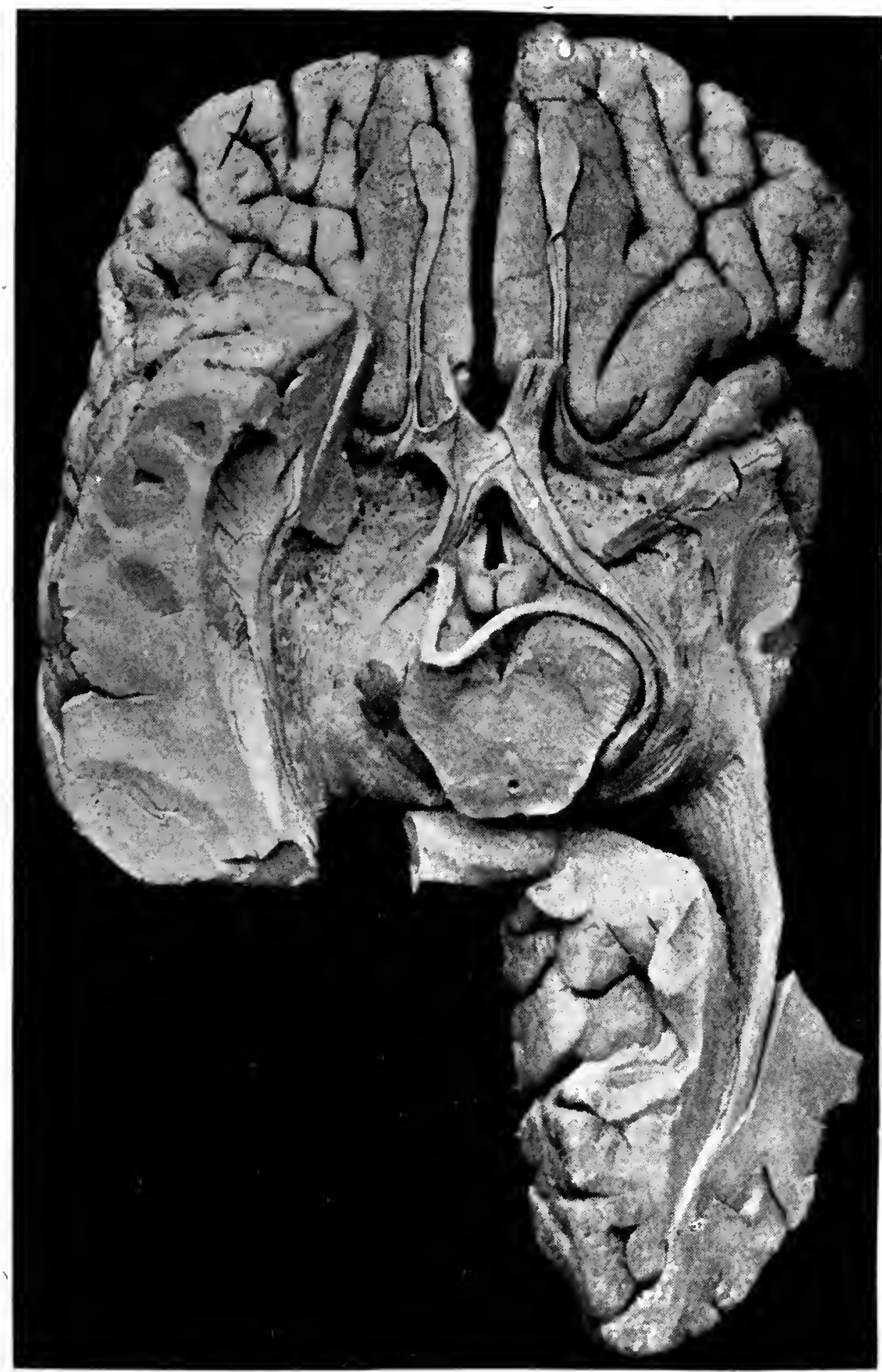

FIG. 187.-Dissection of Brain from inferior aspect to show the Optic Chiasma and Tracts, the Lower Visual Centres, and the Optic Radiation. A flap has been turned aside from the chiasma, and the crossed nasal fibres of the left optic nerve indicated. 'The commissure of Gudden has been dissected away from the back of the chiasma and the continuity of its fibres both here and in the optic tract demonstrated, as far as the medial geniculate body. Lateral and slightly anterior to this body is a swelling, the lateral geniculate body, into which the rest of the fibres of the tract are traceable, and-beyond it, to the pulvinar. From these centres the optic radiation is seen sweeping backwards to the occipital pole. $\times \frac{3}{4}$.

receives the largest number of fibres from the tract (80 per cent, according to Monakow); they partly sink into its 
interior (where most of them end in the grey matter between its lamellae, but some traverse it to enter the pulvinar) and partly spread out over its surface, and similarly either end in its substance or pass on to the pulvinar. Most of the fibres to the pulvinar, therefore, have passed either through or over the lateral geniculate body on their way to it. The fibres to the superior colliculus reach it directly through the superior brachium, which lies, however, in close contact with the lower aspect of the lateral geniculate body.

These bodies; which are known as the "lower visual centres," are situated on the postero-lateral aspect of the mid-brain (Figs. I87, I89). The lateral geniculate body is an oval mass on the lateral side of the thalamus, and is connected to the superior colliculus by a slender band, the superior brachium. The superior colliculus (anterior or superior quadrigeminal body) lies on the dorsal aspect of the mid-brain above the inferior colliculus and below the thalamus and the pineal body. The pulvinar (posterior tubercle of the thalamus) is the prominence on the posterior end of the thalamus. The blood supply is provided. by the posterior cerebral artery (postero-lateral set of basal branches).

The retinal fibres of the optic nerve, chiasma, and tracts have thus been traced from their origin in the retina to their termination in the lower visual centres of the midbrain. In the appendix to this work, which follows, the second part of the path taken by the visual impressions will be traced to its ultimate destination in the occipital cortex, and an analysis of the fibres of the whole tract given. It will add to the interest of this section on the nerves of the orbit if a brief account is also given of their nuclei of origin and their connections, but no attempt will be made here to cover this large and complicated field in detail; the bibliographies given in such papers as are referred to will enable the reader to find the literature bearing upon the subject. 


\section{PART IV.-APPENDIX}

\section{THE CEREBRAL CONNECTIONS OF THE NERVES}

THE Optic Radiation (radiatio occipito-thalamica of Gratiolet) is the second or intra-cerebral link in the pathway of the visual impressions from the retina to the perceptive centre in the cortex, and it extends from the lower visual centres of the mid-brain, runs through the central white matter of the posterior part of the cerebral hemisphere, and ends in the surface of the occipital pole. It consists of a fairly narrow $(3 \mathrm{~mm}$.) but deep band of fibres, which arise as processes of cells mainly of the lateral geniculate body, but also, according to most authors, from the pulvinar of the thalamus; they emerge from the lateral side of these bodies, and passing through the retro-lenticular or posterior part of the internal capsule, bend backwards to run along the lateral wall and round the posterior horn of the lateral ventricle, separated from it by the tapetum of the corpus callosum, to reach the cortex of the occipital pole; here they end in arborisations round the cells of the fifth or granule layer of the cortex in an area which constitutes the " higher visual centre."

The Cortical Visual Centre (higher visual "centre," or visuo-sensory area) is comprised in an extensive area of the cortex on the medial surface and pole of the occipital lobe, chiefly in the region of the cuneus (the wedge-shaped area of cortex lying between the parieto-occipital and calcarine fissures). The extent of this area, as deduced from clinical observations of cases of disease or injury and from experimental investigation, shows much variation and is difficult to define with exactness. The cortex in 
this region is characterised, however, by the presence of a white line in the grey matter, visible to the naked eye and first noticed by Gennari in 1776 , and the region is consequently named the area striata. The appearance, which is illustrated in the figure below, is caused by a thick granule layer of cells with a dense plexus of medullated fibres situated in the fifth cortical layer, wherein the fibres of the optic radiation have been traced to end. The

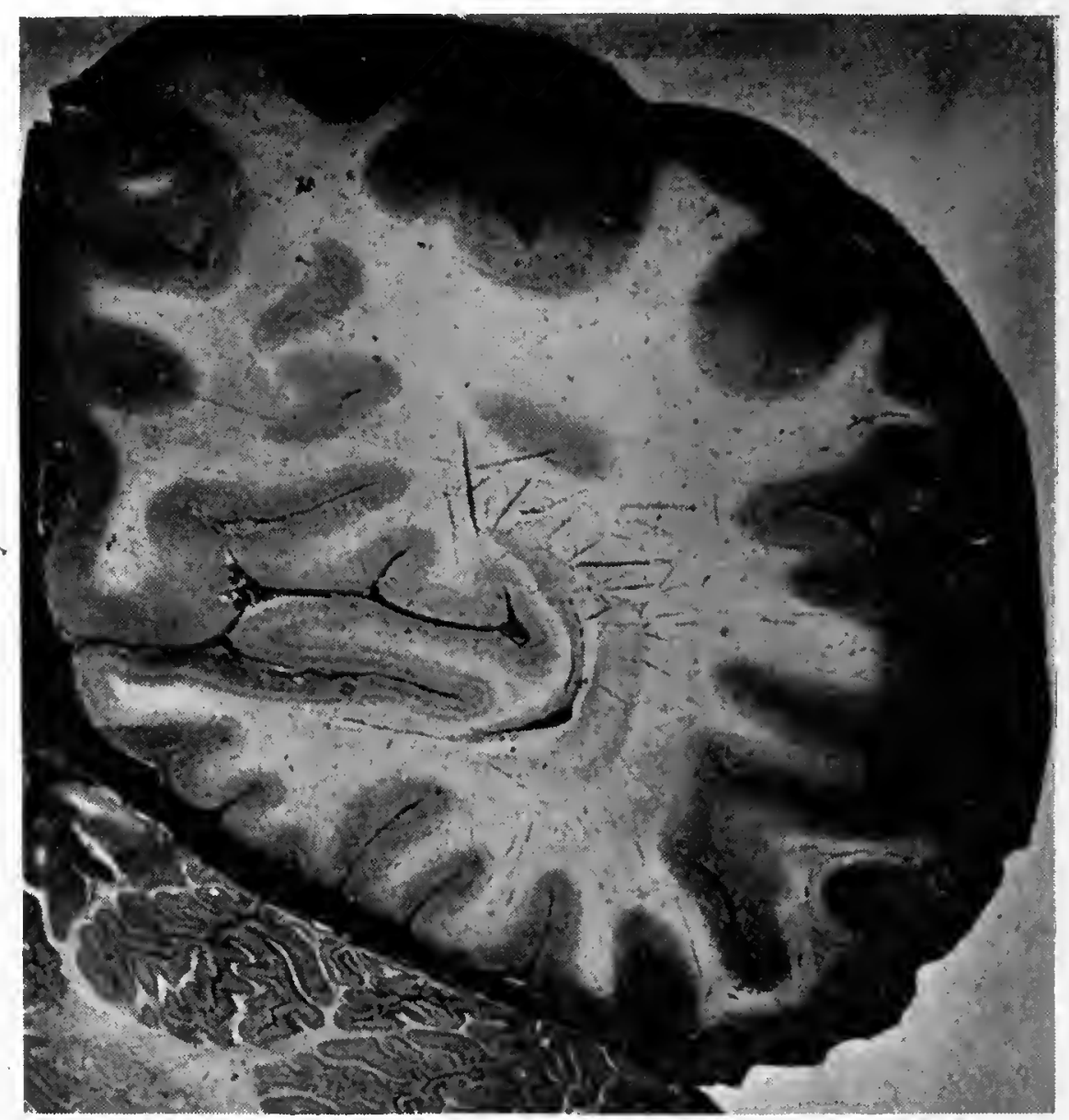

FIG. I88. - Transverse section through the Right Occipital Lobe of the Brain, seen from behind, to show the white line of Gennari in the cortex of the lower lip of the calcarine fissure (marked $\mathrm{C}$ in the figure opposite).

exact limits of the striate area have been mapped out by microscopical examination of this cell layer (Mott, Bolton), by the character of the nerve fibres (Campbell), by the period of myelinisation of the fibres (Flechsig), and by the naked eye differences in the cortex as seen in sections of fresh brains (Elliot Smith, I907). These various deliminations practically coincide, and the account of the region which follows is taken from the description of the author last named. 
If this area of cortex be excised and spread out flat, it will be found to present an elongated ovoid form, which covers about 3000 sq. mm. (varying from 2700 to 4000 in different brains). The narrow end of the ovoid lies just

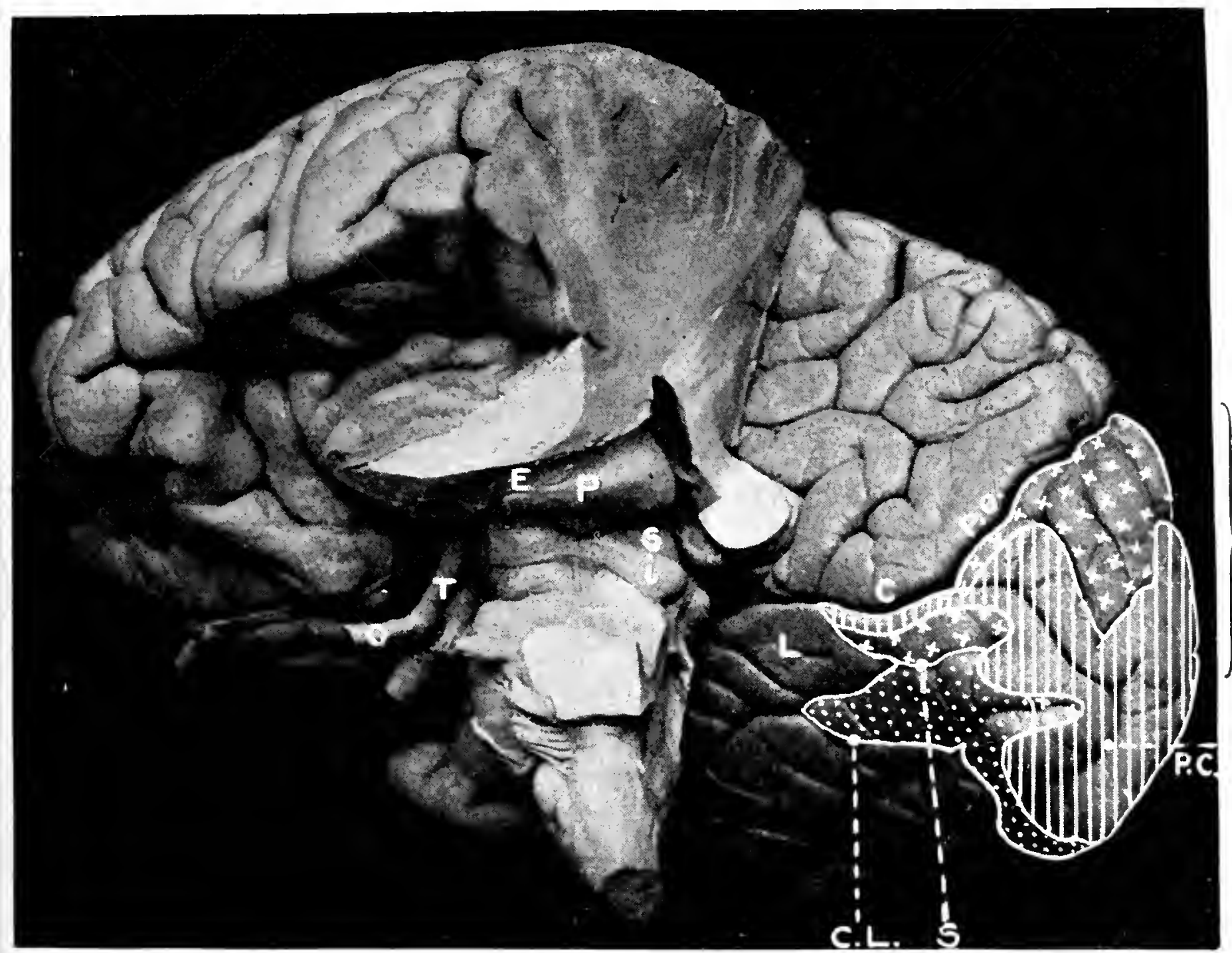

FIG. I89.-Dissection of Brain, viewed from left side and tilted over to show the cortical visual areas, according to the findings of Elliot Smith. Half of the left hemisphere has been cut away to expose the medial surface of the right occipital lobe. $\times$ about $\frac{2}{3}$.

The striate area is marked by vertical lines, the para-striate area by crosses, and the peristriate area by dots. C. =the calcarine sulcus; P.C. $=$ the terminal part of the posterior calcarine sulcus ; P.O. = the parieto-occipital sulcus $;$ C.L. =the collateral sulcus; S. = the sagittal sulcus of the gyrus lingualis (L.). The lower visual centres are also displayed: $\mathrm{O}_{.}=$the optic nerve; $\mathrm{T}_{0}=$ the optic tract, traceable into a small elevation just below $\mathrm{E}$., the lateral geniculate body, and P., the pulvinar. Below P. is the more conspicuous medial geniculate body. Below the hinder end of the pulvinar are seen the colliculi, superior (S.) and inferior (i.); the fourth nerve is seen below the latter. The fifth, sixth, seventh, eighth, ninth, tenth, and eleventh nerves are also seen on the side of the pons and medulla.

behind and below the splenium of the corpus callosum, and the rest of it extends backwards from this point to the occipital pole, and beyond it on to its lateral aspect. In the course of development this area becomes folded along its axis during the sixth month, and so forms the calcarine 
fissure or sulcus (of Huxley). By a fold which develops in the occipital lobe above it (the parieto-occipital sulcus), it is divided into two parts: an anterior, the calcarine fissure proper (sulcus calcarinus), and a posterior (sulcus calcarinus posterior); the area overlapping the lateral surface of the pole may also become folded into a sulcus calcarinus lateralis. The anterior part of the fissure is much deeper, more constant in form and position, and more precocious in development than the posterior part, and phylogenetically it is much older. There is also a fundamental structural difference between them, for the stria of Gennari is found only in the lower wall and lip of the anterior part of the fissure or sulcus calcarinus proper, as is seen in Fig. I88, whereas the striation extends throughout both walls of the posterior calcarine sulcus, and in most cases beyond its lips on to the surface of the cuneus and the gyrus lingualis, that is, over both lips of the fissure. The upper and lower boundaries of the area are often demarked from the adjacent cortex by shallow limiting sulci.

The area striata is held to be the visuo-sensory area or the perceptive "centre" for the retinal impressions, since its destruction entails blindness of parts of each eye. It should, however, be realised that no cortical area can properly be described as an exclusive centre of a particular function, but such "centres" are merely nodal points in an exceedingly complex system of neurones which must act as a whole to perform any function whatsoever (Herrick, I9I8).

This area is surrounded by two peripheral concentric bands of cortex, which again present structural differences, though not so well marked: an inner, called the area para-striata, and an outer, the area peri-striata, which extend considerably on to the lateral aspect of the occipital lobe. These areas are doubtless visuo-psychic or visual memory "centres," since injury to this part of the cortex entails " mind blindness" : the objects are seen, but convey nothing to the patient's mind.

The whole visual area in the occipital lobe appears divisible, therefore, into two parts: an inner, the striate 
area, closely following the calcarine fissure and serving for the reception of primary visual impulses, and an outer, the para- and peri-striate areas, destined for the further elaboration and storing of the visual sensory impressions. Further subdivision of these adjacent regions into areas specially concerned with appreciation of colour, form,

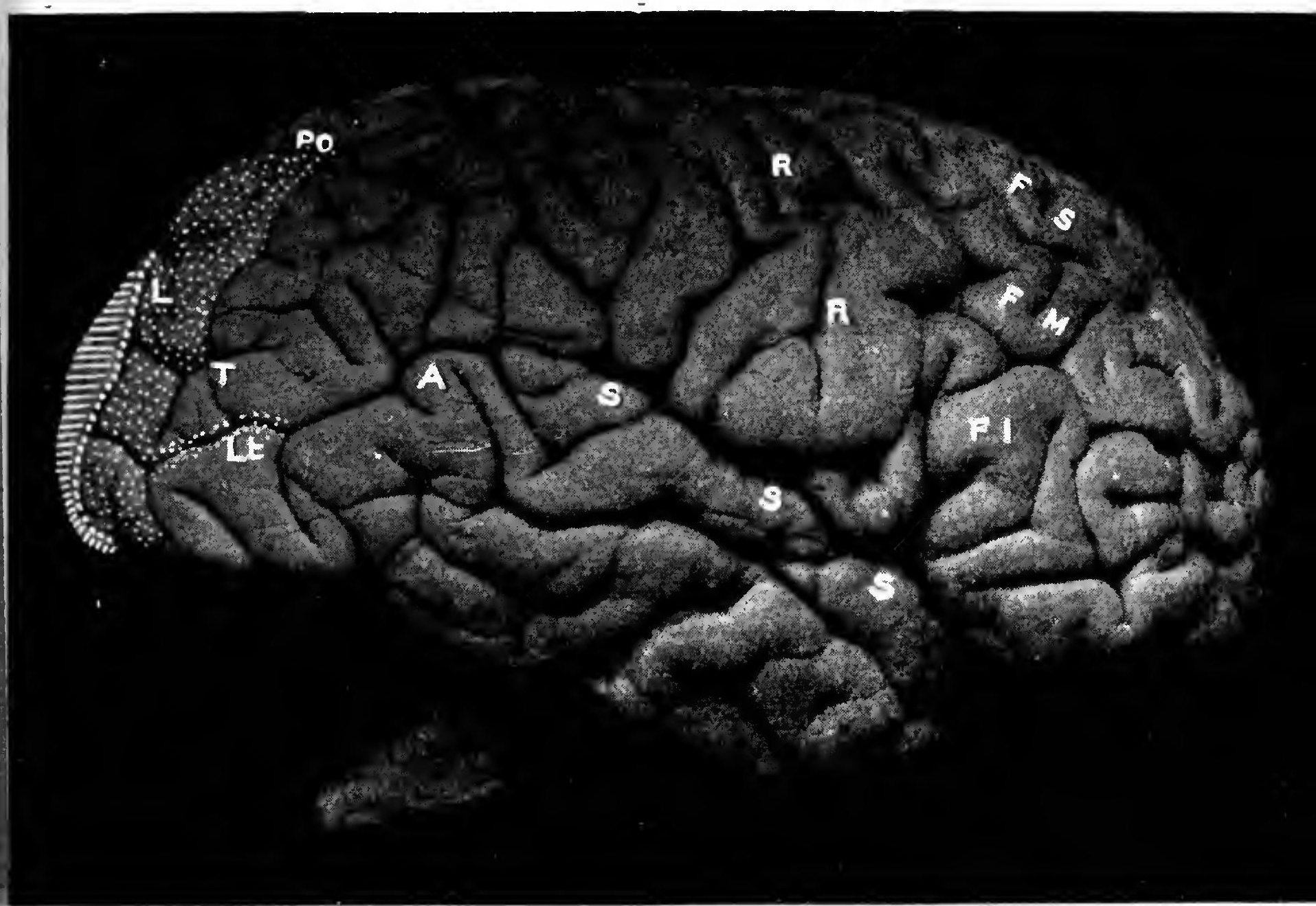

Fia. I go. - I ateral view of Right Hemisphere, to illustrate the approximate position of the "centres" connected with vision (see text).

On the occipital lobe, P.O.=parieto-occipital, $\mathrm{I} .=$ =lunate, T. =transverse occipital, l.t. lateral sulcus; the striate area is indicated by cross-lines, the para-striate and peri-striate ateas by large and small dots respectively. A. is placed on the angular gyrus, which surrounds the upturned end of the superior temporal sulcus. S., S., S. is the lateral cerebral fissure (fissure of Sylvius). R., R. is the central sulcus (fissure of Rolando). F.S., F.M., F.I. are placed on the posterior parts of the superior, middle, and inferior frontal convolutions respectively; between them and the central sulcus are the prae-central sulcus and convolution.

places, names, space, etc., has been suggested, largely on evidence derived from clinico-pathological cases, for a consideration of which, together with that of other " centres," the reader may consult the article by Mills in Posey and Spiller's work (Igo6).

The cortex of the angular gyrus (A. in Fig. Igo) is considered by some authors to be an important, if not the most 
important, visual area; it is said to be concerned with central direct vision of the opposite eye, whilst the occipital pole area is connected only with the peripheral parts of the retina, a view that has not met with general acceptance. Injury of this part of the cortex more certainly entails " word-blindness," the patient being unable to understand printed or written matter, though spoken words are understood; it is therefore termed the visual word memory "centre," and is said to be on the left side of the brain in right-handed subjects. This area is also stated to be directly concerned with winking movements of the eyelids.

The motor centre for eye movements is placed on the frontal lobe, on the posterior part of the middle frontal convolution, and in front of the prae-central sulcus (FM. in Fig. I9o). Stimulation of this area on the right side causes both eyes to turn to the left (" conjugate deviation "), but the angular gyrus is also stated to be the centre for this movement (Grasset, quoted by Testut, IgI4). It is, moreover, found that stimulation of any part of the occipital lobe produces eye movements; thus, stimulation of its upper surface on the right side causes both eyes to turn downwards and to the left; excitation of its posterior part causes both eyes to turn upwards and to the left; and stimulation of the intermediate zone, specially on the mesial surface, causes lateral deviation to the left. Such impulses, no doubt, pass through the descending or corticifugal fibres of the optic radiation. The centre correlated with movements of the eyelids is apparently situated in the praecentral convolution, behind the centre for the ocular movements and just above the centre for the face and mouth.

The visual cortical area is connected with the corresponding area of the opposite hemisphere by commissural fibres which traverse the splenium of the corpus callosum; with the visual memory, visual word, auditory and speech "centres" by association fibres; with the lower visual centres, and possibly also with the lower motor nuclei, by descending or corticifugal fibres of the optic radiation.

As.regards the influence of the cerebellum on the eye movements, Risien Russel (I894) concludes from experi- 
mental investigations that this part of the brain exercises a direct and perfectly independent action on the ocular muscles; the influence of the cerebrum, however, in correction of the phenomena of cerebellar deficiency is to be noted, since it has been found that the severity of the symptoms (atonia, asthenia and astasia in the muscles

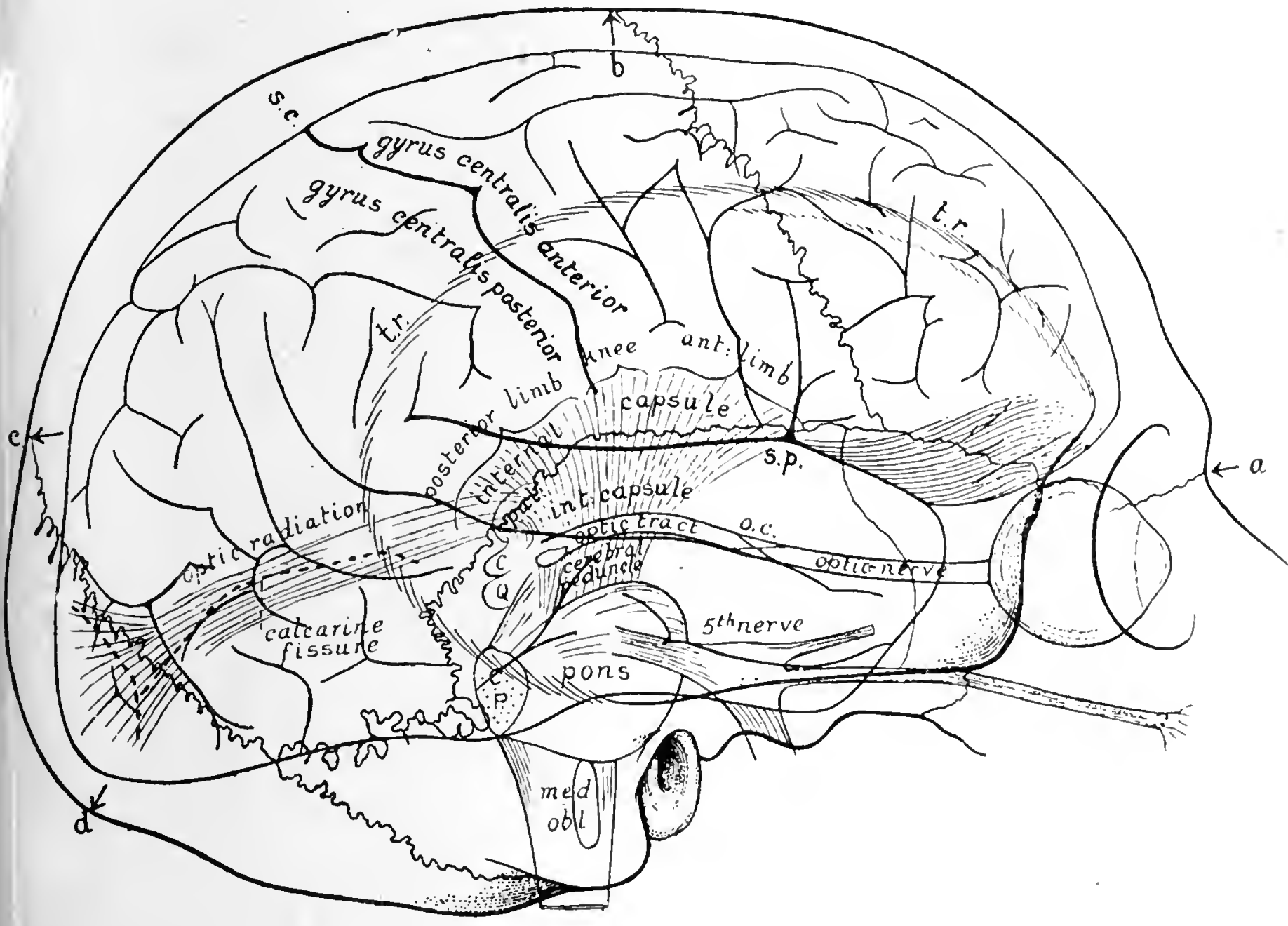

FIG. 19x.-Right lateral aspect of the Skull and Cerebral Hemisphere outlined in black, with orthogonal projection of the medulla oblongata, pons, mid-brain, internal capsule, visual tracts, and fifth cerebral nerve. Man aged fifty-six years. Cephalic index, 7o. (J. Symington.) (From Quain, I908.)

$a .=$ nasion $; b_{0}=$ bregma $; c_{0}=$ lambda $; d_{0}=$ inion $; t_{0} r_{0}=$ temporal ridge $; s_{0} c_{0}=$ sulcus centralis ; s.p. $=$ Sylvian point.

of the same side) occasioned by destruction of one half of the cerebeullm of the dog gradually lessen (Black, I9I6).

The relation of the various parts of the visual pathway to the skull is shown in Fig. Igx. The external occipital protuberance, that is, the point inion, lies on a level with the lower margin of the occipital pole, and therefore just below the cortical visual centre. 


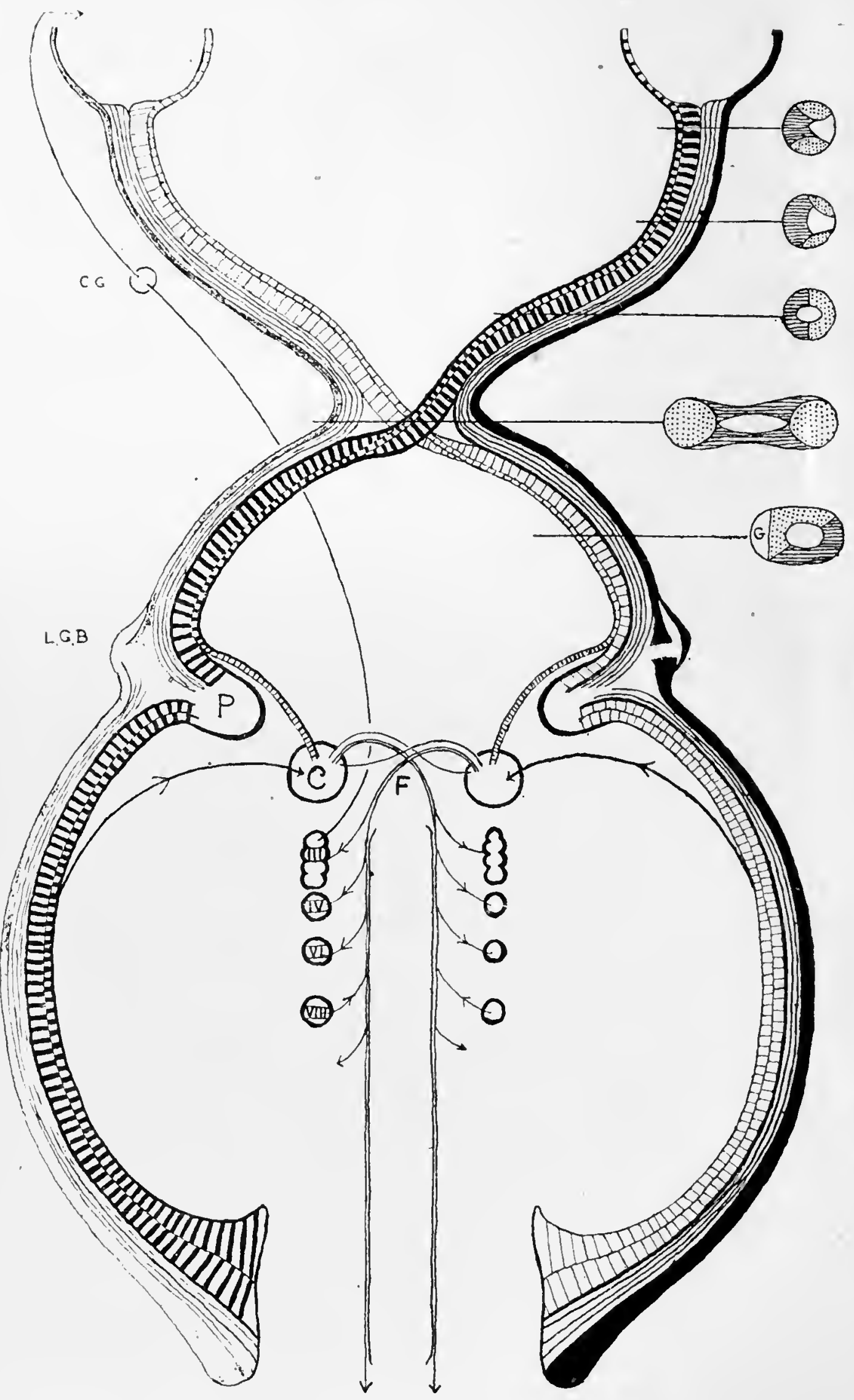

Fig. 192.- Scheme to illustrate the visual paths from the retinae to the occipital poles, those from the right eye being emphasised. The nasal or crossed and the temporal or direct fibres are shown passing into the lower visual centres, but the diagram is not intended to convey the idea that any particular band of fibres of the nerve or tract ends in any particular one of these bodies.

L.G.B.=the lateral geniculate body, and P.=the pulvinar. The relative positions of the bundles in the nerve and tract (from Testut, IgII, after Hensen and Vialet) are shown in the cross-sections, where the crossed fibres are indicated by horizontal lines, the direct fibres by dots, and the macular fibres by the blank area. G. = the position of the commissure of Gudden. The connections of $C_{\text {. }}$, the superior colliculus, with the nuclei of the nerves to the muscles of the eyeball by means of the fountain decussation of Meynert (F.), and also the fibres it receives from the higher or cortical visual centre, are shown. The path of the pupillary reflex froin the oculomotor nucleus (III.) through the ciliary ganglion (C.G.) to the sphincter iridis muscle fibres of the globe are also indicated. 
Analysis of the fibres in the visual paths and their destination.-In the optic nerve the fibres can be divided according to their function into :

(i.) Visual fibres conveying visual impressions to the brain;

(ii.) Pupillary fibres connecting the retina with the pupillomotor centres ;

(iii.) Retino-motor fibres passing from the brain to the retina;

(iv.) Inter-retinal fibres, connecting the two retinae;

though our knowledge regarding the course of the paths taken by these groups becomes less certain in passing from the first to the last.

(i.) The visual fibres are grouped in the nerve in three bundles according to the retinal fields from which they originate :

(a) From the temporal side of the retina; these temporal or " direct" fibres pass through the chiasma into the optic tract and lower visual centres of the same side.

(b) From the nasal side of the retina; these nasal or " crossed" fibres, more voluminous than the last since they come from about the medial two-thirds of the retinal field, pass across in the chiasma to the opposite tract and lower visual centres.

(c) From the cells of the fovea centralis of the macula lutea area of the retina ; these central or " macular " fibres comprise one-third of the total number, and coming from the area of direct and most distinct vision are the most important. In the nerve they lie at first lateral to and a little below the others, but later come to occupy a central position; at the chiasma they are generally considered to undergo partial decussation, half of the fibres passing across to the opposite side, though this is denied by some authors; in the tract they lie centrally, and they terminate like the other visual fibres in the lower visual centres. The relative positions of these bundles in the nerve and tract are shown in the above figure.

(ii.) The "pupillary fibres" of the optic nerve are of finer calibre, and are concerned with contraction of the iris on exposure to light; they probably enter the superior 
colliculus only of the lower visual centre; the whole path of this light-reflex is referred to later on p. 385 .

(iii.) The retino-motor group consists of exceedingly fine fibres, some of which form direct connection between the cortical centre and the retina, whilst others appear to arise in the lower visual centres and pass thence to the retina; they may be vaso-constrictor or trophic in function, or concerned with certain movements of the retinal elements.

(iv.) The inter-retinal fibres are considered to pass from one retina to the other, though the path is not definitely known ; their presence may afford an explanation of sympathetic inflammation of the eyes, or of binocular contrast and after-images.

In the optic chiasma, the two orders of fibres, crossed and indirect, are not clearly separated (though an artificial demonstration can be prepared, as in Fig. I87); the lateral parts of the chiasma, however, are largely occupied by direct fibres and the median part by crossed fibres, the latter taking a somewhat curved course and some of them mounting in the root of the opposite nerve to a height of as much as $3 \mathrm{~mm}$. (Gallemaerts, I900).

In the optic tract of one side, in consequence of the partial decussation of the optic nerves, there are found four bundles of fibres of retinal origin :

Direct or temporal fibres from the eye of the same side,

Crossed or nasal fibres from the opposite eye,

Direct macular, and

Crossed macular fibres, of similar origins.

Each tract therefore contains all the visual fibres from the corresponding sides of both retinae, the right tract, for example, containing the fibres from the right halves of both retinae, including half the macular or central fibres from each eye, and it carries all the impulses originating from stimuli occurring in the left field of vision. The relative positions of the bundles, though by no means sharply defined, are seen in the above figure, from which it appears that the fibres from the different parts of the retina preserve the relative position of their origins in the optic nerve and tract. It may also be said that the different 
regions of the retina correspond point by point to similar regions of a transverse section of the optic nerve of the same side and to similar regions of a transverse section of the optic tract of the opposite side.

Besides these visual fibres and the other groups noted above as constituents of the optic nerve, the tract contains additional fibres, all of which, however, appear to bear no certain relation to vision; they are as follows:

The commissure of Gudden (commissura inferior Guddini) is a well-marked bundle of fibres passing from the medial geniculate body of one side forwards along the optic tract as its medial root, round the back of the chiasma, and down the opposite tract, to end eventually in the inferior colliculus of the opposite side; the bundle can be dissected away completely from the rest of the tract and chiasma, as shown in Fig. I87; it has no connection with vision, but is part of the auditory cerebral mechanism.

The commissure of Meynert (commissura superior Meynerti) is a much smaller tract of fibres lying on the mesial side of Gudden's commissure and pursuing a similar course ; its fibres arise from a small basal optic ganglion in the grey matter between the third ventricle and the chiasma on the side of the tuber cinereum, and end in the sub-thalamic region; they do not appear to have any direct connection with the optic nerve, though they have been called its basal root, and are described as passing to the retina.

The tractus peduncularis transversus, a bundle of fibres occurring constantly in most animals, but only occasionally in man (30 per cent), may be found just in front of the superior colliculus; it passes round the ventral aspect of the cerebral peduncle and enters the tegmentum near the exit of the oculomotor nerve. The origin and termination of its fibres are still obscure, but since it has been found to undergo atrophy after enucleation of the eye or after removal of the cortical visual area it is supposed to be related to the secondary visual paths. It may be an aberrant part of the optic tract.

Finally, Darkschewitsch described some fibres of the optic tract as passing to the ganglion habenulae and the 
pineal peduncle, and thence through the posterior commissure to end in the oculomotor nucleus; he considered them to subserve the reflex changes in the pupil.

Of the lower visual centres, the lateral geniculate body is stated to receive 80 per cent of the retinal fibres of the optic tract. As in the nerve and tract, there appears to be some relative representation of the retinal fields in this body, since investigations suggest that the fibres from the upper and lower parts of the retina end respectively in its upper and lower halves. It is connected, like the pulvinar, with the visual cortical area through the optic radiation, and is mainly concerned with the reception and transmission of visual impulses.

The superior colliculus receives a much smaller proportion of retinal fibres from the optic tract; fibres also enter it from the lemniscus (whereby it is connected with the sensory tracts of the medulla and spinal cord), and from the cerebral cortex, the latter, together with the retinal fibres, entering it through the superior brachium; but, unlike the lateral geniculate body and the pulvinar, it sends no fibres outwards to the cortex. It is joined to its fellow of the opposite side by decussating fibres. Its most important efferent connection is by means of fibres which cross the median plane, forming the "fountain decussation of Meynert" (decussatio tegmenti dorsalis), to enter and descend in the medial (posterior) longitudinal fasciculus, whereby they are connected by terminal arborisations with the nuclei of origin of the oculomotor, trochlear, and abducent nerves, and are continued on in the tectospinal tract to the spinal medulla and cord. These connections afford pathways through which the reflex and possibly also the voluntary optic and ocular mechanisms may be conducted, and the superior colliculus thereby becomes of great importance. It receives impressions from the eye through the optic nerve and tract on the one hand, and impulses from the cerebral cortex through the optic radiation on the other, and it can link up-either path with the nuclei of the motor nerves or with tracts in the medulla and cord by means of the fountain decussation and the medial longitudinal fasciculus. It may therefore be regarded 
as a ganglionic centre for the co-ordination of visual impressions with those of other regions of the body in influencing its movements.

The fibres concerned with the pupillary light-reflex most probably pass through these paths, though the exact course is not known for certain. The reflex of contraction of the pupil may be provoked by light falling upon any part of the retina, though the macular region is the most important, and a bilateral effect upon the pupils is obtained by throwing light upon either eye (consensual reflex). The fibres appear to traverse successively the optic nerve, the chiasma, where they in part decussate, and the optic tract, to enter the superior colliculus. Here, according to most authorities, they end, and fresh fibres are said to convey the impulses through the fountain decussation to either the sub-nucleus or the lateral principal nucleus of the oculomotor nucleus (vide infra), though this last connection has not actually been traced. A third set of fibres convey them along the oculomotor nerve and into the ciliary ganglion by its motor root; and after a final interruption the path to the constrictor iridis muscle is provided by the short ciliary nerves. The whole course as thus outlined is illustrated in Fig. I92. On the other hand, some authors consider that the first axons from the retinal cells pass independently of the colliculus and directly to the oculomotor nucleus; and yet another path is that noted above as given by Darkschewitsch.

The optic radiation is composed mainly of ascending fibres arising from cells of the lateral geniculate body and partly from the pulvinar; none comes from the superior colliculus; it also contains descending fibres passing from the visual cortical area to these lower visual centres, together with commissural and association fibres. It resembles the optic tract of the same side in that it receives impressions from the corresponding lateral halves of both retinae, and probably from the macular field of each. Nothing appears to be definitely known of the relative position of its constituent fibres, though by some authors they are thought to represent the retinal fields in the same manner as do the fibres of the optic tract. 
In the higher visual centre round the calcarine fissure it appears practically certain that similar halves of the retinae, including the halves of the macular areas, of both eyes are related to the cortex on one side, the complete right halves of the retinae to the cortex of the right occipital lobe, and the complete left halves to that of the left lobe; a bilateral representation for each macular area is, however, denied by Holmes and Lister (I9I6). There have been attempts to prove separate cortical regions of representation for the central and peripheral parts of the retinal field, the macular area being associated with the posterior part of the calcarine fissure and cuneus, that is with the extreme end of the occipital pole, and the peripheral parts of the retina with the anterior part of the calcarine fissure and cuneus (Holmes and Lister, I9I6), but on the other hand, the reverse position is by some authors described as the correct representation. It is also held that the macular area is represented in all parts of the visual cortex, and may even extend beyond its usually described limits. The upper half of each retina has been found to be represented in the dorsal and the lower half in the ventral part of each visual field, and even further delimitation has been suggested, namely, that corresponding quadrants of the two retinae are represented in the upper or lower lip of the posterior calcarine fissure on the one or the other side respectively. The whole question is undecided, and much requires to be done in collecting and analysing the evidence, based largely on clinical and pathological observations, and especially in view of the large number of new cases that have been recorded lately as the result of wounds, before such a final generalisation can be made as that the different parts of the retina preserve their relative positions as regards representation, not only in the optic nerve and tract, but also in the cortical visual centre.

The nuclei of origin of the orbital nerves and their connections :-

The oculomotor nucleus (nucleus nervi oculomotorius) 
lies. in the upper part of the cerebral peduncle, parallel to and just beneath the aquaeductus cerebri (aqueduct of Sylvius), below and coextensive with the superior colliculus; it is from 5 to $6 \mathrm{~mm}$. long, and extends nearly the whole length of the floor of the aqueduct from the posterior part of the wall of the third ventricle in front to the anterior part of the fourth ventricle behind, where it is in close

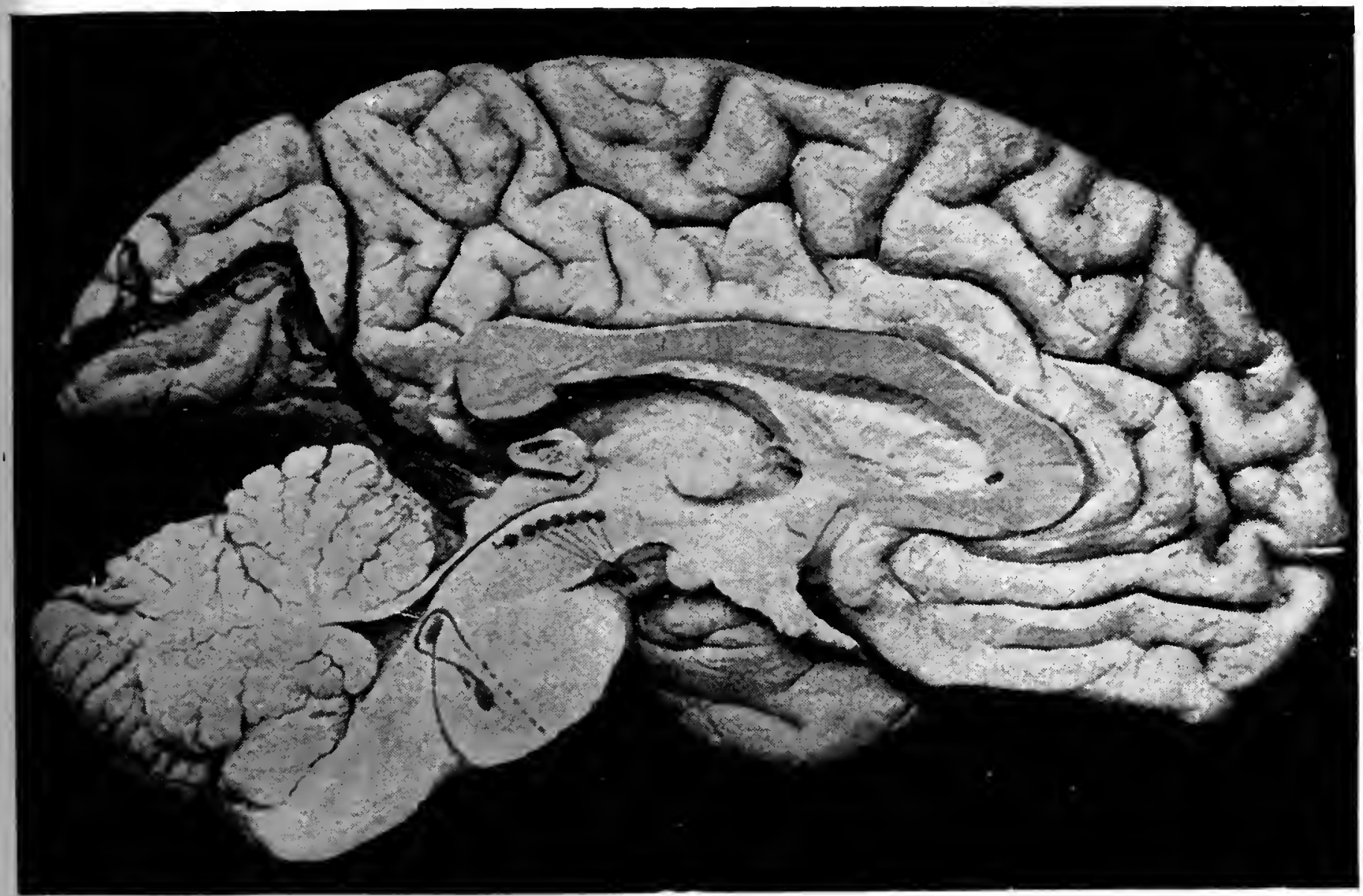

FIG. I93.-Mesial section of Brain, left half viewed from the medial aspect to show, schematically, the nuclei of the third or oculomotor and fourth or trochlear nerves, represented by the row of black dots; the position of the abducent or sixth nerve nucleus is also shown lying within the loop formed by the facial or seventh nerve in the pons; the nucleus of the latter is represented as consisting of a main mass and a secondary, the superior facial nucleus.

The calcarine fissure has been outlined in black. $\times \frac{2}{3}$.

relation with the nucleus of the fourth nerve. The two oculomotor nuclei are separate above but touch below, and their infero-lateral faces are in contact, with the medial longitudinal fasciculus of each side. The subdivision of each mass into smaller centres for the supply of the different muscles of the eyeball is now generally recognised, though their limits are not sharply defined; there appears to be a large-celled lateral "chief nucleus," which supplies the 
extrinsic muscles of the globe, and two smaller-celled subnuclei, one in front (the Edinger-Westphal nucleus) and one behind and below, which is fused with its fellow of the opposite mass to form a central nucleus, and these sub-nuclei are said to supply the intrinsic muscles of the eyeball (Fig. I94, Intr.). Tsuchida finds only the so-called principal

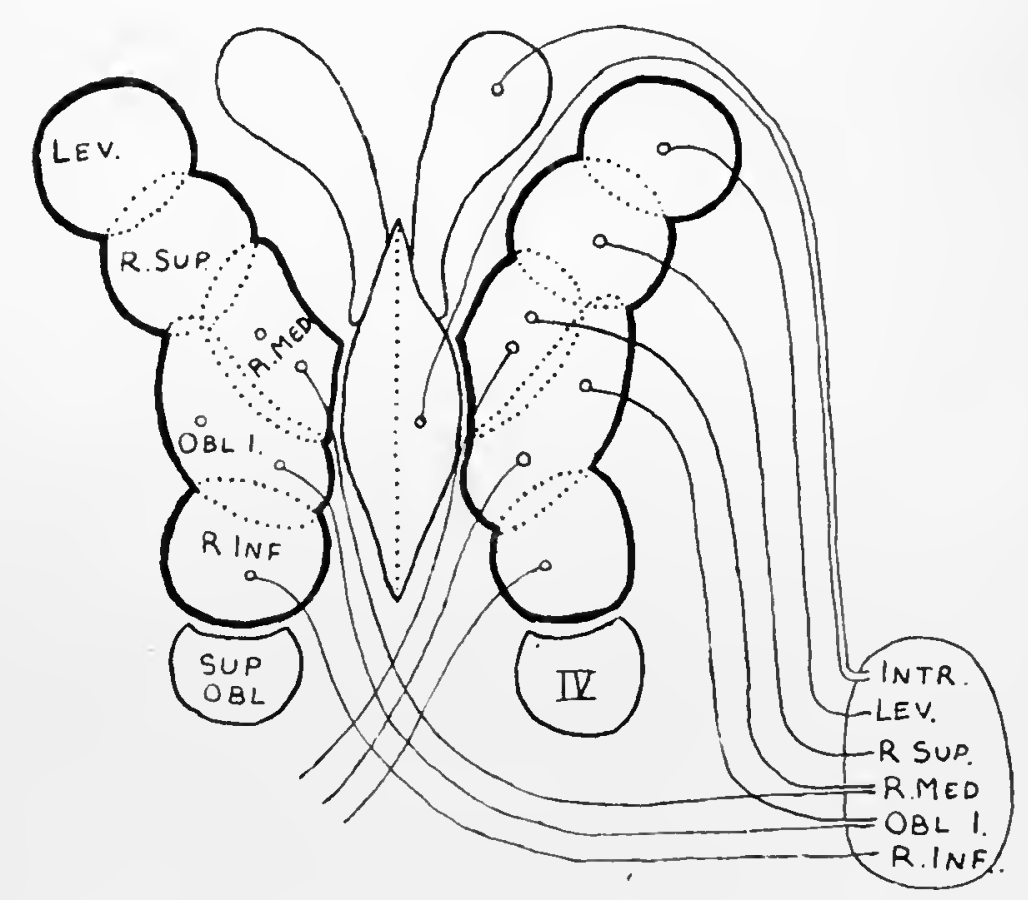

FIG. I94. - Scheme representing the constitution of the Oculomotor Nucleus, modified after Bernheimer (I904). The nuclei of both sides are shown. For description see text.

nuclei anatomically constant, the smaller ones showing remarkable individual variations, and this author locates the innervation of the pupil in the fore end of the lateral chief nucleus and not in the subgroups of cells. Further localisation of the lateral nuclei into centres for each muscle is described, but there is great uncertainty as to their exact topography; for example, the centre for the levator palpebrae superioris is placed at the anterior end by one authority, at the posterior end by another, and it has been pointed out that the results of experiments on monkeys differ from those on rabbits, and one is ignorant of how far such results are applicable to man. The majority of the nerve fibres pass out from that side of the nucleus on which lie the cells from which they originate, but there is a decussation of fibres principally from the posterior parts of the lateral nuclei, so that the inferior rectus muscle appears to receive crossed fibres only, the superior rectus and levator direct fibres only, but the medial rectus and inferior oblique muscles both crossed and direct fibres; the intrinsic muscles of the globe receive their fibres direct. These subdivisions of the nuclei and origin of the nerve fibres have been specially studied 
by Perlia (I889), Bernheimer (I904-9), and Tsuchida (I906), and are represented schematically in the above figure.

The fasciculus so formed by fibres from the nucleus of the same side, but also by some crossed fibres from the opposite nucleus, passes forwards through the medial longitudinal bundle, the tegmentum, the red nucleus, and the substantia nigra, and emerges on the medial side of the cerebral peduncle at the superficial origin of the nerve.

The connections of the oculomotor nucleus are:

(i.) With the superior colliculus, through which impulses reach it from the visual system, as described above.

(ii.) With the cortical motor centre for eye movements.

(iii.) With the cerebellum through the superior cerebellar peduncle.

(iv.) With the trochlear, abducent, and vestibular, and possibly other nuclei by means of the medial longitudinal fasciculus.

As regards these last communications, it has been supposed that some of the fibres

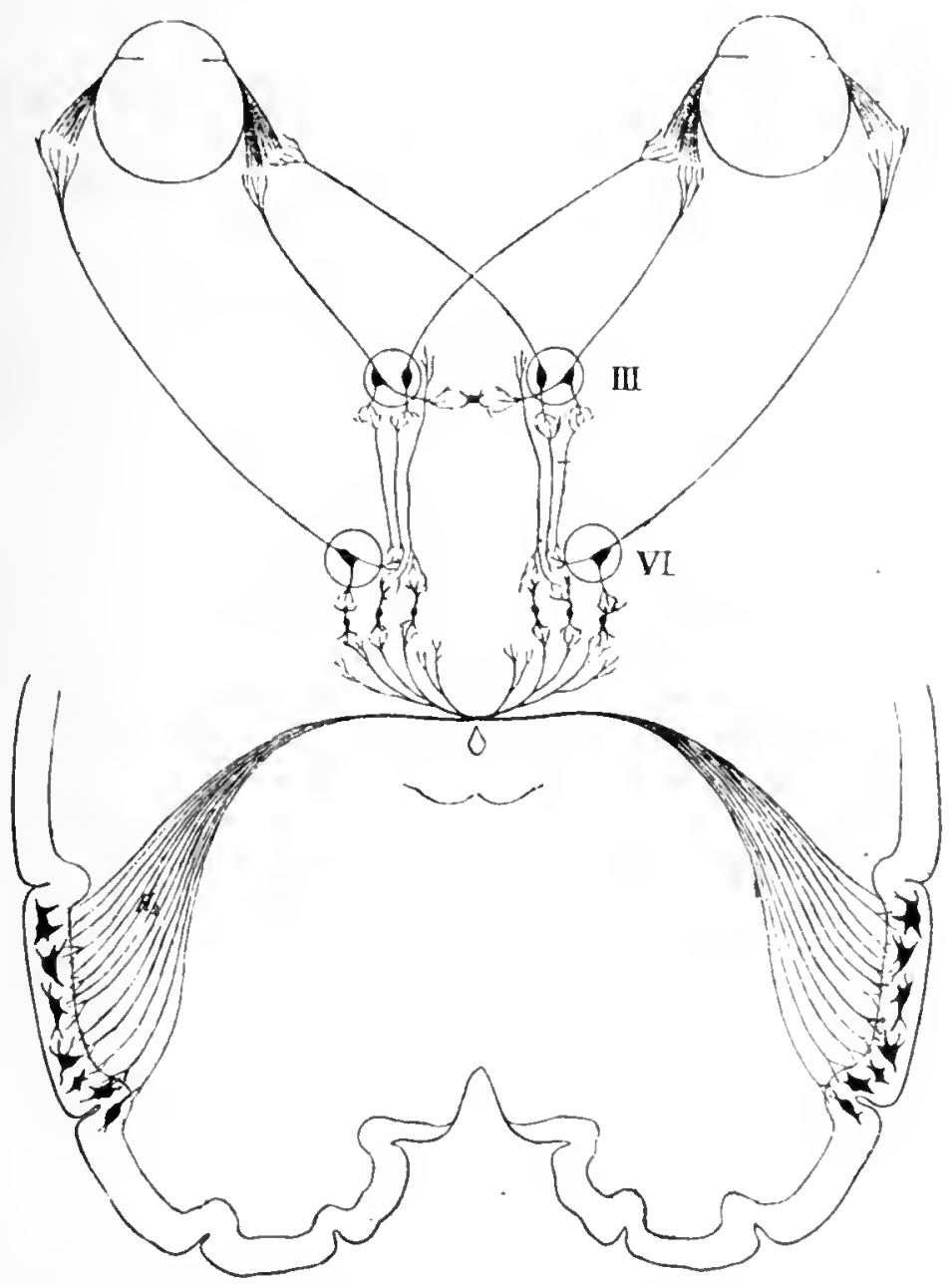

FIG. I95.-Scheme of innervation of lateral and convergent movements of the eyes. (From Bernheimer, 1904.)

III.= oculomotor nucleus; VI. =abducent nucleus. from the oculomotor nucleus pass down the fasciculus into the facial nucleus and then run along with that nerve to supply the orbicularis oculi, corrugator supercilii, and frontalis (see p. I82.).

Another interchange of fibres through the same path is suggested as explanatory of the harmonious action of the lateral rectus (sixth nerve) with the medial rectus (third 
nerve) in producing simultaneous movements of the two eyeballs to the right or left (conjugate deviation), for which several explanations have been given. The sixth nucleus sends no fibres directly into the oculomotor nerve, but it may send fibres, or be connected with fibres, which ascend in the medial longitudinal fasciculus, and establish connections with that part of the oculomotor nucleus from which the crossed fibres to the medial rectus of the opposite side arise (Fig. I95).

The trochlear nucleus (nucleus nervi trochlearis) lies beneath the floor of the Sylvian aqueduct opposite the upper part of the inferior colliculus; it appears to be the detached end of the column of grey matter which forms the oculomotor nucleus (Figs. I93 and I94). The fibres from this origin run lateralwards and downwards through the tegmentum, then turn back and inwards into the upper part of the anterior medullary velum; here they decussate, though not completely, with the corresponding nerve of the opposite side, and emerge from the surface of the brain at the side of the fraenulum veli, just below the inferior colliculus, at its superficial origin (Fig. I89). Its central and cortical relations are similar to those of the oculomotor nucleus.

The abducent nucleus (nucleus nervi abducentis) lies beneath the colliculus facialis (eminentia teres) of the floor of the fourth ventricle close to the mid-line and immediately above the striae medullares. It lies on the ventral side within the loop formed by the facial nerve, but has no connection with it (Fig. I93). (Some of its fibres are said to arise from an accessory or ventral sixth nucleus which lies outside the loop and close to the facial nucleus.) The fibres from these cells pass forwards and downwards on the medial side of the superior olivary nucleus and further on to the lateral side of the pyramidal tract, and after thus traversing the whole thickness of the pons, emerge at the superficial origin at the lower border of this body. The fibres are direct and do not decussate.

The central connections are, like those of the oculo- 
motor nucleus, with the visual and motor cortical areas, with the superior colliculus, and with the nuclei of the third, fourth, and vestibular nerves by means of the medial longitudinal fasciculus, and also with the superior olive. By the medial longitudinal fasciculus a connection is formed between eye movements and equilibration; amongst its constituents are fibres which arise in the lateral vestibular (Deiter's) nucleus of the auditory system and ascend, either on the same or opposite side, to form connections with the nuclei of the third, fourth, and sixth nerves. A reflex arc is formed which is concerned with movements of the head and eyes in response to the vestibular stimulation of the nerve-endings in the semicircular canals of the ears, a stimulation arising from every movement and change of poise of the head. It has been found that strong stimulation of either the semicircular canals, the vestibular nerve, or the nucleus causes an abnormal oscillatory side-to-side movement of the eyes known as nystagmus (a consideration of the mechanism of which will be found in a paper by Wilson and Pike (I9I5). The superior olivary nucleus and its pedicle similarly afford a connection between the cochlear or true auditory nerve and the abducent nucleus, explanatory of the simple auditory reflex of turning the eyes in the direction whence a sound comes.

The trigeminal nucleus of origin is constituted, as previously noted, by the semilunar or Gasserian ganglion (p. 338) which lies outside the brain stem. The centrally directed processes of its cells enter the pons as the sensory root of the nerve; here they bifurcate into ascending and descending branches, the former being short and ending in the upper sensory nucleus, whilst the latter form the descending or spinal trigeminal root which is traceable down through the pons and medulla and into the upper cervical part of the spinal cord. The central connections of this sensory root of the nerve are very extensive, since the impulses collected by its branches from so wide and varied a field are of great importance; they are brought into relation 
with the sensory cerebral cortical areas through the optic thalamus, with the cerebellar cortex through the inferior cerebellar peduncle, and with the nuclei of origin of the motor nerves, notably with the facial nucleus and the nucleus of its own motor root. 


\section{BIBLIOGRAPHY}

AN attempt has been made to give a fairly complete list of the papers and books published since Igoo relating to the anatomy, histology, and embryology of the subject, but other references have been added where deemed of sufficient interest. The list comprises those authors referred to in the text whose names are followed by a date, which is a guide to a particular article where several have been published. The original papers have been consulted in each case, except where an abstract only has been obtainable, as noted.

For the earlier literature the reader should consult the chronological bibliographies given in the Graefe-Saemisch Handbuch der gesamten Augenheilkunde.

ADACHr. "Die Orbita und die Hauptmass des Schädels der Japaner," Zeitschrift für Morphologie und Anthropologie, I904, vii. p. 379.

Afrens. "Die Cribra orbitalia und die Spina trochlearis, etc.," Inaug. Diss. Göttingen (1904), abstracted in Nagel's Jahresbericht für Ophthalmologie, r9o5, p. 39 .

Ambialet. "Orbites et conformations craniennes," Annales d'Oculistique, r905, cxxxiv. p. r76.

Argaud and Falloney. "Sur la structure du tarse palpébral et son indépendance vis-à-vis de la glande de Meibomius," Comptes Rendus de la Société des Biologistes, I9r3, lxxiv. p. ro68.

ArLT. (i.) "Über den Thränenschlauch," Graefe's Archiv für Ophthalmologie, r855, i. 2, p. I35.

(ii.) "Über den Ringmuskeln der Augenlider," Graefe's Archiv für Ophthalmologie, r863, ix. r, p. 64 .

Ask. (i.) "Anthropometrische Studien über die Grösse und Gestaltung der Orbitalmündung bei den Schweden, etc.," Zeitschrift für Augenheilkunde, I906, xvi. p. I46.

(ii.) "Úber die Entwicklung der Caruncula lacrimalis beim Menschen, etc.," Anatomischer Anzeiger, r907, xxx. p. I97.

(iii.) "Über die Entwicklung der Lidränder, etc.," Anatomische Hefte (Merkel und Bonnet), I908, xxxvi. p. I89.

(iv.) " Entwicklung der Drüsenapparatus, der Bindehaut beim Menschen," Anatomische Hefte, rgro, xl. i. p. 489. 
AUbaret. (i.) "Les Replis valvulaires des canalicules et du conduit lacrymo-nasal, etc.," Archives d'Ophtalmologie, I908, xxviii. p. 2 II. (Abstracted in the Ophthalmoscope, I908, vi. p. 900.)

(ii.) "Des rapports des faisceaux lacrymaux de l'orbiculaire des paupières, etc.," Comptes Rendus de la Société de Biologie, I909, lxvii. p. 235.

(iii.) "Morphologie du conduit lacrymo-nasal chez l'homme," Bibliographie Anatomique, I9IO-II, XX. i. p. 97 :

AUBARET et Bonnefon. "Des rapports du conduit lacrymo-nasal avec le méat moyẹn et la gouttière de l'infundibulum," Archives d'Ophtalmologie, 1910, xxx. p. 469.

Augier. "Les Os frontaux accessoires," Comptes-Rendus de l'Association des Anatomistes, I 4e Réunion, I9I2, p. 22.

Axenfeld. (i.) "Bemerkungen zur Histologie und Physiologie der Thränendrüsen" Berichte der 27 Vers. d. Ophthal. Gesellsch. Ophthal. zu Heidelberg, I 898 (abstract in Nagel's Jahresbericht für Ophthalmologie, I 898).

(ii.) "Über die feinere Histologie der Thränendrüsen, etc." (Abstract in Nagel's Jahresbericht für Ophthalmologie, I900, p. 33.)

BACH. "Die Nerven der Augenlider und der Sklera beim Menschen und Kaninchen," Graefe's Archiv für Ophthalmologie, I 895, xli. 3, p. 50.

BAKER. Article on the Eyeball in Norris and Oliver's System of Diseases of the Eye, Philadelphia, I90o, vol. i. p. Iog.

Barraguer. "Anatomie du fond de l'orbite et du sinus sphénoidal," Comptes Rendus de la Société Française de l'Ophtalmologie, I896, xiv. International Congress of Medicine, Madrid, I903. (Abstracted in Annales d'Oculistique, I903, vol. I30, p. I 89.)

Barratt. "Observations on the Structure of the Third, Fourth, and Sixth Cranial Nerves," Journal of Anatomy and Physiology, I90I, XXXv. p. 214.

Bartels. (i.) “Darstellung der Manz'schen Drusen und ihre Beziehung zu den Cysten," Zeitschrift für Augenheilkunde, I908, xx. p. I93.

(ii.) Article on "Das Lymphgefässsystem," in Bardeleben's Handbuch der Anatomie des Menschen, I909, iii. 4, p. IO3.

BeHr. "Besteht beim Menschen ein Abfluss aus dem Glaskörper in der Sehnerven?" Graefe's Archiv für Ophthalmologie, I9I2, Ixxxiii. p. 5 I9.

BERGER. (i.) "Anatomie normale et pathologique de l'œil." Paris, I 893 .

(ii.) "Variétés de la paroi optico-sphénoidale," Archives d'Ophtalmologie, I894, xiv..p. 54.5.

Bernheimer. (i.) "Atiologie und pathologische Anatomie der Augenmuskellähmungen," and "Die Wurzelgebiete der Augennerven," Graefe-Saemisch Handbuch der gesamten Augenheilkunde, 2nd ed., I904, Lief. 39 and I6.

(ii.) "Weitere experimentelle Studien zur Kenntnis der Lage des Sphinkter- und Levatorkerns," Graefe's Archiv für Ophthalmologie, I909, 1xx. p. 539.

Birch-HirschFeld. "Die Krankheiten der Orbita." Article in the Graefe-Saemisch Handbuch der gesammten Augenheilkunde, I909, Lief. I 47 -I 70, S. 261 .

Birnbaum. "Exophthalmometrische Masse bei Normalen und deren Beziehungen zur Grosse der Orbitalöffnung," Archiv für Augenheilkunde, I9I 5, vol. 90, p. 378. 
Bischoғf. " Mikroskopische Analyse der Anastomosen der Kopfnerven." München, r865.

BlAck. "Cerebellar Localisation in the Light of Recent Research," Journal of Laboratory and Clinical Medicine (St. Louis), I9I6, i. p. 467.

Broch. "De l'œil mongoloïde,". etc., Bulletins et mémoires de la Société d'Anthropologie de Paris, I9II, 6e série, II. 3 and 4 , p. 326.

BochDaleK. " "Beitrag zu der anomalen Muskeln der Augenhöhle," Prager Vierteljahrsschrift, I868, Band iv.

Bock. "Zur Kenntniss der Gesunden und kranken Thränendrüsen," Wien, r896 (quoted by Schirmer and Picou).

BoEke. (i.) "Die doppelte (motorische und sympathische) efferente Innervation der quergestreiften Muskelfasern," Anatomischer Anzeiger, I9I 3, xliv. I 5-I6, p. 343 .

(ii.) "On the Termination of the Efferent Nerves in plain MuscleCells and its bearing on the Sympathetic (Accessory) Innervation of the Striated Muscle Fibre." (Abstracted in Ophthalmology (Seattle), I9I 5-I6, vol. I2, p. 696.)

BorN. (i.) “ Über die Nasenhöhlen und den Thränennasengang der Amphibien," Morphologisches Jahrbuch, I876, ii. p. 577.

(ii.) "Über die Nasenhöhlen und den Thränennasengang der amnioten Wirbelthiere," Morphologisches Jahrbuch, I 883, viii. p. I 88.

Boucheron. (i.) "Nerfs de l'hémisphère antérieure de l'œil," Comptes Rendus de la Société de Biologie, I89o, ii. (Mémoires), p. 7 I.

(ii.) "Nerfs ciliaires superficiels," Comptes Rendus, etc., I89I, iii. (Mémoires), p. 59.

Boughton. "The Increase in the Number and Size of the Medullated Fibres in the Oculo-motor Nerve of the White Rat and of the Cat at Different Ages," Journal of Comparative Neurology and Psychology, I906, xvi. p. I53.

Bralley. "Congenital Districhiasis," British Medical Journal, July 2I, I906, p. I42.

Branca. Article on the Skin and its Derivatives in Poirier's Anatomy, I9I2.

Brav. "Oxycephaly and Optic Atrophy," Annals of Ophthalmology of St. Louis, I912, xxi. i. p. I.

Bremer. (i.) "Aberrant Roots and Branches of the Abducent and Hypoglossal Nerves," Journal of Comparative Neurology and Psychology, I908, xviii. p. 6r9.

(ii.) "Recurrent Branches of the Abducens Nerve in Human Embryos," American Journal of Anatomy, I92I, xxviii. 2.

BRIGgs. "Hereditary Congenital Ptosis with report of Sixty-four Cases conforming to the Mendelian Rule of Dominance," American Journal of Ophthalmology, I9I9, vol. 2, p. 4oS.

BrocA. " Recherches sur l'indice orbitaire," Archives d'Ophtalmologie. Paris, I 875. Mémoires d'anthropologie, Paris, I 87 I-77.

Brodman. "Individuelle Variationen der Sehnsphäre und ihre Bedeutung für die Klinik der Hinterhauptschüsse," Medizinische Klinil. Berlin, rgig.

Broman and Ask. Development of Glands of Eyelids in Mammals, Deutsche Südpolen Expedition, I90I-I903, Berlin, I9Io, vol. xii.

BRUCE and PIRIE. "On the Origin of the Facial Nerve," Review of Neurology and Psychiatry, I908, vi. p. 685.

BudGe. "Beschreibung eines neuen Muskels," etc., Zeitschrift für rationelle Medizin, $\mathbf{I} 859,3$, vii. p. 273. 
BurkarD. " Über die Periorbita der Wirbeltiere und ihre muskelosen Elemente," Archiv für Anatomie und Physiologie, Anat. Abt., Suppl. Band, 1902, p. 79. (Abstracted in Nagel's Jahresbericht der Ophthalmologie, 1902, p. 31.)

Cameron. (i.) "The Naso-orbito-alveolar Index," American Journal of Physical Anthropology, 1920, iii. No. I, p. 63.

(ii.) "Contour of Orbital Aperture in representatives of modern and fossil Hominidæ," American Journal of Physical Anthropology, r920, iii. No. 4, Oct.-Dec.

Campos. "Recherches expérimentales et cliniques sur les nerfs sécréteurs des larmes," Archives d'Ophtalmologie, r897, xvii. p. 529.

CARLINI. "Il tessuto elastico in rapporto con le glandule di Moll," Annali di Ottalmologia, I907, xxxvi. p. 231.

Charpy. (i.) "Orifices adipeux de la base de l'orbite," Comptes Rendus de l'Association des Anatomistes, roe Réunion, I908.

(ii.) "Lè Coussinet adipeux du sourcil," Bibliographie Anatomique, I909, xix. I, p. 47.

(iii.) "Capsule de Tenon," Bibliographie Anatomique, I9ro, Xviii. $4-5$, p. I.

(iv.) "Plis et sillons des paupières" Bibliographie Anatomique, IgIo, Xx. P. I.

(v.) "Structure topographique des paupières," Bibliographie Anatomique, I9II, xxi. 2, p. 65.

(vi.) Article on the Muscles of the Eyeball in Poirier's Anatomy, I9I2, vol. ii. p. 539.

Ciaccio. "Über den Bau der Bindehaut des menschlichen Auges," Moleschott's Untersuchungen zur Naturlehre des Menschen und der Thieren, I874, xi. p. 420. (Reference from Virchow.)

Clermont. "Le Muscle releveur de la paupière supérieure et le septum orbitaire," Comptes Rendus de l'Association des Anatomistes, I909, xviii. supplément, xi. p. 264 .

Coffin. "The Development of the Accessory Sinuses of the Nose," The American Journal of the Medical Sciences, r905, cxxix. p. 297. Refers especially to condition in infants and reviews the literature.

Congdon. "The Distribution and Mode of Origin of Septa and Walls of the Sphenoid Sinus," The Anatomical Record, I920, xviii. 2, p. 97.

Contino. (i.) "Über Bau und Entwicklung des Lidrandes beim Menschen," Graefe's Archiv für Ophthalmologie, I907, ]xvi. p. 505.

(ii.) "Úber die Entwicklung der Plica semilunaris beim Menschen," Graefe's Archiv für Ophthalmologie, I gog, lxxi. p. I.

Cope. (i.) "The Pituitary Fossa and the Surgical Methods of Approach to it," The Lancet, March I8, I9I6, p. 60I; also in the British Journal of Surgery, July I9I6, p. I07.

(ii.) "The Internal Structure of the Sphenoidal Sinus," Journal of Anatomy, I9I 7, li. p. I27.

Coppez. "Etudes sur la pigmentation de la conjunctiva," Bulletin de l'Académie Royale de Médecine de Belgique, I905, série 4, xix. p. 443.

CoRnING. "Über die vergleichende Anatomie der Augenmuskelatur," Morphologisches Jahrbuch, I902, xxix. p. 94.

Cosmetratos. "Über einige angeborene Anomalien der Tränenwege," Archiv für Augenheilkunde, r906, 1v. p. 36.

Crevatin. " Beitrag zur Kenntniss der epithelialen Geflechte der Hornhaut der Säugetiere," Ânatomischer Anzeiger, I903, xxiii. p. I 5 I.

Cruveilhier. Anatomie descriptive, r 886 . 
Cryer. "Some Variations in the Frontal Sinus," The Journal of the American Medical Association, I907, xlviii. p. 284. Illustrated by numerous photographs.

Curnow. "Notes of some Irregularities in Muscles and Nerves," Journal of Anatomy and Physiology, I873, vii. p. 304.

Cushing, Harvey. "Strangulation of the Nerxi Abducentes by Lateral Branches of the 13asilar Arteries in Cases of Brain Tumours," Brain, Xxxiii., I9IO-II.

Czermak. "Die topographischen Beziehungen der Augenhöhle, etc." Augenärztliche Unterrichtstafeln (Magnus), ix. Breslau, I895.

DARWIN. "On the Expression of the Emotions in Man and Animals." London, 1872.

DAvida. "Über die Varietäten des Verlaufen der Sutura infraorbitalis sagittalis," Anatomischer Anzeiger, I9I3, xliv. pt. Io, p. 203.

DE Bono and Frisco. "Sulla permeabilità vesso i microarganismi clelle mucose conjunctivali, etc.", Archivio di Ottalmologia, I90 I, viii. p. 40r. (Abstracted in Archives of Ophthalmology (Knapp), Igor, p. II3.)

DEDEKIND. "Beiträge zur Entwicklungsgeschichte der Augengefässe des Menschen," Anatomische Hefte (Merkel und Bonnet), I909, xxxviii. p. I.

DEwEy. "A Contribution to the Study of the Lymphatic System of the Eye," Anatomical Record, I920, xix. p. I25.

DEYL. "Über den Eintritt der Arteria centralis Retinae in den Sehnerven beim Menschen," Anatomischer Anzeiger, I 896, Märch 20, xi. p. 687 .

Dieulafé. "Topographie des voies lacrymales: applications chirurgicales," Bulletin Médical, Feb. I905, p. I79.

Dixon. "On certain Markings due to Nerves and Blood-Vessels upon the Cranial Vault," Journal of Anatomy and Physiology, I904, xxxviii. ; New Series, xviii. p. 377.

Dock. " Oxycephaly and Exophthalmos," The Osler Dedication Volume of Contributions to Medical and Biological Research, I9I9. Hoebner, New York.

Dogrel. (i.) “Die Nervenendigungen in der Tränendrüsen der Säugetiere," Archiv für milkroskopische Anatomie, r893, xlii. p. 632.

(ii.) "Die Nervenendigungen im Lidrande, und in der Conjunctiva palpebrarum des Menschen," Archiv für Mikroskopische Anatomie, I 89.5 , xliv. p. I5.

(iii.) "Die Endigungen der sensiblen Nerven in den Augenmuskeln und deren Sehnen beim Menschen und den Säugetieren," Archiv für mikroskopische Anatomie, lxviii. I906, p. 50 I.

DoNDERs. "Untersuchungen über die Entwicklung und den Wechsel der Cilien," Gracfe's Archiv für Ophthalmologie, I 858, iv. I, p. 286.

Dor. "La Fatigue oculaire." Paris, I899.

Dorfman. "Über Pathogenese und Therapie des Turmschädels," Graefe's Archiv für Ophthalmologie, I908, lxviii. p. 4I 2.

Druault. Article "Organe de la vision" in Poirier's Traité d'anatomie humaine, I9I2, vol. 5 .

Dubreurl. (i.) "Les Glandes lacrymales de l'homme et des manımifères," Revue générale d'Ophtalmologie, I907, xxvi. p. 339.

(ii.) "Les Glandes lacrymales et les glandes annexes de l'œil des vertébrés," Revue générale d'Histologie, r908, viii. p. 695.

Duckworth. Morphology and Anthropology. Cambridge, I904. 
Duverney. "L'Art de disséquer méthodiquement les muscles, etc." Paris, 1749 .

Dwighr. (i.) Article on the "Anatomy of the Orbit" in Norris and Oliver's System of Diseases of the Eye.

(ii.) Article "The Skeleton" in Piersol's Human Anatomy.

EgGeling. "Zur Morphologie der Augenlider der Säuger," Jenaische Zeitschrift für Naturwissenschaft, r904, xxxix. p. I.

EMmert. "Auge und Schädel." Berlin, I880.

Enstin. "Die Histologie der Caruncula lacrymalis des Menschen," Archiv für Augenheilkunde, I905, li. p. 253.

Evans; Howell. "The Applied Topographical Anatomy of the Sinuses accessory to the Nasal Cavities in their Relations to the Orbit and to its more important Contents," The Ophthalmoscope, I908, vi. No. 4, p. 259.

Evart. "A Method for determining the Position of the Eye-socket," Journal of Anatomy and Physiology, r907, xli. p. 304.

FAwCETt. (i.) "The Origin and Intracranial Course of the Ophthalmic Arteries and the Relation they bear to the Optic Nerve," Journal of Anatomy and Physiology, I 896, xxx. p. 49.

(ii.) "The Development of the Human Maxilla, etc.," Journal of Anatomy and Physiology, July I.9 I, xlv. p. 378 .

FEIN. "Ửber Beziehungen zwischen Kieferhöhle und Tränennasengang," Archiv für Laryngologie und Rhinologie, I912, 26, p. 29.

FERroN. "Note sur la constitution des parois du sinus caverneux et les rapports des nerfs, etc.," Journal de Médecine de Bordeaux, I9I3, Feb. 9, No. 6, p. 88.

FEstal. "Recherches anatomiques sur les veines de l'orbite," Thèse. Paris, $x 887$.

Fischer. "Über Pigment in der menschlichen Conjunctiva," Anatomischer Anzeiger, I905, xxvii. p. I 40.

Fisher, J. H. (i.) Ophthalmological Anatomy. Hodder \& Stoughton, London, I904.

(ii.) "Leber's Disease (Hereditary Optic Atrophy), a Suggestion as to its Cause," The Ophthalmoscope, I9I6, xiv. p. 398.

Flecker. "Observations upon Cases of Absence of the Lacrimal Bones and of Existence of Perilacrimal Ossicles," Journal of Anatomy and Physiology, I9I3, xlviii. I, p. 52.

Freischer. (i.) "Beiträge zur Histologie der Tränendrüse, etc.," Anatomische Hefte (Merkel und Bonnet), I904, xxvi. p. ror.

(ii.) "Die Entwicklung der Tränenröhrchen bei den Säugetieren," Graefe's Archiv für Ophthalmologie, rgo6, 1xii. 379.

(iii.) "Musculus retractor bulbi, etc.," Anatomischer Anzeiger, I907, xxx. p. 465 .

Flower. Catalogue of the Museum of the Royal College of Surgeons (2nd ed.), London, r907.

Francis and Gibson. "The Anatomical Relation between the Sphenoidal Sinus and Orbit," The Ophthalmoscope, I9I I, ix. 3, p. I72.

FRIEBERG. (i.) "Untersuchungen über die Mechanik der Tränenableitung," Zeitschrift für Augenheilkunde, I918, vol. 37, pp. 42, 2 II, 324 .

(ii.) "Weitere Untersuchungen über die Mechanik der Tränenableitung," Zeitschrift für Augenheilkunde, I918, vol. 39, p. 266.

Frohse. "Die oberfläche Nerven des Kopfes." Berlin, I895. 
FRUnd. “Die glatte Muskulation der Orbita, etc.,” Beitrag zur klinischen Chirurgie, 73, p. 755. (Abstract in Zeitschrift für Augenheilkunde, I9I 2, Xxviii. p. I64.)

Fuchs. (i.) "Zur Anatomie der Blut- und Lymphgefässe der Augen.lider," Graefe's Archiv für Ophthalmologie, 1878 , xxiv. 3, p. r.

(ii.) "Beiträge zur normalen Anatomie des Augapfels," Graefe's Archiv, etc., I884, xxx. 4, p. I.

(iii.) Text-book of Ophthalmology (Douane's Translation). London and Philadelphia, r917.

Futamura. "Über die Entwickelung der Facialismuskulatur des Menschen," Anatomische Hefte, I906, 9I (30 Band, Heft. 2), p. 435.

Gallemaerts. (i.) " Recherches sur la fente sphénoïdale," Bulletin de l’Académie Royale de Médecine de Belgique, Bruxelles, r897, Séance Fév. 27, 1897.

(ii.) "Sur l'aponévrose orbito-oculaire," ditto, Séance Jan. 28, r 899.

(iii.) "Sur la structure du chiasma optique," ditto, Séance Juin 28 , 1900 .

Gasizell. "On the Relation between the Structure, Function, Distribution, and Origin of the Cranial Nerves, etc.," Journal of Anatomy and Physiology, I889, x. p. I53.

Gayat. "Essais de mensuration de l'orbite," Annales d'Oculistique, I873, lxx. p. I (Volume of the Orbit).

Gegenbaur. (i.). "Über die Pars facialis des Lacrimalis des Menschen," Morphologisches Jahrbuch, I88I, vii. p. I73.

(ii.) "Vergleichende Anatomie der Wirbeltieren," Morpholog. Jahrb., rgor, ii.

Genuchten, VAN. Anatomie du système nerveux de l'homme, rgo6. édition iv., Louvain.

Gérard. (i.) "Les Voies optiques extra-cérébrales," Journal de l'Anatomie et de la Physiologie, I904, xl. i. p. 22.

(ii.) "Des obstacles naturels capables de compliquer le cathétérisme des voies lacrymales," Annales d'Oculistique, 1907, vol. r37, p. r93.

Gerlach. Anatomie des Auges. Leipzig, r88o.

Gerrish. Text-book of Anatomy. Lea Brothers \& Co., Philadelphia, 1902.

Giacomini. Several articles on the Anatomy of the Negro, Le memorie Giorn. della R. Accad. di Med. di Torino, I 882, I884, I887, I 892, I 898.

GoldenbURG. "Tower Skull with Double Optic Nerve Atrophy," American Journal of Ophthalmology, r9r 8, i. p. 760.

Gosselin. "UỦber die Ausführungsgänge der Tränendrüsen," Archives gén. de la Méd., Paris, I843. (Reference from Merkel and Kallius.)

Gowers. " The Movements of the Eyelids," Medico-Chirurgical Transactions, I879, lxii. p. 432 .

Göz. "Untersuchung von Tränendrüsen aus verschiedenen Lebensaltern," Inaugural Dissertation. Tübingen, I908. (Abstract in Nagel's Jahresbericht für Ophthalmologie, I909, p. 28, and quoted by Picou, I912.)

Graefe-SaEmisch. Handbuch der gesamten Augenheilkunde. Engelmann, Leipsig, 2nd ed. Igor-

Grax. Text-book of Anatomy, Descriptive and Applied, 2 ist ed. Longmans, Green \& Co., London, I920.

Green, LeEdham. "Über die Bedeutung der Becherzellen der Conjunctiva," Graefe's Archiv für Ophthalmologie, I894, xl. i. p. r. 
Groyer. (i.) "Zur vergleichenden Anatomie des Musculus orbitalis und der Musculi palpebrales (tarsales)," Sitzungsbericht d. k. Akad. d. Wissenschaften in Wien, 1903, cxii. iii. p. 50.

(ii.) "Zur Anatomie des Musc. palpebral. sup. des Menschen," Zeitschrift für Augenheilkunde. I905, xiv. p. 365 .

(iii.) "Über den Zusammenhang der Mussculi tarsales (palpebrales) mit den geraden Augenmuskeln, etc.," Internat. Monatsschrift für Anatomie und Physiologie, I906, xxiii. p. 2 ro.

GrÜBER, W. Mémoires de l'Académie Impériale de St-Pétersbourg, 1877. Quoted by Ledouble, 1906, p. 148, on the ossiculum infra-orbitale marginale.

Grunert. "Die Lymphbahnen der Aıgenlider," Archiv für Augenheilkunde, r9or, xliv. p. r89.

GrünWald. "Der Recessus ethmolacrymalis," Anatomische Hefte (Merkel und Bonnet), I9Io, xli. p. 373 .

GuRwitsch. "Über die Anastomosen zwischen den Gesichts- und Orbitalvene," Graefe's Archiv für Ophthalmologie, r883, xxix. 4.

Halben. "Beiträge zur Anatomie der Tränenwege," Graefe's Archiv für Ophthalmologie, I903, lvii. p. 6r.

Hamburger. "Die Grundlagen der Prof. Stilling'schen Theorie, etc.," Zeitschrift für Augenheilkunde, I904, xii. p. 35 I.

Hannes. "Über das Vorkommen und die Herkunft von Plasmazellen der menschlichen Tränendrïsen," Arch. Path. Anat. Physiol. I9r r, ccv. p. 4 IO.

Hardy. (i.) Article on Congenital Anomalies of the Eye, with Bibliography, in American Encyclopedia of Ophthalmology, I9I4, vol. iv. p. 2776.

(ii.) "Ocular Disease of Dental Origin," (Abstracts) American Journal of Ophthalmology, April I9r7, xxxiv. 4, p. 97.

HARMAN, Bishop. "The Innervation of the Orbicularis Palpebrarum Muscle," Transactions of the Ophthalmological Society of the United Kingdom, I903, xxiii. p. $35^{8}$.

HEERFORDT. " UỦber das Emphysema der Orbita," Graefe's Archiv für Ophthalmologie, I904, lviii. p. I23.

Heinlein. " Zur mikroskopischen Anatomie der Tränenrührchen," Graefe's Archiv für Ophthalmologie, r875, xxi. 3, p. I.

Henderson. "The Ophthalmic Arteries in the Rabbit and Dog," Royal London Ophthalmic Hospital Report, I903, xv. pt. 3, p. 260.

Henle. Handbuch der topographischen Anatomie. Wien, r853.

Henschen. "Klinische und anatomische Beiträge zur Pathologie des Gehirns." Upsala, r8go.

Herrick. "An Introduction to Neurology." Saunders, Philadelphia, r9r8. Hesser. "Der Bindegewebsapparat und die glatte Muskulatur der Orbita beim Menschen im normalen Zustande," Anatomische Hefte (Merkel und Bonnet), I9r3, xlix. p. I47.

HIwATARI. "Histology of the Corneoscleral Margin." Archives of Ophthalmology (Knapp), Jan. I92 r, 1. r. p. ro.

Hocevar. "Zur Topographie der Tränendrüsen und tubulo-acinöser Drüsen der Augenlider des Menschen,"-Wiener medizinische Wochenschrift, I900, No. 49, p. 2330 , and No. 50, p. 2375.

Holloway. "Cranial Deformities associated with Ocular Changes," Transactions of the American Ophthalmological Society, 194. (Abstracted in Archives of Ophthalmology (Knapp), I9I5, xliv. p. 469.) 
Holmes, C. R. (i.) "The Sphenoidal Cavity and its Relation to the Eye," Archives of Ophthalmology (Knapp), r896, xxv. p. "46o.

(ii.) "Extirpation of the Lacrimal Gland in Epiphora," Archives of Ophthalmology (Knapp), July I919, xlviii. 4, p. 333.

Holmes and Lister. "Disturbances of Vision from Cerebral Lesions, with special Reference to the Cortical Representation of the Macula," Brain, I9r6, xxxix. p. 34, and British Journal of Ophthalmology, July I9I 8 , ii. p. 353 .

Hopkins. "The Innervation of the Muscle Retractor Oculi," The Anatomical Record, 1916, ii. 5, p. 199.

HORNER. "Description of a Small Muscle at the Internal Commissure of the Eyelids," Philadelphia, I824, p. 70.

Hovelacoue. Article on Venous System in third edition of Poirier's Traité d'anatomie humaine. Paris, I920.

Howe. The Muscles of the Eye. Putnam, New York, 1907.

Hueber. "Transverse Orbital Muscles," Nederl. Tijdschr. v. Geneesk, I9I8, i. p. I98. (Abstracts of the American Journal of Ophthal-

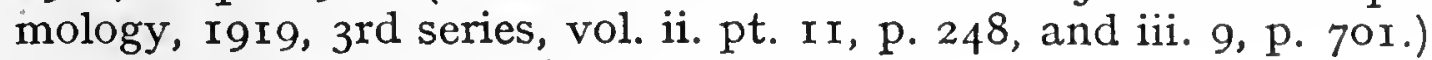

Hyrte. Corrosions Anatomie. Vienna, r873.

Jackson and Connor. "A Wax Model of the Nasal Cavity and Paranasal Sinuses," Annals of Otology, Rhinology, and Laryngology, Sept. I9I7, p. I.

Јасову. "Über die Neuroglia des Sehnerven," Klinische Monatsblätter für Augenheilkunde, r905, xliii. i. p. I29.

Joerss. "Zur Frage der Tränensackdrüsen," Klinische Monatsblätter für Augenheilkunde, r905, xalii. i. p. 392.

Johnson. "Contributions to the Comparative Anatomy of the Mammalian Eye, chiefly based on Ophthalmoscope Examination," Philosophical Transactions of the Royal Society of London, Series B, I90I, cxciv. p. I.

KALT. Article on the Lacrimal Gland in the Encyclopédie Française d'Ophtalmologie. Paris, r9o3.

KeIBer and Mall. Manual of Human Embryology. Lippincott, Philadelphia and London, rgro and rgr2.

Kerth, A. Human Embryology and Morphology. E. Arnold, London, I9r3.

KIDD. "Notes on the Eyebrows of Man," Journal of Anatomy and Physiology, 1904, xxxviii. p. I05 (iii.).

Krllian. Die Nebenhöhle der Nase, etc. (Jena, r903), and Accessory Sinuses of the Nose and their Relations to Neighbouring Organs, Patterson's translation, Chicago, 1904.

Kirchstein. "Über die Tränendrüse des Neugeborenen und die Unterschiede derselben von der des Erwachsenen," Inaugural Dissertation. Berlin, 1894. (Quoted by Picou, Schirmer, Merkel.)

KLODT. "Zur vergleichenden Anatomie der Lidmuskulatur," Archiv für mikroskopische Anatomie, I893, xli. p. r.

KNoTr. "On the Cerebral Sinuses and their Variations," Journal of Anatomy, r882, xvi. p. 27.

Koenigstein. " Notizen zur Anatomie und Physiologie der Orbita," Beiträge zur Augenheilkunde, r896, iii. Heft xxv. p. 399.

KoEPPE. "Klinische Beobachtungen mit der Nernstspaltlampe und dem Hornhaut Mikroskop," Graefe's Archiv für Ophthalmologie, r918, vol. xcvii. pp. 1, 34, 198, 346 . 
KoganeI. "Cribra cranii und cribra orbitalia," Mitteil. aus der med. Fakultät der Kaiserlichen Universität zu Tokyo, I9I3, x. p. II3.

KraUSE, C. Handbuch der Anatomie des Menschen, I842, ii.

KRAUSE, W. (i.) "Über die Drüsen der Conjunctiva," Zeitschrift für rationelle Medizin, I854, iv. p. 337.

(ii.) "Termination of the Nerves in the Conjunctiva," Journal of Anatomy and Physiology, I867, vol. i. p. 346.

(iii.) Handbuch der menschlichen Anatomie, I879, vol. ii. p. 29 (on spinae orbitales).

Krauss, W. (i.) "Beiträge zur Anatomie, Physiologie und Pathologie des orbitalen Venensystems," Archiv für Augenheilkunde, I9Io, lxvi. pp. I63, 285 .

(ii.) "Über die Anatomie der glatten Muskulatur der Orbita und der Lider, speziell die Membrana orbitalis musculosa," Münchene Medizinische Wochenschrift, I9I I, lviii. 38, p. I993. (Abstract given in Zeitschrift für Augenheilkunde, I9I2, xxviii.)

(iii.) " Über die Anatomie der glatten Muskulatur der Orbita und der Lider, speziell die Membrana orbitalis musculosa" (in the newborn), Archiv für Augenheilkunde, I9I2, vol. xxi. p. 977.

Krenbiel. Die Muskulatur der Thränenwege und der Augenlider. Stuttgart, 1878 .

Kuntz. "The Development of the Sympathetic Nervous System in Man," Journal Comparative Neurology, I920, xxxii. p. I 73.

KUSEL. "Contribution à la connaissance des tâches pigmentaires dans la conjonctive humaine." Abstracted in Annales d'Oculistique, I 907 , vol. I37, p. 58 .

LAGRange. Article on the General Anatomy of the Orbit, Encyclopedie Française. Paris, I903.

LANDolt. Article on the Anomalies of the Motor Apparatus of the Eye, in Norris and Oliver's System of Diseases of the Eye.

Landström. “ UUber Morbus Basedowii," Nord. Med. Ark. Stockholm, 1908. (Abstract in Nagel's Jahresbericht für Ophthalmologie, I907, p. 472.$)$

LANG. "Zur Entwicklung des Tränensfuhrapparatus beim Menschen," Anatomischer Anzeiger, I9II, xxxviii. p. 56r.

LANG and Treacher Collins. Article "Congenital Malformations and Abnormalities of the Human Eye," in Norris and Oliver's System of Diseases of the Eye.

LANGER. (i.) " Ủber die Blutgefässe im Augenlider," Medizinisches Jahrbuch, Wien, I878, p. 329.

(ii.) "Der Sinus cavernosus der harten Hirnhaut" Sitzungsb. der math.-nat. Klassen der Kaiser-Akademie der Wissen., Wien, I 885 , xci. iii. p. 307.

LANGLEY and ANDERson. "The Action of Nicotine on the Ciliary Ganglion, etc.," Journal of Physiology, r892, xiii. p. 460.

Lans. Weekbl. v. Geneesk. Aug. rgor. (Abstracted in Annales d'Oculistique, I902.)

LAw. "Comparative Anatomy of the Eye," The Ophthalmic Record, I905, xiv. p. 3I.

LAWRENCE. "On the Position of the Optic Commissure," Proceedings of the Anatomical Society, May I894, in the Journal of Anatomy and Physiology, 1894, xxviii. p. xviii.

LAwson. "The Bacteriology of the Normal Conjunctival Sac," Transactions of the Jenner Institute, I899, 2nd series, p. 56 . 
Leber. "Die Circulations- und Ernährungsverhältnisse des Auges," Graefe-Saemisch Handbuch, I 876 , ii. pt. 2, p. 302.

LeboucQ. "Étude sur les voies lymphatiques de l'œil et de l'orbite," Archives de Biologie, I9 4 $^{-1}$ 5, Xxix. p. I.

LEDERER. " Über Augenbewegungen und Augendrück," Klinische Monatsblätter für Augenheilkunde, I9I2, vol. $5^{\circ}$.

Ledouble. (i.) Traité des variétés du système musculaire de l'homme. Paris, I 897 .

(ii.) Traité des variations des os du crâne de l'homme. Paris, I 903.

(iii.) Traité des variations des os de la face de l'homme. Paris, I 906.

LEPAGE. Thèse doct. Bordeaux, I 909 (Orbicularis). Quoted by Picou.

LEsshaft. "Über den Musculus orbicularis orbitae und seiner Einfluss auf der Mechanismus der Tränenabsonderung," Archiv für Anatomie und Physiologie, r868, p. 265.

Levinsohn. (i.) "Über das Verhalten der Nervenendigungen in den äusseren Augenmuskeln des Menschen," Graefe's Archiv für Ophthalmologie, I90I, liii. p. 295.

(ii.) “Über den Einfluss der äusseren Augenmuskeln auf dem intraokularen Drück," Graefe's Archiv für Ophthalmologie, r9ı, Ixxvi. i. p. I 29.

Lewirsky. "Die Anatomie und Pathologie der Tenon'schen Kapsel," Inaugural Dissertation. Odessa, I910. (Abstracted in Archives of Ophthalmology (Knapp), I9II, xl. p. 34I, and in Nagel's Jahresbericht für Ophthalmologie, I9IO, p. 3o.)

Lrchal. "Anatomy and Histology of the Nasal Tear Duct of the different Domestic Animals," Anatomischer Anzeiger, I9I9, I I-I2, p. 296 and p. $34 \mathrm{I}$.

Liebrecht. (i.) " Schädelbruch und Auge," Archiv für Augenheilkunde, I 906 , vol. lv. p. 36.

(ii.) "Schädelbruch und Sehnerv," Graefe's Archiv für Ophthalmologie, r9 2, lxxxiii. p. 525 .

LINDAHL. "Étude sur l'action bactéricide des larmes," Geneva Ophthalmological Society, 1907, 34. (Abstracted in Revue générale d'Ophtalmologie, I 908.)

Lockwoon. "The Anatomy of the Muscles, Ligaments, Fascia of the Orbit, etc.," Journal of Anatomy and Physiology, I886, xx. p. I.

LOR. " Du méchanisme des mouvements palpébraux," Société Belge d'Ophtalmologie, Séance Avr. I898. (Abstracted in Annales d'Oculistique, 1898 , cxx. p. 46.)

Loeb. "A Study of the Anatomical Relations of the Optic Nerve to the Accessory Cavities of the Nose," Annals of Otology, Rhinology, and Laryngology, June I 909.

Löwental. (i.) "Beiträge zur Kenntnis der Harder'schen Drüse bei den Säugetieren," Anatomischer Anzeiger, I892, vii. p. 546.

(ii.) "Zur Kenntnis der Glandula infraorbitalis einiger Säugetiere," Anatomischer Anzeiger, I894, 2, x. p. I23.

(iii.) "Nouvelles recherches sur les glandes sous-orbitaires, etc.," Bibliographie Anatomique, I909-IO, xix. p. 30I.

Lowman and Lloyd Mrlls. "The Effect of Faulty Skeletal Alignment upon the Eyes," American Journal of Orthopedic Surgery, Dec. I9I8, xvi. p. 459 .

Lutz. "Explanation of Jaw-winking Phenomenon," Archives of Ophthalmology (Knapp), I9r9, vol. xlviii. p. I44. 
Macalister. (i.) "On the Presence of a Lacrymo-jugal Suture in a Human Subject," Proceedings of the Royal Irish Academy, 1874, ii. 2. p. $5^{8}$.

(ii.) "Additional Observations on Muscle Anomalies in Human Anatomy," 3 rd Series (Orbit), Transactions of Royal Irish Academy, I $875, \mathrm{xxv}$. p. 7 .

(iii.) "Notes on the Varieties and Morphology of the Human Lacrimal Bone and its Accessory Ossicles," Proceedings of the Royal Society, I884, vol. xxxvii. p. 229.

(iv.) Text-book of Human Anatomy. Griffin, London, I889.

Maddox. Tests and Studies of the Ocular Muscles, 2nd edition. Keystone Publ. Co., Philadelphia, I907.

Magath. "A Variation in the Distribution of the Nervus Abducens," Archives of Ophthalmology (Knapp), r9r9, xlviii. I. p. 67.

Manz. "Über neue eigentümliche Drüsen am Corneabrande und über den Bau des Limbus conjunctiva," Zeitschrift für rationelle Medizin, $\mathrm{r} 859,3$. v. p. 122 .

MARINESCo. "Sur la nature du ganglion ciliaire," Comptes Rendus de la Société de la Biologie, Paris, I908, i. lxiv. p. 88.

MARTYN. "Notes on a Case of Anomalous Innervation of the Levator Palpebrae Superioris," British Journal of Ophthalmology, r9r9, iii. p. 3 IO.

Matrs. "Entwickelung der Tränenableitungswege," Zeitschrift für Augenheilkunde, I905, xiv. i. p. 222, and I906, xvi. ii. p. 303.

Mazrarski. "Über den Bau und die Einteilung der Drüsen : die Tranendrüse," Anatomische Hefte (Merkel und Bonnet), r902, xviii. p. 215.

MENDEL. "Ứber den Kernursprung des Augen-Facialis," Neurologisches Centralblatt, 1887,23, p. 537.

MERKEL. (i.) "Der Musculus superciliaris," Anatomischer Anzeiger, I 887, I. p. 17.

(ii.) Handbuch der topographischen Anatomie, 2nd ed., Igor.

Merkel and Kallius. Graefe-Saemisch Handbuch der Augenheilkunde, I901, 2. Aufl., I. Bd., I. Kap. (and new ed., I904, i. I, p. I).

MetchnikofF. "Über die Beschaffenheit der Augenlider bei den Mongolen und Kaukasiern," Zeitschrift für Ethnologie, I874, vi. p. I 53 .

MEYER. “Zur Anatomie der Orbitalarterien," Morphologisches Jahrbuch, I887, xii. p. 4I 4 .

Mrlls, Lloyd. "The Effects of Faulty Cranio-spinal Form and Align. ment upon the Eyes," American Journal of Ophthalmology, July I9I9, ii. 7, p. 493 .

Mises, von. "Über die Nerven der menschlichen Augenlider," Sitzungsberichte der Königlichen Akademie der Wissenschaften. Wien, r882, lxxxv. 3, p. I 72.

Moll. "Bemerkungen über den Bau der Augenlider des Menschen," Graefe's Archiv für Ophthalmologie, r857, iii. 2. p. 258.

Monesi. (i.) "Die Morphologie der fötalen Tränenwege beim Menschen," Klinische Monatsblätter für Augenheilkunde, I904, xlii. r. p. I.

(ii.) "Observations on the Comparative Anatomy of the Lacrimal Passages," Bulletino della scienza med., Jan. I905. (Abstracted in Revue générale d'Ophtalmologie, I907, p. 350.)

(iii.) "Embryology and Comparative Anatomy of the Lacrimal Passages," Annali di Ottalmologia, xxxv. 868. (Abstracted in Revue générale d'Ophtalmologie, I907, p. 62.)

Morris. A Treatise on Human Anatomy. Churchill, London, 1898.

Most. "Über die Lymphgefässe und die regionären Lymphdrüsen der 
Bindehaut und der Lider des Auges," Archiv für Anatomie und Physiologie, I905, 2/3, p. 96.

Motais. L'Appareil moteur de l'œil. Paris, I887.

MüLLER, H. (i.) “Über einen glatten Muskel in der Augenhöhle des Menschen und der Säugetiere," Zeitschrift für wissenschaftliche Zoologie, r 858 , ix. p. $54 \mathrm{I}$.

(ii.) "Über glatte-Muskeln an den Augenlidern des Menschen und der Säugetiere," Verhandlungen der P. M. G. in Würzburg, I859, ix. p. 244 .

Nakagawa. "Über echte Papillen in der normalen Conjunctiva," Archiv für Augenheilkunde, I903, xlvii. p. 5I.

Neal. (i.) "The Morphology of the Eye-Muscle Nerves," Journal of Morphology, Philadelphia, I9I4, Xxv. pp. I-I87.

(ii:) "The History of the Eye-Muscles," Journal of Morphology, I9I8, XXX. 2. p. 434 .

NEDDEN. “Experimentelle Untersuchungen baktericider Substanzen im Auge nicht immunisierter Individuellen," Graefe's Archiv für Ophthalmologie, 1907, 1xv. p. 267. (Abstract from Annales d'Oculistique, I908, p. 369.)

Nicholls. "On the Occurrence of an Intra-cranial Ganglion in the Oculomotor Nerve in Scyllium Canicula, etc." (with observations on the ciliary ganglion), Proceedings of the Royal Society, Series B, June I9I 5, vol. Ixxxviii. p. 553 .

Nicola, B. Giorn. d. R. Accad. d. Med. d. Torino, I903. Quoted by Ledouble, I906, p. I6o (on the Zygomatico-frontal Suture).

Noll. " Morphologische Veränderungen der Tränendrüse bei der Sekretion," Archiv für mikroskopische Anatomie, I 9o I, Iviii. p. 487.

Norris and OlIver. System of Diseases of the Eye. Lippincott, Philadelphia, I900.

Nussbaum. "Entwicklungsgeschichte des menschlichen Auges," GraefeSaemisch-Handbuch der gesamten Augenheilkunde, 3rd ed., I9I2, Pt. I., Kap. viii.

OcHI. "Relation of the Ocular Muscles and Sclera in the Etiology of Myopia," American Journal of Ophthalmology, I919, ii. p. 675.

Oetreking. "The Processus Frontosphenoidalis of the Zygoma and its bearing on the Configuration of the Orbit," The Anatomical Record, I9I9, xvii. p. 25.

OGAWA. "Ứber Pigmentierung des Sehnerven."

(i.) Archiv für Augenheilkunde, I905, lii. p. 437.

(ii.) Archiv für Augenheilkunde, I906, 1v. p. ro6.

Onodr. (i.) "Das Verhältnis des Nerven opticus zu der Keilbeinhöhle und ins besondere zu der hintersten Siebbeinzelle," Archiv für Laryngologie, 1903, xiv. 360 .

(ii.) Der Sehnerv und die Nebenhöhlen der Nase. Wien, I907.

(iii.) The Relations of the Lacrymal Organs to the Nose and Nasal Accessory Sinuses. (English trans. by D. Mackenzie.) London, r9I3. OtTley. "On the Attachment of the Eye Muscles in Mammals," Proceedings of the Zoological Society of London, I879, p. I 21.

Parsons, J. H. (i.) "The Nerve Supply of the Lacrimal Gland," Royal London Ophthalmological Hospital Reports (May 1902), I899-I903, xv. p. $8 \mathrm{r}$.

(ii.) "The Ocular Circulation," Arris and Gale Lecture, R.C.S., I903. 
Parsons, J. H.-continued.

(iii.) The Pathology of the Eye. London, I904 and I905.

(iv.) "The Innervation of the Pupil," Royal London Ophthalmological Hospital Reports, I904, xvi. p. 20.

(v.) Diseases of the Eye. London, I907.

Pergens. "Les Dépôts pigmentaires dans la conjunctiva des nègres," Annales d'Oculistique, I898, cxx. p. 42.

Perlia. "Die Anatomie des Oculomotorius Centrums beim Menschen," Graefe's Archiv für Ophthalmologie, I889, xxxv. iv. p. 287.

Perna. “Un musculo trasverso anomalo della cavità orbitaria nell' uomo," Anatomischer Anzeiger, Verhand. 1905, xxvii. p. 215.

PESCHEL. "Congenitaler Epidermisüberzug der Tränenkarunkel," Centralblatt für praktische Augenheilkunde, I903, xvi. p. I48.

PEters. "Epiphora caused by Cornification of Epithelium near Caruncle," (Bibliography) Klinische Monatsblätter für Augenheilkunde, vol. 6I, p. 252. Abstracted in American Journal of Ophthalmology, I919, vol. ii. No. I.

Prcou. "Article on the Annexes of the Eye," in Poirier's Anatomy, I9I2, V. ii.

Piersol. Text-book on Human Anatomy, Ed. 7, r9r9. Lippincott, Philadelphia and London.

Pockley. "Epicanthus and Congenital Ptosis," Medical Journal of Australia, June I9I9, vol. i. sixth year, No. 25, p. 509.

PoIrIer. Traité d'anatomie humaine, éd. iii. I9II, vol. v. fasc. 2 (Les organes du sens). (Ostéologie, vol. i. ed. iii. I9I2.) Masson, Paris.

Pollock, Inglis. "The Persistence of the Nerve Plexus of the Iris after Excision of certain Ganglia" (abstract), British Medical Journal, Nov. I, I9I3, p. I 158.

PONCET. "Recherches sur la terminaison des nerfs dans la conjonctive," Archives de Physiologie. Paris, 1875.

Poole. "The Relations of the Superior Oblique Muscle of the Eye in Mammals," Journal of Anatomy and Physiology, I905, xxxix. p. I 54.

POPOFF. "Contribution à l'étude du repli semilunaire et de la caroncle lacrymale chez l'homme," Thèse de Paris, I9I2. (Abstracted in Nagel's Jahresbericht für Ophthalmologie, I9I2, p. 29.)

Posey and Spiller. "The Eye and Nervous System." Lippincott, Philadelphia and London, I906.

Power, H. (i.) Lectures on Diseases of the Lacrimal Apparatus, Lect. III., Pt. II., Lancet, I886, ii. p. 907.

(ii.) Case of Oxycephaly, Transactions of the Ophthalmological Society of the United Kingdom, I894, xiv. p. 2 I2.

Poyales. "On the Development of the Eye Muscles, Rectus Internus and Externus, in the Human Embryo" (in Spanish), Anatomical Record, I9I7, vol. I3, No. 6, p. 375 .

Puglisi-Allegra. " Sui nervi della glandola lagrimale." Riforma medica Roma, I903, July, xix. p. 795. (Abstracted in Anatomischer Anzeiger, 1903, xxiii. p. 392.)

QUAIN. Elements of Anatomy, 2nd edition, 1908-. Longmans Green, London.

RANGLARET. "Anatomie et pathologie des cellules ethmoidales," Thèse de Paris, I 896.

REICH. "Zur Histologie der Conjunctiva des Menschen," Graefe's Archiv für Ophthalmologie, I875, xxi. I. p. I. 
Reid. “The Presence of Lachrymo-jugal Sutures in Two Skulls," Journal of Anatomy and Physiology, r9ro, April, vol. xliv. p. 249.

Retzrus. "Über das Ganglion ciliäre," Anatomischer Anzeiger, I 894 , ix. 21 . p. 633 .

Rochat and Benjamins. "Einige Bemerkungen über die Anatomie der Tränenwege des Kaninchens," Graefe's Archiv für Ophthalmologie, I9I5, vol. Xci. p. 66.

Rochon-Duvigneaud. (i.) “ Recherches sur le développement des voies lacrymales," Archives d'Ophtalmologie, I900, xx. p. 242.

(ii.) "Note sur l'anatomie de l'orbite, etc.," Archives d'Ophtalmologie, 1903, xxiii. p. 769 .

Rollet. Section on the Accessory Sinuses of the Nose in the Encyclopédie Française d'Ophtalmologie, I903.

Rousseau. "Recherches sur l'étiologie et la pathologénie des iritis nonsyphilitiques," Annales d'Oculistique, I9 16, vol. cliii. p. I62.

Rouviere. "Le Tendon de Zinn, etc.," Bibliographie anatomique, I9I4, xxiv. p. 95 .

Roy. (i.) "Anatomie et physiologie comparées de l'œil et de ses annexes," Archives d'Ophtalmologie, r912, xxxii. p. 494.

(ii.) " The Eyesight of the Negroes of Africa," Archives of Ophthalmology (Knapp), I9I9, xlviii. I. p. 72.

Rush and SchaEfFer. "Supernumerary Orbital Muscles," Archives of Ophthalmology (Knapp), I 917 , xlvi. p. 524.

Russell, Risien. "An Experimental Investigation of Eye Movements," Journal of Physiology, I894, xvii. pp. I, 378.

Rutherford. "The Lacrymal Gland in Surgical Anaesthesia," British Medical Journal, I9I3, June 2I, p. I 3 I 3.

Ryder. Article on the Development of the Eye, Norris and Oliver's System of Diseases of the Eye, I9oo.

Salzmann. Anatomy and Histology of the Human Eyeball in the Normal State. Brown's translation, Chicago, rgr 2.

Santos-Fernandos. "Disposition anatomique du canal nasal chez le nègre," Recueil d'Ophtalmologie, I903, p. 5 OI.

SAPPEY. Anatomie descriptive. Paris, I 867.

SATtLER. (i.) "Beitrag zur Kenntnis der modificirten (Moll'schen) Schweissdrüsen des Lidrandes," Archiv für mikroskopische Anatomie, I 877 , xiii. p. 783 .

(ii.) "Über den sogenannten Landströmschen Muskel und seine Bedeutung für den Exophthalmus bei Morbus Basedowii," Bericht der ophthalmologischen Gesellschaft Heidelberg, I9 I , p. I8I. (Abstracted in Annales d'Oculistique, I9I I, cxlvi. p. 2II.)

(iii.) "Über die Markscheidenentwickelung im Tractus opticus, Chiasma und Nervus opticus," Graefe's Archiv für Ophthalmologie, I9I 5 , Xc. p. 27 I.

Schaeffer. (i.) "The Sinus Maxillaris and its Relations in the Embryo, Child, and Adult Man," American Journal of Anatomy, I910, x. p. 313.

(ii.) "The Lateral Wall of the Cavum Nasi in Man, with special Reference to the various Developmental Stages," Journal of Morphology, I910, xxi. No. 4, p. 6r3.

(iii.) "The Genesis and Development of the Naso-lacrimal Passages in Man," American Journal of Anatomy, I912, xiii. p. I.

(iv.) "The Nose, Paranasal Sinuses, Naso-lacrimal Passages .and Olfactory Organ in Man." Blakiston, Philadelphia, I920. 
SCHAFER. Text-book of Microscopic Anatomy in Quain's Elements of Anatomy, vol. ii. I. Longmans, London, I9I2.

SCHAPRINGER. Centralblatt für praktische Augenheilkunde, 1905, 29th year, P. I 29.

SchIEFFERDECKER. (i.) "Eine Eigentümlichkeit im Bau des Augenmuskels," Sitzungsberichte der Nieder. Gesell. zu Bonn, I904, Oct. etc. (Abstracted by Sobotta in Zeitschrift für Augenheilkunde, I905. xiv. p. I 86.)

(ii.) "Über die Lidmuskulatur des Menschen," Sitzungsbericht der Niederheimschen Gesellschaft zu Bonn, I905, Oct. B. xiv. p. 54.

Schirmer. Section on "Mikroskopische Anatomie und Physiologie der Tränensorgane," Graefe-Saemisch Handbuch der gesamten Augenheilkunde, I904. 2nd ed., Lief. 25 und 76.

Schmidt-Rimpler. (i.) “ Kurzsichtigkeit und Augenhöhlenbau," Graefe's Archiv für Ophthalmologie, I889, xxxv. p. I and p. 200.

(ii.) " Bemerkungen zu Stilling's Aufsatz," Fortschritte der Medizin, I889, p. 573 .

SCHWAlBE. "Lehrbuch der Anatomie des Auges," I887. Erlangen.

SCHWEGEL. "Knochenvarietäten" (On the Supra-Orbital Notch), Zeitschrift für rationelle Medizin, I 859, vol. v. 3, p. 283.

Schweinitz, Wilder, Ball, and Weeks. "The Sympathetic and the Eye," Section of Ophthalmology of the American Medical Association, May 1903. Abstracted in Annales d'Oculistique, 1904, cxxxii. p. 233 .

Scimemi. " Su la conduttura delle lagrime," Annali di Ottalmologia, I 892, xxi. I, p. 222, Archiv für Physiologie, I 892.

Seefelder. "Pathologisch-anatomische Beiträge zur Kenntnis der angeborenen Colobome des Auges," and other papers following. Graefe's Archiv für Ophthalmologie, I908, lxviii. p. 335 ; I909, 1xx. pp: 45,448 ; I9Io, lxxiii. p. 2 I6.

SEgGei. "Über die Abhängigkeit der Myopie vom Orbitalbau," Graefe's Archiv für Ophthalmologie, I890, xxxvi. ii.

Sesemann. “Die Orbitalvenen des Menschen, etc.," Archiv für Anatomie und Physiologie, I 869, p. I 54.

Sharpe. "The Cranial Deformity of Oxcephaly; its Operative Treatment, with a Report of Cases," American Journal of the Medical Sciences, r9r6, vol. cli. p. 840 .

Sherrington. "Observations on the Sensual Rôle of the Proprioceptive Nerve-supply of the Extrinsic Ocular Muscles," Brain, I918, xli. p. 332 .

Sherrington and Toser. "Receptors and Afferents of the Third, Fourth, and Sixth Cranial Nerves," Proceedings of the Royal Society, I9Io, vol. Ixxxii. p. 450.

Shommaker. "A Case of Supernumerary Eyelid," American Journal of Ophthalmology, 1914, xxxi. p. 225.

Sieur et Jacob. Les Fosses et leurs sinus. Paris, I9or.

SKILlERN. The Accessory Sinuses of the Nose. Philadelphia, I9I3.

Smith, Elliot. (i.) "Asymmetry of the Brain and Skull," Journal of Anatomy and Physiology, I907, xli. p. 236.

(ii.) "New Studies on the Folding of the Visual Cortex," etc., Journal of Anatomy and Physiology, 1907, xli. p. I99 and p. 237.

(iii.) The Central Nervous System in Cunningham's Text-book of Anatomy, 4th edition, I9I3. H. Frowde, London.

Speciale-Cirincione. "Ưber die Entwicklung der Tränendrüse beim Menschen," Graefe's Archiv für Ophthalmologie, I908, lxix. 2, p. I93. 
Sperino. "La ossificazione e la posizione della trochlea, etc.," Mem. R. Accad. di Scienze, Ser. III. vol. vi. (Abstracted in Schwalbe's Jahresberichte für Anatomie, I905, p. 85.)

Stanculeanu. (i.) "Recherches sur le développement des voies lacrymales chez l'homme et chez les animaux," Archives d'Ophtalmologie, 1900, $\mathrm{xx} . \mathrm{p} . \mathrm{I} 4 \mathrm{I}$.

(ii.) "Les Rapports anatomiques entre les sinus de la face et l'appareil orbito-oculaire," Arch. d'Ophtal., I902, xxii. p. I08 and p. 248.

Starling. Principles of Human Physiology. Churchill, London, I920, ed. iii.

Steiner. " Les Tâches pigmentaires de la conjunctiva," Annales d'Oculistique, I906, cxxxv. p. 466.

Stephenson. (i.) "Supernumerary carunculae lacrymales," Ophthalmic Review, r896, xv. p. 8.

(ii.) "Congenital Districhiasis," Ophthal. Review, rgor, xx. p. 350.

(iii.) "A Case of Ocular Torticollis," Proceedings Royal Society of Medicine, Sect. Ophth., May 7, 1913, vi. p. 90.

(iv.) "Eye Symptoms as the only Indication of Fractured Base of the Skull," British Journal of Ophthalmology, Nov. r9r9, iii. No. 2.

Stevens. (i). "Anomalies of the Ocular Muscles." Series of Papers in Archives of Ophthalmology (Knapp) for 1887, r 888, r889.

(ii.) "Facial Expressions as influenced by the Ocular Muscles," Journal of the American Medical Association of Chicago, 1892, xix. p. 257.

Stieda. "Ưber die Caruncula lacrymalis des Menschen," Archiv für mikroskopische Anatomie, r890, xxxvi. p. 29I.

Stilling. (i.) Schädelbau und Kurzsichtigkeit, Wiesbaden, x 888.

(ii.) Über das Wachstum der Orbita und dessen Beziehungen zur Refraktion. Archiv für Augenheilkunde, r890, xxii. 3, 4 .

(iii.) See also Fortschritte der Medizin, r889, pp. 444 and 647.

Stopford. "The Arteries of the Pons and Medulla Oblongata," Journal of Anatomy and Physiology, I916, 6, pp. I3I, 255, and 1917, li. p. 250 .

Struthers. "On the Anatomy and Physiology of the Oblique Muscles of the Eye in Man and Vertebrate Animals," Monthly Journal of Medical Science, I849, ix. p. Ir 43.

Sundwall. "The Lachrymal Gland," American Journal of Anatomy, Igr6, vol. $20, \mathrm{p}$. I 47.

Sutron. "The Fascia of the Human Orbit," The Anatomical Record, I920, Xviii. 2.

Swanzy. Article in Norris and Oliver's System of Diseases of the Eye, I900, vol. iv. p. 63I.

SwERSCHEwsky. "Die anatomischen und pathologischen Verhältnisse der Tränenableitungswege," Westn. Ophth. S. 549. (Abstracted in Nagel's Jahresbericht für Ophthalmologie, rgro, p. 37.)

TARTUFERI. "Anatomie pathologique des dacryocystites catarrhales, etc.," Archives d'Ophtalmologie, I902, xxii. p. I66.

TAYLOR. "Changes in the sella turcica in family optic atrophy," British Journal of Ophthalmology, 1919, May, iii. 5. p. I93.

Tenchini. "Di un emissario anomalo dell' orbito frontale," Monit. Zool. ital. 1905, anno xvi. Firenze. (Abstract given in Schwalbe's Jahresberichte für Anatomie, 1905, p. 90.)

Tenon. Mémoires et observations sur l'anatomie. Paris, 1806. 
TEPLiachine. " Recherches sur les nerfs sécrétoires de la glande lacrymale," Archives d'Ophtalmologie, I894, xiv. p. 40 I.

Terson. Section on Anatomy and Physiology of the Eyelids in the Encyclopédie Française d'Ophtalmologie, i. Paris, r903.

Testut. Traité d'anatomie humaine, 5 th edition, Paris, 1905. .

Testut and JAсов. Traité d'anatomie topographique, 3rd ed. Doin, Paris, I9I 4 .

Theobald. (i.) Article on Diseases of the Lacrimal Apparatus in Norris and Oliver's System of Diseases of the Eye.

(ii.) "Chief Functions of the Oblique Muscles of the Eye," Bulletin Johns Hopkins Hospital, I9I8, xxix. p. I 5.

Thomson, A. (i.) "The Orbito-Maxillary Frontal Suture in Man and the Apes, with Notes on the Varieties of the Human Lacrymal Bone," Journal of Anatomy and Physiology, I89o, xxiv. p. 349.

(ii.) "The Anatomy of the Human Eye, as illustrated by Enlarged Stereoscopic Photographs." Clarendon Press, Oxford, I9 I2.

Thomson, E. "Determination of the Influence of the Eye on the Growth of the Orbit by Experimental Enucleation of one Eye in Young Animals," Meeting of the Ophthal. Soc. of the United Kingdom, Nov. Igoo. (Abstracted in Archives of Ophthalmology (Knapp), I90I, XXX. p. 93.)

Thomson, St. Clair. (i.) "Cerebral and Ophthalmic Complications in Sphenoidal Sinusitis," Transactions of the Medical Society of London, I 906, xxix.

(ii.) "The Frequency of Orbital Manifestations of Nasal Sinusitis," The Ophthalmoscope, April igo8.

THоRsсн. "Beziehungen der Tränensackgrube zur Nase und ihren Nebenhöhlen," Klinische Monatsblätter für Augenheilkunde, I909, viii. p. 830.

Topinard. Éléments d'anthropologie générale. Paris, I 885 .

Toser. (i.) "On the Presence of Ganglion Cells in the Roots of the Third, Fourth, and Sixth Cranial Nerves," Physiological Society Proceedings, I9I2, July, p. xv. in the Journal of Physiology, rgr2, vol. xlv.

(ii.) See also under Sherrington.

Traquair. "The Anatomical Relations of the Hypophysis and the Chiasma," The Ophthalmoscope, I9I6, xiv. p. 562.

Tsuchida. " On the Oculomotor Nucleus," Arbeiten aus dem hirnanatomischen Institut in Zürich, I906, ii. (referred to in Piersol: not seen in original).

Tubby. "Is there a Connection between Skeletal Asymmetry and Defects of the Eye?" Transactions of the Ophthalmological Society, I919, p. 335 .

Turner, Aldren. " A Note on the Oculofacial Muscular Group," The Royal London Ophthalmological Hospital Reports, I893, xiii. p. 328.

Turner, Logan (i.) "The Accessory Sinuses of the Nose." Green, London, I90I.

(ii.) "The Relation of Disease of the Nasal Accessory Sinuses to Disease of the Eye," The Lancet, I908, vol. ii. p. 730 ; The British Medical Journal, I908, vol. ii. p. 730.

Turner, Sir W. (i.) "On a Non-striped Muscle connected with the Orbital Periosteum of Man," Natural History Review; I862, p. Io7.

(ii.) "The Infra-orbital Suture," Journal of Anatomy and Physiology, I 885 , vol. xix. p. 2 I 8 . 
UNderwood. "Anatomy and Pathology of the Maxilläy Sinus," Journal of Anatomy and Physiology, I910, xliv. p. 354.

VILLARD. " Recherches sur l'histologie de la conjunctivite normale," N. Montpellier médicale, r896, v. pp. 658, 672, 693.

VILlIGer. Brain and Spinal Cord, edition iii., I9I2. Translated by Piersol. Virchow, H. (i.) “Über Tenon'schen Raum und Tenon'sche Kapsel," Abhandlung der Kgl. Preuss. Akad. der Wissenschaft, I902.

(ii.) "Mikroskopische Anatomie der äusseren Augenhaut und des Lidapparatus," Graefe-Saemisch Handbuch der gesamten Augenheilkunde, 2nd ed., I905-ro.

Waldeyer. Article on Microscopical Anatomy in De Wecker and Landolt's Traité complet d'ophtalmologie, vol. i. p. I69. Paris, I 886.

Wallis. "Some Observations upon the Anatomical Relations of the Optic Nerves and Chiasma to the Sphenoidal Bone," The Practitioner, Jan. I9I 7, vol. xcviii. p. $4 \mathrm{I}$.

WARD, F. O. Outlines of Human Osteology. Renshaw, London, I858, new edition.

Weiss. (i.) Beiträge zur Anatomie der Orbita. Tübingen, -I89o.

(ii.) "Über das Verhalten von M. rectus externus und rect. internus bei wachsender Divergenz der Orbita," Archiv für Augenheilkunde, I 894, xxix. 3, 4, p. 298.

(iii.) "Über die Wachstum des menschlichen Auges," Anatomische Hefte (Merkel und Bonnet), I897, viii. p. I93.

WelCKer. "Cribra orbitalia," Archiv für Anthropologie, I887, xvii. p. I.

WERnCKE. “Ein Beitrag zur Anatomie des Tränensackes speziell zur Frage der Tränensackdrüsen," Klinische Monatsblätter für Augenheilkunde, I905, xliii. I. p. I9I.

West. "Eine Probe zur Feststellung der Funktionsfähigkeit des Tränenröhrchens (eine Canaliculusprobe) und ihre klinische Bedeutung," Zeitschrift für Augenheilkunde, I9I8, vol. xxxix. p. 260.

Whitinall. (i.) "On a Ligament acting as a Check to the Action of the Palpebrae Superioris Muscle," Journal of Anatomy and Physiology, I9ro, xlv. p. r 3 I.

(ii.) "An Instance of the Retractor Bulbi Muscle in Man," Journal of Anatomy and Physiology, I9II, xlvi. p. 36.

(iii.) "On a Tubercle on the Malar Bone," Journal of Anatomy and Physiology, I9I I, xlv. p. 426.

(iv.) "The Relations of the Lacrimal Fossa to the Ethmoidal Cells," The Ophthalmic Review, Nov. I9I I.

(v.) "The Naso-lacrimal Canal: the Extent to which it is formed by the Maxilla and the Influence of this upon its Calibre," The Ophthalmoscope, Oct. I9I2, x. Io.

(vi.) "The Shape of tiue Orbit: its Influence upon the Eyeball (Stilling's theory)," The Ophthalmic Review, Feb. I9I3.

(vii.) "The Relation of the Naso-lacrimal Canal to the Maxillary Antrum: Formation of the Lacrimal Recess," The Ophthalmic Review, Feb. I9I3.

(viii.) "The Ligamentum Palpebrarum Mediale: Why its Removal in Ablation of the Lacrimal Sac does not necessarily entail Ectropia of the Lower Eyelid," The Ophthalmoscope, April I9r3.

(ix.) "The Levator Palpebrae Superioris: the Attachment and Relations of its Aponeurosis," The Ophthalmoscope, May I9I4.

(x.) "Some Instances of Abnormal Ocular Muscles." The Anatomical Record, I92I. 
Wiedersheim. “The Structure of Man." Bernard's Translation, I895. London.

Wilson and Pike. "The Mechanism of Labyrinthine Nystagmus, etc.," Archives of Internal Medicine, Jan. I9I 5, XV. I. p. 3 I.

Wirt. "Ausbreitung der Stirnhöhlen und Siebenzellen über die Orbita," Anatomische Hefte (Merkel und Bonnet), I908, vol. xxxvii. p. I 45.

WolfF. "Ửber die Sehne des Musculus levator palpebrae superioris," Zeitschrift für Augenheilkunde, 1905, xiii. p. 440, and I906, xv. p. 596.

Wolfring. " Untersuchungen über die Drüsen der Bindhaut des Auges," Centrallblatt für die medizinische Wissenschaft, $1872, x$. p. 852 .

Wood, CAsey. American Encyclopedia of Ophthalmology. Cleveland Press, Chicago, I9I3.

ZABEL. "Varietäten und vollständiges Fehlen des Tränenbeines beim Menschen," Anatomische Hefte (Merkel und Bonnet), I900, Band Xv. p. I53.

ZaLuskowski. "Bemerkungen über den Bau der Bindehaut," Archiv für mikroskopische Anatomie, I887, xxx. p. 3i I.

ZANDER. (i.) Beiträge zur Kenntnis der mittleren Schädelgrube mit besonderer Berücksichtigung der Lage des Chiasma opticum," Anatomischer Anzeiger, r 896, xii. p. 457.

(ii.) "Beiträge zur Kenntnis der Hautnerven des Kopfes," Anatomische Hefte (Merkel and Bonnet), I897, ix. p. 59.

ZEIS. "Anatomische Untersuchungen der Meibom'schen Drüsen des Menschen und der Tiere," Von Ammon's Zeitschrift für die Ophthalmologie, I 835 , iv. p. 23 I.

Zeitschmann. (i.) "Vergleichende Histologie: Untersuchungen über den Bau der Augenlider der Haussäugetiere," Graefe's Archiv für Ophthalmologie, I904, lviii. p. IоI.

(ii.) "Zur Frage des Vorkommens eines Tarsus in Lide der Haus" säugetiere," Graefe's Archiv für Ophthalmologie, I904, lix. p. I66.

ZimmermanN. "Beiträge zur Kenntnis einiger Drüsen und Epithelien. Die Tränendrüsen des Menschen," Archiv für mikroskopische Anatomie, I 898 , lii. p. $55^{2}$.

ZINN. Descriptio anatomica oculi humani. Göttingen, I755.

ZojA. "Sopra un soleo men noto dell' ossó frontale." Pavia, I884. Researches quoted by Ledouble, I903, p. I73.

ZuckerkandL. (i.) Normale und pathologische Anatomie der Nasenhöhle. Wien, I882, I892.

(ii.) Bardeleben's Handbuch der Anatomie des Menschen. Jena, I896. (Quoted by Graf Spee.)

(iii.) Atlas der topographischen Anatomie des Menschen. Wien, I 904 , p. II 4 .

ZWEIBACK. “Über die Incisurae supraorbitalis und frontalis des Stirnbein und ihre Varietäten," Inaug. Dissertation. Königsberg, I 9oo. Quoted by Ledouble, 1903, p. 196.

The following authors have been mentioned without reference to particular papers :

ADAMUĚCK.

AGNEW.

Albinus.

ArLoING.
AUERBACH.

BASEDOW.

BeAdi.ES.
Bedell.

BEER.

BELL, Sir C.

BERT. 
Bianchi.

BLUM.

Blumberger.

BocH.

BolTON.

BONNET.

BOURGEOIS.

Bramwell.

BRAUNE.

Brown-Sequard.

Campbell.

Casserius.

Chase.

Chimanowski.

Chudzinski.

COATS.

CoHN.

Colombo.

Contela.

COOPER.

DARKSCHEWITSCh.

DARREL.

Delbet.

DOHN.

Duchenne.

DUPUY.

\section{EDINGER.}

EgeberG.

Elschnig.

Ferrall.

Flechsig.

Foltz.

Frerichs.

Friteau.

Gaillard.

GaLEN.

GedDes.

Geissler.

Generali.

GIAN UZZI

GirARD.

Grasset.

Greeves.

GUdDEN.
HALLIBURTON.

HARDER.

HARTRIDGE.

HARVEY.

HASNER.

HAWLEY.

HENKE.

HEWETT.

HIRSCH.

HoEve.

HUMPHRY.

HUSCHKA.

ISCHREYT.

JoNES, F. WOOD.

Koenig.

Kolmus.

KRankow.

KUHNT.

LAFFAY.

LANGE.

LANGER, VON

LEMKE.

LEWIN.

LEWIS.

Luschka.

MaGaARD.

MAGRON.

MaIER.

MAJEWSKY.

Malaigne.

MATHEWSON.

Meibomius.

Meissner.

MICHEL.

Mitchell.

Molinette.

Monakow.

Motт.

Nance.

Natale.

Nielson.

OPPENHEIMER.
Panas.

Pegler.

Polack.

RADINSKI.

RAUBER.

Recklinghause.

REX.

Richet.

RIOLAN.

RoBertson.

Ruge.

Rupter, DE.

Salus

SALZER.

SANDIFORT.

SCHOUTE.

Shane.

SOMMERING.

STEFFAN.

STROMEYER.

SZILI.

TAIllefer.

TERIINCK

Thane.

ThOMSEN.

Tiedemann.

Tillaux.

TOLdT.

Toti.

Tourneux.

TREves.

VALENTIN.

VERGA.

VEsalius.

VLACOVICH.

Vomus.

Vossius.

WEBER.

WECKER, DE.

WEEKS.

WICHERKIEWICZ.

WINSI_OW. 



\section{N DE X}

Accessory or air-sinuses of nose. See Sinuses, air

Accommodation of lens, accessory muscles of, I 81 action of sympathetic nerves on, 357

Agger nasi, 56

air-cell of, 68

Anaesthesia, behaviour of lacrimal gland under, 2I 7

Angle of eyelids, I I I

of orbit, basic, 92

naso-malar, 9I

walls, 92

trochlear, 273, 276

Ankyloblepharon, 187

Annulus tendineus communis (Zinnii), 260

Antrum, maxillary (Highmorii), $63,57,28,16,44$

Apertures, of orbit, 79

Aponeurosis, epicranial (galea aponeurotica), I07, I09, I 8 I

of levator palpebrae superioris muscle, $135^{\circ}, 136$

orbito-ocularis (Tenon's capsule), 286

Arachnoidea, relation to optic nerve, 362

cisternae of, $324,368,369$

Arc of contact of recti muscles, 270 Arcade, Arcus, marginal (marginale), of septum orbitale, $8 \mathrm{I}, \mathrm{I} 32$

tarsal (tarseus), I69

superciliaris, I2
Area, cortical, of brain. See Centres, cortical macular, of retina, 386 striata, of brain, 374

Areola, palpebral, I I 3

Artery, arteries, arteria, arterialabnormalities, 308

angular, I63, I48, 243

arcades, tarsal (arcus tarseus), I 69

carotid, internal, 301, 316, 368

centralis retinae, 303

ciliary, anterior, 305, I99

posterior, 304

circulus arteriosus iridis, 305, 200

conjunctival, anterior and posterior, I99, I69

of cornea (pericorneal plexus), 200

dorsalis nasi, 307, 167

ethmoidal, 306

of eyelids, I68, 306

facial, I 62,308

frontal, 307,167

infra-orbital, 307, 243, 168, 42, $228,2 \mathrm{I} 3$

lacrimal, 305, I67, 308

maxillary, external (facial), I62 meningeal, middle, 308, 305, 35 nasal (dorsalis nasi), 307, I67, 308

ophthalmic, 300,308

palpebral, lateral, I69, 305

medial, I68, 306

plexus, pericorneal, 200

supra-orbital, 306, I67, I I 
Artery, arteries, arteria, arterial (contd.) -

tarsal (arcades), I69

temporal, superficial, I66, I Io

Atrium (of nasal cavity), 56

Axis of movements of eyeball, 276 optica (visual), 254

orbital, 93

Blepharospasm, I 70,248

Bone. See also under Os, Ossicle concha nasalis, inferior, 56,70 ethmoidal, air-cells of bulla, $57,5^{8,60}$

hiatus semilunaris of, 56,63 , 68

labyrinth, 58, 60

lamina papyracea, or orbital plate of, $47,5^{\mathrm{I}}, 6 \mathrm{O}$

frontal, $9,7,24,47$

accessory, 30

angular process, lateral (zygomatic) of, I2, I3, I9 angular process, medial of,

I 7, 19

sinus of, 59

lacrimal, 48

crests of, 64, 2I, I7

hamular process

facialis) of, 48,70

rudimentary, 75,76

malar. See Zygomatic

maxilla, frontal process of, I6, I 7, 18,49

lamina infra-orbitalis of, 42 sinus of (antrum), 63, 58 sulcus lacrimalis of; 70,75 wall, anterior of, $\mathrm{I} 6$

maxillo-naso-lacrimo-frontal, I9

palatine, 47

air-cell of, 44, 62

orbital process of, 4I

sphenoidal, ala parva of, 24, 25

ala magna of, 33, 35

body of, $47,368,62$

fossa hypophyseos (sella turcica) of, 368,369
Bone (contd.)-

sphenoidal (contd.) -

sinus of, $62,5^{8}$

sulcus chiasmatis of, 367,368

turbinated process of, 47

turbinated inferior, 56,70

Wormian (ossa suturarum), 47 , 34,19

zygomatic, 7, I2, I3, 39

canals, 34, 14

development of, 35

mala bipartita, I3

orbital plate of, 37

orbital tubercle of, I3, 20

process, fronto-sphenoidal of, I2, I3

processus marginalis of, 13,20

spina of, I3

zygoma, relation to brair, 2 I

relation to infra-orbital fissure, 39

optic canal, 26

semilunar ganglion, 338

Bourrelet sénile, I 4

Brain-

relation to eyebrows, I05

lateral wall of orbit, 37

margin of orbit, 2 I

optic canal, 27

optic chiasma, 368

roof of orbit, 30, 32

skull, 379

zygoma, 2I

angular gyrus of, 377,378

areas of. See Centres

cortex, centres in. See Centres

cuneus of, 373

fissure, calcarine, 375

retinal representative in, 386

sulcus orbitalis, 32

Bulb of eye (bulbus oculi). See

Eyeball

Bulla ethmoidalis, 57,58

Canal, Canals-

ethmoidal, 5 I

frontal, Io

infra-orbital, 42 
Canal, Canals (contd.)-

naso-lacrimal (lacrimal), 7o, 90 casts of, 73

incisura of, 44, 48, 70

optic, 26

pterygoid (Vidian), 62

supra-orbital, 9, II

Vidian (pterygoid), 62

zygomatic, 34, I 4

Canaliculi, lacrimal, 222

Canthi of eyelids, II I

Capsule, c. adiposa bulbi (orbital fat), 297

of lacrimal gland, 208

of Tenon (fascia bulbi), 286

injections of, 298

Cartilages, tarsal, I $_{50}$

Caruncle, lacrimal, I 59

development of, I 86

Casts of orbit, 3

of air-sinuses, 55

of lacrimal passages, 73

Cell, Cells, Cellula-

of agger nasi, 68

ethmoidal, 60

abnormal, 44

development of, 57

relation to optic canal of, 27 , 28

relation to orbital roof of, 29

frontal. See Sinus

fronto-ethmo-ungueale, 68

lacrimalis, 68

lacrimo-ethmoidal, 66

orbito-ethmoidal, 29

palatinal, 44, 62

Centre, Centres-

cortical, of brain, 376,174

for eyelids, cortical, 378

for eye movements, cortical, 378

for facial nerve, cortical, I 74

of movement of eyeball, 276 , 254

for pupillary movements, cortical, 354

spinal (centrum ciliospinale inferius), $35^{6}$
Centre, Centres (contd.)-

visual, higher (or cortical), visuo-sensory, $373,376,386$ lower, 384,372

visuo-psychic (visual memory), 376

for winking, 378

for word memory, 378

Cerebellum, relation to ocular movements of, 378,389 , $39 \mathrm{I}$

Chiasma, optic, 367,382

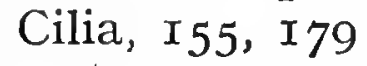

abnormal, $\mathrm{r}_{54}$

development of, $\mathrm{x} 84$

Cisterna basilis (interpeduncularis), 324, 368, 369

Colliculus, inferior, 372,383

superior, 384,372

Coloboma of eyelid, I87

of optic nerve, 360

Commissure of eyelids, II I

of Gudden, 383, 370

of Meynert, 383

optic, $36 \%, 382$

Concha nasalis, inferior, $56,7 \mathrm{I}$

Conchae nasales, 55

Conjugate deviation, $390,336,378$

Conjunctiva, 188

bacteria of, 213

crypts of, 197

dermoid of, 187

development of, 184,202

fornix of, I90, II3

glands of, 195

nerves of, 202

retinacula of, I9I

structure of, 193

vessels of, I99

Conus of optic disc, 365

Cornea, nerves of, 202, 353

vessels of, 199

Corpus adiposum orbitae (orbital fat), 297

quadrigeminum, superior, 384 , $37^{2}$

geniculatum laterale, $384,37 x$, 372 


\section{Corpus (contd.) -}

geniculatum (contd.) -

mediale, 370

Cortical centres. See Centres

Coussinet adipeux du sourcil, ro9

Crrests, lacrimal, 64, 2I, I7

Cribra orbitalia, 3I

Cryptophthalmia, I87

Crypts, conjunctival, I97

Cuneus, of brain, 373

Cysts, dermoid, of eyelids, I87

Dacryon, the point, 86, 66

Decussation, fountain, of Meynert (decussatio tegmenti dorsalis), 384

Dehiscences, of infra-orbital sulcus, 44

of optic canal, 29

of orbital walls, 5I, 29, 44

of sphenoidal sinus, of wall, 62

Dermoid, of conjunctiva, I87

cysts, of eyelids, I87

Development. See under Individual parts

Deviation, conjugate, 390,336 , 378

Diploë, diploic vein and nerve, $31,165,342$

Diplopia, 33I, 335

Disease, Basedow's, 84, 3I 3

dental, in relation to orbit, 16

Distance, axial, of orbital margins, 85

inter-oblique, 102

Duct, Ducts-

ductus lacrimales (canaliculi), 222

excretory (ductuli excretorii), of lacrimal gland, 2 IO

fronto-nasal, 58, 6o, 68

naso-lacrimal (nasal, lacrimal), 233,237

Dura mater, 260, 362, 367, 26

Ectropion, I 78, 232

Epicanthus, II6, II4

Epiphora, 244, 249
Ethmoidal bone. See under Bone

Exophoria, I03

Exophthalmos, 84, 102, 256, 299, 3IO, 3 I 3

Eyeball, 25I

centre, cortical, for movements of, 378

centre of movement of, 276

development of, 360

dimensions of, 254

enucleation of, changes in orbit after, 97

form of, relative to shape of orbit, 102

myopic, oblique muscles in, $28 \mathrm{I}$ point of rotation of, 276,254 position in orbit of, 256,95

Eyebrow, I05

coussinet adipeux of, Io9

injections into, IIO

movements of, I 79

nerves of, IIO

vessels of, I Io

vibrissae of, IO7

Eyelashes. See Cilia

Eyelids, II I

abnormalities of, I87

accessory or third, 203

angles of, III

areola, palpebral of, II 3

canthi of, III

centre, cortical for, 378

commissures of, III

development of, $\mathrm{I} 84$

dissection of, I2O

epicanthus of, II6, II4

epitarsus, 203

fascia, palpebral (septum orbitale) of, 130

folds of, I 13

glands of (marginal), I 57, I53

hernia adiposa of, II 4

injections into, 137

lacus lacrimalis of, I I 8

ligaments of, broad (septum orbitale), I30

palpebral (tendo oculi, or tarsal), I 47 
Eyelids (contd.)-

margin of, I I 8, I 54

development of, 185

nerve plexus of, 176

structure of, I 55

movements of, $\mathrm{r} 8 \mathrm{o}$

muscles of, I26, I 33

action of, I 77

clinical features of, I8I

nerves of, $\mathrm{I} 72$

opening of, $\mathrm{xI} 7$

pigment in, II $3, I 25$

ptosis adiposa, II4

relations, deep, of, II 8

septum orbitale, I30

skin of, I 25

structure of, II9

supernumerary, I88, 203

surface anatomy of, II 2

tarsal plates, $\mathrm{I}_{4} 6$

vessels of, I $6 \mathrm{I}$

Face, development of, 183

vessels of, 162

Fascia, fasciae bulbi (Tenoni), 286

relation to conjunctiva, 192

lacrimal ("deep fascia" of eyelids), 227, I27, I28, 8I

musculares (sheaths of muscles), 29 I

orbitales, 286

orbito-palpebralis (septum orbitale), I 30

palpebral (septum orbitale), I 30

retro-bulbar, 286

sheath of levator palpebrae superioris, I4I

tarso-orbitalis (septum orbitale), 130

Fasciculus, medial (posterior) longitudinal, I74, 384, $389,39 x$

Fissure, Fissura-

calcarine, of brain, $37.5,386$

orbital, inferior, 39

superior, $37,27,260$
Fissure, Fissura (contd.)-

palpebrarum, I I I

sphenoidal (superior orbital), $37,27,260$

spheno-maxillary (inferior orbital), 39

spheno-zygomatic, 40

Fold, Folds-

epicanthus of eyelids, II6, II 4

of eyelids, II 3

falciform, of dura, 26, 367

of muscle sheaths, 293

of lacrimal passages, 239

Mongolian, of eyelids, I 66

Foramen, Foramina-

ethmoidal, $5^{\circ}$

frontal, Io

infra-orbital, I5, I9, 2 I

lacerum anterius. See Superior orbital fissure

meningeal, 35

oculomotorium, 265

opticum, 26

rotundum, 38, 39

spheno-zygomatic, 35

supra-orbital, 9, II

zygomatico-facial, I4, 34

zygomatico-temporal, 34

Form of orbit, 3

Fornix, conjunctival, I90, II3

of lacrimal sac, 229

Fossa glandulae lacrimalis, 24, 96

hypophyseos (sella turcica), 62,369

infra-temporal (zygomatic), 40, 42

origin of inferior oblique muscle of, 43

pterygo - palatine (spheno maxillary), $4^{\circ}$

sacci lacrimalis, 64, 96, 2I, I7

dimensions of, 73

spheno-maxillary, $4^{\circ}$

temporal, $37,4^{\circ}$

zygomatic, 40

Fovea trochlearis, 24

Frontal bone. See under Bone 
Galea aponeurotica, 107, 109, I8I Ganglion, ciliary (lenticular or ophthalmic), $35^{\circ}$

Gasserian, or semilunar, 336, $338,39 \mathrm{I}$ spheno-palatine (Meckel's), $347,214,334$

Geniculate body, lateral, 384, $370,37 \mathrm{I}, 372$ medial, 370

Glabella, I2, I9, I05

Glands of Ciaccio, I96

conjunctival, I95

of eyelid margin, I 57

of Harder, I98

of Henle, I96

infra-orbital, 198

of Krause, 195

lacrimal, 204

accessory, I95

development, 2I 7

nerves, 2 I 4

secretion of, $2 \mathrm{I} 2$

variations, 218

vessels, 2I3, 305, 3I I

of lacrimal sac, 242

of Manz, I97

meibomian, I 53, I86

of Moll, ${ }_{5} 8$

Mucosae Krausei, I96

of plica semilunaris, 198

tarsal, $\mathrm{I} 53, \mathrm{I} 86$

trachoma, I95

of Zeis, I57

Glaucoma, 3II, 354

Globe of eye. See Eyeball

Groove, infra-orbital, 42

supra-orbital, 9

Gyrus, angular, of brain, 377,378

Hamulus lacrimalis, 48, I7

Headache, r8I, I82

Hernia, orbital, 40

palpebral, or adiposa, I I4

Hiatus semilunaris, of ethmoidal

bone, 56,68

Hyperphoria, I 8I
Hypophysis cerebri (pituitary body), 316, 368, 370

Impressiones digitatae, of orbital roof, 30

Incisura lacrimalis, 72

Incisura supra-orbitalis, 9, I9

Index, naso-orbito-alveolar, 88 orbital, 88

Infundibulum, ethmoidale, of frontal sinus, $57,5^{8}, 60$, 66,343

of hypophysis cerebri, 368

- of lacrimal canaliculus, 222

Injections, of eyebrow, IIO of eyelids, 137 of orbit, 298, I9I subarachnoid, of optic nerve sheath, 362

Iris, nerve plexus of, 354

Jaw winking, phenomenon of, I83

Juga cerebralia, of orbital roof, 30

Labyrinth, ethmoidal, 58, 60

Lacertus of lateral rectus muscle, 293

Lacrimal apparatus, 204

bone. See under Bone

canal (naso-lacrimal), 7o

canaliculi, 222

duct (naso-lacrimal), 233, 237

ductules (of gland), 2 ro

fossa, 24,96

gland, 204

passages, membranous, 2 r 9

osseous, 63

protuberance, 63,72

sac, 227

sulcus, 48, 64, 7I, 75

Lacus lacrimalis, II 8

Lagophthalmos, I 87

Lamina infra-orbitalis, 42

papyracea, 47, 5I, 60

Lens, accessory muscles of accommodation of, $\mathrm{I} 8 \mathrm{I}$ 
Ligament, Ligaments, Ligamentum-

broad, of eyelids (septum orbitale), I 30

check, of ocular muscles, 293

of levator palpebrae superioris, $\mathrm{I}_{44}$

canthi externum, 148 intra-capsular of Tenon, 293

of lacrimal gland, 208

of Lockwood; 296

palpebral (septum orbitale), I30

(tarsal), medial, I47, 230

(tarsal), lateral, I48

suspensory of eye (Lockwood), 296

tarsal. See Palpebral

tendo oculi externum, $\mathrm{I}_{4} 8$

internum, 147

Line of Gennari, 374

Linea temporalis, I3, 20

Loge accessoive of lacrimal fossa, 24,204

Lymph channels or vessels. See under Vessels of regions

of optic nerve, 362

of orbit, $320,53,5^{\mathrm{I}}$

Lymph nodules (in conjunctiva), I95

Mala bipartita, I3

Malar bone. See under Bone

Margin, of orbit. See under Orbit

palpebral. See under Eyelids

Membrana orbitalis musculosa, 83,285

Membrane, intermuscular, of recti muscles, 292

Meningeal sheaths of optic nerve, 362

Mensuration of orbit, 84

Muscle, Muscles, Musculusaccessory, of accommodation of lens, r8I

attachments round orbital margin, I3 I
Muscle, Muscles, Musculus (contd.) -

attachments (contd.) at apex of orbit, 259

on to eyeball, 265

caninus, origin of, 16 . capsulo-palpebralis, 296 choanoid, 285

ciliary, of lens, 35I, 353, I8I

of Riolan, I 29

comes obliqui superioris, I44, 284

corrugator supercilii, 108

development (orbital), 282

dilatator pupillae, $334,343,35^{8}$

epicranius, 107

of eyeball, 257

abnormalities of, 283

action of, 276

sheaths of, 29I

structure of, 259

of eyebrows, 107

action of, 179

clinical points of, $\mathrm{I} 8 \mathrm{I}$

of eyelids, I26, I33

action of, 177

clinical points of, I8I

development of, I30, I4I

facial (upper group), I 82

frontalis, 107

gracillimus orbitis, 144 of Horner. See Pars lacrimalis imbalance of, and facial expression, I04

inter-oblique distance of oblique muscle origins, IO2

involuntary, of eyelids, 139,296

of orbit, 82,34

peri-bulbar, 296

lacrimal, anterior, 127

of Landström, 296

levator anguli oris (caninus, origin), $\mathrm{I} 6$

labii superioris, origin, I6 levator palpebrae superioris,

\section{33}

action, 179

nerve, $\mathbf{I} 73$ 
Muscle, Muscles, Musculus (contd.) -

of Müller, in eyelids, I39, 296 in orbit, 82,34

oblique, inferior, $275,280,177$, 83 inferior accessorius, 284 inferior, check ligament, 295 inferior, origins of, abnormal, 284 superior, 272,279 superior, abnormalities, 284 superior, pulley of (trochlea),

274

superior, sheath of, 295 occipito-frontalis (epicranius), I 7

orbicularis oculi (palpebrarum), I 26, I 86

action of, I77, I8o

clinical points of, $\mathrm{I} 8 \mathrm{I}$

in eyebrow, 108

orbitalis (of Müller), 82, 34, 3I 2 origins, on face (Fig. 59), I 3 I

of recti, 259

palpebral, involuntary, I39, 296

pars lacrimalis (Horner), $\mathrm{x} 28$, 224,23 I, 24 I

peri-bulbar, 296

procerus, 107

pyramidalis nasi, Io7

quadrangulus constrictor puncti lacrimalis, 224

quadratus labii superioris, I6, I63

recti, 259

abnormalities of, 283,188

actions of, 276

arc of contact of, 270

development of, 282

fascial sheaths of, 29I, I9I

insertions on to eyeball of, 265

lengths of, 259

ligaments, check of, 293

origin (posterior attachments) of, 259
Muscle, Muscles, Musculus (contd.) recti (contd.) special descriptions :

r. inferior, $27 \mathrm{I}$

r. lateralis, $27 \mathrm{I}$

r. medialis, $27 \mathrm{O}$

r. superior, 272,177

structure of, 259

tendons of, 266

weight of, 259

retractor bulbi, 285,83

superciliaris, 127

tensor tarsi. See Pars lacrimalis

tensor trochleae, I44

transversus glabellae, I 30

transversus orbitis, I 45

zygomaticus (major), I4, I63, I6 4

Myopia, progressive juvenile, 278 Myopic eyeball, position of oblique muscles on, $28 \mathrm{I}$, 273,276

relation of shape of orbit to, IO2

Nasion, the point, $23,26,62$

Nerve, Nerves-

abducent (sixth), 333

nucleus of, 39o, 391, 384, 336 alveolar (dental), 348

anterior superior, $348,16,244$ auditory (connections of), I 74 , 391

carotidus internus, 356 cerebral connections of, 373 ciliary, long, $343,353,35^{8}$

short, 353

to conjunctiva, 202

to cornea, 202, $35^{2}$

ciliary body, of, 353

constrictor pupillae, fibres, $35 \mathrm{I}$, 353

corpuscles, tactile, 202

terminal, $\mathrm{x} 76$

dental. See Alveolar

dilator pupillae, fibres, 357,334 
Nerve, Nerves (contd.) -

ethmoidal, anterior (nasociliary), 343

posterior (Krause), 344

facial, I $72,2 \mathrm{I} 4$

nucleus, I 74, I83

superior, 182

fifth. See Trigeminal

fourth. See Trochlear

frontal, $34^{\circ}$

infra-orbital, $345,42,15$

infra-trochlear, 343, I 75, 202, I6I.

of Kobelt, Io

lacrimal, 339, 2I 4, I 75, 202

mandibular (inferior maxillary); I 74, 338

maxillary (superior maxillary), $345, \quad$ I 74

naso-ciliary (nasal), 342, 353

oculomotor, 324,182

nucleus of, $386,384,182,390$

ophthalmic, $336, \mathrm{I} 74$

optic, $35^{8}, 38 \mathrm{I}, 383$, 3 о I

of orbit, general scheme of, $32 \mathrm{I}$

orbital (zygomatic), 346, 2I4, 2 I 5,340

petrosal, 2I4, 347

plexus, cavernous (sympa-

thetic), $35^{6}$

of ciliary body, 353

of conjunctiva, 202

infra-orbital, I 74, $35^{\circ}$

of iris, 354

marginal (eyelids), I 76

of pterygoid canal (Vidian),

347,2 I 4

pupillo-constrictor fibres, $35 \mathrm{I}$, 353

pupillo-dilator fibres, 357,354 , 356,334

ramus ethmoidalis (of nasociliary), 344

recurrent (of Arnold), 338

seventh. See Facial

sixth. See Abducent

spheno-ethmoidal(of Luschka),
Nerve, Nerves (contd.) -

spheno-palatine, 347

subcutaneus malae (zygomatic), 346

supra-orbital, 34I, I9, I 75

supra-trochlear, 340, I 75

sympathetic system of, 354, 215,176

temporo - malar (zygomatic),

$346,35,20$

tentorii, of ophthalmic, 338

third. See Oculomotor

Tiedemann's, 303

trigeminal (fifth), 338, I 74, 39I, I 82

trochlear (fourth), 332, 390, 384

Vidian, 2I 4, 347

zygomatic (orbital), 346, 214 , 2 I 5,340

zygomatico-facial, 35

zygomatico-temporal, 35, 20

Neuralgia, 344, I 82

Notch, frontal, Io, I9

supra-orbital, 9, I9

supernumerary, I I

Nucleus. See under each Nerve

Olfactory Tract, relation to roof, 32

relation to optic canal, 27

Ophthalmoplegia, I 82

Optic, atrophy, hereditary, 369

chiasma or commissure, 367 , 382

foramen, 26

nerve, $358,38 \mathrm{I}, 30 \mathrm{I}, 383$

radiation (radiatio occipitothalamica), 373

tract, 370,382

Orbit, Orbital-

angle, basic of, 92

naso-malar, $9 \mathrm{I}$

of walls, 92

apertures of, table of, 79

apex, form of, 5

apical point of, 89

-arteries of, 300

abnormalities of, 308 
Orbit, Orbital (contd.)-

asymmetry of, IO2

axis, orbital, 93

birth, features at, 95

casts of, 3

child, features in, 97

contents, general arrangement of, $25 \mathrm{I}$

deformities of, I04

depth, 89

development of, 93, 43

differences, sexual, of, roo, 88, 90

dimensions of, 84

fascia of, 286

fat, 297

floor, 40

form of, 3

relating to eyeball, ro2

general description of, I

growth of, changes in, 97

height of, intra-orbital, I02

index, 88

lymphatics of, 320

margin, angle, basic of, 92

angle, naso-malar of, 9I

arcade, $\mathrm{I}_{32}$

bones forming, 7

dimensions of, 85

distance, axial, of, 85

infra-orbital, I4

lateral, I2, I9

medial, $\mathrm{x} 7,2 \mathrm{I}$

process (processus margi-

nalis), I3, 20

relation to brain, $2 \mathrm{I}$

relation to eyeball, 256,95 , I02

shape of, 6, I3, 97

supra-orbital, 7, I9

surface anatomy of, I9

sutures of, 7

mensuration of, 84

nerves of, 32I

ossification of, 94

roof, 22

relation to fractured skull,
Orbit, Orbital (contd.) -

tubercle (of zygomatic bone), I 3,20

variations in type of skull, IOO

veins of, 308

volume of, 85,3

walls, 22

angles of, 92

bones forming, 22

inferior or lower (floor), 40

lateral, 32

length of, 90

medial, 46

superior or upper (roof), 22

Orifices adipeux of orbital base, 298

Os de la gouttiève lacrymale, 18

hamulus of lacrimal bone, 48 japonicum, I3

maxillo-naso-lacrimal frontal, I9

planum of ethmoidal bone, 47 , $5 \mathrm{I}, 60$

Ossa suturarum (Wormian bones), $47,34,19$

Ossiculum canalis naso-lacrimalis, 48

ethmo-lacrimale, 47

infraorbitale marginale, I5

maxillo-frontale, I 8

peri-lacrimal, 49

Ossification of orbital walls, 94

Osteophyte of orbital roof, 32

Ostium frontale of frontal sinus, 59

Ostium lacrimale of naso-lacrimal duct, 234

Ostium maxillare of maxillary sinus, 63

Oxycephaly, I04

Pain, referred from eye, 182

Palatine bone. See under Bone

Palpebral, Palpebrae. See under Eyelids

Papillae lacrimales, 2I9, II8 
Paralysis, facial nerve, I 82, 249 third, fourth, sixth nerves, 33I,

\section{Passages-}

333,334

lacrimal, membranous, 219 osseous, $63,73,76$

naso-lacrimal, abnormalities of, 246

development, of, 244

dimensions of, 237

mechanism of, 247

nerves of, 244

shape of, 225

structure of, $24 \mathrm{I}$

valves of, 239

vessels of, 243

Pituitary body, 368, 316, 370

Plates, tarsal, I 46

Plexus, caroticus internus (sympathetic), $35^{6}$ cavernous (sympathetic), 356 ciliary, of nerves, 353

conjunctival, sub-epithelial (nervous), 202 infra-orbital (sensori-motor), I 74, $35^{\circ}$

of iris (nervous), 354

marginal, of eyelids (nervous), I 76

peri-corneal (arterial), 200

pterygoid (venous), 3I2, I65, 244,320

Plica lacrimalis (of naso-lacrimal duct), 239

semilunaris, $192,198,203$

Point, apical, of orbit, 89

dacryon, 86, 66

lacrimal (mensuration), 87

(eyelids) punctum (of eyelids), 220, II 8

nasion, $23,26,62$

of rotation of eyeball, 276 ,

254

Process, Processusangular, lateral (zygomatic) of frontal bone, 9, I3, I2, I9 angular, medial of frontal bone, 9 , 19
Process, Processus (contd.) -

frontal, of maxilla, $\mathrm{I} 4, \mathrm{x} 6$

fronto-sphenoidal, of zygomatic bone, 13,20

hamular, of lacrimal bone, 48 , I 7

marginal, of inferior orbital margin, $x_{5}$

marginalis, of zygomatic bone, I3, 20

orbital, of palatine bone, $4 \mathrm{I}$ turbinated, 55

uncinatus, of ethmoidal bone, 57

of ethmoidal bone, cell of, 68 zygomaticus (lateral or ascending) of frontal bone, 9, I3, I2, I9

Protuberance, lacrimal, of nasolacrimal canal, 63, 72

Pterygium, 204

Ptosis, I8o, 33I

adiposa of eyelids, II 4

congenital, I 88

Pulley of superior oblique muscle, 274

Pulvinar, 370,372

Punctum lacrimale, 220, I I 8

Pupil, dilatation of, 357

contraction of, $35 \mathrm{I}, 358,334$

light reflex of, 385

nucleus for innervation of, 388

Quadrigeminal body, inferior, 372,383

superior, 384,372

Radiation, optic (occipito-thalamica of Gratiolet), 373

position of optic fibres in, 385 Raphe palpebrarum lateralis, I 27, I 49

Recess, Recessus-

of Arlt (lacrimal sac), 246

ethmo-lacrimal of hiatus semilunaris; 68

infra-orbital, of maxillary sinus, 72 
Recess, Recessus (contd.)prelacrimal, of maxillary sinus, 63,72

spheno-ethmoidal, of nose, $5^{6}$, 58,62

Reflex, auditory and facial nerve, I74

eyelids, I8I

orbicular, I 74

pain, 182

pupillary light, 385

tear secretion, 216

winking, I 8I

Retina, representation in cortex of, 386

Retinacula of conjunctiva, I9I

Retinaculum oculi lateralis (of recti muscles), I4, 296

Ridge, supra-orbital, I2, I9

temporal, I3, 20

Rima palpebrarum, I I 7

Rivus lacrimalis of lid margin, I 54

Sac, conjunctival, I 88

bacteria of, 213

lacrimal, 227, I 6

dimensions of, 237

glands of, 242

structure of, $24 \mathrm{I}$

Scalp, I Iо, Iо6

Sebum palpebrale, I 53

Sella turcica (of sphenoidal bone), 62,369

Septum orbitale, I 30, I 7, I38, 299

Sheaths of optic nerve, 362,261

Short - sightedness, Stilling's theory of, 102

Sinus, Sinuses-

accessory or air (s. paranasales), 53

cavernous, 315, 62

frontal, $59,29,28$, Iо, 342

infundibulum of, $57,58,60$, 66,343

longitudinal (sagittal), superior, $3 \mathrm{I}, \mathrm{I} 65$

of Maier (lacrimal sac), 224, 246 malaris (zygomaticus), 63, 44
Sinus, Sinuses (contd.) -

maxillary (antrum of Highmore), $63,58,28,16,44$

para-nasales, 53

petrosal (venous), 3I9

sagittal (longitudinal), superior, $3 \mathrm{I}, \mathrm{I} 65$

sphenoidal, 62, 58, 27, 316, 370

spheno-parietal (venous), 3I 8 , 3 Io

supra-orbital, 29

zygomatic (malaris), 63, 44

Skull, asymmetry of, IO2

deformities of, IO4

fracture of, 334, 300

Tower, 104

Space, episcleral, 289

interfascial, of Tenon, 289

sub-arachnoid, 362, 324, 369

supra-vaginal, of optic nerve, $29 \mathrm{I}$

Sphenoidal bone. See under Bone

Spina recti lateralis, 34,260

trochlearis, 24

zygomatica, 13

Spinae orbitales, 34

Spur, lacrimal, anterior, 64

Stilling's theory, I02

Strabismus, 270, 283, 33I, 335

Sulcus, calcarine, of brain, 375 , 386

chiasmatis, 368

infra-orbital, 42

inter-marginal of eyelids, I 55

lacrimal of maxilla, 48, 64, 7I,

of ${ }^{75}$ Verga (naso - lacrimal duct), 235

orbitalis, of brain, 32

sub-tarsal, of eyelids, I9o

Surface anatomy of eyelids, II 2 of orbital margin, I9

Suture, Sutures, Sutura-

abnormal, of orbital roof, 30

canalis infra-orbitalis, 42

ethmo-lacrimal, 47

fronto-ethmoidal, 23 
Suture, Sutures, Sutura (contd.)fronto - malar. See Zygomatico-frontal

fronto-sphenoidal, 25, 34 fronto-zygomatic. See Zygomatico-frontal

infra-orbitalis, $43, \mathrm{I} 7$

verticalis, 16,43

lacrimo-jugal, I 7

lacrimo-maxillary, 64, 66

longitudinalis imperfecta, I 8

notha, $18, \mathrm{I} 6$

orbito-maxillo-frontal, 47

spheno-zygomatic, 34,35

zygomatico-frontal, I2, I3, 33, I 9,24

zygomatico-maxillary, I4, 2I, $4 \mathrm{I}$

Sympathetic nervous system, course to eyeball, 354

in eyelids, $\mathrm{I} 76$

to lacrimal gland, 2I5

Tarsal plates (Tarsus), $\mathbf{I}_{4} 6$ glands, I 53, I 86

Tears, composition of, 212 mechanism of drainage of, 247

Tendon, Tendo-' oculi externus, $\mathrm{I}_{4} 8$ internus, 147

orbitalis inferior (of Zinn), 263 superior (of Zinn), 264

of Zinn (annulus tendineus communis), 260

Tissue, episcleral, 289

Torticollis, ocular, I03

Trachoma, glands, $x 98, x 95$

Tract, olfactory, relations of, 27 , 32

optic, 370,382

Tractus peduncularis transversus, 383

Trochlea, of superior oblique muscle, 274, 21, 24

Trochlear angle, 273, 276

Tubercle, infra-orbital, I4 infra-optic, 263
Tubercle (contd.)-

lacrimal, I8, 64

orbital, I3, 20

Tuberositas malaris, $\mathrm{I}_{4}$

Tunica adventitia of eyeball, 289

Turbinated bone. See under Bone

Valve, Valvesof lacrimal passages, 239

of Hasner, 239

of orbital veins, $3 \mathrm{I}_{4}$

Vein, Veins, Vena-

angular, I63, I6 $5, I 48, I 66$

centralis retinae, $3 \mathrm{II}$

ciliary, 3 II

conjunctival, $20 \mathrm{I}$

development of, 3 I 5

diploic, 165

emissary, ophthalmic, 3I4

of cavernous sinus, 320

ethmoidal, 3II

facial (anterior facial), I63

deep, 165

frontal (frontal-nasal), I6 5

of Gaillard, 44, 3I 5

infra-orbital, 42

lacrimal, 3II

lacrimo-facial, 66

marginal, of eyebrow, IIo, I6 6 maxillary, anterior internal, I 65

meningeal, middle, 35

middle cerebral (Sylvian), 319,

3 I 5

ophthalmic, inferior, $3 \times 2$

superior, 309

ophthalmo-facial, 3I 2

meningeal, 3 Io

orbito-frontal emissary, 30

palpebral, I69

plexus, pterygoid, of, $3 \mathrm{I} 2, \mathrm{I} 65$, I66

supra-orbital, x64

temporal, superficial, 167

temporo-maxillary, 3I 2

valves in, $3 \mathrm{I} 4$ 
Vein, Veins, Vena (contd.)variations of, $3 \mathrm{I} 5$ venae vorticosae, 3 ro

Vestibule of nose, $5^{6}$

Vibrissae, supra-orbital, Io7

Volume of orbit, 85,3

Winking, jaw phenomenon, $x 8_{3}$ reflex arc for, I $8 \mathrm{I}$
Winking (contd.)cortical centre for, 378

Zygomarelation to brain, $2 \mathrm{I}$ inferior orbital fissure, 39 optic canal, 27 semilunar ganglion, 338 Zygomatic bone. See under Bone

THE END 


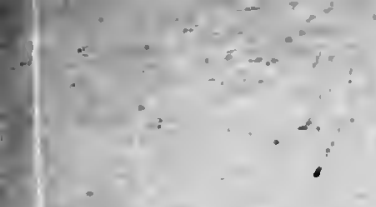

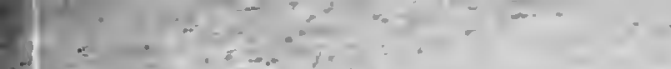

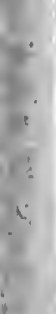







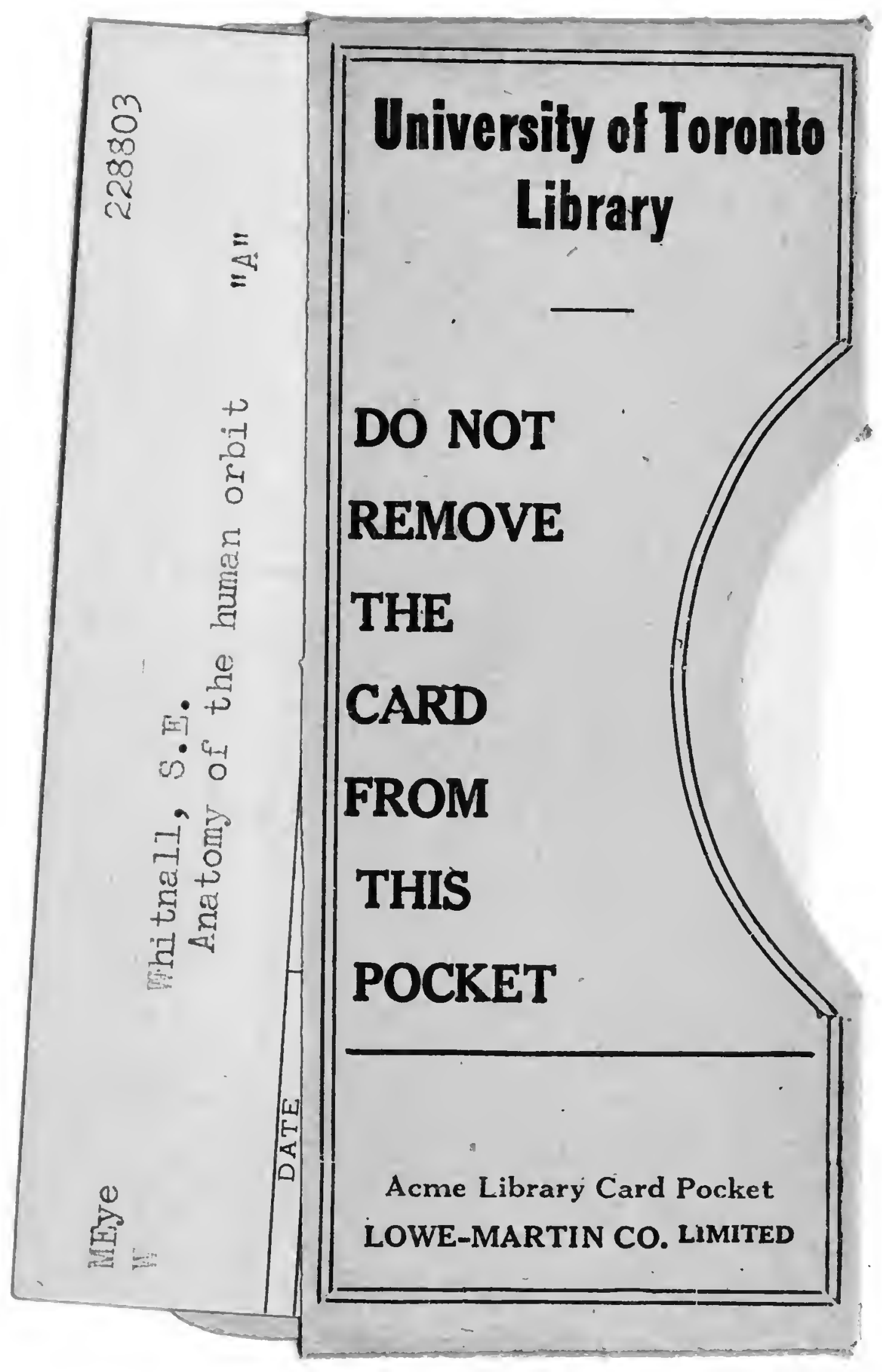


